\title{
Nucleophilicity Prediction via Multivariate Linear Regression Analysis
}

\author{
Manuel Orlandi, ${ }^{*},+,+$ Margarita Escudero-Casao,,,+ Giulia Licini ${ }^{\ddagger},+$ \\ ¥Department of Chemical Sciences, University of Padova, via Marzolo 1, 35131 Padova. \\ +CIRCC - Consorzio Interuniversitario per le Reattività Chimiche e la Catalisi, Padova Unit., via Marzolo 1, 35131 \\ Padova.
}

\section{Supporting Information}

General Information

Nucleophiles

Parameters Acquisition

Single-parameter Correlations and Parameters Analysis

Methyl Cation Affinities (MCA)

Multivariate Linear Regression Analysis

Energies

Geometries of Nucleophiles

Geometries of Protonated Nucleophiles 


\section{General Information}

All computations were performed using the Gaussian $16^{1}$ suite of programs at the $C_{3} P$ facility of the University of Padova. The nucleophiles conformational space was explored manually as most nucleophiles included in this study are small, simple molecules with only few rotatable bonds. Geometry optimizations, vibrational frequencies and intensities were calculated at the M06-2X/def2TZVP level of theory. This functional/basis-set combination was reported to provide a good accuracy for the calculation of properties of organic molecules in quantitative fashion. ${ }^{2}$ Moreover, this method proved solid for parametrization purposes over a wide range of applications in our experience. ${ }^{3}$ All the structures computed in this study are minima as confirmed by vibrational analysis showing no imaginary frequencies. NBO charges were computed using NBO 3.1 implemented in Gaussian 16. ${ }^{3}$ Buried volumes were computed using SambVca 2.1. ${ }^{4}$ Sterimol values were calculated for the M06/2X optimized geometries using Molecular Modeling Pro®. Solvation energies were computed by single point energy calculation on the M06-2X/def2TZVP geometry using M06-2X/def2TZVP[SMD=solvent] method. Multidimensional regression analyses were performed using Matlab®. 


\section{Nucleophiles}

The following charts shows the nucleophiles included in this study. It should be noted that, for some of them, more than one nucleophilicity value $N$ was available in Mayr's database due to measurements in more than one solvent. For the complete list of datapoints including all of the nucleophile/solvent combinations see the parameters table below (Table S1).

Chart 1. List of nucleophiles studied.

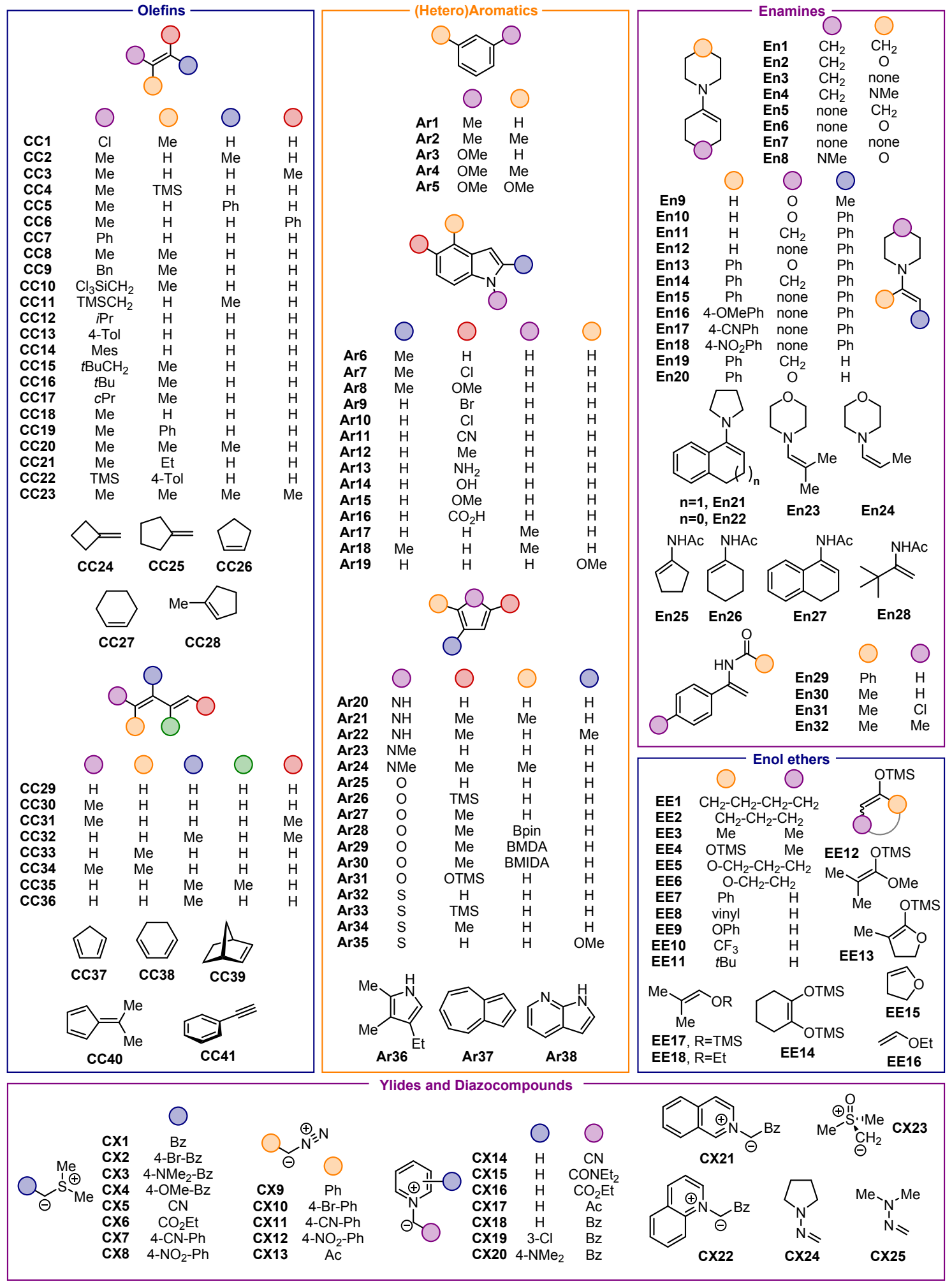




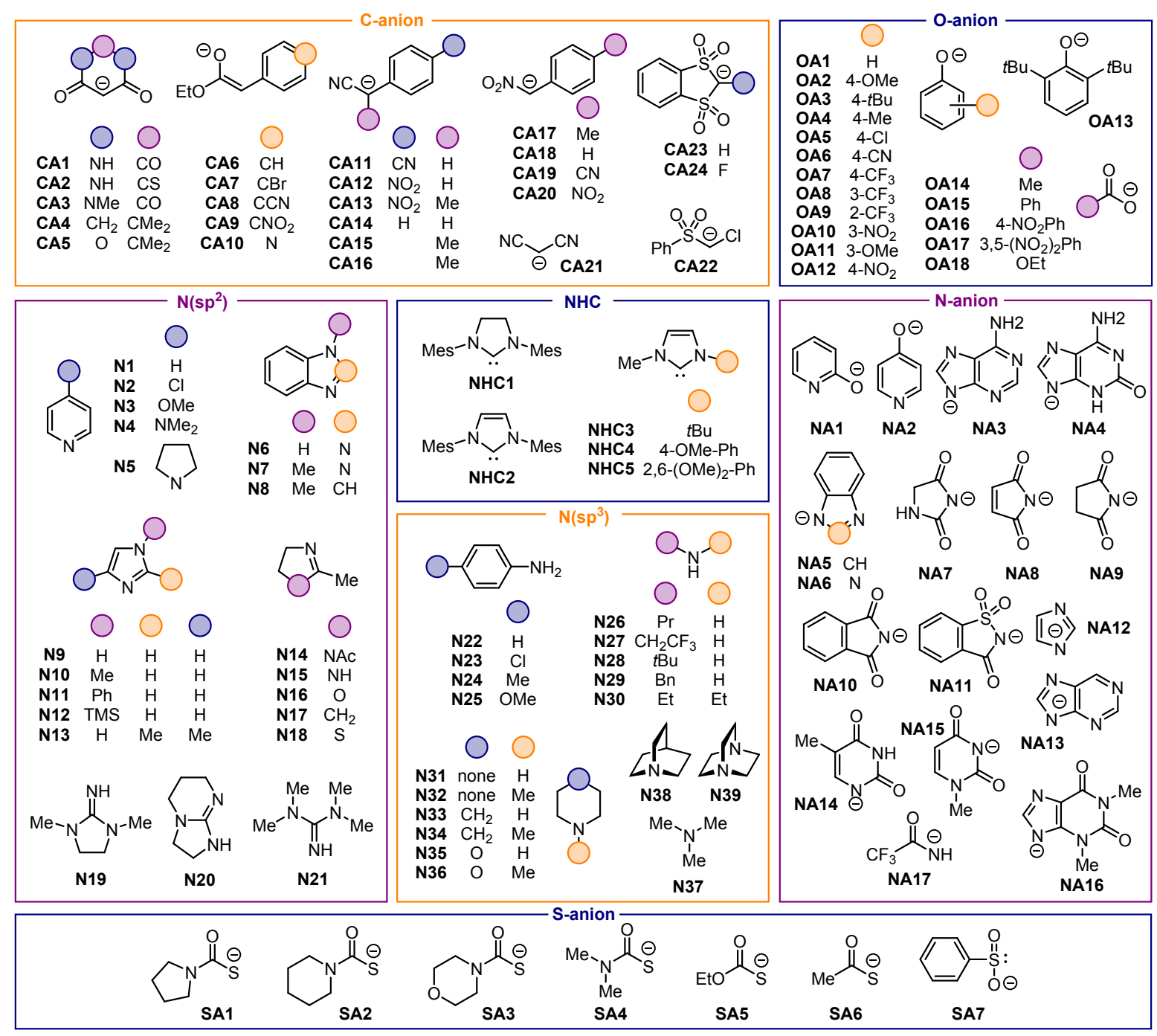




\section{Parameters acquisition}

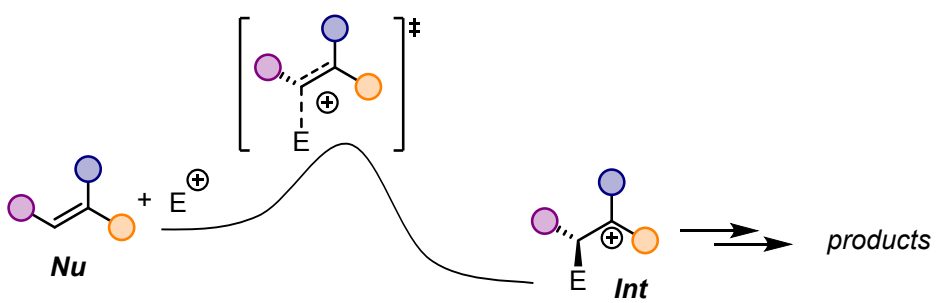

From the gas phase optimization of the nucleophile $\mathbf{N u}$ the following parameters were obtained:

- $\mathbf{e}_{\text {Hомо }}$ and $\mathbf{e}_{\text {LUmo: }}$ energies of the HOMO and LUMO orbital of the nucleophile.

- NBO and MK: NBO and Merz-Kollman charges obtained via the keyword $p o p=(m k, f u l l, n b o)$

- Pol: polarizability of the nucleophile calculated via the keyword polar

To the nucleophile $\mathbf{N u}$ was subsequently added one proton at the nucleophilic atom and the structure was optimized again at the M06-2X/def2TZVP level. From this structure Int, the following parameters were acquired:

- $\mathrm{E}_{\mathrm{PA}}$ : protonation energy calculated from the electronic energy of the nucleophile $\mathbf{N u}$ and the protonated nucleophile Int $\left(\mathrm{E}_{\mathrm{PA}}=\mathrm{E}_{\text {lnt }}-\mathrm{E}_{\mathrm{Nu}}\right)$.

- $\mathbf{e}^{+}$номо and $\mathbf{e}^{+}$Lumo: energies of the HOMO and LUMO orbital of the protonated nucleophile.

- $\% \mathbf{V}$ : buried volume of a sphere centered on the added proton using SambVca 2.1 (https://www.molnac.unisa.it/OMtools/sambvca2.1/index.html). The sphere centered on the reference proton of Int had a radius of $3.5 \AA$. Other protons of the structures were included and unscaled Bondi radii were used for the evaluation of $\% \mathbf{V}$.

- B1, B5, and L: Sterimol descriptors were acquired using the added proton as common connection point to the evaluated group (the nucleophile). In figure $\mathrm{S} 1$ the parameters are depicted for nucleophile N38 (quinuclidine) in order to clarify their meaning:

Figure S1. Representation B1, B5 and L for quinuclidine N38.
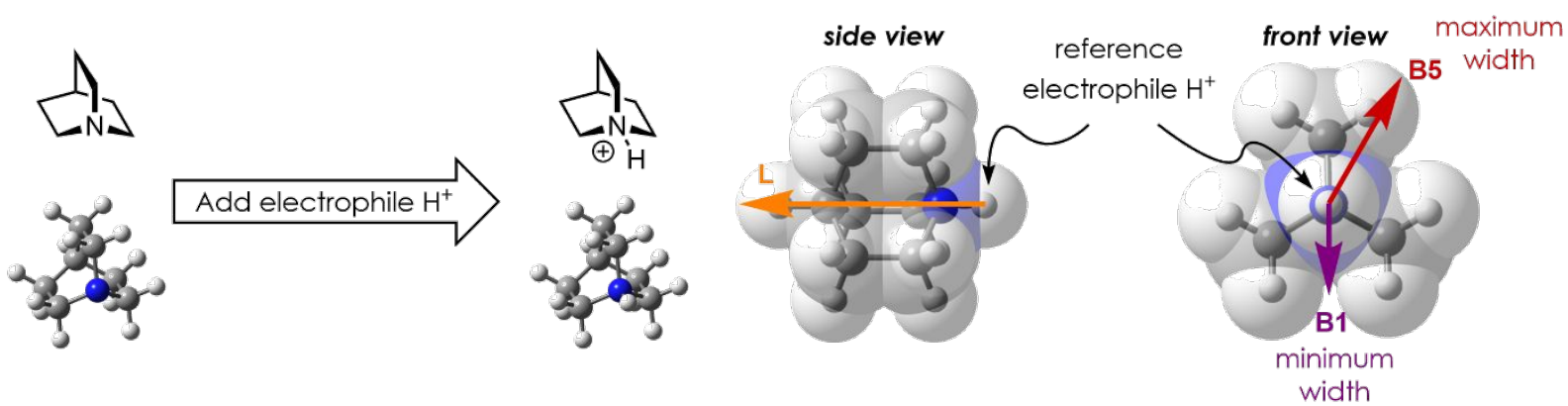

The optimized structures $\mathbf{N u}$ and $\mathbf{I n t}$ were submitted to a single point energy calculation with SMD solvation model to obtain the following parameters:

- $\mathbf{S}_{N u}$ : solvation energy of the nucleophile $\mathbf{N u}$, obtained by subtracting the electronic energy of the nucleophile in the gas phase to the electronic energy calculated in the solvent phase (s): $\mathbf{S}_{N u}=\mathbf{E}_{N u}(\mathbf{s})$ $-\mathrm{E}_{N u}$. 
- $\mathbf{S}_{\text {Int: }}$ solvation energy of the protonated nucleophile Int, obtained by subtracting the electronic energy of the nucleophile in the gas phase to the electronic energy calculated in the solvent phase (s): $\mathbf{S}_{\boldsymbol{l n t}}=$ $\mathrm{E}_{\text {lnt }}(\mathrm{s})-\mathrm{E}_{\text {lnt }}$.

Additional parameters acquired are as follow:

- $\mathbf{S}_{H}$ : solvation energy of the proton calculated at the M06-2X/def2TZVP[SMD=solvent] level of theory.

- $\varepsilon$ : solvent dielectric constant.

- $\mathbf{q}$ : nucleophile total charge. This is a binary parameter that simply distinguishes neutral $(\mathbf{e}=0)$ from anionic $(\mathbf{e}=1)$ nucleophiles.

- MW: molecular weight of the nucleophile $\mathbf{N u}$.

- $\mu$ : chemical potential of the nucleophile $\mathbf{N u}$ according with the Hard and Soft Acids and Bases theory. $\boldsymbol{\mu}=\left(\mathbf{e}_{\text {номо }}+\mathbf{e}_{\text {LUMо }}\right) / 2$

- $\boldsymbol{\mu}^{+}$: chemical potential of the protonated nucleophile Int according with the Hard and Soft Acids and Bases theory. $\boldsymbol{\mu}^{+}=\left(\mathbf{e}^{+}{ }_{\text {номо }}+\mathbf{e}^{+}{ }_{\text {Lumo }}\right) / 2$

- $\mathbf{\eta}$ : hardness of the nucleophile $\mathbf{N u}$ according with the Hard and Soft Acids and Bases theory. $\mathbf{\eta}=\mathbf{e}_{\text {Lumo }}$ - $\mathbf{e}_{\text {номо }}$

The full list of the parameters for all of the nucleophile/solvent combinations (341 datapoints) are listed in Table S1. The $N$ and $s_{N}$ values reported were obtained from Mayr's database at:

\section{https://www.cup.Imu.de/oc/mayr/reaktionsdatenbank2}


Table S1. Collection of nucleophiles parameters.

\begin{tabular}{|c|c|c|c|c|c|c|c|c|c|c|c|c|c|c|c|c|c|c|c|c|c|c|c|c|}
\hline Nu & solvent & $s_{N}$ & $N$ & $\varepsilon$ & (e) & $\begin{array}{c}M W \\
(\mathrm{~g} / \mathrm{mol})\end{array}$ & $\begin{array}{l}\text { еномо } \\
\text { (а.u.) }\end{array}$ & $\begin{array}{l}\text { eumo } \\
\text { (a.u.) }\end{array}$ & $\begin{array}{l}\text { NBO } \\
\text { (a.u.) }\end{array}$ & $\begin{array}{l}\text { MK } \\
\text { (a.u.) }\end{array}$ & $\begin{array}{l}\text { Polar } \\
\text { (a.u.) }\end{array}$ & & & 7VH & (Å) & $\begin{array}{l}31 \\
\AA\end{array}$ & $\begin{array}{l}B 5 \\
\text { (Å) }\end{array}$ & $\begin{array}{c}\mathrm{E}_{\mathrm{PA}} \\
(\mathrm{kcal} / \mathrm{mol})\end{array}$ & $\begin{array}{c}\mathrm{S}_{\mathrm{Nu}} \\
(\mathrm{kcal} / \mathrm{mol})\end{array}$ & $\begin{array}{c}S_{1 \mathrm{n} t} \\
(\mathrm{kcal} / \mathrm{mol})\end{array}$ & $\begin{array}{c}S_{H} \\
(\mathrm{kcal} / \mathrm{mol})\end{array}$ & $\begin{array}{c}\mu \\
\text { (a.u.) }\end{array}$ & $\begin{array}{c}n \\
\text { (a.u.) }\end{array}$ & $\begin{array}{c}\mu^{+} \\
\text {(a.u.) }\end{array}$ \\
\hline 01 & DCM & 1.77 & -4.36 & 93 & 0 & 92.14 & -0.29582 & 0.02389 & .223 & -0.160 & 77.1380 & & & 9.4 & 5.61 & & 5.43 & & -6.70 & & & & & \\
\hline 02 & DCM & ๑8 & .57 & & 0 & 106.17 & 28900 & 2499 & .222 & 391 & 4.6857 & & & & 5.75 & 1 & 5.46 & & & & & & & 893 \\
\hline 03 & $C M$ & & .18 & 3 & 0 & 108.14 & -0.27769 & 02255 & .248 & -0.260 & 1.9990 & -0.51771 & -0.24167 & & .01 & .11 & 5.88 & -203.07 & -6.97 & 2.93 & -122.52 & -0.12757 & .30024 & -0.37969 \\
\hline 04 & DCM & 1.27 & 0.13 & 93 & 0 & 122.17 & -0.27470 & 0.02273 & -0.319 & -0.450 & 95.4633 & -0.49408 & -0.23112 & & 6.00 & 2.11 & 5.87 & -208.04 & -7.33 & -51.45 & -122.52 & -0.125985 & 0.29743 & -0.3626 \\
\hline 05 & DCM & 09 & 2.48 & 93 & 0 & 138.17 & -0.26395 & 03350 & -0.292 & -0.457 & 100.0997 & -0.47374 & -0.22060 & & 5.95 & 2.09 & 5.93 & -215.64 & -7.51 & -49.96 & -122.52 & 5225 & 0.29745 & -0.34717 \\
\hline 06 & AN & & 6.91 & & 0 & & 0.25196 & 2090 & -0.303 & -0.647 & 109.4043 & -0.45300 & & & 4.75 & 2.15 & 5.68 & -224.16 & & & & & 7286 & 2706 \\
\hline 77 & A & & 6 & & 0 & & .26294 & 0826 & .303 & -0.604 & 860 & -0.43 & & & 75 & 2.15 & 6.95 & & & & & & 2712 & -0.321005 \\
\hline 08 & A & & 7. & & 0 & & .24805 & 2135 & .310 & -0.598 & 28.2333 & -0.411 & & & 74 & 2.21 & .57 & -227.46 & & 8.84 & & & 2694 & -0.30148 \\
\hline 09 & A & & 4.38 & & 0 & 196.05 & -0.26879 & 00220 & -0.299 & -0.536 & 117.0187 & -0.43551 & -0.22260 & 30.4 & 4.76 & 2.06 & 7.25 & -212.30 & -11.84 & -66.49 & -134.02 & 33295 & 0.27099 & -0.329055 \\
\hline 10 & A & & 6. & & 0 & 151.59 & -0.26815 & 00357 & -0.301 & -0.493 & 108.6233 & -0.44544 & -0.22 & & 4.76 & 2.05 & 6.95 & & & & & & & \\
\hline 11 & AN & & 2.83 & .5 & 0 & 142.16 & -0.28165 & -0.01330 & -0.292 & -0.487 & 113.0747 & -0.47035 & -0.23694 & 0.3 & 4.77 & 2.05 & 7.60 & -204.61 & -13.85 & -73.63 & -134.02 & 475 & 0.26835 & -0.353645 \\
\hline 12 & & & 6 & & 0 & .18 & 0.25574 & & -0.302 & -0.462 & 283 & -0.44592 & & & 4.76 & 55 & 6.91 & -218.89 & & & & & & -0.329465 \\
\hline 13 & & & 7.2 & & 0 & 132.17 & -0.23704 & 1919 & -0.312 & -0.388 & 105.6340 & -0.39641 & 944 & & 4.75 & 2.05 & 6.82 & -224.77 & & & 4.02 & 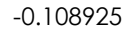 & .25623 & -0.297925 \\
\hline 14 & N & & 6.44 & .5 & 0 & 133.15 & -0.25385 & 01424 & -0.211 & -0.450 & 99.6550 & -0.43121 & -0.21201 & & 4.76 & 2.05 & 6.38 & -218.80 & & -64.80 & -134.02 & 805 & 0.26809 & -0.32161 \\
\hline 15 & DCM & & 6.22 & & 0 & 147.18 & -0.25202 & 01738 & -0.309 & -0.435 & 113.0877 & -0.42112 & 98 & & 4.75 & 2.05 & 7.57 & $-22 C$ & & & & & 94 & \\
\hline 16 & & & 3.97 & & 0 & 161.16 & -0.27116 & -0.00715 & -0.290 & -0.496 & 115.4407 & -0.47111 & -0.2 & & 4.77 & 2.06 & 7.89 & & & -68.53 & -13 & & 0.26401 & -0.34 \\
\hline 17 & DCM & 1. & 5.75 & & 0 & 131.18 & -0.25273 & 0.01627 & -0.303 & -0.536 & 108.0373 & -0.45896 & & & 5.27 & 2.05 & 5.67 & & & -5 & 2.52 & & 269 & -0.33 \\
\hline 18 & $\mathrm{DCM}$ & & 6. & & 0 & 45.21 & -0.24743 & 2053 & -0.304 & -0.597 & 122.1023 & -0.44767 & & & 5.27 & 2.15 & 5.66 & -229.31 & & -54.7 & -122.52 & & 6796 & -0.3 \\
\hline 19 & & & & & 0 & 147.18 & -0.24391 & 02667 & -0.290 & -0.319 & 112.5917 & -0.42740 & & & 4.77 & 2.05 & 6.09 & -21 & & -60.93 & & & & -0.3 \\
\hline 20 & AN & 1 & 4.63 & & 0 & 67.09 & -0.26631 & 0.06146 & -0.079 & -0.257 & 49.1590 & -0.54375 & 02 & & 4.13 & 2.01 & 3.99 & & & -6 & .02 & & & -0.3 \\
\hline 21 & & & & & 0 & & & & -0.303 & -0.353 & & & & & 5.48 & 2.01 & 5.11 & & & & & & & -0.3 \\
\hline 22 & A & & 0.67 & & 0 & .15 & 08 & 6075 & -0.084 & -0.437 & 75.1093 & $-0.5 c$ & & & 4.78 & 2.02 & 5.09 & & & & & & & -0.35 \\
\hline 23 & DCM & & 5.85 & & 0 & .12 & -0.26890 & 06579 & -0.072 & -0.371 & 62.2360 & -0.52233 & -0.22804 & & 4.13 & 2.01 & 4.55 & & & & 2.52 & 555 & 469 & 5185 \\
\hline 24 & & & & & 0 & 109.17 & -0.24181 & 5812 & -0.301 & -0.367 & 87.2550 & -0.47264 & 121 & & 5.47 & 2.01 & 5.12 & -225 & -8. & -5 & -134.02 & -0 & 9993 & -0.331925 \\
\hline 25 & D & & & & 0 & 68.08 & 0.28763 & 4573 & 0.086 & -0.033 & 44.3130 & -0.5 & & & 4.14 & 1.93 & 3.99 & & & & -122 & & & -0.4 \\
\hline 26 & DCM & & 2.16 & & 0 & 140.26 & & 03454 & 0.097 & -0.015 & 106.1360 & & & & 6.03 & 1.98 & 6.88 & & & & & & & \\
\hline 27 & DCM & & & & 0 & & -0.27393 & 05093 & 0.079 & -0.141 & 57.3633 & -0.56533 & -0.25610 & & 4.61 & 1.92 & 5.04 & & & & -122.52 & & & -0.410715 \\
\hline 28 & & & 2.9 & & 0 & 208.06 & -0.27123 & 02005 & -0.088 & 0.012 & 148.1567 & -0.47083 & -0.22169 & & 5.91 & 3.29 & 6.96 & & & & -134.02 & & 128 & -0.34626 \\
\hline 29 & A & & 6.38 & & 0 & 209.05 & -0.25263 & .04840 & -0.014 & -0.209 & 137.5770 & -0.46060 & -0.20083 & & 5.93 & 3.26 & 5.58 & & -15 & -5 & 4.02 & 115 & 30103 & -0.330715 \\
\hline 30 & & & & & 0 & 37.02 & 8001 & 1497 & -0.047 & -0.190 & 39.7333 & -0.48597 & & & 6.09 & 3.33 & 6. & & -22 & & 1.02 & & 498 & -0.35 \\
\hline 31 & & 1 & & & 0 & 156.26 & & & 0.049 & & & & & & & 1.98 & 7.57 & & & & & & & \\
\hline 32 & DCM & 1.1 & -1.01 & & 0 & & -0.29499 & 01842 & -0.433 & -0.244 & & 53752 & & & 4.26 & 2.03 & 4.10 & & & & & & & -0.408 \\
\hline 33 & DCM & 1 & -0.8 & & 0 & 156.32 & -0.28867 & 01216 & -0.427 & -0.185 & 120.1320 & -0.49956 & -0.25168 & 29 & .63 & 2.02 & 7.14 & & -5.78 & -49.67 & -122.52 & 3255 & 083 & -0.37562 \\
\hline 34 & DC & & & & 0 & & & & -0.430 & -0.292 & & & & & & 2. & 28 & & & -5 & -122.52 & 275 & 0.30369 & -0.39348 \\
\hline 35 & & & & & 0 & 4.16 & -0.26850 & 0.02415 & -0.501 & -0.415 & 75.9490 & 3767 & & & 4.26 & 2. & 5.55 & & & & -134.02 & & & -0.365805 \\
\hline 36 & & & 11.63 & & 0 & & 0.24553 & 6021 & -0.077 & -0.392 & 98.7377 & 7777 & & & & 3 & 6.50 & & & & & & & 0.339235 \\
\hline 37 & DCM & 1.02 & 6.66 & .93 & 0 & 128.17 & -0.24455 & 0.05161 & -0.264 & -0.533 & 123.2493 & -0.46788 & -0.23697 & & 5.02 & 2.03 & 6.55 & -227.94 & -9.96 & & & & 19294 & -0.352425 \\
\hline 38 & AN & 1.1 & 3.87 & 37.5 & 0 & 118.14 & -0.27341 & -0.00103 & -0.302 & -0.432 & 89.7660 & -0.47949 & -0.22718 & 30.1 & 4.73 & 2.06 & 5.58 & -211.55 & -10.91 & -65.29 & -134.02 & -0.13722 & .27238 & -0.353335 \\
\hline 18 & DMSO & 0.71 & 18.29 & 46.7 & 1 & 136.13 & -0.07092 & 0.14922 & -0.251 & -0.805 & 116.4223 & -0.31826 & -0.02389 & 35.4 & 4.49 & 2.19 & 6.12 & .06 & & -8.12 & -134.71 & 0.03915 & 0.22014 & -0.171075 \\
\hline 18 & $\mathrm{HZZO}$ & 0.53 & T2.05 & 0.1 & 1 & 136.13 & -0.07092 & 0.14922 & -0.251 & $=0.805$ & 116.4223 & -0.31826 & -0.02009 & 35.4 & 4.49 & 2.19 & 0.12 & -341.06 & -56.85 & -4.93 & -135.03 & 0.03915 & 0.22014 & -0.171075 \\
\hline
\end{tabular}




\begin{tabular}{|c|c|c|c|c|c|c|c|c|c|c|c|c|c|c|c|c|c|c|c|c|c|c|c|c|}
\hline Nu & olvent & $s_{\mathrm{N}}$ & $N$ & $\varepsilon$ & $\begin{array}{c}q \\
(e)\end{array}$ & $\begin{array}{c}\text { MW } \\
\text { (g/mol) }\end{array}$ & & $\begin{array}{l}\text { e uмо } \\
\text { (а.ч.) }\end{array}$ & $\begin{array}{l}\text { NBO } \\
\text { (a.u.) }\end{array}$ & $\begin{array}{c}\text { MK } \\
\text { (a.u.) }\end{array}$ & $\begin{array}{l}\text { Polar } \\
\text { (a.u.) }\end{array}$ & & & \%VH & (̊) & $\begin{array}{l}\text { B1 } \\
(\AA)\end{array}$ & $\begin{array}{l}\text { B5 } \\
(\AA)\end{array}$ & $\begin{array}{c}E_{\mathrm{PA}} \\
(\mathrm{kcal} / \mathrm{mol})\end{array}$ & $\begin{array}{c}\mathbf{S}_{\mathrm{Nu}} \\
(\mathrm{kcal} / \mathrm{mol})\end{array}$ & $\begin{array}{c}\mathrm{S}_{\mathrm{Int}} \\
(\mathrm{kcal} / \mathrm{mol})\end{array}$ & $\begin{array}{c}\mathbf{S}_{\mathrm{H}} \\
(\mathrm{kcal} / \mathrm{mol})\end{array}$ & $\begin{array}{c}\mu \\
\text { (a.u.) }\end{array}$ & $\begin{array}{c}n \\
\text { (a.u.) }\end{array}$ & $\begin{array}{c}\boldsymbol{\mu}^{+} \\
\text {(a.u.) }\end{array}$ \\
\hline A01 & DMSO & 0.8 & 5.59 & 6.7 & 1 & 127.08 & & .20606 & -0.604 & .994 & & & & 33.0 & 5.08 & 2.15 & 4.79 & -330.68 & -52.49 & -16.77 & -134.71 & 0.05118 & 0.30976 & -0.18996 \\
\hline AOZ & & & & & & & & & & & & & & & & & & & & & & & & \\
\hline & & & & & & & & & & & & & & & & & & & & & & & & \\
\hline & & & & & & & & & & & & & & & & & & & & & & & & \\
\hline A04 & 20 & & 77 & .1 & 1 & 9.17 & 08350 & 7308 & -0.577 & .963 & 6.4543 & .32463 & & & .09 & 14 & 6.09 & & & & & & .25658 & -0.16266 \\
\hline A05 & MSO & 86 & .91 & .7 & 1 & 3.12 & .09578 & 18545 & -0.693 & .112 & 7.5340 & 0.36403 & 0.01358 & & 6.23 & 2.09 & 4.51 & -336.89 & -52.63 & -13.58 & & .044835 & .28123 & -0.175225 \\
\hline A05 & & & & & 1 & & & & -0.693 & & & & & & 6.23 & & & & & & & & & \\
\hline A06 & & & & & 1 & & & & & & & & & & & & & & & & & & & \\
\hline 407 & uSO & & & & 1 & 2.09 & & & -0.538 & .215 & 063 & & & & & 24 & & & & & & & & \\
\hline A08 & MSO & & & & 1 & 8.21 & & & -0.515 & .176 & 77.3963 & & & & 4.62 & .24 & & & & & & & & \\
\hline A09 & uSO & .71 & .00 & 6.7 & 1 & 208.19 & -0.0 & 08748 & -0.490 & .092 & 190.2553 & 290 & & & 5.33 & .24 & 7.69 & & & -10.93 & & & .17478 & -0.192505 \\
\hline A 10 & MSC & 0.7 & 27 & & 1 & 164. & & 53 & -0.537 & 350 & 138.8 & & & & & 2.23 & 6.80 & & & & & & & \\
\hline & & & & & 1 & & & & -0.5 & & & & & & & & & & & & & & & \\
\hline 1 & IS & & & & 1 & & & & & & & & & & & & & & & & & & & \\
\hline A1 & MSC & & & & 1 & & & & & .466 & 330 & & & & & & & & & & & & & \\
\hline$A 1$ & IS & & & & 1 & & & & -0.521 & .058 & 3237 & & & & & & & & & & & & & \\
\hline$A 15$ & 15 & & & & 1 & & & & -0.3 & .329 & 777 & & & & .04 & & & & & & & & & \\
\hline $\mathrm{A} 16$ & D & 0.58 & 805 & & 1 & 130 & & & -0.3 & 42 & & & & & 5.09 & & & & & & & & & \\
\hline & & & & & 1 & & & & & & & & & & & & & & & & & & & \\
\hline $\mathbb{A 1}$ & & & & & 1 & & & & & & & & & & & & & & & & & & & \\
\hline Al & US & & & & 1 & & & & -0.249 & & & & & & & & & & & & & & & \\
\hline 1 & & & & & 1 & & & & -0.2 & .862 & & & & & & & & & & & & & & \\
\hline $\mathrm{A} 2 \mathrm{C}$ & ASC & & 20 & & 1 & & & & -0.2 & .797 & & & & & & 19 & & & & & & & & \\
\hline$\Delta ?$ & & & & & 1 & & & & & & & & & & & & & & & & & & & \\
\hline A2 & & & & & 1 & & & & -0.7 & & & & & & & & & & & & & & & \\
\hline $\mathrm{A}$ & & & & & 1 & & & & -0.7 & & & & & & & & & & & & & & & \\
\hline$A 2$ & USC & & 2 & & 1 & & & & -0.9 & & & & & & & & & & & & & & & \\
\hline 12 & 180 & & & & 1 & & & & -1.2 & .891 & & & & & & 15 & & & & & & & & \\
\hline CA24 & & & & & 1 & & & & -0.565 & & & & & & & & & & & & & & & \\
\hline CCO1 & & 1.97 & & & 0 & & & & -0.419 & & & & & & & & & & & & & & & \\
\hline $\mathrm{Cl}$ & & & & & 0 & & & & & & & & & & & & & & & & & & & \\
\hline & & & & & 0 & & & & & & & & & & & & & & & & & & & \\
\hline$=004$ & $C M$ & & 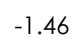 & & 0 & & & 03707 & -0.368 & .543 & & & & & & 70 & & & -3 & & & & & \\
\hline $\mathrm{CO5}$ & & 1.18 & -0.49 & & 0 & & & & & & & .49624 & & & & .06 & & & & & & & & \\
\hline $\mathrm{CO6}$ & & 1.1 & -1.07 & & 0 & & -0.27915 & .00270 & -0.155 & .126 & & -0.49475 & -0.27108 & & & .11 & .73 & & & & & -0.138225 & 0.28185 & 915 \\
\hline $\mathrm{CCO}$ & & & & & 0 & & & & -0 & & & & & & & & & & & & & & & -0.3 \\
\hline & & 0.98 & & & 0 & & & & & & & & & & & & & & & & & & & \\
\hline & & & & & & & & & & & & & & & & & & & & & & & & \\
\hline $\mathrm{CC} 10$ & $C M$ & & 0.57 & 93 & 0 & & & & -0.389 & 0.646 & 103.7080 & 3962 & & & & & & & & & & & & \\
\hline $\mathrm{CC} 11$ & DCM & 0.96 & 4.41 & 8.93 & 0 & 128.29 & -0.28105 & .05131 & -0.203 & -0.028 & 109.9603 & -0.52666 & -0.20193 & 32.3 & 6.85 & 2.23 & 5.48 & & -4.54 & -50.90 & -122.52 & -0.11487 & .33236 & -0.364295 \\
\hline $\mathrm{CC} 12$ & $\mathrm{CM}$ & 1 & 0.65 & & 0 & & -0.30287 & 0.05401 & -0.405 & -0.601 & & -0.57800 & & 27.9 & 5.50 & 1.70 & 5.50 & & -4.00 & & & & 0.35688 & -0.416085 \\
\hline $\mathrm{CC} 13$ & DCM & 1.06 & 1.7 & 8.93 & 0 & 118.18 & -0.27315 & -0.00604 & -0.372 & -0.401 & 106.9627 & -0.49508 & -0.26584 & 23.3 & 7.09 & 1.71 & 7.36 & -211.85 & -7.98 & -52.54 & -122.52 & -0.139595 & 0.26711 & -0.38046 \\
\hline
\end{tabular}




\begin{tabular}{|c|c|c|c|c|c|c|c|c|c|c|c|c|c|c|c|c|c|c|c|c|c|c|c|c|}
\hline Nu & olvent & $s_{\mathrm{N}}$ & $N$ & $\varepsilon$ & q & $\begin{array}{c}\text { MW } \\
\text { (g/mol) }\end{array}$ & $\begin{array}{l}\mathbf{e}_{\text {номо }} \\
\text { (a.u.) }\end{array}$ & $\begin{array}{l}\text { eumo } \\
\text { (a.u.) }\end{array}$ & $\begin{array}{l}\text { NBO } \\
\text { (a.u.) }\end{array}$ & $\begin{array}{c}\text { MK } \\
\text { (a.u.) }\end{array}$ & $\begin{array}{l}\text { Polar } \\
\text { (a.u.) }\end{array}$ & & & \%VH & (Å) & $\begin{array}{l}B 1 \\
\text { (Å) }\end{array}$ & $\begin{array}{l}\text { B5 } \\
\text { (A) }\end{array}$ & $\begin{array}{c}E_{\mathrm{PA}} \\
(\mathrm{kcal} / \mathrm{mol})\end{array}$ & $\begin{array}{c}\mathbf{S}_{\mathrm{Nu}} \\
(\mathrm{kcal} / \mathrm{mol})\end{array}$ & $\begin{array}{c}S_{\mathrm{lnt}} \\
(\mathrm{kcal} / \mathrm{mol})\end{array}$ & $\begin{array}{c}\mathbf{S}_{\mathbf{H}} \\
(\mathrm{kcal} / \mathrm{mol})\end{array}$ & $\begin{array}{c}\mu \\
\text { (a.u.) }\end{array}$ & $\stackrel{n}{n}$ & $\begin{array}{c}\boldsymbol{\mu}^{+} \\
\text {(a.u.) }\end{array}$ \\
\hline $\mathrm{C} 14$ & DCM & 1.09 & 0.68 & 8.93 & 0 & 146.23 & -0.27250 & & -0.372 & .475 & 129.9580 & -0.46592 & & 30.1 & 4.68 & 1.71 & 8.41 & -215.87 & -8.32 & -50.56 & -122.52 & -0.133645 & 0.27771 & 0.359945 \\
\hline & & & & & & & & & 0.400 & .674 & & & & & 6.13 & & & & & & & & & \\
\hline & & & & & & & & & & & & & & & 02 & & & & & & & & & \\
\hline & & & & & & & & & .404 & .528 & & & & & 4.69 & & & & & & & & & \\
\hline $\mathrm{C} 18$ & CM & 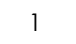 & 41 & 93 & & 2.08 & .31649 & 05113 & -0.401 & .584 & & & -0.29783 & & 4.01 & 70 & 4.57 & & & -63.42 & & & .36762 & 0.48232 \\
\hline C19 & $C M$ & 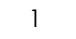 & & 93 & & 118.18 & -0.28144 & .00150 & -0.376 & .584 & 102.9593 & -0.48796 & -0.26537 & & 3.97 & .70 & 7.21 & -211.93 & -7.77 & -53.30 & -122.52 & -0.14147 & 0.27994 & -0.376665 \\
\hline $\mathrm{C2}^{2}$ & $C M$ & & & & & & & 62 & -0.192 & 57 & & & & & 4.00 & & 50 & & & & & & & \\
\hline C21 & & & 1 & & & & & & & & & -0.6 & & & & & & & & & & & & \\
\hline & $\mathrm{CN}$ & & -065 & & & 0.36 & & & -0.342 & .311 & 164.3437 & & & & .22 & 72 & & & & -47.77 & & & & 6046 \\
\hline$C 23$ & $\mathrm{CN}$ & & -1 & & & & & & -0.004 & .190 & & & -0.25806 & & 3.86 & 2.94 & 4.59 & & & -56.26 & & & & \\
\hline C24 & $C M$ & & & 93 & 0 & .12 & -0.30312 & 04385 & -0.413 & .668 & & & -0.21504 & 26.2 & 4.80 & 1.71 & 4.67 & & & -59.56 & & & 0.34697 & -0.4065 \\
\hline$C 25$ & CM & & & & 0 & .15 & -0.297 & 04355 & -0.407 & .660 & 7.8697 & & & & 4.51 & 70 & 5.76 & & & & & & & \\
\hline & & & & & & & & & -0 . & 245 & & & & & 86 & & & & & & & & & \\
\hline & & & & & & & & & & & & & & & & & & & & & & & & \\
\hline 62 & $C_{N}$ & & & & & & & & -0.197 & .368 & & & & & 4.27 & & & & & & & & & \\
\hline$=2$ & $\mathrm{CN}$ & 1 & $-c$ & & & & & 243 & -0.364 & .452 & & & & & & 70 & .09 & & & & & & & \\
\hline 230 & $\mathrm{CN}_{\mathrm{N}}$ & & & & ( & & & & -0.3 & .417 & & & & & 5.64 & .71 & 6.48 & & & & & & & \\
\hline 8 & D & & & & 0 & & & & -0.1 & 0.056 & & & & & 3.51 & 2.09 & 6.83 & & & & & & & \\
\hline & & 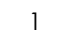 & & & 0 & & & & & & & & & & & & & & & & & & & \\
\hline & & & & & & & & & & & & & & & & & & & & & & & & \\
\hline & & & & & & & & & & .445 & & & & & & & & & & & & & & \\
\hline & & & & & & & & & -0. & .546 & & & & & & & & & & & & & & \\
\hline 36 & $C A$ & & & & 0 & & & & -0.3 & .354 & & & & & 4.75 & 70 & & & & & & & & \\
\hline דים & & & & & 0 & & & & & & & & & & & & & & & & & & & \\
\hline & & & & & 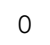 & & & & & & & & & & & & & & & & & & & \\
\hline & & & & & & & 9442 & & -0.2 & .262 & & & & & & & & & & & & & & \\
\hline & & & & & c & 6.17 & & 2723 & -0. & .378 & & & & & & 06 & & & & & & & & \\
\hline & $\mathrm{DCN}$ & & & & C & & & 305 & -0.2 & .312 & & & & & & 70 & .57 & & & & & & & \\
\hline & & & & & 0 & & & & -0.800 & 0.617 & & & & & & .72 & & & & & & & & \\
\hline & & & & & 0 & 259.16 & & -0.00614 & -0.797 & -0.693 & & & & & & & & & & & & & & \\
\hline & & & & & 0 & & & & & & & & & & & & & & & & & & & \\
\hline & & & & & c & & & & & & & & & & & & & & & & & & & \\
\hline CXO & MSO & & & & 0 & 0.29 & -0.2 & .01311 & -0.804 & .790 & 166.1277 & 9705 & & & 5.02 & .64 & & & & -57 & & & 25528 & -0.27 \\
\hline & MSO & & & & 0 & & & 03633 & -0.88 & .659 & 76.305 & & & & & .94 & & & & & & & & \\
\hline CX06 & c & & & & 0 & 8.22 & -0.25199 & 04676 & -0.862 & .809 & 05.3943 & & & & & .29 & 6.84 & & & & & & 0.29875 & \\
\hline & & & & & 0 & & & & -0 & .701 & & & & & & & & & & & & & 2718 & \\
\hline & & & & & 0 & & & & & & & & & & & & & & & & & & & \\
\hline & & & & & $c$ & & & & -0.2 & & & & & & & & & & & & & & & \\
\hline & CM & & & & 0 & & -0.25514 & .01442 & -0.262 & .994 & 122.9997 & -0.43975 & -0.22257 & & & 2.00 & & & -7 & & & & & 0.33116 \\
\hline CX11 & DCM & 0.8 & 7.66 & 8.93 & 0 & 143.15 & -0.27064 & -0.03640 & -0.267 & 0.912 & 121.1133 & -0.47584 & -0.23590 & & 5.60 & 2.02 & 7.78 & & -9.04 & -65.33 & & & .23424 & -0.35587 \\
\hline & DCM & & & & 0 & 152.58 & -0.27640 & -0.05778 & -0.267 & 0.873 & 122.0860 & -0.48934 & & 31 & 6.14 & 2.03 & 7.61 & & -8.36 & -65.05 & -122.52 & & 0.21862 & \\
\hline CX13 & DCM & 0.91 & 3.96 & 8.93 & 0 & 82.11 & -0.29974 & -0.01632 & -0.364 & -0.873 & 55.0903 & -0.54736 & -0.24355 & 30.6 & 3.88 & 1.98 & 4.59 & -205.84 & -4.60 & -61.42 & -122.52 & -0.15803 & 0.28342 & -0.395455 \\
\hline
\end{tabular}




\begin{tabular}{|c|c|c|c|c|c|c|c|c|c|c|c|c|c|c|c|c|c|c|c|c|c|c|c|c|}
\hline Nu & olvent & $s_{N}$ & $N$ & $\varepsilon$ & q & $\begin{array}{c}\text { MW } \\
\text { (g/mol) }\end{array}$ & & $\begin{array}{l}e_{\text {umo }} \\
\text { (a.u.) }\end{array}$ & $\begin{array}{l}\text { NBO } \\
\text { (a.u.) }\end{array}$ & $\begin{array}{c}\text { MK } \\
\text { (a.u.) }\end{array}$ & $\begin{array}{l}\text { Polar } \\
\text { (a.u.) }\end{array}$ & & & \%VH & (̊) & $\begin{array}{l}\text { B1 } \\
(\AA)\end{array}$ & $\begin{array}{l}\text { B5 } \\
(\AA)\end{array}$ & $\begin{array}{c}E_{\mathrm{PA}} \\
(\mathrm{kcal} / \mathrm{mol})\end{array}$ & $\begin{array}{l}\mathbf{S}_{\mathrm{Nu}} \\
(\mathrm{kcal} / \mathrm{mol})\end{array}$ & $\begin{array}{c}\mathrm{S}_{\mathrm{Int}} \\
(\mathrm{kcal} / \mathrm{mol})\end{array}$ & $\begin{array}{c}\mathbf{S}_{\mathrm{H}} \\
(\mathrm{kcal} / \mathrm{mol})\end{array}$ & $\begin{array}{c}\mu \\
\text { (a.u.) }\end{array}$ & $\begin{array}{c}\eta \\
\text { (a.u.) }\end{array}$ & $\begin{array}{c}\boldsymbol{\mu}^{+} \\
\text {(a.u.) }\end{array}$ \\
\hline$X 14$ & DMSO & 0.42 & 5.94 & 6.68 & 0 & & -0.23332 & -0.03737 & -0.311 & & 107.7647 & & -0.22756 & 36.4 & 4.36 & 1.98 & 6.08 & -243.98 & -11.24 & -65.56 & -134.71 & -0.135345 & 0.19595 & -0.38092 \\
\hline$x 15$ & & & & & & & & & -0.287 & & & & & & 5.40 & & & & & & & & & \\
\hline & & & & & & & & & & & & & & & 46 & & & & & & & & & \\
\hline <17 & & & & & & & & & & & & & & & 82 & & & & & & & & & \\
\hline$X 18$ & MSO & 58 & .46 & 6.68 & 0 & 7.24 & 23577 & .04394 & -0.261 & .921 & & .42808 & 19887 & & 5.42 & .06 & 30 & & & -59.14 & & & 19183 & 0.313475 \\
\hline CX19 & MSC & & .98 & 0.68 & 0 & 1.68 & .24339 & .05292 & -0.252 & .963 & 3.4500 & -0.42955 & -0.20524 & & 6.12 & 2.62 & 7.38 & -251.68 & -12.79 & -59.92 & & -0.148155 & .19047 & -0.317395 \\
\hline$\times 20$ & USO & 58 & & 68 & 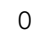 & 2 & & 54 & -0.295 & & & & & & 6.29 & & & & & & & & & \\
\hline X21 & MSO & 57 & .08 & 46.68 & U & 47.3 & 112 & -0.05812 & -0.258 & .811 & 5057 & -0.2 & -0 . & & 5.52 & 2.02 & 7.85 & & & -5 & & & & \\
\hline X22 & MSO & & & 5.68 & & & & & -0.263 & & & & & & 7.54 & 45 & & & & 6.92 & & & & \\
\hline$C \times 23$ & USC & & 1.29 & & & & & & -1.126 & .100 & & & & & 4.64 &.$/ 7$ & 4.92 & & & -65.88 & & & & \\
\hline CX24 & CM & 89 & 7.84 & 93 & 0 & 8.15 & -0.25950 & 05120 & -0.227 & .118 & 75.0000 & & -0.22757 & & 5.22 & .70 & 6.42 & -226.57 & & -55.16 & & & & -0.38 \\
\hline CX25 & $C M$ & & 6.98 & & 0 & 2.11 & -0.27152 & 04629 & -0.206 & .043 & 5339 & & & & 3.99 & 1.70 & 5.42 & & & & & & & \\
\hline EE01 & $C M$ & & & & 0 & 1 & & & -0.271 & & & & & & 20 & & 6. & & & & & & & \\
\hline E02 & & & & & & & & & -0. & & & & & & 28 & & & & & & & & & \\
\hline EE02 & CM & & & & 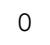 & & & & -0.326 & .457 & & & & & 5.28 & & 7.23 & & & & & & & \\
\hline EE03 & $\mathrm{M}$ & & & & $c$ & 0.26 & & & -0.485 & .727 & & & & & 5.7 & 70 & 7.07 & & & -50 & & & & \\
\hline EE04 & $C N$ & & 0 & & 0 & 32.47 & & 04788 & -0.344 & .196 & 417 & & & & 5.88 & .38 & 7. & & & -4 & & & & \\
\hline EE05 & CN & 86 & 0.61 & & 0 & 36.33 & & & -0.3 & .500 & & & & & 5.13 & 2.01 & 7. & & & & & & & \\
\hline 06 & & & & & 0 & 8.27 & & 05639 & -0 & 487 & & & & & & & & & & & & & & \\
\hline$E 06$ & & & 1 & & ( & 8.27 & & 5639 & -0.406 & 487 & 200 & & & & & & & & & & & & & \\
\hline EE07 & & & & & 0 & & & & -0.462 & & & & & & & & & & & & & & & \\
\hline$=08$ & & & & & C & & & & -0 & 43 & & & & & & & & & & & & & & \\
\hline EEO9 & & & & & 0 & 8.33 & & & -0.522 & .690 & & & & & 6.45 & 70 & 8.21 & & & & & & & \\
\hline$E 10$ & & & & & 0 & & & & & & & & & & & & & & & & & & & \\
\hline 11 & & & & & 0 & & & & & & & & & & & & & & & & & & & \\
\hline$E 12$ & CM & & 9 & & C & 4.32 & -( & 068 & -0.121 & & 4640 & & & & & & & & & -46 & & & & \\
\hline E12 & & & & & 0 & 4.32 & & & -0.121 & & & & & & & & & & & & & & & \\
\hline 13 & $C M$ & & & & c & & & & -0.188 & 47 & & & & & & & 29 & & & & & & & \\
\hline$E 14$ & & & & & 0 & & & & & & & & & & & & & & & & & & & \\
\hline 15 & & & & & 0 & & & & -0.326 & & & & & & & & & & & & & & & \\
\hline 16 & & & & & 0 & & & & & & & & & & & & & & & & & & & \\
\hline 17 & & & & & c & & & & -0. & & 943 & & & & & & & & & -49 & & & & \\
\hline EE18 & $C M$ & & & & 0 & 0.16 & -0.26564 & 06867 & -0.077 & .278 & & 7196 & & & 96 & 88 & 56 & & & -54 & & & & -0.39 \\
\hline EE19 & & & & & 0 & 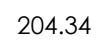 & & 00774 & -0.256 & .298 & 9.0800 & & & & & & & & & & & & & \\
\hline En01 & & & 3.36 & & 0 & 5.28 & -0.24636 & 06322 & -0.283 & 275 & 2.6877 & .50373 & 7114 & & 4.93 & 14 & .64 & & & & & & 0958 & \\
\hline & & & & & 0 & & & & -0.2 & & & & & & & & & & & & & & & \\
\hline & & & & & 0 & & & & & & & & & & & & & & & & & & & \\
\hline n04 & & & 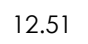 & & 0 & & & 06243 & & & & & & & & & & & & & & & & \\
\hline 10 & $C M$ & 82 & 4.02 & 9 & 0 & & -0.24365 & 06126 & -0.298 & .363 & 21.3360 & 1153 & & & & & & & & & & & & \\
\hline En06 & CM & .82 & 3.41 & 93 & 0 & 153.23 & -0.25033 & 06151 & -0.294 & .353 & 13.0080 & -0.48279 & 7910 & 37.4 & 5.04 & 1.97 & 6.46 & -246.12 & -9.17 & -55.40 & & & & -0.330945 \\
\hline En07 & $C M$ & 0.86 & 15.91 & 8 & 0 & 149.24 & -0.22966 & 05798 & -0.327 & 0.448 & 110.0343 & -0.52874 & -0.17254 & 37.6 & 4.31 & 1.97 & 6.86 & & -8.33 & -52.89 & & & & \\
\hline En08 & $M$ & 0 & 12.03 & & 0 & 182.27 & -0.25467 & .06154 & -0.291 & -0.293 & 134.4080 & -0.41899 & -0.17911 & 39.2 & 4.66 & 2.04 & 6.66 & -242 & -11.11 & -56.00 & -122 & -0.096565 & 0.31621 & -0.29905 \\
\hline
\end{tabular}




\begin{tabular}{|c|c|c|c|c|c|c|c|c|c|c|c|c|c|c|c|c|c|c|c|c|c|c|c|c|}
\hline Nu & olvent & $s_{\mathrm{N}}$ & $N$ & $\varepsilon$ & $\begin{array}{l}q \\
(e)\end{array}$ & $\begin{array}{c}\text { MW } \\
(\mathrm{g} / \mathrm{mol})\end{array}$ & $\begin{array}{l}\mathbf{e}_{\text {номо }} \\
\text { (а.u.) }\end{array}$ & $\begin{array}{l}\text { e uмо } \\
\text { (a.u.) }\end{array}$ & $\begin{array}{l}\text { NBO } \\
\text { (a.u.) }\end{array}$ & $\begin{array}{l}\text { MK } \\
\text { (a.u.) }\end{array}$ & $\begin{array}{l}\text { Polar } \\
\text { (a.u.) }\end{array}$ & $\begin{array}{l}\text { e }^{+} \text {номо } \\
\text { (а.U.) }\end{array}$ & $\begin{array}{l}\mathrm{e}^{+}+\text {Lumo } \\
\text { (a.u.) }\end{array}$ & \%VH & (̊̊) & $\begin{array}{l}\text { B1 } \\
\text { (̊) }\end{array}$ & $\begin{array}{l}\text { B5 } \\
(\AA)\end{array}$ & $\begin{array}{c}\mathrm{E}_{\mathrm{PA}} \\
(\mathrm{kcal} / \mathrm{mol})\end{array}$ & $\begin{array}{c}\mathrm{S}_{\mathrm{Nu}} \\
(\mathrm{kcal} / \mathrm{mol})\end{array}$ & $\begin{array}{c}S_{\text {Int }} \\
(\mathrm{kcal} / \mathrm{mol})\end{array}$ & $\begin{array}{c}\mathbf{S}_{\mathbf{H}} \\
(\mathrm{kcal} / \mathrm{mol})\end{array}$ & $\begin{array}{c}\mu \\
\text { (a.u.) }\end{array}$ & $\begin{array}{c}n \\
\text { (a.u.) }\end{array}$ & $\begin{array}{c}\boldsymbol{\mu}^{+} \\
\text {(a.u.) }\end{array}$ \\
\hline En09 & DCM & 0.8 & 12.06 & 8.93 & 0 & 127.19 & -0.25410 & 0.06412 & -0.285 & -0.026 & 95.3263 & -0.49327 & -0.19803 & 35.3 & 4.40 & 2.07 & 6.68 & -237.46 & -7.99 & -57.00 & -122.52 & -0.09499 & 0.31822 & -0.34565 \\
\hline 110 & DCM & 0.87 & .76 & & 0 & 189.26 & .24286 & 0.00760 & 0.318 & -0.335 & 159.2950 & -0.42 & & & 5.16 & 17 & 6.70 & & -13.29 & -59.38 & & & & -0.309 \\
\hline 10 & AN & 83 & .66 & & & 9.26 & & & .318 & & & & & & 16 & 17 & 70 & & & & & & & -0.309 \\
\hline & & & & & & & & & & & & & & & & & 6.83 & & & & & & & \\
\hline 12 & DCM & 93 & 2.26 & & 0 & 3.26 & & & .352 & .511 & & 42808 & & & 38 & 2.06 & 6.91 & & & & 2.52 & & .23857 & -0.305875 \\
\hline 112 & AN & 0.76 & 3.87 & 7.5 & 0 & 173.26 & -0.22278 & 0.01579 & -0.352 & -0.511 & 159.4293 & -0.42808 & -0.18367 & 4.8 & 38 & 2.06 & 6.91 & -240.04 & -11.91 & -59.46 & -134.02 & -0.103495 & 0.23857 & -0.305875 \\
\hline 13 & AN & 83 & 8.78 & 37.5 & 0 & 265.36 & -0.24650 & -0.00835 & -0.286 & -0.368 & 220.0633 & -0.41405 & -0.17881 & & 7.14 & 2.12 & 6.86 & -239.72 & -14.32 & -60.23 & -134.02 & & 0.23815 & -0.29643 \\
\hline 14 & AN & 0.86 & 9.94 & & 0 & 263.38 & -0.24096 & -0.00 & -0.290 & -0.334 & 229.2227 & & & & 6.90 & 2.13 & 6.98 & & & & & & & \\
\hline 15 & 1 & 82 & 1.66 & & 0 & 249.36 & & & -0.321 & .481 & 627 & & & & 02 & 06 & 6.83 & & & & & & & -0.2 \\
\hline 16 & & 84 & 1.99 & & 0 & 79.38 & 0.22127 & 0.00385 & -0.309 & -0.376 & 239.3180 & -0.37575 & & & .16 & 2.12 & 8.41 & $-24 \varepsilon$ & 17 & & -134.02 & & 0.22512 & -0.273475 \\
\hline 117 & $A$ & 84 & 0.63 & & 0 & 274.37 & -0.24128 & -0.02483 & -0.344 & -0.481 & 246.1423 & -0.42727 & -0.18837 & & 6.74 & 2.39 & 8.09 & -240.54 & -16.66 & -64.18 & -134.02 & & 0.21645 & -0.30782 \\
\hline 118 & AN & .82 & 0.42 & 7.5 & 0 & 294.35 & -0.24436 & -0.04378 & -0.348 & -0.453 & 248.6847 & -0.44438 & -0.18961 & 44. & 6.73 & 2.38 & 7.73 & -239.51 & -15.98 & -63.55 & -134.02 & & 0.20058 & -0.316995 \\
\hline 19 & & & 11.60 & & 0 & 187.29 & -0.2 & 0.00 & -0.480 & .518 & 55.2660 & & & & 5.36 & .70 & 6.93 & & & & & & & \\
\hline 20 & AN & & 10.30 & & 0 & 189.26 & & 0.00 & -0.476 & -0.489 & 146.8910 & & & & 5.61 & 70 & 6.83 & & & & & & & \\
\hline 20 & DCM & 79 & .96 & & 0 & 189.26 & -0.26607 & 00294 & -0.476 & -0.489 & 146.8910 & -0.4 & & & .61 & 1.70 & 6.83 & -242.81 & & & & & 901 & 2086 \\
\hline 21 & & 66 & 4.09 & & 0 & & -0.24055 & 00793 & -0.262 & -0.305 & 164.2300 & -0.43624 & & & 80 & 2.11 & 6.80 & -252 & & & & & & 192 \\
\hline 22 & AN & .93 & 15.27 & & 0 & 185.27 & -0.23566 & 0.01569 & -0.285 & -0.261 & 053 & -0.44109 & & 36. & 6.18 & 2.07 & 6.89 & -253 & & & & & & -0.31 \\
\hline 23 & DCM & .82 & 10.04 & & 0 & & -0.2 & 96 & -0.020 & 0.342 & 106 & & & & 4.32 & 2.90 & 6.80 & & & & & & & -0.3 \\
\hline 24 & & & 12.26 & & 0 & & & & -0.293 & & & & & & 5 & & & & & & & & & \\
\hline 125 & & 0.87 & 7.06 & & 0 & 125.17 & -0.26620 & 0.04269 & -0.238 & -0.384 & 90.9140 & & & & & & & -22 & & & & & 0.30889 & -0.376865 \\
\hline 126 & Al & & 5 & & 0 & & -0.26924 & 03680 & -0.233 & 0.321 & 102.4600 & 1972 & & & 81 & .06 & 5.88 & -224.07 & & & & & 604 & -0.3 \\
\hline 27 & & & 4 & & 0 & 187.24 & -0.26278 & -0.0 & -0.179 & -0.218 & 860 & & & & 18 & 06 & 6.47 & -22 & & & & & & -0.3 \\
\hline 28 & AN & 0.98 & 4.61 & & 0 & 141.21 & -0.28211 & 0.03315 & -0.449 & -0.643 & 105.7877 & & & 35.6 & 5.59 & 1.70 & 6.07 & -22 & & & & & & -0.3 \\
\hline 129 & AN & 1 & 5.44 & & 0 & 223.28 & -0.27780 & -0.01958 & -0.416 & -0.547 & & & & 32.9 & 5.29 & .70 & 9.38 & & & & & & & \\
\hline 30 & & & & & 0 & & & -0.0 & -0.422 & & & & & & & & & & & & & & & \\
\hline 31 & & 1 & & & 0 & & & & -0.416 & & & & & & & & & & & & & & & -0.3 \\
\hline 32 & & & & & 0 & 175.23 & 380 & & -0.422 & -0.614 & 337 & & & & 0.00 & .70 & 36 & & & & & & & -0.3 \\
\hline 01 & D & & & & 0 & & & & -0.414 & -0.618 & & & & & & 77 & 3.15 & & & & & & & -0.3 \\
\hline 101 & & & 1.05 & & 0 & & & 0.00228 & -0.414 & -0.618 & & & & & 5.80 & 77 & 3.15 & & & & & & & -0.3 \\
\hline 102 & DCM & & & & 0 & 113.54 & -0.33572 & -0.00944 & -0.415 & -0.662 & & -0.52115 & -0.22760 & & & 77 & 3.15 & -22 & & & & & 628 & -0.3 \\
\hline 02 & & & & & 0 & & & -0.00944 & -0.415 & .662 & & & & & & & & & & & & & & -0.3 \\
\hline 03 & & & & & 0 & & & & & & & & & & & & & & & & & & & \\
\hline 03 & & & 1.44 & & 0 & 109.13 & 0971 & & -0.441 & -0.665 & 5590 & 849 & & & & .90 & & & -5 & & & & & -0.3 \\
\hline 104 & DCM & & & & 0 & & -0.26430 & 0.02622 & -0.459 & -0.754 & & & & & & .06 & & & -10.13 & & & & & \\
\hline NO4 & & 0.67 & 14.9 & 36.7 & 0 & 122.17 & -0.26430 & 0.02622 & -0.459 & -0.754 & 95.4870 & -0.44126 & -0.16954 & 26.6 & 8.08 & 2.06 & 3.22 & & -8.99 & 5.42 & & & 0.29052 & -0.3054 \\
\hline N04 & DMSO & 0.67 & 14.8 & & 0 & 122.17 & -0.26430 & 0.02622 & -0.459 & -0.754 & 95.4870 & -0.44126 & -0.16954 & & 8.08 & 2.06 & 3.22 & -24 & -7.89 & & -13 & & 29052 & -0.30 \\
\hline 04 & & & & & 0 & & & & -0.459 & & & & & & & & & & & & & & & \\
\hline 04 & & & 1 & & 0 & & & & & & & & & & & & & & & & & & & \\
\hline 104 & & 0.66 & 15.9 & & 0 & & -0.26430 & 0.02622 & -0.459 & -0.754 & 95.4870 & & & & & & & & & & & & & -0.3054 \\
\hline 05 & $\mathrm{DCM}$ & 0.67 & 15.9 & 8.93 & 0 & 148.21 & -0.26003 & 0.02789 & -0.462 & -0.740 & 116.3893 & -0.43301 & -0.16270 & 26.6 & 9.27 & .43 & 3.23 & -249.35 & -10.63 & -52.62 & -122.52 & & 0.28792 & -0.297855 \\
\hline 06 & AN & 0.76 & 7.69 & 37.5 & 0 & 119.13 & -0.30093 & -0.01662 & -0.216 & -0.394 & 83.8313 & -0.48297 & -0.21399 & 26.3 & 6.30 & 1.58 & 5.27 & -224.73 & -10.71 & -61.61 & -134.02 & -0.158775 & 0.28431 & -0.34848 \\
\hline 07 & $\mathrm{~N}$ & 0.76 & 7.77 & 37.5 & 0 & 133.15 & -0.29319 & -0.01436 & -0.222 & -0.405 & 97.4500 & -0.47322 & -0.20315 & 26.3 & 6.30 & 1.96 & 5.26 & -229.74 & -10.08 & -57.60 & -134.02 & -0.153775 & 0.27883 & -0.338 \\
\hline
\end{tabular}




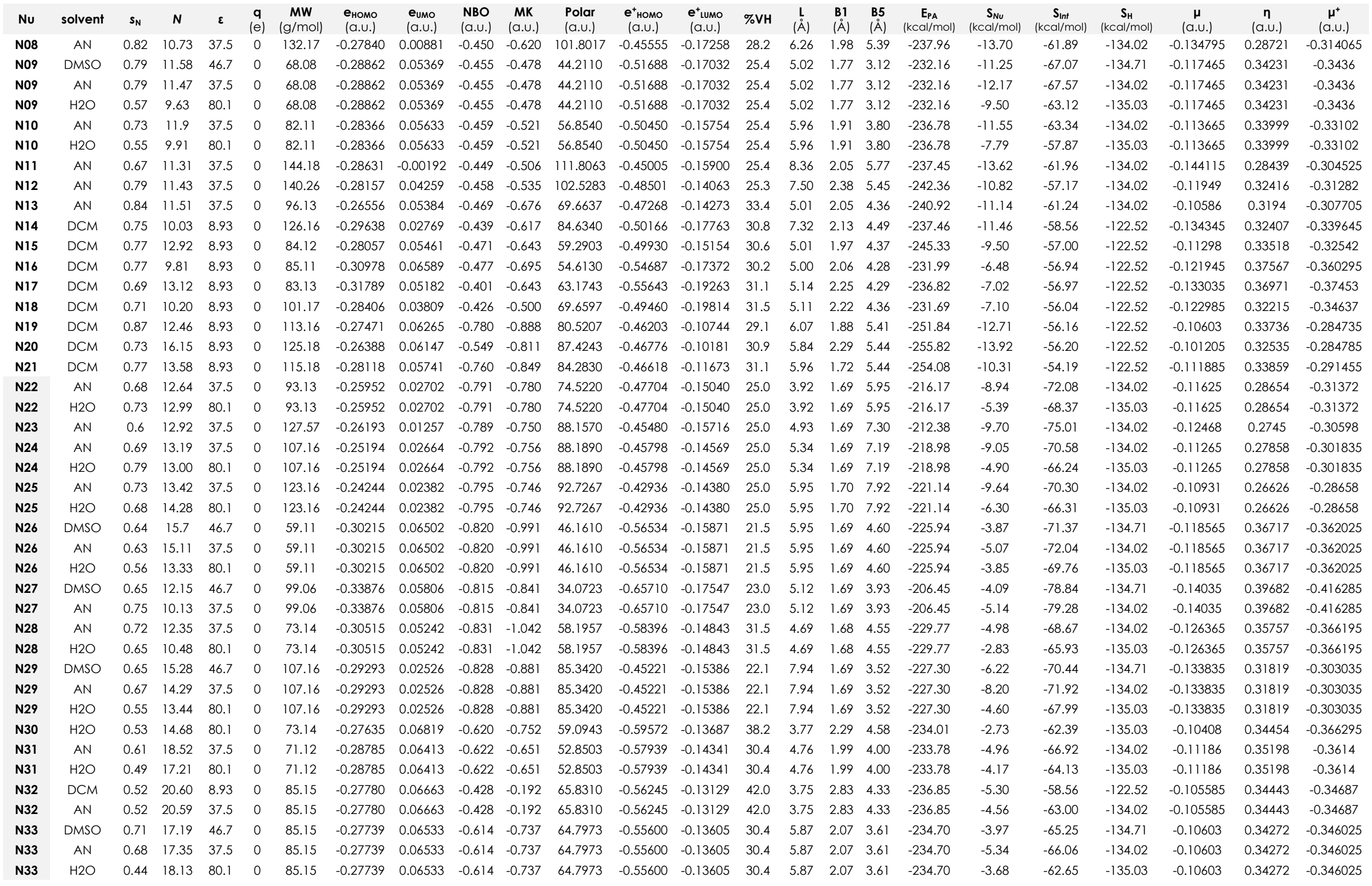




\begin{tabular}{|c|c|c|c|c|c|c|c|c|c|c|c|c|c|c|c|c|c|c|c|c|c|c|c|c|}
\hline Nu & olvent & $s_{N}$ & $N$ & $\varepsilon$ & $\begin{array}{c}q \\
(e)\end{array}$ & $\begin{array}{c}\text { MW } \\
(\mathrm{g} / \mathrm{mol})\end{array}$ & & & $\begin{array}{l}\text { NBO } \\
\text { (a.u.) }\end{array}$ & $\begin{array}{c}\text { MK } \\
\text { (a.u.) }\end{array}$ & $\begin{array}{l}\text { Polar } \\
\text { (a.u.) }\end{array}$ & & & $\% \mathrm{VH}$ & (̊) & $\begin{array}{l}\text { B1 } \\
(\stackrel{\AA}{A})\end{array}$ & $\begin{array}{l}\text { B5 } \\
\text { (Å) }\end{array}$ & $\begin{array}{c}\mathrm{E}_{\mathrm{PA}} \\
(\mathrm{kcal} / \mathrm{mol})\end{array}$ & 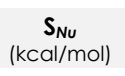 & $\begin{array}{c}\mathbf{S}_{\text {Int }} \\
(\mathrm{kcal} / \mathrm{mol})\end{array}$ & $\begin{array}{c}\mathrm{S}_{\mathrm{H}} \\
\left.\mathrm{Kcal}_{\mathrm{mol}}\right)\end{array}$ & $\begin{array}{c}\mu \\
\text { (a.u.) }\end{array}$ & $\begin{array}{c}\boldsymbol{\eta} \\
\text { (a.u.) }\end{array}$ & $\begin{array}{c}\boldsymbol{\mu}^{+} \\
\text {(a.u.) }\end{array}$ \\
\hline N34 & DCM & 0.52 & 18.90 & .93 & 0 & 99.18 & -0.27685 & 0.06840 & .436 & -0.269 & 77.6010 & & -0.12018 & 45.2 & 3.74 & 2.91 & 5.07 & -238.57 & -5.83 & -57.88 & -122.52 & -0.104225 & 0.34525 & -0.334565 \\
\hline N34 & & & & & 0 & & & & & & & & & & & & .07 & & & & & & & 0 \\
\hline N35 & & & & & & & & & & & & & & & & 04 & 56 & & & & & & & \\
\hline N35 & & & & & c & & & & & & & & & & & & & & & & & & & \\
\hline N35 & & & .62 & .1 & 0 & .12 & .27896 & 06580 & .609 & -0.671 & .9827 & .51408 & .14485 & & 65 & .04 & 56 & -227.72 & -7.05 & -69.20 & & & .34476 & -0.329465 \\
\hline N36 & CM & 52 & .50 & 93 & 0 & 1.15 & .28568 & 07174 & .433 & -0.243 & 9.7200 & 0.50725 & .12802 & & .74 & .89 & 4.56 & -232.03 & -6.33 & -61.25 & -122.52 & -0.10697 & .35742 & -0.317635 \\
\hline N36 & & & .80 & & 0 & & -0.28568 & & 33 & -0.243 & & & & & & 89 & 4.56 & & & & & & & \\
\hline N37 & & & & & 0 & & & & & & & & & & & & & & & & & & & \\
\hline N38 & & & & & 0 & & & & .418 & & & & & & 81 & & & & & & & & & \\
\hline N39 & & & & & 0 & 2.18 & & 05860 & .411 & -0.421 & & & & & & & & & & & & & & \\
\hline NA01 & $\mathrm{H} 2 \mathrm{O}$ & 2 & .47 & 0.1 & 1 & 4.09 & -0.06606 & 19092 & -0.562 & -0.930 & 72.5333 & & -0.01494 & & 5.80 & 1.77 & 3.78 & -355.44 & -65.72 & & & & & \\
\hline NA01 & & & .11 & 5 & 1 & 94.09 & -0.06 & 2 & .562 & -0.930 & & & & & .80 & .77 & 3.78 & & & & & & & \\
\hline NA01 & & & & & 1 & & & & .562 & & & & & & 30 & 77 & & & & & & & & \\
\hline NA02 & & & & & 1 & & & & & & & & & & & & & & & & & & & \\
\hline NAO2 & & & & & 1 & 09 & & 940 & .564 & -0.876 & & & & & & .77 & & & & & & & 83 & \\
\hline NAO2 & 1SC & & & & 1 & 9 & & & .564 & -0.876 & & & & & 5/ & & 11 & & & & & & & \\
\hline NAO3 & & & 3 & 1 & 1 & 134.12 & & 4 & .558 & -0.870 & & & & & 7.31 & 1.77 & 5.25 & & & & & & & \\
\hline NAO3 & 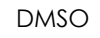 & & .00 & 67 & 1 & 34.12 & & & -0.558 & -0.8 & & & & & 7.31 & 1.77 & & & & & & & & \\
\hline NA04 & & & & & 1 & & & & & & & & & & & & & & & & & & & \\
\hline NA05 & 15 & & & & 1 & & & & & & & & & & & & & & & & & & & \\
\hline NA06 & & & & & 1 & & & & & -0.655 & & & & & 21 & & & & & & & & & \\
\hline VA06 & & & & & 1 & & & & & -0.6 & & & & & 27 & & & & & & & & & \\
\hline NA07 & & & & & 1 & 99.07 & & & .689 & -0.8 & & & & & .97 & 1.89 & & & & & & & & \\
\hline NA08 & & & & & 1 & 96.07 & & & -0.696 & & & & & & & & & & & & & & & \\
\hline NA09 & & & & & 1 & & & & & & & & & & & & & & & & & & & \\
\hline NA1 & & & & & 1 & & & & & & & & & & & & & & & & & & & \\
\hline NA11 & & & & & 1 & 18 & & & .912 & -0.666 & & & & & & .20 & & & & & & & & \\
\hline NA12 & 150 & & & & 1 & & & & .576 & -0.7 & & & & & & & & & & & & & & \\
\hline NA13 & & & & & 1 & & & & -0.556 & -0.797 & & & & & & & & & & & & & & \\
\hline NA13 & DMSO & & & & 1 & & & & -0.556 & -0.797 & & & & & & & & & & & & & & \\
\hline NA1 & & & & & 1 & & & & & & & & & & & & & & & & & & & \\
\hline NA & & & & & 1 & & & & & & & & & & & & & & & & & & & \\
\hline NA15 & & & & 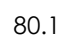 & 1 & 2.09 & -0.1 & 17762 & -0.627 & -0.912 & 0.6173 & & & & 1 & 1.77 & 3.7 & & & & & & & \\
\hline NA15 & DMSO & & & & 1 & & & 7762 & -0.627 & -0.912 & & & & & & & & & & & & & & \\
\hline NA16 & & & & & 1 & & & 17403 & -0.483 & -0.713 & 7.3297 & -0.28256 & -0.00564 & & & 1.90 & 6.46 & & & & & & & \\
\hline NA1 & & & & & 1 & & & & & -0.7 & & & & & & 1.90 & & & & & & & & \\
\hline NA17 & & & & & 1 & & & & & & & & & & & & & & & & & & & \\
\hline & & & & & 0 & & & & & & & & & & & & & & & & & & & \\
\hline HC2 & & & .72 & 7 & 0 & 04.45 & -0.27458 & & 109 & -0.793 & & & & & & & & & & & & & & \\
\hline $\mathrm{NHC3}$ & TII & & 6.54 & 7.58 & 0 & 138.21 & -0.26623 & .06434 & .073 & -0.960 & 6.8777 & -0.48087 & .13393 & & 4.99 & 2.71 & 5.57 & & -12.49 & & 119.28 & -0.100945 & 0.33057 & -0.3074 \\
\hline & THF & & 20.41 & 7.58 & 0 & 188.23 & -0.26159 & 0.01711 & 0.087 & -0.855 & 47.6990 & -0.40182 & & 36.1 & 4.98 & 2.37 & 9.37 & & & & -119.28 & -0.12224 & & -0.27159 \\
\hline NHC5 & THF & 0.46 & 23 & 7.58 & 0 & 218.26 & -0.26172 & 0.02322 & 0.103 & -0.814 & 160.5620 & -0.40091 & -0.13024 & 39.8 & 4.98 & 3.14 & 7.06 & -278.17 & -18.18 & -47.55 & -119.28 & -0.11925 & 0.28494 & -0.26557 \\
\hline
\end{tabular}




\begin{tabular}{|c|c|c|c|c|c|c|c|c|c|c|c|c|c|c|c|c|c|c|c|c|c|c|c|c|}
\hline Nu & solvent & $s_{\mathrm{N}}$ & $N$ & $\varepsilon$ & $\begin{array}{l}q \\
(e)\end{array}$ & $\begin{array}{c}\text { MW } \\
(\mathrm{g} / \mathrm{mol})\end{array}$ & $\begin{array}{l}\mathbf{e}_{\text {номо }} \\
\text { (а.ч.) }\end{array}$ & 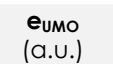 & $\begin{array}{l}\text { NBO } \\
\text { (a.u.) }\end{array}$ & $\begin{array}{l}\text { MK } \\
\text { (a.u.) }\end{array}$ & $\begin{array}{l}\text { Polar } \\
\text { (a.u.) }\end{array}$ & $\begin{array}{l}\text { e }^{+} \text {номо } \\
\text { (а.ч.) }\end{array}$ & $\begin{array}{l}\text { e }^{+} \text {เuмо } \\
\text { (а.u.) }\end{array}$ & \%VH & (À) & $\begin{array}{l}\text { B1 } \\
(\AA)\end{array}$ & $\begin{array}{l}\text { B5 } \\
(\AA)\end{array}$ & $\begin{array}{c}E_{\mathrm{PA}} \\
(\mathrm{kcal} / \mathrm{mol})\end{array}$ & $\begin{array}{c}\mathbf{S}_{\mathrm{Nu}} \\
(\mathrm{kcal} / \mathrm{mol})\end{array}$ & $\begin{array}{c}\mathbf{S}_{\mathrm{Int}} \\
(\mathrm{kcal} / \mathrm{mol})\end{array}$ & $\begin{array}{c}\mathbf{S}_{\mathrm{H}} \\
(\mathrm{kcal} / \mathrm{mol})\end{array}$ & $\begin{array}{c}\mu \\
\text { (a.u.) }\end{array}$ & $\begin{array}{c}n \\
\text { (a.u.) }\end{array}$ & $\begin{array}{c}\boldsymbol{\mu}^{+} \\
\text {(a.u.) }\end{array}$ \\
\hline OA01 & DMF & 0.89 & 18.86 & 36.7 & 1 & 93.11 & -0.04990 & 0.20207 & -0.769 & -0.804 & 78.4493 & -0.28243 & 0.01986 & 23.8 & 5.39 & 1.52 & 5.86 & -357.15 & -56.66 & -7.49 & -134.08 & 0.076085 & 0.25197 & -0.131285 \\
\hline OA01 & DMSO & 71 & 9.86 & 46.7 & 1 & 11 & 04990 & 207 & 769 & .804 & 78.4493 & 3243 & 1986 & & 5.39 & 1.52 & 5.86 & & -56.19 & 68 & & 085 & 197 & 285 \\
\hline OA01 & AN & 0.85 & 18.53 & 37.5 & 1 & 11 & 04990 & 0207 & .769 & -0.804 & 3.4493 & -0.28243 & 01986 & & 5.39 & 1.52 & 5.86 & & -57.79 & -8.23 & 4.02 & 76085 & 25197 & -0.131285 \\
\hline OA02 & DMSO & 0.8 & 20.09 & 46.7 & 1 & 123.14 & -0.04325 & 0.18742 & -0.781 & -0.795 & 96.5880 & -0.25931 & 0.01817 & 23.8 & 5.39 & 1.52 & 8.25 & -359.34 & -55.94 & -7.56 & -134.71 & 0.072085 & 0.23067 & -0.12057 \\
\hline OA02 & AN & 0.69 & 20.62 & 37.5 & 1 & 123.14 & -0.04325 & 0.18742 & -0.781 & -0.795 & 96.5880 & -0.25931 & 0.01817 & 23.8 & 5.39 & 1.52 & 8.25 & -359.34 & -57.66 & -9.05 & -134.02 & .072085 & 0.23067 & -0.12057 \\
\hline OA03 & DMF & 8 & 19.90 & 36.7 & 1 & 149.22 & -0.05370 & 16783 & -0.765 & -0.790 & 130.4110 & -0.27262 & 0.02074 & 3.8 & 6.11 & 1.52 & 8.37 & -356.67 & -55.41 & -8 & 4.08 & 065 & 53 & 12594 \\
\hline OA03 & MSO & 0.79 & 19.67 & 46.7 & 1 & 149.22 & -0.05370 & 0.16783 & -0.765 & -0.790 & 130.4110 & -0.27262 & 0.02074 & 23.8 & 6.11 & 1.52 & 8.37 & -356.67 & -54.56 & -7.17 & -134.71 & . 057065 & 0.22153 & -0.12594 \\
\hline OA04 & DMF & 0.85 & 19.65 & 36.7 & 1 & 107.14 & -0.04745 & 0.19566 & -0.771 & -0.790 & 93.0813 & -0.27239 & 0.02023 & 23.8 & 5.38 & 1.52 & 7.19 & -358.27 & -56.29 & -7.63 & -134.08 & 0.074105 & 0.24311 & -0.12608 \\
\hline OA04 & DMSO & 0.87 & 19.08 & 46.7 & 1 & 107.14 & -0.04745 & 0.19566 & -0.771 & -0.790 & 93.0813 & -0.27239 & 0.02023 & 23.8 & 5.38 & 1.52 & 7.19 & -358.27 & -55 & -6.7 & -134 & . 074105 & 0.24311 & \\
\hline OA05 & DMF & 0.72 & 19.67 & 36.7 & 1 & 127.56 & -0.06199 & 0.18297 & -0.758 & -0.783 & 90.9537 & -0.28208 & 0.00549 & 23.8 & 5.40 & 1.52 & 7.21 & -350.15 & -52.50 & -8.19 & -134.08 & 0.06049 & 0.24496 & 3295 \\
\hline OA05 & DMSO & 0.64 & 20.34 & 46.7 & 1 & 127.56 & -0.06199 & 0.18297 & -0.758 & -0.783 & 90.9537 & -0.28208 & 0.00549 & 23.8 & 5.40 & 1.52 & 7.21 & -350.15 & -51.94 & -7.32 & -134.71 & 0.06049 & 0.24496 & 8295 \\
\hline OA05 & AN & 0.82 & 18.32 & 37.5 & 1 & 127.56 & -0.06199 & 0.18297 & -0.758 & -0.783 & 90.9537 & -0.28208 & 0.00549 & 23.8 & 5.40 & 1.52 & 7.21 & -350.15 & -53.50 & -8.90 & -134.02 & 0.06049 & 0.24496 & 3295 \\
\hline OA06 & DMF & 0.71 & 17.85 & 36.7 & 1 & 118.12 & -0.08616 & 0.16631 & -0.717 & -0.747 & 99.2850 & -0.30409 & -0.01468 & 23.8 & 5.42 & 1.52 & 7.80 & -337.88 & -49.16 & -10.59 & -134.08 & 0.040075 & 0.25247 & \\
\hline OA06 & DMSO & 0.57 & 18.78 & 46.7 & 1 & 118.12 & -0.08616 & 0.16631 & -0.717 & -0.747 & 99.2850 & -0.30409 & -0.01468 & 23.8 & 5.42 & 1.52 & 7.80 & -337.88 & -48.50 & -9.66 & -134.71 & . 040075 & 0.25247 & -0.15 \\
\hline OA06 & AN & 0.59 & 19.58 & 37.5 & 1 & 118.12 & -0.08616 & 16631 & -0.717 & -0.747 & 99.2850 & -0.30409 & -0.01468 & 23.8 & 5.42 & 1.52 & 7.80 & -337.88 & -50 . & -11.50 & -134.02 & 0075 & 0.25247 & \\
\hline OA07 & DMF & 0.53 & 22.05 & 36.7 & 1 & 161.11 & -0.07989 & 0.17873 & -0.734 & -0.761 & 92.1377 & -0.30465 & 0.00047 & 23.8 & 5.41 & 1.52 & 7.64 & -343.75 & -48.54 & -7.42 & -134.08 & 0.04942 & 0.25862 & -0.15209 \\
\hline OA07 & AN & 0.53 & 21.69 & 37.5 & 1 & 161.11 & -0.07989 & 0.17873 & -0.734 & -0.761 & 92.1377 & -0.30465 & 0.00047 & 23.8 & 5.41 & 1.52 & 7.64 & -343.75 & -49.53 & -8.27 & -134.02 & 942 & 0.25862 & 209 \\
\hline OA08 & $A C N$ & 0.69 & 18.39 & 37.5 & 1 & 161.1 & -0.07249 & 0.16920 & -0.748 & -0.775 & 91.0353 & -0.30136 & -0.00527 & 26.5 & 6.97 & 1.52 & 6.01 & & & -8. & -13 & & & \\
\hline OA08 & DMSO & 0.61 & 18.47 & 46.68 & 1 & 161.1 & -0.07249 & 0.16920 & -0.748 & -0.775 & 91.0353 & -0.30136 & -0.00527 & 26.5 & 6.97 & 1.52 & 6.01 & -346.25 & -48.76 & -6.34 & -134.71 & 0.048355 & 0.24169 & -0.153315 \\
\hline OA08 & DMF & 0.58 & 20.37 & 36.71 & 1 & 161.1 & -0.07249 & 0.16920 & -0.748 & -0.775 & 91.0353 & -0.30136 & -0.00527 & 26.5 & 6.97 & 1.52 & 6.01 & -346.25 & -49.41 & -7.28 & -134.08 & 0.048355 & 0.24169 & 3315 \\
\hline OA09 & $A C N$ & 0.69 & 18.39 & 37.5 & 1 & 161.1 & -0.07692 & 0.17160 & -0.729 & 0.713 & 90.1460 & -0.30189 & -0.00390 & 32.0 & 6.48 & 1.98 & 5.85 & -343.63 & -52.02 & -8.73 & -134.02 & 0.04734 & 0.24852 & -0.152895 \\
\hline OA09 & DMSO & 0.61 & 18.47 & 46.68 & 1 & 161.1 & -0.07692 & 0.17160 & -0.729 & 0.713 & 90.1460 & -0.30189 & -0.00390 & 32.0 & 6.48 & 1.98 & 5.85 & -343.63 & -50.29 & -6.78 & -134.71 & 0.04734 & 0.24852 & -0.152895 \\
\hline OA09 & DMF & 0.58 & 20.37 & 36.71 & 1 & 161.1 & -0.07692 & 0.17160 & -0.729 & 0.713 & 90.1460 & -0.30189 & -0.00390 & 32.0 & 6.48 & 1.98 & 5.85 & -343.63 & -50.94 & -7.73 & -134.08 & 0.04734 & 0.24852 & -0.15 \\
\hline OA10 & $A C N$ & 0.43 & 25.51 & 37.5 & 1 & 138.1 & -0.08255 & 0.09401 & -0.741 & -0.767 & 95.9867 & -0.31177 & -0.05521 & 26.4 & 6.98 & 1.52 & 5.84 & -341.18 & -49.42 & -10.29 & -134 & 573 & 0.17656 & $-0.1 \varepsilon$ \\
\hline OA10 & DMSO & 0.54 & 21.29 & 46.68 & 1 & 138.1 & -0.08255 & 0.09401 & -0.741 & -0.767 & 95.9867 & -0.31177 & -0.05521 & 26.4 & 6.98 & 1.52 & 5.84 & -341.18 & -48.02 & -8.82 & -134.71 & 0.00573 & 0.17656 & -0.18349 \\
\hline OA10 & DMF & 0.54 & 22.41 & 36.71 & 1 & 138.1 & -0.08255 & 0.09401 & -0.741 & -0.767 & 95.9867 & -0.31177 & -0.05521 & 26.4 & 6.98 & 1.52 & 5.84 & -341.18 & -48.68 & -9.74 & -134.08 & 0.00573 & 0.17656 & -0.18349 \\
\hline OA11 & $\mathrm{ACN}$ & 0.80 & 18.81 & 37.5 & 1 & 123.13 & -0.05355 & 0.19631 & -0.762 & -0.788 & 95.4590 & -0.27535 & 0.02597 & 26.5 & 7.46 & 1.52 & 6.04 & -355.61 & -57.28 & -9.08 & -134.02 & 0.07138 & 0.24986 & -0.12469 \\
\hline OA12 & DMF & 0. & 15.05 & 36.7 & 1 & 138.11 & -0.09955 & 0.10926 & -0.691 & -0.711 & 105.3497 & -0.31582 & -0.04830 & 23.8 & 5.42 & 1.52 & 7.60 & -333.31 & -46.74 & -10.00 & -134.08 & 355 & 0.20881 & \\
\hline OA12 & DMSO & 0.77 & 14.32 & 46.7 & 1 & 138.11 & -0.09955 & 0.10926 & -0.691 & -0.711 & 105.3497 & -0.31582 & -0.04830 & 23.8 & 5.42 & 1.52 & 7.60 & -333.31 & -46.07 & -9.08 & -134.71 & .004855 & 0.20881 & -0.18206 \\
\hline OA12 & AN & 0.82 & 15.14 & 37.5 & 1 & 138.11 & -0.09955 & 0.10926 & -0.691 & -0.711 & 105.3497 & -0.31582 & -0.04830 & 23.8 & 5.42 & 1.52 & 7.60 & -333.31 & -47.44 & -10.55 & -134.02 & 0.004855 & 0.20881 & -0.18206 \\
\hline OA13 & DMSO & 0.59 & 21.02 & 46.68 & 1 & 205.32 & -0.06756 & 0.18236 & -0.814 & -0.782 & 177.9637 & -0.26980 & 0.02658 & 47.0 & 6.97 & 2.74 & 5.95 & -343.17 & -46.05 & -6.26 & -134.71 & 0.0574 & 0.24992 & -0.12161 \\
\hline OA14 & AN & 0.75 & 16.90 & 37.5 & 1 & 59.05 & -0.09818 & 0.24018 & -0.800 & -0.830 & 35.3567 & -0.36255 & 0.04952 & 21.9 & 4.43 & 1.52 & 3.72 & -356.30 & -60.03 & -6.02 & -134.02 & 0.071 & 0.33836 & -0.156515 \\
\hline OA15 & AN & 0.72 & 16.45 & 37.5 & 1 & 121.12 & -0.12854 & 0.15036 & -0.770 & -0.728 & 86.8737 & -0.32245 & -0.02327 & 23.3 & 7.64 & 1.52 & 4.96 & -347.76 & -58.35 & -8.46 & -134.02 & 0.01091 & .2789 & -0.17286 \\
\hline OA16 & cetone & 0.68 & 18.74 & 20.7 & 1 & 166.12 & -0.15242 & 0.05379 & -0.756 & -0.717 & 106.1783 & -0.35102 & -0.07661 & 23.2 & 9.24 & 1.52 & 4.90 & -335.35 & -50.04 & -10.43 & -131.23 & -0.049315 & 20621 & -0.213815 \\
\hline OA17 & AN & 0.71 & 14.90 & 37.5 & 1 & 211.12 & -0.16856 & 0.03630 & -0.745 & -0.705 & 121.2173 & -0.37963 & -0.08940 & 23.2 & 8.65 & 1.52 & 6.63 & -326.75 & -47.32 & -11.95 & -134.02 & -0.06613 & 0.20486 & -0.234515 \\
\hline OA18 & AN & 0.64 & 16.03 & 37.5 & 1 & 75.05 & -0.11692 & 0.23294 & -0.775 & -0.817 & 38.2407 & -0.38195 & 0.05815 & 21.3 & 5.46 & 1.52 & 4.49 & & & & & 0.05801 & 0.34986 & -0.1619 \\
\hline SA1 & $\mathrm{ACN}$ & 0.63 & 22.40 & 37.5 & 1 & 146.25 & -0.06295 & 0.16825 & -0.409 & -0.598 & 113.5917 & -0.26327 & 0.00606 & 31.3 & 7.15 & 1.80 & 5.75 & -339.01 & -59.47 & -10.59 & -134.02 & 0.05265 & 0.2312 & -0.128605 \\
\hline
\end{tabular}




\begin{tabular}{|c|c|c|c|c|c|c|c|c|c|c|c|c|c|c|c|c|c|c|c|c|c|c|c|c|}
\hline 12 & $\mathrm{ACN}$ & .57 & 3.84 & .5 & 1 & 60.27 & & & 3 & .599 & & & & .8 & 6.62 & 1.80 & 6.48 & & & -10.40 & & & & \\
\hline 13 & $\mathrm{ACN}$ & .64 & .72 & 7.5 & & 2.25 & 6983 & & -0.385 & .566 & 118.3267 & 004 & & .4 & 6.80 & 80 & 6.39 & -304.4 & 6.24 & 0.99 & 34.02 & 705 & 3707 & \\
\hline & CN & 69 & .93 & .5 & & & & & -0.409 & .603 & & 6643 & & & & & & & & & & & & \\
\hline 45 & $\mathrm{ACN}$ & .69 & 19.30 & 37.5 & 1 & 121.19 & -0.07132 & 7575 & -0.334 & -0.623 & 87.9217 & -0.29031 & & 8.5 & .23 & 1.80 & 5.66 & -334 & & -5.80 & & & & 5402 \\
\hline 46 & $\mathrm{ACN}$ & 0.63 & 21.20 & 37.5 & 1 & 75.11 & -0.06112 & 22819 & -0.571 & -0.825 & 52.1640 & -0.33207 & 0.01552 & 27.2 & 4.59 & 1.80 & 4.54 & -342.75 & -57.18 & -5.55 & 134.02 & 5535 & 0.28931 & 158275 \\
\hline A & $\mathrm{ACN}$ & 1.59 & 20.11 & 37.5 & 1 & 41.16 & -0.07770 & 14909 & -1.066 & -0.446 & 6.4460 & -0.33945 & -0.02858 & 32.5 & 3.45 & 2.09 & 6.49 & -330.96 & -57.56 & -10.50 & -134. & 6995 & 0.22679 & -0.184015 \\
\hline A7 & DMSO & 0.60 & 19.60 & 46.68 & & 141.16 & -0.07770 & 0.14909 & -1.066 & -0.446 & 96.4460 & -0.33945 & -0.02858 & 32.50 & 3.45 & 2.09 & 6.49 & -330.96 & -55.93 & -9.97 & -134.71 & 0.035695 & 0.22679 & -0.184015 \\
\hline
\end{tabular}




\section{Single-parameter Correlations and Parameters Analysis}

Preliminary analysis of the dataset was done by single parameter correlation. This allows establishing which parameters provide a fair basis for further multidimensional analysis of the system under study. In Figure S2, $N$ was plotted against those parameters that provided the best trends or that are typically accounted for as descriptors of

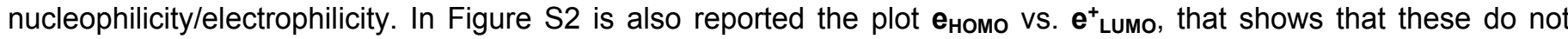
correlate among them (see the main text for a pertinent discussion).

Figure S2. Single parameter correlation between $N$ and selected parameters, and the plot $\mathbf{e}_{\text {номо Vs. }} \mathbf{e}^{+}$Lumo.

\begin{tabular}{|c|c|c|}
\hline - N-anion (27) & Olefins (42) & - $N\left(\mathrm{sp}^{3}\right.$ )-nucleophiles (35) \\
\hline - C-anion (31) & $\mathrm{N}\left(\mathrm{sp}^{2}\right)$-nucleophiles (32) & - (Hetero)aromatics (37) \\
\hline - O-anion (41) & - Enol ethers $(22)$ & - Ylides + NHC + diazocompounds (31) \\
\hline - S-anion (8) & - Enamines (35) & \\
\hline
\end{tabular}
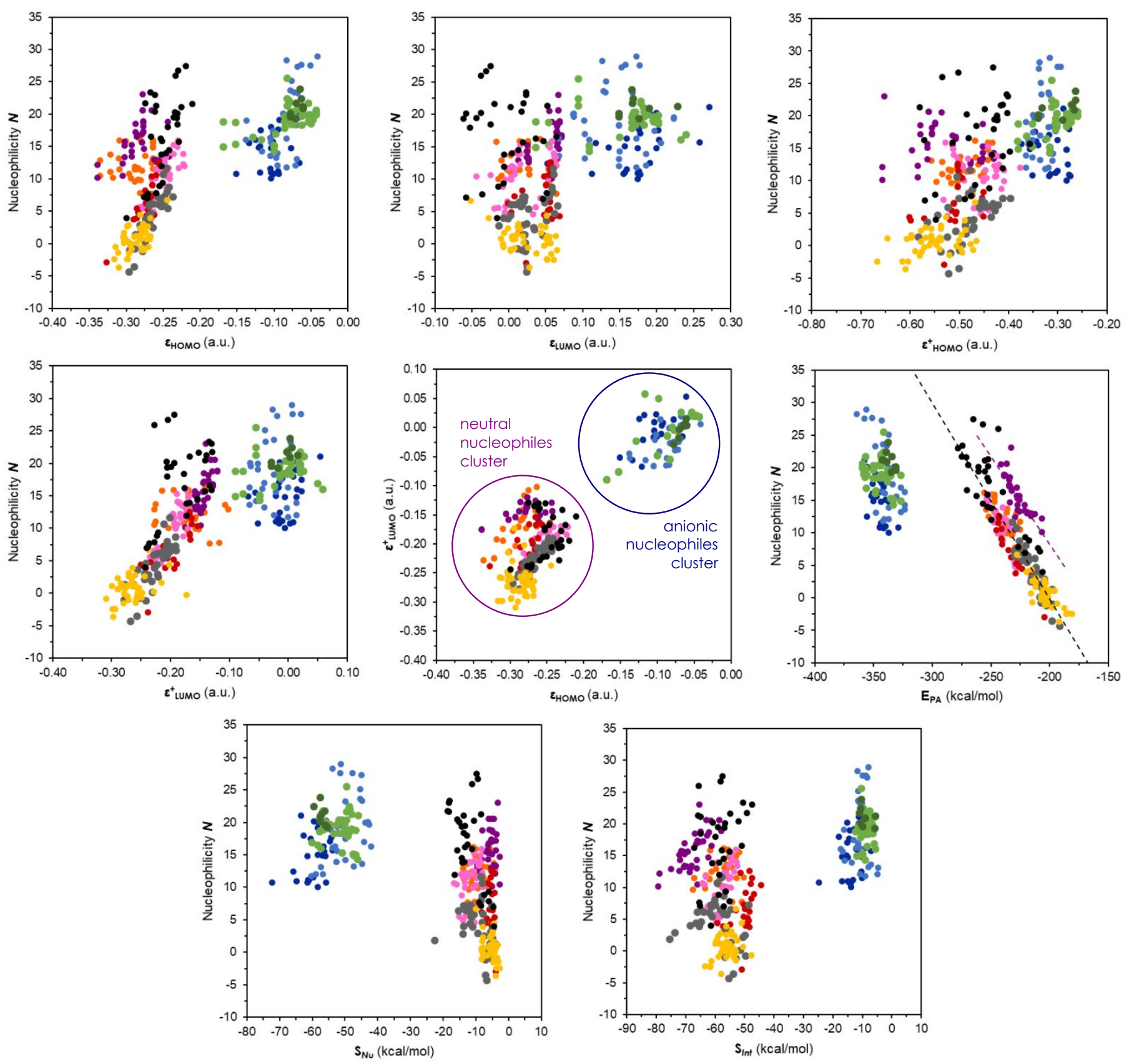

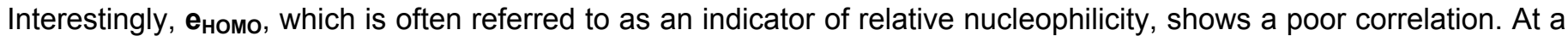
first glance, a slightly better trend was found between $N$ and $\mathbf{e}^{+}$Lumo. We found that these latest parameters do not 
correlate among them even though a trend could have been expected. In fact, the LUMO orbital of Int is obtained from the HOMO of $\mathbf{N u}$ after mixing with a reference electrophile's orbital which is the same for all of the nucleophiles $\left(\mathrm{H}^{+} \mathrm{s}\right.$ $A O)$. The best trend among the parameters computed is given by $E_{P A}$, which is supposed to include contributions from both $\mathbf{e}_{\text {номо }}$ and $\mathbf{e}^{+}$Lumo since these two orbitals are contributors to the wave functions of $\mathbf{N u}$ and Int respectively.

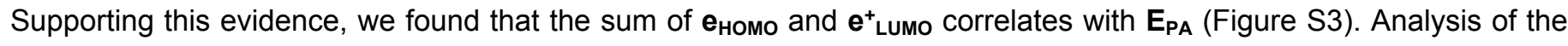
outliers (crosses in the plots) showed that for these nucleophiles (N1-N3, N22-N25, NA8, NA10, NA11, CA22-CA25, OA15-OA17, NHC1-NHC5) either the HOMO of $\mathbf{N u}$ or the LUMO of Int did not correspond to the orbital involved in the protonation reaction. For example, in Figure S3 are reported the LUMO and LUMO+1 of maleimide (the protonated nucleophile NA8). It can be seen that while the LUMO is located on the conjugated $\pi$-system, the LUMO+1 resembles the $\mathrm{N}-\mathrm{H} \sigma^{*}$ orbital from the Valence Bond theory. When for the mentioned outliers the energy of the LUMO+1 was used instead of $\mathbf{e}^{+}$Lumo (or the HOMO-2 instead of the HOMO, for anilines), the correlation in Figure S3 improved giving $R^{2}=0.98$. This suggests that most of the protonation energy (and therefore of the reaction $\Delta \mathrm{G}^{\ddagger}$ contributing to $N$ ) is due to both the energy of the nucleophile HOMO and of the intermediate LUMO (or other MO involved in the reaction).

Figure S3. Graphical depiction of the relevance of $\sigma^{*}$ orbitals for the description of $\mathbf{E}_{\mathrm{PA}}$.
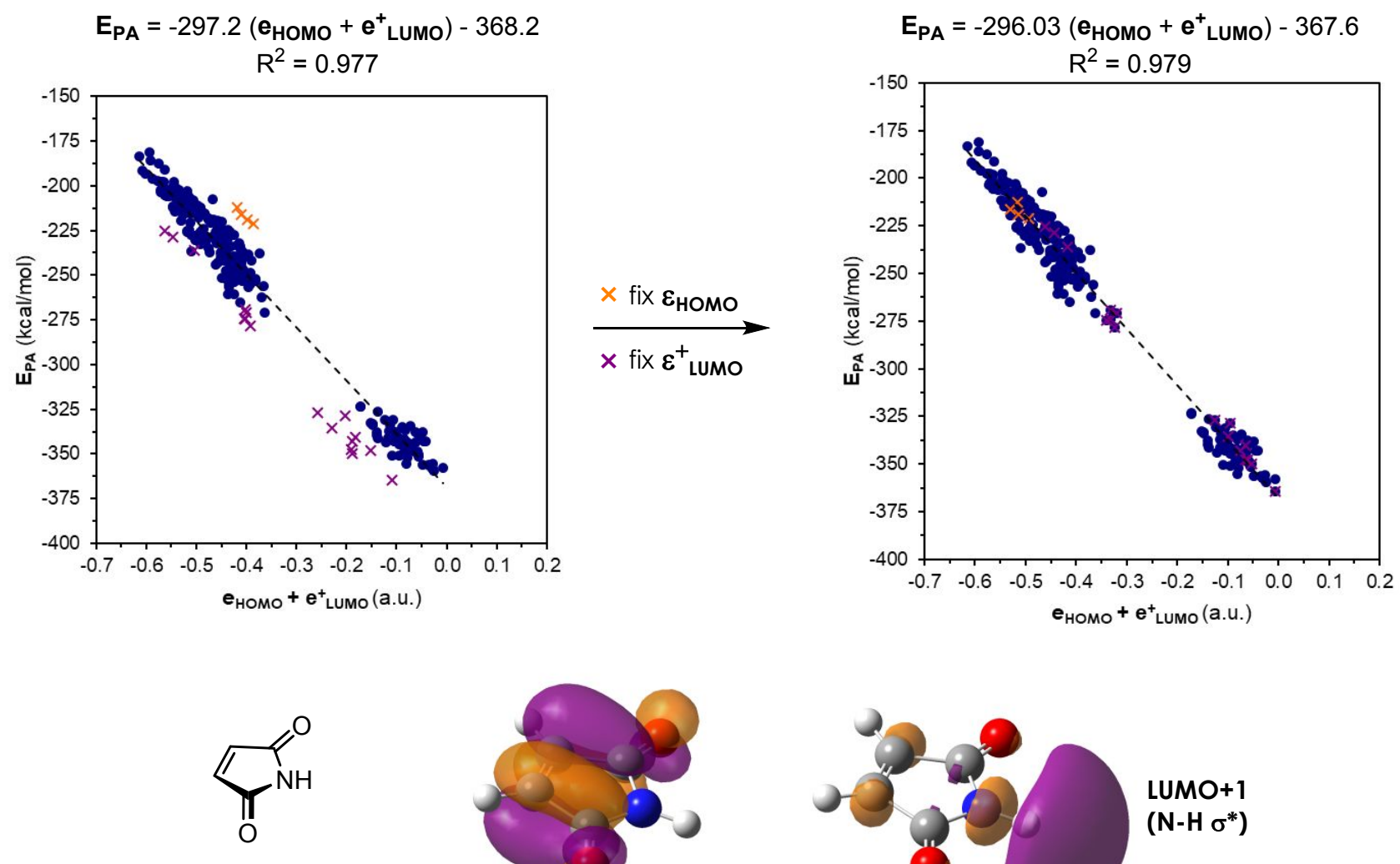

NA8, maleimide
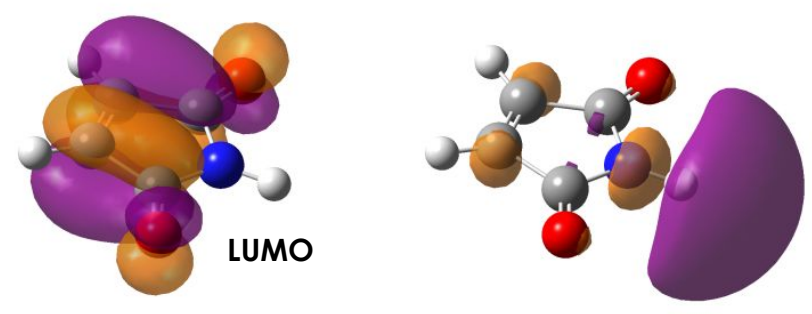

LUMO+1

$\left(\mathrm{N}-\mathrm{H} \sigma^{*}\right)$

In the correlations of figure S2, anions and neutral nucleophiles always result to be divided into two clusters, also in the

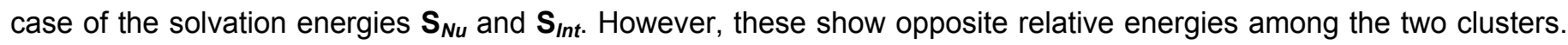
This suggests that these two parameters could be important in providing a balanced compensation for the different behavior of the two clusters. Therefore, $\mathbf{S}_{N u}$ and $\mathbf{S}_{\mathbf{I n t}}$ were added to $\mathbf{E}_{\mathrm{PA}}$ resulting in the correlation of Figure $\mathbf{S} 4 \mathrm{a}$. This plot shows that major differences between anionic and neutral nucleophiles can be accounted for by solvation effects. Moreover, this provides excellent basis for further multidimensional correlation analysis. 
Finally, a correlation matrix showing the $\mathrm{R}^{2}$ values for the correlation among the parameters and with $N$ is reported in Figure S4b. Misleading $\mathrm{R}^{2}$ values resulting from two-point-correlations due to clustering of anions and neutral nucleophiles are highlighted in red.

Figure S4a. Correlation between $N$ and $\mathbf{E}_{\mathrm{PA}}$ with the inclusion of solvation energies.

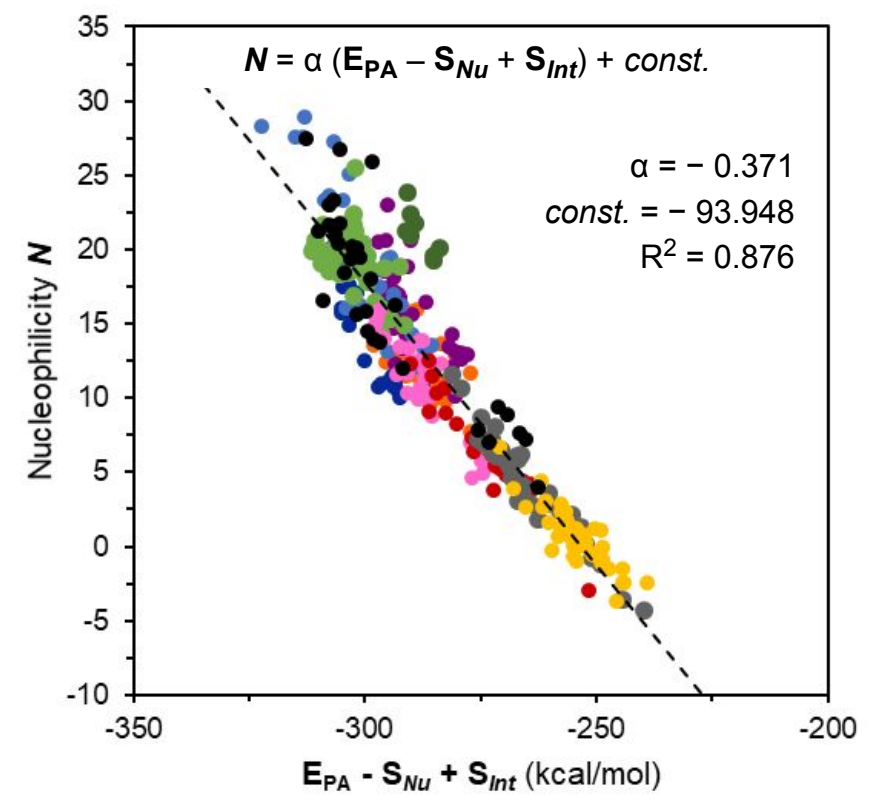

Figure S4b. Correlation matrix.

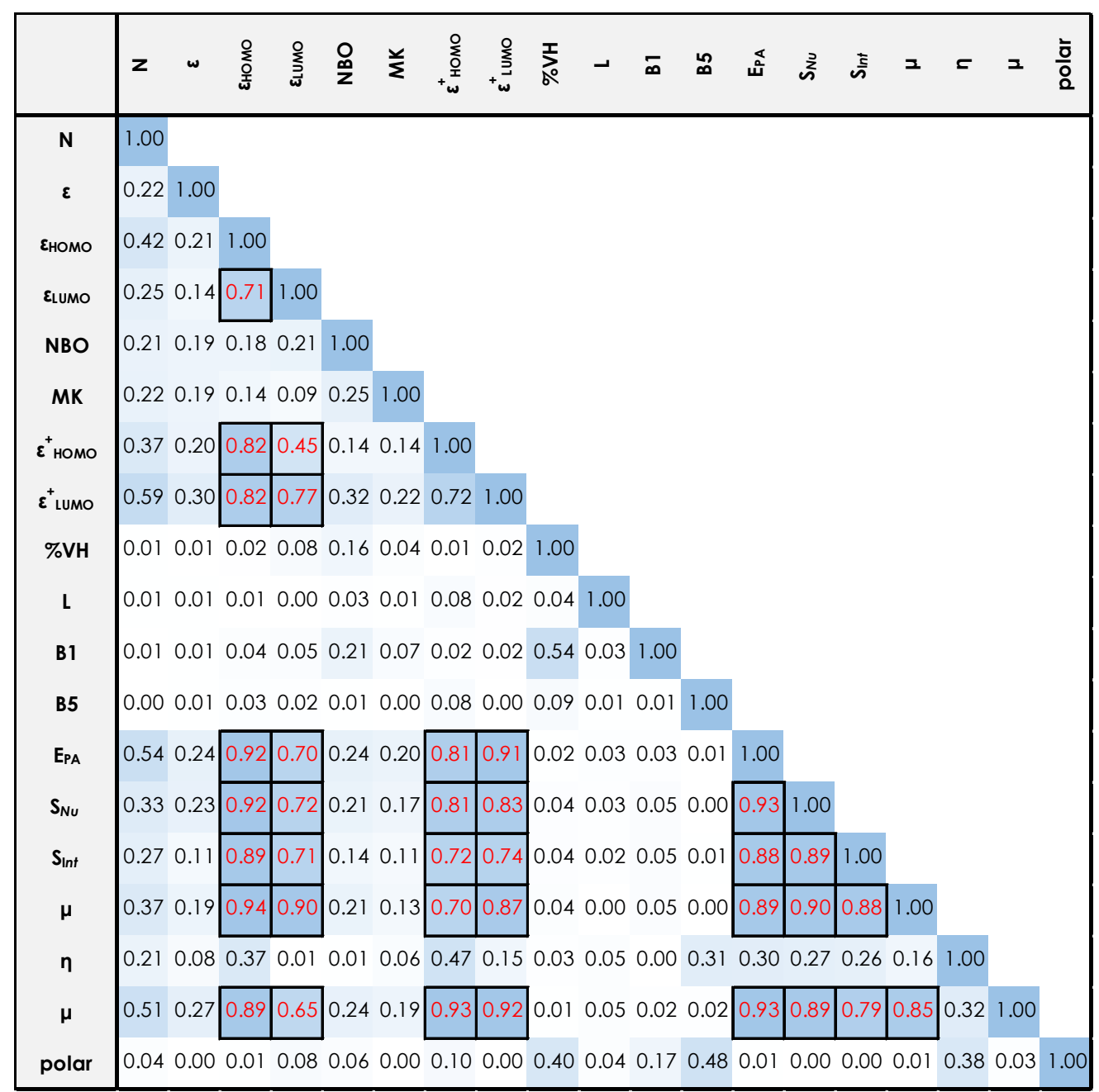




\section{Methyl Cation Affinities (MCA)}

The graph in Figure S4 shows that despite most datapoints correlate with $\mathrm{E}_{\mathrm{PA}}+\mathbf{S}_{\mathrm{Nu}}+\mathbf{S}_{I n t}$, some nucleophiles (especially $\mathrm{S}$-nucleophiles) are outliers. However, S-nucleophiles are known to have reversed $\mathrm{H}$-basicity versus $\mathrm{C}$-basicity with respect to the corresponding O-nucleophiles. That is, they are weaker Brønsted bases but stronger Lewis bases towards C-centered electrophiles than the corresponding O-nucleophiles. Thus we sought to evaluate whether the behavior of S-nucleophiles in Figure S4 could be due to a poor choice of the reference electrophile $\left(\mathrm{H}^{+}\right)$. To this end, we computed the methyl cation affinities (MCA) of 96 nucleophiles from the dataset and the corresponding solvation energies. The results are depicted in Figure S5. The graph on the left shows the correlation between $\mathrm{E}_{\mathrm{PA}}$ and methylation energies $\mathbf{E}_{\text {MCA }}$ computed at the M06-2X/def2TZVP level. The high correlation between the two affinities suggests that the difference between S-nucleophiles (orange crosses) and other classes of nucleophiles is not due to thermodynamic factors. Similarly, the plot on the right shows the correlation between $\mathbf{E}_{\mathrm{PA}}+\mathbf{S}_{N u}+\mathbf{S}_{\text {Int }}$ and $\mathbf{E}_{M C A}+\mathbf{S}_{N u}+\mathbf{S}_{\text {IntMC }}\left(\mathbf{S}_{\text {IntMC }}\right.$ being the solvation energy of the methylated nucleophile). Despite inclusion of solvation factors causes some little scattering of the datapoints, the correlation remains $\left(R_{2}=0.934\right)$, thus suggesting that MCAs are not complementary candidates of $\mathrm{E}_{\mathrm{PA}}$ for the description of S-nucleophiles activity at least from a computational perspective.

Figure S5. Correlation between $\mathrm{E}_{\mathrm{PA}}$ and $\mathrm{MCA}$ with and without inclusion of solvation energies.
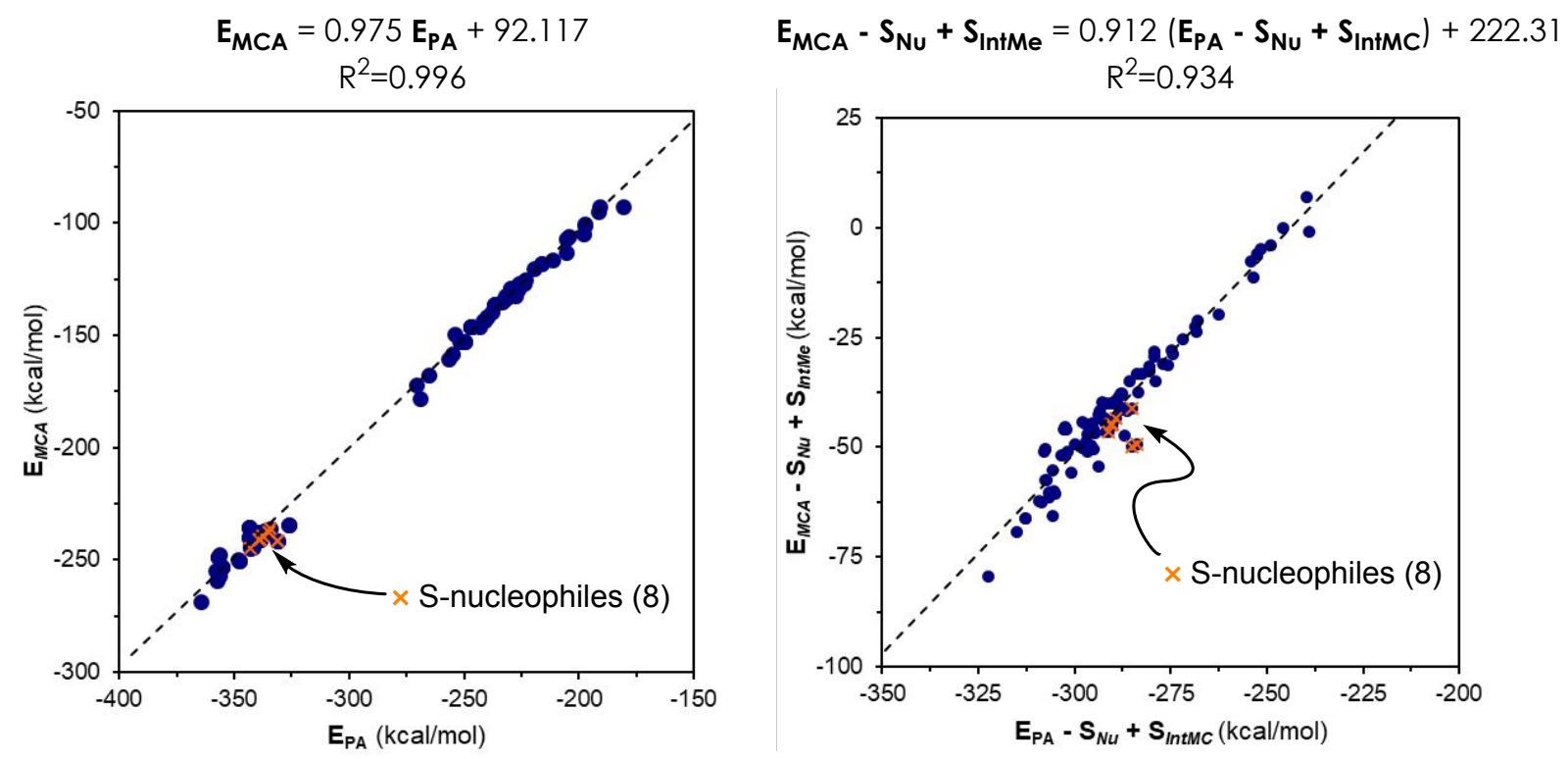

A list of the 96 nucleophiles evaluated and their MCAs are reported in Table S2.

Table S2. MCA, solvation energies of the methylated nucleophiles, and MCA with inclusion of solvation effects.

$\begin{array}{ccccc}\begin{array}{c}\text { Nucleophile } \\ \text { Ar01 }\end{array} & \text { solvent } & E_{M C A} & \boldsymbol{S}_{\text {IntMC }} & E_{M C A}-S_{N u}+S_{\text {IntMC }} \\ \text { Ar06 } & \text { DCM } & -92.71 & -53.61 & 7.07 \\ \text { Ar17 } & \text { AN } & -126.62 & -59.79 & -28.65 \\ \text { Ar20 } & \text { DCM } & -125.18 & -54.84 & -22.66 \\ \text { Ar22 } & \text { AN } & -116.13 & -62.42 & -23.82 \\ \text { Ar25 } & \text { DCM } & -132.22 & -57.54 & -34.98 \\ \text { Ar32 } & \text { DCM } & -104.91 & -57.28 & -11.34 \\ \text { CA04 } & \text { DMSO } & -244.26 & -11.19 & -3.95 \\ \text { CA04 } & \text { H2O } & -244.26 & -8.39 & -55.82 \\ \text { CA05 } & \text { DMSO } & -237.00 & -12.35 & -45.75 \\ \text { CA05 } & \text { H2O } & -237.00 & -9.30 & -50.03\end{array}$




\begin{tabular}{|c|c|c|c|c|}
\hline Nucleophile & solvent & $\mathbf{E}_{M C A}$ & $S_{\text {IntMC }}$ & $E_{M C A}-S_{N u}+S_{I n t M}$ \\
\hline CA06 & DMSO & -259.34 & -8.05 & -69.24 \\
\hline CA10 & DMSO & -250.13 & -9.13 & -62.66 \\
\hline CA11 & DMSO & -243.86 & -7.96 & -60.22 \\
\hline CA16 & DMSO & -256.89 & -7.39 & -66.26 \\
\hline CA17 & DMSO & -250.52 & -8.03 & -60.57 \\
\hline CA17 & $\mathrm{H} 2 \mathrm{O}$ & -250.52 & -4.02 & -50.49 \\
\hline CA19 & DMSO & -234.37 & -10.93 & -54.42 \\
\hline CA19 & $\mathrm{H} 2 \mathrm{O}$ & -234.37 & -6.93 & -47.28 \\
\hline CA22 & DMSO & -268.74 & -11.27 & -79.49 \\
\hline $\mathrm{CCO} 1$ & DCM & -94.78 & -55.87 & 0.00 \\
\hline $\mathrm{CCO} 2$ & DCM & -92.59 & -58.47 & -0.80 \\
\hline $\mathrm{CCO7}$ & DCM & -106.85 & -53.37 & -5.95 \\
\hline CC08 & DCM & -100.15 & -57.55 & -7.75 \\
\hline CC40 & AN & -120.48 & -55.21 & -21.17 \\
\hline CX02 & DCM & -152.58 & -57.31 & -46.60 \\
\hline CX02 & DMSO & -152.58 & -59.20 & -50.95 \\
\hline CX07 & DMSO & -158.50 & -63.19 & -61.32 \\
\hline CX13 & $\mathrm{DCM}$ & -112.90 & -58.32 & -19.92 \\
\hline$C \times 15$ & DMSO & -167.59 & -55.12 & -66.17 \\
\hline $\mathrm{CX} 16$ & DMSO & -160.34 & -55.72 & -59.94 \\
\hline CX24 & $D C M$ & -129.90 & -54.11 & -31.18 \\
\hline EE03 & DCM & -126.92 & -49.54 & -25.45 \\
\hline EE06 & DCM & -146.32 & -47.37 & -41.62 \\
\hline EE10 & DCM & -105.77 & -49.62 & -4.85 \\
\hline En02 & DCM & -143.21 & -54.28 & -41.23 \\
\hline En05 & DCM & -153.00 & -51.94 & -49.52 \\
\hline En12 & DCM & -142.00 & -55.09 & -37.46 \\
\hline En12 & $\mathrm{AN}$ & -142.00 & -58.02 & -41.41 \\
\hline En25 & AN & -126.50 & -61.32 & -30.99 \\
\hline En30 & AN & -128.04 & -58.97 & -27.85 \\
\hline N01 & DCM & -129.84 & -55.44 & -31.64 \\
\hline N01 & $\mathrm{H} 2 \mathrm{O}$ & -129.84 & -54.69 & -33.26 \\
\hline N04 & DCM & -145.94 & -50.53 & -39.65 \\
\hline N04 & $\mathrm{DMF}$ & -145.94 & -52.54 & -42.80 \\
\hline N04 & DMSO & -145.94 & -51.49 & -42.85 \\
\hline N04 & $\mathrm{H} 2 \mathrm{O}$ & -145.94 & -47.86 & -40.10 \\
\hline N04 & AN & -145.94 & -53.41 & -42.74 \\
\hline N04 & THF & -145.94 & -48.03 & -38.73 \\
\hline N09 & DMSO & -133.28 & -62.54 & -37.88 \\
\hline N09 & AN & -133.28 & -63.32 & -37.74 \\
\hline N09 & $\mathrm{H} 2 \mathrm{O}$ & -133.28 & -57.87 & -34.97 \\
\hline N16 & DCM & -132.19 & -54.35 & -33.37 \\
\hline N18 & $D C M$ & -132.81 & -53.64 & -32.66 \\
\hline N21 & DCM & -149.35 & -52.03 & -44.38 \\
\hline N22 & AN & -118.00 & -67.01 & -29.37 \\
\hline N22 & $\mathrm{H} 2 \mathrm{O}$ & -118.00 & -62.27 & -28.19 \\
\hline N28 & $A N$ & -129.08 & -64.19 & -41.59 \\
\hline N28 & $\mathrm{H} 2 \mathrm{O}$ & -129.08 & -60.33 & -39.88 \\
\hline N31 & AN & -134.96 & -63.00 & -46.30 \\
\hline N32 & DCM & -136.50 & -55.59 & -40.10 \\
\hline N32 & AN & -136.50 & -59.44 & -44.68 \\
\hline
\end{tabular}




\begin{tabular}{|c|c|c|c|c|}
\hline Nucleophile & solvent & $\mathbf{E}_{M C A}$ & $S_{\text {IntMC }}$ & $E_{M C A}-S_{N u}+S_{I n t M C}$ \\
\hline N35 & DMSO & -129.86 & -65.91 & -43.76 \\
\hline N35 & AN & -129.86 & -66.53 & -43.35 \\
\hline N35 & $\mathrm{H} 2 \mathrm{O}$ & -129.86 & -63.67 & -39.79 \\
\hline N37 & AN & -134.45 & -61.65 & -46.07 \\
\hline N38 & AN & -143.21 & -58.20 & -48.65 \\
\hline N39 & AN & -139.70 & -61.66 & -46.67 \\
\hline NA01 & $\mathrm{H} 2 \mathrm{O}$ & -253.27 & -8.45 & -49.31 \\
\hline NA01 & AN & -253.27 & -10.31 & -57.41 \\
\hline NA01 & DMSO & -253.27 & -9.01 & -57.54 \\
\hline NA02 & $\mathrm{H} 2 \mathrm{O}$ & -239.78 & -13.34 & -44.96 \\
\hline NA02 & AN & -239.78 & -14.59 & -51.85 \\
\hline NA02 & DMSO & -239.78 & -13.36 & -51.91 \\
\hline NA12 & DMSO & -254.97 & -10.36 & -55.16 \\
\hline NA13 & $\mathrm{H} 2 \mathrm{O}$ & -238.00 & -12.81 & -43.09 \\
\hline NA13 & DMSO & -238.00 & -14.22 & -47.17 \\
\hline NA15 & $\mathrm{H} 2 \mathrm{O}$ & -238.48 & -12.30 & -42.52 \\
\hline NA15 & DMSO & -238.48 & -13.84 & -50.99 \\
\hline NHC3 & THF & -172.08 & -49.29 & -62.20 \\
\hline NHC4 & THF & -178.33 & -49.61 & -65.52 \\
\hline OA01 & DMF & -248.57 & -5.83 & -51.05 \\
\hline OA01 & DMSO & -248.57 & -4.84 & -50.52 \\
\hline OA01 & AN & -248.57 & -6.47 & -50.57 \\
\hline OA07 & DMF & -235.39 & -5.75 & -45.91 \\
\hline OA07 & DMSO & -235.39 & -4.63 & -45.45 \\
\hline OA07 & AN & -235.39 & -6.48 & -45.65 \\
\hline OA14 & AN & -247.91 & -4.73 & -45.92 \\
\hline SA1 & $A C N$ & -241.17 & -9.81 & -44.82 \\
\hline SA2 & $\mathrm{ACN}$ & -239.52 & -9.59 & -44.90 \\
\hline SA3 & $A C N$ & -236.27 & -10.20 & -43.54 \\
\hline SA4 & $A C N$ & -240.96 & -8.20 & -44.85 \\
\hline SA5 & $\mathrm{ACN}$ & -237.49 & -5.88 & -41.19 \\
\hline SA6 & $A C N$ & -245.07 & -5.38 & -46.58 \\
\hline SA7 & $\mathrm{ACN}$ & -241.60 & -11.95 & -49.29 \\
\hline SA7 & DMSO & -241.60 & -10.98 & -49.96 \\
\hline
\end{tabular}




\section{Multivariate Linear Regression Analysis}

Multidimensional regression analyses were performed using MATLAB R2020 ${ }^{6}$ according with previous procedures developed in the Sigman group. ${ }^{7}$ The dataset was divided into two sets: a training set (222 points) and a validation set (119 points). The specific datapoints belonging to each set are reported in the model table (see below). The normalized descriptors $d_{i}^{*}$ were obtained from the parameters $d_{i}$ according with the formula:

$$
d_{i}^{*}=\frac{d_{i}-m_{i}}{s_{i}}
$$

Where $m_{i}$ is the mean of the parameter $d_{i}$ of the $i$-th nucleophile from the training set, and $s_{i}$ is the standard deviation of parameter $d_{i}$ calculated from the training set. The use of the normalized parameters $d_{i}^{*}$ allows for the parameters' coefficients in the model equation to reflect the relevance of each descriptor. The observable array (the experimental $N$ values in our case) is also normalized analogously. Upon definition of the normalized observable array as $Y$ and of the normalized parameters matrix as $\mathrm{X}$, the MATLAB function $N_{\text {pred }}=$ LinearModel.stepwise $\left(X, Y,{ }^{\prime}\right.$ mode $\left.l I^{\prime}\right)$ is used. In our case, model1 = y $\sim 1+\mathrm{x} 1+\mathrm{x} 2+\mathrm{x} 3$, where $\mathrm{x} 1, \mathrm{x} 2$, and $\mathrm{x} 3$ are $\mathbf{E}_{\mathbf{P A}}, \mathbf{S}_{\mathbf{N u}}$, and $\mathbf{S}_{\operatorname{lnt}}$ as these were found to provide an excellent base for building finer models. This procedure rendered a model $N_{\text {pred }}$ of the form:

$$
y \sim 1+x 2+x 3+x 12+x 13+x 16+x 1^{*} x 4+x 1^{*} x 15+x 14^{*} x 21+x 15^{*} x 21
$$

which contained a number of terms characterized by high p-values thus highlighting overfitting issues. In order to reduce the model to the minimum acceptable number of terms, the function

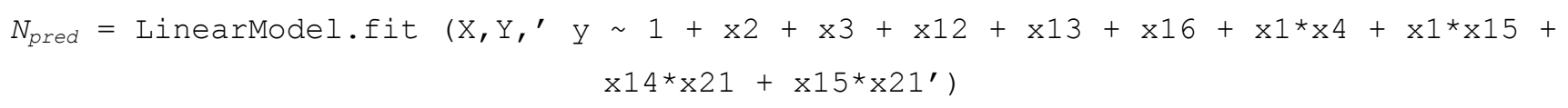

was used where the less relevant terms were deleted from the starting model systematically in an iterative process until all of the remaining terms gave $p$-value $<10^{-5}$. Other criteria used for the elimination of some of the terms is the meaning of the parameter according to chemical intuition. That is, in cases where two parameters have similar p-values, if one of them is a cross-term with no or little physical meaning, this would be deleted first.

After the model is obtained, this is used for predicting the nucleophilicity values of the validation set using excel. In order to do so, the parameters of the training set are normalized using the $m_{i}$ and $s_{i}$ values from the training set so that normalization for the two sets and their reference scales would coincide.

The model obtained for the training set is reported in Figure S6 together with external predictions and the residuals:

$\begin{array}{cccccc} & & \text { Estimate } & \mathrm{SE} & \text { t-stat } & \text { p-value } \\ & \text { (Intercept) } & 0.00 & 0.01741 & 1.11 \mathrm{E}-13 & 1 \\ \boldsymbol{\varepsilon} & \mathrm{x1} & -0.07 & 0.02367 & -3.0127 & 1.00 \mathrm{E}-06 \\ \mathbf{q} & \mathrm{x} 2 & 0.98 & 0.12945 & 7.5783 & 1.07 \mathrm{E}-12 \\ \mathbf{e}_{\text {HOMO }} & \mathrm{x} 4 & 0.38 & 0.07865 & 4.8502 & 2.39 \mathrm{E}-06 \\ \mathbf{\% V} & \mathrm{x} 10 & -0.17 & 0.02958 & -5.5801 & 7.29 \mathrm{E}-08 \\ \mathbf{B 1} & \mathrm{x} 12 & 0.18 & 0.02768 & 6.6096 & 3.07 \mathrm{E}-10 \\ \mathbf{E}_{\mathbf{P A}} & \mathrm{x} 14 & -2.84 & 0.08383 & -33.902 & 1.47 \mathrm{E}-87 \\ \mathbf{S}_{\boldsymbol{N u}} & \mathrm{x} 15 & 1.93 & 0.10032 & 19.271 & 1.72 \mathrm{E}-48 \\ \mathbf{S}_{\boldsymbol{I n t}} & \mathrm{x} 16 & -1.63 & 0.09117 & -17.864 & 3.69 \mathrm{E}-44 \\ \mathbf{S}_{\boldsymbol{H}} & \mathrm{x} 21 & 0.20 & 0.01941 & 10.457 & 6.61 \mathrm{E}-21\end{array}$

The model was validated using three different procedures:

i) external validation by prediction of datapoints outside the training set as explained before; 
ii) $\quad$ ii) K-fold cross-validation (random-2-foldCV), where the training dataset was split randomly in $\mathrm{k}$ subsets (in our case $\mathrm{k}=2$ ). One of the $\mathrm{k}$-subset is used as training set to obtain the model coefficient for that k-subset, and then the other k-subset is used to validate the model as an external prediction. The $\mathrm{R}^{2}$ obtained by the prediction k-subset is defined as $\mathrm{Q}^{2}$. This process was repeated 20 times to get an average $\mathrm{Q}^{2}$ over 20 different random splitting of the traning dataset. The average $\mathrm{Q}^{2}$ is reported in the graph as random-2foldCV, which in our case is 0.922 . Since the training set was composed of about $2 / 3$ of the whole dataset (222 over 341 datapoints), and since in this 2-foldCV the training dataset is split in half, ca. 1/3 of the whole dataset (111 datapoints) is sufficient to obtain excellent predictions of nucleophilicity (average $Q^{2}=0.922$ ) using our model equation.

iii) Leave-One-Out cross validation ( $\mathrm{LOO}$ ) is a $\mathrm{K}$-fold cross validation where $\mathrm{K}$ equals the total number of datapoints (222 in our case). Therefore, a datapoint is left out of the set and the remaining K-1 datapoints are used to predict the excluded point according with the model equation. The process is repeated for all of the datapoints. The collection of the predicted datapoints is plotted against the measured observable to give a $\mathrm{Q}^{2}$ that in our case was 0.927 .

Figure S6. Multidimensional model obtained by the multivariate regression procedure described.
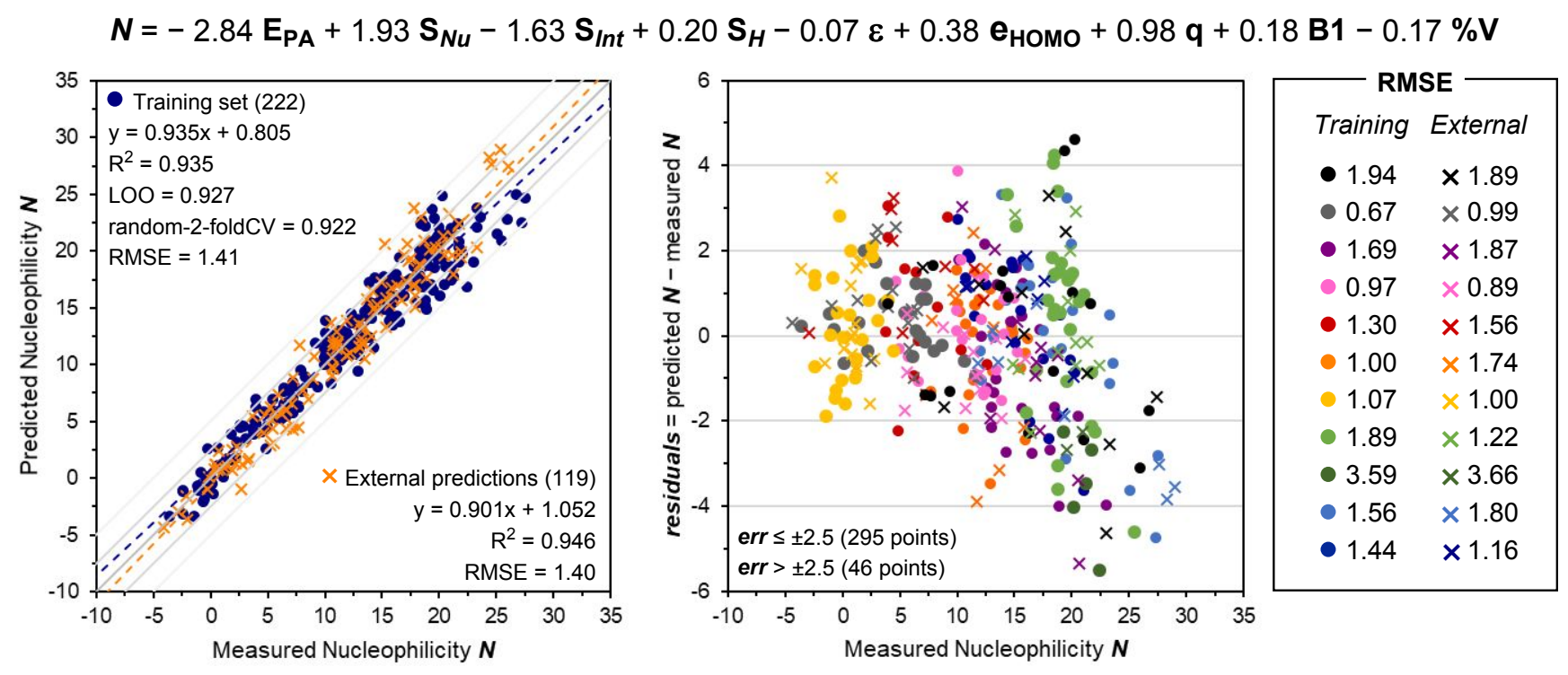

Table S3. Datapoints for the model reported in Figure S6.

$\begin{array}{cccccc} & \text { Nucleophile } & \text { solvent } & \text { N(measured) } & \text { N(predicted) } & \text { rediduals } \\ \text { training } & \text { Ar02 } & \text { DCM } & -3.57 & -3.34 & 0.23 \\ \text { training } & \text { Ar03 } & \text { DCM } & -1.18 & -0.65 & 0.53 \\ \text { training } & \text { Ar04 } & \text { DCM } & 0.13 & -0.52 & -0.65 \\ \text { training } & \text { Ar06 } & \text { AN } & 6.91 & 7.76 & 0.85 \\ \text { training } & \text { Ar08 } & \text { AN } & 7.26 & 8.14 & 0.88 \\ \text { training } & \text { Ar10 } & \text { AN } & 6.08 & 5.59 & -0.49 \\ \text { training } & \text { Ar11 } & \text { AN } & 2.83 & 4.56 & 1.73 \\ \text { training } & \text { Ar13 } & \text { AN } & 7.22 & 7.07 & -0.15 \\ \text { training } & \text { Ar14 } & \text { AN } & 6.44 & 6.57 & 0.13 \\ \text { training } & \text { Ar19 } & \text { AN } & 5.41 & 5.94 & 0.53 \\ \text { training } & \text { Ar21 } & \text { AN } & 8.01 & 7.65 & -0.36 \\ \text { training } & \text { Ar22 } & \text { AN } & 10.67 & 10.08 & -0.59 \\ \text { training } & \text { Ar24 } & \text { AN } & 8.69 & 8.46 & -0.23 \\ \text { training } & \text { Ar26 } & \text { DCM } & 2.16 & 1.80 & -0.36 \\ \text { training } & \text { Ar27 } & \text { DCM } & 3.61 & 4.37 & 0.76 \\ \text { training } & \text { Ar29 } & \text { AN } & 6.38 & 7.62 & 1.24 \\ \text { training } & \text { Ar30 } & \text { AN } & 1.84 & 3.83 & 1.99 \\ \text { training } & \text { Ar31 } & \text { DCM } & 7.22 & 8.41 & 1.19 \\ & & & \text { S23 } & & \end{array}$




\begin{tabular}{|c|c|c|c|c|c|}
\hline training & Ar33 & DCM & -0.80 & -0.65 & 0.16 \\
\hline training & Ar34 & DCM & 1.35 & 1.66 & 0.31 \\
\hline training & Ar36 & $\mathrm{AN}$ & 11.63 & 10.68 & -0.95 \\
\hline training & Ar37 & DCM & 6.66 & 6.67 & 0.01 \\
\hline training & Ar38 & $\mathrm{AN}$ & 3.87 & 5.10 & 1.23 \\
\hline training & CA01 & DMSO & 15.59 & 16.71 & 1.12 \\
\hline training & CAO2 & DMSO & 14.24 & 15.08 & 0.84 \\
\hline training & CAO3 & DMSO & 17.46 & 17.57 & 0.11 \\
\hline training & CA04 & DMSO & 16.27 & 17.94 & 1.67 \\
\hline training & CA05 & DMSO & 13.91 & 17.22 & 3.31 \\
\hline training & CA05 & $\mathrm{H} 2 \mathrm{O}$ & 12.06 & 11.03 & -1.03 \\
\hline training & CA06 & DMSO & 27.54 & 24.72 & -2.82 \\
\hline training & CA08 & DMSO & 23.64 & 23.01 & -0.63 \\
\hline training & CA09 & DMSO & 20.00 & 22.16 & 2.16 \\
\hline training & CA10 & DMSO & 23.27 & 22.15 & -1.12 \\
\hline training & CA11 & DMSO & 27.28 & 22.55 & -4.73 \\
\hline training & CA12 & DMSO & 25.11 & 21.48 & -3.63 \\
\hline training & CA13 & DMSO & 23.35 & 23.84 & 0.49 \\
\hline training & CA14 & DMSO & 20.10 & 20.71 & 0.61 \\
\hline training & CA15 & DMSO & 19.61 & 22.86 & 3.25 \\
\hline training & CA17 & DMSO & 18.31 & 19.68 & 1.37 \\
\hline training & CA18 & DMSO & 18.29 & 17.88 & -0.41 \\
\hline training & CA18 & $\mathrm{H} 2 \mathrm{O}$ & 12.05 & 11.70 & -0.35 \\
\hline training & CA19 & $\mathrm{H} 2 \mathrm{O}$ & 13.23 & 13.21 & -0.02 \\
\hline training & CA20 & DMSO & 16.29 & 17.47 & 1.18 \\
\hline training & CA21 & $\mathrm{H} 2 \mathrm{O}$ & 19.50 & 16.60 & -2.90 \\
\hline training & CA23 & DMSO & 16.06 & 17.79 & 1.73 \\
\hline training & CA24 & DMSO & 19.03 & 18.72 & -0.31 \\
\hline training & $\mathrm{CCO} 2$ & $\mathrm{DCM}$ & -2.45 & -3.16 & -0.71 \\
\hline training & $\mathrm{CCO} 3$ & DCM & -2.44 & -1.04 & 1.40 \\
\hline training & CCO4 & DCM & -1.46 & -3.34 & -1.88 \\
\hline training & CCO5 & DCM & -0.49 & 0.06 & 0.55 \\
\hline training & $\mathrm{CCO} 6$ & DCM & -1.07 & -1.06 & 0.01 \\
\hline training & CCO9 & DCM & 0.25 & -1.34 & -1.59 \\
\hline training & CC10 & DCM & -0.57 & -1.86 & -1.29 \\
\hline training & CC11 & DCM & 4.41 & 4.07 & -0.34 \\
\hline training & $\mathrm{CC} 12$ & DCM & 0.65 & 1.13 & 0.48 \\
\hline training & CC13 & DCM & 1.70 & 1.60 & -0.10 \\
\hline training & CC16 & DCM & 0.06 & 0.02 & -0.04 \\
\hline training & $\mathrm{CC} 17$ & DCM & 2.60 & 4.46 & 1.86 \\
\hline training & CC18 & DCM & -2.41 & -1.20 & 1.21 \\
\hline training & CC22 & DCM & -0.65 & -2.12 & -1.47 \\
\hline training & CC27 & DCM & 1.18 & 0.64 & -0.54 \\
\hline training & CC29 & DCM & -0.87 & 0.50 & 1.37 \\
\hline training & CC32 & DCM & 3.09 & 3.45 & 0.36 \\
\hline training & CC33 & DCM & 0.66 & 2.67 & 2.01 \\
\hline training & CC34 & DCM & 2.60 & 4.70 & 2.10 \\
\hline training & CC36 & DCM & 1.10 & 0.12 & -0.98 \\
\hline training & CC37 & DCM & 2.30 & 3.14 & 0.84 \\
\hline training & CC39 & DCM & -0.25 & 2.58 & 2.83 \\
\hline training & CC40 & $\mathrm{AN}$ & 3.93 & 4.76 & 0.83 \\
\hline training & CC41 & DCM & -0.04 & -1.08 & -1.04 \\
\hline training & CX01 & DMSO & 13.95 & 15.46 & 1.51 \\
\hline training & CX02 & DMSO & 13.78 & 14.96 & 1.18 \\
\hline training & CX04 & DMSO & 14.48 & 15.40 & 0.92 \\
\hline training & CX05 & DMSO & 16.23 & 13.94 & -2.29 \\
\hline training & CX07 & DMSO & 21.07 & 18.63 & -2.44 \\
\hline training & CX08 & DMSO & 18.42 & 17.60 & -0.82 \\
\hline training & CX09 & DCM & 9.35 & 8.04 & -1.31 \\
\hline training & CX11 & DCM & 7.66 & 6.24 & -1.42 \\
\hline training & CX12 & DCM & 7.17 & 5.80 & -1.37 \\
\hline training & $C \times 13$ & $\mathrm{DCM}$ & 3.96 & 4.72 & 0.76 \\
\hline training & CX14 & DMSO & 25.94 & 22.83 & -3.11 \\
\hline training & CX16 & DMSO & 26.71 & 24.96 & -1.75 \\
\hline training & $C \times 17$ & DMSO & 20.24 & 24.87 & 4.63 \\
\hline
\end{tabular}




\begin{tabular}{|c|c|c|c|c|c|}
\hline training & CX20 & DMSO & 21.61 & 22.36 & 0.75 \\
\hline training & CX21 & DMSO & 20.08 & 21.09 & 1.01 \\
\hline training & CX22 & DMSO & 19.38 & 23.73 & 4.35 \\
\hline training & CX24 & $\mathrm{DCM}$ & 7.84 & 9.48 & 1.64 \\
\hline training & EEO & DCM & 10.61 & 10.05 & -0.56 \\
\hline training & EEO2 & $\mathrm{AN}$ & 6.43 & 7.93 & 1.50 \\
\hline training & EEO2 & DCM & 6.57 & 6.46 & -0.11 \\
\hline training & EE03 & DCM & 5.41 & 6.99 & 1.58 \\
\hline training & EE04 & DCM & 10.38 & 10.05 & -0.33 \\
\hline training & EE06 & DCM & 12.56 & 11.89 & -0.67 \\
\hline training & EE07 & DCM & 6.22 & 5.29 & -0.93 \\
\hline training & EE08 & DCM & 4.83 & 2.59 & -2.24 \\
\hline training & EE09 & DCM & 8.23 & 8.91 & 0.68 \\
\hline training & EE11 & DCM & 3.78 & 3.88 & 0.10 \\
\hline training & EE12 & $\mathrm{AN}$ & 9.11 & 11.89 & 2.78 \\
\hline training & EE16 & DCM & 3.92 & 6.98 & 3.06 \\
\hline training & EE17 & $\mathrm{DCM}$ & 3.94 & 6.25 & 2.31 \\
\hline training & En01 & DCM & 13.36 & 12.56 & -0.80 \\
\hline training & En03 & DCM & 14.91 & 15.81 & 0.90 \\
\hline training & En04 & DCM & 12.51 & 11.22 & -1.29 \\
\hline training & En05 & DCM & 14.02 & 14.89 & 0.87 \\
\hline training & En09 & DCM & 12.06 & 12.44 & 0.38 \\
\hline training & En12 & DCM & 12.26 & 10.87 & -1.39 \\
\hline training & En12 & $\mathrm{AN}$ & 13.87 & 12.36 & -1.51 \\
\hline training & En14 & AN & 9.94 & 10.07 & 0.13 \\
\hline training & En 15 & AN & 11.66 & 10.73 & -0.93 \\
\hline training & En18 & AN & 10.42 & 10.33 & -0.09 \\
\hline training & En20 & $\mathrm{AN}$ & 10.30 & 12.08 & 1.78 \\
\hline training & En20 & DCM & 9.96 & 10.57 & 0.61 \\
\hline training & En21 & $\mathrm{AN}$ & 14.09 & 14.13 & 0.04 \\
\hline training & En22 & AN & 15.27 & 14.90 & -0.37 \\
\hline training & En23 & DCM & 10.04 & 13.92 & 3.88 \\
\hline training & En24 & DCM & 12.26 & 13.65 & 1.39 \\
\hline training & En25 & $\mathrm{AN}$ & 7.06 & 8.00 & 0.94 \\
\hline training & En27 & AN & 4.91 & 4.62 & -0.29 \\
\hline training & En28 & AN & 4.61 & 5.90 & 1.29 \\
\hline training & En30 & AN & 5.73 & 5.26 & -0.47 \\
\hline training & En32 & $A N$ & 6.57 & 5.51 & -1.06 \\
\hline training & N01 & DCM & 12.90 & 9.42 & -3.48 \\
\hline training & N01 & $\mathrm{H} 2 \mathrm{O}$ & 11.05 & 9.67 & -1.38 \\
\hline training & NO2 & $\mathrm{H} 2 \mathrm{O}$ & 10.50 & 8.33 & -2.17 \\
\hline training & N03 & $\mathrm{H} 2 \mathrm{O}$ & 11.44 & 10.41 & -1.03 \\
\hline training & N04 & DMF & 14.90 & 14.97 & 0.07 \\
\hline training & N04 & DMSO & 14.80 & 15.00 & 0.20 \\
\hline training & N04 & $\mathrm{AN}$ & 15.51 & 14.75 & -0.76 \\
\hline training & N04 & THF & 15.90 & 13.46 & -2.44 \\
\hline training & N05 & DCM & 15.90 & 15.49 & -0.41 \\
\hline training & N06 & $\mathrm{AN}$ & 7.69 & 6.39 & -1.30 \\
\hline training & N08 & AN & 10.73 & 11.00 & 0.27 \\
\hline training & N09 & DMSO & 11.58 & 12.31 & 0.73 \\
\hline training & N09 & $\mathrm{AN}$ & 11.47 & 12.19 & 0.72 \\
\hline training & N10 & $A N$ & 11.90 & 12.76 & 0.86 \\
\hline training & N10 & $\mathrm{H} 2 \mathrm{O}$ & 9.91 & 11.45 & 1.54 \\
\hline training & N11 & $\mathrm{AN}$ & 11.31 & 11.42 & 0.11 \\
\hline training & N13 & $\mathrm{AN}$ & 11.51 & 13.01 & 1.50 \\
\hline training & N14 & DCM & 10.03 & 10.77 & 0.74 \\
\hline training & N15 & DCM & 12.92 & 14.02 & 1.10 \\
\hline training & N17 & DCM & 13.12 & 12.30 & -0.82 \\
\hline training & N18 & DCM & 10.20 & 10.77 & 0.57 \\
\hline training & N2O & DCM & 16.15 & 16.08 & -0.07 \\
\hline training & N21 & DCM & 13.58 & 14.31 & 0.73 \\
\hline training & N22 & $\mathrm{AN}$ & 12.64 & 11.42 & -1.22 \\
\hline training & N22 & $\mathrm{H} 2 \mathrm{O}$ & 12.99 & 10.84 & -2.15 \\
\hline training & N24 & $\mathrm{AN}$ & 13.19 & 11.85 & -1.34 \\
\hline training & N24 & $\mathrm{H} 2 \mathrm{O}$ & 13.00 & 11.34 & -1.66 \\
\hline
\end{tabular}




\begin{tabular}{|c|c|c|c|c|c|}
\hline training & N25 & $\mathrm{AN}$ & 13.42 & 12.40 & -1.02 \\
\hline training & N25 & $\mathrm{H} 2 \mathrm{O}$ & 14.28 & 11.55 & -2.73 \\
\hline training & N26 & DMSO & 15.70 & 16.92 & 1.22 \\
\hline training & N26 & $\mathrm{AN}$ & 15.11 & 16.71 & 1.60 \\
\hline training & N27 & DMSO & 12.15 & 12.14 & -0.01 \\
\hline training & N27 & $\mathrm{AN}$ & 10.13 & 11.92 & 1.79 \\
\hline training & N28 & AN & 12.35 & 14.51 & 2.16 \\
\hline training & N29 & AN & 14.29 & 15.31 & 1.02 \\
\hline training & N29 & $\mathrm{H} 2 \mathrm{O}$ & 13.44 & 14.64 & 1.20 \\
\hline training & N30 & $\mathrm{H} 2 \mathrm{O}$ & 14.68 & 15.01 & 0.33 \\
\hline training & N31 & $A N$ & 18.52 & 16.86 & -1.66 \\
\hline training & N33 & DMSO & 17.19 & 17.33 & 0.14 \\
\hline training & N33 & $\mathrm{H} 2 \mathrm{O}$ & 18.13 & 15.45 & -2.68 \\
\hline training & N34 & DCM & 18.90 & 14.91 & -3.99 \\
\hline training & N34 & $\mathrm{AN}$ & 18.72 & 16.84 & -1.88 \\
\hline training & N35 & AN & 15.65 & 16.12 & 0.47 \\
\hline training & N35 & $\mathrm{H} 2 \mathrm{O}$ & 15.62 & 13.91 & -1.71 \\
\hline training & N36 & DCM & 16.50 & 13.75 & -2.75 \\
\hline training & N37 & $\mathrm{AN}$ & 23.05 & 19.06 & -3.99 \\
\hline training & N38 & AN & 20.54 & 18.65 & -1.89 \\
\hline training & NA01 & $\mathrm{H} 2 \mathrm{O}$ & 12.47 & 13.68 & 1.21 \\
\hline training & NA01 & DMSO & 19.91 & 19.35 & -0.56 \\
\hline training & NAO2 & AN & 20.22 & 19.37 & -0.85 \\
\hline training & NA02 & DMSO & 18.97 & 19.47 & 0.50 \\
\hline training & NA03 & $\mathrm{H} 2 \mathrm{O}$ & 10.93 & 12.86 & 1.93 \\
\hline training & NA03 & DMSO & 18.00 & 15.59 & -2.41 \\
\hline training & NA06 & $\mathrm{H} 2 \mathrm{O}$ & 11.52 & 11.99 & 0.47 \\
\hline training & NA06 & DMSO & 16.29 & 14.27 & -2.02 \\
\hline training & NA07 & DMSO & 17.52 & 16.98 & -0.54 \\
\hline training & NA08 & DMSO & 14.87 & 16.48 & 1.61 \\
\hline training & NA10 & DMSO & 15.52 & 16.12 & 0.60 \\
\hline training & NA12 & DMSO & 21.09 & 17.45 & -3.64 \\
\hline training & NA13 & DMSO & 15.03 & 14.86 & -0.17 \\
\hline training & NA14 & $\mathrm{H} 2 \mathrm{O}$ & 11.17 & 13.01 & 1.84 \\
\hline training & NA15 & $\mathrm{H} 2 \mathrm{O}$ & 10.75 & 12.11 & 1.36 \\
\hline training & NA16 & $\mathrm{H} 2 \mathrm{O}$ & 10.06 & 12.80 & 2.74 \\
\hline training & NA16 & DMSO & 14.78 & 16.52 & 1.74 \\
\hline training & NA17 & DMSO & 15.70 & 17.49 & 1.79 \\
\hline training & NHC2 & THF & 21.72 & 20.33 & -1.39 \\
\hline training & NHC3 & THF & 16.54 & 19.42 & 2.88 \\
\hline training & NHC4 & THF & 20.41 & 17.08 & -3.33 \\
\hline training & OA01 & $\mathrm{DMF}$ & 18.86 & 20.35 & 1.49 \\
\hline training & OA01 & DMSO & 19.86 & 20.01 & 0.15 \\
\hline training & OA01 & AN & 18.53 & 19.98 & 1.45 \\
\hline training & OA02 & DMSO & 20.09 & 21.57 & 1.48 \\
\hline training & OA02 & $\mathrm{AN}$ & 20.62 & 21.44 & 0.82 \\
\hline training & OA03 & $\mathrm{DMF}$ & 19.90 & 21.28 & 1.38 \\
\hline training & OA03 & DMSO & 19.67 & 20.99 & 1.32 \\
\hline training & OA03 & AN & 21.70 & 20.84 & -0.86 \\
\hline training & OA04 & DMSO & 19.08 & 20.79 & 1.71 \\
\hline training & OA05 & $\mathrm{AN}$ & 18.32 & 20.17 & 1.85 \\
\hline training & OA06 & DMF & 17.85 & 18.68 & 0.83 \\
\hline training & OA06 & $\mathrm{AN}$ & 19.58 & 18.52 & -1.06 \\
\hline training & OA07 & $\mathrm{DMF}$ & 22.05 & 19.79 & -2.26 \\
\hline training & OA07 & DMSO & 18.98 & 19.51 & 0.53 \\
\hline training & OA07 & $A N$ & 21.69 & 19.56 & -2.13 \\
\hline training & OA08 & $\mathrm{ACN}$ & 18.39 & 22.45 & 4.06 \\
\hline training & OA08 & DMSO & 18.47 & 22.72 & 4.25 \\
\hline training & OA09 & $\mathrm{ACN}$ & 18.39 & 18.87 & 0.48 \\
\hline training & OA09 & DMSO & 18.47 & 19.08 & 0.61 \\
\hline training & OA10 & $\mathrm{ACN}$ & 25.51 & 20.91 & -4.60 \\
\hline training & OA11 & $A C N$ & 18.81 & 22.20 & 3.39 \\
\hline training & OA12 & DMSO & 14.32 & 17.64 & 3.32 \\
\hline training & OA12 & AN & 15.14 & 17.71 & 2.57 \\
\hline training & OA13 & DMSO & 21.02 & 21.98 & 0.96 \\
\hline
\end{tabular}




\begin{tabular}{|c|c|c|c|c|c|}
\hline training & OA14 & AN & 16.90 & 16.11 & -0.79 \\
\hline training & OA16 & acetone & 18.74 & 15.69 & -3.05 \\
\hline training & OA17 & acetone & 18.80 & 15.19 & -3.61 \\
\hline training & OA18 & AN & 16.03 & 14.22 & -1.81 \\
\hline training & SA01 & $\mathrm{ACN}$ & 22.40 & 16.90 & -5.50 \\
\hline training & SAO3 & $\mathrm{ACN}$ & 21.72 & 19.03 & -2.69 \\
\hline training & SA05 & $A C N$ & 19.30 & 17.04 & -2.26 \\
\hline training & SA06 & $\mathrm{ACN}$ & 21.20 & 17.73 & -3.47 \\
\hline training & SA07 & $\mathrm{ACN}$ & 20.11 & 16.08 & -4.03 \\
\hline validation & Ar01 & DCM & -4.36 & -4.07 & 0.29 \\
\hline validation & Ar05 & DCM & 2.48 & 1.89 & -0.59 \\
\hline validation & Ar07 & $\mathrm{AN}$ & 6.08 & 6.59 & 0.51 \\
\hline validation & Ar09 & AN & 4.38 & 5.44 & 1.06 \\
\hline validation & Ar12 & $A N$ & 6.00 & 6.61 & 0.61 \\
\hline validation & Ar15 & DCM & 6.22 & 5.24 & -0.98 \\
\hline validation & Ar16 & $\mathrm{AN}$ & 3.97 & 4.64 & 0.67 \\
\hline validation & Ar17 & DCM & 5.75 & 6.06 & 0.31 \\
\hline validation & Ar18 & DCM & 6.54 & 7.13 & 0.59 \\
\hline validation & Ar20 & $\mathrm{AN}$ & 4.63 & 7.19 & 2.56 \\
\hline validation & Ar23 & DCM & 5.85 & 5.54 & -0.31 \\
\hline validation & Ar25 & DCM & 1.33 & 2.18 & 0.85 \\
\hline validation & Ar28 & AN & 2.90 & 5.19 & 2.29 \\
\hline validation & Ar32 & DCM & -1.01 & -0.30 & 0.71 \\
\hline validation & Ar35 & $\mathrm{AN}$ & 3.06 & 5.56 & 2.50 \\
\hline validation & CA04 & $\mathrm{H} 2 \mathrm{O}$ & 11.77 & 11.13 & -0.64 \\
\hline validation & CA07 & DMSO & 27.62 & 24.60 & -3.02 \\
\hline validation & CA16 & DMSO & 28.95 & 25.41 & -3.54 \\
\hline validation & CA17 & $\mathrm{H} 2 \mathrm{O}$ & 13.09 & 13.21 & 0.12 \\
\hline validation & CA19 & DMSO & 16.96 & 17.71 & 0.75 \\
\hline validation & CA2O & $\mathrm{H} 2 \mathrm{O}$ & 13.58 & 12.97 & -0.61 \\
\hline validation & CA21 & DMSO & 19.36 & 17.47 & -1.89 \\
\hline validation & CA22 & DMSO & 28.27 & 24.43 & -3.84 \\
\hline validation & CCO1 & DCM & -3.65 & -2.09 & 1.56 \\
\hline validation & $\mathrm{CCO}$ & DCM & 0.78 & 0.36 & -0.42 \\
\hline validation & CC08 & $\mathrm{DCM}$ & 1.11 & 1.38 & 0.27 \\
\hline validation & CC14 & DCM & 0.68 & 0.62 & -0.06 \\
\hline validation & CC15 & DCM & 0.79 & 0.77 & -0.02 \\
\hline validation & CC 19 & DCM & 2.35 & 0.75 & -1.60 \\
\hline validation & CC2O & DCM & 1.17 & 2.76 & 1.59 \\
\hline validation & CC21 & DCM & 1.00 & 1.07 & 0.07 \\
\hline validation & $\mathrm{CC} 23$ & DCM & -1.00 & 2.71 & 3.71 \\
\hline validation & CC24 & DCM & 1.65 & 3.40 & 1.75 \\
\hline validation & CC25 & DCM & 2.82 & 2.28 & -0.54 \\
\hline validation & CC26 & $\mathrm{DCM}$ & -1.55 & -2.18 & -0.63 \\
\hline validation & CC28 & DCM & 0.08 & -0.21 & -0.29 \\
\hline validation & CC30 & DCM & 1.49 & 3.22 & 1.73 \\
\hline validation & CC31 & $\mathrm{DCM}$ & 1.17 & 0.38 & -0.79 \\
\hline validation & CC35 & $\mathrm{DCM}$ & 1.17 & 0.44 & -0.73 \\
\hline validation & CC38 & DCM & 0.67 & 1.84 & 1.17 \\
\hline validation & CX02 & $\mathrm{DCM}$ & 11.95 & 13.17 & 1.22 \\
\hline validation & CX03 & DMSO & 15.68 & 16.70 & 1.02 \\
\hline validation & CX06 & DMSO & 15.85 & 15.89 & 0.04 \\
\hline validation & $C \times 10$ & $D C M$ & 8.87 & 7.19 & -1.68 \\
\hline validation & $C \times 15$ & DMSO & 27.45 & 26.01 & -1.44 \\
\hline validation & CX18 & DMSO & 19.46 & 21.90 & 2.44 \\
\hline validation & CX19 & DMSO & 17.98 & 21.27 & 3.29 \\
\hline validation & CX23 & DMSO & 21.29 & 20.40 & -0.89 \\
\hline validation & CX25 & DCM & 6.98 & 8.57 & 1.59 \\
\hline validation & EE01 & $\mathrm{DCM}$ & 5.21 & 5.28 & 0.07 \\
\hline validation & EE06 & $A N$ & 12.34 & 13.18 & 0.84 \\
\hline validation & EE10 & DCM & -2.94 & -2.86 & 0.08 \\
\hline validation & EE12 & $\mathrm{DCM}$ & 9.00 & 10.62 & 1.62 \\
\hline validation & EE13 & $\mathrm{DCM}$ & 11.50 & 13.08 & 1.58 \\
\hline validation & EE14 & $D C M$ & 4.46 & 7.69 & 3.23 \\
\hline
\end{tabular}




\begin{tabular}{|c|c|c|c|c|c|}
\hline validation & EE15 & DCM & 4.37 & 6.59 & 2.22 \\
\hline validation & EE18 & DCM & 4.23 & 7.21 & 2.98 \\
\hline validation & EE19 & $\mathrm{AN}$ & 7.32 & 5.93 & -1.39 \\
\hline validation & En02 & DCM & 11.40 & 11.02 & -0.38 \\
\hline validation & En06 & DCM & 13.41 & 13.35 & -0.06 \\
\hline validation & En07 & DCM & 15.91 & 15.38 & -0.53 \\
\hline validation & En08 & DCM & 12.03 & 10.77 & -1.26 \\
\hline validation & En10 & DCM & 10.76 & 9.05 & -1.71 \\
\hline validation & En10 & $\mathrm{AN}$ & 11.66 & 10.73 & -0.93 \\
\hline validation & En11 & AN & 13.84 & 11.90 & -1.94 \\
\hline validation & En13 & AN & 8.78 & 8.99 & 0.21 \\
\hline validation & En16 & AN & 11.99 & 11.11 & -0.88 \\
\hline validation & En17 & AN & 10.63 & 10.68 & 0.05 \\
\hline validation & En19 & AN & 11.60 & 12.94 & 1.34 \\
\hline validation & En26 & AN & 5.64 & 6.16 & 0.52 \\
\hline validation & En29 & AN & 5.44 & 3.68 & -1.76 \\
\hline validation & En31 & $\mathrm{AN}$ & 5.60 & 4.73 & -0.87 \\
\hline validation & N02 & DCM & 11.70 & 7.79 & -3.91 \\
\hline validation & N03 & DCM & 13.70 & 10.54 & -3.16 \\
\hline validation & N04 & DCM & 15.80 & 13.64 & -2.16 \\
\hline validation & N04 & $\mathrm{H} 2 \mathrm{O}$ & 13.19 & 13.38 & 0.19 \\
\hline validation & N07 & $\mathrm{AN}$ & 7.77 & 8.12 & 0.35 \\
\hline validation & N09 & $\mathrm{H} 2 \mathrm{O}$ & 9.63 & 10.69 & 1.06 \\
\hline validation & N12 & $\mathrm{AN}$ & 11.43 & 13.84 & 2.41 \\
\hline validation & N16 & DCM & 9.81 & 10.68 & 0.87 \\
\hline validation & N19 & DCM & 12.46 & 14.05 & 1.59 \\
\hline validation & N23 & $\mathrm{AN}$ & 12.92 & 10.98 & -1.94 \\
\hline validation & N26 & $\mathrm{H} 2 \mathrm{O}$ & 13.33 & 15.36 & 2.03 \\
\hline validation & N28 & $\mathrm{H} 2 \mathrm{O}$ & 10.48 & 13.52 & 3.04 \\
\hline validation & N29 & DMSO & 15.28 & 15.62 & 0.34 \\
\hline validation & N31 & $\mathrm{H} 2 \mathrm{O}$ & 17.21 & 14.98 & -2.23 \\
\hline validation & N32 & DCM & 20.60 & 15.25 & -5.35 \\
\hline validation & N32 & $\mathrm{AN}$ & 20.59 & 17.20 & -3.39 \\
\hline validation & N33 & AN & 17.35 & 17.08 & -0.27 \\
\hline validation & N35 & DMSO & 16.96 & 16.34 & -0.62 \\
\hline validation & N36 & $\mathrm{AN}$ & 16.80 & 15.86 & -0.94 \\
\hline validation & N39 & AN & 18.80 & 18.33 & -0.47 \\
\hline validation & NA01 & AN & 20.11 & 19.15 & -0.96 \\
\hline validation & NA02 & $\mathrm{H} 2 \mathrm{O}$ & 14.76 & 14.61 & -0.15 \\
\hline validation & NA04 & $\mathrm{H} 2 \mathrm{O}$ & 10.77 & 11.92 & 1.15 \\
\hline validation & NA05 & DMSO & 19.13 & 17.30 & -1.83 \\
\hline validation & NA09 & DMSO & 16.03 & 17.91 & 1.88 \\
\hline validation & NA11 & $\mathrm{AN}$ & 10.78 & 11.94 & 1.16 \\
\hline validation & NA13 & $\mathrm{H} 2 \mathrm{O}$ & 11.00 & 12.18 & 1.18 \\
\hline validation & NA14 & DMSO & 17.63 & 18.93 & 1.30 \\
\hline validation & NA15 & DMSO & 17.04 & 17.90 & 0.86 \\
\hline validation & $\mathrm{NHCl}$ & THF & 23.35 & 20.81 & -2.54 \\
\hline validation & NHC5 & THF & 23.00 & 18.37 & -4.63 \\
\hline validation & OA02 & DMF & 19.90 & 21.90 & 2.00 \\
\hline validation & OA04 & DMF & 19.65 & 21.12 & 1.47 \\
\hline validation & OA05 & $\mathrm{DMF}$ & 19.67 & 20.47 & 0.80 \\
\hline validation & OA05 & DMSO & 20.34 & 20.17 & -0.17 \\
\hline validation & OA06 & DMSO & 18.78 & 18.41 & -0.37 \\
\hline validation & OA08 & DMF & 20.37 & 23.30 & 2.93 \\
\hline validation & OA09 & DMF & 20.37 & 19.67 & -0.70 \\
\hline validation & OA10 & DMSO & 21.29 & 21.15 & -0.14 \\
\hline validation & OA10 & DMF & 22.41 & 21.72 & -0.69 \\
\hline validation & OA12 & DMF & 15.05 & 17.91 & 2.86 \\
\hline validation & OA15 & AN & 16.45 & 14.17 & -2.28 \\
\hline validation & OA16 & AN & 15.30 & 14.52 & -0.78 \\
\hline validation & OA17 & AN & 14.90 & 14.22 & -0.68 \\
\hline validation & SA02 & $\mathrm{ACN}$ & 23.84 & 17.77 & -6.07 \\
\hline validation & SA04 & $\mathrm{ACN}$ & 20.93 & 18.68 & -2.25 \\
\hline validation & SA07 & DMSO & 19.60 & 16.93 & -2.67 \\
\hline
\end{tabular}




\section{MRA of $\boldsymbol{N} \cdot \mathbf{s}_{\mathrm{N}}$}

Even though effective, modeling of the nucleophilicity $N$ as shown in the previous section resulted to be more challenging for highly nucleophilic compounds (anions). In order to gain additional insights into the significance of our model we investigated possible reasons for this observation. Even though $N$ provides a ranking of the reactivity of nucleophiles, also the sensitivity parameter $s_{N}$ should be considered for a more comprehensive picture of nucleophilicity. In fact, due to different $s_{N}$ values for different nucleophiles, the relative reactivity of different nucleophiles might switch depending on the reference electrophile employed. Indeed, rearranging Mayr's equation into equation eq. $\$ 1$ as shown by Mayr ${ }^{8}$ results in the definition of a different nucleophilicity parameter $N \cdot \mathrm{s}_{\mathrm{N}}$ that is inclusive of both $N$ and $\mathrm{s}_{\mathrm{N}}$. Correlation between $N$ and $\mathrm{s}_{\mathrm{N}}$, and $N$ and $N \cdot \mathrm{s}_{\mathrm{N}}$ are shown in Figure $\mathrm{S} 7$. These plots suggest that for poor nucleophiles $N$ and $\mathrm{s}_{\mathrm{N}}$ are mostly colinear due to $\mathrm{s}_{\mathrm{N}}$ being around unity and $N$ small. However, as $N$ increases $\mathrm{s}_{\mathrm{N}}$ decreases towards fractional values and causes the parameter $N \cdot \mathbf{s}_{N}$ to deviate significantly from $N$ with substantial scattering for $N>10$.

$$
\begin{array}{lr}
\log (k)=s_{N}(N+E) \quad \text { (Mayr's equation) } \\
\log (k)=N \cdot s_{N}+E \cdot s_{N} & \text { (eq. } \mathrm{S} 1)
\end{array}
$$

Figure S7. Correlations $N$ vs. $s_{N}$, and $N$ vs. $N \cdot s_{N}$.
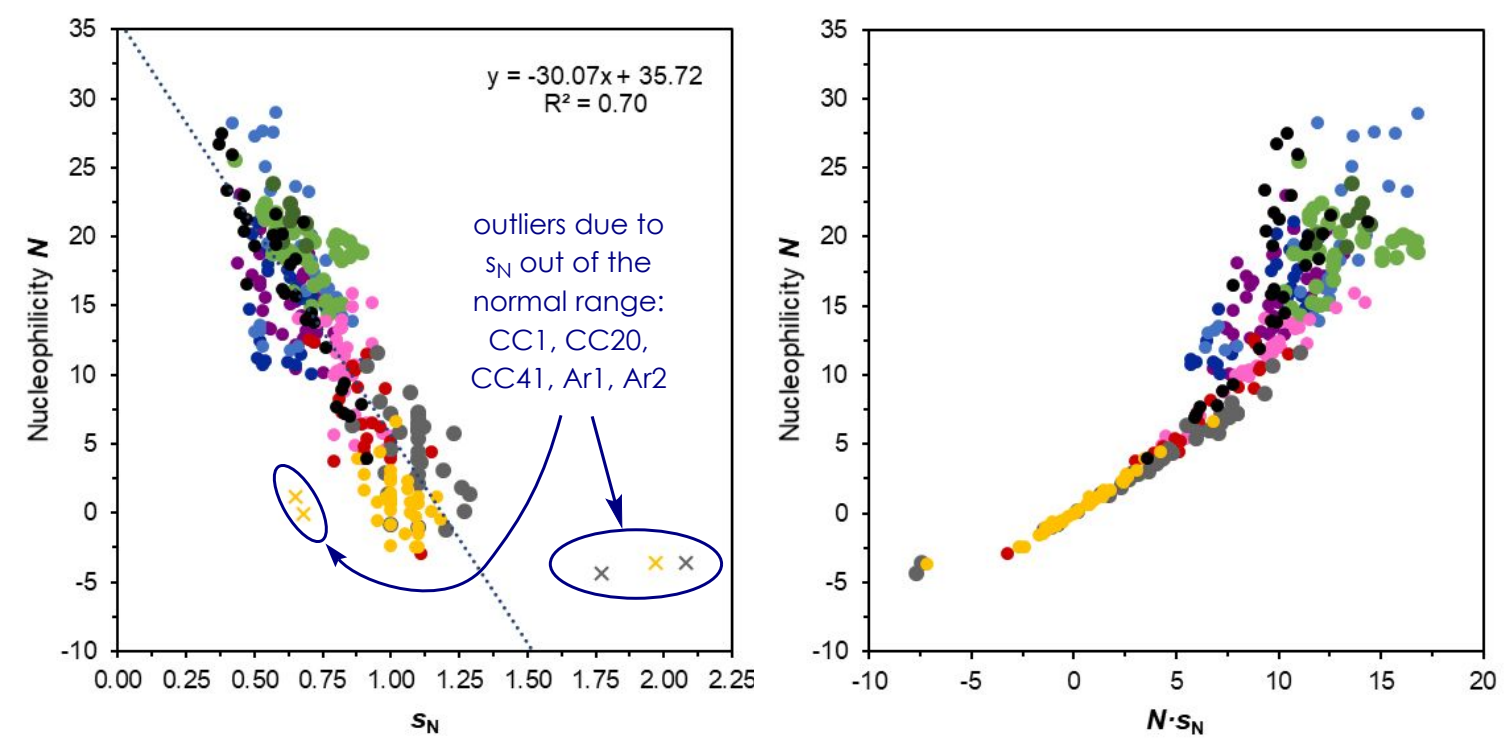

Based on these preliminary observations about the role of $s_{N}$ in the description of highly reactive nucleophiles, we used MRA to obtain a model for the description of $N \cdot \mathrm{S}_{\mathrm{N}}$. The model obtained is reported In Figure S8. However, it should be noted that despite the good model obtained with this parameter (RMSE $=1.04$ ), its utility is limited as $N \cdot \mathrm{s}_{N}$ lacks the ability of ordering the nucleophiles according with their reactivity. This can be seen from the right plot in Figure S7, where some phenolates seem to be more reactive than carbanions or ylides. 
Figure S8. Multidimensional correlation model for $N \cdot s_{N}$

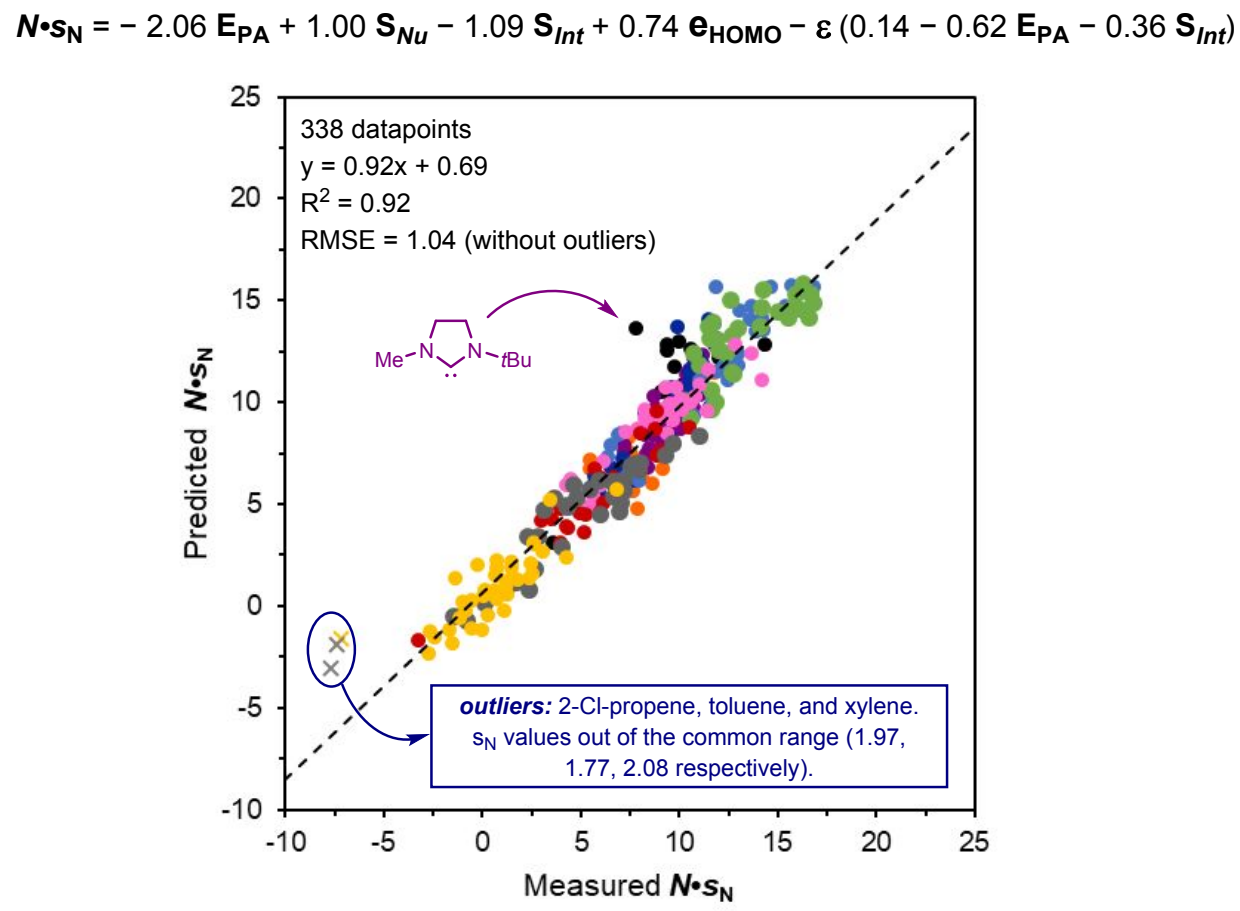

Table S4. Datapoints for the model reported in Figure S8.

$\begin{array}{ccccc}\text { Nucleophile } & \text { solvent } & \mathbf{N} \mathbf{s}_{\mathbf{N}}(\mathbf{m e a s u r e d}) & \mathbf{N} \mathbf{s N} \text { (predicted) } & \text { rediduals } \\ \text { Ar01 } & \text { DCM } & -7.72 & -2.75 & 4.96 \\ \text { Ar02 } & \text { DCM } & -7.43 & -1.55 & 5.88 \\ \text { Ar03 } & \text { DCM } & -1.42 & -0.16 & 1.25 \\ \text { Ar04 } & \text { DCM } & 0.17 & 0.54 & 0.38 \\ \text { Ar05 } & \text { DCM } & 2.70 & 2.16 & -0.54 \\ \text { Ar06 } & \text { AN } & 7.60 & 7.24 & -0.36 \\ \text { Ar07 } & \text { AN } & 6.69 & 6.47 & -0.21 \\ \text { Ar08 } & \text { AN } & 7.99 & 7.48 & -0.50 \\ \text { Ar09 } & \text { AN } & 4.82 & 5.65 & 0.83 \\ \text { Ar10 } & \text { AN } & 6.69 & 5.71 & -0.97 \\ \text { Ar11 } & \text { AN } & 3.11 & 4.98 & 1.87 \\ \text { Ar12 } & \text { AN } & 6.60 & 6.48 & -0.12 \\ \text { Ar13 } & \text { AN } & 7.94 & 7.35 & -0.59 \\ \text { Ar14 } & \text { AN } & 7.08 & 6.63 & -0.46 \\ \text { Ar15 } & \text { DCM } & 6.97 & 5.01 & -1.96 \\ \text { Ar16 } & \text { AN } & 4.37 & 5.34 & 0.97 \\ \text { Ar17 } & \text { DCM } & 7.07 & 5.40 & -1.68 \\ \text { Ar18 } & \text { DCM } & 7.19 & 6.48 & -0.72 \\ \text { Ar19 } & \text { AN } & 5.95 & 6.67 & 0.72 \\ \text { Ar20 } & \text { AN } & 4.63 & 6.09 & 1.46 \\ \text { Ar21 } & \text { AN } & 7.69 & 7.22 & -0.47 \\ \text { Ar22 } & \text { AN } & 9.71 & 8.11 & -1.60 \\ \text { Ar23 } & \text { DCM } & 6.03 & 4.48 & -1.55 \\ \text { Ar24 } & \text { AN } & 9.30 & 7.68 & -1.62 \\ \text { Ar25 } & \text { DCM } & 1.72 & 0.89 & -0.83 \\ \text { Ar26 } & \text { DCM } & 2.38 & 0.96 & -1.42 \\ \text { Ar27 } & \text { DCM } & 4.01 & 2.73 & -1.28 \\ \text { Ar28 } & \text { AN } & 2.84 & 4.20 & 1.36 \\ \text { Ar29 } & \text { AN } & 5.49 & 6.57 & 1.08 \\ \text { Ar30 } & \text { AN } & 2.32 & 4.39 & 2.07 \\ \text { Ar31 } & \text { DCM } & 7.22 & 5.67 & -1.55 \\ \text { Ar32 } & \text { DCM } & -1.11 & -0.54 & 0.57 \\ \text { Ar33 } & \text { DCM } & -0.80 & -0.42 & 0.38 \\ \text { Ar34 } & \text { DCM } & 1.34 & 1.10 & -0.24 \\ \text { Ar35 } & \text { AN } & 3.64 & 5.56 & 1.92 \\ & & & & \end{array}$




\begin{tabular}{|c|c|c|c|c|}
\hline Ar36 & AN & 11.05 & 8.52 & -2.52 \\
\hline Ar37 & DCM & 6.79 & 6.02 & -0.77 \\
\hline Ar38 & AN & 4.26 & 5.31 & 1.06 \\
\hline CA01 & DMSO & 12.47 & 10.84 & -1.63 \\
\hline CA02 & DMSO & 11.68 & 9.83 & -1.84 \\
\hline CA03 & DMSO & 12.57 & 11.24 & -1.33 \\
\hline CA04 & DMSO & 12.53 & 12.19 & -0.34 \\
\hline CAO4 & $\mathrm{H} 2 \mathrm{O}$ & 7.42 & 6.88 & -0.53 \\
\hline CA05 & DMSO & 11.96 & 11.40 & -0.56 \\
\hline CA05 & $\mathrm{H} 2 \mathrm{O}$ & 7.96 & 6.50 & -1.46 \\
\hline CA06 & DMSO & 15.70 & 15.31 & -0.39 \\
\hline CA07 & DMSO & 14.64 & 14.96 & 0.33 \\
\hline CA08 & DMSO & 15.37 & 13.87 & -1.49 \\
\hline CA09 & DMSO & 14.20 & 13.26 & -0.94 \\
\hline CA10 & DMSO & 16.29 & 13.96 & -2.33 \\
\hline CA11 & DMSO & 13.64 & 14.01 & 0.37 \\
\hline CA12 & DMSO & 13.56 & 13.36 & -0.20 \\
\hline CA13 & DMSO & 13.08 & 13.72 & 0.64 \\
\hline CA14 & DMSO & 14.27 & 12.70 & -1.57 \\
\hline CA15 & DMSO & 11.77 & 13.01 & 1.24 \\
\hline CA16 & DMSO & 16.79 & 15.35 & -1.44 \\
\hline CA17 & DMSO & 13.92 & 13.29 & -0.63 \\
\hline CA17 & $\mathrm{H} 2 \mathrm{O}$ & 6.55 & 8.10 & 1.55 \\
\hline CA18 & DMSO & 12.99 & 12.34 & -0.65 \\
\hline CA18 & $\mathrm{H} 2 \mathrm{O}$ & 6.39 & 7.76 & 1.37 \\
\hline CA19 & DMSO & 12.38 & 11.35 & -1.03 \\
\hline CA19 & $\mathrm{H} 2 \mathrm{O}$ & 6.88 & 8.14 & 1.26 \\
\hline CA20 & DMSO & 12.22 & 10.99 & -1.23 \\
\hline CA20 & $\mathrm{H} 2 \mathrm{O}$ & 7.06 & 8.12 & 1.05 \\
\hline CA21 & DMSO & 12.97 & 11.94 & -1.03 \\
\hline CA21 & $\mathrm{H} 2 \mathrm{O}$ & 10.73 & 9.10 & -1.62 \\
\hline CA22 & DMSO & 11.87 & 15.04 & 3.17 \\
\hline CA23 & DMSO & 11.08 & 10.70 & -0.38 \\
\hline CA24 & DMSO & 11.04 & 10.74 & -0.30 \\
\hline CCOI & DCM & -7.19 & -1.79 & 5.41 \\
\hline CCO2 & DCM & -2.70 & -2.58 & 0.12 \\
\hline CCO3 & DCM & -2.66 & -1.49 & 1.17 \\
\hline CCO4 & DCM & -1.53 & -1.84 & -0.31 \\
\hline CCO5 & DCM & -0.58 & 0.56 & 1.14 \\
\hline CCO6 & DCM & -1.18 & -0.16 & 1.02 \\
\hline CCO7 & DCM & 0.74 & 0.60 & -0.14 \\
\hline CC08 & DCM & 1.09 & 0.45 & -0.63 \\
\hline CCO9 & DCM & 0.25 & -0.13 & -0.38 \\
\hline CClO & DCM & -0.54 & -0.95 & -0.41 \\
\hline $\mathrm{CCl1}$ & DCM & 4.23 & 2.38 & -1.85 \\
\hline $\mathrm{CC} 12$ & DCM & 0.65 & 0.56 & -0.09 \\
\hline $\mathrm{CCl3}$ & DCM & 1.80 & 1.64 & -0.17 \\
\hline $\mathrm{CCl} 4$ & DCM & 0.74 & 1.90 & 1.16 \\
\hline CC15 & DCM & 0.85 & 0.64 & -0.20 \\
\hline $\mathrm{CCl} 16$ & DCM & 0.06 & 0.36 & 0.29 \\
\hline $\mathrm{CCl}$ & DCM & 2.60 & 2.90 & 0.30 \\
\hline CC18 & DCM & -2.41 & -1.99 & 0.42 \\
\hline CC19 & DCM & 2.35 & 1.62 & -0.73 \\
\hline CC20 & DCM & 0.76 & 1.92 & 1.16 \\
\hline CC21 & DCM & 1.00 & 0.43 & -0.57 \\
\hline CC22 & DCM & -1.03 & 0.73 & 1.76 \\
\hline CC23 & DCM & -1.40 & 1.27 & 2.67 \\
\hline CC24 & DCM & 1.49 & 1.81 & 0.33 \\
\hline CC25 & DCM & 2.54 & 1.40 & -1.14 \\
\hline CC26 & DCM & -1.71 & -1.32 & 0.39 \\
\hline CC27 & DCM & 1.38 & 1.13 & -0.25 \\
\hline CC28 & DCM & 0.09 & 0.76 & 0.67 \\
\hline CC29 & DCM & -0.87 & -0.42 & 0.45 \\
\hline CC30 & DCM & 1.49 & 1.75 & 0.26 \\
\hline CC31 & DCM & 1.29 & 0.66 & -0.62 \\
\hline
\end{tabular}




\begin{tabular}{|c|c|c|c|c|}
\hline CC32 & DCM & 3.09 & 2.60 & -0.49 \\
\hline CC33 & DCM & 0.66 & 1.42 & 0.76 \\
\hline CC34 & DCM & 2.60 & 2.98 & 0.38 \\
\hline CC35 & DCM & 1.17 & 0.85 & -0.32 \\
\hline CC36 & DCM & 1.08 & -0.34 & -1.42 \\
\hline CC37 & DCM & 2.44 & 1.97 & -0.46 \\
\hline CC38 & DCM & 0.74 & 1.86 & 1.13 \\
\hline CC39 & DCM & -0.27 & 1.90 & 2.17 \\
\hline CC40 & $\mathrm{AN}$ & 3.46 & 5.62 & 2.16 \\
\hline CC41 & DCM & -0.03 & -0.81 & -0.78 \\
\hline CX01 & DMSO & 9.63 & 10.40 & 0.77 \\
\hline CX02 & DCM & 9.08 & 10.88 & 1.79 \\
\hline CX02 & DMSO & 9.92 & 10.04 & 0.12 \\
\hline CX03 & DMSO & 10.19 & 11.29 & 1.10 \\
\hline CX04 & DMSO & 10.28 & 10.64 & 0.36 \\
\hline CX05 & DMSO & 9.74 & 9.86 & 0.12 \\
\hline CX06 & DMSO & 9.67 & 10.56 & 0.89 \\
\hline CX07 & DMSO & 14.33 & 12.58 & -1.75 \\
\hline CX08 & DMSO & 11.97 & 12.04 & 0.07 \\
\hline CX09 & DCM & 7.76 & 6.05 & -1.71 \\
\hline CX10 & DCM & 7.27 & 5.62 & -1.66 \\
\hline CX11 & DCM & 6.13 & 4.91 & -1.22 \\
\hline CX12 & DCM & 5.95 & 4.38 & -1.57 \\
\hline CX13 & $\mathrm{DCM}$ & 3.60 & 2.64 & -0.96 \\
\hline CX14 & DMSO & 10.89 & 11.37 & 0.47 \\
\hline CX15 & DMSO & 10.43 & 13.65 & 3.21 \\
\hline CX16 & DMSO & 9.88 & 12.34 & 2.45 \\
\hline CX17 & DMSO & 12.14 & 11.80 & -0.35 \\
\hline CX18 & DMSO & 11.29 & 11.25 & -0.04 \\
\hline CX19 & DMSO & 11.33 & 10.74 & -0.59 \\
\hline CX2O & DMSO & 12.53 & 12.56 & 0.03 \\
\hline CX21 & DMSO & 11.45 & 11.31 & -0.13 \\
\hline CX22 & DMSO & 9.69 & 11.53 & 1.84 \\
\hline CX23 & DMSO & 10.01 & 12.19 & 2.18 \\
\hline CX24 & DCM & 6.98 & 6.60 & -0.38 \\
\hline CX25 & DCM & 5.93 & 5.71 & -0.22 \\
\hline EEO1 & DCM & 5.21 & 4.64 & -0.57 \\
\hline EE02 & $\mathrm{AN}$ & 5.72 & 6.90 & 1.18 \\
\hline EE02 & DCM & 6.11 & 5.14 & -0.97 \\
\hline EE03 & DCM & 4.92 & 4.43 & -0.50 \\
\hline EEO4 & DCM & 9.03 & 7.60 & -1.43 \\
\hline EE05 & $\mathrm{DCM}$ & 9.12 & 7.67 & -1.45 \\
\hline EE06 & $A N$ & 8.88 & 9.47 & 0.58 \\
\hline EE06 & $\mathrm{DCM}$ & 8.79 & 8.61 & -0.18 \\
\hline EE07 & $D C M$ & 5.97 & 4.94 & -1.03 \\
\hline EE08 & $\mathrm{DCM}$ & 4.35 & 3.84 & -0.51 \\
\hline EE09 & $\mathrm{DCM}$ & 6.67 & 6.52 & -0.15 \\
\hline EE10 & $D C M$ & -3.26 & -1.64 & 1.62 \\
\hline EE11 & $D C M$ & 2.99 & 4.20 & 1.21 \\
\hline EE12 & DCM & 8.82 & 7.33 & -1.49 \\
\hline EE12 & $\mathrm{AN}$ & 8.02 & 8.47 & 0.46 \\
\hline EE13 & $D C M$ & 10.47 & 8.76 & -1.70 \\
\hline EEl4 & DCM & 5.13 & 3.87 & -1.26 \\
\hline EE15 & $D C M$ & 3.93 & 4.38 & 0.45 \\
\hline EEl 6 & DCM & 3.53 & 3.91 & 0.38 \\
\hline EE17 & DCM & 3.94 & 3.22 & -0.72 \\
\hline EE18 & DCM & 4.23 & 3.82 & -0.41 \\
\hline EE19 & $A N$ & 6.00 & 6.81 & 0.81 \\
\hline Enol & DCM & 10.82 & 10.27 & -0.55 \\
\hline $\mathrm{EnO2}$ & $D C M$ & 9.46 & 9.45 & -0.01 \\
\hline EnO3 & $\mathrm{DCM}$ & 12.82 & 12.64 & -0.18 \\
\hline EnO4 & DCM & 10.01 & 9.82 & -0.19 \\
\hline En05 & $D C M$ & 11.50 & 11.53 & 0.04 \\
\hline EnO6 & $D C M$ & 11.00 & 10.74 & -0.26 \\
\hline EnO7 & DCM & 13.68 & 12.30 & -1.39 \\
\hline
\end{tabular}




\begin{tabular}{|c|c|c|c|c|}
\hline En08 & DCM & 9.62 & 9.45 & -0.18 \\
\hline En09 & DCM & 9.65 & 9.39 & -0.26 \\
\hline En10 & DCM & 9.36 & 8.74 & -0.62 \\
\hline En 10 & $\mathrm{AN}$ & 9.68 & 9.30 & -0.38 \\
\hline Enll & AN & 10.10 & 9.87 & -0.24 \\
\hline En12 & DCM & 11.40 & 9.89 & -1.51 \\
\hline En 12 & $\mathrm{AN}$ & 10.54 & 10.28 & -0.26 \\
\hline En13 & AN & 7.29 & 8.99 & 1.70 \\
\hline En 14 & AN & 8.55 & 9.56 & 1.01 \\
\hline En 15 & AN & 9.56 & 10.36 & 0.80 \\
\hline En16 & AN & 10.07 & 10.64 & 0.57 \\
\hline En17 & AN & 8.93 & 9.62 & 0.69 \\
\hline En 18 & $A N$ & 8.54 & 9.36 & 0.81 \\
\hline En19 & AN & 9.28 & 9.73 & 0.45 \\
\hline En20 & $A N$ & 8.24 & 9.20 & 0.96 \\
\hline En20 & DCM & 7.87 & 8.83 & 0.96 \\
\hline En21 & $\mathrm{AN}$ & 9.30 & 10.83 & 1.53 \\
\hline En22 & AN & 14.20 & 11.21 & -3.00 \\
\hline En23 & DCM & 8.23 & 9.42 & 1.19 \\
\hline En24 & DCM & 9.81 & 10.44 & 0.63 \\
\hline En25 & $\mathrm{AN}$ & 6.14 & 7.24 & 1.10 \\
\hline En26 & $A N$ & 4.46 & 6.55 & 2.10 \\
\hline En27 & AN & 4.27 & 6.61 & 2.34 \\
\hline En28 & AN & 4.52 & 6.48 & 1.97 \\
\hline En29 & AN & 5.44 & 5.73 & 0.29 \\
\hline En30 & AN & 5.56 & 6.21 & 0.66 \\
\hline En31 & AN & 5.60 & 5.90 & 0.30 \\
\hline En32 & $A N$ & 5.98 & 6.47 & 0.49 \\
\hline N01 & DCM & 8.64 & 5.63 & -3.01 \\
\hline N01 & $\mathrm{H} 2 \mathrm{O}$ & 8.07 & 6.38 & -1.69 \\
\hline N02 & $D C M$ & 7.84 & 4.38 & -3.46 \\
\hline N02 & $\mathrm{H} 2 \mathrm{O}$ & 7.67 & 5.76 & -1.91 \\
\hline N03 & $\mathrm{DCM}$ & 9.18 & 6.51 & -2.67 \\
\hline N03 & $\mathrm{H} 2 \mathrm{O}$ & 7.78 & 6.78 & -1.00 \\
\hline N04 & DCM & 10.43 & 9.42 & -1.01 \\
\hline N04 & $\mathrm{DMF}$ & 9.98 & 9.63 & -0.36 \\
\hline N04 & DMSO & 9.92 & 9.42 & -0.50 \\
\hline N04 & $\mathrm{H} 2 \mathrm{O}$ & 7.39 & 8.56 & 1.17 \\
\hline N04 & AN & 9.62 & 9.57 & -0.05 \\
\hline NO4 & THF & 10.49 & 9.03 & -1.46 \\
\hline N05 & DCM & 10.65 & 9.97 & -0.69 \\
\hline N06 & $\mathrm{AN}$ & 5.84 & 5.65 & -0.19 \\
\hline N07 & AN & 5.91 & 6.05 & 0.15 \\
\hline N08 & AN & 8.80 & 7.99 & -0.81 \\
\hline N09 & DMSO & 9.15 & 7.96 & -1.19 \\
\hline N09 & AN & 9.06 & 8.17 & -0.89 \\
\hline N09 & $\mathrm{H} 2 \mathrm{O}$ & 5.49 & 6.99 & 1.50 \\
\hline N10 & $A N$ & 8.69 & 8.36 & -0.33 \\
\hline N10 & $\mathrm{H} 2 \mathrm{O}$ & 5.45 & 7.47 & 2.02 \\
\hline N11 & $\mathrm{AN}$ & 7.58 & 7.65 & 0.07 \\
\hline $\mathrm{N} 12$ & AN & 9.03 & 8.22 & -0.81 \\
\hline N13 & $A N$ & 9.67 & 9.34 & -0.32 \\
\hline $\mathrm{N} 14$ & DCM & 7.52 & 7.54 & 0.02 \\
\hline N15 & DCM & 9.95 & 9.84 & -0.11 \\
\hline N16 & DCM & 7.55 & 6.39 & -1.16 \\
\hline N17 & DCM & 9.05 & 7.06 & -1.99 \\
\hline N18 & $D C M$ & 7.24 & 6.88 & -0.36 \\
\hline N19 & $\mathrm{DCM}$ & 10.84 & 10.58 & -0.26 \\
\hline N2O & $\mathrm{DCM}$ & 11.79 & 11.63 & -0.16 \\
\hline N21 & $\mathrm{DCM}$ & 10.46 & 10.77 & 0.31 \\
\hline N22 & $A N$ & 8.60 & 8.34 & -0.25 \\
\hline N22 & $\mathrm{H} 2 \mathrm{O}$ & 9.48 & 8.35 & -1.13 \\
\hline N23 & $A N$ & 7.75 & 8.09 & 0.34 \\
\hline N24 & $A N$ & 9.10 & 8.74 & -0.36 \\
\hline N24 & $\mathrm{H} 2 \mathrm{O}$ & 10.27 & 8.76 & -1.51 \\
\hline
\end{tabular}




\begin{tabular}{|c|c|c|c|c|}
\hline N25 & $\mathrm{AN}$ & 9.80 & 9.25 & -0.55 \\
\hline N25 & $\mathrm{H} 2 \mathrm{O}$ & 9.71 & 8.95 & -0.76 \\
\hline N26 & DMSO & 10.05 & 8.91 & -1.13 \\
\hline N26 & AN & 9.52 & 9.10 & -0.42 \\
\hline N26 & $\mathrm{H} 2 \mathrm{O}$ & 7.46 & 7.73 & 0.27 \\
\hline N27 & DMSO & 7.90 & 6.15 & -1.75 \\
\hline N27 & AN & 7.60 & 6.12 & -1.48 \\
\hline N28 & $A N$ & 8.89 & 8.92 & 0.03 \\
\hline N28 & $\mathrm{H} 2 \mathrm{O}$ & 6.81 & 7.82 & 1.00 \\
\hline N29 & DMSO & 9.93 & 8.79 & -1.14 \\
\hline N29 & AN & 9.57 & 8.97 & -0.61 \\
\hline N29 & $\mathrm{H} 2 \mathrm{O}$ & 7.39 & 7.88 & 0.49 \\
\hline N30 & $\mathrm{H} 2 \mathrm{O}$ & 7.78 & 8.94 & 1.16 \\
\hline N31 & AN & 11.30 & 9.85 & -1.45 \\
\hline N31 & $\mathrm{H} 2 \mathrm{O}$ & 8.43 & 8.30 & -0.14 \\
\hline N32 & DCM & 10.71 & 9.40 & -1.32 \\
\hline N32 & AN & 10.71 & 9.99 & -0.71 \\
\hline N33 & DMSO & 12.20 & 9.95 & -2.26 \\
\hline N33 & AN & 11.80 & 10.13 & -1.67 \\
\hline N33 & $\mathrm{H} 2 \mathrm{O}$ & 7.98 & 8.75 & 0.77 \\
\hline N34 & DCM & 9.83 & 9.50 & -0.33 \\
\hline N34 & AN & 9.73 & 10.03 & 0.30 \\
\hline N35 & DMSO & 11.36 & 9.53 & -1.83 \\
\hline N35 & AN & 11.58 & 9.71 & -1.87 \\
\hline N35 & $\mathrm{H} 2 \mathrm{O}$ & 8.43 & 7.99 & -0.45 \\
\hline N36 & DCM & 8.58 & 8.60 & 0.02 \\
\hline N36 & $\mathrm{AN}$ & 8.74 & 9.36 & 0.62 \\
\hline N37 & AN & 10.37 & 10.12 & -0.25 \\
\hline N38 & AN & 12.32 & 10.60 & -1.73 \\
\hline N39 & $A N$ & 13.16 & 11.09 & -2.07 \\
\hline NA01 & $\mathrm{H} 2 \mathrm{O}$ & 6.48 & 7.22 & 0.73 \\
\hline NAOI & AN & 11.46 & 14.44 & 2.98 \\
\hline NA01 & DMSO & 11.95 & 13.31 & 1.37 \\
\hline NA02 & $\mathrm{H} 2 \mathrm{O}$ & 7.08 & 7.30 & 0.22 \\
\hline NA02 & $A N$ & 9.91 & 13.73 & 3.82 \\
\hline NA02 & DMSO & 11.76 & 12.71 & 0.94 \\
\hline NA03 & $\mathrm{H} 2 \mathrm{O}$ & 6.78 & 5.78 & -1.00 \\
\hline NAO3 & DMSO & 9.90 & 10.77 & 0.87 \\
\hline NA04 & $\mathrm{H} 2 \mathrm{O}$ & 7.00 & 5.08 & -1.92 \\
\hline NA05 & DMSO & 10.52 & 11.73 & 1.21 \\
\hline NA06 & $\mathrm{H} 2 \mathrm{O}$ & 7.72 & 6.35 & -1.37 \\
\hline NA06 & DMSO & 10.59 & 10.37 & -0.22 \\
\hline NA07 & DMSO & 9.64 & 10.93 & 1.29 \\
\hline NA08 & DMSO & 11.30 & 10.76 & -0.54 \\
\hline NA09 & DMSO & 10.58 & 10.86 & 0.29 \\
\hline NA10 & DMSO & 10.40 & 10.26 & -0.14 \\
\hline NA11 & AN & 9.59 & 8.51 & -1.08 \\
\hline NA12 & DMSO & 10.76 & 12.85 & 2.09 \\
\hline NA13 & $\mathrm{H} 2 \mathrm{O}$ & 5.94 & 5.61 & -0.33 \\
\hline NA13 & DMSO & 11.57 & 9.99 & -1.58 \\
\hline NAl 4 & $\mathrm{H} 2 \mathrm{O}$ & 5.70 & 6.53 & 0.83 \\
\hline NA 14 & DMSO & 10.93 & 12.07 & 1.14 \\
\hline NA15 & $\mathrm{H} 2 \mathrm{O}$ & 5.70 & 6.22 & 0.52 \\
\hline NA15 & DMSO & 10.74 & 11.66 & 0.92 \\
\hline NAl 6 & $\mathrm{H} 2 \mathrm{O}$ & 7.14 & 6.53 & -0.61 \\
\hline NA16 & DMSO & 10.49 & 10.96 & 0.47 \\
\hline NA17 & DMSO & 11.15 & 11.84 & 0.70 \\
\hline $\mathrm{NHCl}$ & THF & 9.34 & 13.14 & 3.80 \\
\hline $\mathrm{NHC2}$ & THF & 9.77 & 12.49 & 2.72 \\
\hline $\mathrm{NHC} 3$ & THF & 7.77 & 13.71 & 5.93 \\
\hline $\mathrm{NHC} 4$ & THF & 9.39 & 13.20 & 3.81 \\
\hline $\mathrm{NHC} 5$ & THF & 10.58 & 13.35 & 2.77 \\
\hline OA01 & DMF & 16.79 & 15.24 & -1.55 \\
\hline OA01 & DMSO & 14.10 & 13.91 & -0.19 \\
\hline OA01 & AN & 15.75 & 15.05 & -0.70 \\
\hline
\end{tabular}




\begin{tabular}{|c|c|c|c|c|}
\hline OA02 & DMF & 16.32 & 16.07 & -0.25 \\
\hline OA02 & DMSO & 16.07 & 14.66 & -1.41 \\
\hline OA02 & AN & 14.23 & 15.83 & 1.60 \\
\hline OA03 & DMF & 15.92 & 15.46 & -0.46 \\
\hline OA03 & DMSO & 15.54 & 14.13 & -1.41 \\
\hline OA03 & AN & 12.59 & 15.25 & 2.67 \\
\hline OA04 & DMF & 16.70 & 15.61 & -1.09 \\
\hline OA04 & DMSO & 16.60 & 14.26 & -2.34 \\
\hline OA05 & DMF & 14.16 & 14.70 & 0.54 \\
\hline OA05 & DMSO & 13.02 & 13.52 & 0.51 \\
\hline OA05 & AN & 15.02 & 14.54 & -0.48 \\
\hline OA06 & DMF & 12.67 & 13.05 & 0.37 \\
\hline OA06 & DMSO & 10.70 & 12.09 & 1.39 \\
\hline OA06 & AN & 11.55 & 12.96 & 1.41 \\
\hline OA07 & DMF & 11.69 & 13.70 & 2.01 \\
\hline OA07 & DMSO & 11.96 & 12.70 & 0.75 \\
\hline OA07 & AN & 11.50 & 13.58 & 2.08 \\
\hline OA08 & $\mathrm{ACN}$ & 12.69 & 14.02 & 1.33 \\
\hline OA08 & DMSO & 11.27 & 13.11 & 1.84 \\
\hline OA08 & $\mathrm{DMF}$ & 11.81 & 14.15 & 2.34 \\
\hline OA09 & $\mathrm{ACN}$ & 12.69 & 13.24 & 0.55 \\
\hline OA09 & DMSO & 11.27 & 12.34 & 1.08 \\
\hline OA09 & DMF & 11.81 & 13.34 & 1.53 \\
\hline OA10 & $A C N$ & 10.97 & 13.51 & 2.54 \\
\hline OA10 & DMSO & 11.50 & 12.63 & 1.13 \\
\hline OA10 & DMF & 12.10 & 13.63 & 1.53 \\
\hline OA11 & $\mathrm{ACN}$ & 15.05 & 14.95 & -0.10 \\
\hline OA12 & $\mathrm{DMF}$ & 12.04 & 12.22 & 0.18 \\
\hline OA12 & DMSO & 11.03 & 11.39 & 0.36 \\
\hline OA12 & AN & 12.41 & 12.11 & -0.30 \\
\hline OA13 & DMSO & 12.40 & 13.44 & 1.03 \\
\hline OAl4 & AN & 12.68 & 12.20 & -0.48 \\
\hline OA15 & AN & 11.84 & 10.57 & -1.27 \\
\hline OAl6 & acetone & 12.74 & 11.36 & -1.39 \\
\hline OAl6 & $\mathrm{AN}$ & 11.63 & 9.62 & -2.01 \\
\hline OA17 & acetone & 11.66 & 10.27 & -1.39 \\
\hline OA17 & AN & 10.58 & 8.82 & -1.76 \\
\hline OA18 & AN & 10.26 & 10.69 & 0.43 \\
\hline SAl & $A C N$ & 14.11 & 11.84 & -2.27 \\
\hline SA2 & $A C N$ & 13.59 & 11.97 & -1.62 \\
\hline SA3 & $A C N$ & 13.90 & 11.62 & -2.28 \\
\hline SA4 & $\mathrm{ACN}$ & 14.44 & 11.97 & -2.48 \\
\hline SA5 & $A C N$ & 13.32 & 10.75 & -2.57 \\
\hline SA6 & $\mathrm{ACN}$ & 13.36 & 11.96 & -1.40 \\
\hline SA7 & $\mathrm{ACN}$ & 11.86 & 10.41 & -1.45 \\
\hline SA7 & DMSO & 11.76 & 9.96 & -1.80 \\
\hline
\end{tabular}


Energies

\begin{tabular}{|c|c|c|c|c|c|c|c|}
\hline & \multirow[b]{2}{*}{ solvent } & \multicolumn{2}{|c|}{ Nu } & \multicolumn{2}{|c|}{ Int $\left(\mathrm{NuH}^{+}\right)$} & \multicolumn{2}{|c|}{$\mathrm{NuCH}_{3}{ }^{+}$} \\
\hline & & $E$ (gas) & E (solv) & E (gas) & E (solv) & $E$ (gas) & E (solv) \\
\hline Ar01 & $\mathrm{DCM}$ & -271.5350230 & -271.5457000 & -271.8395880 & -271.9277060 & -311.1491990 & -311.2346270 \\
\hline ArO2 & DCM & -310.8454300 & -310.8567300 & -311.1607380 & -311.2463010 & & \\
\hline Ar03 & DCM & -346.7507020 & -346.7618080 & -347.0743150 & -347.1586620 & & \\
\hline Ar04 & DCM & -386.0611680 & -386.0728540 & -386.3927040 & -386.4746950 & & \\
\hline Ar05 & DCM & -461.2774830 & -461.2894590 & -461.6211360 & -461.7007480 & & \\
\hline Ar06 & AN & -403.1117620 & -403.1294000 & -403.4689850 & -403.5666120 & -442.7799690 & -442.8752550 \\
\hline Ar07 & AN & -862.7162400 & -862.7349730 & -863.0658720 & -863.1676910 & & \\
\hline Ar08 & AN & -517.6357320 & -517.6543860 & -517.9982120 & -518.0935700 & & \\
\hline Ar09 & AN & -2937.3961190 & -2937.4149940 & -2937.7344520 & -2937.8404090 & & \\
\hline Ar10 & AN & -823.4025460 & -823.4206670 & -823.7413180 & -823.8464530 & & \\
\hline Ar11 & AN & -456.0511280 & -456.0732050 & -456.3772060 & -456.4945450 & & \\
\hline $\operatorname{Ar} 12$ & AN & -403.1079550 & -403.1251800 & -403.4567800 & -403.5555370 & & \\
\hline $\operatorname{Ar} 13$ & AN & -419.1549240 & -419.1768100 & -419.5131170 & -419.6108880 & & \\
\hline Ar14 & AN & -439.0286210 & -439.0494420 & -439.3773000 & -439.4805740 & & \\
\hline $\operatorname{Ar} 15$ & DCM & -478.3224830 & -478.3406810 & -478.6736040 & -478.7649500 & & \\
\hline Ar16 & AN & -552.3962330 & -552.4181120 & -552.7321680 & -552.8413720 & & \\
\hline Ar17 & DCM & -403.1012230 & -403.1182080 & -403.4568360 & -403.5461530 & -442.7671350 & -442.8545270 \\
\hline $\operatorname{Ar} 18$ & DCM & -442.4143280 & -442.4321110 & -442.7797630 & -442.8669910 & & \\
\hline Ar19 & AN & -478.3251430 & -478.3432520 & -478.6748270 & -478.7719220 & & \\
\hline Ar20 & AN & -210.1584350 & -210.1712350 & -210.4958690 & -210.5989240 & -249.8099260 & -249.9093990 \\
\hline Ar21 & AN & -288.7818530 & -288.7952640 & -289.1326270 & -289.2285630 & & \\
\hline Ar22 & AN & -288.7797910 & -288.7926700 & -289.1430220 & -289.2372970 & -328.4569310 & -328.5486210 \\
\hline Ar23 & DCM & -249.4600920 & -249.4724870 & -249.8070430 & -249.8979320 & & \\
\hline Ar24 & AN & -328.0837950 & -328.0966760 & -328.4437920 & -328.5347590 & & \\
\hline Ar25 & DCM & -230.0167620 & -230.0233800 & -230.3323130 & -230.4272130 & -269.6503720 & -269.7416560 \\
\hline Ar26 & $\mathrm{DCM}$ & -638.6699010 & -638.6778400 & -639.0039670 & -639.0846150 & & \\
\hline Ar27 & DCM & -269.3315720 & -269.3395320 & -269.6642990 & -269.7543510 & & \\
\hline Ar28 & AN & -680.0161260 & -680.0312950 & -680.3591650 & -680.4468690 & & \\
\hline Ar29 & AN & -696.0259220 & -696.0502390 & -696.3916820 & -696.4845240 & & \\
\hline Ar30 & AN & -844.1416930 & -844.1777380 & -844.4762610 & -844.5964430 & & \\
\hline Ar31 & DCM & -713.9424100 & -713.9506910 & -714.3087470 & -714.3861760 & & \\
\hline Ar32 & DCM & -552.9931960 & -553.0012020 & -553.3081010 & -553.3982580 & -592.6204160 & -592.7077000 \\
\hline Ar33 & DCM & -961.6486670 & -961.6578820 & -961.9784600 & -962.0576170 & & \\
\hline Ar34 & DCM & -592.3038060 & -592.3130050 & -592.6323020 & -592.7192990 & & \\
\hline Ar35 & AN & -667.5161530 & -667.5247560 & -667.8595910 & -667.9500610 & & \\
\hline Ar36 & AN & -367.3967110 & -367.4100260 & -367.7661340 & -367.8579800 & & \\
\hline Ar37 & DCM & -385.7987040 & -385.8145700 & -386.1619480 & -386.2464470 & & \\
\hline Ar38 & AN & -379.8470540 & -379.8644450 & -380.1841870 & -380.2882280 & & \\
\hline CA01 & DMSO & -489.5546010 & -489.6382430 & -490.0815710 & -490.1082950 & & \\
\hline CAO2 & DMSO & -812.5060410 & -812.5856090 & -813.0217470 & -813.0472270 & & \\
\hline CAO3 & DMSO & -568.1551740 & -568.2347380 & -568.6871700 & -568.7078460 & & \\
\hline CA04 & DMSO & -461.9641430 & -462.0485140 & -462.5087950 & -462.5280330 & -501.8198310 & -501.8376710 \\
\hline CA04 & $\mathrm{H} 2 \mathrm{O}$ & -461.9641430 & -462.0600910 & -462.5087950 & -462.5233720 & -501.8198310 & -501.8332040 \\
\hline CA05 & DMSO & -533.8434260 & -533.9272920 & -534.3803060 & -534.4019460 & -573.6875390 & -573.7072250 \\
\hline CA05 & $\mathrm{H} 2 \mathrm{O}$ & -533.8434260 & -533.9374320 & -534.3803060 & -534.3974610 & -573.6875390 & -573.7023520 \\
\hline CA06 & DMSO & -538.1587970 & -538.2408090 & -538.7286670 & -538.7428170 & -578.0385110 & -578.0513470 \\
\hline CA07 & DMSO & -3111.7665390 & -3111.8427040 & -3112.3263500 & -3112.3420850 & & \\
\hline CA08 & DMSO & -630.4344850 & -630.5068620 & -630.9791430 & -630.9973280 & & \\
\hline CA09 & DMSO & -742.7071470 & -742.7768000 & -743.2454340 & -743.2628500 & & \\
\hline CA10 & DMSO & -554.2151030 & -554.2946330 & -554.7704410 & -554.7864340 & -594.0801380 & -594.0946830 \\
\hline CA11 & DMSO & -700.3268270 & -700.3983830 & -700.8735260 & -700.8868910 & -740.1818760 & -740.1945570 \\
\hline CA12 & DMSO & -455.4920520 & -455.5643910 & -456.0294860 & -456.0478410 & & \\
\hline CA13 & DMSO & -494.7984000 & -494.8697610 & -495.3379160 & -495.3554800 & & \\
\hline CA14 & DMSO & -567.7656010 & -567.8341470 & -568.2954810 & -568.3130800 & & \\
\hline CA15 & DMSO & -607.0719890 & -607.1400570 & -607.6039510 & -607.6207530 & & \\
\hline CA16 & DMSO & -402.5216440 & -402.6034310 & -403.0894600 & -403.1020470 & -442.3974480 & -442.4092230 \\
\hline CA17 & DMSO & -514.8050750 & -514.8867880 & -515.3594260 & -515.3728450 & -554.6707310 & -554.6835206 \\
\hline CA17 & $\mathrm{H} 2 \mathrm{O}$ & -514.8050750 & -514.8964750 & -515.3594260 & -515.3668520 & -554.6707310 & -554.6771362 \\
\hline CA18 & DMSO & -475.4963120 & -475.5772600 & -476.0398330 & -476.0527780 & & \\
\hline CA18 & $\mathrm{H} 2 \mathrm{O}$ & -475.4963120 & -475.5869090 & -476.0398330 & -476.0476890 & & \\
\hline CA19 & DMSO & -567.7676970 & -567.8381110 & -568.2880500 & -568.3062150 & -607.6076140 & -607.6250380 \\
\hline
\end{tabular}




\begin{tabular}{|c|c|c|c|c|c|c|c|}
\hline CA19 & $\mathrm{H} 2 \mathrm{O}$ & -567.7676970 & -567.8431030 & -568.2880500 & -568.3005620 & -607.6076140 & -607.6186560 \\
\hline CA20 & DMSO & -680.0387210 & -680.1057610 & -680.5540160 & -680.5714580 & & \\
\hline CA2O & $\mathrm{H} 2 \mathrm{O}$ & -680.0387210 & -680.1102240 & -680.5540160 & -680.5651600 & & \\
\hline CA21 & DMSO & -224.4394360 & -224.5229790 & -224.9780770 & -224.9933040 & & \\
\hline CA21 & $\mathrm{H} 2 \mathrm{O}$ & -224.4394360 & -224.5206050 & -224.9780770 & -224.9899780 & & \\
\hline CA22 & DMSO & -1279.1744950 & -1279.2602820 & -1279.7554280 & -1279.7741550 & -1319.0691840 & -1319.0871520 \\
\hline CA23 & DMSO & -1367.0240510 & -1367.1099450 & -1367.5666010 & -1367.5942840 & & \\
\hline CA24 & DMSO & -1466.2539710 & -1466.3406800 & -1466.8003200 & -1466.8261580 & & \\
\hline $\mathrm{CCO}$ & DCM & -577.4878280 & -577.4941330 & -577.7933060 & -577.8857420 & -617.1052950 & -617.1943330 \\
\hline $\mathrm{CCO} 2$ & DCM & -157.1950080 & -157.2006890 & -157.4834810 & -157.5817760 & -196.8089920 & -196.9021680 \\
\hline $\mathrm{CCO3}$ & DCM & -157.1911410 & -157.1970100 & -157.4872800 & -157.5856560 & & \\
\hline $\mathrm{CCO4}$ & DCM & -526.5326420 & -526.5377770 & -526.8478220 & -526.9320210 & & \\
\hline CCO5 & DCM & -348.9284240 & -348.9388670 & -349.2535220 & -349.3385710 & & \\
\hline $\mathrm{CCO6}$ & DCM & -348.9257820 & -348.9388560 & -349.2503300 & -349.3356890 & & \\
\hline $\mathrm{CCO7}$ & DCM & -309.6168240 & -309.6288960 & -309.9448390 & -310.0314640 & -349.2535220 & -349.3385710 \\
\hline CC08 & DCM & -157.1965950 & -157.2017790 & -157.5114340 & -157.6067830 & -196.8226210 & -196.9143300 \\
\hline CC09 & DCM & -388.2341780 & -388.2473050 & -388.5643760 & -388.6493600 & & \\
\hline $\mathrm{CC} 10$ & DCM & -1826.8646650 & -1826.8758530 & -1827.1870910 & -1827.2754870 & & \\
\hline $\mathrm{CC} 11$ & DCM & -565.8431820 & -565.8504240 & -566.1864960 & -566.2676190 & & \\
\hline $\mathrm{CC} 12$ & DCM & -235.8106060 & -235.8169810 & -236.1345750 & -236.2242290 & & \\
\hline $\mathrm{CC} 13$ & DCM & -348.9274370 & -348.9401620 & -349.2650500 & -349.3487740 & & \\
\hline $\mathrm{CC} 14$ & DCM & -427.5443650 & -427.5576310 & -427.8883780 & -427.9689590 & & \\
\hline $\mathrm{CC} 15$ & DCM & -314.4256330 & -314.4334930 & -314.7538150 & -314.8414850 & & \\
\hline $\mathrm{CC} 16$ & DCM & -275.1185960 & -275.1254490 & -275.4439700 & -275.5319630 & & \\
\hline $\mathrm{CC} 17$ & DCM & -234.5793630 & -234.5879070 & -234.9213200 & -235.0107300 & & \\
\hline $\mathrm{CC} 18$ & DCM & -117.8839270 & -117.8882250 & -118.1762710 & -1 18.2773390 & & \\
\hline $\mathrm{CC} 19$ & DCM & -348.9273770 & -348.9397560 & -349.2651130 & -349.3500470 & & \\
\hline $\mathrm{CC2O}$ & DCM & -196.4991760 & -196.5054100 & -196.8226110 & -196.9158160 & & \\
\hline CC21 & DCM & -196.5025790 & -196.5086680 & -196.8226590 & -196.9144370 & & \\
\hline $\mathrm{CC} 22$ & DCM & -757.5750200 & -757.5875130 & -757.9175100 & -757.9936340 & & \\
\hline $\mathrm{CC} 23$ & DCM & -235.8119630 & -235.8190220 & -236.1345750 & -236.2242290 & & \\
\hline $\mathrm{CC} 24$ & DCM & -195.2652920 & -195.2723540 & -195.5919480 & -195.6868600 & & \\
\hline $\mathrm{CC} 25$ & DCM & -234.6062320 & -234.6140430 & -234.9322060 & -235.0248170 & & \\
\hline $\mathrm{CC} 26$ & DCM & -195.2983460 & -195.3057020 & -195.5974300 & -195.6950900 & & \\
\hline CC27 & DCM & -234.6114940 & -234.6193800 & -234.9322060 & -235.0248180 & & \\
\hline $\mathrm{CC} 28$ & DCM & -273.9263880 & -273.9351710 & -274.2470880 & -274.3386430 & & \\
\hline CC29 & DCM & -155.9682490 & -155.9740070 & -156.2763000 & -156.3701930 & & \\
\hline $\mathrm{CC} 30$ & DCM & -195.2801510 & -195.2876150 & -195.6075680 & -195.6971220 & & \\
\hline CC31 & DCM & -234.5916670 & -234.6006930 & -234.9111490 & -234.9995990 & & \\
\hline $\mathrm{CC} 32$ & DCM & -234.5921340 & -234.6000990 & -234.9293520 & -235.0159650 & & \\
\hline CC33 & DCM & -195.2781140 & -195.2856480 & -195.6026100 & -195.6927500 & & \\
\hline $\mathrm{CC} 34$ & DCM & -234.5906450 & -234.5990280 & -234.9293520 & -235.0159650 & & \\
\hline CC35 & DCM & -234.5912370 & -234.5980650 & -234.9158230 & -235.0037940 & & \\
\hline $\mathrm{CC} 36$ & DCM & -195.2805790 & -195.2869450 & -195.5927920 & -195.6836870 & & \\
\hline CC37 & DCM & -194.0802650 & -194.0892870 & -194.4039690 & -194.4980400 & & \\
\hline CC38 & DCM & -233.3907080 & -233.4001300 & -233.7154590 & -233.8068390 & & \\
\hline CC39 & DCM & -272.6976160 & -272.7067060 & -273.0281200 & -273.1203160 & & \\
\hline CC40 & AN & -310.7931720 & -310.8056290 & -311.1429320 & -311.2325860 & -350.4515920 & -350.5395700 \\
\hline CC41 & $\mathrm{DCM}$ & -308.3733970 & -308.3858050 & -308.6978490 & -308.7821870 & & \\
\hline CX01 & DMSO & -861.6125290 & -861.6340290 & -862.0154920 & -862.1088350 & & \\
\hline $\mathrm{CX02}$ & $\mathrm{DCM}$ & -3435.2110340 & -3435.2374730 & -3435.6093120 & -3435.7026800 & -3474.9206070 & -3475.0119360 \\
\hline CX02 & DMSO & -3435.2110340 & -3435.2335540 & -3435.6093120 & -3435.7064080 & -3474.9206070 & -3475.0149500 \\
\hline CX03 & DMSO & -995.5693270 & -995.5942950 & -995.9873430 & -996.0753200 & & \\
\hline CX04 & DMSO & -976.1393360 & -976.1622810 & -976.5483470 & -976.6393950 & & \\
\hline CX05 & DMSO & -609.4773220 & -609.4986500 & -609.8592970 & -609.9662570 & & \\
\hline CX06 & DMSO & -784.4298030 & -784.4477690 & -784.8333680 & -784.9252640 & & \\
\hline CX07 & DMSO & -840.5156150 & -840.5374100 & -840.9222760 & -841.0258820 & -880.2346290 & -880.3353370 \\
\hline CX08 & DMSO & -952.7837470 & -952.8057060 & -953.1870530 & -953.2905100 & & \\
\hline CX09 & $\mathrm{DCM}$ & -379.7811670 & -379.7908960 & -380.1330440 & -380.2230910 & & \\
\hline CX10 & DCM & -2953.3784370 & -2953.3903060 & -2953.7254810 & -2953.8192880 & & \\
\hline CX11 & DCM & -472.0324570 & -472.0468600 & -472.3673200 & -472.4714270 & & \\
\hline CX12 & DCM & -584.2991260 & -584.3124480 & -584.6313230 & -584.7349940 & & \\
\hline CX13 & $\mathrm{DCM}$ & -301.3915470 & -301.3988840 & -301.7195830 & -301.8174560 & -341.0378870 & -341.1308220 \\
\hline CX14 & DMSO & -379.7725980 & -379.7905120 & -380.1614030 & -380.2658730 & & \\
\hline CX15 & DMSO & -613.4532010 & -613.4689010 & -613.8758080 & -613.9673240 & -653.1867040 & -653.2745480 \\
\hline
\end{tabular}




\begin{tabular}{|c|c|c|c|c|c|c|c|}
\hline CX16 & DMSO & -554.7260030 & -554.7410220 & -555.1350440 & -555.2277220 & -594.4479540 & -594.5367460 \\
\hline CX17 & DMSO & -440.1785930 & -440.1954280 & -440.5807750 & -440.6776000 & & \\
\hline CX18 & DMSO & -631.9095870 & -631.9309700 & -632.3164320 & -632.4106790 & & \\
\hline CX19 & DMSO & -1091.5095930 & -1091.5299740 & -1091.9106760 & -1092.0061710 & & \\
\hline CX2O & DMSO & -765.8704390 & -765.8995840 & -766.3023000 & -766.3898350 & & \\
\hline CX21 & DMSO & -785.5440300 & -785.5680580 & -785.9590220 & -786.0491040 & & \\
\hline CX22 & DMSO & -785.5417860 & -785.5646820 & -785.9571290 & -786.0478430 & & \\
\hline CX23 & DMSO & -592.4257260 & -592.4390640 & -592.8279650 & -592.9329540 & & \\
\hline CX24 & DCM & -305.9734750 & -305.9832490 & -306.3345440 & -306.4224460 & -345.6469050 & -345.7331360 \\
\hline CX25 & DCM & -228.5560260 & -228.5633920 & -228.9075190 & -228.9985460 & & \\
\hline EE01 & DCM & -718.5394330 & -718.5500710 & -718.9035610 & -718.9815160 & & \\
\hline EEO2 & AN & -679.2239900 & -679.2318640 & -679.5883410 & -679.6726520 & & \\
\hline EE03 & DCM & -601.8114010 & -601.8182840 & -602.1713650 & -602.2516610 & -641.4800960 & -641.5590460 \\
\hline EE04 & DCM & -1085.7393750 & -1085.7480970 & -1086.1307770 & -1086.2016560 & & \\
\hline EE05 & DCM & -754.4582930 & -754.4683540 & -754.8427710 & -754.9190410 & & \\
\hline EE06 & $\mathrm{AN}$ & -715.1419700 & -715.1494060 & -715.5297800 & -715.6116950 & & \\
\hline EE06 & DCM & -715.1419700 & -715.1505430 & -715.5297800 & -715.6067310 & -754.8415810 & -754.9170658 \\
\hline EE07 & DCM & -793.5421200 & -793.5553200 & -793.9129860 & -793.9898840 & & \\
\hline EE08 & DCM & -639.8939320 & -639.9019230 & -640.2542150 & -640.3315430 & & \\
\hline EE09 & DCM & -868.7666360 & -868.7796560 & -869.1500420 & -869.2259610 & & \\
\hline EE10 & DCM & -899.5897660 & -899.5959010 & -899.9153270 & -899.9966420 & -939.2247560 & -939.3038360 \\
\hline EE11 & DCM & -719.7360840 & -719.7443260 & -720.1009500 & -720.1781350 & & \\
\hline EE12 & DCM & -755.6489260 & -755.6571410 & -756.0329570 & -756.1074370 & & \\
\hline EE12 & $\mathrm{AN}$ & -755.6489260 & -755.6556700 & -756.0329570 & -756.1119050 & & \\
\hline EE13 & DCM & -754.4533220 & -754.4619300 & -754.8415810 & -754.9170650 & & \\
\hline EE14 & DCM & -1202.4637840 & -1202.4750120 & -1202.8204330 & -1202.8985760 & & \\
\hline EE15 & DCM & -231.2099930 & -231.2171000 & -231.5476830 & -231.6420840 & & \\
\hline EE16 & DCM & -232.4096070 & -232.4166850 & -232.7514910 & -232.8426830 & & \\
\hline EE17 & DCM & -641.1174270 & -641.1254400 & -641.4660200 & -641.5452680 & & \\
\hline EE18 & DCM & -311.0312070 & -311.0395300 & -311.3733190 & -311.4607400 & & \\
\hline EE19 & AN & -831.6565780 & -831.6702030 & -832.0312790 & -832.1115700 & & \\
\hline EE2 & DCM & -679.2239900 & -679.2334410 & -679.5883410 & -679.6676500 & & \\
\hline En01 & DCM & -485.3039680 & -485.3184480 & -485.6978810 & -485.7814910 & & \\
\hline EnO2 & DCM & -521.2107980 & -521.2260510 & -521.5955310 & -521.6835330 & -560.9054530 & -560.9919620 \\
\hline En03 & DCM & -445.9903400 & -446.0042120 & -446.3923080 & -446.4789500 & & \\
\hline En04 & DCM & -540.6424700 & -540.6592660 & -541.0336360 & -541.1188990 & & \\
\hline En05 & DCM & -445.9908970 & -446.0047890 & -446.3919980 & -446.4760150 & -485.7011440 & -485.7839090 \\
\hline En06 & $\mathrm{DCM}$ & -481.8974770 & -481.9120880 & -482.2897040 & -482.3779980 & & \\
\hline En07 & DCM & -406.6777270 & -406.6910060 & -407.0796020 & -407.1638920 & & \\
\hline En08 & DCM & -576.5480460 & -576.5657510 & -576.9338820 & -577.0231210 & & \\
\hline En09 & DCM & -404.4812680 & -404.4939960 & -404.8596880 & -404.9505210 & & \\
\hline En10 & DCM & -596.2166650 & -596.2378470 & -596.5904540 & -596.6850810 & & \\
\hline En10 & AN & -596.2166650 & -596.2367330 & -596.5904540 & -596.6913130 & & \\
\hline En11 & AN & -560.3111100 & -560.3296910 & -560.6932810 & -560.7886000 & & \\
\hline En12 & DCM & -520.9976010 & -521.0182070 & -521.3801370 & -521.4699300 & -560.6903140 & -560.7781010 \\
\hline En12 & $\mathrm{AN}$ & -520.9976010 & -521.0165780 & -521.3801370 & -521.4748960 & -560.6903140 & -560.7827680 \\
\hline En13 & AN & -827.2565890 & -827.2794130 & -827.6386190 & -827.7345980 & & \\
\hline En14 & AN & -791.3504010 & -791.3718210 & -791.7399750 & -791.8315110 & & \\
\hline En15 & AN & -752.0368960 & -752.0589670 & -752.4300340 & -752.5221480 & & \\
\hline En16 & AN & -866.5619970 & -866.5851740 & -866.9582440 & -867.0491430 & & \\
\hline En17 & AN & -844.2906590 & -844.3172020 & -844.6739810 & -844.7762590 & & \\
\hline En18 & AN & -956.5576360 & -956.5831050 & -956.9393190 & -957.0405880 & & \\
\hline En19 & AN & -560.3092800 & -560.3238150 & -560.7039230 & -560.7911230 & & \\
\hline En20 & AN & -596.2154740 & -596.2314140 & -596.6024200 & -596.6948080 & & \\
\hline En20 & DCM & -596.2154740 & -596.2328790 & -596.6024200 & -596.6895520 & & \\
\hline En21 & AN & -598.4192690 & -598.4359320 & -598.8210790 & -598.9079870 & & \\
\hline En22 & AN & -559.1094550 & -559.1264950 & -559.5137590 & -559.6006930 & & \\
\hline En23 & DCM & -443.7876910 & -443.8003460 & -444.1702770 & -444.2594590 & & \\
\hline En24 & DCM & -404.4744300 & -404.4873230 & -404.8595000 & -404.9501640 & & \\
\hline En25 & AN & -403.3227970 & -403.3389520 & -403.6806600 & -403.7804140 & -442.9908170 & -443.0885330 \\
\hline En26 & AN & -442.6363570 & -442.6544520 & -442.9934410 & -443.0912850 & & \\
\hline En27 & AN & -595.0653720 & -595.0875120 & -595.4311140 & -595.5250220 & & \\
\hline En28 & AN & -443.8294780 & -443.8461730 & -444.1923590 & -444.2874840 & & \\
\hline En29 & AN & -709.3682000 & -709.3924290 & -709.7350850 & -709.8277570 & & \\
\hline En30 & AN & -517.6379720 & -517.6578360 & -518.0002660 & -518.0957630 & -557.3084400 & -557.4024180 \\
\hline
\end{tabular}




\begin{tabular}{|c|c|c|c|c|c|c|c|}
\hline En31 & AN & -977.2418070 & -977.2626760 & -977.6006470 & -977.6986630 & & \\
\hline En32 & AN & -556.9487130 & -556.9692010 & -557.3158860 & -557.4090890 & & \\
\hline N01 & DCM & -248.2656600 & -248.2767300 & -248.6300050 & -248.7236720 & -287.9389950 & -288.0273520 \\
\hline N01 & $\mathrm{H} 2 \mathrm{O}$ & -248.2656600 & -248.2729460 & -248.6300050 & -248.7251350 & -287.9389950 & -288.0261490 \\
\hline N02 & DCM & -707.8697100 & -707.8810750 & -708.2287000 & -708.3226730 & & \\
\hline N02 & $\mathrm{H} 2 \mathrm{O}$ & -707.8697100 & -707.8762840 & -708.2287000 & -708.3237810 & & \\
\hline N03 & DCM & -362.7950420 & -362.8075580 & -363.1715460 & -363.2593550 & & \\
\hline N03 & $\mathrm{H} 2 \mathrm{O}$ & -362.7950420 & -362.8040430 & -363.1715460 & -363.2599110 & & \\
\hline N04 & DCM & -382.2274060 & -382.2435570 & -382.6208130 & -382.7049820 & -421.9264120 & -422.0069390 \\
\hline N04 & DMF & -382.2274060 & -382.2417260 & -382.6208130 & -382.7091380 & -421.9264120 & -422.0101340 \\
\hline N04 & DMSO & -382.2274060 & -382.2399860 & -382.6208130 & -382.7077410 & -421.9264120 & -422.0084700 \\
\hline N04 & $\mathrm{H} 2 \mathrm{O}$ & -382.2274060 & -382.2385890 & -382.6208130 & -382.7032580 & -421.9264120 & -422.0026890 \\
\hline N04 & AN & -382.2274060 & -382.2432180 & -382.6208130 & -382.7104080 & -421.9264120 & -422.0115220 \\
\hline N04 & THF & -382.2274060 & -382.2410270 & -382.6208130 & -382.7010400 & -421.9264120 & -422.0029490 \\
\hline N05 & DCM & -459.6494640 & -459.6664000 & -460.0468330 & -460.1306910 & & \\
\hline N06 & AN & -395.8553090 & -395.8723720 & -396.2134460 & -396.3116280 & & \\
\hline N07 & AN & -435.1617730 & -435.1778300 & -435.5278930 & -435.6196810 & & \\
\hline N08 & AN & -419.1568530 & -419.1786870 & -419.5360760 & -419.6347030 & & \\
\hline N09 & DMSO & -226.2122190 & -226.2301490 & -226.5821870 & -226.6890660 & -265.8910500 & -265.9907110 \\
\hline N09 & AN & -226.2122190 & -226.2316210 & -226.5821870 & -226.6898630 & -265.8910500 & -265.9919630 \\
\hline N09 & $\mathrm{H} 2 \mathrm{O}$ & -226.2122190 & -226.2273570 & -226.5821870 & -226.6827800 & -265.8910500 & -265.9832790 \\
\hline N10 & AN & -265.5146290 & -265.5330280 & -265.8919660 & -265.9929000 & & \\
\hline N10 & $\mathrm{H} 2 \mathrm{O}$ & -265.5146290 & -265.5270380 & -265.8919660 & -265.9841830 & & \\
\hline N11 & AN & -457.2480480 & -457.2697600 & -457.6264510 & -457.7251840 & & \\
\hline N12 & AN & -634.8879960 & -634.9052350 & -635.2742200 & -635.3653240 & & \\
\hline N13 & AN & -304.8382320 & -304.8559840 & -305.2221650 & -305.3197540 & & \\
\hline N14 & DCM & -419.3758700 & -419.3941280 & -419.7542830 & -419.8476070 & & \\
\hline N15 & DCM & -266.7155260 & -266.7306610 & -267.1064890 & -267.1973260 & & \\
\hline N16 & DCM & -286.5904800 & -286.6007990 & -286.9601850 & -287.0509270 & -326.2675620 & -326.3541800 \\
\hline N17 & DCM & -250.6650910 & -250.6762840 & -251.0424870 & -251.1332750 & & \\
\hline N18 & DCM & -609.5540890 & -609.5654040 & -609.9233150 & -610.0126180 & -649.2321630 & -649.3176520 \\
\hline N19 & DCM & -361.3766440 & -361.3968950 & -361.7779820 & -361.8674760 & & \\
\hline N2O & DCM & -399.4857160 & -399.5079040 & -399.8933980 & -399.9829610 & & \\
\hline N21 & DCM & -362.5627170 & -362.5791450 & -362.9676280 & -363.0539840 & -402.2671580 & -402.3500690 \\
\hline N22 & AN & -287.5848140 & -287.5990630 & -287.9293000 & -288.0441670 & -327.2392850 & -327.3460670 \\
\hline N22 & $\mathrm{H} 2 \mathrm{O}$ & -287.5848140 & -287.5933980 & -287.9293000 & -288.0382580 & -327.2392850 & -327.3385160 \\
\hline N23 & AN & -747.1887350 & -747.2041960 & -747.5271920 & -747.6467350 & & \\
\hline N24 & AN & -326.8941730 & -326.9085980 & -327.2431420 & -327.3556180 & & \\
\hline N24 & $\mathrm{H} 2 \mathrm{O}$ & -326.8941730 & -326.9019800 & -327.2431420 & -327.3487070 & & \\
\hline N25 & AN & -402.1075320 & -402.1229000 & -402.4599380 & -402.5719690 & & \\
\hline N25 & $\mathrm{H} 2 \mathrm{O}$ & -402.1075320 & -402.1175660 & -402.4599380 & -402.5656080 & & \\
\hline N26 & DMSO & -174.4598040 & -174.4659740 & -174.8198670 & -174.9336090 & & \\
\hline N26 & AN & -174.4598040 & -174.4678770 & -174.8198670 & -174.9346650 & & \\
\hline N26 & $\mathrm{H} 2 \mathrm{O}$ & -174.4598040 & -174.4659330 & -174.8198670 & -174.9310420 & & \\
\hline N27 & DMSO & -432.9439140 & -432.9504350 & -433.2729100 & -433.3985450 & & \\
\hline N27 & AN & -432.9439140 & -432.9521030 & -433.2729100 & -433.3992480 & & \\
\hline N28 & AN & -213.7754870 & -213.7834260 & -214.1416540 & -214.2510870 & -253.4476140 & -253.5499060 \\
\hline N28 & $\mathrm{H} 2 \mathrm{O}$ & -213.7754870 & -213.7800020 & -214.1416540 & -214.2467160 & -253.4476140 & -253.5437500 \\
\hline N29 & DMSO & -326.8827380 & -326.8926430 & -327.2449720 & -327.3572340 & & \\
\hline N29 & AN & -326.8827380 & -326.8958120 & -327.2449720 & -327.3595880 & & \\
\hline N29 & $\mathrm{H} 2 \mathrm{O}$ & -326.8827380 & -326.8900760 & -327.2449720 & -327.3533180 & & \\
\hline N30 & $\mathrm{H} 2 \mathrm{O}$ & -213.7612450 & -213.7655990 & -214.1341710 & -214.2335920 & & \\
\hline N31 & AN & -212.5568170 & -212.5647250 & -212.9293750 & -213.0360270 & -252.2383120 & -252.3387080 \\
\hline N31 & $\mathrm{H} 2 \mathrm{O}$ & -212.5568170 & -212.5634590 & -212.9293750 & -213.0315690 & & \\
\hline N32 & DCM & -251.8608640 & -251.8693070 & -252.2383060 & -252.3316290 & -291.5448130 & -291.6334080 \\
\hline N32 & AN & -251.8608640 & -251.8681380 & -252.2383060 & -252.3387000 & -291.5448130 & -291.6395330 \\
\hline N33 & DMSO & -251.8739700 & -251.8803030 & -252.2479960 & -252.3519750 & & \\
\hline N33 & AN & -251.8739700 & -251.8824820 & -252.2479960 & -252.3532780 & & \\
\hline N33 & $\mathrm{H} 2 \mathrm{O}$ & -251.8739700 & -251.8798390 & -252.2479960 & -252.3478430 & & \\
\hline N34 & DCM & -291.1772540 & -291.1865380 & -291.5574430 & -291.6496790 & & \\
\hline N34 & AN & -291.1772540 & -291.1852450 & -291.5574430 & -291.6565070 & & \\
\hline N35 & DMSO & -287.7799340 & -287.7884100 & -288.1428320 & -288.2548740 & -327.4533080 & -327.5583470 \\
\hline N35 & AN & -287.7799340 & -287.7900400 & -288.1428320 & -288.2555580 & -327.4533080 & -327.5593240 \\
\hline N35 & $\mathrm{H} 2 \mathrm{O}$ & -287.7799340 & -287.7911710 & -288.1428320 & -288.2531150 & -327.4533080 & -327.5547780 \\
\hline N36 & $\mathrm{DCM}$ & -327.0835420 & -327.0936220 & -327.4533090 & -327.5509110 & & \\
\hline
\end{tabular}




\begin{tabular}{|c|c|c|c|c|c|c|c|}
\hline N36 & AN & -327.0835420 & -327.0930820 & -327.4533090 & -327.5593290 & & \\
\hline N37 & AN & -174.4444300 & -174.4497280 & -174.8157830 & -174.9198810 & -214.1251120 & -214.2233530 \\
\hline N38 & AN & -329.2743860 & -329.2840430 & -329.6596490 & -329.7575450 & -368.9690280 & -369.0617750 \\
\hline N39 & AN & -345.3007790 & -345.3135340 & -345.6799150 & -345.7834090 & -384.9898390 & -385.0881020 \\
\hline NA01 & $\mathrm{H} 2 \mathrm{O}$ & -322.9448290 & -323.0495650 & -323.5112690 & -323.5276120 & -362.8148760 & -362.8283460 \\
\hline NA01 & AN & -322.9448290 & -323.0396090 & -323.5112690 & -323.5292900 & -362.8148760 & -362.8313040 \\
\hline NA01 & DMSO & -322.9448290 & -323.0373510 & -323.5112690 & -323.5277440 & -362.8148760 & -362.8292420 \\
\hline NA02 & $\mathrm{H} 2 \mathrm{O}$ & -322.9482720 & -323.0462220 & -323.4958670 & -323.5195540 & -362.7968180 & -362.8180690 \\
\hline NAO2 & AN & -322.9482720 & -323.0372350 & -323.4958670 & -323.5201580 & -362.7968180 & -362.8200720 \\
\hline NA02 & DMSO & -322.9482720 & -323.0351880 & -323.4958670 & -323.5185830 & -362.7968180 & -362.8181080 \\
\hline NA03 & $\mathrm{H} 2 \mathrm{O}$ & -466.7748490 & -466.8781370 & -467.3237580 & -467.3505760 & & \\
\hline NA03 & DMSO & -466.7748490 & -466.8745660 & -467.3237580 & -467.3523050 & & \\
\hline NA04 & $\mathrm{H} 2 \mathrm{O}$ & -542.0201790 & -542.1352880 & -542.5690830 & -542.6085260 & & \\
\hline NA05 & DMSO & -379.2978340 & -379.3908780 & -379.8531780 & -379.8734350 & & \\
\hline NA06 & $\mathrm{H} 2 \mathrm{O}$ & -395.3075200 & -395.4028180 & -395.8553090 & -395.8714230 & & \\
\hline NA06 & DMSO & -395.3075200 & -395.3975590 & -395.8553090 & -395.8694720 & & \\
\hline NA07 & DMSO & -376.1658810 & -376.2623280 & -376.7248920 & -376.7477820 & & \\
\hline NA08 & DMSO & -358.8790650 & -358.9697290 & -359.4367410 & -359.4531760 & & \\
\hline NA09 & DMSO & -360.1108320 & -360.2026330 & -360.6695730 & -360.6888670 & & \\
\hline NA10 & DMSO & -512.5374940 & -512.6272840 & -513.0914700 & -513.1095040 & & \\
\hline NA11 & AN & -947.8325440 & -947.9213300 & -948.3562490 & -948.3794060 & & \\
\hline NA12 & DMSO & -225.6418740 & -225.7430490 & -226.2122190 & -226.2301490 & -265.5146290 & -265.5311450 \\
\hline NA13 & $\mathrm{H} 2 \mathrm{O}$ & -411.4027100 & -411.4999620 & -411.9440120 & -411.9677880 & -451.2484220 & -451.2688300 \\
\hline NA13 & DMSO & -411.4027100 & -411.4957210 & -411.9440120 & -411.9684660 & -451.2484220 & -451.2710890 \\
\hline NA14 & $\mathrm{H} 2 \mathrm{O}$ & -453.5951580 & -453.6929710 & -454.1416600 & -454.1628180 & & \\
\hline NA14 & DMSO & -453.5951580 & -453.6815630 & -454.1416600 & -454.1647860 & & \\
\hline NA15 & $\mathrm{H} 2 \mathrm{O}$ & -414.2847770 & -414.3828720 & -414.8286390 & -414.8507910 & -454.1312440 & -454.1508400 \\
\hline NA15 & DMSO & -414.2847770 & -414.3718530 & -414.8286390 & -414.8528180 & -454.1312440 & -454.1533070 \\
\hline NA16 & $\mathrm{H} 2 \mathrm{O}$ & -640.5321670 & -640.6251430 & -641.0692350 & -641.0914230 & & \\
\hline NA16 & DMSO & -640.5321670 & -640.6213540 & -641.0692350 & -641.0960430 & & \\
\hline NA17 & DMSO & -506.4199140 & -506.5057920 & -506.9806060 & -506.9920310 & & \\
\hline NHCl & THF & -925.2982570 & -925.3271280 & -925.7352280 & -925.8156700 & & \\
\hline NHC2 & THF & -924.1039360 & -924.1337150 & -924.5416200 & -924.6201990 & & \\
\hline NHC3 & THF & -422.7063810 & -422.7262780 & -423.1377610 & -423.2189280 & -462.4470430 & -462.5255990 \\
\hline NHC4 & THF & -611.0351230 & -611.0601790 & -611.4643520 & -611.5471790 & -650.7857380 & -650.8647900 \\
\hline NHC5 & THF & -725.5520560 & -725.5810280 & -725.9953560 & -726.0711330 & & \\
\hline OA01 & DMF & -306.8881470 & -306.9784410 & -307.4573080 & -307.4692470 & -346.7507020 & -346.7599880 \\
\hline OA01 & DMSO & -306.8881470 & -306.9776910 & -307.4573080 & -307.4679570 & -346.7507020 & -346.7584080 \\
\hline OA01 & AN & -306.8881470 & -306.9802350 & -307.4573080 & -307.4704310 & -346.7507020 & -346.7610190 \\
\hline OAO2 & DMF & -421.4077960 & -421.4978070 & -421.9804440 & -421.9938970 & & \\
\hline OA02 & DMSO & -421.4077960 & -421.4969470 & -421.9804440 & -421.9924930 & & \\
\hline OA02 & AN & -421.4077960 & -421.4996840 & -421.9804440 & -421.9948730 & & \\
\hline OA03 & DMF & -464.1213660 & -464.2096630 & -464.6897540 & -464.7030710 & & \\
\hline OA03 & DMSO & -464.1213660 & -464.2083180 & -464.6897540 & -464.7011730 & & \\
\hline OA03 & AN & -464.1213660 & -464.2118730 & -464.6897540 & -464.7045590 & & \\
\hline OA04 & DMF & -346.1959560 & -346.2856660 & -346.7669000 & -346.7790560 & & \\
\hline OA04 & DMSO & -346.1959560 & -346.2847400 & -346.7669000 & -346.7775800 & & \\
\hline OA05 & DMF & -766.5026250 & -766.5862870 & -767.0606250 & -767.0736780 & & \\
\hline OA05 & DMSO & -766.5026250 & -766.5853900 & -767.0606250 & -767.0722950 & & \\
\hline OA05 & AN & -766.5026250 & -766.5878890 & -767.0606250 & -767.0748030 & & \\
\hline OA06 & DMF & -399.1701950 & -399.2485360 & -399.7086410 & -399.7255100 & & \\
\hline OA06 & DMSO & -399.1701950 & -399.2474780 & -399.7086410 & -399.7240430 & & \\
\hline OA06 & AN & -399.1701950 & -399.2500320 & -399.7086410 & -399.7269740 & & \\
\hline OA07 & DMF & -644.0039620 & -644.0813180 & -644.5517650 & -644.5635940 & -683.8455170 & -683.8546790 \\
\hline OA07 & DMSO & -644.0039620 & -644.0802680 & -644.5517650 & -644.5621000 & -683.8455170 & -683.8528960 \\
\hline OA07 & AN & -644.0039620 & -644.0829000 & -644.5517650 & -644.5649480 & -683.8455170 & -683.8558440 \\
\hline OA08 & $\mathrm{ACN}$ & -643.9986080 & -644.0790410 & -644.5503990 & -644.5633620 & & \\
\hline OA08 & DMSO & -643.9986080 & -644.0763180 & -644.5503990 & -644.5605080 & & \\
\hline OA08 & DMF & -643.9986080 & -644.0773530 & -644.5503990 & -644.5620020 & & \\
\hline OA09 & $\mathrm{ACN}$ & -644.0006460 & -644.0835480 & -644.5482630 & -644.5621740 & & \\
\hline OA09 & DMSO & -644.0006460 & -644.0807920 & -644.5482630 & -644.5590640 & & \\
\hline OA09 & DMF & -644.0006460 & -644.0818270 & -644.5482630 & -644.5605790 & & \\
\hline OA10 & $\mathrm{ACN}$ & -511.4287710 & -511.5075300 & -511.9724770 & -511.9888750 & & \\
\hline OA10 & DMSO & -511.4287710 & -511.5052920 & -511.9724770 & -511.9865300 & & \\
\hline OA10 & DMF & -511.4287710 & -511.5063430 & -511.9724770 & -511.9880010 & & \\
\hline
\end{tabular}




$\begin{array}{llrrrrrr}\text { OA11 } & \text { ACN } & -421.4169500 & -421.5082390 & -421.9836580 & -421.9981290 & & \\ \text { OA12 } & \text { DMF } & -511.4441380 & -511.5186230 & -511.9753120 & -511.9912500 & & \\ \text { OA12 } & \text { DMSO } & -511.4441380 & -511.5175490 & -511.9753120 & -511.9897810 & & \\ \text { OA12 } & \text { AN } & -511.4441380 & -511.5197430 & -511.9753120 & -511.9921270 & & \\ \text { OA13 } & \text { DMSO } & -621.3633000 & -621.4366900 & -621.9101880 & -621.9201680 & & \\ \text { OA14 } & \text { AN } & -228.5209360 & -228.6165980 & -229.0887380 & -229.0983390 & -268.3824430 & -268.3899800 \\ \text { OA15 } & \text { AN } & -420.2657920 & -420.3587790 & -420.8199900 & -420.8334710 & & \\ \text { OA16 } & \text { ACetone } & -624.7986850 & -624.8784310 & -625.3330970 & -625.3497260 & & \\ \text { OA16 } & \text { AN } & -624.7986850 & -624.8798680 & -625.3330970 & -625.3490730 & & \\ \text { OA17 } & \text { ACetone } & -829.3202450 & -829.3947680 & -829.8409640 & -829.8607320 & & \\ \text { OA17 } & \text { AN } & -829.3202450 & -829.3956490 & -829.8409640 & -829.8600050 & & \\ \text { OA18 } & \text { AN } & -303.7647750 & -303.8563040 & -304.3199150 & -304.3286690 & & \\ \text { SA01 } & \text { ACN } & -1046.5174610 & -1046.6122260 & -1047.0577160 & -1047.0745900 & -1086.3682220 & -1086.3838480 \\ \text { SA02 } & \text { ACN } & -1085.8279910 & -1085.9196440 & -1086.3663240 & -1086.3829010 & -1125.6761170 & -1125.6914020 \\ \text { SA03 } & \text { ACN } & -1121.7373480 & -1121.8269780 & -1122.2702720 & -1122.2877830 & -1161.5803060 & -1161.5965600 \\ \text { SA04 } & \text { ACN } & -969.0947390 & -969.1865610 & -969.6345550 & -969.6489160 & -1008.9451650 & -1008.9582310 \\ \text { SA05 } & \text { ACN } & -988.9750900 & -989.0635180 & -989.5087190 & -989.5179670 & -1028.8199810 & -1028.8293570 \\ \text { SA06 } & \text { ACN } & -551.4800210 & -551.5711450 & -552.0262370 & -552.0350860 & -591.3370000 & -591.3455730 \\ \text { SA07 } & \text { ACN } & -780.3095400 & -780.4012740 & -780.8369690 & -780.8536960 & -820.1609840 & -820.1800240 \\ \text { SA07 } & \text { DMSO } & -780.3095400 & -780.3986740 & -780.8369690 & -780.8528547 & -820.1609840 & -820.1784870\end{array}$


Geometries of Nucleophiles

CC1

$\begin{array}{lrrr}\mathrm{C} & -0.894 & 1.378 & 0.000 \\ \mathrm{H} & -1.965 & 1.538 & 0.000 \\ \mathrm{H} & -0.245 & 2.240 & 0.000 \\ \mathrm{C} & -0.423 & 0.143 & 0.000 \\ \mathrm{C} & -1.259 & -1.106 & 0.000 \\ \mathrm{H} & -0.639 & -1.998 & -0.002 \\ \mathrm{H} & -1.897 & -1.133 & 0.884 \\ \mathrm{H} & -1.900 & -1.131 & -0.882 \\ \mathrm{Cl} & 1.300 & -0.118 & 0.000\end{array}$

CC2

$\begin{array}{lrrr}\mathrm{C} & 0.532 & 0.395 & 0.000 \\ \mathrm{H} & 0.380 & 1.472 & 0.000 \\ \mathrm{C} & -0.532 & -0.395 & 0.000 \\ \mathrm{H} & -0.380 & -1.472 & 0.000 \\ \mathrm{C} & 1.951 & -0.079 & 0.000 \\ \mathrm{H} & 2.489 & 0.289 & -0.877 \\ \mathrm{H} & 2.488 & 0.288 & 0.878 \\ \mathrm{H} & 2.002 & -1.168 & 0.000 \\ \mathrm{C} & -1.951 & 0.079 & 0.000 \\ \mathrm{H} & -2.002 & 1.168 & 0.000 \\ \mathrm{H} & -2.488 & -0.289 & -0.877 \\ \mathrm{H} & -2.488 & -0.289 & 0.878\end{array}$

cc3

$\begin{array}{lrrr}\mathrm{C} & -0.664 & 0.709 & 0.000 \\ \mathrm{H} & -1.187 & 1.658 & 0.000 \\ \mathrm{C} & 0.664 & 0.709 & 0.000 \\ \mathrm{H} & 1.187 & 1.658 & 0.000 \\ \mathrm{C} & -1.492 & -0.545 & 0.000 \\ \mathrm{H} & -2.557 & -0.323 & 0.002 \\ \mathrm{H} & -1.278 & -1.159 & -0.878 \\ \mathrm{H} & -1.276 & -1.161 & 0.876 \\ \mathrm{C} & 1.492 & -0.545 & 0.000 \\ \mathrm{H} & 1.274 & -1.162 & -0.875 \\ \mathrm{H} & 2.557 & -0.323 & -0.004 \\ \mathrm{H} & 1.280 & -1.158 & 0.879\end{array}$

CC4

$\begin{array}{rrrr}\mathrm{C} & 1.816 & 1.414 & 0.000 \\ \mathrm{H} & 2.894 & 1.553 & 0.000 \\ \mathrm{H} & 1.217 & 2.318 & 0.000 \\ \mathrm{C} & 1.266 & 0.202 & 0.000 \\ \mathrm{C} & 2.123 & -1.038 & 0.000 \\ \mathrm{H} & 1.914 & -1.657 & 0.877 \\ \mathrm{H} & 1.914 & -1.657 & -0.877\end{array}$

$\begin{array}{lrrr}\mathrm{H} & 3.186 & -0.794 & 0.000 \\ \mathrm{Si} & -0.600 & -0.016 & 0.000 \\ \mathrm{C} & -1.442 & 1.656 & 0.001 \\ \mathrm{H} & -1.174 & 2.237 & -0.883 \\ \mathrm{H} & -2.527 & 1.530 & 0.001 \\ \mathrm{H} & -1.174 & 2.236 & 0.885 \\ \mathrm{C} & -1.100 & -0.985 & 1.526 \\ \mathrm{H} & -2.182 & -1.127 & 1.556 \\ \mathrm{H} & -0.636 & -1.974 & 1.536 \\ \mathrm{H} & -0.802 & -0.464 & 2.437 \\ \mathrm{C} & -1.099 & -0.984 & -1.527 \\ \mathrm{H} & -2.181 & -1.126 & -1.557 \\ \mathrm{H} & -0.801 & -0.462 & -2.438 \\ \mathrm{H} & -0.636 & -1.973 & -1.537\end{array}$

CC5

$\mathrm{H}$

C

$\mathrm{H}$

C

$\mathrm{H}$

$\mathrm{H}$

$\mathrm{H}$

C

C

C

C

$\mathrm{H}$

C

$\mathrm{H}$

C

$\mathrm{H}$

$\mathrm{H}$

$\mathrm{H}$

2.389

0.385

0.005

$\begin{array}{lll}2.170 & 1.448 & 0.010\end{array}$

$\begin{array}{lll}1.378 & -0.500 & 0.001\end{array}$

$\begin{array}{lll}1.626 & -1.560 & -0.001\end{array}$

$3.841 \quad-0.006 \quad-0.002$

$\begin{array}{lll}4.489 & 0.863 & 0.091\end{array}$

4.071

$-0.685$

0.821

4.106

$-0.516$

$-0.931$

$-0.064$

$-0.207$

0.000

$-0.581$

1.092

$-0.001$

$-0.966$

$-1.271$

0.000

$-1.948$

1.312

$-0.001$

0.089

1.942

$-0.001$

$-2.337$

$-1.054$

0.000

$-0.583$

$-2.284$

0.001

$-2.834$

0.240

$-0.001$

$-2.327$

2.326

$-0.001$

$-3.015$

$-1.897$

0.000

$-3.902$

0.416

$-0.001$

CC6

$\mathrm{H}$

C

C

$\mathrm{H}$

C

$\mathrm{H}$

$\mathrm{H}$

$\mathrm{H}$

C

C

C

C

$\mathrm{H}$

$\begin{array}{rrr}2.562 & 0.434 & -0.103 \\ 3.461 & 0.997 & -0.331 \\ 1.399 & 1.005 & -0.415 \\ 1.427 & 2.005 & -0.837 \\ 2.778 & -0.887 & 0.568 \\ 3.592 & -0.811 & 1.289 \\ 1.885 & -1.230 & 1.090 \\ 3.065 & -1.658 & -0.153 \\ 0.049 & 0.449 & -0.220 \\ -0.993 & 1.299 & 0.158 \\ -0.244 & -0.899 & -0.435 \\ -2.276 & 0.814 & 0.352 \\ -0.784 & 2.352 & 0.308\end{array}$




$\begin{array}{lrrr}\mathrm{C} & -1.530 & -1.386 & -0.247 \\ \mathrm{H} & 0.537 & -1.564 & -0.779 \\ \mathrm{C} & -2.549 & -0.533 & 0.155 \\ \mathrm{H} & -3.067 & 1.489 & 0.654 \\ \mathrm{H} & -1.738 & -2.433 & -0.424 \\ \mathrm{H} & -3.551 & -0.913 & 0.301\end{array}$

$\begin{array}{lrrr}\text { CC7 } & & & \\ \text { C } & 1.349 & 1.323 & 0.000 \\ \text { C } & -0.014 & 1.083 & 0.000 \\ \text { C } & -0.509 & -0.223 & 0.000 \\ \text { C } & 0.406 & -1.275 & 0.000 \\ \text { C } & 1.773 & -1.038 & 0.000 \\ \text { C } & 2.249 & 0.264 & 0.000 \\ \text { H } & 1.714 & 2.342 & 0.000 \\ \text { H } & -0.699 & 1.921 & 0.000 \\ \text { H } & 0.036 & -2.294 & 0.000 \\ \text { H } & 2.464 & -1.871 & 0.000 \\ \text { H } & 3.315 & 0.455 & 0.000 \\ \text { C } & -1.948 & -0.532 & 0.000 \\ \text { H } & -2.183 & -1.593 & 0.001 \\ \text { C } & -2.952 & 0.337 & 0.000 \\ \text { H } & -2.795 & 1.409 & -0.001 \\ \text { H } & -3.978 & -0.003 & 0.000\end{array}$

$\begin{array}{lrrr}\text { CC8 } & & & \\ \text { C } & 0.000 & 0.125 & 0.000 \\ \text { C } & 0.001 & 1.453 & 0.000 \\ \text { H } & -0.923 & 2.017 & 0.000 \\ \text { H } & 0.926 & 2.016 & 0.000 \\ \text { C } & 1.268 & -0.677 & 0.000 \\ \text { H } & 2.150 & -0.039 & 0.001 \\ \text { H } & 1.309 & -1.329 & 0.877 \\ \text { H } & 1.310 & -1.327 & -0.878 \\ \text { C } & -1.269 & -0.676 & 0.000 \\ \text { H } & -1.311 & -1.327 & -0.877 \\ \text { H } & -1.311 & -1.326 & 0.878 \\ \text { H } & -2.150 & -0.036 & 0.000\end{array}$

\section{Cc9}

$\begin{array}{lrrr}\text { C } & 1.955 & -1.364 & -0.346 \\ \text { C } & 0.671 & -1.142 & 0.125 \\ \text { C } & 0.273 & 0.129 & 0.537 \\ \text { C } & 1.188 & 1.171 & 0.458 \\ \text { C } & 2.477 & 0.954 & -0.015 \\ \text { C } & 2.863 & -0.315 & -0.418 \\ \text { H } & 2.249 & -2.358 & -0.660 \\ \text { H } & -0.042 & -1.958 & 0.170 \\ \text { H } & 0.890 & 2.165 & 0.772\end{array}$

$\begin{array}{lrrr}\mathrm{H} & 3.177 & 1.778 & -0.069 \\ \mathrm{H} & 3.866 & -0.488 & -0.787 \\ \mathrm{C} & -1.137 & 0.352 & 1.038 \\ \mathrm{H} & -1.237 & 1.394 & 1.356 \\ \mathrm{H} & -1.319 & -0.275 & 1.914 \\ \mathrm{C} & -2.175 & 0.053 & -0.015 \\ \mathrm{C} & -3.079 & -0.905 & 0.149 \\ \mathrm{H} & -3.109 & -1.504 & 1.052 \\ \mathrm{H} & -3.821 & -1.117 & -0.611 \\ \mathrm{C} & -2.106 & 0.899 & -1.252 \\ \mathrm{H} & -2.197 & 1.958 & -0.996 \\ \mathrm{H} & -1.139 & 0.775 & -1.746 \\ \mathrm{H} & -2.895 & 0.642 & -1.957\end{array}$

CC10

$\begin{array}{lrrr}\mathrm{C} & -3.020 & 1.221 & 0.306 \\ \mathrm{H} & -3.983 & 1.092 & -0.172 \\ \mathrm{H} & -2.745 & 2.225 & 0.605 \\ \mathrm{C} & -2.213 & 0.187 & 0.512 \\ \mathrm{C} & -2.571 & -1.207 & 0.088 \\ \mathrm{H} & -2.429 & -1.914 & 0.909 \\ \mathrm{H} & -3.605 & -1.262 & -0.248 \\ \mathrm{H} & -1.929 & -1.543 & -0.731 \\ \mathrm{C} & -0.871 & 0.368 & 1.168 \\ \mathrm{H} & -0.759 & -0.306 & 2.025 \\ \mathrm{H} & -0.756 & 1.390 & 1.538 \\ \mathrm{Si} & 0.593 & 0.046 & 0.070 \\ \mathrm{Cl} & 0.309 & 0.840 & -1.781 \\ \mathrm{Cl} & 2.255 & 0.892 & 0.899 \\ \mathrm{Cl} & 0.963 & -1.951 & -0.139\end{array}$

CC11

$\begin{array}{lrrr}\mathrm{C} & 2.450 & -0.344 & 0.346 \\ \mathrm{H} & 2.339 & -1.376 & 0.673 \\ \mathrm{C} & 1.460 & 0.508 & 0.588 \\ \mathrm{C} & 0.155 & 0.165 & 1.231 \\ \mathrm{H} & -0.138 & 0.938 & 1.948 \\ \mathrm{H} & 0.234 & -0.776 & 1.782 \\ \mathrm{Si} & -1.225 & -0.020 & -0.049 \\ \mathrm{H} & 1.577 & 1.539 & 0.257 \\ \mathrm{C} & 3.730 & 0.005 & -0.346 \\ \mathrm{H} & 4.592 & -0.191 & 0.295 \\ \mathrm{H} & 3.866 & -0.592 & -1.251 \\ \mathrm{H} & 3.748 & 1.059 & -0.628 \\ \mathrm{C} & -2.858 & -0.256 & 0.839 \\ \mathrm{H} & -3.095 & 0.609 & 1.463 \\ \mathrm{H} & -3.676 & -0.389 & 0.128 \\ \mathrm{H} & -2.831 & -1.136 & 1.484 \\ \mathrm{C} & -1.294 & 1.521 & -1.113 \\ \mathrm{H} & -2.112 & 1.460 & -1.834\end{array}$




$\begin{array}{lrrr}\mathrm{H} & -1.451 & 2.415 & -0.506 \\ \mathrm{H} & -0.366 & 1.652 & -1.672 \\ \mathrm{C} & -0.849 & -1.511 & -1.116 \\ \mathrm{H} & 0.128 & -1.405 & -1.590 \\ \mathrm{H} & -0.837 & -2.427 & -0.522 \\ \mathrm{H} & -1.596 & -1.632 & -1.903\end{array}$

\section{CC12}

$\begin{array}{lrrr}\text { C } & -0.811 & -0.089 & 0.080 \\ \text { C } & -1.751 & -0.882 & 0.581 \\ \text { H } & -2.803 & -0.694 & 0.408 \\ \text { H } & -1.496 & -1.748 & 1.182 \\ \text { C } & -1.160 & 1.123 & -0.738 \\ \text { H } & -0.866 & 2.039 & -0.221 \\ \text { H } & -0.640 & 1.118 & -1.699 \\ \text { H } & -2.230 & 1.169 & -0.929 \\ \text { C } & 0.658 & -0.373 & 0.311 \\ \text { C } & 1.347 & -0.825 & -0.980 \\ \text { H } & 1.353 & -0.025 & -1.724 \\ \text { H } & 2.385 & -1.095 & -0.781 \\ \text { H } & 0.842 & -1.689 & -1.412 \\ \text { C } & 1.386 & 0.831 & 0.909 \\ \text { H } & 0.886 & 1.189 & 1.811 \\ \text { H } & 2.411 & 0.565 & 1.169 \\ \text { H } & 1.431 & 1.656 & 0.194 \\ \text { H } & 0.715 & -1.197 & 1.027\end{array}$

$\begin{array}{lrrr}\text { CC13 } & & & \\ \text { C } & -0.937 & -1.182 & -0.002 \\ \text { C } & 0.436 & -1.039 & -0.001 \\ \text { C } & 1.025 & 0.230 & 0.000 \\ \text { C } & 0.180 & 1.336 & 0.000 \\ \text { C } & -1.201 & 1.189 & -0.001 \\ \text { C } & -1.783 & -0.071 & -0.002 \\ \text { H } & -1.370 & -2.177 & -0.003 \\ \text { H } & 1.060 & -1.924 & -0.001 \\ \text { H } & 0.612 & 2.330 & 0.000 \\ \text { H } & -1.833 & 2.069 & -0.002 \\ \text { C } & 2.480 & 0.438 & 0.001 \\ \text { H } & 2.788 & 1.480 & 0.001 \\ \text { C } & 3.422 & -0.499 & 0.001 \\ \text { H } & 3.191 & -1.556 & 0.001 \\ \text { H } & 4.469 & -0.230 & 0.002 \\ \text { C } & -3.276 & -0.250 & 0.003 \\ \text { H } & -3.599 & -0.852 & -0.848 \\ \text { H } & -3.604 & -0.763 & 0.908 \\ \text { H } & -3.788 & 0.710 & -0.046\end{array}$

$\begin{array}{lrrr}\text { C } & -1.152 & -1.262 & 0.029 \\ \text { C } & 0.234 & -1.132 & 0.106 \\ \text { C } & 0.796 & 0.153 & 0.109 \\ \text { C } & -0.051 & 1.277 & 0.053 \\ \text { C } & -1.422 & 1.101 & -0.034 \\ \text { C } & -1.996 & -0.167 & -0.051 \\ \text { H } & -1.581 & -2.259 & 0.039 \\ \text { H } & -2.064 & 1.974 & -0.088 \\ \text { C } & 2.251 & 0.390 & 0.195 \\ \text { H } & 2.557 & 1.200 & 0.851 \\ \text { C } & 3.199 & -0.258 & -0.473 \\ \text { H } & 2.968 & -1.049 & -1.174 \\ \text { H } & 4.242 & 0.001 & -0.349 \\ \text { C } & -3.487 & -0.330 & -0.153 \\ \text { H } & -3.781 & -1.370 & -0.015 \\ \text { H } & -4.000 & 0.273 & 0.597 \\ \text { H } & -3.846 & -0.006 & -1.132 \\ \text { C } & 1.057 & -2.389 & 0.211 \\ \text { H } & 1.409 & -2.720 & -0.769 \\ \text { H } & 1.935 & -2.246 & 0.838 \\ \text { H } & 0.454 & -3.195 & 0.628 \\ \text { C } & 0.521 & 2.669 & 0.067 \\ \text { H } & 1.006 & 2.890 & 1.021 \\ \text { H } & 1.272 & 2.798 & -0.713 \\ \text { H } & -0.264 & 3.408 & -0.086\end{array}$

\section{CC15}

$\begin{array}{lrrr}\mathrm{C} & -2.338 & -1.168 & 0.054 \\ \mathrm{H} & -3.329 & -1.006 & 0.461 \\ \mathrm{H} & -2.032 & -2.195 & -0.106 \\ \mathrm{C} & -1.534 & -0.152 & -0.238 \\ \mathrm{C} & -0.171 & -0.397 & -0.836 \\ \mathrm{H} & -0.100 & -1.461 & -1.073 \\ \mathrm{H} & -0.097 & 0.144 & -1.787 \\ \mathrm{C} & 1.065 & -0.024 & 0.019 \\ \mathrm{C} & 1.319 & 1.485 & 0.037 \\ \mathrm{H} & 0.526 & 2.035 & 0.541 \\ \mathrm{H} & 2.252 & 1.700 & 0.561 \\ \mathrm{H} & 1.409 & 1.875 & -0.980 \\ \mathrm{C} & 2.280 & -0.699 & -0.623 \\ \mathrm{H} & 3.194 & -0.441 & -0.086 \\ \mathrm{H} & 2.175 & -1.786 & -0.612 \\ \mathrm{H} & 2.398 & -0.382 & -1.662 \\ \mathrm{C} & 0.897 & -0.542 & 1.446 \\ \mathrm{H} & 0.064 & -0.051 & 1.952 \\ \mathrm{H} & 0.697 & -1.616 & 1.449 \\ \mathrm{H} & 1.804 & -0.362 & 2.027 \\ \mathrm{C} & -1.971 & 1.269 & -0.003 \\ \mathrm{H} & -1.519 & 1.674 & 0.905 \\ \mathrm{H} & -1.668 & 1.917 & -0.829 \\ \mathrm{H} & -3.052 & 1.328 & 0.111 \\ & & & \end{array}$




$\begin{array}{lrrr}\text { CC16 } & & & \\ \text { C } & 0.922 & 0.214 & 0.003 \\ \text { C } & 1.468 & 1.426 & 0.011 \\ \text { H } & 2.544 & 1.546 & -0.008 \\ \text { H } & 0.882 & 2.334 & 0.039 \\ \text { C } & 1.806 & -1.006 & -0.002 \\ \text { H } & 1.750 & -1.531 & 0.955 \\ \text { H } & 1.513 & -1.719 & -0.774 \\ \text { H } & 2.845 & -0.728 & -0.171 \\ \text { C } & -0.584 & -0.026 & 0.003 \\ \text { C } & -1.004 & -0.613 & -1.354 \\ \text { H } & -0.522 & -1.571 & -1.550 \\ \text { H } & -2.084 & -0.776 & -1.369 \\ \text { H } & -0.749 & 0.072 & -2.164 \\ \text { C } & -0.951 & -1.021 & 1.112 \\ \text { H } & -0.620 & -0.657 & 2.087 \\ \text { H } & -2.034 & -1.156 & 1.147 \\ \text { H } & -0.500 & -2.000 & 0.941 \\ \text { C } & -1.372 & 1.264 & 0.229 \\ \text { H } & -1.101 & 1.733 & 1.176 \\ \text { H } & -1.201 & 1.983 & -0.574 \\ \text { H } & -2.440 & 1.041 & 0.252\end{array}$

$\begin{array}{rrrr}\mathrm{H} & 1.165 & -1.250 & 0.000 \\ \mathrm{H} & 1.797 & 0.151 & -0.878 \\ \mathrm{H} & 1.797 & 0.151 & 0.877\end{array}$

CC19

$\begin{array}{lrrr}\text { C } & -0.203 & 0.057 & -0.009 \\ \text { C } & 0.569 & 1.200 & 0.213 \\ \text { C } & 0.460 & -1.155 & -0.204 \\ \text { C } & 1.953 & 1.138 & 0.212 \\ \text { C } & 1.846 & -1.219 & -0.210 \\ \text { H } & -0.109 & -2.061 & -0.366 \\ \text { C } & 2.599 & -0.073 & -0.005 \\ \text { H } & 2.530 & 2.035 & 0.392 \\ \text { H } & 2.338 & -2.169 & -0.374 \\ \text { H } & 3.680 & -0.122 & -0.004 \\ \text { H } & 0.077 & 2.142 & 0.415 \\ \text { C } & -1.687 & 0.125 & -0.027 \\ \text { C } & -2.331 & 1.229 & -0.398 \\ \text { H } & -1.808 & 2.112 & -0.737 \\ \text { H } & -3.413 & 1.270 & -0.392 \\ \text { C } & -2.440 & -1.116 & 0.371 \\ \text { H } & -2.320 & -1.904 & -0.375 \\ \text { H } & -2.072 & -1.510 & 1.320 \\ \text { H } & -3.504 & -0.906 & 0.464 \\ & & & \\ \text { CC20 } & & & \end{array}$

CC17

$\begin{array}{lrrr}\mathrm{C} & 1.137 & 1.414 & -0.175 \\ \mathrm{H} & 2.140 & 1.676 & -0.488 \\ \mathrm{H} & 0.419 & 2.220 & -0.093 \\ \mathrm{C} & 0.812 & 0.158 & 0.104 \\ \mathrm{C} & 1.805 & -0.967 & 0.023 \\ \mathrm{H} & 2.788 & -0.615 & -0.285 \\ \mathrm{H} & 1.901 & -1.462 & 0.993 \\ \mathrm{H} & 1.462 & -1.724 & -0.687 \\ \mathrm{C} & -0.559 & -0.261 & 0.505 \\ \mathrm{C} & -1.774 & 0.546 & 0.184 \\ \mathrm{C} & -1.517 & -0.733 & -0.557 \\ \mathrm{H} & -0.594 & -0.827 & 1.430 \\ \mathrm{H} & -1.641 & 1.468 & -0.363 \\ \mathrm{H} & -2.574 & 0.562 & 0.909 \\ \mathrm{H} & -2.142 & -1.589 & -0.344 \\ \mathrm{H} & -1.183 & -0.650 & -1.582\end{array}$

CC20

$\begin{array}{lrrr}\text { C } & 0.463 & -0.026 & 0.000 \\ \text { C } & -0.672 & -0.721 & 0.000 \\ \text { C } & -2.057 & -0.155 & 0.000 \\ \text { H } & -2.616 & -0.492 & -0.876 \\ \text { H } & -2.616 & -0.491 & 0.877 \\ \text { H } & -2.057 & 0.934 & 0.000 \\ \text { C } & 0.477 & 1.486 & 0.000 \\ \text { H } & -0.029 & 1.891 & 0.878 \\ \text { H } & 1.495 & 1.871 & 0.001 \\ \text { H } & -0.028 & 1.891 & -0.879 \\ \text { C } & 1.794 & -0.747 & 0.000 \\ \text { H } & 1.899 & -1.382 & 0.881 \\ \text { H } & 1.896 & -1.388 & -0.877 \\ \text { H } & 2.630 & -0.049 & -0.004 \\ \text { H } & -0.604 & -1.806 & 0.000\end{array}$

CC18

CC21

$\begin{array}{lrrr}\mathrm{C} & -1.272 & -0.222 & 0.000 \\ \mathrm{H} & -1.281 & -1.306 & 0.000 \\ \mathrm{H} & -2.231 & 0.280 & 0.000 \\ \mathrm{C} & -0.135 & 0.456 & 0.000 \\ \mathrm{H} & -0.170 & 1.542 & -0.001 \\ \mathrm{C} & 1.227 & -0.162 & 0.000\end{array}$

C

$\begin{array}{lll}0.569 & 1.200 & 0.213\end{array}$

$$
-3.504-0.906
$$

0.464 


$\begin{array}{lrrr}\mathrm{H} & 0.293 & -1.853 & -0.724 \\ \mathrm{C} & -0.797 & 0.508 & 0.524 \\ \mathrm{H} & -0.870 & 1.596 & 0.546 \\ \mathrm{H} & -0.959 & 0.157 & 1.549 \\ \mathrm{H} & 1.282 & 2.080 & -0.209 \\ \mathrm{C} & -1.893 & -0.066 & -0.374 \\ \mathrm{H} & -1.913 & -1.156 & -0.333 \\ \mathrm{H} & -2.874 & 0.293 & -0.063 \\ \mathrm{H} & -1.735 & 0.230 & -1.412\end{array}$

CC22

\begin{tabular}{|c|c|c|c|}
\hline C & 2.388 & -1.029 & 0.635 \\
\hline C & 3.255 & -0.188 & -0.054 \\
\hline C & 2.740 & 0.999 & -0.569 \\
\hline C & 1.407 & 1.336 & -0.397 \\
\hline C & 0.537 & 0.493 & 0.298 \\
\hline C & 1.050 & -0.701 & 0.802 \\
\hline $\mathrm{H}$ & 2.764 & -1.958 & 1.048 \\
\hline $\mathrm{H}$ & 3.393 & 1.667 & -1.119 \\
\hline $\mathrm{H}$ & 1.022 & 2.257 & -0.817 \\
\hline $\mathrm{H}$ & 0.402 & -1.367 & 1.357 \\
\hline C & -0.891 & 0.859 & 0.474 \\
\hline C & -1.215 & 2.047 & 0.987 \\
\hline $\mathrm{H}$ & -0.455 & 2.747 & 1.324 \\
\hline $\mathrm{H}$ & -2.246 & 2.363 & 1.099 \\
\hline C & 4.709 & -0.534 & -0.226 \\
\hline $\mathrm{H}$ & 5.330 & 0.058 & 0.449 \\
\hline $\mathrm{H}$ & 4.892 & -1.586 & -0.011 \\
\hline $\mathrm{H}$ & 5.044 & -0.329 & -1.243 \\
\hline Si & -2.225 & -0.306 & -0.173 \\
\hline C & -1.883 & -0.598 & -1.991 \\
\hline $\mathrm{H}$ & -0.883 & -1.014 & -2.129 \\
\hline $\mathrm{H}$ & -2.603 & -1.297 & -2.420 \\
\hline $\mathrm{H}$ & -1.938 & 0.335 & -2.554 \\
\hline C & -2.217 & -1.956 & 0.721 \\
\hline $\mathrm{H}$ & -3.109 & -2.523 & 0.445 \\
\hline $\mathrm{H}$ & -1.348 & -2.559 & 0.451 \\
\hline $\mathrm{H}$ & -2.224 & -1.828 & 1.805 \\
\hline C & -3.901 & 0.498 & 0.057 \\
\hline $\mathrm{H}$ & -3.964 & 1.451 & -0.470 \\
\hline $\mathrm{H}$ & -4.682 & -0.154 & -0.336 \\
\hline $\mathrm{H}$ & -4.118 & 0.677 & 1.112 \\
\hline
\end{tabular}

CC23

$\begin{array}{rrrr}\text { C } & -0.668 & 0.000 & 0.000 \\ \mathrm{C} & 0.668 & 0.000 & 0.000 \\ \mathrm{C} & 1.511 & -1.248 & -0.031 \\ \mathrm{H} & 2.313 & -1.130 & -0.765 \\ \mathrm{H} & 1.997 & -1.409 & 0.936 \\ \mathrm{H} & 0.959 & -2.146 & -0.290\end{array}$

$\begin{array}{lrrr}\mathrm{C} & 1.511 & 1.248 & 0.031 \\ \mathrm{H} & 2.312 & 1.130 & 0.765 \\ \mathrm{H} & 1.998 & 1.409 & -0.936 \\ \mathrm{H} & 0.959 & 2.146 & 0.289 \\ \mathrm{C} & -1.511 & 1.248 & -0.031 \\ \mathrm{H} & -0.959 & 2.146 & -0.290 \\ \mathrm{H} & -2.313 & 1.130 & -0.765 \\ \mathrm{H} & -1.997 & 1.409 & 0.936 \\ \mathrm{C} & -1.511 & -1.248 & 0.031 \\ \mathrm{H} & -1.997 & -1.410 & -0.936 \\ \mathrm{H} & -0.959 & -2.146 & 0.290 \\ \mathrm{H} & -2.313 & -1.129 & 0.765\end{array}$

CC24

$\begin{array}{lrrr}\mathrm{C} & 0.430 & 1.097 & 0.000 \\ \mathrm{C} & 0.431 & -1.097 & 0.000 \\ \mathrm{C} & 1.530 & 0.000 & 0.001 \\ \mathrm{H} & 0.417 & 1.733 & -0.885 \\ \mathrm{H} & 0.416 & 1.735 & 0.883 \\ \mathrm{H} & 0.417 & -1.734 & -0.885 \\ \mathrm{H} & 0.416 & -1.735 & 0.883 \\ \mathrm{H} & 2.165 & 0.000 & -0.882 \\ \mathrm{H} & 2.163 & 0.000 & 0.885 \\ \mathrm{C} & -0.618 & 0.000 & 0.000 \\ \mathrm{C} & -1.938 & 0.000 & 0.000 \\ \mathrm{H} & -2.501 & -0.926 & 0.001 \\ \mathrm{H} & -2.501 & 0.926 & 0.001\end{array}$

CC25

$\begin{array}{lrrr}\text { C } & -0.014 & -1.224 & 0.129 \\ \text { C } & -1.411 & -0.723 & -0.255 \\ \text { C } & -1.412 & 0.722 & 0.254 \\ \text { C } & -0.014 & 1.224 & -0.128 \\ \text { H } & -0.018 & -1.554 & 1.172 \\ \text { H } & 0.337 & -2.059 & -0.476 \\ \text { H } & -1.521 & -0.726 & -1.342 \\ \text { H } & -2.214 & -1.328 & 0.164 \\ \text { H } & -2.214 & 1.328 & -0.165 \\ \text { H } & -1.523 & 0.725 & 1.341 \\ \text { H } & -0.017 & 1.555 & -1.171 \\ \text { H } & 0.337 & 2.058 & 0.478 \\ \text { C } & 0.873 & 0.000 & 0.000 \\ \text { C } & 2.197 & 0.000 & 0.000 \\ \text { H } & 2.762 & 0.921 & -0.078 \\ \text { H } & 2.762 & -0.921 & 0.077\end{array}$

\section{CC26}

$\begin{array}{llll}\text { C } & 0.000 & -1.217 & -0.149 \\ \text { C } & 1.226 & -0.320 & 0.113\end{array}$




$\begin{array}{lrrr}\text { C } & 0.663 & 1.070 & -0.051 \\ \text { C } & -0.663 & 1.070 & -0.051 \\ \text { C } & -1.226 & -0.320 & 0.113 \\ \text { H } & 0.000 & -2.123 & 0.456 \\ \text { H } & 2.051 & -0.529 & -0.570 \\ \text { H } & 1.616 & -0.450 & 1.128 \\ \text { H } & 1.284 & 1.954 & -0.113 \\ \text { H } & -1.284 & 1.954 & -0.113 \\ \text { H } & -2.051 & -0.529 & -0.570 \\ \text { H } & -1.616 & -0.450 & 1.128 \\ \text { H } & 0.000 & -1.520 & -1.197\end{array}$

CC27

$\begin{array}{lrrr}\text { C } & -1.504 & 0.709 & 0.170 \\ \mathrm{C} & -0.072 & 1.183 & -0.135 \\ \mathrm{C} & 0.752 & -0.076 & 0.004 \\ \mathrm{C} & -0.026 & -1.153 & 0.027 \\ \mathrm{C} & -1.488 & -0.810 & -0.094 \\ \mathrm{H} & -2.260 & 1.234 & -0.411 \\ \mathrm{H} & 0.263 & 1.977 & 0.537 \\ \mathrm{H} & 0.023 & 1.575 & -1.154 \\ \mathrm{H} & 0.341 & -2.171 & 0.077 \\ \mathrm{H} & -2.117 & -1.360 & 0.608 \\ \mathrm{H} & -1.854 & -1.050 & -1.098 \\ \mathrm{H} & -1.719 & 0.883 & 1.225 \\ \mathrm{C} & 2.243 & -0.030 & 0.041 \\ \mathrm{H} & 2.591 & 0.562 & 0.891 \\ \mathrm{H} & 2.672 & -1.028 & 0.115 \\ \mathrm{H} & 2.636 & 0.450 & -0.859\end{array}$

\section{CC30}

$\begin{array}{lrrr}\mathrm{C} & 1.105 & 0.418 & 0.000 \\ \mathrm{H} & 1.075 & 1.505 & 0.000 \\ \mathrm{C} & -0.049 & -0.246 & 0.000 \\ \mathrm{H} & -0.041 & -1.334 & 0.000 \\ \mathrm{C} & 2.457 & -0.216 & 0.000 \\ \mathrm{H} & 3.032 & 0.091 & -0.877 \\ \mathrm{H} & 3.032 & 0.091 & 0.877 \\ \mathrm{H} & 2.383 & -1.303 & 0.000 \\ \mathrm{C} & -1.355 & 0.397 & 0.000 \\ \mathrm{H} & -1.357 & 1.484 & 0.000 \\ \mathrm{C} & -2.511 & -0.261 & 0.000 \\ \mathrm{H} & -3.460 & 0.257 & 0.000 \\ \mathrm{H} & -2.538 & -1.345 & 0.000\end{array}$

$\begin{array}{lrrr}\text { CC28 } & & & \\ \text { C } & 1.146 & -1.206 & -0.315 \\ \text { C } & 1.831 & -0.022 & 0.359 \\ \text { C } & 1.198 & 1.286 & -0.106 \\ \text { C } & -0.304 & 1.217 & -0.095 \\ \text { C } & -1.001 & 0.088 & 0.004 \\ \text { C } & -0.327 & -1.260 & 0.077 \\ \text { H } & 1.528 & 2.113 & 0.528 \\ \text { H } & 1.713 & -0.114 & 1.443 \\ \text { H } & 2.902 & -0.021 & 0.150 \\ \text { H } & 1.226 & -1.092 & -1.400 \\ \text { H } & 1.640 & -2.143 & -0.054 \\ \text { H } & -0.841 & 2.158 & -0.167 \\ \text { H } & -0.432 & -1.650 & 1.097 \\ \text { H } & -0.867 & -1.962 & -0.565 \\ \text { H } & 1.545 & 1.528 & -1.117 \\ \text { C } & -2.500 & 0.069 & 0.054 \\ \text { H } & -2.915 & 1.076 & 0.040 \\ \text { H } & -2.909 & -0.487 & -0.793 \\ \text { H } & -2.847 & -0.435 & 0.960\end{array}$

\section{CC31}

$\begin{array}{lrrr}\text { C } & -1.790 & 0.432 & 0.000 \\ \text { H } & -1.710 & 1.516 & 0.000 \\ \text { C } & -0.669 & -0.287 & 0.000 \\ \text { H } & -0.733 & -1.372 & 0.000 \\ \text { C } & -3.171 & -0.139 & 0.000 \\ \text { H } & -3.732 & 0.192 & 0.877 \\ \text { H } & -3.732 & 0.192 & -0.877 \\ \text { H } & -3.146 & -1.228 & 0.000 \\ \text { C } & 0.669 & 0.287 & 0.000 \\ \text { H } & 0.733 & 1.373 & 0.000 \\ \text { C } & 1.790 & -0.432 & 0.000 \\ \text { H } & 1.710 & -1.516 & 0.000 \\ \text { C } & 3.171 & 0.139 & 0.000 \\ \text { H } & 3.732 & -0.192 & 0.877 \\ \text { H } & 3.732 & -0.192 & -0.877 \\ \text { H } & 3.146 & 1.228 & 0.000\end{array}$

\section{CC32}

$\begin{array}{llll}\text { C } & 2.072 & -1.066 & 0.000\end{array}$




$\begin{array}{lrrrrrrr}H & 3.122 & -0.803 & 0.000 & \mathrm{C} & 1.009 & 1.529 & 0.000 \\ \mathrm{H} & 1.824 & -2.121 & 0.000 & \mathrm{H} & 2.041 & 1.855 & 0.000 \\ \mathrm{C} & 1.118 & -0.134 & 0.000 & \mathrm{H} & 0.254 & 2.303 & 0.000 \\ \mathrm{C} & -0.293 & -0.529 & 0.000 & \mathrm{C} & 0.703 & 0.231 & 0.000 \\ \mathrm{H} & -0.483 & -1.600 & 0.000 & \mathrm{C} & -0.703 & -0.231 & 0.000 \\ \mathrm{C} & -1.330 & 0.306 & 0.000 & \mathrm{C} & -1.009 & -1.529 & 0.000 \\ \mathrm{H} & -1.149 & 1.377 & 0.000 & \mathrm{H} & -2.041 & -1.855 & 0.000 \\ \mathrm{C} & -2.762 & -0.124 & 0.000 & \mathrm{H} & -0.254 & -2.303 & 0.000 \\ \mathrm{H} & -3.286 & 0.263 & -0.877 & \mathrm{C} & -1.790 & 0.808 & 0.000 \\ \mathrm{H} & -2.848 & -1.210 & 0.000 & \mathrm{H} & -1.717 & 1.451 & 0.880 \\ \mathrm{H} & -3.286 & 0.263 & 0.877 & \mathrm{H} & -1.718 & 1.451 & -0.880 \\ \mathrm{C} & 1.449 & 1.331 & 0.000 & \mathrm{H} & -2.771 & 0.336 & 0.000 \\ \mathrm{H} & 1.028 & 1.823 & -0.879 & \mathrm{C} & 1.790 & -0.808 & 0.000 \\ \mathrm{H} & 1.028 & 1.823 & 0.879 & \mathrm{H} & 1.718 & -1.451 & 0.880 \\ \mathrm{H} & 2.526 & 1.485 & 0.000 & \mathrm{H} & 1.717 & -1.451 & -0.879 \\ & & & & \mathrm{H} & 2.771 & -0.336 & 0.000\end{array}$

CC33

$\begin{array}{lrrr}\mathrm{C} & 1.226 & 0.638 & 0.000 \\ \mathrm{H} & 1.791 & 1.565 & 0.000 \\ \mathrm{C} & -0.105 & 0.733 & 0.000 \\ \mathrm{H} & -0.548 & 1.724 & 0.000 \\ \mathrm{C} & 2.048 & -0.611 & 0.000 \\ \mathrm{H} & 2.699 & -0.638 & -0.877 \\ \mathrm{H} & 2.699 & -0.637 & 0.876 \\ \mathrm{H} & 1.445 & -1.516 & 0.000 \\ \mathrm{C} & -1.055 & -0.373 & 0.000 \\ \mathrm{H} & -0.660 & -1.383 & 0.000 \\ \mathrm{C} & -2.374 & -0.199 & 0.000 \\ \mathrm{H} & -2.808 & 0.794 & 0.000 \\ \mathrm{H} & -3.058 & -1.037 & 0.000\end{array}$

cC36

$\begin{array}{lrrr}\text { C } & -1.588 & -0.893 & 0.000 \\ \text { H } & -1.486 & -1.971 & 0.000 \\ \text { H } & -2.593 & -0.490 & 0.000 \\ \text { C } & -0.517 & -0.099 & 0.000 \\ \text { C } & 0.826 & -0.688 & 0.000 \\ \text { H } & 0.862 & -1.773 & 0.000 \\ \mathrm{C} & 1.964 & -0.001 & 0.000 \\ \text { H } & 1.980 & 1.082 & 0.000 \\ \text { H } & 2.922 & -0.504 & 0.000 \\ \text { C } & -0.636 & 1.397 & 0.000 \\ \text { H } & -0.149 & 1.824 & 0.879 \\ \text { H } & -0.148 & 1.824 & -0.879 \\ \text { H } & -1.680 & 1.704 & 0.000\end{array}$

CC34

$\begin{array}{lrrr}\mathrm{C} & 2.797 & -0.217 & 0.000 \\ \mathrm{H} & 2.946 & -1.290 & 0.000 \\ \mathrm{H} & 3.681 & 0.406 & 0.000 \\ \mathrm{C} & 1.573 & 0.309 & 0.000 \\ \mathrm{H} & 1.472 & 1.388 & 0.000 \\ \mathrm{C} & 0.359 & -0.495 & 0.000 \\ \mathrm{H} & 0.520 & -1.570 & 0.000 \\ \mathrm{C} & -0.903 & -0.053 & 0.000 \\ \mathrm{C} & -1.306 & 1.392 & 0.000 \\ \mathrm{H} & -1.923 & 1.608 & 0.877 \\ \mathrm{H} & -0.464 & 2.078 & 0.000 \\ \mathrm{H} & -1.922 & 1.608 & -0.877 \\ \mathrm{C} & -2.055 & -1.014 & 0.000 \\ \mathrm{H} & -1.719 & -2.050 & 0.000 \\ \mathrm{H} & -2.689 & -0.855 & 0.877 \\ \mathrm{H} & -2.689 & -0.855 & -0.877\end{array}$

CC37

$\begin{array}{lrrr}\text { C } & 1.174 & -0.280 & 0.000 \\ \text { C } & 0.734 & 0.986 & 0.000 \\ \text { C } & -0.734 & 0.985 & 0.000 \\ \text { C } & -1.174 & -0.280 & 0.000 \\ \text { H } & 2.203 & -0.605 & 0.000 \\ \text { H } & 1.348 & 1.874 & 0.000 \\ \text { H } & -1.349 & 1.874 & 0.000 \\ \text { H } & -2.203 & -0.605 & 0.000 \\ \text { C } & 0.000 & -1.211 & 0.000 \\ \text { H } & 0.000 & -1.869 & -0.876 \\ \text { H } & 0.000 & -1.869 & 0.876\end{array}$

CC38

$\begin{array}{lrrr}C & 1.187 & -0.714 & -0.260 \\ C & 1.182 & 0.722 & 0.260 \\ C & -0.115 & 1.415 & -0.063\end{array}$




$\begin{array}{lrrr}\text { C } & -1.251 & 0.720 & -0.115 \\ \text { C } & -1.246 & -0.728 & 0.115 \\ \text { C } & -0.105 & -1.415 & 0.063 \\ \text { H } & 1.313 & -0.709 & -1.351 \\ \text { H } & 1.307 & 0.717 & 1.351 \\ \text { H } & -0.118 & 2.490 & -0.196 \\ \text { H } & -2.195 & 1.215 & -0.305 \\ \text { H } & -2.187 & -1.230 & 0.305 \\ \text { H } & -0.101 & -2.491 & 0.195 \\ \text { H } & 2.038 & -1.260 & 0.148 \\ \text { H } & 2.029 & 1.274 & -0.147\end{array}$

\section{CC39}

$\begin{array}{lrrr}\text { C } & -1.273 & -0.666 & -0.499 \\ \text { C } & -0.086 & -1.122 & 0.325 \\ \text { C } & -0.085 & 1.123 & 0.324 \\ \text { C } & -1.272 & 0.666 & -0.499 \\ \text { H } & -1.913 & -1.319 & -1.075 \\ \text { H } & -1.912 & 1.319 & -1.076 \\ \text { C } & -0.029 & 0.001 & 1.375 \\ \text { H } & 0.898 & 0.001 & 1.953 \\ \text { H } & -0.891 & 0.001 & 2.041 \\ \text { C } & 1.177 & -0.777 & -0.521 \\ \text { H } & 2.073 & -1.170 & -0.040 \\ \text { H } & 1.120 & -1.200 & -1.523 \\ \text { C } & 1.178 & 0.775 & -0.521 \\ \text { H } & 2.074 & 1.168 & -0.040 \\ \text { H } & 1.121 & 1.198 & -1.523 \\ \text { H } & -0.117 & -2.146 & 0.690 \\ \text { H } & -0.115 & 2.147 & 0.688\end{array}$

$\begin{array}{lrrr}\text { CC40 } & & & \\ \text { C } & 0.967 & 1.170 & 0.000 \\ \text { C } & 2.241 & 0.732 & 0.000 \\ \text { C } & 2.241 & -0.732 & 0.000 \\ \text { C } & 0.967 & -1.170 & 0.000 \\ \text { H } & 0.649 & 2.200 & -0.001 \\ \text { H } & 3.127 & 1.349 & 0.000 \\ \text { H } & 3.127 & -1.349 & 0.000 \\ \text { H } & 0.649 & -2.200 & 0.000 \\ \text { C } & 0.079 & 0.000 & 0.000 \\ \text { C } & -1.268 & 0.000 & 0.000 \\ \text { C } & -2.084 & 1.256 & 0.000 \\ \text { H } & -2.737 & 1.274 & -0.876 \\ \text { H } & -2.738 & 1.273 & 0.876 \\ \text { H } & -1.479 & 2.157 & 0.001 \\ \text { C } & -2.084 & -1.256 & 0.000 \\ \text { H } & -2.737 & -1.274 & -0.876 \\ \text { H } & -1.479 & -2.157 & 0.000 \\ \text { H } & -2.737 & -1.274 & 0.876\end{array}$

\section{CC41}

$\begin{array}{lrrr}\text { C } & -1.504 & 1.202 & 0.000 \\ \text { C } & -2.200 & 0.000 & 0.000 \\ \text { C } & -1.504 & -1.202 & 0.000 \\ \text { C } & -0.119 & -1.206 & 0.000 \\ \text { C } & 0.585 & 0.000 & 0.000 \\ \text { C } & -0.119 & 1.206 & 0.000 \\ \text { H } & -2.043 & 2.140 & 0.000 \\ \text { H } & -3.282 & 0.000 & 0.000 \\ \text { H } & -2.043 & -2.140 & 0.000 \\ \text { H } & 0.430 & -2.138 & 0.000 \\ \text { H } & 0.430 & 2.138 & 0.000 \\ \text { C } & 2.017 & 0.000 & 0.000 \\ \text { C } & 3.217 & 0.000 & 0.000 \\ \text { H } & 4.280 & 0.000 & 0.001\end{array}$

Ar1

$\begin{array}{lrrr}\text { C } & -1.893 & 0.000 & 0.008 \\ \text { C } & -1.193 & -1.199 & 0.002 \\ \text { C } & 0.194 & -1.195 & -0.009 \\ \text { C } & 0.908 & 0.001 & -0.012 \\ \text { C } & 0.194 & 1.196 & -0.009 \\ \text { C } & -1.194 & 1.198 & 0.002 \\ \text { H } & -2.975 & -0.001 & 0.014 \\ \text { H } & -1.729 & -2.140 & 0.002 \\ \text { H } & 0.734 & -2.135 & -0.018 \\ \text { H } & 0.733 & 2.136 & -0.018 \\ \text { H } & -1.730 & 2.139 & 0.002 \\ \text { C } & 2.411 & 0.000 & 0.009 \\ \text { H } & 2.783 & -0.025 & 1.036 \\ \text { H } & 2.813 & -0.871 & -0.507 \\ \text { H } & 2.813 & 0.896 & -0.465\end{array}$

Ar2

$\begin{array}{lrrr}\text { C } & 1.201 & -1.128 & 0.001 \\ \text { C } & 1.221 & 0.261 & 0.001 \\ \text { C } & 0.005 & 0.944 & 0.001 \\ \text { C } & -1.212 & 0.277 & 0.000 \\ \text { C } & -1.205 & -1.118 & -0.001 \\ \text { C } & -0.009 & -1.814 & 0.000 \\ \text { H } & 2.134 & -1.677 & 0.001 \\ \text { H } & 0.012 & 2.030 & 0.001 \\ \text { H } & -2.146 & -1.656 & -0.001 \\ \text { H } & -0.014 & -2.896 & 0.000 \\ \text { C } & -2.514 & 1.030 & 0.000 \\ \text { H } & -3.109 & 0.778 & 0.880 \\ \text { H } & -2.349 & 2.106 & -0.001 \\ \text { H } & -3.110 & 0.777 & -0.879\end{array}$




$\begin{array}{llll}\mathrm{C} & 2.517 & 1.024 & -0.001 \\ \mathrm{H} & 2.601 & 1.648 & -0.892 \\ \mathrm{H} & 2.582 & 1.684 & 0.866 \\ \mathrm{H} & 3.372 & 0.350 & 0.022\end{array}$

Ar3

$\begin{array}{lrrr}\mathrm{C} & -2.266 & 0.336 & 0.000 \\ \mathrm{C} & -1.843 & -0.990 & 0.000 \\ \mathrm{C} & -0.497 & -1.298 & 0.000 \\ \mathrm{C} & 0.453 & -0.276 & 0.000 \\ \mathrm{C} & 0.041 & 1.052 & 0.000 \\ \mathrm{C} & -1.320 & 1.345 & 0.000 \\ \mathrm{H} & -3.321 & 0.575 & 0.000 \\ \mathrm{H} & -0.149 & -2.323 & 0.000 \\ \mathrm{H} & 0.759 & 1.859 & 0.000 \\ \mathrm{H} & -1.633 & 2.382 & 0.000 \\ \mathrm{H} & -2.571 & -1.791 & 0.000 \\ \mathrm{O} & 1.751 & -0.674 & 0.000 \\ \mathrm{C} & 2.743 & 0.326 & 0.000 \\ \mathrm{H} & 3.698 & -0.193 & 0.000 \\ \mathrm{H} & 2.672 & 0.955 & -0.892 \\ \mathrm{H} & 2.672 & 0.955 & 0.891\end{array}$

\section{Ar4}

$\begin{array}{lrrr}\text { C } & -1.654 & 1.108 & 0.000 \\ \mathrm{C} & -1.635 & -0.286 & 0.000 \\ \mathrm{C} & -0.411 & -0.938 & 0.000 \\ \mathrm{C} & 0.783 & -0.222 & 0.000 \\ \mathrm{C} & 0.761 & 1.169 & 0.000 \\ \mathrm{C} & -0.467 & 1.820 & 0.000 \\ \mathrm{H} & -2.602 & 1.632 & 0.000 \\ \mathrm{H} & -0.358 & -2.020 & 0.000 \\ \mathrm{H} & 1.674 & 1.746 & 0.000 \\ \mathrm{H} & -0.489 & 2.902 & 0.000 \\ \mathrm{O} & 1.919 & -0.966 & 0.000 \\ \mathrm{C} & 3.151 & -0.283 & 0.000 \\ \mathrm{H} & 3.923 & -1.047 & 0.000 \\ \mathrm{H} & 3.258 & 0.341 & -0.892 \\ \mathrm{H} & 3.258 & 0.341 & 0.891 \\ \mathrm{C} & -2.911 & -1.083 & 0.000 \\ \mathrm{H} & -2.967 & -1.727 & -0.879 \\ \mathrm{H} & -2.969 & -1.725 & 0.880 \\ \mathrm{H} & -3.782 & -0.429 & -0.001\end{array}$

\section{Ar5}

$\begin{array}{lrrr}C & -1.020 & 1.650 & 0.000 \\ C & -1.307 & 0.281 & 0.000 \\ C & -0.282 & -0.648 & 0.000 \\ C & 1.046 & -0.210 & 0.000\end{array}$

$\begin{array}{lrrr}\mathrm{C} & 1.346 & 1.144 & 0.000 \\ \mathrm{C} & 0.294 & 2.060 & 0.000 \\ \mathrm{H} & -1.841 & 2.354 & 0.000 \\ \mathrm{H} & -0.459 & -1.713 & 0.000 \\ \mathrm{H} & 2.366 & 1.497 & 0.000 \\ \mathrm{H} & 0.520 & 3.119 & 0.000 \\ \mathrm{O} & 1.975 & -1.199 & 0.000 \\ \mathrm{C} & 3.332 & -0.821 & 0.000 \\ \mathrm{H} & 3.905 & -1.744 & 0.001 \\ \mathrm{H} & 3.581 & -0.239 & -0.891 \\ \mathrm{H} & 3.580 & -0.238 & 0.892 \\ \mathrm{O} & -2.622 & -0.048 & 0.000 \\ \mathrm{C} & -2.957 & -1.417 & 0.000 \\ \mathrm{H} & -2.571 & -1.918 & 0.892 \\ \mathrm{H} & -4.043 & -1.465 & 0.000 \\ \mathrm{H} & -2.570 & -1.918 & -0.891\end{array}$

Ar6

$\begin{array}{lrrr}\text { C } & -0.233 & 0.758 & 0.000 \\ \mathrm{C} & -0.207 & -0.653 & 0.000 \\ \mathrm{C} & -1.369 & -1.420 & 0.000 \\ \mathrm{C} & -2.576 & -0.748 & 0.000 \\ \mathrm{C} & -2.626 & 0.654 & 0.000 \\ \mathrm{C} & -1.471 & 1.409 & 0.000 \\ \mathrm{C} & 1.132 & 1.197 & 0.000 \\ \mathrm{C} & 1.920 & 0.084 & 0.000 \\ \mathrm{H} & -1.330 & -2.502 & 0.000 \\ \mathrm{H} & -3.498 & -1.313 & 0.000 \\ \mathrm{H} & -3.590 & 1.147 & 0.000 \\ \mathrm{H} & -1.520 & 2.491 & 0.000 \\ \mathrm{H} & 1.490 & 2.213 & 0.000 \\ \mathrm{H} & 1.446 & -1.981 & 0.000 \\ \mathrm{~N} & 1.114 & -1.034 & 0.000 \\ \mathrm{C} & 3.403 & -0.047 & 0.000 \\ \mathrm{H} & 3.753 & -0.586 & 0.883 \\ \mathrm{H} & 3.753 & -0.585 & -0.883 \\ \mathrm{H} & 3.861 & 0.940 & 0.000\end{array}$

Ar7

$\begin{array}{lrrr}\text { C } & 0.450 & -0.578 & 0.000 \\ \text { C } & 0.733 & 0.805 & 0.000 \\ \text { C } & -0.272 & 1.768 & 0.000 \\ \text { C } & -1.581 & 1.333 & 0.000 \\ \text { C } & -1.869 & -0.039 & 0.000 \\ \text { C } & -0.883 & -1.000 & 0.000 \\ \text { C } & 1.711 & -1.258 & 0.000 \\ \text { C } & 2.688 & -0.307 & 0.000 \\ \text { H } & -0.043 & 2.826 & 0.000 \\ \text { H } & -2.397 & 2.041 & 0.000 \\ \text { H } & 1.876 & -2.323 & 0.000\end{array}$




$\begin{array}{lrrr}\mathrm{H} & 2.601 & 1.809 & 0.000 \\ \mathrm{~N} & 2.100 & 0.938 & 0.000 \\ \mathrm{H} & -1.143 & -2.050 & 0.000 \\ \mathrm{Cl} & -3.537 & -0.532 & 0.000 \\ \mathrm{C} & 4.170 & -0.450 & 0.000 \\ \mathrm{H} & 4.612 & 0.016 & -0.883 \\ \mathrm{H} & 4.612 & 0.016 & 0.883 \\ \mathrm{H} & 4.439 & -1.504 & 0.000\end{array}$

$\begin{array}{lrrr}\text { Ar8 } & & & \\ \text { C } & 0.389 & -0.490 & 0.000 \\ \text { C } & 0.792 & 0.857 & 0.000 \\ \text { C } & -0.132 & 1.900 & 0.000 \\ \text { C } & -1.469 & 1.574 & 0.000 \\ \text { C } & -1.892 & 0.230 & 0.000 \\ \text { C } & -0.980 & -0.806 & 0.000 \\ \text { C } & 1.585 & -1.278 & 0.000 \\ \text { C } & 2.645 & -0.417 & 0.000 \\ \text { H } & 0.183 & 2.935 & 0.000 \\ \text { H } & -2.230 & 2.342 & 0.000 \\ \text { H } & 1.657 & -2.354 & 0.000 \\ \text { H } & 2.743 & 1.697 & 0.000 \\ \text { N } & 2.170 & 0.873 & 0.000 \\ \text { H } & -1.293 & -1.840 & 0.000 \\ \text { C } & 4.108 & -0.693 & 0.000 \\ \text { H } & 4.592 & -0.270 & -0.883 \\ \text { H } & 4.592 & -0.270 & 0.883 \\ \text { H } & 4.281 & -1.767 & 0.000 \\ \text { O } & -3.246 & 0.061 & 0.000 \\ \text { C } & -3.732 & -1.257 & 0.000 \\ \text { H } & -3.405 & -1.802 & 0.891 \\ \text { H } & -4.817 & -1.185 & 0.000 \\ \text { H } & -3.404 & -1.802 & -0.891\end{array}$

\section{Ar9}

$\begin{array}{lrrr}\mathrm{C} & -1.467 & -0.672 & 0.000 \\ \mathrm{C} & -1.906 & 0.669 & 0.000 \\ \mathrm{C} & -1.016 & 1.741 & 0.000 \\ \mathrm{C} & 0.333 & 1.458 & 0.000 \\ \mathrm{C} & 0.774 & 0.126 & 0.000 \\ \mathrm{C} & -0.093 & -0.941 & 0.000 \\ \mathrm{C} & -2.639 & -1.495 & 0.000 \\ \mathrm{C} & -3.710 & -0.655 & 0.000 \\ \mathrm{H} & -1.364 & 2.766 & 0.000 \\ \mathrm{H} & 1.059 & 2.258 & 0.000 \\ \mathrm{H} & -2.678 & -2.571 & 0.000 \\ \mathrm{H} & -3.872 & 1.458 & 0.000 \\ \mathrm{~N} & -3.278 & 0.649 & 0.000 \\ \mathrm{H} & -4.764 & -0.880 & 0.000 \\ \mathrm{H} & 0.280 & -1.956 & 0.000\end{array}$

$\mathrm{Br}$

2.646

$-0.200$

0.000

Ar10

$\begin{array}{lrrr}\mathrm{C} & -0.857 & -0.682 & 0.000 \\ \mathrm{C} & -1.229 & 0.679 & 0.000 \\ \mathrm{C} & -0.287 & 1.705 & 0.000 \\ \mathrm{C} & 1.046 & 1.354 & 0.000 \\ \mathrm{C} & 1.422 & 0.003 & 0.000 \\ \mathrm{C} & 0.502 & -1.019 & 0.000 \\ \mathrm{C} & -2.069 & -1.447 & 0.000 \\ \mathrm{C} & -3.097 & -0.554 & 0.000 \\ \mathrm{H} & -0.583 & 2.746 & 0.000 \\ \mathrm{H} & 1.815 & 2.114 & 0.000 \\ \mathrm{H} & -2.161 & -2.519 & 0.000 \\ \mathrm{H} & -3.154 & 1.564 & 0.000 \\ \mathrm{~N} & -2.600 & 0.727 & 0.000 \\ \mathrm{H} & -4.160 & -0.727 & 0.000 \\ \mathrm{H} & 0.828 & -2.050 & 0.000 \\ \mathrm{Cl} & 3.119 & -0.379 & 0.000\end{array}$

Ar11

$\begin{array}{lrrr}\text { C } & -0.742 & -0.696 & 0.000 \\ \text { C } & -1.117 & 0.667 & 0.000 \\ \text { C } & -0.179 & 1.701 & 0.000 \\ \text { C } & 1.151 & 1.355 & 0.000 \\ \text { C } & 1.545 & 0.000 & 0.000 \\ \text { C } & 0.613 & -1.025 & 0.000 \\ \text { C } & -1.953 & -1.462 & 0.000 \\ \text { C } & -2.981 & -0.572 & 0.000 \\ \text { H } & -0.484 & 2.739 & 0.000 \\ \text { H } & 1.915 & 2.120 & 0.000 \\ \text { H } & -3.038 & 1.548 & 0.000 \\ \text { N } & -2.483 & 0.711 & 0.000 \\ \text { H } & 0.940 & -2.057 & 0.000 \\ \text { H } & -4.044 & -0.743 & 0.000 \\ \text { H } & -2.044 & -2.535 & 0.000 \\ \text { C } & 2.944 & -0.316 & 0.000 \\ \text { N } & 4.065 & -0.565 & 0.000\end{array}$

Ar12

$\begin{array}{lrrr}C & -0.537 & -0.694 & 0.000 \\ C & -0.828 & 0.684 & 0.000 \\ C & 0.178 & 1.650 & 0.000 \\ C & 1.482 & 1.209 & 0.000 \\ C & 1.812 & -0.163 & 0.000 \\ C & 0.804 & -1.105 & 0.000 \\ C & -1.793 & -1.385 & 0.000 \\ C & -2.768 & -0.433 & 0.000 \\ \text { H } & -0.055 & 2.707 & 0.000\end{array}$




$\begin{array}{lrrr}\mathrm{H} & 2.286 & 1.936 & 0.000 \\ \mathrm{H} & -2.697 & 1.684 & -0.001 \\ \mathrm{~N} & -2.196 & 0.815 & 0.000 \\ \mathrm{H} & 1.048 & -2.161 & 0.000 \\ \mathrm{H} & -3.840 & -0.542 & -0.001 \\ \mathrm{H} & -1.950 & -2.450 & 0.001 \\ \mathrm{C} & 3.261 & -0.572 & 0.000 \\ \mathrm{H} & 3.779 & -0.183 & -0.879 \\ \mathrm{H} & 3.778 & -0.185 & 0.880 \\ \mathrm{H} & 3.363 & -1.656 & -0.001\end{array}$

\section{Ar13}

$\begin{array}{lrrr}\text { C } & 0.524 & -0.693 & 0.004 \\ \text { C } & 0.805 & 0.688 & -0.001 \\ \text { C } & -0.210 & 1.640 & 0.000 \\ \text { C } & -1.513 & 1.194 & 0.008 \\ \text { C } & -1.825 & -0.183 & 0.009 \\ \text { C } & -0.810 & -1.121 & 0.006 \\ \text { C } & 1.785 & -1.372 & 0.000 \\ \text { C } & 2.753 & -0.412 & -0.006 \\ \text { H } & 0.009 & 2.701 & -0.003 \\ \text { H } & -2.325 & 1.911 & 0.022 \\ \text { H } & 1.951 & -2.436 & 0.003 \\ \text { H } & 2.671 & 1.702 & -0.009 \\ \text { N } & 2.175 & 0.830 & -0.007 \\ \text { H } & 3.826 & -0.515 & -0.009 \\ \text { H } & -1.048 & -2.178 & 0.010 \\ \text { H } & -3.808 & 0.076 & -0.363 \\ \text { H } & -3.341 & -1.513 & -0.250 \\ \text { N } & -3.173 & -0.573 & 0.077\end{array}$

$\begin{array}{lrrr}\text { Ar14 } & & & \\ \text { C } & -0.511 & -0.694 & 0.000 \\ \text { C } & -0.786 & 0.687 & 0.000 \\ \text { C } & 0.230 & 1.641 & 0.000 \\ \text { C } & 1.532 & 1.197 & 0.000 \\ \text { C } & 1.824 & -0.179 & 0.000 \\ \text { C } & 0.823 & -1.125 & 0.000 \\ \text { C } & -1.774 & -1.369 & 0.000 \\ \text { C } & -2.739 & -0.406 & 0.000 \\ \text { H } & 0.009 & 2.701 & 0.000 \\ \text { H } & 2.360 & 1.893 & 0.000 \\ \text { H } & -2.647 & 1.709 & -0.001 \\ \text { N } & -2.155 & 0.834 & 0.000 \\ \text { H } & 1.064 & -2.182 & 0.000 \\ \text { O } & 3.152 & -0.509 & 0.000 \\ \text { H } & -1.944 & -2.432 & 0.000 \\ \text { H } & -3.812 & -0.505 & 0.000 \\ \text { H } & 3.242 & -1.466 & 0.001\end{array}$

Ar15

$\begin{array}{lrrr}\text { C } & 0.768 & -0.651 & 0.000 \\ \text { C } & 1.310 & 0.647 & 0.000 \\ \text { C } & 0.499 & 1.783 & 0.000 \\ \text { C } & -0.862 & 1.599 & 0.000 \\ \text { C } & -1.425 & 0.305 & 0.000 \\ \text { C } & -0.628 & -0.821 & 0.000 \\ \text { C } & 1.871 & -1.562 & 0.000 \\ \text { C } & 3.008 & -0.809 & 0.000 \\ \text { H } & 0.922 & 2.779 & 0.000 \\ \text { H } & -1.539 & 2.441 & 0.000 \\ \text { H } & 3.335 & 1.282 & 0.000 \\ \text { N } & 2.680 & 0.522 & 0.000 \\ \text { H } & -1.048 & -1.816 & 0.000 \\ \text { O } & -2.788 & 0.282 & 0.000 \\ \text { C } & -3.413 & -0.978 & 0.000 \\ \text { H } & -3.145 & -1.554 & 0.891 \\ \text { H } & -4.483 & -0.791 & -0.001 \\ \text { H } & -3.144 & -1.554 & -0.891 \\ \text { H } & 1.827 & -2.638 & 0.000 \\ \text { H } & 4.041 & -1.118 & -0.001\end{array}$

Ar16

$\begin{array}{lrrr}\text { C } & -1.143 & -0.672 & 0.000 \\ \mathrm{C} & -1.529 & 0.688 & 0.000 \\ \mathrm{C} & -0.599 & 1.729 & 0.000 \\ \mathrm{C} & 0.735 & 1.392 & 0.000 \\ \mathrm{C} & 1.141 & 0.042 & 0.000 \\ \mathrm{C} & 0.215 & -0.986 & 0.000 \\ \mathrm{C} & -2.349 & -1.449 & 0.000 \\ \mathrm{C} & -3.383 & -0.568 & 0.000 \\ \mathrm{H} & -0.913 & 2.765 & 0.000 \\ \mathrm{H} & 1.489 & 2.165 & 0.000 \\ \mathrm{H} & -2.430 & -2.522 & 0.000 \\ \mathrm{H} & -3.457 & 1.554 & 0.000 \\ \mathrm{~N} & -2.896 & 0.721 & 0.000 \\ \mathrm{H} & -4.445 & -0.747 & 0.000 \\ \mathrm{H} & 0.565 & -2.011 & 0.000 \\ \mathrm{C} & 2.574 & -0.329 & 0.000 \\ \mathrm{O} & 2.999 & -1.454 & 0.000 \\ \mathrm{O} & 3.400 & 0.739 & 0.000 \\ \mathrm{H} & 4.301 & 0.386 & 0.000\end{array}$

\section{Ar17}

$\begin{array}{lrrr}C & -0.385 & 0.982 & 0.000 \\ C & 0.152 & -0.325 & 0.000 \\ C & -0.653 & -1.464 & 0.000 \\ C & -2.020 & -1.275 & 0.000 \\ C & -2.576 & 0.016 & 0.000\end{array}$




$\begin{array}{lrrr}\text { C } & -1.776 & 1.139 & 0.000 \\ \mathrm{C} & 0.729 & 1.882 & 0.000 \\ \mathrm{C} & 1.853 & 1.113 & 0.000 \\ \mathrm{H} & -0.225 & -2.459 & 0.000 \\ \mathrm{H} & -2.677 & -2.135 & 0.000 \\ \mathrm{H} & -3.653 & 0.125 & 0.000 \\ \mathrm{H} & -2.215 & 2.129 & 0.000 \\ \mathrm{H} & 0.697 & 2.958 & -0.001 \\ \mathrm{H} & 2.891 & 1.404 & 0.001 \\ \mathrm{~N} & 1.519 & -0.220 & 0.000 \\ \mathrm{C} & 2.432 & -1.335 & 0.000 \\ \mathrm{H} & 2.288 & -1.954 & 0.888 \\ \mathrm{H} & 2.286 & -1.955 & -0.887 \\ \mathrm{H} & 3.451 & -0.957 & -0.001\end{array}$

\section{Ar18}

$\begin{array}{lrrr}\text { C } & 0.548 & -0.957 & 0.000 \\ \text { C } & 0.305 & 0.433 & 0.000 \\ \text { C } & 1.334 & 1.372 & 0.000 \\ \text { C } & 2.630 & 0.894 & 0.000 \\ \text { C } & 2.896 & -0.483 & 0.000 \\ \text { C } & 1.871 & -1.409 & 0.000 \\ \text { C } & -0.735 & -1.592 & 0.000 \\ \text { C } & -1.680 & -0.607 & 0.000 \\ \text { H } & 1.129 & 2.436 & 0.000 \\ \text { H } & 3.456 & 1.594 & 0.000 \\ \text { H } & 3.924 & -0.822 & 0.000 \\ \text { H } & 2.088 & -2.470 & 0.000 \\ \text { H } & -0.940 & -2.650 & 0.000 \\ \text { N } & -1.056 & 0.626 & 0.000 \\ \text { C } & -1.682 & 1.924 & 0.001 \\ \text { H } & -1.394 & 2.491 & 0.888 \\ \text { H } & -1.392 & 2.492 & -0.885 \\ \text { H } & -2.762 & 1.808 & -0.001 \\ \text { C } & -3.164 & -0.732 & -0.001 \\ \text { H } & -3.610 & -0.267 & 0.882 \\ \text { H } & -3.609 & -0.267 & -0.883 \\ \text { H } & -3.435 & -1.785 & -0.001\end{array}$

\section{Ar19}

$\begin{array}{lrrr}\text { C } & -0.364 & -0.464 & 0.000 \\ \text { C } & -1.349 & 0.537 & 0.000 \\ \text { C } & -1.040 & 1.902 & 0.000 \\ \text { C } & 0.294 & 2.233 & 0.000 \\ \text { C } & 1.310 & 1.258 & 0.000 \\ C & 0.990 & -0.086 & 0.000 \\ \text { C } & -1.032 & -1.729 & 0.000 \\ \text { C } & -2.367 & -1.462 & 0.000 \\ \text { H } & -1.812 & 2.660 & 0.000 \\ \text { H } & 0.582 & 3.276 & 0.000\end{array}$

$\begin{array}{rrrr}\mathrm{H} & 2.341 & 1.580 & 0.000 \\ \mathrm{H} & -0.573 & -2.702 & 0.000 \\ \mathrm{H} & -3.457 & 0.358 & 0.000 \\ \mathrm{~N} & -2.564 & -0.100 & 0.000 \\ \mathrm{H} & -3.208 & -2.135 & 0.000 \\ \mathrm{O} & 1.886 & -1.103 & 0.000 \\ \mathrm{C} & 3.253 & -0.763 & 0.000 \\ \mathrm{H} & 3.801 & -1.701 & 0.000 \\ \mathrm{H} & 3.516 & -0.187 & -0.891 \\ \mathrm{H} & 3.515 & -0.187 & 0.891\end{array}$

Ar20

$\begin{array}{lrrr}\text { C } & 0.330 & -1.118 & 0.000 \\ \text { C } & -0.978 & -0.710 & 0.000 \\ \text { C } & -0.978 & 0.710 & 0.000 \\ \text { C } & 0.330 & 1.118 & 0.000 \\ \text { N } & 1.115 & 0.000 & 0.000 \\ \text { H } & 2.119 & 0.000 & 0.000 \\ \text { H } & 0.763 & -2.104 & 0.000 \\ \text { H } & -1.839 & -1.357 & 0.000 \\ \text { H } & -1.838 & 1.357 & 0.000 \\ \text { H } & 0.763 & 2.104 & 0.000\end{array}$

Ar21

$\begin{array}{lrrr}\mathrm{C} & 1.129 & 0.022 & 0.000 \\ \mathrm{C} & 0.712 & -1.282 & 0.000 \\ \mathrm{C} & -0.712 & -1.282 & 0.000 \\ \mathrm{C} & -1.129 & 0.022 & 0.000 \\ \mathrm{~N} & 0.000 & 0.802 & 0.000 \\ \mathrm{H} & 0.000 & 1.808 & 0.000 \\ \mathrm{H} & 1.362 & -2.142 & 0.000 \\ \mathrm{H} & -1.362 & -2.142 & 0.000 \\ \mathrm{C} & -2.495 & 0.616 & 0.000 \\ \mathrm{H} & -2.667 & 1.236 & 0.883 \\ \mathrm{H} & -2.667 & 1.236 & -0.883 \\ \mathrm{H} & -3.238 & -0.178 & 0.000 \\ \mathrm{C} & 2.495 & 0.616 & 0.000 \\ \mathrm{H} & 2.667 & 1.236 & -0.883 \\ \mathrm{H} & 2.667 & 1.236 & 0.883 \\ \mathrm{H} & 3.238 & -0.178 & 0.000\end{array}$

Ar22

$\begin{array}{lrrr}\text { C } & 1.107 & -0.164 & 0.000 \\ \text { C } & -0.019 & -0.946 & 0.000 \\ \text { C } & -1.159 & -0.089 & 0.000 \\ \text { C } & -0.681 & 1.192 & 0.000 \\ \text { N } & 0.691 & 1.138 & 0.000 \\ \text { H } & 1.302 & 1.934 & 0.000 \\ \text { H } & -0.028 & -2.025 & 0.000\end{array}$




$\begin{array}{lrrr}\mathrm{H} & -1.204 & 2.134 & 0.000 \\ \mathrm{C} & 2.552 & -0.529 & 0.000 \\ \mathrm{H} & 3.065 & -0.143 & -0.884 \\ \mathrm{H} & 3.066 & -0.142 & 0.883 \\ \mathrm{H} & 2.655 & -1.612 & 0.001 \\ \mathrm{C} & -2.595 & -0.506 & 0.000 \\ \mathrm{H} & -2.834 & -1.107 & -0.879 \\ \mathrm{H} & -2.834 & -1.106 & 0.880 \\ \mathrm{H} & -3.252 & 0.363 & -0.001\end{array}$

$\begin{array}{lrrr}\mathrm{C} & -0.716 & 0.953 & 0.000 \\ \mathrm{C} & -1.087 & -0.347 & 0.000 \\ \mathrm{O} & 0.000 & -1.150 & 0.000 \\ \mathrm{H} & 2.042 & -0.843 & 0.000 \\ \mathrm{H} & 1.372 & 1.806 & 0.000 \\ \mathrm{H} & -1.372 & 1.806 & 0.000 \\ \mathrm{H} & -2.042 & -0.843 & 0.000\end{array}$

Ar23

$\begin{array}{lrrr}\text { C } & -0.172 & -1.112 & -0.013 \\ \text { C } & -1.483 & -0.709 & 0.014 \\ \text { C } & -1.483 & 0.709 & 0.014 \\ \text { C } & -0.173 & 1.112 & -0.012 \\ \text { N } & 0.619 & 0.000 & -0.034 \\ \text { H } & 0.265 & -2.096 & -0.022 \\ \text { H } & -2.343 & -1.358 & 0.020 \\ \text { H } & -2.343 & 1.358 & 0.020 \\ \text { H } & 0.265 & 2.096 & -0.021 \\ \text { C } & 2.063 & 0.000 & 0.023 \\ \text { H } & 2.445 & 0.887 & -0.479 \\ \text { H } & 2.420 & -0.005 & 1.055 \\ \text { H } & 2.446 & -0.881 & -0.489\end{array}$

\section{Ar24}$$
\text { C }
$$

$\mathrm{C}$

C

C

N

$\mathrm{H}$

$\mathrm{H}$

C

$\mathrm{H}$

$\mathrm{H}$

$\mathrm{H}$

C

$\mathrm{H}$

$\mathrm{H}$

$\mathrm{H}$

C

$\mathrm{H}$

$\mathrm{H}$

$\mathrm{H}$

$1.124-0.301$

$-0.007$

$0.710-1.606$

0.015

$-0.711-1.606$

0.015

$-1.125$

$-0.300$

$-0.007$

0.000

0.491

$-0.029$

1.361

$-2.465$

0.025

$-1.363$

$-2.464$

1.934

0.024

0.028

0.001

2.323

$-0.472$

0.007

2.296

1.059

0.880

2.322

$-0.482$

$-2.498$

0.277

$-0.012$

$-2.667$

0.944

0.838

$-2.705$

0.843

$-0.924$

$-3.225$

$-0.532$

0.049

2.498

0.275

$-0.012$

2.706

0.841

$-0.924$

2.667

0.943

0.837

3.224

$-0.533$

0.050

Ar26

$\begin{array}{lrrr}\text { C } & -0.665 & 0.116 & 0.000 \\ \text { C } & -1.534 & 1.164 & 0.000 \\ \text { C } & -2.856 & 0.620 & 0.000 \\ \text { C } & -2.697 & -0.722 & 0.000 \\ \text { O } & -1.389 & -1.047 & 0.000 \\ \text { H } & -1.260 & 2.206 & 0.000 \\ \text { H } & -3.791 & 1.155 & 0.000 \\ \text { H } & -3.392 & -1.545 & 0.000 \\ \text { Si } & 1.202 & 0.007 & 0.000 \\ \mathrm{C} & 1.759 & -0.913 & 1.530 \\ \text { H } & 1.312 & -1.908 & 1.560 \\ \text { H } & 2.844 & -1.030 & 1.542 \\ \text { H } & 1.462 & -0.383 & 2.436 \\ \mathrm{C} & 1.759 & -0.913 & -1.530 \\ \text { H } & 1.462 & -0.383 & -2.436 \\ \text { H } & 2.844 & -1.029 & -1.542 \\ \text { H } & 1.312 & -1.908 & -1.560 \\ \text { C } & 1.834 & 1.766 & 0.000 \\ \text { H } & 2.925 & 1.782 & 0.000 \\ \text { H } & 1.491 & 2.307 & -0.884 \\ \text { H } & 1.491 & 2.307 & 0.884\end{array}$

Ar27

\section{Ar25}

C

$\begin{array}{lrr}1.087 & -0.347 & 0.000 \\ 0.716 & 0.953 & 0.000\end{array}$

$\begin{array}{rrr}0.634 & 0.104 & 0.000 \\ -0.216 & 1.158 & 0.000 \\ -1.542 & 0.612 & 0.000 \\ -1.389 & -0.729 & 0.000 \\ -0.073 & -1.054 & 0.000 \\ 0.066 & 2.197 & 0.000 \\ -2.474 & 1.150 & 0.000 \\ -2.080 & -1.552 & 0.000 \\ 2.112 & -0.016 & 0.000 \\ 2.460 & -0.556 & 0.882 \\ 2.460 & -0.556 & -0.882 \\ 2.559 & 0.975 & 0.000\end{array}$

\section{Ar28}

$\begin{array}{lrrr}C & -3.640 & 0.178 & -0.033 \\ C & -3.631 & -1.168 & 0.156 \\ C & -2.259 & -1.547 & 0.215\end{array}$




\begin{tabular}{lrrr}
$\mathrm{C}$ & -1.525 & -0.412 & 0.059 \\
$\mathrm{O}$ & -2.380 & 0.646 & -0.094 \\
$\mathrm{H}$ & -4.498 & -1.801 & 0.242 \\
$\mathrm{H}$ & -1.853 & -2.535 & 0.356 \\
$\mathrm{C}$ & -4.733 & 1.171 & -0.178 \\
$\mathrm{H}$ & -4.675 & 1.927 & 0.606 \\
$\mathrm{H}$ & -4.666 & 1.682 & -1.140 \\
$\mathrm{H}$ & -5.697 & 0.671 & -0.113 \\
$\mathrm{C}$ & 2.196 & -0.671 & -0.099 \\
$\mathrm{C}$ & 1.998 & 0.868 & 0.078 \\
$\mathrm{~B}$ & -0.005 & -0.176 & 0.032 \\
$\mathrm{O}$ & 0.592 & 1.030 & -0.209 \\
$\mathrm{O}$ & 0.894 & -1.188 & 0.248 \\
$\mathrm{C}$ & 2.461 & -1.069 & -1.546 \\
$\mathrm{H}$ & 3.457 & -0.761 & -1.866 \\
$\mathrm{H}$ & 2.387 & -2.152 & -1.630 \\
$\mathrm{H}$ & 1.724 & -0.621 & -2.214 \\
$\mathrm{C}$ & 2.802 & 1.725 & -0.879 \\
$\mathrm{H}$ & 2.605 & 2.778 & -0.677 \\
$\mathrm{H}$ & 3.871 & 1.544 & -0.743 \\
$\mathrm{H}$ & 2.538 & 1.520 & -1.914 \\
$\mathrm{C}$ & 2.213 & 1.329 & 1.515 \\
$\mathrm{H}$ & 3.268 & 1.290 & 1.789 \\
$\mathrm{H}$ & 1.864 & 2.356 & 1.608 \\
$\mathrm{H}$ & 1.647 & 0.710 & 2.212 \\
$\mathrm{C}$ & 3.235 & -1.286 & 0.818 \\
$\mathrm{H}$ & 3.309 & -2.355 & 0.618 \\
$\mathrm{H}$ & 4.213 & -0.835 & 0.638 \\
$\mathrm{H}$ & 2.971 & -1.151 & 1.864 \\
& & & \\
\hline & & &
\end{tabular}

\section{Ar29}

$\begin{array}{lrrr}\text { C } & 2.951 & -0.007 & 0.035 \\ \text { C } & 2.930 & -1.360 & 0.072 \\ \text { C } & 1.572 & -1.747 & -0.176 \\ \text { C } & 0.855 & -0.610 & -0.354 \\ \text { O } & 1.706 & 0.464 & -0.218 \\ \text { H } & 3.780 & -1.999 & 0.244 \\ \text { H } & 1.171 & -2.745 & -0.231 \\ \text { C } & 4.029 & 1.000 & 0.198 \\ \text { H } & 4.138 & 1.605 & -0.705 \\ \text { H } & 3.818 & 1.676 & 1.029 \\ \text { H } & 4.975 & 0.497 & 0.394 \\ \mathrm{C} & -1.108 & 1.746 & 0.700 \\ \mathrm{C} & -1.046 & 2.050 & -0.816 \\ \mathrm{H} & -1.843 & 2.348 & 1.238 \\ \mathrm{H} & -0.130 & 1.876 & 1.161 \\ \mathrm{H} & -1.942 & 2.585 & -1.147 \\ \mathrm{H} & -0.181 & 2.686 & -1.024 \\ \mathrm{O} & -0.960 & 0.829 & -1.491 \\ \mathrm{O} & -1.455 & -1.478 & -0.840 \\ \mathrm{C} & -2.775 & -1.313 & -0.396\end{array}$

$\begin{array}{lrrr}\mathrm{H} & -3.476 & -1.260 & -1.233 \\ \mathrm{H} & -3.054 & -2.172 & 0.222 \\ \mathrm{C} & -2.839 & 0.002 & 0.415 \\ \mathrm{H} & -3.214 & 0.815 & -0.202 \\ \mathrm{H} & -3.450 & -0.073 & 1.315 \\ \mathrm{~N} & -1.432 & 0.312 & 0.769 \\ \mathrm{C} & -1.025 & -0.344 & 2.014 \\ \mathrm{H} & 0.039 & -0.184 & 2.171 \\ \mathrm{H} & -1.595 & 0.050 & 2.858 \\ \mathrm{H} & -1.202 & -1.415 & 1.913 \\ \mathrm{~B} & -0.679 & -0.301 & -0.628\end{array}$

Ar30

$\begin{array}{lrrr}\text { C } & 3.171 & -0.254 & 0.004 \\ \text { C } & 3.080 & -1.575 & -0.277 \\ \text { C } & 1.700 & -1.837 & -0.555 \\ \text { C } & 1.041 & -0.659 & -0.432 \\ \text { O } & 1.943 & 0.317 & -0.081 \\ \text { H } & 3.900 & -2.274 & -0.295 \\ \text { H } & 1.250 & -2.777 & -0.827 \\ \text { C } & 4.303 & 0.640 & 0.349 \\ \text { H } & 4.413 & 1.431 & -0.394 \\ \text { H } & 4.151 & 1.113 & 1.321 \\ \text { H } & 5.226 & 0.065 & 0.385 \\ \text { C } & -0.871 & 1.702 & 0.950 \\ \text { H } & -1.625 & 2.247 & 1.515 \\ \text { H } & 0.119 & 1.906 & 1.361 \\ \text { O } & -0.705 & 1.022 & -1.280 \\ \text { O } & -1.359 & -1.305 & -0.902 \\ \text { C } & -2.518 & -0.127 & 0.755 \\ \text { H } & -3.065 & 0.707 & 0.313 \\ \text { H } & -2.995 & -0.415 & 1.689 \\ \text { N } & -1.097 & 0.247 & 0.946 \\ \text { C } & -0.510 & -0.459 & 2.100 \\ \text { H } & 0.555 & -0.247 & 2.139 \\ \text { H } & -0.999 & -0.135 & 3.019 \\ \text { H } & -0.651 & -1.531 & 1.961 \\ \text { B } & -0.464 & -0.224 & -0.542 \\ \text { C } & -0.869 & 2.098 & -0.528 \\ \text { C } & -2.515 & -1.279 & -0.253 \\ \text { O } & -0.996 & 3.218 & -0.917 \\ \text { O } & -3.428 & -2.026 & -0.416\end{array}$

Ar31

$\begin{array}{rrrr}\text { C } & 2.717 & -0.940 & 0.002 \\ \text { C } & 3.322 & 0.263 & 0.003 \\ \text { C } & 2.282 & 1.253 & -0.003 \\ \text { C } & 1.123 & 0.554 & -0.008 \\ \text { O } & 1.358 & -0.778 & -0.005 \\ \text { H } & 3.069 & -1.955 & 0.005\end{array}$




$\begin{array}{lrrr}\mathrm{H} & 4.385 & 0.438 & 0.007 \\ \mathrm{H} & 2.375 & 2.323 & -0.004 \\ \mathrm{O} & -0.142 & 0.946 & -0.015 \\ \mathrm{Si} & -1.523 & -0.016 & 0.001 \\ \mathrm{C} & -1.539 & -1.049 & 1.549 \\ \mathrm{H} & -2.471 & -1.615 & 1.618 \\ \mathrm{H} & -0.710 & -1.757 & 1.556 \\ \mathrm{H} & -1.462 & -0.421 & 2.438 \\ \mathrm{C} & -1.552 & -1.082 & -1.525 \\ \mathrm{H} & -2.485 & -1.648 & -1.574 \\ \mathrm{H} & -1.482 & -0.472 & -2.427 \\ \mathrm{H} & -0.724 & -1.790 & -1.524 \\ \mathrm{C} & -2.906 & 1.224 & -0.007 \\ \mathrm{H} & -2.858 & 1.854 & -0.896 \\ \mathrm{H} & -3.875 & 0.722 & 0.001 \\ \mathrm{H} & -2.853 & 1.871 & 0.870\end{array}$

Ar34

$\begin{array}{lrrr}\text { C } & -0.774 & 0.218 & 0.000 \\ \text { C } & -0.015 & 1.349 & 0.000 \\ \text { C } & 1.384 & 1.088 & 0.000 \\ \text { C } & 1.663 & -0.241 & 0.000 \\ \text { S } & 0.230 & -1.178 & 0.000 \\ \text { H } & -0.447 & 2.340 & -0.001 \\ \text { H } & 2.144 & 1.856 & 0.001 \\ \text { H } & 2.627 & -0.722 & 0.000 \\ \text { C } & -2.265 & 0.106 & 0.000 \\ \text { H } & -2.628 & -0.425 & -0.881 \\ \text { H } & -2.628 & -0.425 & 0.881 \\ \text { H } & -2.705 & 1.103 & 0.000\end{array}$

Ar35

Ar32

$\begin{array}{lrrr}\text { C } & -0.003 & -1.229 & 0.000 \\ \text { C } & -1.262 & -0.711 & 0.000 \\ \text { C } & -1.262 & 0.711 & 0.000 \\ \text { C } & -0.003 & 1.229 & 0.000 \\ \text { S } & 1.182 & 0.000 & 0.000 \\ \text { H } & 0.289 & -2.267 & 0.000 \\ \text { H } & -2.155 & -1.318 & 0.000 \\ \text { H } & -2.155 & 1.318 & 0.000 \\ \text { H } & 0.289 & 2.267 & 0.000\end{array}$

Ar33

$\begin{array}{lrrr}\mathrm{C} & 0.452 & 0.200 & 0.000 \\ \mathrm{C} & 1.192 & 1.355 & 0.000 \\ \mathrm{C} & 2.596 & 1.140 & 0.000 \\ \mathrm{C} & 2.912 & -0.185 & 0.000 \\ \mathrm{~S} & 1.508 & -1.155 & 0.000 \\ \mathrm{H} & 0.740 & 2.338 & 0.000 \\ \mathrm{H} & 3.333 & 1.930 & 0.000 \\ \mathrm{H} & 3.892 & -0.635 & 0.000 \\ \mathrm{Si} & -1.411 & 0.030 & 0.000 \\ \mathrm{C} & -1.944 & -0.907 & 1.529 \\ \mathrm{H} & -3.028 & -1.031 & 1.552 \\ \mathrm{H} & -1.491 & -1.901 & 1.552 \\ \mathrm{H} & -1.640 & -0.380 & 2.435 \\ \mathrm{C} & -1.944 & -0.909 & -1.528 \\ \mathrm{H} & -3.028 & -1.033 & -1.551 \\ \mathrm{H} & -1.640 & -0.384 & -2.434 \\ \mathrm{H} & -1.491 & -1.903 & -1.550 \\ \mathrm{C} & -2.128 & 1.757 & -0.001 \\ \mathrm{H} & -3.218 & 1.715 & -0.001 \\ \mathrm{H} & -1.815 & 2.315 & 0.883 \\ \mathrm{H} & -1.814 & 2.314 & -0.886\end{array}$

Ar36

$\begin{array}{lrrr}\text { C } & -1.250 & -0.014 & 0.078 \\ \text { C } & 0.098 & -0.204 & 0.267 \\ \text { C } & 0.744 & 1.054 & 0.051 \\ \text { C } & -0.235 & 1.960 & -0.254 \\ \text { H } & -0.171 & 3.012 & -0.475 \\ \text { N } & -1.439 & 1.305 & -0.237 \\ \text { H } & -2.331 & 1.729 & -0.419 \\ \text { C } & -2.390 & -0.970 & 0.151 \\ \text { H } & -3.162 & -0.628 & 0.843 \\ \text { H } & -2.857 & -1.119 & -0.826 \\ \text { H } & -2.039 & -1.941 & 0.498 \\ \text { C } & 2.211 & 1.319 & 0.162 \\ \text { H } & 2.577 & 1.122 & 1.171 \\ \text { H } & 2.786 & 0.688 & -0.520 \\ \text { H } & 2.436 & 2.359 & -0.076 \\ \text { C } & 0.791 & -1.494 & 0.578 \\ \text { H } & 0.147 & -2.130 & 1.190 \\ \text { H } & 1.676 & -1.287 & 1.186\end{array}$




$\begin{array}{llll}\mathrm{C} & 1.210 & -2.255 & -0.682 \\ \mathrm{H} & 1.741 & -3.176 & -0.436 \\ \mathrm{H} & 0.333 & -2.512 & -1.279 \\ \mathrm{H} & 1.861 & -1.639 & -1.304\end{array}$

Ar37

$\begin{array}{lrrr}\text { C } & 2.486 & 0.000 & 0.000 \\ \text { C } & 1.899 & -1.260 & 0.000 \\ \text { C } & 0.549 & -1.587 & 0.000 \\ \text { C } & 1.899 & 1.260 & 0.000 \\ \text { C } & -0.550 & -0.745 & 0.000 \\ \text { C } & 0.549 & 1.587 & 0.000 \\ \text { C } & -0.550 & 0.745 & 0.000 \\ \text { H } & 3.571 & 0.000 & 0.000 \\ \text { H } & 2.587 & -2.097 & 0.000 \\ \text { H } & 0.317 & -2.649 & 0.000 \\ \text { H } & 2.587 & 2.097 & 0.000 \\ \text { H } & 0.317 & 2.649 & 0.000 \\ \text { C } & -1.890 & -1.145 & 0.000 \\ \text { H } & -2.232 & -2.168 & -0.001 \\ \text { C } & -2.692 & 0.000 & 0.000 \\ \text { H } & -3.772 & -0.001 & 0.000 \\ \text { C } & -1.890 & 1.145 & 0.000 \\ \text { H } & -2.232 & 2.169 & 0.000\end{array}$

Ar38

En2

$\begin{array}{lrrr}\mathrm{C} & 0.459 & 0.661 & 0.000 \\ \mathrm{C} & 0.000 & -0.677 & 0.000 \\ \mathrm{C} & -2.159 & -0.116 & 0.000 \\ \mathrm{C} & -1.842 & 1.250 & 0.000 \\ \mathrm{C} & -0.520 & 1.653 & 0.000 \\ \mathrm{C} & 1.890 & 0.609 & 0.000 \\ \mathrm{C} & 2.232 & -0.710 & 0.000 \\ \mathrm{H} & -3.197 & -0.427 & 0.000 \\ \mathrm{H} & -2.642 & 1.976 & 0.000 \\ \mathrm{H} & -0.257 & 2.704 & 0.000 \\ \mathrm{H} & 2.576 & 1.439 & 0.000 \\ \mathrm{H} & 1.061 & -2.494 & 0.000 \\ \mathrm{H} & 3.208 & -1.165 & 0.000 \\ \mathrm{~N} & 1.100 & -1.490 & 0.000 \\ \mathrm{~N} & -1.259 & -1.089 & 0.000\end{array}$

$\begin{array}{lrrr}\text { En1 } & & & \\ \text { C } & 0.712 & 0.053 & -0.044 \\ \text { C } & 1.387 & 1.180 & 0.214 \\ \text { C } & 1.438 & -1.257 & -0.268 \\ \text { C } & 2.888 & 1.273 & 0.275 \\ \text { H } & 0.852 & 2.108 & 0.371 \\ \text { C } & 2.900 & -1.045 & -0.644\end{array}$

$\begin{array}{lrrr}\text { H } & 1.391 & -1.870 & 0.638 \\ \mathrm{H} & 0.916 & -1.811 & -1.052 \\ \mathrm{C} & 3.559 & -0.094 & 0.346 \\ \mathrm{H} & 3.178 & 1.879 & 1.137 \\ \mathrm{H} & 3.255 & 1.813 & -0.606 \\ \mathrm{H} & 3.418 & -2.006 & -0.668 \\ \mathrm{H} & 2.960 & -0.618 & -1.649 \\ \mathrm{H} & 4.628 & -0.002 & 0.150 \\ \mathrm{H} & 3.449 & -0.501 & 1.356 \\ \mathrm{C} & -1.343 & -1.065 & 0.576 \\ \mathrm{C} & -1.404 & 1.251 & -0.125 \\ \mathrm{C} & -2.783 & -1.278 & 0.133 \\ \mathrm{H} & -1.326 & -0.807 & 1.650 \\ \mathrm{H} & -0.786 & -1.992 & 0.458 \\ \mathrm{C} & -2.849 & 1.100 & -0.580 \\ \mathrm{H} & -1.379 & 1.655 & 0.902 \\ \mathrm{H} & -0.893 & 1.967 & -0.769 \\ \mathrm{C} & -3.570 & 0.023 & 0.219 \\ \mathrm{H} & -3.237 & -2.053 & 0.753 \\ \mathrm{H} & -2.783 & -1.638 & -0.900 \\ \mathrm{H} & -3.354 & 2.063 & -0.483 \\ \mathrm{H} & -2.856 & 0.832 & -1.640 \\ \mathrm{H} & -4.590 & -0.114 & -0.144 \\ \mathrm{H} & -3.640 & 0.334 & 1.267 \\ \mathrm{~N} & -0.688 & -0.011 & -0.198\end{array}$

\section{C}

C

C

C

$\mathrm{H}$

C

$\mathrm{H}$

$\mathrm{H}$

C

$\mathrm{H}$

$\mathrm{H}$

$\mathrm{H}$

$\mathrm{H}$

H

$\mathrm{H}$

$\mathrm{H}$

C

C

C

$\mathrm{H}$

$\mathrm{H}$

C

$\mathrm{H}$

$\mathrm{H}$

$\mathrm{H}$

$\begin{array}{rrr}0.702 & 0.054 & -0.064 \\ 1.372 & 1.190 & 0.168 \\ 1.425 & -1.261 & -0.253 \\ 2.872 & 1.283 & 0.241 \\ 0.834 & 2.120 & 0.295 \\ 2.896 & -1.064 & -0.602 \\ 1.352 & -1.858 & 0.662 \\ 0.918 & -1.825 & -1.039 \\ 3.538 & -0.082 & 0.370 \\ 3.153 & 1.919 & 1.084 \\ 3.251 & 1.791 & -0.654 \\ 3.412 & -2.025 & -0.585 \\ 2.978 & -0.669 & -1.619 \\ 4.611 & 0.000 & 0.191 \\ 3.407 & -0.456 & 1.390 \\ -1.359 & -1.067 & 0.549 \\ -1.425 & 1.237 & -0.114 \\ -2.811 & -1.189 & 0.131 \\ -1.309 & -0.847 & 1.627 \\ -0.862 & -2.020 & 0.374 \\ -2.878 & 1.034 & -0.504 \\ -1.373 & 1.643 & 0.909 \\ -0.977 & 1.965 & -0.792 \\ -3.326 & -1.918 & 0.757\end{array}$




$\begin{array}{rrrr}\mathrm{H} & -2.862 & -1.518 & -0.916 \\ \mathrm{H} & -3.442 & 1.952 & -0.349 \\ \mathrm{H} & -2.933 & 0.757 & -1.565 \\ \mathrm{~N} & -0.698 & -0.014 & -0.219 \\ \mathrm{O} & -3.493 & 0.035 & 0.280\end{array}$

En3

$\begin{array}{lrrr}\text { N } & 0.973 & -0.003 & -0.173 \\ \mathrm{C} & 1.772 & 1.201 & -0.075 \\ \mathrm{C} & 1.766 & -1.190 & 0.150 \\ \mathrm{C} & 3.178 & 0.699 & -0.371 \\ \mathrm{H} & 1.418 & 1.946 & -0.790 \\ \mathrm{C} & 3.193 & -0.656 & 0.337 \\ \mathrm{H} & 1.414 & -1.680 & 1.063 \\ \mathrm{H} & 3.300 & 0.569 & -1.448 \\ \mathrm{H} & 3.946 & -1.339 & -0.051 \\ \mathrm{H} & 1.719 & 1.650 & 0.929 \\ \mathrm{H} & 3.954 & 1.375 & -0.016 \\ \mathrm{H} & 3.393 & -0.508 & 1.399 \\ \mathrm{H} & 1.711 & -1.924 & -0.660 \\ \mathrm{C} & -0.403 & 0.044 & -0.006 \\ \mathrm{C} & -1.070 & 1.181 & 0.253 \\ \mathrm{C} & -1.120 & -1.266 & -0.228 \\ \mathrm{C} & -2.571 & 1.284 & 0.275 \\ \mathrm{H} & -0.517 & 2.094 & 0.431 \\ \mathrm{C} & -2.593 & -1.204 & 0.165 \\ \mathrm{H} & -1.019 & -1.537 & -1.285 \\ \mathrm{H} & -0.625 & -2.060 & 0.335 \\ \mathrm{C} & -3.235 & 0.070 & -0.365 \\ \mathrm{H} & -2.883 & 2.195 & -0.242 \\ \mathrm{H} & -2.929 & 1.389 & 1.307 \\ \mathrm{H} & -3.109 & -2.091 & -0.206 \\ \mathrm{H} & -2.676 & -1.216 & 1.256 \\ \mathrm{H} & -4.309 & 0.075 & -0.170 \\ \mathrm{H} & -3.101 & 0.113 & -1.451\end{array}$

$\begin{array}{lrrr}\text { En4 } & & & \\ \text { C } & -1.119 & 0.056 & 0.051 \\ \text { C } & -1.802 & 1.184 & -0.182 \\ \text { C } & -1.831 & -1.263 & 0.257 \\ \text { C } & -3.303 & 1.266 & -0.237 \\ \text { H } & -1.273 & 2.117 & -0.325 \\ \text { C } & -3.298 & -1.073 & 0.626 \\ \text { H } & -1.768 & -1.865 & -0.656 \\ \text { H } & -1.308 & -1.820 & 1.038 \\ \text { C } & -3.961 & -0.105 & -0.345 \\ \text { H } & -3.600 & 1.891 & -1.083 \\ \text { H } & -3.675 & 1.779 & 0.658 \\ \text { H } & -3.807 & -2.039 & 0.626 \\ \text { H } & -3.367 & -0.670 & 1.641\end{array}$

$\begin{array}{lrrr}\text { H } & -5.032 & -0.029 & -0.151 \\ \mathrm{H} & -3.842 & -0.488 & -1.364 \\ \mathrm{C} & 0.945 & -1.048 & -0.576 \\ \mathrm{C} & 0.995 & 1.259 & 0.090 \\ \mathrm{C} & 2.396 & -1.188 & -0.159 \\ \mathrm{H} & 0.901 & -0.820 & -1.653 \\ \mathrm{H} & 0.438 & -1.997 & -0.410 \\ \mathrm{C} & 2.448 & 1.088 & 0.493 \\ \mathrm{H} & 0.946 & 1.661 & -0.935 \\ \mathrm{H} & 0.526 & 1.979 & 0.760 \\ \mathrm{H} & 2.880 & -1.942 & -0.783 \\ \mathrm{H} & 2.437 & -1.536 & 0.889 \\ \mathrm{H} & 2.972 & 2.036 & 0.357 \\ \mathrm{H} & 2.494 & 0.826 & 1.566 \\ \mathrm{~N} & 0.282 & 0.001 & 0.193 \\ \mathrm{~N} & 3.098 & 0.072 & -0.313 \\ \mathrm{C} & 4.497 & -0.059 & 0.034 \\ \mathrm{H} & 4.968 & -0.807 & -0.605 \\ \mathrm{H} & 5.006 & 0.893 & -0.119 \\ \mathrm{H} & 4.640 & -0.363 & 1.084\end{array}$

En5

C

C

C

$\mathrm{H}$

$\mathrm{H}$

C

$\mathrm{H}$

$\mathrm{H}$

$\mathrm{H}$

$\mathrm{H}$

$\mathrm{H}$

$\mathrm{H}$

N

C

C

C

C

$\mathrm{H}$

$\mathrm{H}$

C

$\mathrm{H}$

$\mathrm{H}$

$\mathrm{H}$

$\mathrm{H}$

$\mathrm{H}$

C

$\mathrm{H}$

$\mathrm{H}$

$\begin{array}{rrr}-1.040 & -1.172 & 0.363 \\ -1.075 & 1.248 & 0.071 \\ -2.488 & -1.272 & -0.094 \\ -1.009 & -1.130 & 1.465 \\ -0.500 & -2.062 & 0.052 \\ -2.529 & 1.194 & -0.374 \\ -1.020 & 1.487 & 1.147 \\ -0.559 & 2.044 & -0.465 \\ -2.949 & -2.142 & 0.377 \\ -2.506 & -1.434 & -1.176 \\ -3.017 & 2.131 & -0.103 \\ -2.560 & 1.112 & -1.464 \\ -0.377 & 0.001 & -0.203 \\ 1.007 & 0.057 & -0.062 \\ 1.868 & -1.155 & -0.362 \\ 1.767 & 1.131 & 0.197 \\ 3.272 & -0.709 & 0.075 \\ 1.555 & -2.055 & 0.166 \\ 1.813 & -1.377 & -1.433 \\ 3.235 & 0.829 & 0.062 \\ 1.398 & 2.124 & 0.405 \\ 4.059 & -1.124 & -0.552 \\ 3.449 & -1.051 & 1.096 \\ 3.837 & 1.261 & 0.863 \\ 3.631 & 1.228 & -0.878 \\ -3.250 & 0.003 & 0.242 \\ -4.280 & -0.055 & -0.116 \\ -3.293 & 0.124 & 1.330\end{array}$




$\begin{array}{lrrr}\text { En6 } & & & \\ \text { C } & -1.058 & -1.166 & 0.342 \\ \text { C } & -1.097 & 1.235 & 0.078 \\ \text { C } & -2.516 & -1.184 & -0.078 \\ \text { H } & -0.998 & -1.159 & 1.441 \\ \text { H } & -0.577 & -2.072 & -0.023 \\ \text { C } & -2.557 & 1.116 & -0.317 \\ \text { H } & -1.017 & 1.480 & 1.149 \\ \text { H } & -0.640 & 2.046 & -0.490 \\ \text { H } & -3.039 & -2.005 & 0.410 \\ \text { H } & -2.580 & -1.315 & -1.166 \\ \text { H } & -3.107 & 2.001 & 0.001 \\ \text { H } & -2.633 & 1.023 & -1.408 \\ \text { N } & -0.388 & 0.002 & -0.220 \\ \text { O } & -3.173 & 0.006 & 0.297 \\ \text { C } & 0.996 & 0.059 & -0.076 \\ \text { C } & 1.851 & -1.162 & -0.340 \\ \text { C } & 1.752 & 1.138 & 0.165 \\ \text { C } & 3.253 & -0.709 & 0.098 \\ \text { H } & 1.527 & -2.045 & 0.210 \\ \text { H } & 1.804 & -1.412 & -1.405 \\ \text { C } & 3.221 & 0.829 & 0.050 \\ \text { H } & 1.381 & 2.136 & 0.347 \\ \text { H } & 4.044 & -1.139 & -0.514 \\ \text { H } & 3.422 & -1.028 & 1.127 \\ \text { H } & 3.816 & 1.277 & 0.847 \\ \text { H } & 3.626 & 1.207 & -0.895\end{array}$

\section{En7}

$\begin{array}{lrrr}\text { C } & -2.955 & -0.696 & -0.161 \\ \mathrm{C} & -1.519 & -1.196 & 0.066 \\ \mathrm{C} & -0.697 & 0.065 & -0.053 \\ \mathrm{C} & -1.468 & 1.163 & -0.012 \\ \mathrm{C} & -2.924 & 0.813 & 0.153 \\ \mathrm{H} & -3.689 & -1.241 & 0.431 \\ \mathrm{H} & -1.223 & -1.954 & -0.662 \\ \mathrm{H} & -1.385 & -1.637 & 1.059 \\ \mathrm{H} & -1.103 & 2.180 & -0.048 \\ \mathrm{H} & -3.581 & 1.375 & -0.513 \\ \mathrm{H} & -3.268 & 1.015 & 1.174 \\ \mathrm{H} & -3.213 & -0.834 & -1.212 \\ \mathrm{~N} & 0.666 & -0.002 & -0.185 \\ \mathrm{C} & 1.460 & 1.214 & -0.110 \\ \mathrm{C} & 1.442 & -1.163 & 0.222 \\ \mathrm{C} & 2.890 & 0.727 & 0.167 \\ \mathrm{H} & 1.376 & 1.772 & -1.047 \\ \mathrm{C} & 2.854 & -0.763 & -0.187 \\ \mathrm{H} & 1.390 & -1.334 & 1.307 \\ \mathrm{H} & 3.637 & 1.281 & -0.399 \\ \mathrm{H} & 2.970 & -0.901 & -1.263\end{array}$

$\begin{array}{rrrr}\mathrm{H} & 1.095 & 1.868 & 0.692 \\ \mathrm{H} & 3.122 & 0.846 & 1.226 \\ \mathrm{H} & 3.623 & -1.341 & 0.322 \\ \mathrm{H} & 1.091 & -2.064 & -0.280\end{array}$

\section{En8}

$\begin{array}{rrr}-0.289 & 0.048 & 0.078 \\ -0.973 & 1.173 & -0.143 \\ -1.023 & -1.258 & 0.257 \\ -2.471 & 1.222 & -0.204 \\ -0.470 & 2.121 & -0.270 \\ -2.481 & -1.017 & 0.612 \\ -0.976 & -1.848 & -0.662 \\ -0.537 & -1.834 & 1.048 \\ -2.783 & 1.829 & -1.059 \\ -2.857 & 1.735 & 0.699 \\ -3.034 & -1.957 & 0.575 \\ -2.556 & -0.629 & 1.644 \\ 1.766 & -1.077 & -0.542 \\ 1.829 & 1.237 & 0.100 \\ 3.223 & -1.187 & -0.136 \\ 1.704 & -0.868 & -1.621 \\ 1.273 & -2.029 & -0.351 \\ 3.287 & 1.043 & 0.475 \\ 1.761 & 1.634 & -0.926 \\ 1.384 & 1.966 & 0.778 \\ 3.735 & -1.920 & -0.759 \\ 3.286 & -1.504 & 0.914 \\ 3.846 & 1.962 & 0.304 \\ 3.356 & 0.778 & 1.538 \\ 1.109 & -0.018 & 0.223 \\ 3.896 & 0.039 & -0.305 \\ -3.074 & -0.093 & -0.336 \\ -4.511 & -0.016 & -0.188 \\ -4.919 & 0.679 & -0.922 \\ -4.954 & -0.998 & -0.360 \\ -4.811 & 0.331 & 0.816 \\ & & \end{array}$

En9

$\begin{array}{lrrr}\text { C } & 1.430 & -0.483 & -0.216 \\ \text { C } & 2.409 & 0.369 & 0.088 \\ \text { C } & 3.857 & -0.012 & 0.059 \\ \text { H } & 2.177 & 1.393 & 0.358 \\ \text { H } & 4.334 & 0.169 & 1.025 \\ \text { H } & 4.410 & 0.568 & -0.683 \\ \text { C } & -0.787 & -1.281 & 0.227 \\ \text { C } & -0.377 & 1.096 & 0.108 \\ \text { C } & -2.234 & -1.008 & -0.133 \\ \text { H } & -0.695 & -1.346 & 1.322 \\ \text { H } & -0.470 & -2.234 & -0.199\end{array}$




$\begin{array}{lrrr}\mathrm{C} & -1.848 & 1.276 & -0.224 \\ \mathrm{H} & -0.213 & 1.246 & 1.187 \\ \mathrm{H} & 0.204 & 1.846 & -0.430 \\ \mathrm{H} & -2.890 & -1.747 & 0.328 \\ \mathrm{H} & -2.356 & -1.051 & -1.223 \\ \mathrm{H} & -2.213 & 2.221 & 0.177 \\ \mathrm{H} & -1.976 & 1.279 & -1.314 \\ \mathrm{~N} & 0.064 & -0.223 & -0.302 \\ \mathrm{O} & -2.640 & 0.256 & 0.344 \\ \mathrm{H} & 1.679 & -1.513 & -0.460 \\ \mathrm{H} & 3.979 & -1.068 & -0.185\end{array}$

$\begin{array}{lrrr}\text { C } & 4.332 & -0.977 & 0.428 \\ \text { H } & 2.580 & -2.183 & 0.673 \\ \text { C } & 3.967 & 1.243 & -0.391 \\ \text { H } & 1.948 & 1.766 & -0.847 \\ \mathrm{C} & 4.840 & 0.259 & 0.054 \\ \mathrm{H} & 5.000 & -1.758 & 0.768 \\ \mathrm{H} & 4.352 & 2.205 & -0.706 \\ \mathrm{H} & 5.904 & 0.450 & 0.096 \\ \mathrm{C} & -2.186 & -1.120 & -0.197 \\ \mathrm{C} & -2.489 & 1.160 & 0.647 \\ \mathrm{C} & -3.641 & -1.156 & -0.645 \\ \mathrm{H} & -2.081 & -1.601 & 0.789 \\ \mathrm{H} & -1.572 & -1.681 & -0.901 \\ \mathrm{C} & -3.937 & 1.172 & 0.185 \\ \mathrm{H} & -2.450 & 0.874 & 1.711 \\ \mathrm{H} & -2.048 & 2.154 & 0.561 \\ \mathrm{H} & -3.999 & -2.187 & -0.601 \\ \mathrm{H} & -3.694 & -0.836 & -1.689 \\ \mathrm{H} & -4.513 & 1.839 & 0.828 \\ \mathrm{H} & -3.983 & 1.570 & -0.833 \\ \mathrm{~N} & -1.682 & 0.240 & -0.147 \\ \mathrm{H} & -0.032 & 1.459 & 0.046 \\ \mathrm{C} & -4.510 & -0.240 & 0.208 \\ \mathrm{H} & -5.541 & -0.246 & -0.150 \\ \mathrm{H} & -4.526 & -0.608 & 1.239\end{array}$

$\begin{array}{lrrr}\text { En10 } & & & \\ \text { C } & 0.327 & -0.435 & -0.059 \\ \text { C } & -0.624 & 0.509 & -0.094 \\ \text { H } & -0.349 & 1.557 & -0.116 \\ \text { C } & -2.061 & 0.225 & -0.065 \\ \text { C } & -2.947 & 1.223 & 0.352 \\ \text { C } & -2.601 & -1.009 & -0.441 \\ \text { C } & -4.312 & 0.992 & 0.419 \\ \text { H } & -2.551 & 2.191 & 0.637 \\ \text { C } & -3.964 & -1.244 & -0.367 \\ \text { H } & -1.951 & -1.787 & -0.821 \\ \text { C } & -4.829 & -0.247 & 0.066 \\ \text { H } & -4.974 & 1.782 & 0.749 \\ \text { H } & -4.357 & -2.208 & -0.664 \\ \text { H } & -5.894 & -0.430 & 0.116 \\ \text { C } & 2.198 & 1.103 & -0.203 \\ \text { C } & 2.514 & -1.148 & 0.642 \\ \text { C } & 3.667 & 1.099 & -0.586 \\ \text { H } & 2.063 & 1.595 & 0.772 \\ \text { H } & 1.637 & 1.667 & -0.950 \\ \text { C } & 3.961 & -1.063 & 0.197 \\ \text { H } & 2.449 & -0.879 & 1.706 \\ \text { H } & 2.145 & -2.169 & 0.524 \\ \text { H } & 4.082 & 2.103 & -0.502 \\ \text { H } & 3.770 & 0.758 & -1.625 \\ \text { H } & 4.598 & -1.656 & 0.852 \\ \text { H } & 4.054 & -1.439 & -0.830 \\ \text { N } & 1.692 & -0.256 & -0.166 \\ \text { O } & 4.426 & 0.267 & 0.263 \\ \text { H } & 0.045 & -1.477 & 0.051 \\ & & & \end{array}$

\section{En12}

$\begin{array}{lrrr}\text { En11 } & & & \\ \text { C } & -0.317 & 0.415 & -0.051 \\ \text { C } & 0.638 & -0.526 & -0.075 \\ \text { H } & 0.370 & -1.576 & -0.076 \\ \text { C } & 2.073 & -0.233 & -0.058 \\ \text { C } & 2.968 & -1.218 & 0.371 \\ \text { C } & 2.605 & 0.999 & -0.456\end{array}$

$\begin{array}{rrr}-2.025 & 0.223 & -0.057 \\ -2.591 & -1.103 & -0.200 \\ -3.033 & 1.255 & 0.121 \\ -4.093 & -0.840 & -0.311 \\ -2.184 & -1.606 & -1.082 \\ -4.281 & 0.454 & 0.485 \\ -4.363 & -0.675 & -1.355 \\ -5.203 & 0.979 & 0.243 \\ -2.346 & -1.721 & 0.674 \\ -4.691 & -1.668 & 0.066 \\ -4.278 & 0.237 & 1.555 \\ -3.190 & 1.822 & -0.805 \\ -0.688 & 0.450 & -0.016 \\ 0.278 & -0.485 & -0.098 \\ -0.002 & -1.529 & -0.173 \\ -2.738 & 1.957 & 0.903 \\ 1.713 & -0.207 & -0.052 \\ 2.605 & -1.263 & 0.165 \\ 2.258 & 1.072 & -0.222 \\ 3.973 & -1.053 & 0.230 \\ 2.209 & -2.264 & 0.293 \\ 3.625 & 1.283 & -0.151 \\ 1.610 & 1.913 & -0.434 \\ 4.495 & 0.223 & 0.077\end{array}$




$\begin{array}{rrrr}\mathrm{H} & 4.635 & -1.893 & 0.401 \\ \mathrm{H} & 4.017 & 2.283 & -0.287 \\ \mathrm{H} & -0.427 & 1.496 & 0.107 \\ \mathrm{H} & 5.562 & 0.391 & 0.127\end{array}$

En13

$\begin{array}{lrrr}\text { C } & 0.448 & -0.320 & -0.001 \\ \mathrm{C} & -0.438 & -1.333 & -0.033 \\ \mathrm{H} & -0.055 & -2.342 & -0.127 \\ \mathrm{C} & 0.046 & 1.113 & -0.041 \\ \mathrm{C} & 0.555 & 2.008 & 0.898 \\ \mathrm{C} & -0.828 & 1.580 & -1.019 \\ \mathrm{C} & 0.177 & 3.342 & 0.874 \\ \mathrm{H} & 1.247 & 1.643 & 1.648 \\ \mathrm{C} & -1.202 & 2.915 & -1.046 \\ \mathrm{H} & -1.219 & 0.885 & -1.751 \\ \mathrm{C} & -0.705 & 3.798 & -0.097 \\ \mathrm{H} & 0.569 & 4.027 & 1.615 \\ \mathrm{H} & -1.883 & 3.266 & -1.811 \\ \mathrm{H} & -0.999 & 4.840 & -0.118 \\ \mathrm{C} & -1.904 & -1.246 & 0.054 \\ \mathrm{C} & -2.677 & -2.158 & -0.669 \\ \mathrm{C} & -2.569 & -0.325 & 0.869 \\ \mathrm{C} & -4.062 & -2.131 & -0.612 \\ \mathrm{H} & -2.179 & -2.892 & -1.292 \\ \mathrm{C} & -3.953 & -0.298 & 0.928 \\ \mathrm{H} & -1.994 & 0.370 & 1.467 \\ \mathrm{C} & -4.708 & -1.196 & 0.184 \\ \mathrm{H} & -4.638 & -2.844 & -1.188 \\ \mathrm{H} & -4.446 & 0.423 & 1.567 \\ \mathrm{H} & -5.789 & -1.174 & 0.234 \\ \mathrm{C} & 2.302 & -1.872 & 0.363 \\ \mathrm{C} & 2.674 & 0.157 & -0.867 \\ \mathrm{C} & 3.766 & -1.837 & 0.768 \\ \mathrm{H} & 2.188 & -2.503 & -0.532 \\ \mathrm{H} & 1.713 & -2.311 & 1.170 \\ \mathrm{C} & 4.121 & 0.124 & -0.416 \\ \mathrm{H} & 2.587 & -0.343 & -1.843 \\ \mathrm{H} & 2.346 & 1.189 & -0.981 \\ \mathrm{H} & 4.151 & -2.850 & 0.879 \\ \mathrm{H} & 3.863 & -1.313 & 1.728 \\ \mathrm{H} & 4.767 & 0.556 & -1.179 \\ \mathrm{H} & 4.226 & 0.703 & 0.512 \\ \mathrm{~N} & 1.831 & -0.521 & 0.119 \\ \mathrm{O} & 4.561 & -1.200 & -0.206\end{array}$

En14

$\begin{array}{llll}C & 0.448 & -0.320 & -0.001 \\ C & -0.438 & -1.333 & -0.033 \\ H & -0.055 & -2.342 & -0.127\end{array}$

$\begin{array}{lrrr}\text { C } & 0.046 & 1.113 & -0.041 \\ \mathrm{C} & 0.555 & 2.008 & 0.898 \\ \mathrm{C} & -0.828 & 1.580 & -1.019 \\ \mathrm{C} & 0.177 & 3.342 & 0.874 \\ \mathrm{H} & 1.247 & 1.643 & 1.648 \\ \mathrm{C} & -1.202 & 2.915 & -1.046 \\ \mathrm{H} & -1.219 & 0.885 & -1.751 \\ \mathrm{C} & -0.705 & 3.798 & -0.097 \\ \mathrm{H} & 0.569 & 4.027 & 1.615 \\ \mathrm{H} & -1.883 & 3.266 & -1.811 \\ \mathrm{H} & -0.999 & 4.840 & -0.118 \\ \mathrm{C} & -1.904 & -1.246 & 0.054 \\ \mathrm{C} & -2.677 & -2.158 & -0.669 \\ \mathrm{C} & -2.569 & -0.325 & 0.869 \\ \mathrm{C} & -4.062 & -2.131 & -0.612 \\ \mathrm{H} & -2.179 & -2.892 & -1.292 \\ \mathrm{C} & -3.953 & -0.298 & 0.928 \\ \mathrm{H} & -1.994 & 0.370 & 1.467 \\ \mathrm{C} & -4.708 & -1.196 & 0.184 \\ \mathrm{H} & -4.638 & -2.844 & -1.188 \\ \mathrm{H} & -4.446 & 0.423 & 1.567 \\ \mathrm{H} & -5.789 & -1.174 & 0.234 \\ \mathrm{C} & 2.302 & -1.872 & 0.363 \\ \mathrm{C} & 2.674 & 0.157 & -0.867 \\ \mathrm{C} & 3.766 & -1.837 & 0.768 \\ \mathrm{H} & 2.188 & -2.503 & -0.532 \\ \mathrm{H} & 1.713 & -2.311 & 1.170 \\ \mathrm{C} & 4.121 & 0.124 & -0.416 \\ \mathrm{H} & 2.587 & -0.343 & -1.843 \\ \mathrm{H} & 2.346 & 1.189 & -0.981 \\ \mathrm{H} & 4.151 & -2.850 & 0.879 \\ \mathrm{H} & 3.863 & -1.313 & 1.728 \\ \mathrm{H} & 4.767 & 0.556 & -1.179 \\ \mathrm{H} & 4.226 & 0.703 & 0.512 \\ \mathrm{~N} & 1.831 & -0.521 & 0.119 \\ \mathrm{O} & 4.561 & -1.200 & -0.206\end{array}$

\section{En15}

$\begin{array}{lrrr}\text { N } & 2.023 & -0.726 & 0.209 \\ \mathrm{C} & 2.467 & -2.099 & 0.368 \\ \mathrm{C} & 3.081 & 0.111 & -0.376 \\ \mathrm{C} & 3.984 & -1.974 & 0.393 \\ \mathrm{H} & 2.044 & -2.532 & 1.277 \\ \mathrm{C} & 4.226 & -0.871 & -0.637 \\ \mathrm{H} & 4.314 & -1.649 & 1.382 \\ \mathrm{H} & 5.203 & -0.399 & -0.551 \\ \mathrm{H} & 2.149 & -2.723 & -0.480 \\ \mathrm{H} & 4.487 & -2.909 & 0.151 \\ \mathrm{H} & 4.138 & -1.285 & -1.643 \\ \mathrm{H} & 3.385 & 0.893 & 0.324 \\ \mathrm{C} & 0.684 & -0.425 & 0.080\end{array}$




$\begin{array}{lrrr}\text { C } & -0.279 & -1.374 & 0.076 \\ \text { H } & 0.034 & -2.411 & 0.052 \\ \text { H } & 2.739 & 0.602 & -1.288 \\ \text { C } & 0.386 & 1.030 & -0.027 \\ \text { C } & 0.855 & 1.915 & 0.941 \\ \text { C } & -0.360 & 1.523 & -1.093 \\ \text { C } & 0.567 & 3.269 & 0.854 \\ \text { H } & 1.435 & 1.530 & 1.771 \\ \text { C } & -0.643 & 2.877 & -1.185 \\ \text { H } & -0.729 & 0.831 & -1.840 \\ \text { C } & -0.183 & 3.753 & -0.210 \\ \text { H } & 0.925 & 3.947 & 1.618 \\ \text { H } & -1.227 & 3.249 & -2.017 \\ \text { H } & -0.408 & 4.810 & -0.280 \\ \text { C } & -1.734 & -1.177 & 0.105 \\ \text { C } & -2.548 & -2.109 & -0.549 \\ \text { C } & -2.366 & -0.136 & 0.794 \\ \text { C } & -3.929 & -1.990 & -0.547 \\ \text { H } & -2.081 & -2.934 & -1.074 \\ \text { C } & -3.746 & -0.014 & 0.794 \\ \text { H } & -1.770 & 0.579 & 1.345 \\ \text { C } & -4.538 & -0.936 & 0.120 \\ \text { H } & -4.531 & -2.724 & -1.067 \\ \text { H } & -4.208 & 0.801 & 1.336 \\ \text { H } & -5.615 & -0.840 & 0.125\end{array}$

\section{En16}

$\begin{array}{lrrr}\text { N } & 2.630 & -0.893 & 0.206 \\ \mathrm{C} & 2.916 & -2.305 & 0.383 \\ \mathrm{C} & 3.757 & -0.203 & -0.441 \\ \mathrm{C} & 4.437 & -2.352 & 0.386 \\ \mathrm{H} & 2.459 & -2.671 & 1.304 \\ \mathrm{C} & 4.784 & -1.313 & -0.681 \\ \mathrm{H} & 4.815 & -2.038 & 1.361 \\ \mathrm{H} & 5.809 & -0.950 & -0.619 \\ \mathrm{H} & 2.518 & -2.904 & -0.450 \\ \mathrm{H} & 4.828 & -3.344 & 0.167 \\ \mathrm{H} & 4.638 & -1.747 & -1.672 \\ \mathrm{H} & 4.160 & 0.573 & 0.214 \\ \mathrm{C} & 1.329 & -0.444 & 0.080 \\ \mathrm{C} & 0.266 & -1.278 & 0.100 \\ \mathrm{H} & 0.459 & -2.344 & 0.091 \\ \mathrm{H} & 3.447 & 0.280 & -1.369 \\ \mathrm{C} & 1.196 & 1.033 & -0.035 \\ \mathrm{C} & 1.794 & 1.867 & 0.907 \\ \mathrm{C} & 0.477 & 1.601 & -1.084 \\ \mathrm{C} & 1.660 & 3.245 & 0.814 \\ \mathrm{H} & 2.352 & 1.423 & 1.723 \\ \mathrm{C} & 0.348 & 2.979 & -1.181 \\ \mathrm{H} & 0.009 & 0.952 & -1.812 \\ \mathrm{C} & 0.936 & 3.804 & -0.231\end{array}$

$\begin{array}{lrrr}\text { H } & 2.118 & 3.883 & 1.559 \\ \mathrm{H} & -0.216 & 3.409 & -1.999 \\ \mathrm{H} & 0.831 & 4.878 & -0.305 \\ \mathrm{C} & -1.158 & -0.915 & 0.148 \\ \mathrm{C} & -2.088 & -1.734 & -0.487 \\ \mathrm{C} & -1.658 & 0.193 & 0.848 \\ \mathrm{C} & -3.453 & -1.463 & -0.468 \\ \mathrm{H} & -1.738 & -2.609 & -1.022 \\ \mathrm{C} & -3.007 & 0.478 & 0.874 \\ \mathrm{H} & -0.977 & 0.834 & 1.390 \\ \mathrm{C} & -3.917 & -0.344 & 0.211 \\ \mathrm{H} & -4.131 & -2.129 & -0.982 \\ \mathrm{H} & -3.387 & 1.332 & 1.419 \\ \mathrm{O} & -5.225 & 0.019 & 0.295 \\ \mathrm{C} & -6.170 & -0.800 & -0.351 \\ \mathrm{H} & -6.160 & -1.816 & 0.056 \\ \mathrm{H} & -7.142 & -0.349 & -0.169 \\ \mathrm{H} & -5.987 & -0.845 & -1.429\end{array}$

En17

$N$

C

C

C

$\mathrm{H}$

C

$\mathrm{H}$

$\mathrm{H}$

$\mathrm{H}$

$\mathrm{H}$

$\mathrm{H}$

$\mathrm{H}$

C

C

$\mathrm{H}$

$\mathrm{H}$

C

C

C

C

$\mathrm{H}$

C

$\mathrm{H}$

C

$\mathrm{H}$

$\mathrm{H}$

$\mathrm{H}$

C

C

C

C

$\begin{array}{rrr}2.510 & -0.837 & 0.176 \\ 2.865 & -2.239 & 0.327 \\ 3.653 & -0.036 & -0.284 \\ 4.386 & -2.211 & 0.396 \\ 2.391 & -2.656 & 1.217 \\ 4.731 & -1.080 & -0.573 \\ 4.708 & -1.954 & 1.407 \\ 5.736 & -0.685 & -0.440 \\ 2.526 & -2.825 & -0.539 \\ 4.834 & -3.166 & 0.125 \\ 4.637 & -1.436 & -1.601 \\ 3.979 & 0.657 & 0.497 \\ 1.206 & -0.436 & 0.061 \\ 0.175 & -1.319 & 0.044 \\ 0.416 & -2.373 & -0.006 \\ 3.393 & 0.554 & -1.162 \\ 1.010 & 1.036 & -0.036 \\ 1.476 & 1.875 & 0.973 \\ 0.356 & 1.587 & -1.133 \\ 1.273 & 3.244 & 0.896 \\ 1.981 & 1.443 & 1.829 \\ 0.159 & 2.957 & -1.215 \\ -0.011 & 0.930 & -1.912 \\ 0.613 & 3.788 & -0.199 \\ 1.625 & 3.888 & 1.691 \\ -0.353 & 3.377 & -2.070 \\ 0.455 & 4.857 & -0.261 \\ -1.257 & -1.030 & 0.072 \\ -2.131 & -1.961 & -0.509 \\ -1.828 & 0.094 & 0.688 \\ -3.498 & -1.772 & -0.513\end{array}$




$\begin{array}{lrrr}\text { H } & -1.717 & -2.847 & -0.974 \\ \mathrm{C} & -3.194 & 0.294 & 0.687 \\ \mathrm{H} & -1.196 & 0.813 & 1.190 \\ \mathrm{C} & -4.042 & -0.633 & 0.080 \\ \mathrm{H} & -4.153 & -2.499 & -0.974 \\ \mathrm{H} & -3.618 & 1.165 & 1.169 \\ \mathrm{C} & -5.459 & -0.424 & 0.078 \\ \mathrm{~N} & -6.595 & -0.255 & 0.076\end{array}$

\section{En18}

\begin{tabular}{|c|c|c|c|}
\hline $\mathrm{N}$ & 2.844 & -0.870 & 0.181 \\
\hline C & 3.168 & -2.277 & 0.357 \\
\hline C & 4.012 & -0.098 & -0.265 \\
\hline C & 4.689 & -2.282 & 0.436 \\
\hline $\mathrm{H}$ & 2.679 & -2.670 & 1.251 \\
\hline C & 5.067 & -1.168 & -0.539 \\
\hline $\mathrm{H}$ & 5.009 & -2.024 & 1.447 \\
\hline $\mathrm{H}$ & 6.080 & -0.795 & -0.403 \\
\hline $\mathrm{H}$ & 2.822 & -2.869 & -0.502 \\
\hline $\mathrm{H}$ & 5.117 & -3.249 & 0.178 \\
\hline $\mathrm{H}$ & 4.971 & -1.529 & -1.565 \\
\hline $\mathrm{H}$ & 4.345 & 0.588 & 0.520 \\
\hline C & 1.553 & -0.440 & 0.063 \\
\hline C & 0.500 & -1.299 & 0.057 \\
\hline $\mathrm{H}$ & 0.718 & -2.359 & 0.017 \\
\hline $\mathrm{H}$ & 3.775 & 0.499 & -1.146 \\
\hline C & 1.392 & 1.035 & -0.058 \\
\hline C & 1.864 & 1.878 & 0.945 \\
\hline C & 0.762 & 1.583 & -1.170 \\
\hline C & 1.691 & 3.250 & 0.846 \\
\hline $\mathrm{H}$ & 2.350 & 1.448 & 1.813 \\
\hline C & 0.596 & 2.956 & -1.274 \\
\hline $\mathrm{H}$ & 0.389 & 0.923 & -1.944 \\
\hline C & 1.056 & 3.792 & -0.265 \\
\hline $\mathrm{H}$ & 2.048 & 3.897 & 1.636 \\
\hline $\mathrm{H}$ & 0.102 & 3.375 & -2.141 \\
\hline $\mathrm{H}$ & 0.920 & 4.862 & -0.343 \\
\hline C & -0.923 & -0.979 & 0.078 \\
\hline C & -1.816 & -1.913 & -0.473 \\
\hline C & -1.471 & 0.175 & 0.662 \\
\hline C & -3.179 & -1.700 & -0.483 \\
\hline $\mathrm{H}$ & -1.418 & -2.820 & -0.911 \\
\hline C & -2.833 & 0.403 & 0.657 \\
\hline $\mathrm{H}$ & -0.825 & 0.896 & 1.143 \\
\hline C & -3.672 & -0.534 & 0.077 \\
\hline $\mathrm{H}$ & -3.863 & -2.415 & -0.917 \\
\hline $\mathrm{H}$ & -3.256 & 1.289 & 1.108 \\
\hline$N$ & -5.117 & -0.293 & 0.070 \\
\hline 0 & -5.520 & 0.738 & 0.565 \\
\hline 0 & -5.828 & -1.137 & -0.430 \\
\hline
\end{tabular}

\begin{tabular}{lrrr} 
C & -1.385 & 0.400 & -0.073 \\
C & -1.524 & -0.751 & -0.849 \\
C & -2.472 & 0.836 & 0.680 \\
C & -2.728 & -1.437 & -0.884 \\
C & -3.678 & 0.150 & 0.645 \\
H & -2.361 & 1.712 & 1.307 \\
$\mathrm{C}$ & -3.810 & -0.988 & -0.139 \\
H & -2.824 & -2.323 & -1.499 \\
H & -4.513 & 0.500 & 1.238 \\
H & -4.749 & -1.525 & -0.164 \\
H & -0.675 & -1.095 & -1.428 \\
$\mathrm{C}$ & -0.102 & 1.156 & -0.077 \\
$\mathrm{C}$ & -0.107 & 2.495 & -0.119 \\
$\mathrm{H}$ & -1.043 & 3.026 & -0.205 \\
$\mathrm{H}$ & 0.799 & 3.082 & -0.106 \\
$\mathrm{C}$ & 2.304 & 1.058 & -0.398 \\
$\mathrm{C}$ & 1.188 & -0.614 & 0.954 \\
$\mathrm{C}$ & 3.406 & 0.069 & -0.757 \\
$\mathrm{H}$ & 2.615 & 1.659 & 0.473 \\
$\mathrm{H}$ & 2.136 & 1.746 & -1.227 \\
$\mathrm{C}$ & 2.247 & -1.654 & 0.622 \\
$\mathrm{H}$ & 1.462 & -0.097 & 1.888 \\
$\mathrm{H}$ & 0.223 & -1.090 & 1.118 \\
$\mathrm{C}$ & 3.584 & -0.981 & 0.334 \\
$\mathrm{H}$ & 4.335 & 0.616 & -0.926 \\
$\mathrm{H}$ & 3.139 & -0.423 & -1.697 \\
$\mathrm{H}$ & 2.335 & -2.356 & 1.454 \\
$\mathrm{H}$ & 1.922 & -2.221 & -0.255 \\
$\mathrm{H}$ & 4.335 & -1.716 & 0.042 \\
$\mathrm{H}$ & 3.946 & -0.496 & 1.247 \\
$\mathrm{~N}$ & 1.055 & 0.367 & -0.126 \\
& & & \\
\hline
\end{tabular}

En20

$\begin{array}{lrrr}\text { C } & 1.375 & 0.401 & 0.082 \\ \text { C } & 1.537 & -0.745 & 0.860 \\ \text { C } & 2.446 & 0.847 & -0.689 \\ \text { C } & 2.748 & -1.419 & 0.878 \\ \text { C } & 3.658 & 0.172 & -0.672 \\ \text { H } & 2.316 & 1.720 & -1.317 \\ \text { C } & 3.814 & -0.962 & 0.113 \\ \text { H } & 2.863 & -2.302 & 1.494 \\ \text { H } & 4.480 & 0.528 & -1.280 \\ \text { H } & 4.758 & -1.490 & 0.125 \\ \text { H } & 0.703 & -1.094 & 1.456 \\ \text { C } & 0.087 & 1.146 & 0.102 \\ \text { C } & 0.074 & 2.483 & 0.157 \\ \text { H } & 1.004 & 3.026 & 0.241 \\ \text { H } & -0.839 & 3.059 & 0.156 \\ \text { C } & -2.325 & 1.022 & 0.383\end{array}$




$\begin{array}{lrrr}\mathrm{C} & -1.193 & -0.642 & -0.924 \\ \mathrm{C} & -3.411 & -0.002 & 0.661 \\ \mathrm{H} & -2.613 & 1.636 & -0.486 \\ \mathrm{H} & -2.217 & 1.681 & 1.246 \\ \mathrm{C} & -2.312 & -1.611 & -0.596 \\ \mathrm{H} & -1.419 & -0.138 & -1.875 \\ \mathrm{H} & -0.256 & -1.185 & -1.040 \\ \mathrm{H} & -4.378 & 0.490 & 0.761 \\ \mathrm{H} & -3.181 & -0.528 & 1.597 \\ \mathrm{H} & -2.470 & -2.305 & -1.421 \\ \mathrm{H} & -2.048 & -2.182 & 0.305 \\ \mathrm{~N} & -1.063 & 0.344 & 0.149 \\ \mathrm{O} & -3.531 & -0.933 & -0.391\end{array}$

\section{En21}

$\begin{array}{lrrr}\text { C } & -0.232 & 1.953 & -0.343 \\ \text { C } & -0.351 & 0.617 & -0.245 \\ \text { C } & 0.885 & -0.200 & -0.156 \\ \text { C } & 2.061 & 0.400 & 0.317 \\ \text { C } & 2.012 & 1.852 & 0.708 \\ \text { C } & 1.116 & 2.616 & -0.261 \\ \text { H } & 0.039 & -1.958 & -1.035 \\ \text { H } & -1.100 & 2.577 & -0.503 \\ \text { C } & 0.928 & -1.519 & -0.603 \\ \text { C } & 3.229 & -0.346 & 0.385 \\ \text { H } & 1.593 & 1.942 & 1.716 \\ \text { H } & 1.592 & 2.656 & -1.249 \\ \mathrm{C} & 3.254 & -1.672 & -0.028 \\ \mathrm{C} & 2.103 & -2.254 & -0.536 \\ \text { H } & 4.133 & 0.124 & 0.754 \\ \text { H } & 4.174 & -2.240 & 0.029 \\ \text { H } & 2.119 & -3.276 & -0.889 \\ \text { H } & 3.020 & 2.268 & 0.732 \\ \text { H } & 0.999 & 3.648 & 0.072 \\ \text { N } & -1.563 & -0.071 & -0.319 \\ \mathrm{C} & -1.844 & -1.137 & 0.666 \\ \mathrm{C} & -2.773 & 0.702 & -0.555 \\ \mathrm{C} & -3.337 & -0.994 & 0.979 \\ \mathrm{H} & -1.636 & -2.121 & 0.242 \\ \mathrm{C} & -3.886 & -0.291 & -0.263 \\ \mathrm{H} & -2.850 & 1.552 & 0.139 \\ \mathrm{H} & -3.809 & -1.953 & 1.185 \\ \mathrm{H} & -3.987 & -0.996 & -1.090 \\ \mathrm{H} & -1.221 & -1.025 & 1.556 \\ \mathrm{H} & -3.479 & -0.353 & 1.850 \\ \mathrm{H} & -4.848 & 0.190 & -0.097 \\ \mathrm{H} & -2.779 & 1.096 & -1.573\end{array}$

\section{En22}

$\begin{array}{llll}\text { C } & -0.990 & -0.117 & -0.079\end{array}$

$\begin{array}{lrrr}\text { C } & -2.030 & 0.803 & 0.103 \\ \text { C } & -3.629 & -0.971 & 0.016 \\ \text { C } & -2.602 & -1.884 & -0.187 \\ \text { C } & -1.276 & -1.468 & -0.240 \\ \text { C } & 0.281 & 0.637 & -0.100 \\ \text { C } & 0.011 & 1.956 & 0.037 \\ \text { H } & -4.654 & -1.315 & 0.056 \\ \text { H } & -2.836 & -2.934 & -0.310 \\ \text { H } & -0.496 & -2.194 & -0.416 \\ \text { H } & -1.871 & 2.821 & -0.621 \\ \text { H } & 0.741 & 2.750 & 0.039 \\ \text { H } & -4.146 & 1.101 & 0.296 \\ \mathrm{C} & -3.346 & 0.384 & 0.156 \\ \mathrm{C} & -1.464 & 2.192 & 0.178 \\ \text { H } & -1.720 & 2.682 & 1.123 \\ \mathrm{C} & 2.680 & 0.934 & -0.283 \\ \mathrm{C} & 1.855 & -1.271 & 0.219 \\ \mathrm{C} & 3.850 & -0.039 & -0.284 \\ \mathrm{H} & 2.695 & 1.559 & 0.623 \\ \mathrm{H} & 2.661 & 1.601 & -1.147 \\ \mathrm{C} & 3.328 & -1.156 & 0.620 \\ \mathrm{H} & 1.727 & -2.025 & -0.563 \\ \mathrm{H} & 1.221 & -1.546 & 1.064 \\ \mathrm{H} & 4.020 & -0.415 & -1.294 \\ \mathrm{H} & 4.772 & 0.412 & 0.077 \\ \mathrm{H} & 3.864 & -2.097 & 0.506 \\ \mathrm{H} & 3.405 & -0.846 & 1.664 \\ \mathrm{~N} & 1.516 & 0.063 & -0.290\end{array}$

\section{En23}

$\begin{array}{lrrr}\text { C } & -1.146 & -1.357 & 0.135 \\ \text { C } & -0.655 & 0.814 & -0.749 \\ \text { C } & -1.999 & 1.290 & -0.232 \\ \text { C } & -2.479 & -0.820 & 0.621 \\ \text { H } & 0.064 & 1.633 & -0.725 \\ \text { H } & -1.280 & -1.810 & -0.861 \\ \text { H } & -0.790 & -2.130 & 0.818 \\ \text { H } & -1.873 & 1.706 & 0.777 \\ \text { H } & -2.413 & 2.058 & -0.884 \\ \text { H } & -2.372 & -0.467 & 1.655 \\ \text { H } & -3.242 & -1.598 & 0.589 \\ \text { H } & -0.765 & 0.484 & -1.794 \\ \text { O } & -2.935 & 0.234 & -0.199 \\ \text { N } & -0.170 & -0.278 & 0.092 \\ \text { C } & 1.136 & -0.720 & -0.224 \\ \text { H } & 1.205 & -1.672 & -0.752 \\ \text { C } & 2.248 & -0.046 & 0.066 \\ \text { C } & 2.230 & 1.244 & 0.829 \\ \text { H } & 2.412 & 2.098 & 0.168 \\ \text { H } & 1.272 & 1.391 & 1.328 \\ \text { H } & 3.024 & 1.251 & 1.578\end{array}$




$\begin{array}{llrr}\mathrm{C} & 3.593 & -0.548 & -0.367 \\ \mathrm{H} & 4.082 & 0.172 & -1.030 \\ \mathrm{H} & 4.251 & -0.679 & 0.496 \\ \mathrm{H} & 3.518 & -1.498 & -0.894\end{array}$

En24

$\begin{array}{lrrr}\mathrm{C} & 1.376 & -1.047 & -0.265 \\ \mathrm{C} & 2.603 & -0.538 & -0.113 \\ \mathrm{C} & 3.071 & 0.849 & 0.238 \\ \mathrm{H} & 3.396 & -1.253 & -0.297 \\ \mathrm{H} & 3.208 & 1.474 & -0.649 \\ \mathrm{H} & 4.041 & 0.788 & 0.732 \\ \mathrm{C} & -0.941 & -1.280 & 0.349 \\ \mathrm{C} & -0.047 & 0.943 & 0.064 \\ \mathrm{C} & -2.299 & -0.747 & -0.064 \\ \mathrm{H} & -0.871 & -1.277 & 1.447 \\ \mathrm{H} & -0.827 & -2.309 & 0.005 \\ \mathrm{C} & -1.442 & 1.392 & -0.338 \\ \mathrm{H} & 0.099 & 1.143 & 1.136 \\ \mathrm{H} & 0.688 & 1.515 & -0.501 \\ \mathrm{H} & -3.095 & -1.289 & 0.445 \\ \mathrm{H} & -2.423 & -0.862 & -1.149 \\ \mathrm{H} & -1.611 & 2.424 & -0.035 \\ \mathrm{H} & -1.547 & 1.317 & -1.428 \\ \mathrm{~N} & 0.113 & -0.466 & -0.238 \\ \mathrm{O} & -2.436 & 0.612 & 0.290 \\ \mathrm{H} & 1.314 & -2.106 & -0.496 \\ \mathrm{H} & 2.399 & 1.370 & 0.915\end{array}$

\section{En25}

$\begin{array}{lrrr}\text { C } & -2.806 & -0.494 & -0.196 \\ \mathrm{C} & -1.523 & -1.273 & 0.132 \\ \mathrm{C} & -0.456 & -0.213 & 0.013 \\ \mathrm{C} & -0.961 & 1.020 & -0.010 \\ \mathrm{C} & -2.464 & 0.981 & 0.093 \\ \mathrm{H} & -3.670 & -0.850 & 0.362 \\ \mathrm{H} & -1.356 & -2.114 & -0.547 \\ \mathrm{H} & -1.534 & -1.677 & 1.150 \\ \mathrm{H} & -0.377 & 1.924 & -0.040 \\ \mathrm{H} & -2.953 & 1.660 & -0.607 \\ \mathrm{H} & -2.787 & 1.278 & 1.096 \\ \mathrm{H} & -3.027 & -0.607 & -1.258 \\ \mathrm{~N} & 0.876 & -0.631 & 0.006 \\ \mathrm{C} & 1.980 & 0.176 & -0.014 \\ \mathrm{H} & 1.034 & -1.624 & -0.028 \\ \mathrm{O} & 1.911 & 1.365 & -0.014 \\ \mathrm{C} & 3.295 & -0.570 & -0.020 \\ \mathrm{H} & 3.423 & -1.114 & 0.917 \\ \mathrm{H} & 3.332 & -1.289 & -0.839 \\ \mathrm{H} & 4.101 & 0.149 & -0.130\end{array}$

En26

$\begin{array}{lrrr}\text { N } & 1.185 & -0.619 & 0.016 \\ \mathrm{C} & 2.303 & 0.168 & -0.009 \\ \mathrm{H} & 1.342 & -1.612 & -0.003 \\ \mathrm{O} & 2.278 & 1.379 & 0.009 \\ \mathrm{C} & 3.614 & -0.589 & -0.064 \\ \mathrm{H} & 3.499 & -1.671 & -0.053 \\ \mathrm{H} & 4.141 & -0.292 & -0.970 \\ \mathrm{H} & 4.221 & -0.282 & 0.787 \\ \mathrm{C} & -0.170 & -0.226 & 0.038 \\ \mathrm{C} & -0.596 & 1.034 & 0.126 \\ \mathrm{C} & -1.101 & -1.409 & -0.061 \\ \mathrm{C} & -2.055 & 1.397 & 0.090 \\ \mathrm{H} & 0.124 & 1.832 & 0.214 \\ \mathrm{C} & -2.537 & -1.044 & 0.294 \\ \mathrm{H} & -1.054 & -1.814 & -1.079 \\ \mathrm{H} & -0.742 & -2.201 & 0.604 \\ \mathrm{C} & -2.936 & 0.253 & -0.400 \\ \mathrm{H} & -2.187 & 2.273 & -0.549 \\ \mathrm{H} & -2.376 & 1.708 & 1.091 \\ \mathrm{H} & -3.206 & -1.861 & 0.018 \\ \mathrm{H} & -2.618 & -0.910 & 1.376 \\ \mathrm{H} & -3.988 & 0.479 & -0.221 \\ \mathrm{H} & -2.809 & 0.134 & -1.480\end{array}$

En27

$\begin{array}{lrrr}\text { N } & -1.645 & -0.537 & 0.202 \\ \mathrm{C} & -2.977 & -0.298 & -0.005 \\ \mathrm{H} & -1.410 & -1.422 & 0.619 \\ \mathrm{O} & -3.415 & 0.740 & -0.448 \\ \mathrm{C} & -3.889 & -1.442 & 0.382 \\ \mathrm{H} & -3.370 & -2.392 & 0.495 \\ \mathrm{H} & -4.374 & -1.190 & 1.326 \\ \mathrm{H} & -4.663 & -1.534 & -0.377 \\ \mathrm{C} & -0.568 & 0.351 & 0.016 \\ \mathrm{C} & -0.694 & 1.670 & -0.164 \\ \mathrm{C} & 0.529 & 2.526 & -0.344 \\ \mathrm{H} & -1.673 & 2.118 & -0.211 \\ \mathrm{C} & 1.688 & 1.985 & 0.484 \\ \mathrm{H} & 0.305 & 3.552 & -0.051 \\ \mathrm{H} & 0.810 & 2.557 & -1.404 \\ \mathrm{H} & 2.608 & 2.536 & 0.288 \\ \mathrm{H} & 1.449 & 2.113 & 1.546 \\ \mathrm{C} & 0.768 & -0.287 & -0.010 \\ \mathrm{C} & 1.895 & 0.520 & 0.211 \\ \mathrm{C} & 0.946 & -1.643 & -0.277 \\ \mathrm{C} & 3.158 & -0.053 & 0.185 \\ \mathrm{C} & 2.216 & -2.204 & -0.301 \\ \mathrm{H} & 0.095 & -2.271 & -0.509\end{array}$




$\begin{array}{lrrr}\mathrm{C} & 3.325 & -1.409 & -0.063 \\ \mathrm{H} & 4.023 & 0.576 & 0.358 \\ \mathrm{H} & 2.335 & -3.258 & -0.515 \\ \mathrm{H} & 4.319 & -1.839 & -0.081\end{array}$

En28

$\begin{array}{lrrr}\text { N } & 0.844 & -0.289 & -0.012 \\ \mathrm{C} & 2.175 & 0.038 & -0.010 \\ \mathrm{H} & 0.644 & -1.273 & -0.015 \\ \mathrm{O} & 2.597 & 1.172 & -0.009 \\ \mathrm{C} & 3.115 & -1.149 & 0.011 \\ \mathrm{H} & 3.597 & -1.186 & 0.988 \\ \mathrm{H} & 2.624 & -2.103 & -0.178 \\ \mathrm{H} & 3.889 & -0.984 & -0.735 \\ \mathrm{C} & -0.277 & 0.566 & 0.001 \\ \mathrm{C} & -0.180 & 1.894 & 0.011 \\ \mathrm{H} & -1.075 & 2.495 & 0.021 \\ \mathrm{H} & 0.775 & 2.391 & 0.010 \\ \mathrm{C} & -1.595 & -0.206 & 0.000 \\ \mathrm{C} & -1.688 & -1.076 & -1.264 \\ \mathrm{H} & -0.904 & -1.833 & -1.315 \\ \mathrm{H} & -2.646 & -1.599 & -1.281 \\ \mathrm{H} & -1.616 & -0.457 & -2.159 \\ \mathrm{C} & -1.678 & -1.097 & 1.250 \\ \mathrm{H} & -1.598 & -0.493 & 2.155 \\ \mathrm{H} & -2.635 & -1.620 & 1.266 \\ \mathrm{H} & -0.894 & -1.855 & 1.282 \\ \mathrm{C} & -2.794 & 0.739 & 0.013 \\ \mathrm{H} & -2.803 & 1.383 & -0.867 \\ \mathrm{H} & -3.714 & 0.152 & 0.012 \\ \mathrm{H} & -2.796 & 1.368 & 0.904\end{array}$

En29

$\begin{array}{lrrr}\mathrm{N} & -0.013 & 0.549 & -0.105 \\ \mathrm{C} & 1.219 & 1.127 & 0.044 \\ \mathrm{H} & -0.035 & -0.453 & -0.197 \\ \mathrm{O} & 1.385 & 2.308 & 0.256 \\ \mathrm{C} & -2.400 & 0.225 & 0.013 \\ \mathrm{C} & -3.556 & 0.416 & -0.742 \\ \mathrm{C} & -2.332 & -0.874 & 0.870 \\ \mathrm{C} & -4.622 & -0.463 & -0.633 \\ \mathrm{C} & -3.399 & -1.754 & 0.977 \\ \mathrm{H} & -1.451 & -1.023 & 1.485 \\ \mathrm{C} & -4.547 & -1.551 & 0.226 \\ \mathrm{H} & -5.511 & -0.304 & -1.230 \\ \mathrm{H} & -3.335 & -2.594 & 1.657 \\ \mathrm{H} & -5.379 & -2.239 & 0.307 \\ \mathrm{H} & -3.604 & 1.251 & -1.429 \\ \mathrm{C} & -1.267 & 1.182 & -0.089 \\ \mathrm{C} & -1.458 & 2.501 & -0.144\end{array}$

$\begin{array}{lrrr}\mathrm{H} & -2.469 & 2.876 & -0.085 \\ \mathrm{H} & -0.641 & 3.198 & -0.213 \\ \mathrm{C} & 2.380 & 0.178 & -0.052 \\ \mathrm{C} & 3.554 & 0.545 & 0.599 \\ \mathrm{C} & 2.340 & -1.012 & -0.774 \\ \mathrm{C} & 4.665 & -0.279 & 0.552 \\ \mathrm{H} & 3.572 & 1.486 & 1.134 \\ \mathrm{C} & 3.458 & -1.832 & -0.831 \\ \mathrm{H} & 1.455 & -1.292 & -1.333 \\ \mathrm{C} & 4.618 & -1.470 & -0.162 \\ \mathrm{H} & 5.572 & 0.008 & 1.068 \\ \mathrm{H} & 3.424 & -2.748 & -1.405 \\ \mathrm{H} & 5.489 & -2.112 & -0.204\end{array}$

En30

$\begin{array}{lrrr}\text { N } & -1.405 & -0.264 & 0.211 \\ \text { C } & -2.770 & -0.217 & 0.066 \\ \text { H } & -1.026 & -1.099 & 0.629 \\ \text { O } & -3.363 & 0.737 & -0.380 \\ \text { C } & -3.470 & -1.484 & 0.508 \\ \text { H } & -3.161 & -2.325 & -0.115 \\ \text { H } & -3.227 & -1.720 & 1.544 \\ \text { H } & -4.541 & -1.338 & 0.410 \\ \text { C } & 0.938 & 0.252 & -0.041 \\ \text { C } & 1.940 & 1.054 & 0.505 \\ \text { C } & 1.283 & -1.004 & -0.541 \\ \text { C } & 3.254 & 0.615 & 0.540 \\ \text { C } & 2.598 & -1.443 & -0.505 \\ \text { H } & 0.521 & -1.629 & -0.993 \\ \text { C } & 3.588 & -0.635 & 0.035 \\ \text { H } & 4.019 & 1.247 & 0.972 \\ \text { H } & 2.851 & -2.415 & -0.909 \\ \text { H } & 4.614 & -0.978 & 0.066 \\ \text { H } & 1.675 & 2.018 & 0.920 \\ \text { C } & -0.466 & 0.736 & -0.098 \\ \text { C } & -0.768 & 1.991 & -0.432 \\ \text { H } & 0.038 & 2.659 & -0.698 \\ \text { H } & -1.784 & 2.345 & -0.477\end{array}$

En31

$\begin{array}{lrrr}\text { N } & -2.203 & -0.327 & 0.195 \\ \text { C } & -3.567 & -0.418 & 0.057 \\ \text { H } & -1.740 & -1.114 & 0.619 \\ \text { O } & -4.253 & 0.467 & -0.394 \\ \text { C } & -4.155 & -1.724 & 0.545 \\ \text { H } & -3.448 & -2.551 & 0.510 \\ \text { H } & -4.487 & -1.591 & 1.576 \\ \text { H } & -5.026 & -1.956 & -0.063 \\ \text { C } & 0.068 & 0.445 & -0.053 \\ \text { C } & 0.981 & 1.339 & 0.505\end{array}$




$\begin{array}{lrrr}\mathrm{C} & 0.547 & -0.756 & -0.576 \\ \mathrm{C} & 2.335 & 1.051 & 0.532 \\ \mathrm{C} & 1.900 & -1.056 & -0.555 \\ \mathrm{H} & -0.141 & -1.454 & -1.038 \\ \mathrm{C} & 2.786 & -0.148 & 0.001 \\ \mathrm{H} & 3.040 & 1.743 & 0.972 \\ \mathrm{H} & 2.270 & -1.982 & -0.973 \\ \mathrm{H} & 0.619 & 2.262 & 0.939 \\ \mathrm{C} & -1.379 & 0.775 & -0.098 \\ \mathrm{C} & -1.816 & 1.995 & -0.411 \\ \mathrm{H} & -1.088 & 2.750 & -0.670 \\ \mathrm{H} & -2.864 & 2.237 & -0.450 \\ \mathrm{Cl} & 4.477 & -0.518 & 0.040\end{array}$

\section{En32}

$\begin{array}{lrrr}\text { N } & -1.831 & -0.310 & 0.188 \\ \text { C } & -3.196 & -0.355 & 0.058 \\ \text { H } & -1.390 & -1.119 & 0.593 \\ \text { O } & -3.859 & 0.558 & -0.377 \\ \text { C } & -3.826 & -1.646 & 0.533 \\ \text { H } & -3.136 & -2.489 & 0.525 \\ \text { H } & -4.189 & -1.500 & 1.551 \\ \text { H } & -4.682 & -1.863 & -0.102 \\ \text { C } & 0.466 & 0.384 & -0.053 \\ \text { C } & 1.411 & 1.247 & 0.501 \\ \text { C } & 0.907 & -0.833 & -0.568 \\ \text { C } & 2.751 & 0.905 & 0.526 \\ \text { C } & 2.253 & -1.171 & -0.539 \\ \text { H } & 0.198 & -1.511 & -1.030 \\ \text { C } & 3.197 & -0.310 & 0.008 \\ \text { H } & 3.468 & 1.590 & 0.965 \\ \text { H } & 2.575 & -2.116 & -0.960 \\ \text { H } & 1.081 & 2.186 & 0.928 \\ \text { C } & -0.969 & 0.764 & -0.102 \\ \text { C } & -1.366 & 1.998 & -0.411 \\ \text { H } & -0.614 & 2.731 & -0.665 \\ \text { H } & -2.406 & 2.275 & -0.449 \\ \text { C } & 4.654 & -0.677 & 0.062 \\ \text { H } & 5.277 & 0.142 & -0.298 \\ \text { H } & 4.961 & -0.894 & 1.088 \\ \text { H } & 4.862 & -1.557 & -0.544\end{array}$

\section{EE1}

$\begin{array}{lrrr}C & -0.697 & -0.114 & -0.545 \\ C & -1.223 & 1.107 & -0.500 \\ C & -2.640 & 1.368 & -0.070 \\ C & -3.249 & 0.159 & 0.635 \\ C & -2.964 & -1.114 & -0.155 \\ C & -1.462 & -1.367 & -0.234 \\ \text { H } & -2.667 & 2.239 & 0.590\end{array}$

$\begin{array}{lrrr}\mathrm{H} & -0.606 & 1.948 & -0.795 \\ \mathrm{H} & -2.809 & 0.065 & 1.633 \\ \mathrm{H} & -4.323 & 0.300 & 0.768 \\ \mathrm{H} & -3.466 & -1.973 & 0.292 \\ \mathrm{H} & -3.363 & -1.000 & -1.167 \\ \mathrm{H} & -1.087 & -1.780 & 0.710 \\ \mathrm{H} & -1.224 & -2.104 & -1.004 \\ \mathrm{H} & -3.250 & 1.633 & -0.941 \\ \mathrm{O} & 0.602 & -0.342 & -0.915 \\ \mathrm{Si} & 1.902 & 0.002 & 0.066 \\ \mathrm{C} & 2.139 & 1.846 & 0.240 \\ \mathrm{H} & 2.205 & 2.327 & -0.737 \\ \mathrm{H} & 1.315 & 2.302 & 0.791 \\ \mathrm{H} & 3.063 & 2.058 & 0.784 \\ \mathrm{C} & 1.595 & -0.747 & 1.749 \\ \mathrm{H} & 1.506 & -1.833 & 1.690 \\ \mathrm{H} & 2.410 & -0.512 & 2.436 \\ \mathrm{H} & 0.672 & -0.352 & 2.181 \\ \mathrm{C} & 3.364 & -0.772 & -0.782 \\ \mathrm{H} & 4.280 & -0.608 & -0.210 \\ \mathrm{H} & 3.222 & -1.848 & -0.894 \\ \mathrm{H} & 3.505 & -0.345 & -1.776\end{array}$

EE2

$\begin{array}{lrrr}\text { O } & 0.226 & -0.834 & 0.031 \\ \mathrm{Si} & 1.668 & 0.006 & -0.009 \\ \mathrm{C} & 1.776 & 1.014 & -1.575 \\ \mathrm{H} & 1.655 & 0.373 & -2.450 \\ \mathrm{H} & 1.015 & 1.793 & -1.622 \\ \mathrm{H} & 2.754 & 1.495 & -1.644 \\ \mathrm{C} & 1.816 & 1.090 & 1.502 \\ \mathrm{H} & 1.721 & 0.493 & 2.411 \\ \mathrm{H} & 2.795 & 1.574 & 1.520 \\ \mathrm{H} & 1.056 & 1.870 & 1.531 \\ \mathrm{C} & 2.969 & -1.322 & 0.006 \\ \mathrm{H} & 3.970 & -0.886 & -0.016 \\ \mathrm{H} & 2.886 & -1.935 & 0.905 \\ \mathrm{H} & 2.865 & -1.976 & -0.860 \\ \mathrm{C} & -1.025 & -0.330 & 0.032 \\ \mathrm{C} & -2.164 & -1.306 & 0.133 \\ \mathrm{C} & -1.436 & 0.938 & 0.000 \\ \mathrm{C} & -3.385 & -0.433 & -0.193 \\ \mathrm{H} & -2.035 & -2.146 & -0.551 \\ \mathrm{H} & -2.200 & -1.719 & 1.146 \\ \mathrm{C} & -2.938 & 1.019 & 0.089 \\ \mathrm{H} & -0.794 & 1.807 & -0.026 \\ \mathrm{H} & -4.274 & -0.716 & 0.368 \\ \mathrm{H} & -3.619 & -0.531 & -1.254 \\ \mathrm{H} & -3.371 & 1.722 & -0.625 \\ \mathrm{H} & -3.252 & 1.348 & 1.085\end{array}$




$\begin{array}{lrrr}\text { EE3 } & & & \\ \text { C } & -1.695 & 0.116 & -0.288 \\ \text { C } & -2.093 & 1.363 & -0.066 \\ \text { C } & -2.518 & -1.105 & -0.032 \\ \text { H } & -1.455 & 2.203 & -0.302 \\ \text { H } & -2.008 & -1.754 & 0.684 \\ \text { H } & -2.630 & -1.670 & -0.959 \\ \text { O } & -0.468 & -0.173 & -0.819 \\ \text { Si } & 0.967 & -0.033 & 0.016 \\ \text { C } & 1.356 & 1.756 & 0.374 \\ \text { H } & 1.339 & 2.354 & -0.539 \\ \text { H } & 0.640 & 2.185 & 1.078 \\ \text { H } & 2.351 & 1.844 & 0.815 \\ \text { C } & 0.814 & -0.974 & 1.621 \\ \text { H } & 0.641 & -2.037 & 1.443 \\ \text { H } & 1.722 & -0.875 & 2.219 \\ \text { H } & -0.017 & -0.586 & 2.215 \\ \text { C } & 2.247 & -0.779 & -1.107 \\ \text { H } & 3.236 & -0.747 & -0.646 \\ \text { H } & 2.008 & -1.819 & -1.330 \\ \text { H } & 2.296 & -0.234 & -2.051 \\ \text { H } & -3.077 & 1.558 & 0.335 \\ \text { H } & -3.501 & -0.848 & 0.355\end{array}$

EE4

$\begin{array}{lrrr}\mathrm{C} & 0.349 & 2.351 & -0.315 \\ \mathrm{H} & 1.271 & 2.548 & -0.845 \\ \mathrm{C} & -0.002 & 1.075 & -0.182 \\ \mathrm{C} & -0.460 & 3.500 & 0.198 \\ \mathrm{H} & -1.355 & 3.145 & 0.705 \\ \mathrm{H} & 0.113 & 4.105 & 0.904 \\ \mathrm{H} & -0.766 & 4.162 & -0.615 \\ \mathrm{O} & -1.120 & 0.680 & 0.459 \\ \mathrm{O} & 0.728 & 0.037 & -0.681 \\ \mathrm{Si} & -2.115 & -0.579 & -0.026 \\ \mathrm{Si} & 2.145 & -0.488 & 0.045 \\ \mathrm{C} & 1.810 & -0.772 & 1.856 \\ \mathrm{H} & 1.368 & 0.121 & 2.303 \\ \mathrm{H} & 1.121 & -1.604 & 2.008 \\ \mathrm{H} & 2.735 & -0.995 & 2.392 \\ \mathrm{C} & 3.488 & 0.787 & -0.168 \\ \mathrm{H} & 4.442 & 0.389 & 0.186 \\ \mathrm{H} & 3.606 & 1.063 & -1.217 \\ \mathrm{H} & 3.270 & 1.692 & 0.402 \\ \mathrm{C} & 2.584 & -2.055 & -0.855 \\ \mathrm{H} & 2.705 & -1.864 & -1.922 \\ \mathrm{H} & 3.525 & -2.462 & -0.478 \\ \mathrm{H} & 1.815 & -2.819 & -0.732 \\ \mathrm{C} & -3.722 & -0.243 & 0.851 \\ \mathrm{H} & -4.144 & 0.713 & 0.537 \\ & & & \end{array}$

$\begin{array}{llll}\mathrm{H} & -4.453 & -1.025 & 0.636 \\ \mathrm{H} & -3.572 & -0.208 & 1.931 \\ \mathrm{C} & -2.307 & -0.517 & -1.877 \\ \mathrm{H} & -3.003 & -1.285 & -2.220 \\ \mathrm{H} & -2.690 & 0.454 & -2.196 \\ \mathrm{H} & -1.346 & -0.682 & -2.367 \\ \mathrm{C} & -1.420 & -2.219 & 0.522 \\ \mathrm{H} & -0.470 & -2.427 & 0.028 \\ \mathrm{H} & -1.260 & -2.235 & 1.602 \\ \mathrm{H} & -2.113 & -3.026 & 0.275\end{array}$

EE5

$\begin{array}{lrrr}\text { C } & -2.843 & -1.213 & 0.168 \\ \text { C } & -3.413 & 0.175 & -0.113 \\ \text { C } & -2.411 & 1.225 & 0.331 \\ \text { C } & -0.709 & -0.140 & -0.509 \\ \text { C } & -1.390 & -1.248 & -0.216 \\ \text { H } & -2.770 & 2.238 & 0.161 \\ \text { H } & -3.590 & 0.299 & -1.184 \\ \text { H } & -4.361 & 0.330 & 0.403 \\ \text { H } & -2.974 & -1.465 & 1.225 \\ \text { H } & -3.397 & -1.966 & -0.398 \\ \text { H } & -0.873 & -2.191 & -0.324 \\ \text { H } & -2.182 & 1.102 & 1.396 \\ \text { O } & -1.196 & 1.120 & -0.404 \\ \text { O } & 0.555 & -0.122 & -0.968 \\ \text { Si } & 1.877 & -0.002 & 0.052 \\ \text { C } & 2.189 & -1.674 & 0.815 \\ \text { H } & 1.327 & -1.994 & 1.404 \\ \text { H } & 2.373 & -2.427 & 0.046 \\ \text { H } & 3.057 & -1.646 & 1.477 \\ \text { C } & 3.285 & 0.520 & -1.041 \\ \text { H } & 3.085 & 1.494 & -1.491 \\ \text { H } & 4.215 & 0.591 & -0.474 \\ \text { H } & 3.434 & -0.201 & -1.846 \\ \text { C } & 1.500 & 1.249 & 1.380 \\ \text { H } & 1.287 & 2.227 & 0.947 \\ \text { H } & 0.627 & 0.940 & 1.959 \\ \text { H } & 2.340 & 1.350 & 2.070\end{array}$

EE6

$\begin{array}{lrrr}\mathrm{O} & -0.222 & -0.948 & 0.205 \\ \mathrm{Si} & -1.586 & 0.015 & -0.014 \\ \mathrm{C} & -1.478 & 0.867 & -1.667 \\ \mathrm{H} & -0.653 & 1.580 & -1.681 \\ \mathrm{H} & -1.322 & 0.143 & -2.469 \\ \mathrm{H} & -2.402 & 1.409 & -1.879 \\ \mathrm{C} & -2.980 & -1.215 & 0.026 \\ \mathrm{H} & -2.996 & -1.751 & 0.976 \\ \mathrm{H} & -3.942 & -0.713 & -0.096\end{array}$




$\begin{array}{lrrr}\mathrm{H} & -2.876 & -1.949 & -0.775 \\ \mathrm{C} & -1.734 & 1.241 & 1.377 \\ \mathrm{H} & -1.740 & 0.732 & 2.343 \\ \mathrm{H} & -0.900 & 1.942 & 1.364 \\ \mathrm{H} & -2.665 & 1.806 & 1.289 \\ \mathrm{C} & 1.042 & -0.567 & 0.114 \\ \mathrm{C} & 2.152 & -1.291 & -0.006 \\ \mathrm{C} & 2.627 & 1.000 & -0.143 \\ \mathrm{C} & 3.330 & -0.355 & 0.047 \\ \mathrm{H} & 3.001 & 1.795 & 0.497 \\ \mathrm{H} & 2.672 & 1.321 & -1.187 \\ \mathrm{H} & 4.065 & -0.524 & -0.740 \\ \mathrm{H} & 3.856 & -0.410 & 1.005 \\ \mathrm{O} & 1.243 & 0.785 & 0.184 \\ \mathrm{H} & 2.184 & -2.366 & -0.044\end{array}$

\section{EE7}

$\begin{array}{lrrr}\mathrm{C} & -0.170 & 2.417 & -0.670 \\ \mathrm{H} & -1.140 & 2.821 & -0.921 \\ \mathrm{H} & 0.643 & 3.103 & -0.486 \\ \mathrm{C} & 0.019 & 1.100 & -0.640 \\ \mathrm{O} & -0.994 & 0.240 & -0.959 \\ \mathrm{Si} & -2.080 & -0.334 & 0.173 \\ \mathrm{C} & -1.142 & -0.942 & 1.665 \\ \mathrm{H} & -1.830 & -1.304 & 2.432 \\ \mathrm{H} & -0.545 & -0.139 & 2.102 \\ \mathrm{H} & -0.463 & -1.756 & 1.404 \\ \mathrm{C} & -2.984 & -1.708 & -0.693 \\ \mathrm{H} & -3.745 & -2.145 & -0.045 \\ \mathrm{H} & -2.294 & -2.501 & -0.987 \\ \mathrm{H} & -3.478 & -1.339 & -1.594 \\ \mathrm{C} & -3.239 & 1.042 & 0.664 \\ \mathrm{H} & -3.983 & 0.684 & 1.378 \\ \mathrm{H} & -3.767 & 1.438 & -0.205 \\ \mathrm{H} & -2.691 & 1.861 & 1.133 \\ \mathrm{C} & 1.308 & 0.439 & -0.323 \\ \mathrm{C} & 1.549 & -0.858 & -0.773 \\ \mathrm{C} & 2.292 & 1.083 & 0.428 \\ \mathrm{C} & 2.753 & -1.489 & -0.496 \\ \mathrm{H} & 0.786 & -1.363 & -1.350 \\ \mathrm{C} & 3.492 & 0.451 & 0.706 \\ \mathrm{H} & 2.106 & 2.076 & 0.817 \\ \mathrm{C} & 3.729 & -0.837 & 0.243 \\ \mathrm{H} & 2.928 & -2.493 & -0.859 \\ \mathrm{H} & 4.243 & 0.962 & 1.296 \\ \mathrm{H} & 4.666 & -1.331 & 0.464\end{array}$

$\begin{array}{llll}\text { EE8 } & & & \\ \text { C } & 1.513 & 1.996 & -0.133 \\ \text { H } & 0.711 & 2.659 & -0.426\end{array}$

$\begin{array}{lrrr}\mathrm{H} & 2.419 & 2.423 & 0.270 \\ \mathrm{C} & 1.407 & 0.676 & -0.288 \\ \mathrm{O} & 0.293 & 0.096 & -0.815 \\ \mathrm{Si} & -1.131 & -0.113 & 0.030 \\ \mathrm{C} & -0.753 & -0.934 & 1.661 \\ \mathrm{H} & -1.654 & -1.009 & 2.274 \\ \mathrm{H} & -0.015 & -0.353 & 2.220 \\ \mathrm{H} & -0.351 & -1.938 & 1.521 \\ \mathrm{C} & -2.174 & -1.189 & -1.070 \\ \mathrm{H} & -3.144 & -1.392 & -0.612 \\ \mathrm{H} & -1.681 & -2.145 & -1.257 \\ \mathrm{H} & -2.348 & -0.705 & -2.032 \\ \mathrm{C} & -1.948 & 1.537 & 0.329 \\ \mathrm{H} & -2.931 & 1.397 & 0.784 \\ \mathrm{H} & -2.085 & 2.084 & -0.606 \\ \mathrm{H} & -1.353 & 2.156 & 1.003 \\ \mathrm{C} & 2.489 & -0.247 & 0.069 \\ \mathrm{H} & 3.378 & 0.213 & 0.484 \\ \mathrm{C} & 2.423 & -1.563 & -0.098 \\ \mathrm{H} & 1.543 & -2.024 & -0.528 \\ \mathrm{H} & 3.247 & -2.206 & 0.177\end{array}$

EE9

$$
\text { C }
$$

$\mathrm{H}$

$\mathrm{H}$

C

O

0

C

C

C

C

C

C

$\mathrm{H}$

$\mathrm{H}$

$\mathrm{H}$

$\mathrm{H}$

$\mathrm{H}$

$\mathrm{Si}$

C

$\mathrm{H}$

$\mathrm{H}$

$\mathrm{H}$

C

$\mathrm{H}$

$\mathrm{H}$

$\mathrm{H}$

C

$\mathrm{H}$

$\begin{array}{rrr}0.317 & 2.356 & -0.119 \\ -0.269 & 3.246 & -0.284 \\ 1.392 & 2.406 & -0.137 \\ -0.320 & 1.214 & 0.119 \\ -1.644 & 1.099 & 0.190 \\ 0.275 & 0.000 & 0.347 \\ 1.639 & -0.106 & 0.159 \\ 4.358 & -0.425 & -0.193 \\ 2.467 & -0.130 & 1.267 \\ 2.146 & -0.236 & -1.124 \\ 3.513 & -0.397 & -1.296 \\ 3.834 & -0.293 & 1.085 \\ 2.033 & -0.021 & 2.252 \\ 1.467 & -0.205 & -1.967 \\ 3.918 & -0.502 & -2.293 \\ 4.490 & -0.316 & 1.946 \\ 5.423 & -0.553 & -0.331 \\ -2.565 & -0.294 & -0.016 \\ -2.030 & -1.164 & -1.576 \\ -2.738 & -1.956 & -1.832 \\ -1.045 & -1.616 & -1.457 \\ -1.992 & -0.466 & -2.414 \\ -4.296 & 0.365 & -0.172 \\ -5.013 & -0.450 & -0.286 \\ -4.388 & 1.022 & -1.038 \\ -4.573 & 0.937 & 0.716 \\ -2.411 & -1.402 & 1.471 \\ -3.096 & -2.249 & 1.385\end{array}$




$\begin{array}{lrrrrrrr}\mathrm{H} & -2.661 & -0.860 & 2.384 & \mathrm{H} & -3.251 & 1.602 & -1.170 \\ \mathrm{H} & -1.396 & -1.788 & 1.567 & \mathrm{C} & -3.268 & -0.668 & 0.348 \\ & & & & \mathrm{H} & -3.091 & -1.213 & 1.277 \\ \text { EE10 } & & & & \mathrm{H} & -4.142 & -0.030 & 0.493 \\ \mathrm{C} & & & & \mathrm{H} & -3.503 & -1.387 & -0.438 \\ \mathrm{H} & 0.916 & 2.018 & -0.131 & \mathrm{C} & -1.784 & 1.192 & 1.100 \\ \mathrm{H} & 0.079 & 2.664 & -0.355 & \mathrm{H} & -2.663 & 1.816 & 1.275 \\ \mathrm{C} & 1.837 & 2.455 & 0.223 & \mathrm{H} & -1.545 & 0.668 & 2.028 \\ \mathrm{O} & 0.821 & 0.710 & -0.307 & \mathrm{H} & -0.948 & 1.843 & 0.843\end{array}$

\section{EE12}

$\begin{array}{lrrr}\text { C } & 0.990 & 0.217 & -0.180 \\ \text { C } & 2.021 & -0.617 & -0.088 \\ \text { C } & 3.323 & -0.200 & 0.527 \\ \text { H } & 3.547 & -0.816 & 1.403 \\ \text { H } & 4.144 & -0.349 & -0.181 \\ \text { H } & 3.311 & 0.842 & 0.835 \\ \mathrm{C} & 1.929 & -2.031 & -0.575 \\ \text { H } & 2.697 & -2.229 & -1.326 \\ \text { H } & 2.104 & -2.731 & 0.248 \\ \text { H } & 0.957 & -2.247 & -1.014 \\ \text { O } & 1.012 & 1.473 & 0.358 \\ \text { O } & -0.188 & -0.101 & -0.776 \\ \mathrm{C} & 0.858 & 2.514 & -0.597 \\ \text { H } & -0.074 & 2.404 & -1.153 \\ \text { H } & 0.844 & 3.449 & -0.042 \\ \text { H } & 1.700 & 2.511 & -1.295 \\ \text { Si } & -1.621 & -0.231 & 0.083 \\ \text { C } & -2.395 & 1.453 & 0.295 \\ \text { H } & -1.740 & 2.109 & 0.870 \\ \text { H } & -2.594 & 1.922 & -0.670 \\ \text { H } & -3.346 & 1.373 & 0.827 \\ \text { C } & -1.229 & -0.989 & 1.738 \\ \text { H } & -2.138 & -1.151 & 2.321 \\ \text { H } & -0.727 & -1.951 & 1.615 \\ \text { H } & -0.568 & -0.337 & 2.313 \\ \text { C } & -2.703 & -1.327 & -0.959 \\ \text { H } & -3.687 & -1.452 & -0.504 \\ \text { H } & -2.843 & -0.898 & -1.953 \\ \text { H } & -2.256 & -2.315 & -1.078\end{array}$

EE13

$\begin{array}{lrrr}\mathrm{O} & 0.292 & 0.736 & 0.305 \\ \mathrm{Si} & 1.792 & 0.046 & -0.020 \\ \mathrm{C} & 1.764 & -0.694 & -1.730 \\ \mathrm{H} & 1.068 & -1.533 & -1.775 \\ \mathrm{H} & 1.459 & 0.047 & -2.470 \\ \mathrm{H} & 2.755 & -1.059 & -2.007 \\ \mathrm{C} & 2.958 & 1.491 & 0.077 \\ \mathrm{H} & 2.915 & 1.957 & 1.062\end{array}$




$\begin{array}{lrrr}\mathrm{H} & 3.986 & 1.171 & -0.101 \\ \mathrm{H} & 2.705 & 2.248 & -0.667 \\ \mathrm{C} & 2.203 & -1.231 & 1.268 \\ \mathrm{H} & 2.167 & -0.798 & 2.269 \\ \mathrm{H} & 1.497 & -2.060 & 1.228 \\ \mathrm{H} & 3.210 & -1.624 & 1.109 \\ \mathrm{C} & -0.890 & 0.146 & 0.166 \\ \mathrm{C} & -2.096 & 0.689 & 0.027 \\ \mathrm{C} & -2.183 & -1.656 & -0.154 \\ \mathrm{C} & -3.099 & -0.437 & 0.026 \\ \mathrm{H} & -2.440 & -2.508 & 0.471 \\ \mathrm{H} & -2.151 & -1.970 & -1.200 \\ \mathrm{H} & -3.825 & -0.366 & -0.786 \\ \mathrm{H} & -3.663 & -0.475 & 0.964 \\ \mathrm{O} & -0.861 & -1.224 & 0.211 \\ \mathrm{C} & -2.432 & 2.138 & 0.000 \\ \mathrm{H} & -3.066 & 2.415 & 0.847 \\ \mathrm{H} & -1.525 & 2.740 & 0.045 \\ \mathrm{H} & -2.976 & 2.407 & -0.909\end{array}$

$\begin{array}{lrrr}\mathrm{C} & -2.226 & -0.244 & 1.835 \\ \mathrm{H} & -1.232 & -0.266 & 2.284 \\ \mathrm{H} & -2.569 & 0.793 & 1.813 \\ \mathrm{H} & -2.907 & -0.809 & 2.475 \\ \mathrm{C} & -1.516 & -2.720 & 0.170 \\ \mathrm{H} & -2.155 & -3.353 & 0.790 \\ \mathrm{H} & -1.479 & -3.161 & -0.828 \\ \mathrm{H} & -0.512 & -2.733 & 0.595 \\ \mathrm{C} & -3.894 & -0.999 & -0.635 \\ \mathrm{H} & -3.853 & -1.362 & -1.663 \\ \mathrm{H} & -4.569 & -1.649 & -0.074 \\ \mathrm{H} & -4.323 & 0.004 & -0.650\end{array}$

\section{EE14}

$\begin{array}{lrrr}\mathrm{C} & -0.137 & 3.534 & -0.667 \\ \mathrm{C} & -1.093 & 2.376 & -0.936 \\ \mathrm{C} & -0.549 & 1.068 & -0.448 \\ \mathrm{C} & 0.521 & 0.970 & 0.342 \\ \mathrm{C} & 1.230 & 2.162 & 0.919 \\ \mathrm{C} & 0.415 & 3.441 & 0.751 \\ \mathrm{H} & -2.061 & 2.556 & -0.452 \\ \mathrm{H} & -1.301 & 2.278 & -2.005 \\ \mathrm{H} & 0.694 & 3.489 & -1.377 \\ \mathrm{H} & -0.646 & 4.485 & -0.826 \\ \mathrm{H} & 1.414 & 1.955 & 1.976 \\ \mathrm{H} & 2.214 & 2.275 & 0.453 \\ \mathrm{H} & -0.421 & 3.430 & 1.459 \\ \mathrm{H} & 1.028 & 4.311 & 0.990 \\ \mathrm{O} & -1.244 & -0.032 & -0.869 \\ \mathrm{O} & 0.983 & -0.257 & 0.760 \\ \mathrm{Si} & 2.118 & -1.101 & -0.128 \\ \mathrm{Si} & -2.190 & -0.983 & 0.124 \\ \mathrm{C} & 2.576 & -2.565 & 0.927 \\ \mathrm{H} & 3.348 & -3.159 & 0.435 \\ \mathrm{H} & 2.965 & -2.236 & 1.892 \\ \mathrm{H} & 1.721 & -3.216 & 1.111 \\ \mathrm{C} & 1.418 & -1.612 & -1.777 \\ \mathrm{H} & 0.608 & -2.332 & -1.667 \\ \mathrm{H} & 1.018 & -0.743 & -2.304 \\ \mathrm{H} & 2.198 & -2.061 & -2.396 \\ \mathrm{C} & 3.611 & -0.015 & -0.421 \\ \mathrm{H} & 3.365 & 0.826 & -1.072 \\ \mathrm{H} & 4.011 & 0.379 & 0.515 \\ \mathrm{H} & 4.401 & -0.590 & -0.910\end{array}$

EE15

$\begin{array}{lrrr}\text { C } & -0.994 & -0.638 & 0.093 \\ \text { C } & -0.875 & 0.887 & -0.079 \\ \text { C } & 0.619 & 1.069 & 0.054 \\ \text { C } & 1.190 & -0.125 & 0.012 \\ \text { O } & 0.329 & -1.176 & -0.090 \\ \text { H } & -1.318 & -0.897 & 1.103 \\ \text { H } & -1.230 & 1.224 & -1.056 \\ \text { H } & 1.136 & 2.012 & 0.108 \\ \text { H } & 2.235 & -0.395 & 0.029 \\ \text { H } & -1.446 & 1.420 & 0.681 \\ \text { H } & -1.653 & -1.116 & -0.629\end{array}$

\section{EE16}

$\begin{array}{lrrr}\mathrm{C} & 1.274 & 0.345 & 0.000 \\ \mathrm{H} & 1.213 & 1.431 & -0.001 \\ \mathrm{C} & 2.433 & -0.293 & 0.000 \\ \mathrm{H} & 3.354 & 0.270 & 0.000 \\ \mathrm{H} & 2.476 & -1.373 & 0.001 \\ \mathrm{O} & 0.085 & -0.300 & -0.001 \\ \mathrm{C} & -1.063 & 0.535 & 0.001 \\ \mathrm{H} & -1.046 & 1.177 & 0.888 \\ \mathrm{H} & -1.046 & 1.179 & -0.885 \\ \mathrm{C} & -2.286 & -0.349 & 0.000 \\ \mathrm{H} & -2.293 & -0.987 & 0.883 \\ \mathrm{H} & -3.191 & 0.259 & 0.001 \\ \mathrm{H} & -2.293 & -0.985 & -0.885\end{array}$

\section{EE17}

$\begin{array}{lrrr}\text { C } & 2.369 & 0.005 & -0.004 \\ \text { C } & 1.102 & -0.394 & -0.014 \\ \text { O } & 0.048 & 0.468 & -0.024 \\ \text { Si } & -1.547 & -0.003 & 0.001 \\ \text { C } & -1.905 & -1.054 & -1.499 \\ \text { H } & -2.955 & -1.351 & -1.522 \\ \text { H } & -1.304 & -1.965 & -1.503\end{array}$




$\begin{array}{lrrr}\text { H } & -1.689 & -0.506 & -2.418 \\ \mathrm{C} & -2.521 & 1.579 & -0.029 \\ \mathrm{H} & -3.594 & 1.378 & -0.016 \\ \mathrm{H} & -2.295 & 2.155 & -0.928 \\ \mathrm{H} & -2.281 & 2.197 & 0.837 \\ \mathrm{C} & -1.878 & -0.980 & 1.556 \\ \mathrm{H} & -2.926 & -1.283 & 1.608 \\ \mathrm{H} & -1.653 & -0.386 & 2.444 \\ \mathrm{H} & -1.270 & -1.886 & 1.596 \\ \mathrm{H} & 0.853 & -1.454 & -0.017 \\ \mathrm{C} & 2.753 & 1.453 & -0.001 \\ \mathrm{H} & 1.877 & 2.097 & -0.007 \\ \mathrm{H} & 3.355 & 1.687 & 0.881 \\ \mathrm{H} & 3.367 & 1.688 & -0.875 \\ \mathrm{C} & 3.485 & -0.995 & 0.004 \\ \mathrm{H} & 4.129 & -0.865 & -0.870 \\ \mathrm{H} & 4.118 & -0.865 & 0.886 \\ \mathrm{H} & 3.112 & -2.019 & 0.001\end{array}$

\section{EE18}

$\begin{array}{lrrr}\mathrm{C} & -0.319 & -0.576 & 0.105 \\ \mathrm{H} & -0.204 & -1.656 & 0.155 \\ \mathrm{C} & -1.513 & -0.004 & 0.006 \\ \mathrm{O} & 0.839 & 0.138 & 0.192 \\ \mathrm{C} & 2.001 & -0.575 & -0.193 \\ \mathrm{H} & 2.081 & -1.491 & 0.404 \\ \mathrm{H} & 1.921 & -0.867 & -1.246 \\ \mathrm{C} & 3.199 & 0.317 & 0.031 \\ \mathrm{H} & 3.267 & 0.601 & 1.081 \\ \mathrm{H} & 4.114 & -0.202 & -0.252 \\ \mathrm{H} & 3.113 & 1.223 & -0.567 \\ \mathrm{C} & -1.689 & 1.482 & -0.064 \\ \mathrm{H} & -0.732 & 1.997 & -0.096 \\ \mathrm{H} & -2.268 & 1.756 & -0.950 \\ \mathrm{H} & -2.248 & 1.843 & 0.804 \\ \mathrm{C} & -2.759 & -0.838 & -0.017 \\ \mathrm{H} & -3.335 & -0.647 & -0.926 \\ \mathrm{H} & -2.534 & -1.904 & 0.027 \\ \mathrm{H} & -3.407 & -0.592 & 0.828\end{array}$

\section{EE19}

$\begin{array}{lrrr}\text { C } & -1.254 & -0.090 & -0.326 \\ C & -2.256 & 0.814 & 0.034 \\ C & -3.783 & -1.007 & 0.255 \\ C & -2.781 & -1.902 & -0.106 \\ C & -1.500 & -1.451 & -0.402 \\ C & -0.027 & 0.675 & -0.569 \\ C & -0.249 & 1.983 & -0.372 \\ H & -4.774 & -1.379 & 0.482 \\ H & -3.005 & -2.960 & -0.157\end{array}$

$\begin{array}{lrrr}\mathrm{H} & -0.713 & -2.138 & -0.691 \\ \mathrm{H} & -2.209 & 2.852 & -0.688 \\ \mathrm{H} & 0.481 & 2.769 & -0.496 \\ \mathrm{H} & -4.314 & 1.051 & 0.609 \\ \mathrm{C} & -3.527 & 0.361 & 0.328 \\ \mathrm{C} & -1.684 & 2.209 & 0.024 \\ \mathrm{H} & -1.772 & 2.686 & 1.005 \\ \mathrm{O} & 1.118 & 0.066 & -0.954 \\ \mathrm{Si} & 2.375 & -0.239 & 0.105 \\ \mathrm{C} & 3.106 & 1.376 & 0.683 \\ \mathrm{H} & 2.378 & 1.952 & 1.256 \\ \mathrm{H} & 3.971 & 1.197 & 1.326 \\ \mathrm{H} & 3.434 & 1.983 & -0.163 \\ \mathrm{C} & 3.599 & -1.228 & -0.882 \\ \mathrm{H} & 3.945 & -0.662 & -1.748 \\ \mathrm{H} & 4.470 & -1.485 & -0.276 \\ \mathrm{H} & 3.149 & -2.154 & -1.241 \\ \mathrm{C} & 1.703 & -1.194 & 1.559 \\ \mathrm{H} & 0.886 & -0.646 & 2.034 \\ \mathrm{H} & 1.318 & -2.167 & 1.251 \\ \mathrm{H} & 2.478 & -1.358 & 2.310\end{array}$

cX1

$\begin{array}{lrrr}\text { C } & -1.049 & -0.559 & 0.044 \\ \text { H } & -0.926 & -1.458 & -0.538 \\ \text { C } & -0.082 & 0.435 & 0.298 \\ \text { C } & 1.371 & 0.101 & 0.112 \\ \text { C } & 2.277 & 1.153 & 0.021 \\ \text { C } & 1.837 & -1.209 & 0.036 \\ \text { C } & 3.629 & 0.901 & -0.156 \\ \text { H } & 1.894 & 2.162 & 0.099 \\ \text { C } & 3.190 & -1.462 & -0.137 \\ \text { H } & 1.144 & -2.034 & 0.140 \\ \text { C } & 4.088 & -0.408 & -0.238 \\ \text { H } & 4.327 & 1.725 & -0.228 \\ \text { H } & 3.546 & -2.484 & -0.187 \\ \text { O } & -0.405 & 1.580 & 0.656 \\ \text { S } & -2.619 & 0.011 & 0.365 \\ \text { C } & -3.138 & 1.042 & -1.031 \\ \text { H } & -2.455 & 1.889 & -1.012 \\ \text { H } & -3.031 & 0.477 & -1.955 \\ \text { H } & -4.164 & 1.374 & -0.876 \\ \text { C } & -3.610 & -1.442 & 0.035 \\ \text { H } & -3.364 & -1.844 & -0.947 \\ \text { H } & -3.383 & -2.171 & 0.809 \\ \text { H } & -4.660 & -1.161 & 0.079 \\ \text { H } & 5.143 & -0.606 & -0.373\end{array}$

\section{CX2}

$\begin{array}{llll}C & 2.572 & -0.556 & -0.075\end{array}$




$\begin{array}{lrrr}\mathrm{H} & 2.353 & -1.465 & 0.460 \\ \mathrm{C} & 1.698 & 0.519 & -0.328 \\ \mathrm{C} & 0.218 & 0.291 & -0.218 \\ \mathrm{C} & -0.610 & 1.405 & -0.124 \\ \mathrm{C} & -0.353 & -0.978 & -0.217 \\ \mathrm{C} & -1.984 & 1.262 & -0.015 \\ \mathrm{H} & -0.152 & 2.385 & -0.145 \\ \mathrm{C} & -1.726 & -1.139 & -0.112 \\ \mathrm{H} & 0.275 & -1.853 & -0.324 \\ \mathrm{C} & -2.528 & -0.013 & -0.007 \\ \mathrm{H} & -2.628 & 2.127 & 0.062 \\ \mathrm{H} & -2.172 & -2.124 & -0.119 \\ \mathrm{O} & 2.119 & 1.650 & -0.621 \\ \mathrm{~S} & 4.197 & -0.101 & -0.302 \\ \mathrm{C} & 4.724 & 0.833 & 1.157 \\ \mathrm{H} & 4.110 & 1.732 & 1.141 \\ \mathrm{H} & 4.528 & 0.244 & 2.051 \\ \mathrm{H} & 5.779 & 1.087 & 1.063 \\ \mathrm{C} & 5.053 & -1.640 & 0.012 \\ \mathrm{H} & 4.730 & -2.057 & 0.964 \\ \mathrm{H} & 4.808 & -2.319 & -0.801 \\ \mathrm{H} & 6.123 & -1.443 & 0.025 \\ \mathrm{Br} & -4.407 & -0.223 & 0.143 \\ & & & \end{array}$

\section{CX3}

$\begin{array}{lrrr}\text { C } & -2.236 & 0.551 & -0.067 \\ \text { H } & -2.042 & 1.451 & 0.492 \\ \mathrm{C} & -1.340 & -0.519 & -0.298 \\ \mathrm{C} & 0.127 & -0.278 & -0.163 \\ \mathrm{C} & 0.983 & -1.374 & -0.103 \\ \mathrm{C} & 0.694 & 0.991 & -0.111 \\ \mathrm{C} & 2.349 & -1.219 & 0.024 \\ \mathrm{H} & 0.546 & -2.363 & -0.165 \\ \mathrm{C} & 2.060 & 1.169 & 0.012 \\ \mathrm{H} & 0.062 & 1.866 & -0.198 \\ \mathrm{C} & 2.925 & 0.063 & 0.098 \\ \mathrm{H} & 2.972 & -2.100 & 0.062 \\ \mathrm{H} & 2.455 & 2.174 & 0.029 \\ \mathrm{O} & -1.756 & -1.652 & -0.597 \\ \mathrm{~S} & -3.844 & 0.075 & -0.329 \\ \mathrm{C} & -4.417 & -0.839 & 1.128 \\ \mathrm{H} & -3.797 & -1.733 & 1.148 \\ \mathrm{H} & -4.255 & -0.235 & 2.018 \\ \mathrm{H} & -5.467 & -1.104 & 1.005 \\ \mathrm{C} & -4.728 & 1.608 & -0.065 \\ \mathrm{H} & -4.432 & 2.048 & 0.886 \\ \mathrm{H} & -4.472 & 2.275 & -0.885 \\ \mathrm{H} & -5.796 & 1.398 & -0.071 \\ \mathrm{~N} & 4.287 & 0.228 & 0.251 \\ \mathrm{C} & 5.154 & -0.916 & 0.078 \\ \mathrm{H} & 6.186 & -0.603 & 0.215\end{array}$

$\begin{array}{lrrr}\mathrm{H} & 4.938 & -1.684 & 0.823 \\ \mathrm{H} & 5.056 & -1.369 & -0.916 \\ \mathrm{C} & 4.854 & 1.547 & 0.084 \\ \mathrm{H} & 4.661 & 1.965 & -0.912 \\ \mathrm{H} & 4.451 & 2.240 & 0.825 \\ \mathrm{H} & 5.929 & 1.493 & 0.233\end{array}$

\section{CX4}

C

$\mathrm{H}$

C

C

C

C

C

$\mathrm{H}$

C

$\mathrm{H}$

C

$\mathrm{H}$

$\mathrm{H}$

O

$\mathrm{S}$

C

$\mathrm{H}$

$\mathrm{H}$

$\mathrm{H}$

C

$\mathrm{H}$

$\mathrm{H}$

$\mathrm{H}$

C

$\mathrm{H}$

$\mathrm{H}$

$\mathrm{H}$

O

CX5

$\begin{array}{lrrr}\text { S } & 0.529 & 0.000 & -0.388 \\ \mathrm{C} & 1.637 & 1.355 & 0.066 \\ \mathrm{H} & 1.861 & 1.291 & 1.130 \\ \mathrm{H} & 2.549 & 1.295 & -0.526 \\ \mathrm{H} & 1.103 & 2.277 & -0.150 \\ \mathrm{C} & 1.637 & -1.355 & 0.066 \\ \mathrm{H} & 1.862 & -1.291 & 1.130 \\ \mathrm{H} & 1.102 & -2.277 & -0.148 \\ \mathrm{H} & 2.548 & -1.296 & -0.526 \\ \mathrm{C} & -0.748 & 0.000 & 0.671 \\ \mathrm{H} & -0.576 & 0.001 & 1.737 \\ \mathrm{C} & -2.044 & 0.000 & 0.136\end{array}$




\section{CX6}

$\begin{array}{lrrr}\text { S } & 1.933 & 0.000 & -0.299 \\ \mathrm{C} & 2.920 & 1.354 & 0.377 \\ \mathrm{H} & 2.923 & 1.289 & 1.464 \\ \mathrm{H} & 3.934 & 1.298 & -0.016 \\ \mathrm{H} & 2.440 & 2.275 & 0.058 \\ \mathrm{C} & 2.918 & -1.354 & 0.381 \\ \mathrm{H} & 2.921 & -1.287 & 1.468 \\ \mathrm{H} & 2.437 & -2.275 & 0.064 \\ \mathrm{H} & 3.932 & -1.301 & -0.012 \\ \mathrm{C} & 0.470 & 0.002 & 0.488 \\ \mathrm{H} & 0.423 & 0.004 & 1.565 \\ \mathrm{C} & -0.700 & 0.000 & -0.333 \\ \mathrm{O} & -0.755 & -0.002 & -1.547 \\ \mathrm{O} & -1.826 & 0.002 & 0.432 \\ \mathrm{C} & -3.054 & 0.000 & -0.291 \\ \mathrm{H} & -3.092 & -0.880 & -0.936 \\ \mathrm{H} & -3.094 & 0.878 & -0.938 \\ \mathrm{C} & -4.174 & 0.000 & 0.723 \\ \mathrm{H} & -5.139 & -0.002 & 0.214 \\ \mathrm{H} & -4.116 & -0.883 & 1.359 \\ \mathrm{H} & -4.118 & 0.884 & 1.357\end{array}$

CX7

$\begin{array}{lrrr}\text { S } & -2.561 & -0.433 & 0.001 \\ \mathrm{C} & -3.713 & -0.092 & 1.355 \\ \mathrm{H} & -4.035 & 0.946 & 1.291 \\ \mathrm{H} & -4.566 & -0.766 & 1.291 \\ \mathrm{H} & -3.164 & -0.257 & 2.278 \\ \mathrm{C} & -3.713 & -0.096 & -1.355 \\ \mathrm{H} & -4.037 & 0.942 & -1.292 \\ \mathrm{H} & -3.164 & -0.262 & -2.278 \\ \mathrm{H} & -4.566 & -0.771 & -1.290 \\ \mathrm{C} & -1.390 & 0.740 & -0.001 \\ \mathrm{H} & -1.716 & 1.772 & -0.002 \\ \mathrm{C} & 0.008 & 0.422 & -0.001 \\ \mathrm{C} & 0.941 & 1.480 & -0.001 \\ \mathrm{C} & 0.534 & -0.887 & 0.000 \\ \mathrm{C} & 2.299 & 1.251 & -0.001 \\ \mathrm{H} & 0.576 & 2.499 & -0.002 \\ \mathrm{C} & 1.891 & -1.118 & 0.001 \\ \mathrm{H} & -0.136 & -1.738 & 0.001 \\ \mathrm{C} & 2.797 & -0.054 & 0.000 \\ \mathrm{H} & 2.990 & 2.084 & -0.001 \\ \mathrm{H} & 2.267 & -2.133 & 0.001 \\ \mathrm{C} & 4.205 & -0.298 & 0.000 \\ \mathrm{~N} & 5.338 & -0.495 & 0.001\end{array}$

$\begin{array}{rrr}0.129 & -0.389 & 0.000 \\ -0.160 & 0.979 & 0.000 \\ -0.934 & -1.297 & 0.000 \\ -1.473 & 1.419 & 0.000 \\ -2.244 & -0.850 & 0.000 \\ -0.724 & -2.360 & 0.000 \\ -2.523 & 0.511 & 0.000 \\ -1.677 & 2.482 & 0.000 \\ -3.052 & -1.569 & 0.000 \\ -3.547 & 0.859 & 0.000 \\ 0.645 & 1.704 & 0.000 \\ 1.489 & -0.899 & 0.000 \\ 1.736 & -1.948 & 0.000 \\ 3.348 & 0.653 & 0.000 \\ 2.498 & -0.084 & 0.000\end{array}$

\section{CX10}

$\begin{array}{rrrr}C & 0.982 & 0.068 & 0.000 \\ C & 0.124 & -1.021 & 0.000 \\ C & -1.245 & -0.815 & 0.000 \\ C & -1.775 & 0.479 & 0.000 \\ C & -0.889 & 1.560 & 0.000\end{array}$




$\begin{array}{lrrr}\mathrm{C} & 0.481 & 1.360 & 0.000 \\ \mathrm{H} & -1.904 & -1.674 & 0.000 \\ \mathrm{H} & -1.278 & 2.571 & 0.000 \\ \mathrm{H} & 1.156 & 2.204 & 0.000 \\ \mathrm{H} & 0.524 & -2.025 & 0.000 \\ \mathrm{C} & -3.203 & 0.739 & 0.000 \\ \mathrm{H} & -3.633 & 1.727 & 0.000 \\ \mathrm{Br} & 2.854 & -0.214 & 0.000 \\ \mathrm{~N} & -4.049 & -0.245 & 0.000 \\ \mathrm{~N} & -4.754 & -1.120 & 0.000\end{array}$

$\begin{array}{lrrr}\text { C } & 0.937 & -0.128 & 0.012 \\ \text { O } & 1.983 & -0.731 & -0.053 \\ \text { C } & 0.896 & 1.384 & 0.025 \\ \text { H } & 1.042 & 1.724 & -1.001 \\ \text { H } & -0.037 & 1.791 & 0.409 \\ \text { H } & 1.737 & 1.739 & 0.616 \\ \text { C } & -0.320 & -0.867 & 0.069 \\ \text { H } & -0.345 & -1.944 & 0.063 \\ \text { N } & -1.466 & -0.247 & 0.007 \\ \text { N } & -2.440 & 0.277 & -0.050\end{array}$

CX11

CX14

$\begin{array}{lrrr}\mathrm{C} & 1.808 & -0.061 & 0.000 \\ \mathrm{C} & 0.895 & -1.116 & 0.000 \\ \mathrm{C} & -0.461 & -0.860 & 0.000 \\ \mathrm{C} & -0.939 & 0.456 & 0.000 \\ \mathrm{C} & -0.013 & 1.507 & 0.000 \\ \mathrm{C} & 1.343 & 1.254 & 0.000 \\ \mathrm{H} & -1.154 & -1.692 & 0.000 \\ \mathrm{H} & -0.368 & 2.530 & 0.000 \\ \mathrm{H} & 2.051 & 2.071 & 0.000 \\ \mathrm{H} & 1.258 & -2.135 & 0.000 \\ \mathrm{C} & -2.351 & 0.771 & 0.000 \\ \mathrm{H} & -2.746 & 1.773 & 0.000 \\ \mathrm{~N} & -3.235 & -0.184 & 0.000 \\ \mathrm{~N} & -3.972 & -1.029 & 0.000 \\ \mathrm{C} & 3.216 & -0.328 & 0.000 \\ \mathrm{~N} & 4.345 & -0.541 & 0.000\end{array}$

$\begin{array}{lrrr}\mathrm{C} & -2.466 & -0.580 & 0.000 \\ \mathrm{C} & -1.365 & -1.424 & 0.000 \\ \mathrm{C} & -2.211 & 0.786 & 0.000 \\ \mathrm{C} & -0.085 & -0.926 & 0.000 \\ \mathrm{H} & -1.483 & -2.498 & 0.000 \\ \mathrm{C} & -0.927 & 1.263 & 0.000 \\ \mathrm{~N} & 0.159 & 0.424 & 0.000 \\ \mathrm{H} & 0.792 & -1.555 & 0.000 \\ \mathrm{H} & -0.691 & 2.315 & 0.000 \\ \mathrm{C} & 1.422 & 0.937 & 0.000 \\ \mathrm{H} & 1.528 & 2.006 & 0.000 \\ \mathrm{H} & -3.474 & -0.965 & 0.000 \\ \mathrm{H} & -3.016 & 1.508 & 0.000 \\ \mathrm{C} & 2.522 & 0.082 & 0.001 \\ \mathrm{~N} & 3.411 & -0.658 & 0.000\end{array}$

CX15

\section{CX12}

$\begin{array}{lrr}\text { C } & 1.387 & 0.003 \\ \text { C } & 0.514 & -1.073 \\ \text { C } & -0.845 & -0.832 \\ \text { C } & -1.339 & 0.480 \\ \text { C } & -0.427 & 1.545 \\ \text { C } & 0.933 & 1.311 \\ \text { H } & -1.529 & -1.672 \\ \text { H } & -0.796 & 2.562 \\ \text { H } & 1.646 & 2.123 \\ \text { H } & 0.908 & -2.078 \\ \text { C } & -2.752 & 0.777 \\ \text { H } & -3.161 & 1.774 \\ \text { N } & -3.625 & -0.190 \\ \text { N } & -4.352 & -1.043 \\ \text { N } & 2.830 & -0.252 \\ \text { O } & 3.569 & 0.708 \\ \text { O } & 3.198 & -1.406\end{array}$

C

C

C

C

$\mathrm{H}$

C

$\mathrm{N}$

$\mathrm{H}$

$\mathrm{H}$

C

$\mathrm{H}$

C

O

$\mathrm{H}$

$\mathrm{H}$

C

C

$\mathrm{H}$

$\mathrm{H}$

$\mathrm{H}$

$\mathrm{H}$

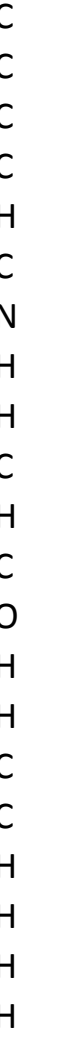

$\begin{array}{rrr}4.433 & -0.221 & -0.225 \\ 3.490 & -1.230 & -0.304 \\ 3.955 & 1.065 & 0.008 \\ 2.144 & -0.978 & -0.158 \\ 3.783 & -2.255 & -0.481 \\ 2.615 & 1.295 & 0.147 \\ 1.677 & 0.292 & 0.068 \\ 1.381 & -1.736 & -0.200 \\ 2.214 & 2.281 & 0.325 \\ 0.354 & 0.623 & 0.206 \\ 0.195 & 1.667 & 0.394 \\ -0.707 & -0.340 & 0.247 \\ -0.522 & -1.566 & 0.259 \\ 5.489 & -0.419 & -0.336 \\ 4.624 & 1.911 & 0.084 \\ -2.403 & 1.520 & 0.219 \\ -3.588 & 1.749 & -0.714 \\ -1.569 & 2.101 & -0.163 \\ -2.644 & 1.905 & 1.218 \\ -3.822 & 2.813 & -0.755 \\ -3.350 & 1.405 & -1.721\end{array}$




$\begin{array}{lrrr}\mathrm{H} & -4.481 & 1.225 & -0.375 \\ \mathrm{C} & -3.030 & -0.845 & 0.657 \\ \mathrm{C} & -3.567 & -1.632 & -0.532 \\ \mathrm{H} & -3.836 & -0.305 & 1.159 \\ \mathrm{H} & -2.598 & -1.540 & 1.377 \\ \mathrm{H} & -4.317 & -2.353 & -0.203 \\ \mathrm{H} & -4.026 & -0.974 & -1.271 \\ \mathrm{H} & -2.748 & -2.171 & -1.004 \\ \mathrm{~N} & -2.006 & 0.128 & 0.299\end{array}$

\section{CX16}

$\begin{array}{lrrr}\mathrm{C} & -3.941 & 0.256 & 0.000 \\ \mathrm{C} & -2.975 & 1.248 & 0.000 \\ \mathrm{C} & -3.492 & -1.060 & 0.000 \\ \mathrm{C} & -1.632 & 0.946 & 0.000 \\ \mathrm{H} & -3.249 & 2.294 & 0.000 \\ \mathrm{C} & -2.152 & -1.339 & 0.000 \\ \mathrm{~N} & -1.197 & -0.353 & 0.000 \\ \mathrm{H} & -0.850 & 1.687 & 0.000 \\ \mathrm{H} & -1.768 & -2.347 & 0.001 \\ \mathrm{C} & 0.124 & -0.715 & 0.000 \\ \mathrm{H} & 0.324 & -1.771 & 0.000 \\ \mathrm{C} & 1.198 & 0.210 & 0.000 \\ \mathrm{O} & 1.150 & 1.432 & 0.000 \\ \mathrm{H} & -4.995 & 0.491 & 0.000 \\ \mathrm{H} & -4.182 & -1.892 & 0.000 \\ \mathrm{O} & 2.388 & -0.449 & 0.001 \\ \mathrm{C} & 3.547 & 0.379 & 0.000 \\ \mathrm{C} & 4.752 & -0.531 & -0.001 \\ \mathrm{H} & 3.530 & 1.025 & 0.880 \\ \mathrm{H} & 3.528 & 1.026 & -0.879 \\ \mathrm{H} & 5.667 & 0.061 & -0.002 \\ \mathrm{H} & 4.753 & -1.169 & 0.883\end{array}$

$\begin{array}{llll}\text { C } & -3.506 & -0.534 & 0.000 \\ H & -4.054 & -0.194 & 0.879 \\ H & -3.461 & -1.622 & 0.000 \\ H & -4.054 & -0.195 & -0.880\end{array}$

CX18

$\begin{array}{lrrr}\text { C } & -4.651 & 0.006 & -0.028 \\ \text { C } & -3.819 & -1.076 & 0.199 \\ \text { C } & -4.045 & 1.236 & -0.267 \\ \text { C } & -2.447 & -0.942 & 0.189 \\ \text { H } & -4.223 & -2.060 & 0.391 \\ \text { C } & -2.681 & 1.345 & -0.271 \\ \text { N } & -1.862 & 0.271 & -0.044 \\ \text { H } & -1.754 & -1.750 & 0.354 \\ \text { H } & -2.173 & 2.279 & -0.450 \\ \text { C } & -0.494 & 0.463 & -0.060 \\ \text { H } & -0.196 & 1.473 & -0.276 \\ \text { C } & 0.456 & -0.567 & 0.100 \\ \text { O } & 0.200 & -1.771 & 0.250 \\ \text { C } & 1.902 & -0.145 & 0.047 \\ \text { C } & 2.348 & 1.130 & 0.388 \\ \text { C } & 2.834 & -1.105 & -0.338 \\ \text { C } & 3.698 & 1.443 & 0.329 \\ \text { H } & 1.646 & 1.878 & 0.733 \\ \text { C } & 4.182 & -0.791 & -0.407 \\ \text { H } & 2.475 & -2.098 & -0.574 \\ \text { C } & 4.618 & 0.485 & -0.075 \\ \text { H } & 4.034 & 2.433 & 0.609 \\ \text { H } & 4.896 & -1.544 & -0.716 \\ \text { H } & 5.671 & 0.731 & -0.123 \\ \text { H } & -5.726 & -0.097 & -0.022 \\ \text { H } & -4.629 & 2.126 & -0.453\end{array}$

\section{CX17}

$\begin{array}{lrrr}\text { C } & 2.997 & 0.345 & 0.000 \\ \text { C } & 2.002 & 1.305 & 0.000 \\ \text { C } & 2.597 & -0.988 & 0.000 \\ \text { C } & 0.668 & 0.957 & 0.000 \\ \text { H } & 2.242 & 2.359 & 0.000 \\ \text { C } & 1.267 & -1.309 & 0.000 \\ \text { N } & 0.285 & -0.355 & 0.000 \\ \text { H } & -0.150 & 1.661 & 0.000 \\ \text { H } & 0.915 & -2.329 & 0.000 \\ \text { C } & -1.038 & -0.754 & 0.000 \\ \text { H } & -1.178 & -1.821 & -0.001 \\ \text { C } & -2.141 & 0.127 & 0.000 \\ \text { O } & -2.086 & 1.364 & 0.000 \\ \text { H } & 4.043 & 0.616 & 0.000 \\ \text { H } & 3.317 & -1.793 & 0.000\end{array}$

CX19

$\begin{array}{lrrr}\text { C } & 3.878 & 0.993 & 0.090 \\ \text { C } & 2.872 & 1.935 & 0.214 \\ \text { C } & 3.472 & -0.327 & -0.058 \\ \text { C } & 1.540 & 1.586 & 0.192 \\ \text { H } & 3.113 & 2.982 & 0.332 \\ \text { C } & 2.145 & -0.666 & -0.078 \\ \text { N } & 1.162 & 0.278 & 0.047 \\ \text { H } & 0.724 & 2.284 & 0.279 \\ \text { H } & 1.816 & -1.686 & -0.191 \\ \text { C } & -0.152 & -0.132 & 0.021 \\ \text { H } & -0.280 & -1.190 & -0.118 \\ \text { C } & -1.263 & 0.742 & 0.082 \\ \text { O } & -1.203 & 1.977 & 0.139 \\ \text { C } & -2.619 & 0.089 & 0.036 \\ \text { C } & -2.863 & -1.213 & 0.467 \\ \text { C } & -3.681 & 0.855 & -0.440\end{array}$




$\begin{array}{lrrr}\mathrm{C} & -4.144 & -1.744 & 0.409 \\ \mathrm{H} & -2.062 & -1.810 & 0.882 \\ \mathrm{C} & -4.957 & 0.321 & -0.509 \\ \mathrm{H} & -3.479 & 1.872 & -0.747 \\ \mathrm{C} & -5.192 & -0.981 & -0.086 \\ \mathrm{H} & -4.324 & -2.752 & 0.758 \\ \mathrm{H} & -5.773 & 0.922 & -0.889 \\ \mathrm{H} & -6.190 & -1.397 & -0.134 \\ \mathrm{Cl} & 4.636 & -1.589 & -0.221 \\ \mathrm{H} & 4.925 & 1.254 & 0.105\end{array}$

cx20

$\begin{array}{lrrr}\text { C } & -3.437 & 0.079 & -0.012 \\ \text { C } & -2.582 & -1.019 & 0.168 \\ \text { C } & -2.794 & 1.321 & -0.200 \\ \text { C } & -1.216 & -0.879 & 0.161 \\ \text { H } & -2.970 & -2.014 & 0.317 \\ \text { C } & -1.432 & 1.406 & -0.198 \\ \text { H } & -3.353 & 2.231 & -0.349 \\ \text { N } & -0.619 & 0.331 & -0.020 \\ \text { H } & -0.525 & -1.699 & 0.289 \\ \text { H } & -0.926 & 2.348 & -0.339 \\ \mathrm{C} & 0.771 & 0.512 & -0.027 \\ \text { H } & 1.074 & 1.529 & -0.198 \\ \mathrm{C} & 1.689 & -0.534 & 0.084 \\ \mathrm{O} & 1.412 & -1.748 & 0.185 \\ \mathrm{C} & 3.146 & -0.144 & 0.042 \\ \mathrm{C} & 3.626 & 1.107 & 0.423 \\ \mathrm{C} & 4.055 & -1.112 & -0.379 \\ \mathrm{C} & 4.983 & 1.391 & 0.365 \\ \mathrm{H} & 2.943 & 1.858 & 0.799 \\ \mathrm{C} & 5.410 & -0.827 & -0.445 \\ \mathrm{H} & 3.670 & -2.088 & -0.643 \\ \mathrm{C} & 5.879 & 0.427 & -0.076 \\ \mathrm{H} & 5.343 & 2.364 & 0.675 \\ \mathrm{H} & 6.105 & -1.587 & -0.782 \\ \mathrm{H} & 6.938 & 0.648 & -0.121 \\ \mathrm{C} & -5.395 & -1.341 & 0.176 \\ \mathrm{H} & -5.111 & -1.781 & 1.136 \\ \mathrm{H} & -5.101 & -2.034 & -0.619 \\ \mathrm{C} & -5.618 & 1.130 & -0.217 \\ \mathrm{H} & -5.428 & 1.586 & -1.193 \\ \mathrm{H} & -5.446 & 1.885 & 0.555 \\ \mathrm{H} & -6.476 & -1.239 & 0.155 \\ \mathrm{H} & -6.664 & 0.838 & -0.177 \\ \mathrm{~N} & -4.792 & -0.039 & -0.006\end{array}$

$\begin{array}{lrrr}\text { C } & -3.386 & 0.661 & -0.084 \\ \text { C } & -2.926 & -0.664 & 0.097 \\ \text { C } & -3.871 & -1.711 & 0.250 \\ \text { C } & -5.207 & -1.432 & 0.221 \\ \text { H } & -2.687 & 2.693 & -0.372 \\ \text { H } & -6.729 & 0.090 & 0.019 \\ \text { H } & -5.115 & 1.936 & -0.250 \\ \mathrm{C} & -2.397 & 1.660 & -0.231 \\ \mathrm{C} & -1.551 & -0.910 & 0.122 \\ \mathrm{H} & -3.519 & -2.726 & 0.390 \\ \mathrm{H} & -5.927 & -2.232 & 0.338 \\ \mathrm{C} & -1.075 & 1.369 & -0.200 \\ \mathrm{H} & -1.164 & -1.910 & 0.258 \\ \mathrm{H} & -0.280 & 2.091 & -0.298 \\ \mathrm{~N} & -0.631 & 0.060 & -0.022 \\ \mathrm{C} & 0.714 & -0.249 & 0.001 \\ \mathrm{C} & 1.770 & 0.688 & -0.067 \\ \mathrm{O} & 1.657 & 1.921 & -0.124 \\ \mathrm{C} & 3.159 & 0.103 & -0.030 \\ \mathrm{C} & 4.181 & 0.915 & 0.455 \\ \mathrm{C} & 3.471 & -1.178 & -0.481 \\ \mathrm{C} & 5.484 & 0.448 & 0.515 \\ \mathrm{H} & 3.927 & 1.917 & 0.776 \\ \mathrm{C} & 4.778 & -1.642 & -0.432 \\ \mathrm{H} & 2.701 & -1.808 & -0.905 \\ \mathrm{C} & 5.786 & -0.834 & 0.073 \\ \mathrm{H} & 6.269 & 1.085 & 0.903 \\ \mathrm{H} & 5.010 & -2.635 & -0.797 \\ \mathrm{H} & 6.805 & -1.198 & 0.114 \\ \mathrm{H} & 0.916 & -1.295 & 0.146\end{array}$

\section{CX22}

$\begin{array}{lrrr}\text { CX21 } & & & \\ \text { C } & -5.666 & -0.107 & 0.039 \\ \text { C } & -4.773 & 0.918 & -0.110\end{array}$

$\begin{array}{rrr}0.287 & -0.115 & 0.008 \\ 1.450 & -0.904 & -0.132 \\ 1.490 & -2.134 & -0.291 \\ 2.757 & -0.156 & -0.052 \\ 2.905 & 1.182 & -0.412 \\ 3.874 & -0.866 & 0.380 \\ 4.143 & 1.802 & -0.325 \\ 2.059 & 1.737 & -0.797 \\ 5.109 & -0.245 & 0.477 \\ 3.750 & -1.911 & 0.629 \\ 5.247 & 1.092 & 0.127 \\ 4.248 & 2.838 & -0.620 \\ 5.969 & -0.805 & 0.822 \\ 6.212 & 1.577 & 0.196 \\ -2.102 & 0.285 & 0.014 \\ -1.255 & -1.926 & -0.113 \\ -1.921 & 1.681 & -0.019 \\ -3.415 & -0.234 & 0.074 \\ -2.548 & -2.447 & -0.094\end{array}$




$\begin{array}{rrrrrrrr}\text { H } & -0.372 & -2.544 & -0.168 & \mathrm{C} & 1.865 & -0.200 & -0.085 \\ \mathrm{C} & -2.999 & 2.524 & 0.054 & \mathrm{H} & 2.035 & 0.873 & -0.117 \\ \mathrm{H} & -0.940 & 2.112 & -0.130 & \mathrm{H} & 2.716 & -0.859 & -0.186 \\ \mathrm{C} & -4.501 & 0.667 & 0.160 & \mathrm{~N} & 0.732 & -0.753 & 0.085 \\ \mathrm{C} & -3.631 & -1.629 & 0.024 & \mathrm{~N} & -0.383 & -0.016 & 0.279 \\ \mathrm{H} & -2.652 & -3.521 & -0.155 & \mathrm{C} & -0.354 & 1.394 & -0.031 \\ \mathrm{C} & -4.305 & 2.017 & 0.160 & \mathrm{H} & -0.033 & 1.578 & -1.066 \\ \mathrm{H} & -2.836 & 3.592 & 0.018 & \mathrm{H} & -1.354 & 1.799 & 0.109 \\ \mathrm{H} & -5.500 & 0.251 & 0.218 & \mathrm{H} & 0.324 & 1.922 & 0.641 \\ \mathrm{H} & -4.640 & -2.016 & 0.066 & \mathrm{C} & -1.585 & -0.726 & -0.107 \\ \mathrm{H} & -5.146 & 2.695 & 0.224 & \mathrm{H} & -2.448 & -0.272 & 0.379 \\ \mathrm{~N} & -1.006 & -0.603 & -0.031 & \mathrm{H} & -1.743 & -0.713 & -1.193 \\ \mathrm{H} & 0.389 & 0.919 & 0.259 & \mathrm{H} & -1.489 & -1.758 & 0.219\end{array}$

\section{CX23}

$\begin{array}{lrrr}\text { S } & 0.000 & 0.087 & 0.156 \\ \text { O } & 0.001 & -0.480 & 1.502 \\ \mathrm{C} & 1.406 & -0.616 & -0.678 \\ \mathrm{H} & 1.461 & -1.667 & -0.403 \\ \mathrm{H} & 1.320 & -0.470 & -1.750 \\ \mathrm{H} & 2.277 & -0.090 & -0.290 \\ \mathrm{C} & -1.406 & -0.618 & -0.677 \\ \mathrm{H} & -1.460 & -1.668 & -0.402 \\ \mathrm{H} & -2.277 & -0.091 & -0.291 \\ \mathrm{H} & -1.320 & -0.473 & -1.750 \\ \mathrm{C} & -0.001 & 1.661 & -0.244 \\ \mathrm{H} & 0.925 & 2.174 & -0.019 \\ \mathrm{H} & -0.927 & 2.172 & -0.019\end{array}$

\section{CX24}

$\begin{array}{lrrr}\mathrm{C} & 2.535 & 0.261 & -0.171 \\ \mathrm{H} & 2.371 & 1.335 & -0.125 \\ \mathrm{H} & 3.532 & -0.107 & -0.369 \\ \mathrm{~N} & 1.625 & -0.612 & 0.022 \\ \mathrm{C} & -0.111 & 1.111 & 0.034 \\ \mathrm{C} & -0.678 & -1.241 & 0.182 \\ \mathrm{C} & -1.625 & 0.954 & 0.131 \\ \mathrm{H} & 0.202 & 1.436 & -0.970 \\ \mathrm{H} & 0.289 & 1.827 & 0.755 \\ \mathrm{C} & -1.857 & -0.463 & -0.401 \\ \mathrm{H} & -0.924 & -1.647 & 1.168 \\ \mathrm{H} & -0.340 & -2.059 & -0.451 \\ \mathrm{H} & -1.937 & 1.018 & 1.175 \\ \mathrm{H} & -2.159 & 1.719 & -0.431 \\ \mathrm{H} & -2.821 & -0.877 & -0.114 \\ \mathrm{H} & -1.796 & -0.460 & -1.491 \\ \mathrm{~N} & 0.376 & -0.234 & 0.292\end{array}$

\section{NHC1}

$\begin{array}{lrrr}\text { C } & 0.000 & 0.000 & -0.242 \\ \text { C } & -0.757 & 0.089 & 2.005 \\ \text { C } & 0.757 & -0.089 & 2.004 \\ \text { H } & -1.063 & 1.082 & 2.348 \\ \text { H } & 1.278 & 0.657 & 2.604 \\ \text { C } & 2.417 & 0.002 & 0.121 \\ \text { C } & 3.248 & 1.106 & 0.306 \\ \text { C } & 2.895 & -1.170 & -0.474 \\ \text { C } & 4.573 & 1.020 & -0.115 \\ \text { C } & 4.220 & -1.211 & -0.883 \\ \text { C } & 5.075 & -0.127 & -0.711 \\ \text { H } & 5.221 & 1.879 & 0.019 \\ \text { H } & 4.598 & -2.117 & -1.346 \\ \text { C } & -2.417 & -0.002 & 0.121 \\ \text { C } & -2.895 & 1.170 & -0.474 \\ \text { C } & -3.248 & -1.106 & 0.306 \\ \text { C } & -4.220 & 1.211 & -0.883 \\ \text { C } & -4.573 & -1.020 & -0.115 \\ \text { C } & -5.075 & 0.126 & -0.711 \\ \text { H } & -4.599 & 2.117 & -1.346 \\ \text { H } & -5.221 & -1.879 & 0.019 \\ \text { C } & -2.732 & -2.372 & 0.932 \\ \text { H } & -1.691 & -2.547 & 0.658 \\ \text { H } & -2.787 & -2.328 & 2.023 \\ \text { H } & -3.326 & -3.226 & 0.611 \\ \text { C } & -1.990 & 2.353 & -0.674 \\ \text { H } & -1.456 & 2.602 & 0.246 \\ \text { H } & -1.233 & 2.133 & -1.427 \\ \text { H } & -2.563 & 3.225 & -0.988 \\ \text { C } & 2.732 & 2.372 & 0.932 \\ \text { H } & 1.692 & 2.547 & 0.658 \\ & & & \end{array}$




$\begin{array}{lrrr}\mathrm{H} & 2.787 & 2.328 & 2.023 \\ \mathrm{H} & 3.327 & 3.226 & 0.611 \\ \mathrm{C} & 1.990 & -2.353 & -0.674 \\ \mathrm{H} & 1.456 & -2.601 & 0.246 \\ \mathrm{H} & 1.232 & -2.133 & -1.427 \\ \mathrm{H} & 2.562 & -3.225 & -0.988 \\ \mathrm{C} & -6.501 & 0.198 & -1.186 \\ \mathrm{H} & -6.974 & 1.127 & -0.867 \\ \mathrm{H} & -6.547 & 0.166 & -2.277 \\ \mathrm{H} & -7.088 & -0.635 & -0.801 \\ \mathrm{C} & 6.501 & -0.198 & -1.186 \\ \mathrm{H} & 6.547 & -0.167 & -2.277 \\ \mathrm{H} & 7.088 & 0.635 & -0.802 \\ \mathrm{H} & 6.974 & -1.127 & -0.866 \\ \mathrm{~N} & -1.068 & -0.061 & 0.571 \\ \mathrm{~N} & 1.068 & 0.061 & 0.571 \\ \mathrm{H} & -1.278 & -0.656 & 2.604 \\ \mathrm{H} & 1.063 & -1.082 & 2.348\end{array}$

$\begin{array}{lrrr}\mathrm{H} & 2.279 & 2.832 & 0.959 \\ \mathrm{H} & 3.168 & 3.311 & -0.489 \\ \mathrm{C} & 2.151 & -2.446 & 0.348 \\ \mathrm{H} & 1.637 & -2.353 & 1.306 \\ \mathrm{H} & 1.380 & -2.570 & -0.414 \\ \mathrm{H} & 2.775 & -3.338 & 0.370 \\ \mathrm{C} & -6.510 & -0.344 & -1.051 \\ \mathrm{H} & -7.087 & 0.548 & -0.809 \\ \mathrm{H} & -6.566 & -0.493 & -2.131 \\ \mathrm{H} & -6.985 & -1.202 & -0.575 \\ \mathrm{C} & 6.509 & -0.348 & -1.052 \\ \mathrm{H} & 6.566 & -0.492 & -2.133 \\ \mathrm{H} & 7.088 & 0.542 & -0.805 \\ \mathrm{H} & 6.983 & -1.209 & -0.580 \\ \mathrm{~N} & -1.055 & 0.180 & 0.549 \\ \mathrm{~N} & 1.055 & 0.179 & 0.549\end{array}$

NHC3

$\begin{array}{lrrr}\text { NHC2 } & & & \\ \text { C } & 0.000 & -0.083 & -0.270 \\ \text { C } & -0.674 & 0.581 & 1.820 \\ \text { C } & 0.674 & 0.581 & 1.820 \\ \text { H } & -1.386 & 0.816 & 2.592 \\ \text { H } & 1.386 & 0.815 & 2.592 \\ \text { C } & 2.420 & 0.047 & 0.144 \\ \text { C } & 3.153 & 1.196 & -0.143 \\ \text { C } & 2.977 & -1.227 & 0.056 \\ \text { C } & 4.484 & 1.046 & -0.516 \\ \text { C } & 4.311 & -1.329 & -0.323 \\ \text { C } & 5.078 & -0.206 & -0.611 \\ \text { H } & 5.067 & 1.931 & -0.744 \\ \text { H } & 4.761 & -2.313 & -0.393 \\ \text { C } & -2.420 & 0.049 & 0.144 \\ \text { C } & -3.148 & 1.197 & -0.156 \\ \text { C } & -2.982 & -1.224 & 0.070 \\ \text { C } & -4.479 & 1.048 & -0.529 \\ \text { C } & -4.315 & -1.326 & -0.309 \\ \text { C } & -5.078 & -0.203 & -0.611 \\ \text { H } & -5.059 & 1.933 & -0.768 \\ \text { H } & -4.770 & -2.309 & -0.368 \\ \text { C } & -2.160 & -2.442 & 0.376 \\ \text { H } & -1.384 & -2.573 & -0.380 \\ \text { H } & -1.652 & -2.343 & 1.338 \\ \text { H } & -2.785 & -3.333 & 0.401 \\ \text { C } & -2.499 & 2.551 & -0.100 \\ \text { H } & -2.255 & 2.836 & 0.926 \\ \text { H } & -1.565 & 2.550 & -0.663 \\ \text { H } & -3.159 & 3.311 & -0.515 \\ \text { C } & 2.510 & 2.552 & -0.071 \\ \text { H } & 1.568 & 2.558 & -0.623\end{array}$

$\begin{array}{lrrr}\text { C } & 0.860 & 0.867 & 0.000 \\ \mathrm{C} & 1.791 & -1.218 & 0.000 \\ \mathrm{C} & 0.453 & -1.378 & 0.000 \\ \mathrm{H} & 2.587 & -1.943 & 0.000 \\ \mathrm{H} & -0.137 & -2.278 & 0.000 \\ \mathrm{~N} & 2.011 & 0.144 & 0.000 \\ \mathrm{~N} & -0.091 & -0.104 & 0.000 \\ \mathrm{C} & 3.325 & 0.749 & 0.000 \\ \mathrm{H} & 3.884 & 0.453 & -0.888 \\ \mathrm{H} & 3.188 & 1.827 & 0.000 \\ \mathrm{H} & 3.884 & 0.454 & 0.889 \\ \mathrm{C} & -1.547 & 0.144 & 0.000 \\ \mathrm{C} & -2.150 & -0.486 & -1.256 \\ \mathrm{H} & -1.996 & -1.566 & -1.277 \\ \mathrm{H} & -3.224 & -0.297 & -1.287 \\ \mathrm{H} & -1.694 & -0.054 & -2.148 \\ \mathrm{C} & -2.150 & -0.486 & 1.256 \\ \mathrm{H} & -1.694 & -0.056 & 2.148 \\ \mathrm{H} & -3.224 & -0.298 & 1.286 \\ \mathrm{H} & -1.996 & -1.566 & 1.276 \\ \mathrm{C} & -1.811 & 1.643 & 0.000 \\ \mathrm{H} & -1.375 & 2.117 & -0.878 \\ \mathrm{H} & -2.890 & 1.804 & 0.000 \\ \mathrm{H} & -1.375 & 2.116 & 0.878\end{array}$

$\mathrm{NCH} 4$

$\begin{array}{lrrr}\text { C } & 2.322 & 0.806 & -0.263 \\ \text { C } & 3.406 & -1.126 & 0.295 \\ \text { C } & 2.087 & -1.371 & 0.412 \\ \text { H } & 4.257 & -1.760 & 0.473 \\ \text { H } & 1.564 & -2.252 & 0.736 \\ \text { C } & 0.036 & -0.022 & 0.053\end{array}$




$\begin{array}{lrrr}\text { C } & -0.801 & -1.090 & -0.214 \\ \text { C } & -0.512 & 1.235 & 0.303 \\ \text { C } & -2.184 & -0.925 & -0.218 \\ \text { C } & -1.879 & 1.407 & 0.289 \\ \text { C } & -2.727 & 0.328 & 0.033 \\ \text { H } & -2.813 & -1.776 & -0.432 \\ \text { H } & -2.321 & 2.375 & 0.481 \\ \text { N } & 3.518 & 0.190 & -0.113 \\ \text { N } & 1.447 & -0.190 & 0.070 \\ \text { C } & 4.783 & 0.848 & -0.364 \\ \text { H } & 5.324 & 0.343 & -1.165 \\ \text { H } & 4.567 & 1.870 & -0.660 \\ \text { H } & 5.397 & 0.851 & 0.537 \\ \text { H } & -0.385 & -2.061 & -0.446 \\ \text { H } & 0.155 & 2.063 & 0.498 \\ \text { O } & -4.057 & 0.599 & 0.050 \\ \text { C } & -4.943 & -0.465 & -0.208 \\ \text { H } & -5.945 & -0.049 & -0.148 \\ \text { H } & -4.783 & -0.881 & -1.207 \\ \text { H } & -4.835 & -1.260 & 0.535\end{array}$

$\begin{array}{lrrr}\text { NHC5 } & & & \\ \text { C } & 1.743 & 0.162 & -0.857 \\ \text { C } & 2.854 & -0.374 & 1.072 \\ \text { C } & 1.539 & -0.397 & 1.360 \\ \text { H } & 3.714 & -0.565 & 1.690 \\ \text { H } & 1.012 & -0.616 & 2.272 \\ \text { C } & -0.521 & 0.011 & 0.059 \\ \text { C } & -1.275 & -1.158 & -0.042 \\ \text { C } & -1.149 & 1.257 & 0.025 \\ \text { C } & -2.659 & -1.085 & -0.189 \\ \text { C } & -2.533 & 1.333 & -0.130 \\ \text { C } & -3.264 & 0.161 & -0.236 \\ \text { H } & -3.258 & -1.979 & -0.274 \\ \text { H } & -3.036 & 2.287 & -0.166 \\ \text { N } & 2.948 & -0.036 & -0.267 \\ \text { N } & 0.888 & -0.069 & 0.183 \\ \text { H } & -4.338 & 0.220 & -0.356 \\ \text { O } & -0.577 & -2.312 & 0.015 \\ \text { O } & -0.343 & 2.328 & 0.160 \\ \text { C } & -1.286 & -3.520 & -0.144 \\ \text { H } & -2.019 & -3.658 & 0.655 \\ \text { H } & -1.793 & -3.554 & -1.112 \\ \text { H } & -0.545 & -4.313 & -0.096 \\ \text { C } & -0.921 & 3.609 & 0.052 \\ \text { H } & -1.404 & 3.741 & -0.920 \\ \text { H } & -1.651 & 3.784 & 0.847 \\ \text { H } & -0.103 & 4.317 & 0.149 \\ \text { C } & 4.203 & 0.094 & -0.975 \\ \text { H } & 4.755 & -0.846 & -0.951 \\ \text { H } & 3.972 & 0.354 & -2.004\end{array}$

\section{CA1}

$\begin{array}{lrrr}\mathrm{C} & 0.000 & -1.391 & 0.000 \\ \mathrm{~N} & 1.140 & -0.644 & 0.000 \\ \mathrm{C} & 1.233 & 0.777 & 0.000 \\ \mathrm{C} & 0.000 & 1.453 & 0.000 \\ \mathrm{C} & -1.233 & 0.777 & 0.000 \\ \mathrm{~N} & -1.140 & -0.644 & 0.000 \\ \mathrm{H} & 0.000 & 2.531 & 0.000 \\ \mathrm{O} & 2.372 & 1.251 & 0.000 \\ \mathrm{O} & -2.372 & 1.251 & 0.000 \\ \mathrm{O} & 0.000 & -2.617 & 0.000 \\ \mathrm{H} & 2.014 & -1.146 & 0.000 \\ \mathrm{H} & -2.014 & -1.146 & 0.000\end{array}$

\section{CA2}

$\begin{array}{lrrr}\mathrm{C} & 0.989 & 0.000 & 0.000 \\ \mathrm{~N} & 0.263 & 1.130 & 0.000 \\ \mathrm{C} & -1.164 & 1.232 & 0.000 \\ \mathrm{C} & -1.839 & 0.000 & 0.000 \\ \mathrm{C} & -1.164 & -1.232 & 0.000 \\ \mathrm{~N} & 0.263 & -1.130 & 0.000 \\ \mathrm{H} & -2.917 & 0.000 & 0.000 \\ \mathrm{O} & -1.626 & 2.372 & 0.000 \\ \mathrm{O} & -1.626 & -2.372 & 0.000 \\ \mathrm{H} & 0.766 & 2.003 & 0.000 \\ \mathrm{H} & 0.766 & -2.003 & 0.000 \\ \mathrm{~S} & 2.675 & 0.000 & 0.000\end{array}$

\section{CA3}

$\begin{array}{lrrr}\mathrm{C} & 0.000 & -1.076 & 0.000 \\ \mathrm{~N} & 1.168 & -0.361 & 0.000 \\ \mathrm{C} & 1.238 & 1.070 & 0.000 \\ \mathrm{C} & 0.000 & 1.730 & 0.000 \\ \mathrm{C} & -1.238 & 1.070 & 0.000 \\ \mathrm{~N} & -1.168 & -0.361 & 0.000 \\ \mathrm{H} & 0.000 & 2.808 & 0.000 \\ \mathrm{O} & 2.357 & 1.591 & 0.000 \\ \mathrm{O} & -2.357 & 1.591 & 0.000 \\ \mathrm{O} & 0.000 & -2.305 & 0.000 \\ \mathrm{C} & 2.393 & -1.133 & 0.000 \\ \mathrm{H} & 2.440 & -1.774 & -0.881 \\ \mathrm{H} & 3.214 & -0.423 & 0.001 \\ \mathrm{H} & 2.439 & -1.775 & 0.881 \\ \mathrm{C} & -2.393 & -1.133 & 0.000 \\ \mathrm{H} & -2.439 & -1.775 & -0.880 \\ \mathrm{H} & -2.439 & -1.774 & 0.881 \\ \mathrm{H} & -3.214 & -0.423 & 0.000\end{array}$




\section{CA4}

$\begin{array}{lrrr}\text { C } & -1.063 & -1.247 & -0.099 \\ \text { C } & -1.706 & 0.000 & 0.046 \\ \text { C } & -1.063 & 1.247 & -0.099 \\ \text { H } & -2.763 & 0.000 & 0.286 \\ \text { O } & -1.606 & -2.358 & 0.015 \\ \text { O } & -1.605 & 2.358 & 0.015 \\ \mathrm{C} & 0.428 & 1.237 & -0.460 \\ \text { H } & 0.866 & 2.152 & -0.051 \\ \text { H } & 0.493 & 1.311 & -1.553 \\ \mathrm{C} & 0.427 & -1.237 & -0.460 \\ \mathrm{H} & 0.493 & -1.311 & -1.553 \\ \mathrm{H} & 0.866 & -2.152 & -0.051 \\ \mathrm{C} & 1.195 & 0.000 & 0.005 \\ \mathrm{C} & 1.317 & 0.000 & 1.531 \\ \mathrm{H} & 1.859 & -0.886 & 1.872 \\ \mathrm{H} & 1.860 & 0.886 & 1.872 \\ \mathrm{H} & 0.330 & 0.000 & 1.995 \\ \mathrm{C} & 2.600 & 0.000 & -0.597 \\ \mathrm{H} & 3.160 & 0.886 & -0.280 \\ \mathrm{H} & 3.159 & -0.886 & -0.280 \\ \mathrm{H} & 2.555 & 0.000 & -1.689\end{array}$

$\begin{array}{lrrr}\mathrm{C} & 3.078 & 1.252 & 0.000 \\ \mathrm{H} & 1.000 & 1.787 & 0.000 \\ \mathrm{C} & 3.576 & -1.081 & 0.000 \\ \mathrm{H} & 1.897 & -2.400 & 0.000 \\ \mathrm{C} & 4.032 & 0.236 & 0.000 \\ \mathrm{H} & 3.403 & 2.288 & 0.000 \\ \mathrm{H} & 4.290 & -1.899 & 0.000 \\ \mathrm{H} & 5.091 & 0.462 & 0.000 \\ \mathrm{C} & -1.214 & 0.198 & 0.000 \\ \mathrm{O} & -1.231 & 1.431 & 0.000 \\ \mathrm{O} & -2.453 & -0.473 & 0.000 \\ \mathrm{C} & -3.581 & 0.366 & 0.000 \\ \mathrm{H} & -3.571 & 1.021 & -0.876 \\ \mathrm{H} & -3.571 & 1.020 & 0.878 \\ \mathrm{C} & -4.808 & -0.523 & 0.000 \\ \mathrm{H} & -5.720 & 0.078 & 0.000 \\ \mathrm{H} & -4.814 & -1.164 & -0.884 \\ \mathrm{H} & -4.814 & -1.165 & 0.882\end{array}$

\section{CA5}

$\begin{array}{rrrr}\text { C } & -0.971 & -1.238 & -0.090 \\ \text { C } & -1.605 & 0.000 & 0.134 \\ \text { C } & -0.970 & 1.238 & -0.089 \\ \text { H } & -2.661 & 0.000 & 0.344 \\ \text { O } & -1.455 & -2.356 & -0.085 \\ \text { O } & -1.454 & 2.356 & -0.085 \\ \text { C } & 1.078 & 0.000 & 0.005 \\ \text { C } & 1.306 & -0.001 & 1.517 \\ \text { H } & 1.867 & -0.892 & 1.801 \\ \text { H } & 1.866 & 0.890 & 1.802 \\ \text { H } & 0.348 & -0.002 & 2.035 \\ \text { C } & 2.392 & 0.000 & -0.752 \\ \text { H } & 2.965 & 0.892 & -0.497 \\ \text { H } & 2.965 & -0.891 & -0.499 \\ \text { H } & 2.181 & 0.001 & -1.821 \\ \text { O } & 0.398 & 1.170 & -0.384 \\ \text { O } & 0.398 & -1.170 & -0.385\end{array}$

CA7

$\begin{array}{lrrr}\text { C } & 1.851 & 0.780 & 0.000 \\ \text { H } & 2.171 & 1.813 & 0.000 \\ \mathrm{C} & 0.459 & 0.509 & 0.000 \\ \mathrm{C} & -0.108 & -0.794 & 0.000 \\ \mathrm{C} & -0.473 & 1.579 & 0.000 \\ \mathrm{C} & -1.476 & -0.994 & 0.000 \\ \mathrm{H} & 0.562 & -1.642 & 0.000 \\ \mathrm{C} & -1.840 & 1.382 & 0.000 \\ \mathrm{H} & -0.092 & 2.594 & 0.000 \\ \mathrm{C} & -2.343 & 0.089 & 0.000 \\ \mathrm{H} & -1.874 & -2.001 & 0.000 \\ \mathrm{H} & -2.516 & 2.228 & 0.000 \\ \mathrm{C} & 2.862 & -0.190 & 0.000 \\ \mathrm{O} & 2.793 & -1.419 & 0.000 \\ \mathrm{O} & 4.137 & 0.398 & 0.000 \\ \mathrm{C} & 5.210 & -0.511 & 0.000 \\ \mathrm{H} & 5.158 & -1.163 & -0.877 \\ \mathrm{H} & 5.157 & -1.163 & 0.877 \\ \mathrm{C} & 6.491 & 0.299 & 0.000 \\ \mathrm{H} & 7.362 & -0.360 & 0.000 \\ \mathrm{H} & 6.537 & 0.938 & -0.883 \\ \mathrm{H} & 6.537 & 0.938 & 0.883 \\ \mathrm{Br} & -4.236 & -0.197 & 0.000\end{array}$

\section{CA6}

\section{CA8}

$\begin{array}{lrrr}\mathrm{C} & -0.147 & -0.704 & 0.000 \\ \mathrm{H} & -0.402 & -1.755 & 0.000 \\ \mathrm{C} & 1.232 & -0.351 & 0.000 \\ \mathrm{C} & 1.721 & 0.982 & 0.000 \\ \mathrm{C} & 2.224 & -1.365 & 0.000\end{array}$

$\begin{array}{lrrr}\mathrm{C} & 0.908 & 0.746 & 0.000 \\ \mathrm{H} & 1.209 & 1.784 & 0.000 \\ \mathrm{C} & -0.466 & 0.450 & 0.000 \\ \mathrm{C} & -1.014 & -0.868 & 0.000 \\ \mathrm{C} & -1.419 & 1.514 & 0.000\end{array}$




$\begin{array}{lrrrrrrr}\mathrm{C} & -2.370 & -1.082 & 0.000 & \mathrm{C} & 1.228 & -0.354 & 0.000 \\ \mathrm{H} & -0.330 & -1.704 & 0.000 & \mathrm{C} & 1.734 & 0.973 & 0.000 \\ \mathrm{C} & -2.771 & 1.293 & 0.000 & \mathrm{C} & 2.243 & -1.349 & 0.000 \\ \mathrm{H} & -1.047 & 2.532 & 0.000 & \mathrm{C} & 3.095 & 1.195 & 0.000 \\ \mathrm{C} & -3.286 & -0.015 & 0.000 & \mathrm{H} & 1.043 & 1.803 & 0.000 \\ \mathrm{H} & -2.752 & -2.096 & 0.000 & \mathrm{C} & 3.575 & -1.004 & 0.000 \\ \mathrm{H} & -3.459 & 2.130 & 0.000 & \mathrm{H} & 1.962 & -2.396 & 0.000 \\ \mathrm{C} & 1.948 & -0.207 & 0.000 & \mathrm{H} & 3.458 & 2.221 & 0.000 \\ \mathrm{O} & 1.904 & -1.432 & 0.000 & \mathrm{H} & 4.327 & -1.791 & 0.000 \\ \mathrm{O} & 3.199 & 0.413 & 0.000 & \mathrm{C} & -1.208 & 0.196 & 0.000 \\ \mathrm{C} & 4.301 & -0.465 & 0.000 & \mathrm{O} & -1.215 & 1.425 & 0.000 \\ \mathrm{H} & 4.264 & -1.117 & -0.877 & \mathrm{O} & -2.442 & -0.471 & 0.000 \\ \mathrm{H} & 4.264 & -1.116 & 0.877 & \mathrm{C} & -3.572 & 0.368 & 0.000 \\ \mathrm{C} & 5.557 & 0.382 & 0.000 & \mathrm{H} & -3.560 & 1.022 & -0.877 \\ \mathrm{H} & 6.446 & -0.251 & 0.000 & \mathrm{H} & -3.560 & 1.021 & 0.877 \\ \mathrm{H} & 5.585 & 1.022 & -0.883 & \mathrm{C} & -4.798 & -0.522 & 0.000 \\ \mathrm{H} & 5.584 & 1.022 & 0.883 & \mathrm{H} & -5.709 & 0.080 & 0.000 \\ \mathrm{C} & -4.688 & -0.249 & 0.000 & \mathrm{H} & -4.803 & -1.163 & -0.883 \\ \mathrm{~N} & -5.827 & -0.436 & 0.000 & \mathrm{H} & -4.803 & -1.163 & 0.883 \\ & & & & \mathrm{~N} & 4.046 & 0.250 & 0.000\end{array}$

CA9

$\begin{array}{lrrr}\mathrm{C} & 1.343 & 0.754 & 0.000 \\ \mathrm{H} & 1.653 & 1.790 & 0.000 \\ \mathrm{C} & -0.025 & 0.468 & 0.000 \\ \mathrm{C} & -0.583 & -0.851 & 0.000 \\ \mathrm{C} & -0.970 & 1.545 & 0.000 \\ \mathrm{C} & -1.936 & -1.054 & 0.000 \\ \mathrm{H} & 0.094 & -1.691 & 0.000 \\ \mathrm{C} & -2.320 & 1.339 & 0.000 \\ \mathrm{H} & -0.586 & 2.559 & 0.000 \\ \mathrm{C} & -2.825 & 0.031 & 0.000 \\ \mathrm{H} & -2.345 & -2.055 & 0.000 \\ \mathrm{H} & -3.015 & 2.166 & 0.000 \\ \mathrm{C} & 2.387 & -0.206 & 0.000 \\ \mathrm{O} & 2.334 & -1.428 & 0.000 \\ \mathrm{O} & 3.632 & 0.411 & 0.000 \\ \mathrm{C} & 4.737 & -0.467 & 0.000 \\ \mathrm{H} & 4.698 & -1.118 & -0.877 \\ \mathrm{H} & 4.698 & -1.117 & 0.878 \\ \mathrm{C} & 5.991 & 0.381 & 0.000 \\ \mathrm{H} & 6.880 & -0.252 & 0.000 \\ \mathrm{H} & 6.019 & 1.021 & -0.883 \\ \mathrm{H} & 6.019 & 1.021 & 0.883 \\ \mathrm{~N} & -4.227 & -0.190 & 0.000 \\ \mathrm{O} & -4.982 & 0.781 & 0.000 \\ \mathrm{O} & -4.649 & -1.344 & 0.000\end{array}$

\section{CA11}

$\begin{array}{lrrr}\text { C } & -3.168 & 0.765 & 0.014 \\ \text { C } & -1.790 & 0.517 & -0.003 \\ \text { C } & -1.232 & -0.798 & -0.022 \\ \text { C } & -0.842 & 1.584 & -0.006 \\ \text { C } & 0.124 & -1.009 & -0.041 \\ \text { H } & -1.909 & -1.643 & -0.025 \\ \text { C } & 0.512 & 1.361 & -0.025 \\ \text { H } & -1.215 & 2.602 & 0.003 \\ \text { C } & 1.028 & 0.061 & -0.048 \\ \text { H } & 0.505 & -2.024 & -0.058 \\ \text { H } & 1.194 & 2.204 & -0.029 \\ \text { C } & -4.120 & -0.260 & 0.016 \\ \text { N } & -4.909 & -1.113 & 0.018 \\ \text { H } & -3.534 & 1.782 & 0.026 \\ \text { C } & 2.482 & -0.182 & 0.000 \\ \text { F } & 2.857 & -1.293 & -0.672 \\ \text { F } & 2.976 & -0.362 & 1.256 \\ \text { F } & 3.207 & 0.835 & -0.511\end{array}$

\section{CA12}

CA10

$\begin{array}{llll}C & -0.137 & -0.712 & 0.000 \\ H & -0.392 & -1.763 & 0.000\end{array}$

$\begin{array}{rrr}2.361 & 0.782 & 0.000 \\ 0.998 & 0.484 & 0.000 \\ 0.488 & -0.853 & 0.000 \\ 0.012 & 1.520 & 0.000 \\ -0.856 & -1.113 & 0.000 \\ 1.196 & -1.673 & 0.000 \\ -1.329 & 1.250 & 0.000 \\ 0.350 & 2.550 & 0.000\end{array}$




$\begin{array}{lrrr}\mathrm{C} & -1.806 & -0.074 & 0.000 \\ \mathrm{H} & -1.204 & -2.140 & 0.000 \\ \mathrm{H} & -2.045 & 2.065 & 0.000 \\ \mathrm{C} & 3.352 & -0.209 & 0.000 \\ \mathrm{~N} & 4.172 & -1.031 & 0.000 \\ \mathrm{C} & -3.198 & -0.350 & 0.000 \\ \mathrm{~N} & -4.332 & -0.572 & 0.000 \\ \mathrm{H} & 2.691 & 1.811 & 0.000\end{array}$

\section{CA13}

$\begin{array}{lrrr}\mathrm{C} & 2.133 & 0.315 & 0.000 \\ \mathrm{C} & 0.743 & 0.180 & 0.000 \\ \mathrm{C} & 0.082 & -1.092 & 0.000 \\ \mathrm{C} & -0.121 & 1.321 & 0.000 \\ \mathrm{C} & -1.279 & -1.203 & 0.000 \\ \mathrm{H} & 0.693 & -1.987 & 0.000 \\ \mathrm{C} & -1.488 & 1.198 & 0.000 \\ \mathrm{H} & 0.313 & 2.312 & 0.000 \\ \mathrm{C} & -2.109 & -0.060 & 0.000 \\ \mathrm{H} & -1.740 & -2.183 & 0.000 \\ \mathrm{H} & -2.108 & 2.088 & 0.000 \\ \mathrm{C} & -3.523 & -0.178 & 0.000 \\ \mathrm{~N} & -4.674 & -0.273 & 0.000 \\ \mathrm{C} & 2.799 & 1.665 & 0.000 \\ \mathrm{H} & 2.527 & 2.261 & -0.880 \\ \mathrm{H} & 2.527 & 2.261 & 0.881 \\ \mathrm{H} & 3.883 & 1.555 & 0.000 \\ \mathrm{C} & 2.953 & -0.826 & 0.000 \\ \mathrm{~N} & 3.642 & -1.760 & 0.000\end{array}$

CA14

$\begin{array}{lrrr}\mathrm{C} & -2.757 & 0.786 & 0.000 \\ \mathrm{C} & -1.397 & 0.506 & 0.000 \\ \mathrm{C} & -0.871 & -0.828 & 0.000 \\ \mathrm{C} & -0.426 & 1.562 & 0.000 \\ \mathrm{C} & 0.472 & -1.071 & 0.000 \\ \mathrm{H} & -1.570 & -1.655 & 0.000 \\ \mathrm{C} & 0.915 & 1.315 & 0.000 \\ \mathrm{H} & -0.783 & 2.585 & 0.000 \\ \mathrm{C} & 1.390 & -0.007 & 0.000 \\ \mathrm{H} & 0.854 & -2.083 & 0.000 \\ \mathrm{H} & 1.633 & 2.122 & 0.000 \\ \mathrm{C} & -3.741 & -0.216 & 0.000 \\ \mathrm{~N} & -4.557 & -1.039 & 0.000 \\ \mathrm{H} & -3.100 & 1.811 & 0.000 \\ \mathrm{~N} & 2.781 & -0.264 & 0.000 \\ \mathrm{O} & 3.563 & 0.687 & 0.000 \\ \mathrm{O} & 3.173 & -1.430 & 0.000\end{array}$

$\begin{array}{lrrr}\mathrm{C} & 2.523 & 0.316 & 0.000 \\ \mathrm{C} & 1.138 & 0.191 & 0.000 \\ \mathrm{C} & 0.469 & -1.081 & 0.000 \\ \mathrm{C} & 0.283 & 1.345 & 0.000 \\ \mathrm{C} & -0.890 & -1.183 & 0.000 \\ \mathrm{H} & 1.075 & -1.978 & 0.000 \\ \mathrm{C} & -1.080 & 1.236 & 0.000 \\ \mathrm{H} & 0.729 & 2.330 & 0.000 \\ \mathrm{C} & -1.691 & -0.025 & 0.000 \\ \mathrm{H} & -1.376 & -2.148 & 0.000 \\ \mathrm{H} & -1.709 & 2.115 & 0.000 \\ \mathrm{C} & 3.207 & 1.658 & 0.000 \\ \mathrm{H} & 2.942 & 2.254 & -0.880 \\ \mathrm{H} & 2.942 & 2.254 & 0.881 \\ \mathrm{H} & 4.290 & 1.534 & 0.000 \\ \mathrm{C} & 3.337 & -0.833 & 0.000 \\ \mathrm{~N} & 4.025 & -1.766 & 0.000 \\ \mathrm{~N} & -3.101 & -0.131 & 0.000 \\ \mathrm{O} & -3.778 & 0.897 & 0.000 \\ \mathrm{O} & -3.614 & -1.250 & 0.000\end{array}$

\section{CA16}

$\begin{array}{lrrr}\text { C } & 1.386 & 0.383 & 0.000 \\ \text { C } & -0.006 & 0.137 & 0.000 \\ \text { C } & -0.562 & -1.176 & 0.000 \\ \text { C } & -0.954 & 1.198 & 0.000 \\ \text { C } & -1.921 & -1.392 & 0.000 \\ \text { H } & 0.119 & -2.020 & 0.000 \\ \text { C } & -2.318 & 0.960 & 0.000 \\ \text { H } & -0.601 & 2.222 & 0.000 \\ \text { C } & -2.834 & -0.331 & 0.000 \\ \text { H } & -2.289 & -2.414 & 0.000 \\ \text { H } & -2.997 & 1.808 & 0.000 \\ \text { C } & 1.926 & 1.787 & 0.000 \\ \text { H } & 1.603 & 2.362 & -0.879 \\ \text { H } & 1.603 & 2.362 & 0.880 \\ \text { H } & 3.016 & 1.776 & 0.000 \\ \text { C } & 2.293 & -0.679 & 0.000 \\ \text { N } & 3.056 & -1.559 & 0.000 \\ \text { H } & -3.901 & -0.510 & 0.000\end{array}$

\section{CA17}

$\begin{array}{lrrr}\mathrm{C} & -1.666 & 0.744 & 0.001 \\ \mathrm{H} & -1.948 & 1.784 & 0.002 \\ \mathrm{C} & -0.287 & 0.377 & -0.001 \\ \mathrm{C} & 0.243 & -0.932 & -0.007 \\ \mathrm{C} & 0.665 & 1.425 & -0.003 \\ \mathrm{C} & 1.613 & -1.147 & -0.010 \\ \mathrm{H} & -0.443 & -1.764 & -0.011 \\ \mathrm{C} & 2.024 & 1.190 & -0.007\end{array}$




$\begin{array}{rrrr}\mathrm{H} & 0.304 & 2.448 & -0.005 \\ \mathrm{C} & 2.538 & -0.108 & -0.008 \\ \mathrm{H} & 1.977 & -2.171 & -0.016 \\ \mathrm{H} & 2.709 & 2.034 & -0.010 \\ \mathrm{O} & -2.573 & -1.339 & 0.002 \\ \mathrm{O} & -3.884 & 0.399 & 0.005 \\ \mathrm{~N} & -2.727 & -0.093 & 0.003 \\ \mathrm{C} & 4.022 & -0.361 & 0.016 \\ \mathrm{H} & 4.454 & -0.156 & 1.000 \\ \mathrm{H} & 4.243 & -1.402 & -0.227 \\ \mathrm{H} & 4.548 & 0.269 & -0.705\end{array}$

\section{CA18}

$\begin{array}{lrrr}\mathrm{C} & 1.145 & -0.764 & 0.000 \\ \mathrm{H} & 1.369 & -1.817 & 0.000 \\ \mathrm{C} & -0.210 & -0.321 & 0.000 \\ \mathrm{C} & -0.663 & 1.019 & 0.000 \\ \mathrm{C} & -1.218 & -1.318 & 0.000 \\ \mathrm{C} & -2.017 & 1.314 & 0.000 \\ \mathrm{H} & 0.072 & 1.808 & 0.000 \\ \mathrm{C} & -2.563 & -1.009 & 0.000 \\ \mathrm{H} & -0.911 & -2.358 & 0.000 \\ \mathrm{C} & -2.988 & 0.318 & 0.000 \\ \mathrm{H} & -2.321 & 2.355 & 0.000 \\ \mathrm{H} & -3.292 & -1.812 & 0.000 \\ \mathrm{O} & 2.174 & 1.264 & 0.000 \\ \mathrm{O} & 3.379 & -0.547 & 0.000 \\ \mathrm{~N} & 2.254 & 0.012 & 0.000 \\ \mathrm{H} & -4.042 & 0.566 & 0.000\end{array}$

CA19

$\begin{array}{lrrr}\mathrm{C} & 1.927 & 0.730 & 0.000 \\ \mathrm{H} & 2.240 & 1.760 & 0.000 \\ \mathrm{C} & 0.558 & 0.395 & 0.000 \\ \mathrm{C} & -0.002 & -0.912 & 0.000 \\ \mathrm{C} & -0.373 & 1.474 & 0.000 \\ \mathrm{C} & -1.365 & -1.101 & 0.000 \\ \mathrm{H} & 0.665 & -1.758 & 0.000 \\ \mathrm{C} & -1.729 & 1.277 & 0.000 \\ \mathrm{H} & 0.015 & 2.486 & 0.000 \\ \mathrm{C} & -2.259 & -0.023 & 0.000 \\ \mathrm{H} & -1.762 & -2.109 & 0.000 \\ \mathrm{H} & -2.403 & 2.125 & 0.000 \\ \mathrm{O} & 2.799 & -1.374 & 0.000 \\ \mathrm{O} & 4.135 & 0.333 & 0.000 \\ \mathrm{C} & -3.667 & -0.235 & 0.000 \\ \mathrm{~N} & -4.808 & -0.403 & 0.000 \\ \mathrm{~N} & 2.982 & -0.141 & 0.000\end{array}$

$\begin{array}{lrrr}\mathrm{C} & 2.329 & 0.736 & 0.000 \\ \mathrm{H} & 2.652 & 1.763 & 0.000 \\ \mathrm{C} & 0.965 & 0.412 & 0.000 \\ \mathrm{C} & 0.394 & -0.895 & 0.000 \\ \mathrm{C} & 0.042 & 1.504 & 0.000 \\ \mathrm{C} & -0.967 & -1.074 & 0.000 \\ \mathrm{H} & 1.053 & -1.746 & 0.000 \\ \mathrm{C} & -1.313 & 1.321 & 0.000 \\ \mathrm{H} & 0.441 & 2.511 & 0.000 \\ \mathrm{C} & -1.832 & 0.022 & 0.000 \\ \mathrm{H} & -1.390 & -2.069 & 0.000 \\ \mathrm{H} & -1.993 & 2.160 & 0.000 \\ \mathrm{O} & 3.197 & -1.372 & 0.000 \\ \mathrm{O} & 4.535 & 0.329 & 0.000 \\ \mathrm{~N} & 3.385 & -0.144 & 0.000 \\ \mathrm{~N} & -3.247 & -0.179 & 0.000 \\ \mathrm{O} & -3.980 & 0.803 & 0.000 \\ \mathrm{O} & -3.681 & -1.324 & 0.000\end{array}$

CA21

$\begin{array}{lrrr}\mathrm{C} & 0.000 & 0.718 & 0.000 \\ \mathrm{C} & 1.222 & 0.048 & 0.000 \\ \mathrm{~N} & 2.259 & -0.478 & 0.000 \\ \mathrm{H} & 0.001 & 1.797 & 0.000 \\ \mathrm{C} & -1.222 & 0.049 & 0.000 \\ \mathrm{~N} & -2.259 & -0.478 & 0.000\end{array}$

\section{CA22}

$\begin{array}{lrrr}\text { C } & -1.407 & -0.212 & -1.182 \\ \text { C } & -0.701 & -0.263 & 0.008 \\ \text { C } & -1.307 & 0.103 & 1.203 \\ \text { C } & -2.630 & 0.512 & 1.205 \\ \text { C } & -3.347 & 0.567 & 0.013 \\ \text { C } & -2.734 & 0.207 & -1.178 \\ \text { H } & -0.900 & -0.497 & -2.094 \\ \text { H } & -0.714 & 0.075 & 2.109 \\ \text { H } & -3.106 & 0.799 & 2.135 \\ \text { H } & -4.379 & 0.895 & 0.015 \\ \text { H } & -3.287 & 0.256 & -2.109 \\ \text { S } & 0.998 & -0.865 & 0.028 \\ \text { O } & 1.289 & -1.094 & -1.380 \\ \text { O } & 0.991 & -2.041 & 0.885 \\ \text { C } & 1.839 & 0.349 & 0.861 \\ \text { H } & 2.772 & -0.088 & 1.208 \\ \text { Cl } & 2.184 & 1.758 & -0.195\end{array}$

\section{CA23}

$\begin{array}{llll}\text { C } & 0.695 & -0.689 & -0.026 \\ \text { C } & 0.695 & 0.689 & -0.026\end{array}$




$\begin{array}{lrrr}\mathrm{C} & 1.882 & 1.403 & -0.008 \\ \mathrm{C} & 3.077 & 0.697 & -0.014 \\ \mathrm{C} & 3.077 & -0.697 & -0.014 \\ \mathrm{C} & 1.882 & -1.403 & -0.008 \\ \mathrm{C} & -1.859 & 0.000 & -0.420 \\ \mathrm{H} & 1.864 & 2.486 & -0.006 \\ \mathrm{H} & 4.019 & 1.232 & -0.022 \\ \mathrm{H} & 4.019 & -1.232 & -0.022 \\ \mathrm{H} & 1.864 & -2.486 & -0.006 \\ \mathrm{H} & -2.060 & 0.000 & -1.484 \\ \mathrm{~S} & -0.969 & 1.389 & -0.006 \\ \mathrm{~S} & -0.969 & -1.389 & -0.006 \\ \mathrm{O} & -1.145 & 1.844 & 1.353 \\ \mathrm{O} & -1.067 & 2.389 & -1.050 \\ \mathrm{O} & -1.145 & -1.844 & 1.352 \\ \mathrm{O} & -1.067 & -2.389 & -1.050\end{array}$

\section{CA24}

$\begin{array}{lrrr}\text { C } & 0.825 & -0.690 & 0.046 \\ \text { C } & 0.825 & 0.690 & 0.046 \\ \text { C } & 2.007 & 1.403 & -0.054 \\ \text { C } & 3.198 & 0.696 & -0.156 \\ \text { C } & 3.198 & -0.697 & -0.157 \\ \text { C } & 2.007 & -1.404 & -0.054 \\ \text { C } & -1.793 & 0.000 & -0.342 \\ \text { H } & 1.988 & 2.486 & -0.070 \\ \text { H } & 4.135 & 1.231 & -0.249 \\ \text { H } & 4.135 & -1.233 & -0.249 \\ \text { H } & 1.987 & -2.486 & -0.070 \\ \text { S } & -0.840 & 1.379 & 0.198 \\ \text { S } & -0.841 & -1.379 & 0.198 \\ \text { O } & -1.036 & 1.631 & 1.601 \\ \text { O } & -0.949 & 2.477 & -0.729 \\ \text { O } & -1.037 & -1.630 & 1.601 \\ \text { O } & -0.950 & -2.477 & -0.728 \\ \text { F } & -1.684 & 0.000 & -1.736\end{array}$

$\begin{array}{lrrr}\text { N1 } & & & \\ \text { C } & -1.136 & -0.719 & 0.000 \\ \text { C } & -1.191 & 0.669 & 0.000 \\ \text { C } & 0.000 & 1.376 & 0.000 \\ \text { C } & 1.191 & 0.669 & 0.000 \\ \text { C } & 1.136 & -0.719 & 0.000 \\ \text { N } & 0.000 & -1.409 & 0.000 \\ \text { H } & 0.000 & 2.459 & 0.000 \\ \text { H } & -2.050 & -1.303 & 0.000 \\ \text { H } & -2.147 & 1.176 & 0.000 \\ \text { H } & 2.147 & 1.176 & 0.000 \\ \text { H } & 2.050 & -1.303 & 0.000\end{array}$

$\begin{array}{lrrr}\mathrm{C} & -1.581 & -1.135 & 0.000 \\ \mathrm{C} & -0.194 & -1.197 & 0.000 \\ \mathrm{C} & 0.500 & 0.000 & 0.000 \\ \mathrm{C} & -0.194 & 1.197 & 0.000 \\ \mathrm{C} & -1.581 & 1.135 & 0.000 \\ \mathrm{~N} & -2.273 & 0.000 & 0.000 \\ \mathrm{H} & -2.160 & -2.051 & 0.000 \\ \mathrm{H} & 0.326 & -2.144 & 0.000 \\ \mathrm{H} & 0.326 & 2.144 & 0.000 \\ \mathrm{H} & -2.160 & 2.051 & 0.000 \\ \mathrm{Cl} & 2.228 & 0.000 & 0.000\end{array}$

N3$$
\text { C }
$$

C

C

C

C

$\mathrm{N}$

$\mathrm{H}$

$\mathrm{H}$

$\mathrm{H}$

$\mathrm{H}$

O

C

$\mathrm{H}$

$\mathrm{H}$

$\mathrm{H}$

N4

$$
\text { C }
$$

C

C

C

C

N

$\mathrm{H}$

H

H

$\mathrm{H}$

C

$\mathrm{H}$

$\mathrm{H}$

$\mathrm{H}$

N

C

$\mathrm{H}$

$\mathrm{H}$

$\begin{array}{rrr}-1.343 & 1.292 & 0.000 \\ 0.025 & 1.044 & 0.000 \\ 0.446 & -0.281 & 0.000 \\ -0.523 & -1.283 & 0.000 \\ -1.852 & -0.912 & 0.000 \\ -2.278 & 0.352 & 0.000 \\ -1.695 & 2.318 & 0.000 \\ 0.719 & 1.871 & 0.000 \\ -0.222 & -2.321 & 0.000 \\ -2.623 & -1.676 & 0.000 \\ 1.733 & -0.679 & 0.000 \\ 2.731 & 0.321 & 0.000 \\ 3.683 & -0.202 & 0.001 \\ 2.660 & 0.947 & -0.892 \\ 2.659 & 0.948 & 0.892\end{array}$

$\begin{array}{rrr}1.944 & 1.126 & 0.000 \\ 0.563 & 1.193 & 0.000 \\ -0.181 & 0.000 & 0.000 \\ 0.563 & -1.193 & 0.000 \\ 1.944 & -1.127 & 0.000 \\ 2.653 & 0.000 & 0.000 \\ 2.516 & 2.049 & 0.000 \\ 0.082 & 2.159 & 0.000 \\ 0.082 & -2.159 & 0.000 \\ 2.516 & -2.049 & 0.000 \\ -2.264 & 1.254 & 0.000 \\ -3.332 & 1.054 & -0.001 \\ -2.029 & 1.852 & 0.886 \\ -2.028 & 1.852 & -0.886 \\ -1.546 & 0.000 & 0.000 \\ -2.264 & -1.254 & 0.000 \\ -2.029 & -1.852 & -0.885 \\ -2.028 & -1.852 & 0.886\end{array}$




\begin{tabular}{lrrr} 
H & -3.332 & -1.054 & \multicolumn{1}{l}{0.001} \\
N5 & & & \\
C & & & \\
C & -2.649 & 1.124 & 0.087 \\
C & -1.268 & 1.190 & 0.093 \\
C & -0.527 & 0.000 & 0.000 \\
C & -1.268 & -1.190 & -0.093 \\
N & -2.649 & -1.124 & -0.087 \\
H & -3.357 & 0.000 & 0.000 \\
H & -3.222 & 2.043 & 0.162 \\
H & -0.778 & 2.149 & 0.180 \\
H & -0.778 & -2.149 & -0.180 \\
N & -3.222 & -2.043 & -0.162 \\
C & 0.831 & 0.000 & 0.000 \\
C & 1.635 & 1.212 & -0.011 \\
C & 1.635 & -1.212 & 0.011 \\
H & 3.041 & 0.697 & -0.315 \\
C & 1.276 & 1.910 & -0.770 \\
H & 3.041 & -0.697 & 0.314 \\
H & 1.277 & -1.909 & 0.771 \\
H & 3.818 & 1.348 & 0.078 \\
H & 3.176 & -0.617 & 1.394 \\
H & 1.594 & 1.721 & 0.960 \\
H & 3.176 & 0.617 & -1.395 \\
H & 3.818 & -1.349 & -0.079 \\
& 1.594 & -1.722 & -0.959
\end{tabular}

N6

$\begin{array}{lrrr}\text { C } & 0.274 & 0.687 & 0.000 \\ \text { C } & 0.272 & -0.710 & 0.000 \\ \text { C } & -0.931 & -1.424 & 0.000 \\ \text { C } & -2.096 & -0.694 & 0.000 \\ \text { C } & -2.080 & 0.718 & 0.000 \\ \text { C } & -0.906 & 1.436 & 0.000 \\ \text { H } & -0.929 & -2.505 & 0.000 \\ \text { H } & -3.050 & -1.204 & 0.000 \\ \text { H } & -3.022 & 1.250 & 0.000 \\ \text { H } & -0.903 & 2.517 & 0.000 \\ \text { H } & 2.059 & 1.883 & 0.000 \\ \text { N } & 1.577 & -1.147 & 0.000 \\ \text { N } & 2.345 & -0.129 & 0.000 \\ \text { N } & 1.599 & 0.989 & 0.000\end{array}$

N7

$\begin{array}{lrrr}C & -0.160 & -0.364 & 0.000 \\ C & 0.361 & 0.933 & 0.000 \\ C & 1.742 & 1.147 & 0.000 \\ C & 2.551 & 0.035 & 0.000 \\ C & 2.013 & -1.271 & 0.000\end{array}$

N10

$\begin{array}{rrrr}\text { C } & 0.196 & 1.079 & 0.000 \\ \text { C } & 1.495 & -0.603 & 0.000 \\ \text { C } & 0.225 & -1.107 & 0.000 \\ \text { N } & -0.605 & -0.016 & 0.000\end{array}$




$\begin{array}{lrrr}\mathrm{H} & -0.212 & 2.078 & 0.000 \\ \mathrm{H} & 2.424 & -1.149 & 0.000 \\ \mathrm{H} & -0.159 & -2.112 & 0.000 \\ \mathrm{~N} & 1.465 & 0.765 & 0.000 \\ \mathrm{C} & -2.051 & -0.032 & 0.000 \\ \mathrm{H} & -2.427 & -0.536 & -0.890 \\ \mathrm{H} & -2.428 & -0.540 & 0.887 \\ \mathrm{H} & -2.411 & 0.994 & 0.002\end{array}$

$\begin{array}{lrrr}\text { N11 } & & & \\ \text { C } & -1.103 & -1.168 & -0.281 \\ \text { C } & -0.409 & 0.005 & -0.011 \\ \text { C } & -1.100 & 1.179 & 0.265 \\ \text { C } & -2.486 & 1.176 & 0.265 \\ \text { C } & -3.185 & 0.004 & 0.007 \\ \text { C } & -2.489 & -1.166 & -0.261 \\ \text { H } & -0.556 & -2.069 & -0.528 \\ \text { H } & -0.550 & 2.081 & 0.499 \\ \text { H } & -3.021 & 2.091 & 0.480 \\ \text { H } & -4.267 & 0.004 & 0.014 \\ \text { H } & -3.026 & -2.081 & -0.471 \\ \text { N } & 1.005 & 0.004 & -0.014 \\ \text { C } & 1.821 & -0.991 & 0.443 \\ \text { C } & 1.834 & 1.011 & -0.455 \\ \text { H } & 1.422 & -1.892 & 0.879 \\ \text { C } & 3.103 & 0.562 & -0.246 \\ \text { H } & 1.444 & 1.911 & -0.898 \\ \text { H } & 4.028 & 1.063 & -0.475 \\ \text { N } & 3.083 & -0.687 & 0.321\end{array}$

\section{N12}

$\begin{array}{lrrr}\mathrm{C} & 1.474 & 1.079 & 0.000 \\ \mathrm{C} & 1.451 & -1.102 & 0.000 \\ \mathrm{C} & 2.730 & -0.633 & 0.000 \\ \mathrm{~N} & 2.733 & 0.738 & 0.000 \\ \mathrm{H} & 1.105 & 2.093 & 0.000 \\ \mathrm{H} & 1.058 & -2.105 & 0.000 \\ \mathrm{H} & 3.646 & -1.201 & 0.000 \\ \mathrm{~N} & 0.625 & 0.006 & 0.000 \\ \mathrm{Si} & -1.150 & 0.000 & 0.000 \\ \mathrm{C} & -1.715 & -0.895 & -1.532 \\ \mathrm{H} & -1.339 & -1.919 & -1.550 \\ \mathrm{H} & -2.806 & -0.939 & -1.574 \\ \mathrm{H} & -1.359 & -0.390 & -2.431 \\ \mathrm{C} & -1.715 & -0.891 & 1.534 \\ \mathrm{H} & -1.359 & -0.385 & 2.432 \\ \mathrm{H} & -2.806 & -0.936 & 1.576 \\ \mathrm{H} & -1.339 & -1.916 & 1.555 \\ \mathrm{C} & -1.687 & 1.782 & -0.002 \\ \mathrm{H} & -1.328 & 2.309 & -0.888\end{array}$

$\begin{array}{llll}\mathrm{H} & -2.777 & 1.836 & -0.002 \\ \mathrm{H} & -1.329 & 2.310 & 0.883\end{array}$

N13

$\begin{array}{lrrr}\mathrm{C} & -1.045 & -0.154 & 0.000 \\ \mathrm{C} & 1.107 & -0.068 & 0.000 \\ \mathrm{C} & 0.677 & 1.227 & 0.000 \\ \mathrm{~N} & -0.697 & 1.159 & 0.000 \\ \mathrm{H} & -1.331 & 1.939 & 0.000 \\ \mathrm{H} & 1.205 & 2.164 & 0.000 \\ \mathrm{~N} & 0.021 & -0.914 & 0.000 \\ \mathrm{C} & -2.457 & -0.626 & 0.000 \\ \mathrm{H} & -2.994 & -0.280 & 0.885 \\ \mathrm{H} & -2.995 & -0.277 & -0.883 \\ \mathrm{H} & -2.454 & -1.712 & -0.002 \\ \mathrm{C} & 2.504 & -0.598 & 0.000 \\ \mathrm{H} & 3.058 & -0.275 & -0.883 \\ \mathrm{H} & 3.059 & -0.272 & 0.880 \\ \mathrm{H} & 2.469 & -1.686 & 0.002\end{array}$

N14

$\begin{array}{lrrr}\mathrm{C} & 1.939 & -1.299 & 0.061 \\ \mathrm{C} & 0.424 & -1.559 & -0.065 \\ \mathrm{H} & 2.339 & -1.668 & 1.007 \\ \mathrm{H} & 2.506 & -1.774 & -0.739 \\ \mathrm{H} & 0.165 & -2.027 & -1.017 \\ \mathrm{H} & 0.034 & -2.179 & 0.741 \\ \mathrm{~N} & 2.115 & 0.150 & 0.004 \\ \mathrm{~N} & -0.116 & -0.199 & 0.002 \\ \mathrm{C} & 0.975 & 0.703 & -0.009 \\ \mathrm{C} & -1.456 & 0.116 & 0.014 \\ \mathrm{O} & -1.867 & 1.254 & 0.049 \\ \mathrm{C} & -2.391 & -1.070 & -0.009 \\ \mathrm{H} & -2.268 & -1.672 & 0.893 \\ \mathrm{H} & -3.409 & -0.695 & -0.058 \\ \mathrm{H} & -2.191 & -1.714 & -0.867 \\ \mathrm{C} & 0.777 & 2.178 & -0.036 \\ \mathrm{H} & 0.188 & 2.472 & -0.904 \\ \mathrm{H} & 0.221 & 2.506 & 0.840 \\ \mathrm{H} & 1.758 & 2.643 & -0.063\end{array}$

N15

$\begin{array}{rrrr}\text { C } & 1.441 & 0.734 & 0.127 \\ \mathrm{C} & 1.405 & -0.794 & -0.074 \\ \mathrm{C} & -0.679 & -0.096 & -0.010 \\ \mathrm{~N} & 0.056 & 1.074 & -0.199 \\ \mathrm{H} & -0.328 & 1.924 & 0.185 \\ \mathrm{H} & 2.146 & 1.237 & -0.532 \\ \mathrm{H} & 1.741 & -1.066 & -1.079\end{array}$




$\begin{array}{lrrr}\mathrm{C} & -2.167 & -0.023 & 0.028 \\ \mathrm{H} & -2.501 & 0.614 & 0.850 \\ \mathrm{H} & -2.543 & 0.407 & -0.901 \\ \mathrm{H} & -2.574 & -1.021 & 0.158 \\ \mathrm{~N} & -0.006 & -1.172 & 0.068 \\ \mathrm{H} & 2.025 & -1.331 & 0.641 \\ \mathrm{H} & 1.680 & 0.991 & 1.164\end{array}$

$\begin{array}{lrrr}\text { N16 } & & & \\ \text { C } & -1.389 & -0.775 & 0.000 \\ \text { C } & -1.426 & 0.768 & 0.000 \\ \text { C } & 0.667 & 0.110 & 0.000 \\ \text { H } & -1.837 & -1.219 & -0.888 \\ \text { H } & -1.925 & 1.175 & -0.880 \\ \text { C } & 2.149 & 0.006 & 0.000 \\ \text { H } & 2.480 & -0.545 & 0.880 \\ \text { H } & 2.480 & -0.545 & -0.880 \\ \text { H } & 2.582 & 1.001 & 0.000 \\ \text { N } & -0.018 & 1.170 & 0.000 \\ \text { H } & -1.925 & 1.174 & 0.880 \\ \text { H } & -1.838 & -1.219 & 0.888 \\ \text { O } & 0.013 & -1.083 & 0.000\end{array}$

$\begin{array}{lrrr}\text { N17 } & & & \\ \text { C } & 1.524 & 0.658 & 0.161 \\ \text { C } & 1.377 & -0.856 & -0.083 \\ \text { C } & -0.713 & -0.078 & -0.001 \\ \text { H } & 2.295 & 1.116 & -0.457 \\ \text { H } & 1.708 & -1.146 & -1.084 \\ \text { C } & -2.204 & -0.035 & 0.047 \\ \text { H } & -2.539 & 0.543 & 0.911 \\ \text { H } & -2.596 & 0.465 & -0.842 \\ \text { H } & -2.610 & -1.042 & 0.104 \\ \text { N } & -0.053 & -1.158 & 0.026 \\ \text { H } & 1.938 & -1.462 & 0.627 \\ \text { H } & 1.780 & 0.842 & 1.205 \\ \text { C } & 0.116 & 1.184 & -0.132 \\ \text { H } & -0.216 & 1.971 & 0.547 \\ \text { H } & 0.015 & 1.575 & -1.149\end{array}$

$\begin{array}{lrrr}\text { N18 } & & & \\ \text { C } & -1.649 & -0.287 & 0.231 \\ \text { C } & -1.276 & 1.156 & -0.141 \\ \text { C } & 0.799 & 0.268 & 0.012 \\ \text { H } & -2.474 & -0.670 & -0.364 \\ \text { H } & -1.518 & 1.360 & -1.188 \\ \text { C } & 2.287 & 0.166 & 0.067 \\ \text { H } & 2.595 & -0.385 & 0.956 \\ \text { H } & 2.656 & -0.379 & -0.804\end{array}$

$\begin{array}{rrrr}\mathrm{H} & 2.717 & 1.164 & 0.083 \\ \mathrm{~N} & 0.158 & 1.353 & 0.023 \\ \mathrm{H} & -1.822 & 1.874 & 0.470 \\ \mathrm{H} & -1.897 & -0.379 & 1.288 \\ \mathrm{~S} & -0.146 & -1.242 & -0.101\end{array}$

N19

$\begin{array}{lrrr}\mathrm{C} & 0.770 & 1.486 & -0.124 \\ \mathrm{C} & -0.735 & 1.498 & 0.112 \\ \mathrm{H} & 1.003 & 1.698 & -1.177 \\ \mathrm{H} & 1.306 & 2.196 & 0.505 \\ \mathrm{H} & -0.962 & 1.704 & 1.167 \\ \mathrm{H} & -1.262 & 2.220 & -0.511 \\ \mathrm{~N} & 1.096 & 0.120 & 0.225 \\ \mathrm{~N} & -1.090 & 0.135 & -0.246 \\ \mathrm{C} & 0.003 & -0.697 & -0.008 \\ \mathrm{H} & -0.837 & -2.393 & -0.142 \\ \mathrm{C} & 2.427 & -0.383 & -0.004 \\ \mathrm{H} & 2.697 & -0.349 & -1.067 \\ \mathrm{H} & 3.144 & 0.214 & 0.560 \\ \mathrm{H} & 2.468 & -1.416 & 0.332 \\ \mathrm{C} & -2.423 & -0.328 & 0.045 \\ \mathrm{H} & -2.584 & -0.486 & 1.119 \\ \mathrm{H} & -3.140 & 0.409 & -0.313 \\ \mathrm{H} & -2.620 & -1.262 & -0.480 \\ \mathrm{~N} & 0.070 & -1.968 & 0.005\end{array}$

N20

$\begin{array}{lrrr}\text { C } & -0.204 & -0.727 & -0.019 \\ \mathrm{C} & 2.056 & -0.803 & 0.288 \\ \mathrm{C} & 2.113 & 0.565 & -0.392 \\ \mathrm{C} & 0.958 & 1.438 & 0.091 \\ \mathrm{C} & -1.558 & 1.160 & -0.037 \\ \mathrm{C} & -2.427 & -0.090 & 0.114 \\ \mathrm{H} & 2.882 & -1.427 & -0.053 \\ \mathrm{H} & 2.030 & 0.411 & -1.470 \\ \mathrm{H} & 1.207 & 1.910 & 1.046 \\ \mathrm{H} & -1.612 & 1.558 & -1.058 \\ \mathrm{H} & -3.315 & -0.057 & -0.515 \\ \mathrm{H} & -2.732 & -0.214 & 1.159 \\ \mathrm{H} & -1.705 & -2.091 & -0.106 \\ \mathrm{~N} & -0.240 & 0.630 & 0.248 \\ \mathrm{~N} & 0.807 & -1.494 & 0.001 \\ \mathrm{~N} & -1.502 & -1.127 & -0.318 \\ \mathrm{H} & 2.178 & -0.676 & 1.372 \\ \mathrm{H} & 3.060 & 1.068 & -0.196 \\ \mathrm{H} & 0.757 & 2.240 & -0.627 \\ \mathrm{H} & -1.835 & 1.950 & 0.662\end{array}$




$\begin{array}{lrrr}\mathrm{C} & -0.011 & -0.544 & 0.011 \\ \mathrm{~N} & 1.156 & 0.217 & -0.121 \\ \mathrm{~N} & -1.169 & 0.215 & 0.135 \\ \mathrm{~N} & -0.082 & -1.819 & 0.014 \\ \mathrm{H} & 0.834 & -2.247 & 0.074 \\ \mathrm{C} & 2.369 & -0.497 & -0.446 \\ \mathrm{H} & 2.192 & -1.175 & -1.280 \\ \mathrm{H} & 3.130 & 0.224 & -0.743 \\ \mathrm{H} & 2.762 & -1.074 & 0.403 \\ \mathrm{C} & 1.374 & 1.322 & 0.799 \\ \mathrm{H} & 1.968 & 2.098 & 0.312 \\ \mathrm{H} & 0.424 & 1.746 & 1.111 \\ \mathrm{H} & 1.912 & 0.988 & 1.695 \\ \mathrm{C} & -1.374 & 1.328 & -0.778 \\ \mathrm{H} & -2.027 & 2.067 & -0.311 \\ \mathrm{H} & -0.428 & 1.800 & -1.028 \\ \mathrm{H} & -1.846 & 0.991 & -1.710 \\ \mathrm{C} & -2.385 & -0.532 & 0.396 \\ \mathrm{H} & -2.717 & -1.092 & -0.486 \\ \mathrm{H} & -2.214 & -1.243 & 1.199 \\ \mathrm{H} & -3.164 & 0.173 & 0.686\end{array}$

N22

$\begin{array}{rrrr}\mathrm{H} & 3.649 & 0.837 & 0.273 \\ \mathrm{H} & 3.649 & -0.836 & 0.274 \\ \mathrm{Cl} & -2.712 & 0.000 & 0.006\end{array}$

N24

$\begin{array}{lrrr}\text { C } & 0.717 & 1.197 & -0.009 \\ \text { C } & -0.668 & 1.189 & -0.011 \\ \text { C } & -1.392 & 0.000 & -0.009 \\ \text { C } & -0.668 & -1.189 & -0.011 \\ \text { C } & 0.717 & -1.197 & -0.009 \\ \text { C } & 1.432 & 0.000 & -0.005 \\ \text { H } & 1.254 & 2.138 & -0.019 \\ \text { H } & -1.200 & 2.134 & -0.016 \\ \text { H } & -1.200 & -2.134 & -0.016 \\ \text { H } & 1.253 & -2.139 & -0.019 \\ \text { N } & 2.827 & 0.000 & -0.064 \\ \text { H } & 3.264 & 0.834 & 0.295 \\ \text { H } & 3.264 & -0.834 & 0.296 \\ \text { C } & -2.896 & 0.000 & 0.023 \\ \text { H } & -3.269 & -0.011 & 1.050 \\ \text { H } & -3.300 & 0.887 & -0.464 \\ \text { H } & -3.301 & -0.877 & -0.482\end{array}$

$\begin{array}{lrrr}\text { C } & -0.220 & -1.201 & -0.006 \\ \text { C } & 1.165 & -1.196 & 0.004 \\ \text { C } & 1.871 & 0.000 & 0.008 \\ \text { C } & 1.165 & 1.196 & 0.004 \\ \text { C } & -0.220 & 1.201 & -0.006 \\ \text { C } & -0.931 & 0.000 & -0.009 \\ \text { H } & -0.762 & -2.140 & -0.014 \\ \text { H } & 1.697 & -2.138 & 0.007 \\ \text { H } & 2.952 & 0.000 & 0.016 \\ \text { H } & 1.697 & 2.138 & 0.008 \\ \text { H } & -0.762 & 2.140 & -0.014 \\ \text { N } & -2.323 & 0.000 & -0.074 \\ \text { H } & -2.767 & -0.836 & 0.270 \\ \text { H } & -2.767 & 0.836 & 0.271\end{array}$

N25

$\begin{array}{lrrr}\text { C } & 0.960 & -1.189 & 0.007 \\ \text { C } & -0.419 & -1.000 & 0.002 \\ \text { C } & -0.944 & 0.285 & -0.001 \\ \text { C } & -0.072 & 1.372 & 0.001 \\ \text { C } & 1.293 & 1.177 & 0.007 \\ \text { C } & 1.835 & -0.112 & 0.008 \\ \text { H } & 1.356 & -2.198 & 0.015 \\ \text { H } & -1.064 & -1.867 & 0.001 \\ \text { H } & -0.492 & 2.369 & -0.002 \\ \text { H } & 1.957 & 2.034 & 0.016 \\ \text { N } & 3.222 & -0.303 & 0.075 \\ \text { H } & 3.533 & -1.190 & -0.288 \\ \text { H } & 3.761 & 0.456 & -0.315 \\ \text { O } & -2.275 & 0.580 & -0.008 \\ \text { C } & -3.175 & -0.501 & -0.004 \\ \text { H } & -3.051 & -1.119 & 0.890 \\ \text { H } & -4.173 & -0.069 & -0.007 \\ \text { H } & -3.049 & -1.127 & -0.892\end{array}$

$\begin{array}{lrrr}\text { N23 } & & & \\ \text { C } & 1.099 & 1.199 & -0.006 \\ \text { C } & -0.286 & 1.201 & -0.003 \\ \text { C } & -0.976 & 0.000 & 0.000 \\ \text { C } & -0.286 & -1.201 & -0.003 \\ \text { C } & 1.099 & -1.199 & -0.006 \\ \text { C } & 1.813 & 0.000 & -0.007 \\ \text { H } & 1.635 & 2.140 & -0.013 \\ \text { H } & -0.832 & 2.134 & 0.000 \\ \text { H } & -0.832 & -2.134 & -0.001 \\ \text { H } & 1.635 & -2.141 & -0.013 \\ \text { N } & 3.203 & 0.000 & -0.068\end{array}$

N26

$\begin{array}{llll}\mathrm{N} & -1.884 & 0.170 & -0.089 \\ \mathrm{H} & -2.641 & -0.501 & -0.053 \\ \mathrm{H} & -2.019 & 0.811 & 0.684 \\ \mathrm{C} & -0.589 & -0.494 & 0.026 \\ \mathrm{H} & -0.498 & -1.199 & -0.805\end{array}$




$\begin{array}{lrrr}\mathrm{H} & -0.475 & -1.078 & 0.951 \\ \mathrm{C} & 0.539 & 0.521 & -0.059 \\ \mathrm{H} & 0.433 & 1.078 & -0.992 \\ \mathrm{H} & 0.425 & 1.246 & 0.753 \\ \mathrm{C} & 1.912 & -0.132 & 0.024 \\ \mathrm{H} & 2.054 & -0.844 & -0.791 \\ \mathrm{H} & 2.709 & 0.608 & -0.038 \\ \mathrm{H} & 2.033 & -0.676 & 0.963\end{array}$

$\begin{array}{lrrr}\mathrm{H} & -0.141 & 2.309 & -0.060 \\ \mathrm{H} & -2.553 & 1.831 & -0.252 \\ \mathrm{C} & 1.897 & 0.559 & 0.294 \\ \mathrm{H} & 2.138 & 0.585 & 1.361 \\ \mathrm{H} & 2.085 & 1.567 & -0.095 \\ \mathrm{~N} & 2.729 & -0.474 & -0.314 \\ \mathrm{H} & 2.594 & -0.489 & -1.319 \\ \mathrm{H} & 3.709 & -0.296 & -0.139\end{array}$

$\begin{array}{lrrr}\text { C } & 0.358 & 0.237 & 0.000 \\ \text { C } & -1.156 & 0.239 & 0.000 \\ \text { H } & -1.475 & 0.797 & 0.881 \\ \text { H } & -1.475 & 0.797 & -0.881 \\ \text { N } & -1.753 & -1.074 & 0.000 \\ \text { H } & -1.479 & -1.602 & -0.819 \\ \text { H } & -1.479 & -1.602 & 0.819 \\ \text { F } & 0.851 & -0.391 & -1.077 \\ \text { F } & 0.851 & -0.391 & 1.077 \\ \text { F } & 0.851 & 1.480 & 0.000\end{array}$

N28

$\begin{array}{lrrr}\mathrm{C} & 0.000 & 0.005 & 0.016 \\ \mathrm{C} & -1.250 & 0.801 & -0.346 \\ \mathrm{H} & -1.255 & 1.753 & 0.184 \\ \mathrm{H} & -1.291 & 0.992 & -1.419 \\ \mathrm{H} & -2.151 & 0.249 & -0.067 \\ \mathrm{C} & 0.000 & -1.330 & -0.735 \\ \mathrm{H} & 0.001 & -1.180 & -1.817 \\ \mathrm{H} & 0.885 & -1.915 & -0.474 \\ \mathrm{H} & -0.884 & -1.916 & -0.474 \\ \mathrm{C} & 1.249 & 0.801 & -0.345 \\ \mathrm{H} & 1.254 & 1.754 & 0.185 \\ \mathrm{H} & 2.151 & 0.250 & -0.066 \\ \mathrm{H} & 1.291 & 0.992 & -1.419 \\ \mathrm{~N} & 0.000 & -0.175 & 1.473 \\ \mathrm{H} & -0.815 & -0.709 & 1.758 \\ \mathrm{H} & 0.815 & -0.709 & 1.759\end{array}$

N29

$\begin{array}{lrrr}\text { C } & -2.304 & -0.295 & -0.091 \\ \text { C } & -1.392 & -1.331 & 0.058 \\ \text { C } & -0.036 & -1.061 & 0.171 \\ \text { C } & 0.427 & 0.252 & 0.136 \\ \text { C } & -0.493 & 1.284 & -0.020 \\ \text { C } & -1.850 & 1.016 & -0.129 \\ \text { H } & -3.362 & -0.508 & -0.181 \\ \text { H } & -1.740 & -2.356 & 0.086 \\ \text { H } & 0.681 & -1.864 & 0.281\end{array}$

N

$\mathrm{H}$

C

$\mathrm{H}$

$\mathrm{H}$

C

$\mathrm{H}$

$\mathrm{H}$

$\mathrm{H}$

C

$\mathrm{H}$

$\mathrm{H}$

C

$\mathrm{H}$

$\mathrm{H}$

$\mathrm{H}$

N31

C

C

C

C

$\mathrm{H}$

$\mathrm{H}$

$\mathrm{H}$

$\mathrm{H}$

$\mathrm{H}$

$\mathrm{H}$

$\mathrm{H}$

$\mathrm{H}$

N

$\mathrm{H}$

N32

$\begin{array}{lrrr}\text { C } & -1.488 & 0.774 & -0.055 \\ \text { C } & -0.019 & 1.146 & 0.161 \\ \text { C } & -0.019 & -1.146 & 0.161 \\ \text { C } & -1.488 & -0.774 & -0.056 \\ \text { H } & -2.127 & 1.193 & 0.721\end{array}$

$\begin{array}{rrr}0.773 & 1.024 & -0.058 \\ 1.153 & -0.444 & 0.176 \\ -1.152 & -0.446 & 0.176 \\ -0.775 & 1.022 & -0.058 \\ 1.192 & 1.674 & 0.709 \\ 1.152 & 1.361 & -1.022 \\ 1.291 & -0.624 & 1.253 \\ 2.069 & -0.735 & -0.338 \\ -2.068 & -0.740 & -0.337 \\ -1.289 & -0.626 & 1.253 \\ -1.154 & 1.358 & -1.022 \\ -1.195 & 1.672 & 0.708 \\ 0.001 & -1.162 & -0.359 \\ 0.002 & -2.140 & -0.098\end{array}$




$\begin{array}{lrrr}\mathrm{H} & -1.838 & 1.155 & -1.013 \\ \mathrm{H} & 0.188 & 1.275 & 1.239 \\ \mathrm{H} & 0.281 & 2.062 & -0.350 \\ \mathrm{H} & 0.281 & -2.062 & -0.349 \\ \mathrm{H} & 0.188 & -1.275 & 1.239 \\ \mathrm{H} & -1.838 & -1.155 & -1.014 \\ \mathrm{H} & -2.127 & -1.193 & 0.720 \\ \mathrm{~N} & 0.708 & 0.000 & -0.363 \\ \mathrm{C} & 2.110 & 0.000 & -0.020 \\ \mathrm{H} & 2.596 & -0.884 & -0.437 \\ \mathrm{H} & 2.596 & 0.884 & -0.437 \\ \mathrm{H} & 2.269 & 0.000 & 1.073\end{array}$

N33

C

C

C

C

$\mathrm{H}$

$\mathrm{H}$

$\mathrm{H}$

$\mathrm{H}$

$\mathrm{H}$

$\mathrm{H}$

$\mathrm{H}$

$\mathrm{H}$

N

$\mathrm{H}$

C

$\mathrm{H}$

$\mathrm{H}$

N34

$\begin{array}{lrrr}\text { C } & -0.316 & -1.200 & 0.179 \\ \mathrm{C} & -0.316 & 1.200 & 0.179 \\ \mathrm{C} & 1.150 & 1.248 & -0.228 \\ \mathrm{C} & 1.150 & -1.249 & -0.228 \\ \mathrm{H} & -0.849 & 2.068 & -0.214 \\ \mathrm{H} & -0.391 & -1.242 & 1.283 \\ \mathrm{H} & -0.849 & -2.068 & -0.214 \\ \mathrm{H} & 1.207 & 1.308 & -1.318 \\ \mathrm{H} & 1.612 & 2.150 & 0.178 \\ \mathrm{H} & 1.207 & -1.308 & -1.318 \\ \mathrm{H} & 1.612 & -2.150 & 0.178 \\ \mathrm{H} & -0.391 & 1.242 & 1.283 \\ \mathrm{~N} & -0.965 & 0.000 & -0.327 \\ \mathrm{C} & 1.880 & 0.000 & 0.255 \\ \mathrm{H} & 2.916 & 0.000 & -0.089 \\ \mathrm{H} & 1.906 & 0.000 & 1.350 \\ \mathrm{C} & -2.375 & 0.000 & -0.002\end{array}$

$\begin{array}{rrrr}\mathrm{H} & -2.853 & 0.885 & -0.425 \\ \mathrm{H} & -2.853 & -0.884 & -0.425 \\ \mathrm{H} & -2.553 & 0.000 & 1.087\end{array}$

N35

$\begin{array}{lrrr}\mathrm{C} & 1.196 & 0.715 & -0.201 \\ \mathrm{C} & -1.199 & 0.710 & -0.201 \\ \mathrm{C} & -1.165 & -0.754 & 0.197 \\ \mathrm{C} & 1.168 & -0.749 & 0.197 \\ \mathrm{H} & -2.085 & 1.186 & 0.222 \\ \mathrm{H} & 1.256 & 0.776 & -1.298 \\ \mathrm{H} & 2.080 & 1.196 & 0.222 \\ \mathrm{H} & -1.199 & -0.832 & 1.292 \\ \mathrm{H} & -2.011 & -1.295 & -0.225 \\ \mathrm{H} & 1.203 & -0.827 & 1.292 \\ \mathrm{H} & 2.017 & -1.286 & -0.226 \\ \mathrm{H} & -1.260 & 0.771 & -1.298 \\ \mathrm{O} & 0.003 & -1.383 & -0.290 \\ \mathrm{~N} & -0.003 & 1.357 & 0.325 \\ \mathrm{H} & -0.005 & 2.344 & 0.105\end{array}$

N36

$\begin{array}{lrrr}\text { C } & -0.286 & -1.189 & 0.176 \\ \text { C } & -0.286 & 1.189 & 0.176 \\ \text { C } & 1.185 & 1.162 & -0.194 \\ \text { C } & 1.185 & -1.162 & -0.194 \\ \text { H } & -0.760 & 2.074 & -0.255 \\ \text { H } & -0.380 & -1.253 & 1.275 \\ \text { H } & -0.760 & -2.074 & -0.255 \\ \text { H } & 1.285 & 1.197 & -1.287 \\ \text { H } & 1.708 & 2.014 & 0.238 \\ \text { H } & 1.285 & -1.197 & -1.287 \\ \text { H } & 1.708 & -2.014 & 0.238 \\ \text { H } & -0.380 & 1.253 & 1.275 \\ \text { O } & 1.812 & 0.000 & 0.305 \\ \text { N } & -0.949 & 0.000 & -0.335 \\ \text { C } & -2.358 & 0.000 & -0.007 \\ \text { H } & -2.836 & 0.885 & -0.430 \\ \text { H } & -2.836 & -0.885 & -0.430 \\ \text { H } & -2.533 & 0.000 & 1.082\end{array}$

N37

$\begin{array}{lrrr}\mathrm{N} & 0.000 & 0.000 & -0.387 \\ \mathrm{C} & 1.193 & -0.687 & 0.062 \\ \mathrm{H} & 1.197 & -1.709 & -0.317 \\ \mathrm{H} & 2.079 & -0.177 & -0.317 \\ \mathrm{H} & 1.263 & -0.727 & 1.163 \\ \mathrm{C} & -1.192 & -0.690 & 0.062 \\ \mathrm{H} & -2.079 & -0.182 & -0.317\end{array}$




$\begin{array}{lrrr}\mathrm{H} & -1.193 & -1.713 & -0.317 \\ \mathrm{H} & -1.261 & -0.730 & 1.163 \\ \mathrm{C} & -0.002 & 1.377 & 0.062 \\ \mathrm{H} & 0.882 & 1.891 & -0.317 \\ \mathrm{H} & -0.887 & 1.889 & -0.317 \\ \mathrm{H} & -0.002 & 1.457 & 1.163\end{array}$

N38

$\begin{array}{lrrr}\mathrm{C} & 0.794 & 0.381 & -1.325 \\ \mathrm{C} & -1.288 & 0.002 & 0.001 \\ \mathrm{C} & -0.758 & 0.399 & -1.381 \\ \mathrm{H} & 1.206 & 1.363 & -1.561 \\ \mathrm{H} & 1.204 & -0.325 & -2.049 \\ \mathrm{H} & -1.127 & 1.391 & -1.650 \\ \mathrm{H} & -1.129 & -0.299 & -2.135 \\ \mathrm{C} & 0.797 & 0.955 & 0.992 \\ \mathrm{H} & 1.207 & 0.667 & 1.962 \\ \mathrm{H} & 1.209 & 1.934 & 0.744 \\ \mathrm{C} & -0.756 & 0.999 & 1.035 \\ \mathrm{H} & -1.126 & 0.737 & 2.029 \\ \mathrm{H} & -1.124 & 2.002 & 0.808 \\ \mathrm{H} & -2.378 & 0.004 & 0.001 \\ \mathrm{C} & -0.760 & -1.394 & 0.347 \\ \mathrm{H} & -1.130 & -1.698 & 1.329 \\ \mathrm{H} & -1.132 & -2.122 & -0.377 \\ \mathrm{C} & 0.792 & -1.339 & 0.331 \\ \mathrm{H} & 1.204 & -1.614 & 1.304 \\ \mathrm{H} & 1.201 & -2.036 & -0.402 \\ \mathrm{~N} & 1.283 & -0.002 & -0.001\end{array}$

$\begin{array}{lrrr}\text { C } & -1.040 & -0.032 & 0.000 \\ \text { C } & -0.266 & 1.198 & 0.000 \\ \text { C } & 1.101 & 1.181 & 0.000 \\ \text { C } & 1.784 & -0.045 & 0.000 \\ \text { C } & 0.990 & -1.184 & 0.000 \\ \text { N } & -0.332 & -1.219 & 0.000 \\ \text { H } & -0.822 & 2.128 & 0.000 \\ \text { H } & 2.864 & -0.110 & 0.000 \\ \text { H } & 1.481 & -2.160 & 0.000 \\ \text { H } & 1.656 & 2.116 & 0.000 \\ \text { O } & -2.283 & -0.019 & 0.000\end{array}$

NA2

$\begin{array}{lrrr}\mathrm{C} & -1.113 & -1.126 & 0.000 \\ \mathrm{C} & 0.261 & -1.194 & 0.000 \\ \mathrm{C} & 1.068 & 0.000 & -0.001 \\ \mathrm{C} & 0.261 & 1.194 & 0.000 \\ \mathrm{C} & -1.113 & 1.126 & 0.000 \\ \mathrm{~N} & -1.845 & 0.000 & 0.000 \\ \mathrm{H} & -1.686 & -2.052 & 0.001 \\ \mathrm{H} & 0.765 & -2.154 & 0.001 \\ \mathrm{H} & 0.765 & 2.154 & 0.000 \\ \mathrm{H} & -1.686 & 2.052 & 0.000 \\ \mathrm{O} & 2.320 & 0.000 & 0.000\end{array}$

$\begin{array}{lrrr}\text { N39 } & & & \\ \text { C } & 0.778 & -1.014 & 0.929 \\ \text { C } & -0.778 & -1.014 & 0.929 \\ \text { H } & 1.176 & -1.983 & 0.621 \\ \text { H } & 1.177 & -0.793 & 1.921 \\ \text { H } & -1.177 & -1.983 & 0.622 \\ \text { H } & -1.176 & -0.792 & 1.921 \\ \text { C } & 0.778 & -0.297 & -1.343 \\ \text { H } & 1.176 & 0.454 & -2.028 \\ \text { H } & 1.177 & -1.267 & -1.647 \\ \text { C } & -0.778 & -0.298 & -1.343 \\ \text { H } & -1.177 & 0.452 & -2.028 \\ \text { H } & -1.176 & -1.268 & -1.646 \\ \text { C } & -0.778 & 1.312 & 0.413 \\ \text { H } & -1.176 & 2.060 & -0.275 \\ \text { H } & -1.177 & 1.530 & 1.406 \\ \text { C } & 0.778 & 1.312 & 0.414 \\ \text { H } & 1.177 & 2.060 & -0.273 \\ \text { H } & 1.176 & 1.529 & 1.407 \\ \text { N } & -1.279 & 0.000 & 0.000\end{array}$

NA3

$\begin{array}{lrrr}\text { C } & -0.219 & 0.503 & -0.022 \\ \mathrm{C} & -0.775 & -0.796 & -0.007 \\ \mathrm{C} & 1.279 & -1.673 & 0.008 \\ \mathrm{C} & 1.168 & 0.607 & -0.021 \\ \mathrm{C} & -2.299 & 0.647 & 0.011 \\ \mathrm{H} & -3.297 & 1.073 & 0.027 \\ \mathrm{~N} & 1.923 & -0.487 & 0.002 \\ \mathrm{~N} & -0.013 & -1.917 & 0.002 \\ \mathrm{~N} & -1.219 & 1.434 & -0.006 \\ \mathrm{~N} & -2.124 & -0.689 & 0.011 \\ \mathrm{H} & 1.933 & -2.542 & 0.022 \\ \mathrm{~N} & 1.795 & 1.845 & -0.071 \\ \mathrm{H} & 1.197 & 2.596 & 0.241 \\ \mathrm{H} & 2.717 & 1.840 & 0.335\end{array}$

\section{NA4}

$\begin{array}{lrrr}C & -0.890 & 0.463 & 0.012 \\ C & -0.576 & -0.913 & -0.006 \\ C & 1.630 & -0.550 & -0.015\end{array}$




$\begin{array}{lrrr}\mathrm{C} & 0.144 & 1.440 & 0.002 \\ \mathrm{C} & -2.656 & -0.659 & 0.006 \\ \mathrm{H} & -3.709 & -0.913 & 0.008 \\ \mathrm{~N} & 1.415 & 0.801 & -0.011 \\ \mathrm{~N} & 0.706 & -1.436 & -0.001 \\ \mathrm{O} & 0.121 & 2.664 & -0.007 \\ \mathrm{~N} & -2.249 & 0.605 & 0.020 \\ \mathrm{~N} & -1.708 & -1.629 & -0.009 \\ \mathrm{H} & 2.196 & 1.428 & -0.124 \\ \mathrm{~N} & 2.988 & -0.926 & -0.071 \\ \mathrm{H} & 3.536 & -0.481 & 0.655 \\ \mathrm{H} & 3.035 & -1.932 & 0.030\end{array}$

$\begin{array}{lrrr}\text { NA5 } & & & \\ \text { C } & 0.000 & 0.781 & 0.000 \\ \text { C } & 0.578 & -0.525 & 0.000 \\ \text { C } & -0.241 & -1.659 & 0.000 \\ \text { C } & -1.615 & -1.485 & 0.000 \\ \text { C } & -2.185 & -0.198 & 0.000 \\ \text { C } & -1.391 & 0.936 & 0.000 \\ \text { C } & 2.097 & 0.929 & 0.000 \\ \text { H } & 0.199 & -2.650 & 0.000 \\ \text { H } & -2.267 & -2.352 & 0.000 \\ \text { H } & -3.265 & -0.098 & 0.000 \\ \text { H } & -1.829 & 1.928 & 0.000 \\ \text { H } & 3.090 & 1.369 & 0.000 \\ \text { N } & 1.006 & 1.706 & 0.000 \\ \text { N } & 1.939 & -0.401 & 0.000\end{array}$

NA6

$\begin{array}{rrrr}\text { C } & 0.000 & 0.706 & 0.327 \\ \text { C } & 0.000 & -0.706 & 0.327 \\ \text { C } & 0.000 & -1.423 & -0.881 \\ \text { C } & 0.000 & -0.708 & -2.059 \\ \text { C } & 0.000 & 0.708 & -2.059 \\ \text { C } & 0.000 & 1.423 & -0.881 \\ \text { H } & 0.000 & -2.507 & -0.880 \\ \text { H } & 0.000 & -1.233 & -3.008 \\ \text { H } & 0.000 & 1.233 & -3.008 \\ \text { H } & 0.000 & 2.507 & -0.880 \\ \text { N } & 0.000 & 1.106 & 1.621 \\ \text { N } & 0.000 & -1.106 & 1.621 \\ \text { N } & 0.000 & 0.000 & 2.347\end{array}$

\section{NA7}

$\begin{array}{lrrr}\mathrm{C} & -1.101 & -0.253 & 0.000 \\ \mathrm{C} & 0.698 & 1.216 & 0.000 \\ \mathrm{C} & 1.101 & -0.283 & 0.000 \\ \mathrm{~N} & 0.006 & -1.057 & 0.000\end{array}$

$\begin{array}{lrrr}\mathrm{H} & 1.110 & 1.704 & -0.889 \\ \mathrm{~N} & -0.725 & 1.095 & 0.000 \\ \mathrm{O} & 2.283 & -0.612 & 0.000 \\ \mathrm{O} & -2.279 & -0.586 & 0.000 \\ \mathrm{H} & -1.408 & 1.828 & 0.000 \\ \mathrm{H} & 1.110 & 1.704 & 0.889\end{array}$

NA8

$\begin{array}{lrrr}\text { C } & -1.091 & -0.235 & 0.000 \\ \text { C } & -0.661 & 1.237 & 0.000 \\ \text { C } & 0.660 & 1.238 & 0.000 \\ \text { C } & 1.091 & -0.235 & 0.000 \\ \text { N } & 0.000 & -1.037 & 0.000 \\ \text { H } & 1.363 & 2.060 & 0.000 \\ \text { O } & -2.272 & -0.556 & 0.000 \\ \text { O } & 2.273 & -0.556 & 0.000 \\ \text { H } & -1.365 & 2.059 & 0.000\end{array}$

NA9

$\begin{array}{lrrr}\mathrm{C} & 1.107 & -0.286 & 0.000 \\ \mathrm{C} & 0.758 & 1.221 & 0.000 \\ \mathrm{C} & -0.758 & 1.221 & 0.000 \\ \mathrm{C} & -1.107 & -0.286 & 0.000 \\ \mathrm{~N} & 0.000 & -1.061 & 0.000 \\ \mathrm{H} & 1.209 & 1.684 & 0.879 \\ \mathrm{H} & -1.209 & 1.684 & -0.879 \\ \mathrm{O} & 2.274 & -0.658 & 0.000 \\ \mathrm{O} & -2.274 & -0.658 & 0.000 \\ \mathrm{H} & 1.209 & 1.684 & -0.879 \\ \mathrm{H} & -1.209 & 1.684 & 0.879\end{array}$

NA10

$\begin{array}{lrrr}\text { C } & 0.000 & -0.690 & -0.144 \\ \text { C } & 0.000 & 0.690 & -0.144 \\ \text { C } & 0.000 & 1.412 & -1.318 \\ \text { C } & 0.000 & 0.696 & -2.518 \\ \text { C } & 0.000 & -0.696 & -2.518 \\ \text { C } & 0.000 & -1.412 & -1.318 \\ \text { C } & 0.000 & -1.107 & 1.322 \\ \text { C } & 0.000 & 1.107 & 1.322 \\ \text { H } & 0.000 & 2.495 & -1.300 \\ \text { H } & 0.000 & 1.226 & -3.463 \\ \text { H } & 0.000 & -1.226 & -3.463 \\ \text { H } & 0.000 & -2.495 & -1.300 \\ \text { O } & 0.000 & -2.281 & 1.666 \\ \text { O } & 0.000 & 2.281 & 1.666 \\ \text { N } & 0.000 & 0.000 & 2.108\end{array}$




$\begin{array}{rrrr}\text { C } & 0.297 & -0.599 & 0.000 \\ \text { C } & 0.778 & 0.691 & 0.000 \\ \text { C } & 2.139 & 0.935 & 0.000 \\ \text { C } & 3.003 & -0.155 & 0.000 \\ \text { C } & 2.506 & -1.458 & 0.000 \\ \text { C } & 1.135 & -1.697 & 0.000 \\ \text { C } & -0.357 & 1.708 & 0.000 \\ \text { H } & 2.498 & 1.956 & 0.000 \\ \text { H } & 4.075 & 0.004 & 0.000 \\ \text { H } & 3.197 & -2.293 & 0.000 \\ \text { H } & 0.737 & -2.704 & 0.000 \\ \text { S } & -1.486 & -0.476 & 0.000 \\ \text { N } & -1.568 & 1.118 & 0.000 \\ \text { O } & -0.113 & 2.907 & 0.000 \\ \text { O } & -1.991 & -1.061 & 1.220 \\ \text { O } & -1.991 & -1.061 & -1.220\end{array}$

\section{NA12}

$\begin{array}{lrrr}\mathrm{C} & -1.096 & 0.002 & 0.000 \\ \mathrm{C} & 0.898 & 0.690 & 0.000 \\ \mathrm{C} & 0.895 & -0.694 & 0.000 \\ \mathrm{~N} & -0.393 & -1.139 & 0.000 \\ \mathrm{H} & -2.181 & 0.005 & 0.000 \\ \mathrm{H} & 1.740 & 1.371 & 0.000 \\ \mathrm{H} & 1.735 & -1.377 & 0.000 \\ \mathrm{~N} & -0.389 & 1.141 & 0.000\end{array}$

NA13

$\begin{array}{lrrr}\mathrm{C} & 0.524 & 0.572 & 0.000 \\ \mathrm{C} & 0.000 & -0.758 & 0.000 \\ \mathrm{C} & -2.078 & 0.069 & 0.000 \\ \mathrm{C} & -0.393 & 1.607 & 0.000 \\ \mathrm{C} & 2.093 & -0.807 & 0.000 \\ \mathrm{H} & 3.100 & -1.211 & 0.000 \\ \mathrm{~N} & -1.705 & 1.359 & 0.000 \\ \mathrm{~N} & -1.319 & -1.013 & 0.000 \\ \mathrm{~N} & 1.890 & 0.512 & 0.000 \\ \mathrm{~N} & 1.029 & -1.635 & 0.000 \\ \mathrm{H} & -0.081 & 2.649 & 0.000 \\ \mathrm{H} & -3.153 & -0.100 & 0.000\end{array}$

NA14

$\begin{array}{lrrr}\mathrm{C} & 1.666 & -0.090 & 0.000 \\ \mathrm{~N} & 0.739 & 0.966 & 0.000 \\ \mathrm{C} & -0.642 & 0.879 & 0.000 \\ \mathrm{C} & -1.119 & -0.471 & 0.000 \\ \mathrm{C} & -0.169 & -1.466 & 0.000 \\ \mathrm{~N} & 1.161 & -1.347 & 0.000 \\ \mathrm{H} & -0.531 & -2.497 & 0.000\end{array}$

$\begin{array}{lrrr}\mathrm{H} & 1.132 & 1.896 & 0.000 \\ \mathrm{O} & -1.335 & 1.901 & 0.000 \\ \mathrm{O} & 2.861 & 0.199 & 0.000 \\ \mathrm{C} & -2.594 & -0.717 & 0.000 \\ \mathrm{H} & -3.077 & -0.273 & 0.875 \\ \mathrm{H} & -3.077 & -0.273 & -0.875 \\ \mathrm{H} & -2.802 & -1.790 & 0.000\end{array}$

NA15

$\begin{array}{lrrr}\mathrm{C} & -1.250 & -0.298 & 0.000 \\ \mathrm{~N} & 0.006 & -0.926 & 0.000 \\ \mathrm{C} & 1.259 & -0.329 & 0.000 \\ \mathrm{C} & 1.173 & 1.098 & 0.000 \\ \mathrm{C} & -0.077 & 1.672 & 0.000 \\ \mathrm{~N} & -1.262 & 1.061 & 0.000 \\ \mathrm{H} & 2.081 & 1.681 & 0.000 \\ \mathrm{H} & -0.134 & 2.762 & 0.000 \\ \mathrm{H} & -0.010 & -1.935 & 0.000 \\ \mathrm{O} & 2.276 & -1.025 & 0.000 \\ \mathrm{O} & -2.248 & -1.014 & 0.000\end{array}$

NA16

$\begin{array}{lrrr}\text { C } & -0.958 & -0.888 & 0.000 \\ \text { C } & -1.090 & 0.496 & 0.000 \\ \text { C } & 1.269 & 0.836 & 0.000 \\ \text { C } & 0.323 & -1.510 & 0.000 \\ \text { C } & -2.990 & -0.361 & 0.000 \\ \text { H } & -4.070 & -0.428 & 0.000 \\ \text { N } & 1.389 & -0.545 & 0.000 \\ \text { N } & 0.005 & 1.349 & 0.000 \\ \text { O } & 0.599 & -2.698 & 0.000 \\ \text { O } & 2.259 & 1.563 & 0.000 \\ \text { C } & 2.755 & -1.037 & 0.000 \\ \text { H } & 3.287 & -0.676 & -0.881 \\ \text { H } & 3.287 & -0.676 & 0.881 \\ \text { H } & 2.707 & -2.121 & 0.000 \\ \mathrm{C} & -0.158 & 2.786 & 0.000 \\ \text { H } & 0.312 & 3.223 & 0.882 \\ \text { H } & 0.312 & 3.223 & -0.882 \\ \text { H } & -1.226 & 2.986 & 0.000 \\ \text { N } & -2.221 & -1.433 & 0.000 \\ \text { N } & -2.371 & 0.859 & 0.000\end{array}$

NA17

$\begin{array}{lrrr}\mathrm{C} & -1.029 & 0.051 & 0.001 \\ \mathrm{O} & -1.521 & 1.197 & 0.000 \\ \mathrm{~N} & -1.536 & -1.147 & 0.000 \\ \mathrm{H} & -2.550 & -1.032 & -0.001 \\ \mathrm{C} & 0.529 & -0.008 & 0.000\end{array}$




$\begin{array}{lrrr}\text { F } & 1.031 & -0.648 & -1.077 \\ \text { F } & 1.099 & 1.208 & -0.002 \\ \text { F } & 1.032 & -0.646 & 1.078 \\ & & & \\ \text { OA1 } & & & \\ \text { C } & 1.820 & 0.000 & 0.000 \\ \text { C } & 1.094 & 1.194 & 0.000 \\ \text { C } & -0.286 & 1.206 & 0.000 \\ \text { C } & -1.075 & 0.000 & 0.000 \\ \text { C } & -0.286 & -1.206 & 0.000 \\ \text { C } & 1.094 & -1.194 & 0.000 \\ \text { H } & 2.903 & 0.000 & 0.000 \\ \text { H } & 1.630 & 2.140 & 0.000 \\ \text { H } & -0.830 & 2.145 & 0.000 \\ \text { H } & -0.830 & -2.145 & 0.000 \\ \text { H } & 1.630 & -2.140 & 0.000 \\ \text { O } & -2.334 & 0.000 & 0.000\end{array}$

\section{OA2}

$\begin{array}{lrrr}\text { C } & 0.878 & 0.287 & 0.000 \\ \mathrm{C} & 0.356 & -1.001 & 0.000 \\ \mathrm{C} & -1.019 & -1.209 & 0.000 \\ \mathrm{C} & -1.978 & -0.144 & 0.000 \\ \mathrm{C} & -1.373 & 1.164 & 0.000 \\ \mathrm{C} & -0.007 & 1.362 & 0.000 \\ \mathrm{H} & 1.012 & -1.864 & 0.000 \\ \mathrm{H} & -1.408 & -2.222 & 0.000 \\ \mathrm{H} & -2.046 & 2.014 & 0.000 \\ \mathrm{H} & 0.408 & 2.365 & 0.000 \\ \mathrm{O} & -3.227 & -0.325 & 0.000 \\ \mathrm{O} & 2.240 & 0.598 & 0.000 \\ \mathrm{C} & 3.115 & -0.485 & 0.000 \\ \mathrm{H} & 2.983 & -1.117 & -0.888 \\ \mathrm{H} & 4.127 & -0.079 & 0.000 \\ \mathrm{H} & 2.983 & -1.117 & 0.888\end{array}$

OA3

$\begin{array}{lrrr}\text { C } & -0.307 & 0.036 & 0.000 \\ \mathrm{C} & 0.427 & -1.161 & 0.000 \\ \mathrm{C} & 1.805 & -1.194 & 0.000 \\ \mathrm{C} & 2.614 & -0.002 & 0.000 \\ \mathrm{C} & 1.836 & 1.206 & 0.000 \\ \mathrm{C} & 0.452 & 1.211 & 0.000 \\ \mathrm{H} & -0.107 & -2.109 & 0.000 \\ \mathrm{H} & 2.330 & -2.144 & 0.000 \\ \mathrm{H} & 2.383 & 2.143 & 0.000 \\ \mathrm{H} & -0.047 & 2.174 & 0.000 \\ \mathrm{O} & 3.872 & -0.022 & 0.000 \\ \mathrm{C} & -1.833 & 0.004 & 0.000\end{array}$

$\begin{array}{lrrr}\mathrm{C} & -2.351 & -0.730 & 1.247 \\ \mathrm{H} & -2.018 & -0.216 & 2.151 \\ \mathrm{H} & -3.446 & -0.773 & 1.251 \\ \mathrm{H} & -1.968 & -1.750 & 1.285 \\ \mathrm{C} & -2.351 & -0.729 & -1.247 \\ \mathrm{H} & -3.446 & -0.773 & -1.252 \\ \mathrm{H} & -2.018 & -0.215 & -2.151 \\ \mathrm{H} & -1.968 & -1.750 & -1.286 \\ \mathrm{C} & -2.438 & 1.409 & 0.000 \\ \mathrm{H} & -2.130 & 1.971 & 0.884 \\ \mathrm{H} & -2.130 & 1.971 & -0.883 \\ \mathrm{H} & -3.530 & 1.343 & 0.000\end{array}$

OA4

$\begin{array}{lrrr}\text { C } & -1.335 & 0.000 & -0.017 \\ \text { C } & -0.597 & -1.187 & -0.014 \\ \text { C } & 0.785 & -1.203 & -0.003 \\ \text { C } & 1.576 & 0.000 & 0.006 \\ \text { C } & 0.785 & 1.203 & -0.003 \\ \text { C } & -0.597 & 1.187 & -0.014 \\ \text { H } & -1.134 & -2.134 & -0.024 \\ \text { H } & 1.324 & -2.144 & -0.009 \\ \text { H } & 1.325 & 2.144 & -0.009 \\ \text { H } & -1.134 & 2.135 & -0.023 \\ \text { O } & 2.836 & 0.000 & 0.015 \\ \text { C } & -2.840 & 0.000 & 0.022 \\ \text { H } & -3.250 & -0.879 & -0.484 \\ \text { H } & -3.239 & -0.006 & 1.044 \\ \text { H } & -3.249 & 0.884 & -0.474\end{array}$

OA5

$\begin{array}{lrrr}\mathrm{C} & 0.909 & 0.000 & 0.000 \\ \mathrm{C} & 0.211 & -1.202 & 0.000 \\ \mathrm{C} & -1.169 & -1.207 & 0.000 \\ \mathrm{C} & -1.958 & 0.000 & 0.000 \\ \mathrm{C} & -1.169 & 1.207 & 0.000 \\ \mathrm{C} & 0.211 & 1.202 & 0.000 \\ \mathrm{H} & 0.764 & -2.135 & 0.000 \\ \mathrm{H} & -1.709 & -2.147 & 0.000 \\ \mathrm{H} & -1.709 & 2.147 & 0.000 \\ \mathrm{H} & 0.764 & 2.135 & 0.000 \\ \mathrm{O} & -3.214 & 0.000 & 0.000 \\ \mathrm{Cl} & 2.670 & 0.000 & 0.000\end{array}$

OA6

$\begin{array}{lrrr}C & -1.038 & 0.000 & 0.000 \\ C & -0.311 & -1.207 & 0.000 \\ C & 1.058 & -1.216 & 0.000 \\ C & 1.844 & 0.000 & 0.000\end{array}$




$\begin{array}{lrrr}\mathrm{C} & 1.058 & 1.216 & 0.000 \\ \mathrm{C} & -0.311 & 1.207 & 0.000 \\ \mathrm{H} & -0.860 & -2.143 & 0.000 \\ \mathrm{H} & 1.605 & -2.151 & 0.000 \\ \mathrm{H} & 1.605 & 2.151 & 0.000 \\ \mathrm{H} & -0.860 & 2.143 & 0.000 \\ \mathrm{O} & 3.092 & 0.000 & 0.000 \\ \mathrm{C} & -2.456 & 0.000 & 0.000 \\ \mathrm{~N} & -3.612 & 0.000 & 0.000\end{array}$

OA7

$\begin{array}{lrrr}\text { C } & -0.326 & 0.000 & -0.051 \\ \text { C } & 0.394 & -1.202 & -0.031 \\ \text { C } & 1.766 & -1.212 & -0.006 \\ \text { C } & 2.553 & 0.000 & 0.009 \\ \text { C } & 1.766 & 1.212 & -0.006 \\ \text { C } & 0.394 & 1.203 & -0.030 \\ \text { H } & -0.151 & -2.141 & -0.043 \\ \text { H } & 2.311 & -2.149 & 0.000 \\ \text { H } & 2.312 & 2.149 & 0.000 \\ \text { H } & -0.150 & 2.142 & -0.043 \\ \text { O } & 3.804 & 0.000 & 0.031 \\ \text { C } & -1.796 & 0.000 & -0.004 \\ \text { F } & -2.352 & 1.085 & -0.591 \\ \text { F } & -2.352 & -1.078 & -0.603 \\ \text { F } & -2.325 & -0.007 & 1.255\end{array}$

OA8

$\begin{array}{lrrr}\text { C } & 2.120 & -0.629 & -0.002 \\ \text { C } & 0.698 & -0.871 & -0.027 \\ \text { C } & -0.219 & 0.159 & -0.040 \\ \text { C } & 0.160 & 1.503 & -0.023 \\ \text { C } & 1.528 & 1.774 & 0.003 \\ \text { C } & 2.471 & 0.768 & 0.014 \\ \text { H } & -0.579 & 2.293 & -0.040 \\ \text { H } & 1.858 & 2.809 & 0.013 \\ \text { H } & 3.529 & 1.004 & 0.032 \\ \text { C } & -1.680 & -0.158 & -0.004 \\ \text { F } & -2.201 & -0.081 & 1.243 \\ \text { F } & -2.421 & 0.690 & -0.748 \\ \text { F } & -1.977 & -1.393 & -0.443 \\ \text { H } & 0.371 & -1.903 & -0.043 \\ \text { O } & 2.968 & -1.554 & 0.005\end{array}$

OA9

$\begin{array}{lrrr}C & -2.166 & 0.961 & 0.000 \\ C & -0.726 & 1.075 & 0.000 \\ C & -0.046 & -0.203 & 0.000 \\ C & -0.729 & -1.411 & 0.000\end{array}$

$\begin{array}{lrrr}\text { C } & -2.115 & -1.463 & 0.000 \\ \text { C } & -2.816 & -0.249 & 0.000 \\ \text { H } & -2.718 & 1.893 & 0.000 \\ \text { H } & -0.159 & -2.333 & 0.000 \\ \text { H } & -2.635 & -2.412 & 0.000 \\ \text { H } & -3.902 & -0.263 & 0.000 \\ \text { C } & 1.439 & -0.187 & 0.000 \\ \text { F } & 1.980 & 0.420 & 1.074 \\ \text { F } & 1.972 & -1.440 & 0.000 \\ \text { F } & 1.980 & 0.421 & -1.074 \\ \text { O } & -0.129 & 2.173 & 0.000\end{array}$

\section{OA10}

$\begin{array}{lrrr}\text { C } & 1.141 & 1.784 & 0.000 \\ \text { C } & -0.524 & 0.113 & 0.000 \\ \text { C } & 2.120 & 0.812 & 0.000 \\ \text { H } & 1.434 & 2.829 & 0.000 \\ \text { C } & 0.413 & -0.897 & 0.000 \\ \text { C } & 1.824 & -0.599 & 0.000 \\ \text { H } & 3.169 & 1.087 & 0.000 \\ \text { H } & 0.103 & -1.931 & 0.000 \\ \text { C } & -0.217 & 1.470 & 0.000 \\ \text { O } & 2.707 & -1.488 & 0.000 \\ \text { N } & -1.951 & -0.264 & 0.000 \\ \text { O } & -2.785 & 0.624 & 0.000 \\ \text { O } & -2.246 & -1.442 & 0.000 \\ \text { H } & -0.994 & 2.216 & 0.000\end{array}$

\section{OA11}

$\begin{array}{lrrr}\text { C } & 1.249 & 1.672 & 0.000 \\ \mathrm{C} & -0.756 & 0.384 & 0.000 \\ \mathrm{C} & 2.017 & 0.526 & 0.000 \\ \mathrm{H} & 1.746 & 2.639 & -0.001 \\ \mathrm{C} & -0.024 & -0.785 & 0.000 \\ \mathrm{C} & 1.421 & -0.784 & 0.000 \\ \mathrm{H} & 3.099 & 0.578 & -0.001 \\ \mathrm{H} & -0.493 & -1.760 & 0.000 \\ \mathrm{C} & -0.145 & 1.641 & 0.000 \\ \mathrm{O} & 2.090 & -1.849 & 0.000 \\ \mathrm{H} & -0.752 & 2.535 & 0.000 \\ \mathrm{O} & -2.141 & 0.409 & 0.000 \\ \mathrm{C} & -2.799 & -0.824 & 0.000 \\ \mathrm{H} & -2.550 & -1.417 & 0.886 \\ \mathrm{H} & -2.549 & -1.416 & -0.888 \\ \mathrm{H} & -3.868 & -0.609 & -0.001\end{array}$

\section{OA12}

$\begin{array}{lrrr}C & -0.658 & 0.000 & 0.000 \\ C & 0.050 & -1.216 & 0.000\end{array}$




$\begin{array}{lrrr}\mathrm{C} & 1.413 & -1.222 & 0.000 \\ \mathrm{C} & 2.198 & 0.000 & 0.000 \\ \mathrm{C} & 1.413 & 1.222 & 0.000 \\ \mathrm{C} & 0.050 & 1.216 & 0.000 \\ \mathrm{H} & -0.516 & -2.138 & 0.000 \\ \mathrm{H} & 1.965 & -2.155 & 0.000 \\ \mathrm{H} & 1.965 & 2.155 & 0.000 \\ \mathrm{H} & -0.516 & 2.138 & 0.000 \\ \mathrm{O} & 3.441 & 0.000 & 0.000 \\ \mathrm{~N} & -2.070 & 0.000 & 0.000 \\ \mathrm{O} & -2.671 & -1.075 & 0.000 \\ \mathrm{O} & -2.671 & 1.075 & 0.000\end{array}$

OA14

OA13

$\begin{array}{lrrr}\mathrm{C} & -1.234 & 0.452 & 0.000 \\ \mathrm{C} & 0.000 & -0.308 & 0.000 \\ \mathrm{C} & 1.234 & 0.452 & 0.000 \\ \mathrm{C} & 1.195 & 1.837 & 0.000 \\ \mathrm{C} & 0.000 & 2.549 & 0.000 \\ \mathrm{C} & -1.195 & 1.837 & 0.000 \\ \mathrm{H} & 2.121 & 2.401 & 0.000 \\ \mathrm{H} & 0.000 & 3.632 & 0.000 \\ \mathrm{H} & -2.121 & 2.401 & 0.000 \\ \mathrm{C} & -2.561 & -0.311 & 0.000 \\ \mathrm{C} & -2.660 & -1.195 & -1.254 \\ \mathrm{C} & -2.660 & -1.194 & 1.254 \\ \mathrm{C} & -3.774 & 0.622 & 0.000 \\ \mathrm{H} & -2.649 & -0.572 & -2.152 \\ \mathrm{H} & -1.816 & -1.881 & -1.288 \\ \mathrm{H} & -3.595 & -1.765 & -1.243 \\ \mathrm{H} & -2.649 & -0.571 & 2.152 \\ \mathrm{H} & -3.596 & -1.764 & 1.243 \\ \mathrm{H} & -1.816 & -1.881 & 1.289 \\ \mathrm{H} & -4.689 & 0.022 & 0.000 \\ \mathrm{H} & -3.791 & 1.262 & 0.885 \\ \mathrm{H} & -3.791 & 1.262 & -0.886 \\ \mathrm{C} & 2.561 & -0.311 & 0.000 \\ \mathrm{C} & 2.660 & -1.194 & 1.254 \\ \mathrm{C} & 2.660 & -1.195 & -1.254 \\ \mathrm{C} & 3.774 & 0.622 & 0.000 \\ \mathrm{H} & 2.649 & -0.571 & 2.152 \\ \mathrm{H} & 1.816 & -1.881 & 1.289 \\ \mathrm{H} & 3.596 & -1.764 & 1.243 \\ \mathrm{H} & 2.649 & -0.572 & -2.152 \\ \mathrm{H} & 3.595 & -1.765 & -1.243 \\ \mathrm{H} & 1.816 & -1.881 & -1.289 \\ \mathrm{H} & 4.689 & 0.022 & 0.000 \\ \mathrm{H} & 3.791 & 1.262 & -0.886 \\ \mathrm{H} & 3.791 & 1.262 & 0.885 \\ \mathrm{O} & 0.000 & -1.580 & 0.000\end{array}$

$\begin{array}{lrrr}\mathrm{C} & 0.212 & 0.001 & 0.000 \\ \mathrm{O} & 0.800 & -1.098 & 0.000 \\ \mathrm{O} & 0.694 & 1.152 & 0.000 \\ \mathrm{C} & -1.344 & -0.052 & 0.000 \\ \mathrm{H} & -1.721 & 0.477 & 0.878 \\ \mathrm{H} & -1.721 & 0.474 & -0.880 \\ \mathrm{H} & -1.710 & -1.079 & 0.001\end{array}$

OA15

$\begin{array}{lrrr}\text { C } & 1.826 & 0.000 & 0.000 \\ \text { O } & 2.354 & -1.127 & 0.001 \\ \text { O } & 2.354 & 1.127 & -0.001 \\ \mathrm{C} & 0.276 & 0.000 & 0.000 \\ \mathrm{C} & -0.434 & 1.197 & 0.000 \\ \mathrm{C} & -0.434 & -1.197 & 0.000 \\ \mathrm{C} & -1.823 & 1.201 & 0.000 \\ \mathrm{H} & 0.143 & 2.113 & 0.001 \\ \mathrm{C} & -1.823 & -1.201 & 0.000 \\ \mathrm{H} & 0.143 & -2.113 & -0.001 \\ \mathrm{C} & -2.524 & 0.000 & 0.000 \\ \mathrm{H} & -2.365 & 2.141 & 0.000 \\ \mathrm{H} & -2.364 & -2.141 & 0.000 \\ \mathrm{H} & -3.608 & 0.000 & 0.000\end{array}$

OA16

$\begin{array}{lrrr}\mathrm{C} & -2.961 & 0.000 & 0.000 \\ \mathrm{O} & -3.478 & -1.129 & 0.000 \\ \mathrm{O} & -3.478 & 1.129 & 0.000 \\ \mathrm{C} & -1.407 & 0.000 & 0.000 \\ \mathrm{C} & -0.702 & 1.201 & 0.000 \\ \mathrm{C} & -0.702 & -1.201 & 0.000 \\ \mathrm{C} & 0.683 & 1.215 & 0.000 \\ \mathrm{H} & -1.281 & 2.115 & 0.000 \\ \mathrm{C} & 0.683 & -1.215 & 0.000 \\ \mathrm{H} & -1.281 & -2.115 & 0.000 \\ \mathrm{C} & 1.354 & 0.000 & 0.000 \\ \mathrm{H} & 1.248 & 2.135 & 0.000 \\ \mathrm{H} & 1.248 & -2.135 & 0.000 \\ \mathrm{~N} & 2.818 & 0.000 & 0.000 \\ \mathrm{O} & 3.394 & -1.070 & 0.000 \\ \mathrm{O} & 3.394 & 1.070 & 0.000\end{array}$

OA17

$\begin{array}{lrrr}\mathrm{C} & 2.976 & 0.000 & 0.000 \\ \mathrm{O} & 3.483 & -1.130 & 0.000 \\ \mathrm{O} & 3.483 & 1.131 & -0.001 \\ \mathrm{C} & 1.418 & 0.000 & 0.000\end{array}$




$\begin{array}{lrrr}\text { C } & 0.717 & 1.199 & 0.000 \\ \text { C } & 0.718 & -1.199 & 0.000 \\ \text { C } & -0.669 & 1.174 & 0.000 \\ \text { H } & 1.277 & 2.124 & 0.000 \\ \text { C } & -0.668 & -1.174 & 0.000 \\ \text { H } & 1.277 & -2.124 & 0.000 \\ \text { C } & -1.398 & 0.000 & 0.000 \\ \text { H } & -2.476 & 0.000 & 0.000 \\ \text { N } & -1.415 & -2.445 & 0.000 \\ \text { O } & -0.780 & -3.474 & 0.000 \\ \text { O } & -2.628 & -2.390 & 0.000 \\ \text { N } & -1.416 & 2.445 & 0.000 \\ \text { O } & -2.629 & 2.389 & 0.000 \\ \text { O } & -0.781 & 3.474 & 0.000\end{array}$

\section{OA18}

$\begin{array}{rrrr}\text { C } & -0.651 & 0.063 & 0.000 \\ \mathrm{O} & -1.678 & -0.612 & 0.000 \\ \mathrm{O} & -0.452 & 1.285 & 0.000 \\ \mathrm{O} & 0.537 & -0.748 & 0.000 \\ \mathrm{C} & 1.740 & -0.036 & 0.000 \\ \mathrm{H} & 1.836 & 0.608 & 0.880 \\ \mathrm{H} & 2.549 & -0.773 & 0.000 \\ \mathrm{H} & 1.836 & 0.607 & -0.880\end{array}$

$\begin{array}{lrrr}\text { SA1 } & & & \\ \text { C } & -1.008 & 0 & 0 \\ \text { S } & -1.793 & -1.513 & -0.079 \\ \text { S } & -1.793 & 1.512 & 0.079 \\ \text { C } & 1.171 & -1.208 & 0.008 \\ \text { C } & 1.171 & 1.208 & -0.007 \\ \text { C } & 2.576 & -0.694 & 0.319 \\ \text { H } & 1.124 & -1.71 & -0.966 \\ \text { H } & 0.788 & -1.915 & 0.742 \\ \text { C } & 2.576 & 0.694 & -0.319 \\ \text { H } & 1.125 & 1.71 & 0.966 \\ \text { H } & 0.787 & 1.916 & -0.741 \\ \text { H } & 2.703 & -0.601 & 1.4 \\ \text { H } & 3.359 & -1.352 & -0.06 \\ \text { H } & 3.359 & 1.352 & 0.059 \\ \text { H } & 2.702 & 0.601 & -1.401 \\ \text { N } & 0.358 & 0 & 0\end{array}$

\section{SA2}

$\begin{array}{lrrr}C & -1.156 & 0 & -0.125 \\ \mathrm{~S} & -1.897 & -1.496 & 0.22 \\ \mathrm{~S} & -1.897 & 1.497 & 0.22 \\ \mathrm{C} & 0.918 & -1.199 & -0.776\end{array}$

$\begin{array}{lrrr}\mathrm{C} & 0.918 & 1.198 & -0.776 \\ \mathrm{C} & 1.841 & -1.262 & 0.441 \\ \mathrm{H} & 1.52 & -1.148 & -1.693 \\ \mathrm{H} & 0.266 & -2.065 & -0.822 \\ \mathrm{C} & 1.841 & 1.261 & 0.441 \\ \mathrm{H} & 1.52 & 1.147 & -1.693 \\ \mathrm{H} & 0.267 & 2.065 & -0.823 \\ \mathrm{C} & 2.701 & 0 & 0.527 \\ \mathrm{H} & 2.471 & -2.155 & 0.394 \\ \mathrm{H} & 1.203 & -1.35 & 1.326 \\ \mathrm{H} & 2.471 & 2.154 & 0.393 \\ \mathrm{H} & 1.204 & 1.35 & 1.326 \\ \mathrm{H} & 3.296 & 0 & 1.445 \\ \mathrm{H} & 3.411 & 0 & -0.31 \\ \mathrm{~N} & 0.099 & 0 & -0.711\end{array}$

\section{SA3}

$\begin{array}{lrrr}\text { C } & -1.259 & 0 & -0.012 \\ \text { S } & -2.063 & -1.497 & 0.103 \\ \text { S } & -2.063 & 1.497 & 0.103 \\ \text { C } & 0.885 & -1.198 & -0.461 \\ \text { C } & 0.885 & 1.198 & -0.461 \\ \text { C } & 2.185 & -1.162 & 0.321 \\ \text { H } & 1.114 & -1.262 & -1.536 \\ \text { H } & 0.289 & -2.063 & -0.182 \\ \text { C } & 2.185 & 1.162 & 0.321 \\ \text { H } & 1.114 & 1.262 & -1.536 \\ \text { H } & 0.289 & 2.063 & -0.182 \\ \text { H } & 2.814 & -2.014 & 0.06 \\ \text { H } & 1.956 & -1.202 & 1.395 \\ \text { H } & 2.814 & 2.014 & 0.06 \\ \text { H } & 1.956 & 1.202 & 1.395 \\ \text { N } & 0.116 & 0 & -0.195 \\ \text { O } & 2.946 & 0 & 0.045\end{array}$

\section{SA4}

$\begin{array}{lrrr}\mathrm{C} & -0.37 & 0 & 0 \\ \mathrm{~S} & -1.166 & -1.509 & 0 \\ \mathrm{~N} & 1.003 & 0 & 0 \\ \mathrm{C} & 1.743 & 1.246 & 0 \\ \mathrm{H} & 1.493 & 1.85 & 0.875 \\ \mathrm{H} & 2.81 & 1.023 & 0 \\ \mathrm{H} & 1.494 & 1.85 & -0.875 \\ \mathrm{C} & 1.743 & -1.246 & 0 \\ \mathrm{H} & 1.493 & -1.85 & -0.875 \\ \mathrm{H} & 2.81 & -1.024 & 0 \\ \mathrm{H} & 1.493 & -1.85 & 0.875 \\ \mathrm{~S} & -1.166 & 1.509 & 0\end{array}$




\section{SA5}

$\begin{array}{lrrr}\mathrm{C} & -0.641 & -0.036 & 0 \\ \mathrm{~S} & -1.831 & -1.22 & 0 \\ \mathrm{~S} & -0.849 & 1.638 & 0 \\ \mathrm{O} & 0.63 & -0.552 & -0.001 \\ \mathrm{C} & 1.75 & 0.31 & -0.001 \\ \mathrm{C} & 2.98 & -0.574 & 0.001 \\ \mathrm{H} & 1.719 & 0.962 & 0.876 \\ \mathrm{H} & 1.721 & 0.961 & -0.878 \\ \mathrm{H} & 3.886 & 0.036 & 0 \\ \mathrm{H} & 2.99 & -1.214 & 0.884 \\ \mathrm{H} & 2.99 & -1.216 & -0.881\end{array}$

\section{SA6}

$\begin{array}{lrrr}\mathrm{C} & 1.291 & -0.992 & -0.001 \\ \mathrm{H} & 1.067 & -1.632 & -0.855 \\ \mathrm{H} & 1.118 & -1.584 & 0.9 \\ \mathrm{H} & 2.33 & -0.66 & -0.037 \\ \mathrm{C} & 0.351 & 0.225 & -0.002 \\ \mathrm{O} & 0.875 & 1.334 & 0 \\ \mathrm{~S} & -1.335 & -0.137 & 0\end{array}$

SA7

$\begin{array}{lrrr}\mathrm{O} & -2.128 & 1.253 & 0.375 \\ \mathrm{~S} & -1.762 & 0 & -0.349 \\ \mathrm{O} & -2.128 & -1.253 & 0.375 \\ \mathrm{C} & 0.071 & 0 & -0.126 \\ \mathrm{C} & 0.767 & -1.2 & -0.07 \\ \mathrm{C} & 0.766 & 1.2 & -0.07 \\ \mathrm{C} & 2.152 & -1.202 & 0.038 \\ \mathrm{H} & 0.194 & -2.121 & -0.078 \\ \mathrm{C} & 2.152 & 1.202 & 0.038 \\ \mathrm{H} & 0.194 & 2.121 & -0.078 \\ \mathrm{C} & 2.849 & 0 & 0.087 \\ \mathrm{H} & 2.693 & -2.141 & 0.094 \\ \mathrm{H} & 2.692 & 2.141 & 0.094 \\ \mathrm{H} & 3.929 & 0 & 0.173\end{array}$




\begin{tabular}{lrrr}
\multicolumn{4}{l}{ Geometries of Protonated Nucleoph } \\
CC1 & & & \\
C & 1.049 & 1.275 & -0.015 \\
H & 2.018 & 1.176 & -0.506 \\
H & 0.455 & 2.098 & -0.404 \\
C & 0.340 & 0.000 & 0.000 \\
C & 1.049 & -1.275 & 0.015 \\
H & 0.456 & -2.098 & 0.405 \\
H & 1.265 & -1.485 & -1.049 \\
H & 2.018 & -1.176 & 0.506 \\
Cl & -1.301 & 0.000 & 0.000
\end{tabular}

\section{CC2}

$\begin{array}{lrrr}\mathrm{C} & -0.558 & -0.459 & 0.000 \\ \mathrm{H} & -0.371 & -1.162 & -0.837 \\ \mathrm{C} & 0.591 & 0.398 & 0.000 \\ \mathrm{H} & 0.438 & 1.480 & 0.000 \\ \mathrm{C} & -1.953 & 0.157 & 0.000 \\ \mathrm{H} & -2.511 & -0.157 & -0.879 \\ \mathrm{H} & -2.510 & -0.155 & 0.880 \\ \mathrm{H} & -1.906 & 1.245 & -0.001 \\ \mathrm{C} & 1.957 & -0.058 & 0.000 \\ \mathrm{H} & 2.081 & -1.137 & 0.000 \\ \mathrm{H} & 2.465 & 0.410 & -0.858 \\ \mathrm{H} & 2.465 & 0.410 & 0.858 \\ \mathrm{H} & -0.371 & -1.163 & 0.837\end{array}$

CC3

$\begin{array}{lrrr}\mathrm{C} & -0.742 & 0.657 & 0.000 \\ \mathrm{H} & -1.249 & 1.622 & 0.000 \\ \mathrm{C} & 0.693 & 0.691 & 0.000 \\ \mathrm{H} & 0.950 & 1.370 & 0.840 \\ \mathrm{C} & -1.531 & -0.547 & 0.000 \\ \mathrm{H} & -2.603 & -0.395 & 0.003 \\ \mathrm{H} & -1.207 & -1.159 & -0.858 \\ \mathrm{H} & -1.202 & -1.164 & 0.854 \\ \mathrm{C} & 1.469 & -0.608 & 0.000 \\ \mathrm{H} & 1.244 & -1.204 & -0.885 \\ \mathrm{H} & 2.536 & -0.399 & -0.002 \\ \mathrm{H} & 1.247 & -1.202 & 0.887 \\ \mathrm{H} & 0.949 & 1.369 & -0.840\end{array}$

\section{CC4}

$\begin{array}{rrrr}\mathrm{C} & 1.949 & 1.283 & -0.106 \\ \mathrm{H} & 3.007 & 1.196 & -0.350 \\ \mathrm{H} & 1.442 & 2.080 & -0.650 \\ \mathrm{C} & 1.215 & 0.033 & -0.077 \\ \mathrm{C} & 1.956 & -1.213 & -0.038\end{array}$

$\begin{array}{lrrr}\mathrm{H} & 1.418 & -2.049 & 0.406 \\ \mathrm{H} & 2.012 & -1.440 & -1.126 \\ \mathrm{H} & 2.985 & -1.126 & 0.311 \\ \mathrm{Si} & -0.730 & -0.003 & 0.001 \\ \mathrm{C} & -1.348 & 1.650 & -0.563 \\ \mathrm{H} & -1.063 & 1.867 & -1.594 \\ \mathrm{H} & -2.439 & 1.657 & -0.521 \\ \mathrm{H} & -0.997 & 2.462 & 0.077 \\ \mathrm{C} & -0.978 & -0.313 & 1.824 \\ \mathrm{H} & -2.054 & -0.368 & 2.008 \\ \mathrm{H} & -0.539 & -1.256 & 2.152 \\ \mathrm{H} & -0.583 & 0.495 & 2.441 \\ \mathrm{C} & -1.246 & -1.424 & -1.078 \\ \mathrm{H} & -2.335 & -1.508 & -1.042 \\ \mathrm{H} & -0.967 & -1.268 & -2.121 \\ \mathrm{H} & -0.837 & -2.377 & -0.742 \\ \mathrm{H} & 1.885 & 1.586 & 0.963\end{array}$

CC5

$\begin{array}{lrrr}\mathrm{C} & -2.442 & 0.270 & -0.520 \\ \mathrm{H} & -2.183 & 1.318 & -0.636 \\ \mathrm{C} & -1.278 & -0.602 & -0.379 \\ \mathrm{H} & -1.498 & -1.669 & -0.388 \\ \mathrm{C} & -3.368 & 0.062 & 0.707 \\ \mathrm{H} & -4.262 & 0.665 & 0.566 \\ \mathrm{H} & -3.666 & -0.981 & 0.805 \\ \mathrm{H} & -2.872 & 0.377 & 1.623 \\ \mathrm{C} & 0.051 & -0.256 & -0.190 \\ \mathrm{C} & 0.493 & 1.097 & -0.142 \\ \mathrm{C} & 1.001 & -1.306 & -0.037 \\ \mathrm{C} & 1.820 & 1.373 & 0.052 \\ \mathrm{H} & -0.217 & 1.905 & -0.256 \\ \mathrm{C} & 2.329 & -1.017 & 0.154 \\ \mathrm{H} & 0.658 & -2.333 & -0.074 \\ \mathrm{C} & 2.732 & 0.319 & 0.198 \\ \mathrm{H} & 2.170 & 2.395 & 0.092 \\ \mathrm{H} & 3.056 & -1.808 & 0.269 \\ \mathrm{H} & 3.780 & 0.549 & 0.348 \\ \mathrm{H} & -2.992 & -0.057 & -1.409\end{array}$

\section{CC6}

$\begin{array}{lrrr}\mathrm{C} & -2.591 & -0.542 & 0.000 \\ \mathrm{H} & -3.068 & -1.055 & 0.852 \\ \mathrm{C} & -1.220 & -1.052 & 0.000 \\ \mathrm{H} & -1.183 & -2.141 & 0.000 \\ \mathrm{C} & -2.925 & 0.938 & 0.000 \\ \mathrm{H} & -4.007 & 1.051 & 0.000 \\ \mathrm{H} & -2.545 & 1.440 & 0.890 \\ \mathrm{H} & -2.545 & 1.440 & -0.890 \\ \mathrm{C} & 0.026 & -0.441 & 0.000\end{array}$




$\begin{array}{lrrr}\text { C } & 1.160 & -1.307 & 0.000 \\ \text { C } & 0.237 & 0.969 & 0.000 \\ \text { C } & 2.433 & -0.795 & 0.000 \\ \text { H } & 1.001 & -2.378 & 0.000 \\ \text { C } & 1.514 & 1.467 & 0.000 \\ \text { H } & -0.601 & 1.646 & 0.000 \\ \text { C } & 2.605 & 0.590 & 0.000 \\ \text { H } & 3.292 & -1.451 & 0.000 \\ \text { H } & 1.683 & 2.535 & 0.000 \\ \text { H } & 3.608 & 0.998 & 0.000 \\ \text { H } & -3.068 & -1.055 & -0.852\end{array}$

$\begin{array}{lrrr}\text { C } & 0.096 & 0.000 & -0.558 \\ \text { C } & 0.754 & -1.214 & -0.302 \\ \text { C } & 2.043 & -1.211 & 0.188 \\ \text { C } & 2.683 & 0.000 & 0.440 \\ \text { H } & 2.554 & 2.145 & 0.371 \\ \text { H } & 0.263 & 2.155 & -0.514 \\ \text { H } & 0.263 & -2.155 & -0.514 \\ \text { H } & 2.554 & -2.146 & 0.371 \\ \text { H } & 3.692 & 0.000 & 0.831 \\ \text { C } & -1.258 & 0.000 & -1.353 \\ \text { H } & -1.373 & -0.911 & -1.927 \\ \text { H } & -1.373 & 0.912 & -1.927 \\ \text { C } & -1.727 & 0.000 & -0.001 \\ \text { C } & -2.113 & 1.263 & 0.691 \\ \text { H } & -1.825 & 2.159 & 0.152 \\ \text { H } & -3.207 & 1.244 & 0.750 \\ \text { C } & -2.114 & -1.263 & 0.690 \\ \text { H } & -3.207 & -1.244 & 0.750 \\ \text { H } & -1.826 & -2.159 & 0.151 \\ \text { H } & -1.739 & -1.295 & 1.713 \\ \text { H } & -1.739 & 1.294 & 1.713\end{array}$

CC10

$\begin{array}{lrrr}\text { CC7 } & & & \\ \text { C } & 1.354 & 1.367 & 0.000 \\ \text { C } & 0.010 & 1.104 & 0.000 \\ \text { C } & -0.447 & -0.246 & 0.000 \\ \text { C } & 0.506 & -1.307 & 0.000 \\ \text { C } & 1.850 & -1.030 & 0.000 \\ \text { C } & 2.267 & 0.303 & 0.000 \\ \text { H } & 1.716 & 2.385 & 0.000 \\ \text { H } & -0.700 & 1.919 & 0.000 \\ \text { H } & 0.152 & -2.330 & 0.000 \\ \text { H } & 2.579 & -1.828 & 0.000 \\ \text { H } & 3.328 & 0.523 & 0.000 \\ \text { C } & -1.789 & -0.580 & 0.000 \\ \text { H } & -2.016 & -1.645 & 0.000 \\ \text { C } & -2.950 & 0.306 & 0.000 \\ \text { H } & -2.725 & 1.366 & 0.000 \\ \text { H } & -3.573 & 0.053 & -0.866 \\ \text { H } & -3.573 & 0.053 & 0.867\end{array}$

$\begin{array}{lrrr}\text { CC8 } & & & \\ \text { C } & 0.000 & 0.006 & 0.021 \\ \text { C } & -0.006 & -1.448 & -0.019 \\ \text { H } & -0.919 & -1.868 & -0.439 \\ \text { H } & 0.901 & -1.876 & -0.442 \\ \text { C } & 1.269 & 0.720 & 0.017 \\ \text { H } & 2.090 & 0.124 & 0.414 \\ \text { H } & 1.487 & 0.846 & -1.061 \\ \text { H } & 1.216 & 1.715 & 0.452 \\ \text { C } & -1.263 & 0.731 & 0.017 \\ \text { H } & -1.200 & 1.728 & 0.445 \\ \text { H } & -1.486 & 0.850 & -1.061 \\ \text { H } & -2.086 & 0.144 & 0.422 \\ \text { H } & -0.005 & -1.716 & 1.057\end{array}$

\section{CC9}

$\begin{array}{llll}\text { C } & 2.043 & 1.211 & 0.188 \\ C & 0.754 & 1.214 & -0.302\end{array}$

$\begin{array}{lrrr}\mathrm{C} & -2.708 & -1.245 & -0.246 \\ \mathrm{H} & -3.469 & -1.378 & -1.032 \\ \mathrm{H} & -2.071 & -2.126 & -0.255 \\ \mathrm{C} & -1.998 & -0.013 & -0.617 \\ \mathrm{C} & -2.579 & 1.273 & -0.209 \\ \mathrm{H} & -2.124 & 2.134 & -0.688 \\ \mathrm{H} & -3.662 & 1.259 & -0.359 \\ \mathrm{H} & -2.441 & 1.351 & 0.880 \\ \mathrm{C} & -0.776 & -0.077 & -1.321 \\ \mathrm{H} & -0.533 & 0.802 & -1.920 \\ \mathrm{H} & -0.573 & -1.029 & -1.814 \\ \mathrm{Si} & 0.684 & -0.009 & 0.000 \\ \mathrm{Cl} & 0.369 & -1.462 & 1.351 \\ \mathrm{Cl} & 2.330 & -0.312 & -1.090 \\ \mathrm{Cl} & 0.649 & 1.810 & 0.848 \\ \mathrm{H} & -3.249 & -1.142 & 0.694\end{array}$

\section{CC1}

$\begin{array}{lrrr}\text { C } & 2.225 & 0.599 & 0.320 \\ \text { H } & 2.993 & 1.374 & 0.172 \\ \text { C } & 1.109 & 1.042 & -0.546 \\ \text { C } & -0.014 & 1.687 & -0.137 \\ \text { H } & -0.665 & 2.157 & -0.866 \\ \text { H } & -0.112 & 2.009 & 0.897 \\ \text { Si } & -1.051 & -0.231 & 0.035 \\ \text { H } & 1.224 & 0.847 & -1.612 \\ \text { C } & 2.821 & -0.755 & -0.061\end{array}$




$\begin{array}{lrrr}\mathrm{H} & 3.713 & -0.949 & 0.531 \\ \mathrm{H} & 2.121 & -1.571 & 0.116 \\ \mathrm{H} & 3.107 & -0.772 & -1.114 \\ \mathrm{C} & -2.745 & 0.512 & 0.048 \\ \mathrm{H} & -3.000 & 0.970 & -0.907 \\ \mathrm{H} & -3.455 & -0.300 & 0.231 \\ \mathrm{H} & -2.868 & 1.249 & 0.843 \\ \mathrm{C} & -0.712 & -1.283 & -1.448 \\ \mathrm{H} & -1.402 & -2.131 & -1.422 \\ \mathrm{H} & -0.901 & -0.740 & -2.375 \\ \mathrm{H} & 0.302 & -1.682 & -1.472 \\ \mathrm{C} & -0.516 & -0.912 & 1.668 \\ \mathrm{H} & 0.496 & -1.312 & 1.667 \\ \mathrm{H} & -0.607 & -0.172 & 2.465 \\ \mathrm{H} & -1.193 & -1.736 & 1.913 \\ \mathrm{H} & 1.942 & 0.654 & 1.373\end{array}$

\section{CC12}

$\begin{array}{lrrr}\mathrm{C} & -0.681 & 0.124 & 0.105 \\ \mathrm{C} & -1.826 & -0.698 & 0.493 \\ \mathrm{H} & -2.500 & -0.853 & -0.354 \\ \mathrm{H} & -1.556 & -1.634 & 0.974 \\ \mathrm{C} & -0.917 & 1.365 & -0.631 \\ \mathrm{H} & -0.741 & 2.173 & 0.099 \\ \mathrm{H} & -0.162 & 1.524 & -1.404 \\ \mathrm{H} & -1.924 & 1.455 & -1.026 \\ \mathrm{C} & 0.665 & -0.301 & 0.432 \\ \mathrm{C} & 0.927 & -1.286 & -0.782 \\ \mathrm{H} & 0.935 & -0.746 & -1.728 \\ \mathrm{H} & 1.921 & -1.694 & -0.602 \\ \mathrm{H} & 0.212 & -2.104 & -0.824 \\ \mathrm{C} & 1.745 & 0.766 & 0.507 \\ \mathrm{H} & 1.521 & 1.504 & 1.277 \\ \mathrm{H} & 2.693 & 0.294 & 0.761 \\ \mathrm{H} & 1.879 & 1.280 & -0.445 \\ \mathrm{H} & 0.644 & -0.931 & 1.324 \\ \mathrm{H} & -2.405 & -0.082 & 1.200\end{array}$

$\begin{array}{lrrr}\text { C } & -0.961 & -1.207 & -0.002 \\ \text { C } & 0.393 & -1.052 & -0.001 \\ \text { C } & 0.962 & 0.259 & 0.000 \\ \text { C } & 0.084 & 1.385 & 0.000 \\ \text { C } & -1.272 & 1.212 & -0.002 \\ \text { C } & -1.819 & -0.083 & -0.003 \\ \text { H } & -1.397 & -2.198 & -0.004 \\ \text { H } & 1.035 & -1.921 & -0.001 \\ \text { H } & 0.511 & 2.381 & 0.000 \\ \text { H } & -1.934 & 2.067 & -0.003 \\ \text { C } & 2.319 & 0.490 & 0.001\end{array}$

CC15

$\begin{array}{lrrr}\text { C } & -1.983 & -1.292 & 0.037 \\ \text { H } & -3.019 & -1.306 & -0.327 \\ \text { H } & -1.431 & -2.142 & -0.351 \\ \text { C } & -1.400 & 0.006 & -0.305 \\ \text { C } & -0.155 & 0.106 & -1.013 \\ \text { H } & -0.009 & -0.728 & -1.698 \\ \text { H } & -0.046 & 1.070 & -1.508 \\ \text { C } & 1.084 & 0.008 & 0.044 \\ \text { C } & 0.976 & 1.093 & 1.107 \\ \text { H } & 0.138 & 0.932 & 1.789 \\ \text { H } & 1.886 & 1.086 & 1.709\end{array}$




$\begin{array}{lrrr}\mathrm{H} & 0.887 & 2.087 & 0.665 \\ \mathrm{C} & 2.317 & 0.244 & -0.828 \\ \mathrm{H} & 3.205 & 0.184 & -0.197 \\ \mathrm{H} & 2.406 & -0.512 & -1.609 \\ \mathrm{H} & 2.298 & 1.230 & -1.292 \\ \mathrm{C} & 1.150 & -1.376 & 0.677 \\ \mathrm{H} & 0.333 & -1.563 & 1.374 \\ \mathrm{H} & 1.165 & -2.165 & -0.077 \\ \mathrm{H} & 2.077 & -1.450 & 1.247 \\ \mathrm{C} & -2.124 & 1.217 & 0.083 \\ \mathrm{H} & -2.795 & 1.074 & 0.927 \\ \mathrm{H} & -1.466 & 2.075 & 0.211 \\ \mathrm{H} & -2.749 & 1.444 & -0.799 \\ \mathrm{H} & -2.079 & -1.356 & 1.128\end{array}$

$\begin{array}{lrrr}\text { CC16 } & & & \\ \text { C } & 2.525 & 0.324 & -0.020 \\ \text { H } & 3.437 & -0.227 & -0.315 \\ \text { H } & 2.354 & 1.128 & -0.731 \\ \text { C } & 1.484 & -0.686 & -0.008 \\ \text { H } & 1.542 & -1.438 & 0.777 \\ \text { C } & 0.392 & -0.740 & -0.879 \\ \text { H } & 0.483 & -0.153 & -1.788 \\ \text { H } & -0.040 & -1.727 & -1.015 \\ \text { C } & -0.797 & 0.071 & 0.050 \\ \text { C } & -1.009 & -0.629 & 1.384 \\ \text { H } & -0.203 & -0.437 & 2.093 \\ \text { H } & -1.929 & -0.244 & 1.827 \\ \text { H } & -1.131 & -1.706 & 1.262 \\ \text { C } & -2.019 & -0.091 & -0.851 \\ \text { H } & -2.849 & 0.426 & -0.365 \\ \text { H } & -1.867 & 0.361 & -1.831 \\ \text { H } & -2.297 & -1.137 & -0.975 \\ \text { C } & -0.433 & 1.536 & 0.208 \\ \text { H } & 0.394 & 1.699 & 0.898 \\ \text { H } & -0.202 & 2.004 & -0.749 \\ \text { H } & -1.301 & 2.052 & 0.623 \\ \text { H } & 2.747 & 0.691 & 0.986\end{array}$

\section{CC17}

$\begin{array}{lrrr}\mathrm{C} & 0.884 & 1.431 & -0.026 \\ \mathrm{H} & 1.660 & 1.709 & -0.743 \\ \mathrm{H} & -0.032 & 1.977 & -0.231 \\ \mathrm{C} & 0.743 & -0.037 & 0.012 \\ \mathrm{C} & 1.988 & -0.829 & 0.014 \\ \mathrm{H} & 2.798 & -0.306 & 0.523 \\ \mathrm{H} & 1.853 & -1.832 & 0.411 \\ \mathrm{H} & 2.292 & -0.922 & -1.039 \\ \mathrm{C} & -0.483 & -0.692 & 0.038 \\ \mathrm{C} & -1.717 & 0.039 & 0.704\end{array}$

$\begin{array}{lrrr}\mathrm{C} & -1.713 & -0.059 & -0.727 \\ \mathrm{H} & -0.487 & -1.769 & 0.106 \\ \mathrm{H} & -1.487 & 0.979 & 1.184 \\ \mathrm{H} & -2.301 & -0.660 & 1.287 \\ \mathrm{H} & -2.292 & -0.833 & -1.211 \\ \mathrm{H} & -1.483 & 0.806 & -1.331 \\ \mathrm{H} & 1.267 & 1.740 & 0.955\end{array}$

CC18

$\begin{array}{lrrr}\mathrm{C} & 1.272 & -0.197 & -0.024 \\ \mathrm{H} & 1.237 & -1.263 & -0.232 \\ \mathrm{H} & 2.038 & 0.351 & -0.575 \\ \mathrm{C} & 0.000 & 0.462 & 0.000 \\ \mathrm{H} & 0.000 & 1.552 & 0.000 \\ \mathrm{C} & -1.272 & -0.197 & 0.024 \\ \mathrm{H} & -1.237 & -1.263 & 0.232 \\ \mathrm{H} & -2.038 & 0.351 & 0.575 \\ \mathrm{H} & -1.572 & -0.068 & -1.045 \\ \mathrm{H} & 1.572 & -0.068 & 1.045\end{array}$

CC19

$\begin{array}{lrrr}\text { C } & -0.163 & 0.000 & 0.000 \\ \text { C } & 0.564 & 1.220 & 0.042 \\ \text { C } & 0.564 & -1.220 & -0.042 \\ \text { C } & 1.937 & 1.214 & 0.056 \\ \text { C } & 1.937 & -1.214 & -0.056 \\ \text { H } & 0.042 & -2.164 & -0.090 \\ \text { C } & 2.622 & 0.000 & 0.000 \\ \text { H } & 2.486 & 2.145 & 0.106 \\ \text { H } & 2.486 & -2.144 & -0.106 \\ \text { H } & 3.706 & 0.000 & 0.000 \\ \text { H } & 0.042 & 2.164 & 0.090 \\ \text { C } & -1.572 & 0.000 & 0.000 \\ \text { C } & -2.371 & 1.240 & -0.099 \\ \text { H } & -1.864 & 2.075 & -0.569 \\ \text { H } & -3.313 & 1.037 & -0.611 \\ \text { C } & -2.372 & -1.240 & 0.099 \\ \text { H } & -2.645 & -1.525 & -0.927 \\ \text { H } & -1.864 & -2.076 & 0.567 \\ \text { H } & -3.312 & -1.037 & 0.612 \\ \text { H } & -2.644 & 1.526 & 0.927\end{array}$

CC20

$\begin{array}{lrrr}\text { C } & 0.528 & -0.002 & -0.016 \\ \text { C } & -0.709 & -0.769 & -0.099 \\ \text { C } & -2.034 & -0.054 & 0.083 \\ H & -2.850 & -0.757 & -0.070 \\ H & -2.128 & 0.349 & 1.091 \\ H & -2.159 & 0.759 & -0.632\end{array}$




$\begin{array}{lrrr}\mathrm{C} & 0.505 & 1.455 & -0.019 \\ \mathrm{H} & -0.234 & 1.824 & 0.699 \\ \mathrm{H} & 1.479 & 1.912 & 0.124 \\ \mathrm{H} & 0.097 & 1.755 & -0.998 \\ \mathrm{C} & 1.805 & -0.703 & 0.049 \\ \mathrm{H} & 2.137 & -0.569 & 1.094 \\ \mathrm{H} & 1.738 & -1.767 & -0.161 \\ \mathrm{H} & 2.568 & -0.203 & -0.552 \\ \mathrm{H} & -0.621 & -1.207 & -1.115 \\ \mathrm{H} & -0.599 & -1.660 & 0.534\end{array}$

CC21

$\begin{array}{lrrr}\mathrm{C} & 0.460 & 0.002 & 0.179 \\ \mathrm{C} & 1.237 & 1.177 & -0.203 \\ \mathrm{H} & 1.998 & 0.978 & -0.954 \\ \mathrm{C} & 1.006 & -1.330 & -0.068 \\ \mathrm{H} & 2.037 & -1.365 & 0.305 \\ \mathrm{H} & 0.411 & -2.137 & 0.350 \\ \mathrm{H} & 1.109 & -1.453 & -1.155 \\ \mathrm{C} & -0.860 & 0.166 & 0.750 \\ \mathrm{H} & -1.016 & 1.156 & 1.173 \\ \mathrm{H} & -1.105 & -0.623 & 1.460 \\ \mathrm{H} & 0.609 & 2.032 & -0.453 \\ \mathrm{C} & -1.794 & -0.003 & -0.509 \\ \mathrm{H} & -1.717 & -1.004 & -0.927 \\ \mathrm{H} & -2.809 & 0.156 & -0.153 \\ \mathrm{H} & -1.571 & 0.737 & -1.275 \\ \mathrm{H} & 1.758 & 1.453 & 0.732\end{array}$

\section{CC22}

$\begin{array}{lrrr}\text { C } & 2.271 & -1.220 & -0.002 \\ \text { C } & 3.289 & -0.255 & -0.006 \\ \text { C } & 2.940 & 1.109 & -0.008 \\ \text { C } & 1.630 & 1.498 & -0.005 \\ \text { C } & 0.576 & 0.534 & -0.001 \\ \text { C } & 0.955 & -0.840 & 0.000 \\ \text { H } & 2.535 & -2.269 & -0.002 \\ \text { H } & 3.727 & 1.852 & -0.014 \\ \text { H } & 1.404 & 2.553 & -0.008 \\ \text { H } & 0.190 & -1.602 & 0.002 \\ \text { C } & -0.778 & 0.887 & 0.001 \\ \text { C } & -1.224 & 2.296 & 0.007 \\ \text { H } & -0.450 & 3.055 & 0.008 \\ \text { H } & -1.884 & 2.454 & -0.854 \\ \text { C } & 4.725 & -0.651 & 0.006 \\ \text { H } & 5.169 & -0.374 & 0.966 \\ \text { H } & 4.856 & -1.720 & -0.140 \\ \text { H } & 5.276 & -0.110 & -0.764 \\ \text { Si } & -2.248 & -0.403 & 0.000 \\ \text { C } & -2.119 & -1.406 & -1.568\end{array}$

$\begin{array}{lrrr}\mathrm{H} & -1.261 & -2.075 & -1.606 \\ \mathrm{H} & -3.020 & -2.018 & -1.655 \\ \mathrm{H} & -2.082 & -0.757 & -2.444 \\ \mathrm{C} & -2.125 & -1.396 & 1.575 \\ \mathrm{H} & -3.021 & -2.015 & 1.658 \\ \mathrm{H} & -1.261 & -2.057 & 1.624 \\ \mathrm{H} & -2.101 & -0.741 & 2.448 \\ \mathrm{C} & -3.833 & 0.572 & -0.008 \\ \mathrm{H} & -3.948 & 1.194 & -0.897 \\ \mathrm{H} & -4.662 & -0.140 & -0.006 \\ \mathrm{H} & -3.952 & 1.202 & 0.875 \\ \mathrm{H} & -1.880 & 2.447 & 0.872\end{array}$

CC23

$\begin{array}{lrrr}\text { C } & 0.696 & 0.053 & 0.304 \\ \text { C } & -0.730 & -0.013 & 0.065 \\ \text { C } & -1.557 & 1.184 & 0.062 \\ \text { H } & -1.929 & 1.274 & -0.972 \\ \text { H } & -2.457 & 1.016 & 0.660 \\ \text { H } & -1.048 & 2.103 & 0.342 \\ \mathrm{C} & -1.379 & -1.302 & -0.150 \\ \text { H } & -2.459 & -1.237 & -0.233 \\ \text { H } & -0.945 & -1.742 & -1.060 \\ \text { H } & -1.092 & -1.999 & 0.646 \\ \text { C } & 1.485 & -1.216 & 0.009 \\ \text { H } & 1.081 & -2.098 & 0.505 \\ \text { H } & 1.515 & -1.401 & -1.066 \\ \text { H } & 2.509 & -1.085 & 0.350 \\ \text { C } & 1.394 & 1.318 & -0.214 \\ \text { H } & 1.390 & 1.324 & -1.304 \\ \text { H } & 0.947 & 2.243 & 0.149 \\ \text { H } & 2.428 & 1.297 & 0.119 \\ \text { H } & 0.616 & 0.168 & 1.414\end{array}$

CC24

$\begin{array}{lrrr}\text { C } & 0.539 & -1.157 & -0.327 \\ \text { C } & 0.538 & 1.157 & -0.328 \\ \text { C } & 1.016 & 0.000 & 0.709 \\ \text { H } & 0.104 & -2.073 & 0.052 \\ \text { H } & 1.341 & -1.278 & -1.044 \\ \text { H } & 0.104 & 2.073 & 0.051 \\ \text { H } & 1.341 & 1.278 & -1.045 \\ \text { H } & 0.595 & 0.000 & 1.703 \\ \text { H } & 2.099 & 0.000 & 0.670 \\ \text { C } & -0.302 & 0.000 & -0.388 \\ \text { C } & -1.698 & 0.000 & 0.133 \\ \text { H } & -2.220 & 0.891 & -0.210 \\ \text { H } & -2.219 & -0.894 & -0.204 \\ \text { H } & -1.707 & 0.004 & 1.226\end{array}$




$\begin{array}{lrrr}\text { CC25 } & & & \\ \text { C } & 0.058 & -1.182 & -0.132 \\ \text { C } & 1.479 & -0.742 & 0.244 \\ \text { C } & 1.504 & 0.721 & -0.211 \\ \text { C } & 0.074 & 1.193 & 0.090 \\ \text { H } & 0.012 & -1.343 & -1.233 \\ \text { H } & -0.368 & -2.084 & 0.310 \\ \text { H } & 1.607 & -0.815 & 1.326 \\ \text { H } & 2.240 & -1.356 & -0.228 \\ \text { H } & 2.253 & 1.320 & 0.299 \\ \text { H } & 1.690 & 0.789 & -1.284 \\ \text { H } & -0.019 & 1.408 & 1.177 \\ \text { H } & -0.313 & 2.080 & -0.413 \\ \text { C } & -0.772 & 0.010 & -0.027 \\ \text { C } & -2.227 & 0.007 & 0.003 \\ \text { H } & -2.669 & 0.948 & -0.314 \\ \text { H } & -2.650 & -0.852 & -0.517 \\ \text { H } & -2.478 & -0.135 & 1.069\end{array}$

$\begin{array}{rrrr}\mathrm{H} & -2.478 & 0.134 & -1.070 \\ \mathrm{H} & -2.669 & -0.948 & 0.314 \\ \mathrm{H} & -2.650 & 0.853 & 0.516 \\ \mathrm{H} & -0.019 & -1.409 & -1.177\end{array}$

CC28

CC26

$\begin{array}{lrrr}\text { C } & -0.736 & -1.002 & -0.211 \\ \text { C } & 0.739 & -1.000 & 0.211 \\ \text { C } & 1.183 & 0.439 & -0.098 \\ \text { C } & -0.002 & 1.245 & 0.000 \\ \text { C } & -1.184 & 0.435 & 0.098 \\ \text { H } & -1.335 & -1.743 & 0.312 \\ \text { H } & 1.340 & -1.738 & -0.311 \\ \text { H } & 0.841 & -1.176 & 1.283 \\ \text { H } & 2.097 & 0.851 & 0.328 \\ \text { H } & -0.004 & 2.333 & 0.000 \\ \text { H } & -2.100 & 0.844 & -0.329 \\ \text { H } & -1.312 & 0.547 & 1.210 \\ \text { H } & -0.837 & -1.179 & -1.283 \\ \text { H } & 1.310 & 0.550 & -1.210\end{array}$

CC27

$\begin{array}{lrrr}\text { C } & 1.479 & 0.742 & -0.245 \\ \text { C } & 0.058 & 1.182 & 0.132 \\ \text { C } & -0.772 & -0.010 & 0.027 \\ \text { C } & 0.074 & -1.193 & -0.090 \\ \text { C } & 1.504 & -0.721 & 0.211 \\ \text { H } & 2.240 & 1.356 & 0.228 \\ \text { H } & -0.369 & 2.084 & -0.309 \\ \text { H } & 0.012 & 1.343 & 1.234 \\ \text { H } & -0.313 & -2.080 & 0.414 \\ \text { H } & 2.253 & -1.320 & -0.299 \\ \text { H } & 1.690 & -0.789 & 1.284 \\ \text { H } & 1.607 & 0.815 & -1.326 \\ \mathrm{C} & -2.226 & -0.007 & -0.004\end{array}$

$\begin{array}{lrrr}\text { C } & -1.168 & 1.252 & -0.260 \\ \text { C } & -1.870 & 0.000 & 0.249 \\ \mathrm{C} & -1.167 & -1.252 & -0.260 \\ \mathrm{C} & 0.308 & -1.267 & 0.148 \\ \mathrm{C} & 1.024 & 0.000 & 0.049 \\ \mathrm{C} & 0.307 & 1.267 & 0.148 \\ \mathrm{H} & -1.642 & -2.155 & 0.120 \\ \mathrm{H} & -1.887 & 0.000 & 1.344 \\ \mathrm{H} & -2.908 & 0.000 & -0.081 \\ \mathrm{H} & -1.228 & 1.298 & -1.350 \\ \mathrm{H} & -1.642 & 2.155 & 0.120 \\ \mathrm{H} & 0.387 & -1.442 & 1.244 \\ \mathrm{H} & 0.387 & 1.441 & 1.244 \\ \mathrm{H} & 0.895 & 2.078 & -0.288 \\ \mathrm{H} & -1.228 & -1.297 & -1.350 \\ \mathrm{C} & 2.476 & 0.000 & -0.090 \\ \mathrm{H} & 2.945 & -0.908 & 0.286 \\ \mathrm{H} & 2.621 & 0.000 & -1.187 \\ \mathrm{H} & 2.945 & 0.908 & 0.285 \\ \mathrm{H} & 0.895 & -2.078 & -0.289\end{array}$

CC29

$\begin{array}{lrrr}\text { C } & -1.893 & 0.130 & 0.000 \\ \text { H } & -1.999 & 1.210 & 0.000 \\ \text { H } & -2.804 & -0.458 & 0.000 \\ \text { C } & -0.665 & -0.455 & 0.000 \\ \text { H } & -0.550 & -1.532 & 0.000 \\ \text { C } & 0.459 & 0.371 & 0.000 \\ \text { H } & 0.290 & 1.447 & 0.000 \\ \text { C } & 1.835 & -0.085 & 0.000 \\ \text { H } & 1.947 & -1.165 & -0.001 \\ \text { H } & 2.348 & 0.367 & -0.862 \\ \text { H } & 2.348 & 0.366 & 0.863\end{array}$

CC30

$\begin{array}{lrrr}\mathrm{C} & 1.186 & 0.388 & 0.000 \\ \mathrm{H} & 1.129 & 1.475 & 0.000 \\ \mathrm{C} & 0.000 & -0.313 & 0.000 \\ \mathrm{H} & 0.000 & -1.397 & 0.001 \\ \mathrm{C} & 2.518 & -0.205 & 0.000 \\ \mathrm{H} & 3.071 & 0.175 & -0.869 \\ \mathrm{H} & 3.075 & 0.180 & 0.863 \\ \mathrm{H} & 2.512 & -1.291 & 0.003\end{array}$




$\begin{array}{lrrr}\mathrm{C} & -1.186 & 0.388 & 0.000 \\ \mathrm{H} & -1.129 & 1.475 & 0.000 \\ \mathrm{C} & -2.518 & -0.205 & 0.000 \\ \mathrm{H} & -3.072 & 0.176 & -0.867 \\ \mathrm{H} & -2.512 & -1.291 & 0.001 \\ \mathrm{H} & -3.074 & 0.178 & 0.86\end{array}$

$\begin{array}{rrrr}\text { H } & -0.466 & 1.769 & 0.000 \\ \mathrm{C} & 2.101 & -0.627 & 0.000 \\ \mathrm{H} & 2.773 & -0.597 & -0.866 \\ \mathrm{H} & 2.774 & -0.596 & 0.865 \\ \mathrm{H} & 1.538 & -1.553 & 0.001 \\ \mathrm{C} & -0.926 & -0.307 & 0.000 \\ \mathrm{H} & -0.515 & -1.312 & 0.000 \\ \mathrm{C} & -2.380 & -0.197 & 0.000 \\ \mathrm{H} & -2.741 & 0.827 & 0.000 \\ \mathrm{H} & -2.773 & -0.746 & -0.866 \\ \mathrm{H} & -2.773 & -0.745 & 0.866\end{array}$

\section{CC34}

$\begin{array}{lrrr}\mathrm{C} & 2.805 & -0.176 & 0.003 \\ \mathrm{H} & 2.890 & -1.259 & 0.028 \\ \mathrm{H} & 3.331 & 0.227 & -0.871 \\ \mathrm{C} & 1.419 & 0.298 & -0.015 \\ \mathrm{H} & 1.280 & 1.374 & -0.043 \\ \mathrm{C} & 0.323 & -0.517 & 0.008 \\ \mathrm{H} & 0.473 & -1.591 & 0.038 \\ \mathrm{C} & -0.993 & -0.042 & 0.007 \\ \mathrm{C} & -1.380 & 1.381 & 0.001 \\ \mathrm{H} & -2.225 & 1.527 & -0.676 \\ \mathrm{H} & -1.766 & 1.609 & 1.003 \\ \mathrm{H} & -0.588 & 2.082 & -0.237 \\ \mathrm{C} & -2.097 & -1.020 & 0.004 \\ \mathrm{H} & -2.397 & -1.138 & -1.049 \\ \mathrm{H} & -1.802 & -1.998 & 0.375 \\ \mathrm{H} & -2.975 & -0.643 & 0.528 \\ \mathrm{H} & 3.319 & 0.264 & 0.866\end{array}$

$\begin{array}{lrrr}\text { CC32 } & & & \\ \text { C } & -2.097 & -1.020 & -0.004 \\ \text { H } & -2.976 & -0.642 & -0.527 \\ \text { H } & -1.802 & -1.998 & -0.377 \\ \text { C } & -0.993 & -0.042 & -0.007 \\ \text { C } & 0.323 & -0.517 & -0.007 \\ \text { H } & 0.473 & -1.591 & -0.037 \\ \text { C } & 1.419 & 0.298 & 0.015 \\ \text { H } & 1.281 & 1.374 & 0.042 \\ \text { C } & 2.805 & -0.176 & -0.003 \\ \text { H } & 3.330 & 0.227 & 0.871 \\ \text { H } & 2.890 & -1.258 & -0.027 \\ \text { H } & 3.320 & 0.264 & -0.866 \\ \text { C } & -1.380 & 1.381 & -0.001 \\ \text { H } & -0.588 & 2.082 & 0.236 \\ \text { H } & -1.768 & 1.609 & -1.002 \\ \text { H } & -2.223 & 1.527 & 0.678 \\ \text { H } & -2.395 & -1.140 & 1.049\end{array}$

CC33

$\begin{array}{lrrr}\mathrm{C} & 1.313 & 0.604 & 0.000 \\ \mathrm{H} & 1.899 & 1.521 & 0.000 \\ \mathrm{C} & -0.060 & 0.765 & 0.000\end{array}$

CC35

$\begin{array}{lrrr}\text { C } & -1.101 & -1.455 & -0.057 \\ \text { H } & -2.164 & -1.557 & -0.256 \\ \text { H } & -0.506 & -2.042 & -0.760 \\ \text { C } & -0.656 & -0.056 & -0.004 \\ \text { C } & 0.747 & 0.216 & 0.013 \\ \text { C } & 1.160 & 1.505 & -0.047 \\ \text { H } & 2.218 & 1.739 & -0.078 \\ \text { H } & 0.483 & 2.346 & -0.086 \\ \text { C } & 1.735 & -0.919 & 0.043 \\ \text { H } & 1.525 & -1.606 & 0.862 \\ \text { H } & 1.707 & -1.489 & -0.887 \\ \text { H } & 2.744 & -0.538 & 0.172 \\ \text { C } & -1.718 & 0.959 & 0.048 \\ \text { H } & -1.405 & 1.979 & 0.229 \\ \text { H } & -2.269 & 0.902 & -0.901 \\ \text { H } & -2.445 & 0.654 & 0.807 \\ \text { H } & -0.888 & -1.890 & 0.932\end{array}$




$\begin{array}{lrrr}\text { CC36 } & & & \\ \text { C } & 1.604 & -0.950 & 0.000 \\ \text { H } & 1.435 & -2.021 & 0.000 \\ \text { H } & 2.635 & -0.613 & 0.000 \\ \text { C } & 0.563 & -0.066 & 0.000 \\ \text { C } & -0.702 & -0.671 & 0.000 \\ \text { H } & -0.734 & -1.758 & 0.000 \\ \text { C } & -1.978 & 0.019 & 0.000 \\ \text { H } & -1.909 & 1.102 & -0.001 \\ \text { H } & -2.562 & -0.337 & -0.861 \\ \text { C } & 0.738 & 1.424 & 0.000 \\ \text { H } & 0.275 & 1.867 & -0.883 \\ \text { H } & 0.275 & 1.867 & 0.882 \\ \text { H } & 1.793 & 1.688 & 0.000 \\ \text { H } & -2.560 & -0.336 & 0.863\end{array}$

cc37

$\begin{array}{lrrr}\text { C } & 0.954 & 0.766 & 0.000 \\ \mathrm{C} & -0.472 & 1.113 & 0.000 \\ \mathrm{C} & -1.285 & -0.006 & 0.000 \\ \mathrm{C} & -0.462 & -1.117 & 0.000 \\ \mathrm{H} & 1.453 & 1.215 & 0.868 \\ \mathrm{H} & -0.833 & 2.136 & 0.000 \\ \mathrm{H} & -2.365 & -0.010 & 0.000 \\ \mathrm{H} & -0.813 & -2.143 & 0.000 \\ \mathrm{C} & 0.961 & -0.758 & 0.000 \\ \mathrm{H} & 1.463 & -1.202 & -0.869 \\ \mathrm{H} & 1.463 & -1.202 & 0.869 \\ \mathrm{H} & 1.453 & 1.216 & -0.868\end{array}$

CC38

$\begin{array}{lrrr}\text { C } & -1.379 & 0.000 & -0.310 \\ \text { C } & -0.663 & -1.252 & 0.176 \\ \text { C } & 0.794 & -1.181 & 0.016 \\ \text { C } & 1.489 & 0.000 & -0.154 \\ \text { C } & 0.794 & 1.181 & 0.016 \\ \text { C } & -0.663 & 1.252 & 0.176 \\ \text { H } & -1.386 & 0.000 & -1.402 \\ \text { H } & -0.820 & -1.429 & 1.257 \\ \text { H } & 1.359 & -2.107 & 0.100 \\ \text { H } & 2.565 & 0.000 & -0.260 \\ \text { H } & 1.359 & 2.107 & 0.100 \\ \text { H } & -1.036 & 2.165 & -0.296 \\ \text { H } & -2.416 & 0.000 & 0.019 \\ \text { H } & -1.037 & -2.165 & -0.296 \\ \text { H } & -0.820 & 1.429 & 1.257\end{array}$

$\begin{array}{lrrr}\text { C } & -1.121 & 0.020 & -0.695 \\ \text { C } & -1.121 & 0.018 & 0.694 \\ \text { C } & 0.985 & 0.660 & 0.001 \\ \text { C } & 0.035 & 0.848 & -1.192 \\ \text { H } & -1.942 & -0.338 & -1.301 \\ \text { H } & 0.434 & 0.512 & -2.147 \\ \text { C } & 0.035 & 0.845 & 1.194 \\ \text { H } & 0.434 & 0.506 & 2.148 \\ \text { H } & -0.306 & 1.879 & 1.299 \\ \text { C } & -0.186 & -1.386 & -0.001 \\ \text { H } & -0.503 & -1.969 & 0.865 \\ \text { H } & -0.503 & -1.969 & -0.868 \\ \text { C } & 1.240 & -0.853 & -0.001 \\ \text { H } & 1.782 & -1.180 & 0.884 \\ \text { H } & 1.782 & -1.178 & -0.886 \\ \text { H } & -1.942 & -0.340 & 1.300 \\ \text { H } & 1.869 & 1.288 & 0.002 \\ \text { H } & -0.306 & 1.882 & -1.294\end{array}$

CC40

$\begin{array}{lrrr}\text { C } & -0.882 & 1.154 & 0.000 \\ \text { C } & -2.245 & 0.783 & 0.000 \\ \text { C } & -2.314 & -0.579 & 0.000 \\ \text { C } & -0.963 & -1.182 & 0.000 \\ \text { H } & -0.536 & 2.178 & 0.000 \\ \text { H } & -3.079 & 1.468 & 0.000 \\ \text { H } & -3.225 & -1.163 & 0.000 \\ \text { H } & -0.824 & -1.826 & -0.876 \\ \text { C } & -0.059 & 0.020 & 0.000 \\ \text { C } & 1.318 & 0.016 & 0.000 \\ \text { C } & 2.137 & 1.253 & 0.000 \\ \text { H } & 2.796 & 1.242 & 0.873 \\ \text { H } & 2.796 & 1.241 & -0.873 \\ \text { H } & 1.565 & 2.174 & 0.000 \\ \text { C } & 2.076 & -1.259 & 0.000 \\ \text { H } & 2.736 & -1.285 & 0.872 \\ \text { H } & 1.447 & -2.144 & 0.000 \\ \text { H } & 2.736 & -1.285 & -0.872 \\ \text { H } & -0.824 & -1.826 & 0.876\end{array}$

CC41

CC39

$\begin{array}{lrrr}\text { C } & -1.549 & 1.226 & 0.000 \\ \text { C } & -2.224 & 0.000 & 0.000 \\ \text { C } & -1.549 & -1.226 & 0.000 \\ \text { C } & -0.179 & -1.240 & 0.000 \\ \text { C } & 0.528 & 0.000 & 0.000 \\ \text { C } & -0.179 & 1.240 & 0.000 \\ \text { H } & -2.108 & 2.152 & 0.000 \\ \text { H } & -3.307 & 0.000 & 0.000 \\ \text { H } & -2.108 & -2.151 & 0.000\end{array}$




$\begin{array}{rrrr}\mathrm{H} & 0.381 & -2.166 & 0.000 \\ \mathrm{H} & 0.381 & 2.166 & 0.000 \\ \mathrm{C} & 1.882 & 0.000 & 0.000 \\ \mathrm{C} & 3.159 & 0.000 & 0.000 \\ \mathrm{H} & 3.713 & 0.000 & 0.937 \\ \mathrm{H} & 3.713 & 0.000 & -0.937\end{array}$

$\begin{array}{lrrr}\text { Ar1 } & & & \\ \text { C } & -1.886 & -0.001 & 0.007 \\ \text { C } & -1.111 & -1.244 & 0.001 \\ \text { C } & 0.243 & -1.232 & -0.009 \\ \text { C } & 0.945 & 0.001 & -0.016 \\ \text { C } & 0.242 & 1.233 & -0.009 \\ \text { C } & -1.113 & 1.243 & 0.001 \\ \text { H } & -2.597 & -0.001 & -0.838 \\ \text { H } & -1.654 & -2.182 & 0.004 \\ \text { H } & 0.808 & -2.155 & -0.017 \\ \text { H } & 0.804 & 2.157 & -0.017 \\ \text { H } & -1.657 & 2.180 & 0.004 \\ \text { C } & 2.424 & 0.001 & 0.001 \\ \text { H } & 2.738 & -0.026 & 1.054 \\ \text { H } & 2.836 & -0.885 & -0.478 \\ \text { H } & 2.839 & 0.906 & -0.437 \\ \text { H } & -2.578 & -0.001 & 0.867\end{array}$

Ar2

$\begin{array}{lrrr}\text { C } & 1.257 & 1.114 & -0.014 \\ \mathrm{C} & 1.223 & -0.310 & -0.001 \\ \mathrm{C} & 0.001 & -1.004 & 0.012 \\ \mathrm{C} & -1.194 & -0.339 & 0.010 \\ \mathrm{C} & -1.201 & 1.137 & 0.016 \\ \mathrm{C} & 0.104 & 1.813 & -0.008 \\ \mathrm{H} & 2.217 & 1.615 & -0.027 \\ \mathrm{H} & 0.017 & -2.087 & 0.018 \\ \mathrm{H} & -1.831 & 1.507 & -0.807 \\ \mathrm{H} & 0.111 & 2.896 & -0.013 \\ \mathrm{C} & -2.497 & -1.051 & -0.013 \\ \mathrm{H} & -2.972 & -0.899 & -0.987 \\ \mathrm{H} & -2.377 & -2.118 & 0.150 \\ \mathrm{H} & -3.178 & -0.641 & 0.735 \\ \mathrm{C} & 2.508 & -1.048 & 0.001 \\ \mathrm{H} & 3.085 & -0.761 & 0.885 \\ \mathrm{H} & 2.375 & -2.126 & -0.011 \\ \mathrm{H} & 3.105 & -0.741 & -0.862 \\ \mathrm{H} & -1.761 & 1.481 & 0.902\end{array}$

\section{Ar3}

$\begin{array}{rrrr}C & 2.265 & 0.330 & 0.000 \\ C & 1.768 & -1.058 & 0.000\end{array}$

$\begin{array}{lrrr}\text { C } & 0.456 & -1.344 & 0.000 \\ \mathrm{C} & -0.498 & -0.277 & 0.000 \\ \mathrm{C} & -0.088 & 1.095 & 0.000 \\ \mathrm{C} & 1.229 & 1.376 & 0.000 \\ \mathrm{H} & 2.938 & 0.485 & 0.858 \\ \mathrm{H} & 0.075 & -2.356 & 0.000 \\ \mathrm{H} & -0.825 & 1.884 & 0.000 \\ \mathrm{H} & 1.559 & 2.408 & 0.000 \\ \mathrm{H} & 2.499 & -1.857 & 0.000 \\ \mathrm{O} & -1.730 & -0.645 & 0.000 \\ \mathrm{C} & -2.815 & 0.306 & 0.000 \\ \mathrm{H} & -3.716 & -0.296 & 0.000 \\ \mathrm{H} & -2.766 & 0.917 & 0.899 \\ \mathrm{H} & -2.767 & 0.917 & -0.899 \\ \mathrm{H} & 2.938 & 0.485 & -0.858\end{array}$

\section{Ar4}

$\begin{array}{lrrr}\text { C } & -1.663 & 1.112 & 0.000 \\ \text { C } & -1.596 & -0.367 & 0.000 \\ \text { C } & -0.395 & -0.990 & 0.000 \\ \text { C } & 0.810 & -0.237 & 0.000 \\ \text { C } & 0.804 & 1.198 & 0.000 \\ \text { C } & -0.379 & 1.834 & 0.000 \\ \text { H } & -2.267 & 1.444 & -0.858 \\ \text { H } & -0.307 & -2.069 & 0.000 \\ \text { H } & 1.734 & 1.747 & 0.000 \\ \text { H } & -0.412 & 2.917 & 0.000 \\ \text { O } & 1.896 & -0.934 & 0.000 \\ \text { C } & 3.199 & -0.319 & 0.000 \\ \text { H } & 3.900 & -1.145 & 0.000 \\ \text { H } & 3.323 & 0.283 & -0.899 \\ \text { H } & 3.323 & 0.283 & 0.899 \\ \text { C } & -2.878 & -1.131 & 0.000 \\ \text { H } & -2.928 & -1.780 & -0.877 \\ \text { H } & -2.930 & -1.776 & 0.879 \\ \text { H } & -3.744 & -0.473 & -0.002 \\ \text { H } & -2.267 & 1.444 & 0.858\end{array}$

Ar5

$\begin{array}{lrrr}\text { C } & -1.033 & 1.671 & 0.000 \\ \text { C } & -1.297 & 0.209 & 0.000 \\ \text { C } & -0.277 & -0.707 & 0.000 \\ \text { C } & 1.051 & -0.252 & 0.000 \\ \text { C } & 1.384 & 1.153 & 0.000 \\ \text { C } & 0.395 & 2.054 & 0.000 \\ \text { H } & -1.540 & 2.118 & -0.865 \\ \text { H } & -0.445 & -1.774 & 0.000 \\ \text { H } & 2.418 & 1.462 & 0.000 \\ \text { H } & 0.622 & 3.113 & 0.000 \\ \text { O } & 1.956 & -1.179 & 0.000\end{array}$




$\begin{array}{lrrr}\mathrm{C} & 3.363 & -0.873 & 0.000 \\ \mathrm{H} & 3.860 & -1.836 & 0.000 \\ \mathrm{H} & 3.623 & -0.316 & -0.899 \\ \mathrm{H} & 3.622 & -0.316 & 0.899 \\ \mathrm{O} & -2.566 & -0.079 & 0.000 \\ \mathrm{C} & -2.989 & -1.451 & 0.000 \\ \mathrm{H} & -2.624 & -1.950 & 0.897 \\ \mathrm{H} & -4.072 & -1.421 & 0.000 \\ \mathrm{H} & -2.624 & -1.950 & -0.897 \\ \mathrm{H} & -1.540 & 2.118 & 0.865\end{array}$

Ar6

$\begin{array}{lrrr}\text { C } & 0.287 & -0.752 & 0.000 \\ \text { C } & 0.254 & 0.636 & 0.000 \\ \text { C } & 1.375 & 1.439 & 0.000 \\ \text { C } & 2.596 & 0.776 & 0.000 \\ \text { C } & 2.659 & -0.615 & 0.000 \\ \text { C } & 1.508 & -1.397 & 0.000 \\ \text { C } & -1.130 & -1.249 & 0.000 \\ \text { C } & -1.928 & 0.015 & 0.000 \\ \text { H } & 1.316 & 2.519 & 0.000 \\ \text { H } & 3.511 & 1.352 & 0.000 \\ \text { H } & 3.627 & -1.099 & 0.000 \\ \text { H } & 1.574 & -2.477 & 0.000 \\ \text { H } & -1.390 & -1.852 & 0.876 \\ \text { H } & -1.426 & 1.991 & 0.000 \\ \text { N } & -1.117 & 1.024 & 0.000 \\ \text { C } & -3.399 & 0.125 & 0.000 \\ \text { H } & -3.799 & -0.386 & 0.878 \\ \text { H } & -3.735 & 1.160 & 0.000 \\ \text { H } & -3.800 & -0.387 & -0.877 \\ \text { H } & -1.390 & -1.851 & -0.878\end{array}$

$\begin{array}{llll}\mathrm{C} & -3.239 & -2.668 & 0.000 \\ \mathrm{H} & -3.864 & -2.517 & 0.883 \\ \mathrm{H} & -3.864 & -2.517 & -0.883 \\ \mathrm{H} & -2.871 & -3.690 & 0.000\end{array}$

Ar8

$\begin{array}{lrrr}\text { C } & 0.337 & -0.480 & 0.000 \\ \text { C } & 0.735 & 0.847 & 0.000 \\ \text { C } & -0.156 & 1.908 & 0.000 \\ \text { C } & -1.495 & 1.586 & 0.000 \\ \text { C } & -1.927 & 0.246 & 0.000 \\ \text { C } & -1.005 & -0.807 & 0.000 \\ \text { C } & 1.576 & -1.331 & 0.000 \\ \text { C } & 2.679 & -0.320 & 0.000 \\ \text { H } & 0.170 & 2.939 & 0.000 \\ \text { H } & -2.252 & 2.357 & 0.000 \\ \text { H } & 1.667 & -1.979 & -0.878 \\ \text { H } & 2.704 & 1.718 & 0.000 \\ \text { N } & 2.155 & 0.864 & 0.000 \\ \text { H } & -1.326 & -1.838 & 0.000 \\ \text { C } & 4.129 & -0.595 & 0.000 \\ \text { H } & 4.720 & 0.318 & 0.000 \\ \text { H } & 4.386 & -1.191 & 0.879 \\ \text { H } & 4.386 & -1.193 & -0.877 \\ \text { O } & -3.251 & 0.078 & 0.000 \\ \text { C } & -3.776 & -1.241 & 0.000 \\ \text { H } & -3.464 & -1.782 & 0.895 \\ \text { H } & -4.856 & -1.131 & 0.000 \\ \text { H } & -3.463 & -1.782 & -0.895 \\ \text { H } & 1.666 & -1.979 & 0.877\end{array}$

Ar9

Ar7

$\begin{array}{lrrr}\mathrm{C} & 0.388 & 1.502 & 0.000 \\ \mathrm{C} & -0.990 & 1.685 & 0.000 \\ \mathrm{C} & -1.906 & 0.652 & 0.000 \\ \mathrm{C} & -1.382 & -0.631 & 0.000 \\ \mathrm{C} & 0.000 & -0.836 & 0.000 \\ \mathrm{C} & 0.905 & 0.223 & 0.000 \\ \mathrm{C} & 1.039 & 2.855 & 0.000 \\ \mathrm{C} & -0.131 & 3.767 & 0.000 \\ \mathrm{H} & -2.975 & 0.819 & 0.000 \\ \mathrm{H} & -2.045 & -1.484 & 0.000 \\ \mathrm{H} & 1.668 & 3.051 & 0.876 \\ \mathrm{H} & -2.152 & 3.502 & 0.000 \\ \mathrm{~N} & -1.225 & 3.087 & 0.000 \\ \mathrm{H} & -0.132 & 4.849 & 0.000 \\ \mathrm{H} & 1.970 & 0.035 & 0.000 \\ \mathrm{H} & 1.668 & 3.051 & -0.876 \\ \mathrm{Br} & 0.658 & -2.592 & 0.000\end{array}$




$\begin{array}{lrrr}\text { Ar10 } & & & \\ \text { C } & 0.000 & -1.037 & 0.000 \\ \text { C } & -1.265 & -0.459 & 0.000 \\ \text { C } & -1.492 & 0.903 & 0.000 \\ \text { C } & -0.367 & 1.710 & 0.000 \\ \text { C } & 0.913 & 1.149 & 0.000 \\ \text { C } & 1.118 & -0.229 & 0.000 \\ \text { C } & -0.168 & -2.529 & 0.000 \\ \text { C } & -1.644 & -2.680 & 0.000 \\ \text { H } & -2.487 & 1.328 & 0.000 \\ \text { H } & -0.469 & 2.786 & 0.000 \\ \text { H } & 0.262 & -3.029 & 0.876 \\ \text { H } & -3.215 & -1.381 & 0.000 \\ \text { N } & -2.209 & -1.522 & 0.000 \\ \text { H } & -2.220 & -3.596 & 0.000 \\ \text { H } & 2.122 & -0.630 & 0.000 \\ \text { Cl } & 2.273 & 2.190 & 0.000 \\ \text { H } & 0.262 & -3.029 & -0.876\end{array}$

\section{Ar11}

$\begin{array}{lrrr}\text { C } & 0.000 & -0.962 & 0.000 \\ \text { C } & -1.213 & -0.282 & 0.000 \\ \text { C } & -1.339 & 1.092 & 0.000 \\ \text { C } & -0.154 & 1.809 & 0.000 \\ \text { C } & 1.082 & 1.151 & 0.000 \\ \text { C } & 1.176 & -0.243 & 0.000 \\ \text { C } & -0.287 & -2.435 & 0.000 \\ \text { C } & -1.769 & -2.465 & 0.000 \\ \text { H } & -2.298 & 1.591 & 0.000 \\ \text { H } & -0.174 & 2.890 & 0.000 \\ \text { H } & -3.234 & -1.046 & 0.000 \\ \text { N } & -2.242 & -1.267 & 0.000 \\ \text { H } & 2.145 & -0.723 & 0.000 \\ \text { H } & -2.416 & -3.333 & 0.000 \\ \text { H } & 0.099 & -2.969 & 0.876 \\ \text { C } & 2.288 & 1.931 & 0.000 \\ \text { N } & 3.252 & 2.550 & 0.000 \\ \text { H } & 0.099 & -2.969 & -0.876\end{array}$

\section{Ar12}

$\begin{array}{lrrr}\text { C } & 0.492 & -0.667 & 0.000 \\ C & 0.754 & 0.696 & 0.000 \\ C & -0.229 & 1.668 & 0.000 \\ C & -1.533 & 1.207 & 0.000 \\ C & -1.845 & -0.161 & 0.000 \\ C & -0.818 & -1.105 & 0.000 \\ C & 1.807 & -1.394 & 0.000 \\ C & 2.785 & -0.278 & 0.000 \\ \text { H } & -0.001 & 2.726 & 0.000\end{array}$

$\begin{array}{lrrr}\mathrm{H} & -2.344 & 1.925 & 0.000 \\ \mathrm{H} & 2.634 & 1.754 & 0.000 \\ \mathrm{~N} & 2.166 & 0.852 & 0.000 \\ \mathrm{H} & -1.053 & -2.162 & 0.000 \\ \mathrm{H} & 1.967 & -2.033 & -0.876 \\ \mathrm{H} & 3.865 & -0.337 & 0.000 \\ \mathrm{H} & 1.967 & -2.033 & 0.876 \\ \mathrm{C} & -3.283 & -0.589 & 0.000 \\ \mathrm{H} & -3.797 & -0.198 & 0.879 \\ \mathrm{H} & -3.798 & -0.198 & -0.879 \\ \mathrm{H} & -3.379 & -1.672 & 0.000\end{array}$

Ar13

$\begin{array}{lrrr}\text { C } & 0.476 & -0.666 & 0.000 \\ \mathrm{C} & 0.732 & 0.705 & 0.000 \\ \mathrm{C} & -0.271 & 1.660 & 0.000 \\ \mathrm{C} & -1.567 & 1.199 & 0.000 \\ \mathrm{C} & -1.866 & -0.185 & 0.000 \\ \mathrm{C} & -0.816 & -1.127 & 0.000 \\ \mathrm{C} & 1.800 & -1.380 & 0.000 \\ \mathrm{C} & 2.771 & -0.253 & 0.000 \\ \mathrm{H} & -0.060 & 2.721 & 0.000 \\ \mathrm{H} & -2.385 & 1.907 & 0.000 \\ \mathrm{H} & 1.959 & -2.017 & 0.877 \\ \mathrm{H} & 2.591 & 1.775 & 0.000 \\ \mathrm{~N} & 2.134 & 0.869 & 0.000 \\ \mathrm{H} & 3.850 & -0.299 & 0.001 \\ \mathrm{H} & -1.039 & -2.186 & 0.000 \\ \mathrm{H} & -3.909 & 0.064 & 0.000 \\ \mathrm{H} & -3.389 & -1.572 & 0.000 \\ \mathrm{~N} & -3.153 & -0.596 & 0.000 \\ \mathrm{H} & 1.959 & -2.017 & -0.877\end{array}$

\section{Ar14}

$\begin{array}{lrrr}\text { C } & 0.466 & -0.667 & 0.000 \\ \text { C } & 0.711 & 0.701 & 0.000 \\ \text { C } & -0.290 & 1.659 & 0.000 \\ \text { C } & -1.586 & 1.192 & 0.000 \\ \text { C } & -1.861 & -0.186 & 0.000 \\ \text { C } & -0.832 & -1.132 & 0.000 \\ \text { C } & 1.791 & -1.375 & 0.000 \\ \text { C } & 2.755 & -0.245 & 0.000 \\ \text { H } & -0.078 & 2.719 & 0.000 \\ \text { H } & -2.425 & 1.875 & 0.000 \\ \text { H } & 2.571 & 1.784 & 0.000 \\ \text { N } & 2.118 & 0.875 & 0.000 \\ \text { H } & -1.056 & -2.192 & 0.000 \\ \text { O } & -3.155 & -0.524 & 0.000 \\ \text { H } & 1.957 & -2.012 & -0.876 \\ \text { H } & 3.836 & -0.289 & 0.000\end{array}$




$\begin{array}{lrrr}\text { H } & -3.281 & -1.480 & -0.001 \\ \text { H } & 1.957 & -2.012 & 0.876 \\ \text { Ar15 } & & & \\ \text { C } & & & \\ \text { C } & 0.724 & -0.616 & 0.000 \\ \text { C } & 1.238 & 0.673 & 0.000 \\ \text { C } & 0.447 & 1.813 & 0.000 \\ \text { C } & -0.913 & 1.611 & 0.000 \\ \text { C } & -1.465 & 0.314 & 0.000 \\ \text { C } & -0.642 & -0.820 & 0.000 \\ \text { C } & 1.879 & -1.576 & 0.000 \\ \text { H } & 3.052 & -0.663 & 0.000 \\ \text { H } & 0.866 & 2.810 & 0.000 \\ \text { H } & -1.599 & 2.447 & 0.000 \\ \text { H } & 1.913 & -2.232 & -0.877 \\ \text { N } & 3.276 & 1.362 & -0.001 \\ \text { H } & 2.650 & 0.562 & -0.001 \\ \text { O } & -1.056 & -1.817 & 0.001 \\ \text { C } & -2.795 & 0.267 & 0.001 \\ \text { H } & -3.443 & -0.998 & -0.001 \\ \text { H } & -3.183 & -1.565 & 0.895 \\ \text { H } & -4.507 & -0.786 & -0.002 \\ \text { H } & -3.180 & -1.564 & -0.896 \\ \text { H } & 1.913 & -2.232 & 0.877 \\ & 4.101 & -0.923 & 0.001\end{array}$

$\begin{array}{lrrr}\text { C } & 0.409 & 0.954 & 0.000 \\ \text { C } & -0.100 & -0.339 & 0.000 \\ \text { C } & 0.677 & -1.479 & 0.000 \\ \text { C } & 2.051 & -1.276 & 0.000 \\ \text { C } & 2.589 & 0.009 & 0.000 \\ \text { C } & 1.778 & 1.140 & 0.000 \\ \text { C } & -0.748 & 1.907 & 0.000 \\ \text { C } & -1.909 & 0.983 & 0.000 \\ \text { H } & 0.254 & -2.474 & 0.000 \\ \text { H } & 2.712 & -2.131 & 0.000 \\ \text { H } & 3.664 & 0.129 & 0.000 \\ \text { H } & 2.212 & 2.131 & 0.000 \\ \text { H } & -0.787 & 2.563 & -0.876 \\ \text { H } & -2.959 & 1.244 & 0.000 \\ \text { N } & -1.527 & -0.247 & 0.000 \\ \text { C } & -2.389 & -1.425 & 0.000 \\ \text { H } & -2.173 & -2.013 & -0.891 \\ \text { H } & -2.172 & -2.013 & 0.890 \\ \text { H } & -3.428 & -1.109 & 0.000 \\ \text { H } & -0.786 & 2.563 & 0.877\end{array}$

\section{Ar16}

$\begin{array}{lrrr}\mathrm{C} & 1.092 & -0.639 & 0.000 \\ \mathrm{C} & 1.451 & 0.705 & 0.000 \\ \mathrm{C} & 0.555 & 1.754 & 0.000 \\ \mathrm{C} & -0.788 & 1.402 & 0.000 \\ \mathrm{C} & -1.176 & 0.061 & 0.000 \\ \mathrm{C} & -0.245 & -0.975 & 0.000 \\ \mathrm{C} & 2.348 & -1.460 & 0.000 \\ \mathrm{C} & 3.404 & -0.420 & 0.000 \\ \mathrm{H} & 0.869 & 2.789 & 0.000 \\ \mathrm{H} & -1.547 & 2.170 & 0.000 \\ \mathrm{H} & 2.461 & -2.110 & -0.876 \\ \mathrm{H} & 3.408 & 1.619 & 0.000 \\ \mathrm{~N} & 2.874 & 0.755 & 0.000 \\ \mathrm{H} & 4.476 & -0.562 & 0.000 \\ \mathrm{H} & -0.596 & -1.999 & 0.000 \\ \mathrm{C} & -2.623 & -0.335 & 0.000 \\ \mathrm{O} & -2.986 & -1.474 & 0.000 \\ \mathrm{O} & -3.440 & 0.720 & 0.000 \\ \mathrm{H} & -4.354 & 0.398 & 0.000 \\ \mathrm{H} & 2.461 & -2.110 & 0.876\end{array}$

\section{Ar18}

$\begin{array}{lrrr}\text { C } & 0.593 & -0.941 & 0.000 \\ \text { C } & 0.345 & 0.424 & 0.000 \\ \text { C } & 1.333 & 1.387 & 0.000 \\ \text { C } & 2.642 & 0.918 & 0.000 \\ \text { C } & 2.918 & -0.446 & 0.000 \\ \text { C } & 1.898 & -1.393 & 0.000 \\ \text { C } & -0.730 & -1.641 & 0.000 \\ \text { C } & -1.707 & -0.511 & 0.000 \\ \text { H } & 1.116 & 2.446 & 0.000 \\ \text { H } & 3.458 & 1.627 & 0.000 \\ \text { H } & 3.948 & -0.777 & 0.000 \\ \text { H } & 2.126 & -2.451 & 0.000 \\ \text { H } & -0.901 & -2.275 & -0.877 \\ \text { N } & -1.072 & 0.619 & 0.000 \\ \text { C } & -1.657 & 1.955 & 0.000 \\ \text { H } & -1.319 & 2.483 & 0.890 \\ \text { H } & -1.317 & 2.484 & -0.889 \\ \text { H } & -2.739 & 1.885 & -0.001 \\ \text { C } & -3.182 & -0.654 & 0.000 \\ \text { H } & -3.615 & -0.180 & 0.883 \\ \text { H } & -3.615 & -0.180 & -0.883 \\ \text { H } & -3.454 & -1.706 & 0.000 \\ \text { H } & -0.901 & -2.275 & 0.877\end{array}$

\section{Ar19}

\begin{tabular}{|c|c|c|}
\hline C & 0.337 & -0.416 \\
\hline C & 1.288 & 0.589 \\
\hline C & 1.008 & 1.941 \\
\hline
\end{tabular}




$\begin{array}{lrrr}\text { C } & -0.344 & 2.256 & 0.000 \\ \mathrm{C} & -1.337 & 1.278 & 0.000 \\ \mathrm{C} & -1.013 & -0.081 & 0.000 \\ \mathrm{C} & 1.025 & -1.742 & 0.000 \\ \mathrm{C} & 2.453 & -1.344 & 0.000 \\ \mathrm{H} & 1.775 & 2.702 & 0.000 \\ \mathrm{H} & -0.641 & 3.296 & 0.000 \\ \mathrm{H} & -2.371 & 1.588 & 0.000 \\ \mathrm{H} & 0.801 & -2.365 & -0.874 \\ \mathrm{H} & 3.442 & 0.440 & 0.000 \\ \mathrm{~N} & 2.557 & -0.059 & 0.000 \\ \mathrm{H} & 0.801 & -2.365 & 0.874 \\ \mathrm{H} & 3.323 & -1.986 & 0.000 \\ \mathrm{O} & -1.879 & -1.097 & 0.000 \\ \mathrm{C} & -3.272 & -0.797 & 0.000 \\ \mathrm{H} & -3.782 & -1.755 & 0.000 \\ \mathrm{H} & -3.543 & -0.235 & 0.895 \\ \mathrm{H} & -3.543 & -0.235 & -0.895\end{array}$

Ar20

$\begin{array}{lrrr}\text { C } & 0.083 & -1.176 & 0.000 \\ \text { C } & -1.166 & -0.465 & 0.000 \\ \text { C } & -0.867 & 0.847 & 0.000 \\ \text { C } & 0.604 & 1.029 & 0.000 \\ \text { N } & 1.078 & -0.345 & 0.000 \\ \text { H } & 2.055 & -0.610 & 0.000 \\ \text { H } & 0.241 & -2.247 & 0.000 \\ \text { H } & -2.137 & -0.932 & 0.000 \\ \text { H } & -1.560 & 1.676 & 0.000 \\ \text { H } & 0.968 & 1.560 & 0.886 \\ \text { H } & 0.968 & 1.560 & -0.886\end{array}$

Ar21

$\begin{array}{lrrr}\text { C } & -1.107 & -0.114 & 0.000 \\ \text { C } & -0.715 & 1.317 & 0.000 \\ \text { C } & 0.775 & 1.266 & 0.000 \\ \text { C } & 1.173 & -0.005 & 0.000 \\ \text { N } & -0.017 & -0.814 & 0.000 \\ \text { H } & 0.006 & -1.829 & 0.000 \\ \text { H } & -1.146 & 1.821 & -0.874 \\ \text { H } & 1.421 & 2.129 & 0.000 \\ \text { C } & 2.502 & -0.664 & 0.000 \\ \text { H } & 2.629 & -1.289 & 0.885 \\ \text { H } & 2.629 & -1.290 & -0.885 \\ \text { H } & 3.282 & 0.093 & 0.000 \\ \text { C } & -2.476 & -0.678 & 0.000 \\ \text { H } & -2.627 & -1.303 & -0.883 \\ \text { H } & -2.627 & -1.303 & 0.882 \\ \text { H } & -3.216 & 0.118 & 0.000 \\ \text { H } & -1.146 & 1.821 & 0.874\end{array}$

$\begin{array}{lrrr}\text { C } & 1.119 & -0.144 & -0.003 \\ \text { C } & -0.051 & -0.968 & 0.002 \\ \text { C } & -1.133 & -0.172 & 0.004 \\ \text { C } & -0.668 & 1.240 & 0.003 \\ \text { N } & 0.770 & 1.098 & -0.003 \\ \text { H } & 1.418 & 1.873 & -0.006 \\ \text { H } & -0.027 & -2.046 & 0.004 \\ \text { H } & -1.006 & 1.781 & 0.893 \\ \text { C } & 2.531 & -0.584 & -0.001 \\ \text { H } & 3.069 & -0.133 & -0.837 \\ \text { H } & 3.015 & -0.261 & 0.923 \\ \text { H } & 2.597 & -1.665 & -0.074 \\ \text { C } & -2.569 & -0.547 & -0.001 \\ \text { H } & -2.819 & -1.037 & -0.945 \\ \text { H } & -2.779 & -1.258 & 0.798 \\ \text { H } & -3.215 & 0.321 & 0.118 \\ \text { H } & -1.014 & 1.786 & -0.879\end{array}$

Ar23

$\begin{array}{lrrr}\text { C } & 0.111 & 1.107 & 0.000 \\ \text { C } & 1.506 & 0.745 & 0.000 \\ \text { C } & 1.560 & -0.597 & 0.000 \\ \text { C } & 0.181 & -1.145 & 0.000 \\ \text { N } & -0.645 & 0.057 & 0.000 \\ \text { H } & -0.317 & 2.102 & 0.000 \\ \text { H } & 2.322 & 1.449 & 0.000 \\ \text { H } & 2.443 & -1.218 & 0.000 \\ \text { H } & -0.040 & -1.748 & -0.886 \\ \text { C } & -2.103 & 0.015 & 0.000 \\ \text { H } & -2.447 & -0.510 & -0.890 \\ \text { H } & -2.447 & -0.510 & 0.890 \\ \text { H } & -2.487 & 1.031 & 0.000 \\ \text { H } & -0.041 & -1.750 & 0.884\end{array}$

Ar24

$\begin{array}{lrrr}\text { C } & 1.113 & -0.183 & 0.000 \\ \text { C } & 0.751 & -1.621 & 0.000 \\ \text { C } & -0.737 & -1.598 & 0.001 \\ \text { C } & -1.153 & -0.333 & 0.000 \\ \text { N } & 0.020 & 0.515 & 0.000 \\ \text { H } & 1.187 & -2.119 & -0.874 \\ \text { H } & -1.370 & -2.470 & 0.001 \\ \text { C } & -0.078 & 1.971 & 0.001 \\ \text { H } & -0.627 & 2.291 & -0.883 \\ \text { H } & -0.612 & 2.291 & 0.894 \\ \text { H } & 0.916 & 2.405 & -0.008 \\ \text { C } & -2.508 & 0.273 & -0.001\end{array}$




$\begin{array}{lrrr}\mathrm{H} & -2.668 & 0.895 & 0.881 \\ \mathrm{H} & -2.670 & 0.887 & -0.887 \\ \mathrm{H} & -3.251 & -0.521 & 0.004 \\ \mathrm{C} & 2.486 & 0.374 & 0.000 \\ \mathrm{H} & 2.651 & 0.995 & -0.883 \\ \mathrm{H} & 2.651 & 0.995 & 0.883 \\ \mathrm{H} & 3.215 & -0.432 & 0.000 \\ \mathrm{H} & 1.187 & -2.118 & 0.875\end{array}$

Ar25

$\begin{array}{lrrr}\mathrm{C} & 1.411 & -0.743 & 0.000 \\ \mathrm{O} & 0.006 & -1.042 & 0.000 \\ \mathrm{H} & -0.152 & 2.224 & 0.000 \\ \mathrm{H} & 2.369 & 1.304 & 0.000 \\ \mathrm{H} & 1.852 & -1.201 & -0.890 \\ \mathrm{C} & -2.132 & -0.015 & 0.000 \\ \mathrm{H} & -2.461 & -0.576 & -0.878 \\ \mathrm{H} & -2.461 & -0.576 & 0.878 \\ \mathrm{H} & -2.573 & 0.977 & 0.000 \\ \mathrm{H} & 1.852 & -1.200 & 0.890\end{array}$

$\begin{array}{lrrr}\text { C } & -1.165 & 0.164 & 0.000 \\ \mathrm{C} & -0.106 & 1.188 & 0.000 \\ \mathrm{C} & 1.099 & 0.574 & 0.000 \\ \mathrm{C} & 0.811 & -0.814 & 0.000 \\ \mathrm{O} & -0.437 & -1.078 & 0.000 \\ \mathrm{H} & -1.802 & 0.176 & -0.890 \\ \mathrm{H} & -0.304 & 2.251 & 0.000 \\ \mathrm{H} & 2.085 & 1.010 & 0.000 \\ \mathrm{H} & 1.489 & -1.662 & 0.000 \\ \mathrm{H} & -1.802 & 0.176 & 0.890\end{array}$

Ar28

Ar26

$\begin{array}{lrrr}\mathrm{C} & 0.628 & 0.093 & 0.000 \\ \mathrm{C} & 1.517 & 1.221 & 0.000 \\ \mathrm{C} & 2.773 & 0.734 & 0.000 \\ \mathrm{C} & 2.710 & -0.737 & 0.000 \\ \mathrm{O} & 1.299 & -1.011 & 0.000 \\ \mathrm{H} & 1.199 & 2.251 & 0.000 \\ \mathrm{H} & 3.699 & 1.293 & 0.000 \\ \mathrm{H} & 3.144 & -1.204 & 0.889 \\ \mathrm{Si} & -1.323 & -0.002 & 0.000 \\ \mathrm{C} & -1.721 & -0.931 & -1.557 \\ \mathrm{H} & -1.267 & -1.923 & -1.554 \\ \mathrm{H} & -2.803 & -1.061 & -1.626 \\ \mathrm{H} & -1.394 & -0.396 & -2.449 \\ \mathrm{C} & -1.721 & -0.931 & 1.557 \\ \mathrm{H} & -1.394 & -0.397 & 2.449 \\ \mathrm{H} & -2.803 & -1.061 & 1.627 \\ \mathrm{H} & -1.267 & -1.923 & 1.554 \\ \mathrm{C} & -1.871 & 1.770 & 0.000 \\ \mathrm{H} & -2.962 & 1.813 & 0.000 \\ \mathrm{H} & -1.527 & 2.304 & 0.888 \\ \mathrm{H} & -1.527 & 2.304 & -0.888 \\ \mathrm{H} & 3.144 & -1.204 & -0.889\end{array}$

Ar27

Ar29

$\begin{array}{lrrr}C & -0.666 & 0.055 & 0.000 \\ C & 0.190 & 1.202 & 0.000 \\ C & 1.451 & 0.732 & 0.000\end{array}$

$\begin{array}{rrrr}C & 2.759 & 0.165 & 0.014 \\ C & 2.628 & -1.222 & 0.194 \\ C & 1.496 & -1.580 & -0.488\end{array}$




$\begin{array}{lrrr}\mathrm{C} & 0.901 & -0.408 & -1.053 \\ \mathrm{O} & 1.793 & 0.643 & -0.713 \\ \mathrm{H} & 3.307 & -1.847 & 0.751 \\ \mathrm{H} & 1.080 & -2.571 & -0.604 \\ \mathrm{C} & 3.769 & 1.103 & 0.542 \\ \mathrm{H} & 3.969 & 1.890 & -0.184 \\ \mathrm{H} & 3.385 & 1.573 & 1.452 \\ \mathrm{H} & 4.685 & 0.574 & 0.793 \\ \mathrm{C} & -1.766 & 1.655 & 0.681 \\ \mathrm{C} & -2.131 & 1.756 & -0.797 \\ \mathrm{H} & -2.589 & 1.827 & 1.374 \\ \mathrm{H} & -0.950 & 2.341 & 0.905 \\ \mathrm{H} & -3.096 & 1.284 & -1.008 \\ \mathrm{H} & -2.168 & 2.789 & -1.135 \\ \mathrm{O} & -1.086 & 1.074 & -1.454 \\ \mathrm{O} & -1.455 & -1.315 & -1.006 \\ \mathrm{C} & -1.901 & -1.917 & 0.184 \\ \mathrm{H} & -2.731 & -2.588 & -0.026 \\ \mathrm{H} & -1.101 & -2.503 & 0.659 \\ \mathrm{C} & -2.314 & -0.747 & 1.063 \\ \mathrm{H} & -3.254 & -0.334 & 0.700 \\ \mathrm{H} & -2.402 & -0.971 & 2.126 \\ \mathrm{~N} & -1.237 & 0.265 & 0.826 \\ \mathrm{C} & -0.200 & 0.178 & 1.873 \\ \mathrm{H} & 0.635 & 0.820 & 1.602 \\ \mathrm{H} & -0.611 & 0.507 & 2.827 \\ \mathrm{H} & 0.141 & -0.852 & 1.964 \\ \mathrm{~B} & -0.773 & -0.098 & -0.746 \\ \mathrm{H} & 0.759 & -0.419 & -2.140\end{array}$

$\begin{array}{lrrr}\mathrm{C} & -0.194 & -0.578 & 1.955 \\ \mathrm{H} & 0.857 & -0.302 & 1.906 \\ \mathrm{H} & -0.582 & -0.358 & 2.949 \\ \mathrm{H} & -0.305 & -1.643 & 1.750 \\ \mathrm{~B} & -0.540 & -0.097 & -0.625 \\ \mathrm{C} & -0.938 & 2.198 & -0.347 \\ \mathrm{C} & -2.442 & -1.335 & -0.174 \\ \mathrm{O} & -1.100 & 3.336 & -0.627 \\ \mathrm{O} & -3.285 & -2.157 & -0.277 \\ \mathrm{H} & 0.948 & -0.483 & -2.094\end{array}$

Ar31

$\begin{array}{lrrr}\text { C } & 2.790 & -0.945 & 0.000 \\ \text { C } & 3.289 & 0.455 & 0.000 \\ \text { C } & 2.254 & 1.298 & 0.000 \\ \text { C } & 1.064 & 0.473 & 0.000 \\ \text { O } & 1.356 & -0.799 & 0.000 \\ \text { H } & 3.072 & -1.509 & -0.890 \\ \text { H } & 4.340 & 0.706 & 0.000 \\ \text { H } & 2.236 & 2.375 & 0.000 \\ \text { O } & -0.120 & 0.868 & -0.001 \\ \text { S } & -1.660 & -0.057 & 0.000 \\ \mathrm{C} & -1.604 & -1.052 & 1.555 \\ \text { H } & -2.556 & -1.574 & 1.677 \\ \text { H } & -0.817 & -1.806 & 1.535 \\ \text { H } & -1.467 & -0.416 & 2.432 \\ \mathrm{C} & -1.606 & -1.049 & -1.557 \\ \text { H } & -2.558 & -1.573 & -1.678 \\ \text { H } & -1.472 & -0.411 & -2.433 \\ \text { H } & -0.817 & -1.801 & -1.540 \\ \text { C } & -2.888 & 1.314 & 0.002 \\ \text { H } & -2.783 & 1.943 & -0.882 \\ \text { H } & -3.900 & 0.903 & 0.002 \\ \text { H } & -2.783 & 1.941 & 0.889 \\ \text { H } & 3.072 & -1.509 & 0.891\end{array}$

Ar32

$\begin{array}{lrrr}\text { C } & 0.083 & 1.214 & 0.000 \\ \text { C } & -1.268 & 0.783 & 0.000 \\ \text { C } & -1.354 & -0.568 & 0.000 \\ \text { C } & -0.056 & -1.261 & 0.000 \\ \text { S } & 1.212 & 0.012 & 0.000 \\ \text { H } & 0.402 & 2.251 & 0.000 \\ \text { H } & -2.101 & 1.470 & 0.000 \\ \text { H } & -2.280 & -1.129 & 0.000 \\ \text { H } & 0.081 & -1.900 & -0.881 \\ \text { H } & 0.081 & -1.900 & 0.881\end{array}$




$\begin{array}{lrrr}\text { C } & 0.429 & 0.175 & 0.000 \\ \text { C } & 1.182 & 1.394 & 0.000 \\ \text { C } & 2.519 & 1.203 & 0.000 \\ \text { C } & 2.933 & -0.210 & 0.000 \\ \text { S } & 1.407 & -1.162 & 0.000 \\ \text { H } & 0.700 & 2.362 & 0.000 \\ \text { H } & 3.257 & 1.996 & 0.000 \\ \text { H } & 3.527 & -0.477 & -0.881 \\ \text { Si } & -1.513 & 0.027 & 0.000 \\ \text { C } & -1.914 & -0.910 & 1.554 \\ \text { H } & -2.997 & -1.023 & 1.635 \\ \text { H } & -1.478 & -1.911 & 1.546 \\ \text { H } & -1.567 & -0.387 & 2.446 \\ \text { C } & -1.914 & -0.915 & -1.551 \\ \text { H } & -2.997 & -1.028 & -1.632 \\ \text { H } & -1.567 & -0.395 & -2.444 \\ \text { H } & -1.478 & -1.916 & -1.540 \\ \text { C } & -2.135 & 1.775 & -0.003 \\ \text { H } & -3.227 & 1.767 & -0.003 \\ \text { H } & -1.816 & 2.325 & 0.884 \\ \text { H } & -1.816 & 2.322 & -0.891 \\ \text { H } & 3.527 & -0.477 & 0.881 \\ & & & \end{array}$

\section{Ar34}

$\begin{array}{lrrr}\text { C } & 0.788 & 0.192 & 0.000 \\ \text { C } & 0.010 & 1.390 & 0.000 \\ \text { C } & -1.317 & 1.151 & 0.000 \\ \text { C } & -1.682 & -0.278 & 0.000 \\ \text { S } & -0.138 & -1.196 & 0.000 \\ \text { H } & 0.471 & 2.367 & 0.000 \\ \text { H } & -2.083 & 1.916 & 0.000 \\ \text { H } & -2.269 & -0.559 & -0.881 \\ \text { C } & 2.270 & 0.146 & 0.000 \\ \text { H } & 2.628 & -0.396 & 0.878 \\ \text { H } & 2.628 & -0.396 & -0.878 \\ \text { H } & 2.688 & 1.149 & 0.000 \\ \text { H } & -2.268 & -0.558 & 0.882\end{array}$

Ar35

$\begin{array}{lrrr}\text { C } & 0.544 & -1.180 & 0.000 \\ \text { C } & -0.577 & -0.200 & 0.000 \\ \text { C } & -0.173 & 1.132 & 0.000 \\ \text { C } & 1.205 & 1.221 & 0.000 \\ \text { S } & 2.068 & -0.219 & 0.000 \\ \text { H } & 0.504 & -1.820 & 0.885 \\ \text { H } & -0.828 & 1.989 & 0.000 \\ \text { H } & 1.755 & 2.155 & 0.000 \\ \text { O } & -1.767 & -0.680 & 0.000 \\ \text { C } & -2.896 & 0.224 & 0.000 \\ \text { H } & -2.865 & 0.837 & 0.900\end{array}$

$\begin{array}{lrrr}\mathrm{H} & -2.864 & 0.837 & -0.899 \\ \mathrm{H} & -3.774 & -0.411 & 0.000 \\ \mathrm{H} & 0.504 & -1.820 & -0.885\end{array}$

Ar36

$\begin{array}{lrrr}\text { C } & -0.345 & 1.208 & -0.059 \\ \text { C } & 0.253 & -0.091 & -0.270 \\ \text { C } & -0.722 & -1.013 & -0.085 \\ \text { C } & -2.000 & -0.321 & 0.251 \\ \text { H } & -2.382 & -0.602 & 1.237 \\ \text { N } & -1.610 & 1.076 & 0.234 \\ \text { H } & -2.239 & 1.845 & 0.418 \\ \text { C } & 0.336 & 2.515 & -0.168 \\ \text { H } & -0.304 & 3.343 & 0.127 \\ \text { H } & 1.237 & 2.508 & 0.448 \\ \text { H } & 0.658 & 2.660 & -1.203 \\ \text { C } & -0.626 & -2.491 & -0.170 \\ \text { H } & -1.342 & -2.882 & -0.895 \\ \text { H } & 0.373 & -2.809 & -0.458 \\ \text { H } & -0.868 & -2.942 & 0.794 \\ \text { C } & 1.703 & -0.301 & -0.577 \\ \text { H } & 2.080 & 0.549 & -1.148 \\ \text { H } & 1.808 & -1.173 & -1.224 \\ \text { C } & 2.534 & -0.490 & 0.695 \\ \text { H } & 3.585 & -0.628 & 0.447 \\ \text { H } & 2.454 & 0.375 & 1.355 \\ \text { H } & 2.196 & -1.365 & 1.250 \\ \text { H } & -2.785 & -0.509 & -0.486\end{array}$

\section{Ar37}

$\begin{array}{lrrr}\text { C } & 2.538 & -0.024 & 0.000 \\ \text { C } & 1.929 & -1.264 & 0.000 \\ \text { C } & 0.564 & -1.565 & 0.000 \\ \text { C } & 1.950 & 1.245 & 0.000 \\ \text { C } & -0.500 & -0.691 & 0.000 \\ \text { C } & 0.613 & 1.585 & 0.000 \\ \text { C } & -0.492 & 0.729 & 0.000 \\ \text { H } & 3.622 & -0.033 & 0.000 \\ \text { H } & 2.596 & -2.118 & 0.000 \\ \text { H } & 0.313 & -2.621 & 0.000 \\ \text { H } & 2.645 & 2.077 & 0.000 \\ \text { H } & 0.387 & 2.646 & 0.000 \\ \mathrm{C} & -1.930 & -1.143 & 0.000 \\ \text { H } & -2.153 & -1.763 & 0.876 \\ \mathrm{C} & -2.695 & 0.133 & 0.000 \\ \mathrm{H} & -3.775 & 0.177 & 0.000 \\ \mathrm{C} & -1.866 & 1.189 & 0.000 \\ \mathrm{H} & -2.148 & 2.232 & 0.000 \\ \mathrm{H} & -2.153 & -1.763 & -0.876\end{array}$




$\begin{array}{lrrr}\mathrm{C} & 0.344 & 0.697 & 0.000 \\ \mathrm{C} & 0.000 & -0.645 & 0.000 \\ \mathrm{C} & -2.184 & -0.317 & 0.000 \\ \mathrm{C} & -1.989 & 1.066 & 0.000 \\ \mathrm{C} & -0.705 & 1.600 & 0.000 \\ \mathrm{C} & 1.839 & 0.795 & 0.000 \\ \mathrm{C} & 2.249 & -0.635 & 0.000 \\ \mathrm{H} & -3.183 & -0.732 & 0.000 \\ \mathrm{H} & -2.850 & 1.719 & 0.000 \\ \mathrm{H} & -0.545 & 2.670 & 0.000 \\ \mathrm{H} & 2.260 & 1.302 & 0.876 \\ \mathrm{H} & 1.227 & -2.419 & 0.000 \\ \mathrm{H} & 3.256 & -1.030 & 0.000 \\ \mathrm{~N} & 1.216 & -1.401 & 0.000 \\ \mathrm{~N} & -1.180 & -1.196 & 0.000 \\ \mathrm{H} & 2.260 & 1.302 & -0.876\end{array}$

\section{En1}

\begin{tabular}{lrrr} 
C & -0.583 & 0.000 & -0.281 \\
$\mathrm{C}$ & -1.331 & 1.304 & -0.184 \\
$\mathrm{C}$ & -1.331 & -1.304 & -0.184 \\
$\mathrm{C}$ & -2.721 & 1.232 & 0.450 \\
$\mathrm{H}$ & -0.726 & 2.039 & 0.345 \\
$\mathrm{C}$ & -2.721 & -1.232 & 0.450 \\
$\mathrm{H}$ & -1.421 & -1.664 & -1.217 \\
$\mathrm{H}$ & -0.726 & -2.039 & 0.345 \\
$\mathrm{C}$ & -3.488 & 0.000 & 0.003 \\
$\mathrm{H}$ & -3.251 & 2.149 & 0.197 \\
$\mathrm{H}$ & -2.623 & 1.215 & 1.538 \\
$\mathrm{H}$ & -3.251 & -2.150 & 0.197 \\
$\mathrm{H}$ & -2.623 & -1.215 & 1.538 \\
$\mathrm{H}$ & -4.487 & 0.000 & 0.439 \\
$\mathrm{H}$ & -3.613 & 0.000 & -1.085 \\
$\mathrm{C}$ & 1.519 & -1.215 & -0.670 \\
$\mathrm{C}$ & 1.519 & 1.215 & -0.669 \\
$\mathrm{C}$ & 2.499 & -1.259 & 0.500 \\
$\mathrm{H}$ & 2.055 & -1.119 & -1.616 \\
$\mathrm{H}$ & 0.899 & -2.100 & -0.729 \\
$\mathrm{C}$ & 2.499 & 1.259 & 0.500 \\
$\mathrm{H}$ & 2.055 & 1.119 & -1.616 \\
$\mathrm{H}$ & 0.899 & 2.100 & -0.729 \\
$\mathrm{C}$ & 3.361 & 0.000 & 0.531 \\
$\mathrm{H}$ & 3.117 & -2.152 & 0.398 \\
$\mathrm{H}$ & 1.934 & -1.360 & 1.431 \\
$\mathrm{H}$ & 3.117 & 2.151 & 0.398 \\
$\mathrm{H}$ & 1.934 & 1.360 & 1.431 \\
$\mathrm{H}$ & 3.994 & 0.000 & 1.418 \\
$\mathrm{H}$ & 4.029 & 0.000 & -0.335 \\
$\mathrm{~N}$ & 0.682 & 0.000 & -0.544 \\
& & & \\
\hline
\end{tabular}

En2

$\begin{array}{lrrr}\text { C } & -0.596 & 0.000 & -0.190 \\ \text { C } & -1.342 & 1.307 & -0.118 \\ \text { C } & -1.342 & -1.307 & -0.118 \\ \text { C } & -2.779 & 1.232 & 0.401 \\ \text { H } & -0.775 & 2.021 & 0.477 \\ \text { C } & -2.779 & -1.232 & 0.401 \\ \text { H } & -1.344 & -1.696 & -1.145 \\ \text { H } & -0.775 & -2.021 & 0.477 \\ \text { C } & -3.506 & 0.000 & -0.111 \\ \text { H } & -3.287 & 2.149 & 0.108 \\ \text { H } & -2.770 & 1.212 & 1.493 \\ \text { H } & -3.287 & -2.149 & 0.108 \\ \text { H } & -2.770 & -1.212 & 1.493 \\ \text { H } & -4.537 & 0.000 & 0.240 \\ \text { H } & -3.539 & 0.000 & -1.205 \\ \text { C } & 1.515 & -1.209 & -0.577 \\ \text { C } & 1.515 & 1.209 & -0.577 \\ \text { C } & 2.634 & -1.162 & 0.460 \\ \text { H } & 1.941 & -1.171 & -1.581 \\ \text { H } & 0.924 & -2.111 & -0.482 \\ \text { C } & 2.634 & 1.162 & 0.460 \\ \text { H } & 1.941 & 1.171 & -1.581 \\ \text { H } & 0.924 & 2.111 & -0.482 \\ \text { H } & 3.295 & -2.015 & 0.316 \\ \text { H } & 2.206 & -1.212 & 1.470 \\ \text { H } & 3.295 & 2.015 & 0.316 \\ \text { H } & 2.206 & 1.212 & 1.470 \\ \text { N } & 0.676 & 0.000 & -0.425 \\ \text { O } & 3.404 & 0.000 & 0.306 \\ \text { H } & -1.344 & 1.696 & -1.145\end{array}$

En3

$\begin{array}{lrrr}\text { N } & -0.942 & -0.001 & -0.039 \\ \mathrm{C} & -1.805 & -1.216 & 0.035 \\ \mathrm{C} & -1.791 & 1.226 & 0.026 \\ \mathrm{C} & -3.207 & -0.682 & -0.219 \\ \mathrm{H} & -1.474 & -1.945 & -0.699 \\ \mathrm{C} & -3.167 & 0.704 & 0.417 \\ \mathrm{H} & -1.372 & 1.923 & 0.746 \\ \mathrm{H} & -3.389 & -0.605 & -1.292 \\ \mathrm{H} & -3.958 & 1.361 & 0.067 \\ \mathrm{H} & -1.709 & -1.640 & 1.036 \\ \mathrm{H} & -3.966 & -1.331 & 0.209 \\ \mathrm{H} & -3.243 & 0.627 & 1.503 \\ \mathrm{H} & -1.795 & 1.688 & -0.962 \\ \mathrm{C} & 0.356 & -0.013 & -0.192 \\ \mathrm{C} & 1.113 & -1.307 & -0.241\end{array}$




$\begin{array}{lrrr}\mathrm{C} & 1.124 & 1.254 & -0.418 \\ \mathrm{C} & 2.494 & -1.222 & 0.423 \\ \mathrm{H} & 0.531 & -2.114 & 0.197 \\ \mathrm{C} & 2.504 & 1.265 & 0.250 \\ \mathrm{H} & 1.258 & 1.306 & -1.508 \\ \mathrm{H} & 0.545 & 2.129 & -0.136 \\ \mathrm{C} & 3.278 & -0.009 & -0.053 \\ \mathrm{H} & 3.029 & -2.146 & 0.210 \\ \mathrm{H} & 2.367 & -1.173 & 1.508 \\ \mathrm{H} & 3.042 & 2.146 & -0.097 \\ \mathrm{H} & 2.380 & 1.373 & 1.331 \\ \mathrm{H} & 4.251 & 0.021 & 0.435 \\ \mathrm{H} & 3.463 & -0.083 & -1.130 \\ \mathrm{H} & 1.239 & -1.541 & -1.306\end{array}$

En5

$\begin{array}{lrrr}\text { En4 } & & & \\ \text { C } & -0.990 & 0.000 & -0.264 \\ \text { C } & -1.731 & 1.305 & -0.130 \\ \text { C } & -1.731 & -1.305 & -0.130 \\ \text { C } & -3.100 & 1.232 & 0.549 \\ \text { H } & -1.107 & 2.033 & 0.386 \\ \text { C } & -3.100 & -1.232 & 0.549 \\ \text { H } & -1.853 & -1.675 & -1.157 \\ \text { H } & -1.107 & -2.033 & 0.386 \\ \text { C } & -3.882 & 0.000 & 0.126 \\ \text { H } & -3.638 & 2.149 & 0.314 \\ \text { H } & -2.967 & 1.214 & 1.633 \\ \text { H } & -3.638 & -2.149 & 0.314 \\ \text { H } & -2.967 & -1.214 & 1.633 \\ \text { H } & -4.866 & 0.000 & 0.592 \\ \text { H } & -4.041 & 0.000 & -0.958 \\ \text { C } & 1.084 & -1.208 & -0.808 \\ \text { C } & 1.084 & 1.208 & -0.808 \\ \text { C } & 2.242 & -1.191 & 0.180 \\ \text { H } & 1.468 & -1.151 & -1.827 \\ \text { H } & 0.489 & -2.106 & -0.710 \\ \text { C } & 2.242 & 1.191 & 0.180 \\ \text { H } & 1.468 & 1.151 & -1.827 \\ \text { H } & 0.489 & 2.106 & -0.709 \\ \text { H } & 2.860 & -2.071 & -0.001 \\ \text { H } & 1.843 & -1.266 & 1.208 \\ \text { H } & 2.860 & 2.071 & 0.000 \\ \text { H } & 1.843 & 1.266 & 1.208 \\ \text { N } & 0.258 & 0.000 & -0.601 \\ \text { N } & 3.042 & 0.000 & -0.008 \\ \text { C } & 4.220 & 0.000 & 0.847 \\ \text { H } & 4.822 & -0.883 & 0.635 \\ \text { H } & 4.822 & 0.883 & 0.635 \\ \text { H } & 3.959 & 0.000 & 1.915 \\ \text { H } & -1.853 & 1.675 & -1.157\end{array}$

$\begin{array}{lrrr}\mathrm{C} & 1.169 & 1.282 & 0.538 \\ \mathrm{C} & 1.180 & -1.172 & 0.717 \\ \mathrm{C} & 2.245 & 1.224 & -0.543 \\ \mathrm{H} & 1.620 & 1.330 & 1.532 \\ \mathrm{H} & 0.515 & 2.137 & 0.409 \\ \mathrm{C} & 2.251 & -1.279 & -0.363 \\ \mathrm{H} & 1.640 & -1.052 & 1.701 \\ \mathrm{H} & 0.529 & -2.039 & 0.749 \\ \mathrm{H} & 2.845 & 2.131 & -0.472 \\ \mathrm{H} & 1.761 & 1.232 & -1.524 \\ \mathrm{H} & 2.854 & -2.164 & -0.161 \\ \mathrm{H} & 1.766 & -1.431 & -1.332 \\ \mathrm{~N} & 0.367 & 0.042 & 0.492 \\ \mathrm{C} & -0.884 & 0.014 & 0.210 \\ \mathrm{C} & -1.745 & 1.225 & -0.012 \\ \mathrm{C} & -1.703 & -1.236 & 0.049 \\ \mathrm{C} & -3.174 & 0.666 & -0.008 \\ \mathrm{H} & -1.569 & 2.007 & 0.725 \\ \mathrm{H} & -1.494 & 1.638 & -0.996 \\ \mathrm{C} & -3.009 & -0.740 & -0.584 \\ \mathrm{H} & -1.187 & -2.003 & -0.526 \\ \mathrm{H} & -3.859 & 1.288 & -0.578 \\ \mathrm{H} & -3.544 & 0.612 & 1.017 \\ \mathrm{H} & -3.846 & -1.397 & -0.365 \\ \mathrm{H} & -2.895 & -0.692 & -1.669 \\ \mathrm{C} & 3.113 & -0.021 & -0.393 \\ \mathrm{H} & 3.832 & -0.077 & -1.209 \\ \mathrm{H} & 3.689 & 0.046 & 0.535 \\ \mathrm{H} & -1.890 & -1.644 & 1.050\end{array}$

En6

$\begin{array}{lrrr}\mathrm{C} & -1.179 & 1.266 & -0.469 \\ \mathrm{C} & -1.190 & -1.174 & -0.642 \\ \mathrm{C} & -2.339 & 1.133 & 0.515 \\ \mathrm{H} & -1.560 & 1.362 & -1.486 \\ \mathrm{H} & -0.561 & 2.125 & -0.226 \\ \mathrm{C} & -2.347 & -1.185 & 0.351 \\ \mathrm{H} & -1.579 & -1.103 & -1.659 \\ \mathrm{H} & -0.574 & -2.064 & -0.555 \\ \mathrm{H} & -2.992 & 1.998 & 0.421 \\ \mathrm{H} & -1.952 & 1.092 & 1.542 \\ \mathrm{H} & -3.005 & -2.023 & 0.132 \\ \mathrm{H} & -1.963 & -1.294 & 1.374 \\ \mathrm{~N} & -0.371 & 0.032 & -0.414 \\ \mathrm{O} & -3.104 & -0.009 & 0.231 \\ \mathrm{C} & 0.888 & 0.010 & -0.161 \\ \mathrm{C} & 1.748 & 1.226 & 0.028 \\ \mathrm{C} & 1.717 & -1.236 & -0.032 \\ \mathrm{C} & 3.178 & 0.676 & -0.056\end{array}$




$\begin{array}{rrrr}\mathrm{H} & 1.525 & 2.014 & -0.689 \\ \mathrm{H} & 1.549 & 1.624 & 1.030 \\ \mathrm{C} & 3.054 & -0.731 & 0.528 \\ \mathrm{H} & 1.237 & -2.000 & 0.577 \\ \mathrm{H} & 3.890 & 1.302 & 0.475 \\ \mathrm{H} & 3.491 & 0.625 & -1.101 \\ \mathrm{H} & 3.882 & -1.383 & 0.262 \\ \mathrm{H} & 3.001 & -0.684 & 1.617 \\ \mathrm{H} & 1.850 & -1.652 & -1.038\end{array}$

\section{En7}

$\begin{array}{lrrr}\text { C } & 2.899 & 0.731 & -0.229 \\ \text { C } & 1.492 & 1.223 & 0.136 \\ \text { C } & 0.641 & 0.000 & 0.000 \\ \text { C } & 1.492 & -1.223 & -0.136 \\ \text { C } & 2.899 & -0.731 & 0.229 \\ \text { H } & 3.679 & 1.326 & 0.239 \\ \text { H } & 1.128 & 2.061 & -0.457 \\ \text { H } & 1.447 & 1.532 & 1.189 \\ \text { H } & 1.128 & -2.061 & 0.458 \\ \text { H } & 3.679 & -1.326 & -0.239 \\ \text { H } & 3.038 & -0.787 & 1.310 \\ \text { H } & 3.038 & 0.787 & -1.310 \\ \text { N } & -0.638 & 0.000 & 0.000 \\ \mathrm{C} & -1.482 & -1.216 & -0.122 \\ \mathrm{C} & -1.482 & 1.216 & 0.122 \\ \mathrm{C} & -2.876 & -0.723 & 0.247 \\ \mathrm{H} & -1.429 & -1.557 & -1.158 \\ \mathrm{C} & -2.876 & 0.723 & -0.247 \\ \mathrm{H} & -1.428 & 1.557 & 1.158 \\ \mathrm{H} & -3.652 & -1.332 & -0.210 \\ \mathrm{H} & -3.009 & 0.757 & -1.329 \\ \mathrm{H} & -1.097 & -1.998 & 0.529 \\ \mathrm{H} & -3.009 & -0.757 & 1.329 \\ \mathrm{H} & -3.652 & 1.332 & 0.210 \\ \mathrm{H} & -1.097 & 1.997 & -0.529 \\ \mathrm{H} & 1.447 & -1.532 & -1.188\end{array}$

\section{En8}

$\begin{array}{lrrr}\text { C } & -0.199 & 0.000 & -0.188 \\ \text { C } & -0.947 & 1.300 & -0.107 \\ \text { C } & -0.947 & -1.300 & -0.107 \\ \text { C } & -2.394 & 1.174 & 0.362 \\ \text { H } & -0.413 & 2.002 & 0.534 \\ \text { C } & -2.394 & -1.174 & 0.363 \\ \text { H } & -0.935 & -1.715 & -1.121 \\ \text { H } & -0.413 & -2.002 & 0.534 \\ \text { H } & -2.933 & 2.064 & 0.042 \\ \text { H } & -2.431 & 1.149 & 1.464 \\ \text { H } & -2.933 & -2.064 & 0.043\end{array}$

$\begin{array}{lrrr}\text { H } & -2.431 & -1.149 & 1.464 \\ \mathrm{C} & 1.906 & -1.211 & -0.572 \\ \mathrm{C} & 1.906 & 1.211 & -0.572 \\ \mathrm{C} & 3.032 & -1.162 & 0.458 \\ \mathrm{H} & 2.327 & -1.181 & -1.579 \\ \mathrm{H} & 1.312 & -2.109 & -0.466 \\ \mathrm{C} & 3.032 & 1.162 & 0.458 \\ \mathrm{H} & 2.326 & 1.181 & -1.579 \\ \mathrm{H} & 1.312 & 2.109 & -0.466 \\ \mathrm{H} & 3.692 & -2.015 & 0.312 \\ \mathrm{H} & 2.610 & -1.209 & 1.471 \\ \mathrm{H} & 3.692 & 2.015 & 0.312 \\ \mathrm{H} & 2.610 & 1.209 & 1.471 \\ \mathrm{~N} & 1.072 & 0.000 & -0.422 \\ \mathrm{O} & 3.801 & 0.000 & 0.297 \\ \mathrm{~N} & -3.015 & 0.000 & -0.210 \\ \mathrm{C} & -4.456 & 0.000 & 0.003 \\ \mathrm{H} & -4.892 & 0.883 & -0.462 \\ \mathrm{H} & -4.892 & -0.883 & -0.462 \\ \mathrm{H} & -4.716 & 0.000 & 1.071 \\ \mathrm{H} & -0.935 & 1.716 & -1.121\end{array}$

En9

$\begin{array}{lrrr}\text { C } & -1.279 & 0.508 & 0.067 \\ \mathrm{C} & -2.365 & -0.503 & -0.056 \\ \mathrm{C} & -3.721 & 0.146 & -0.310 \\ \mathrm{H} & -2.103 & -1.187 & -0.870 \\ \mathrm{H} & -4.488 & -0.620 & -0.399 \\ \mathrm{H} & -3.714 & 0.722 & -1.235 \\ \mathrm{C} & 0.964 & 1.329 & 0.453 \\ \mathrm{C} & 0.487 & -1.079 & 0.613 \\ \mathrm{C} & 2.110 & 0.975 & -0.490 \\ \mathrm{H} & 1.322 & 1.350 & 1.483 \\ \mathrm{H} & 0.506 & 2.282 & 0.200 \\ \mathrm{C} & 1.663 & -1.300 & -0.339 \\ \mathrm{H} & 0.837 & -1.099 & 1.647 \\ \mathrm{H} & -0.288 & -1.826 & 0.472 \\ \mathrm{H} & 2.914 & 1.698 & -0.367 \\ \mathrm{H} & 1.762 & 1.005 & -1.530 \\ \mathrm{H} & 2.138 & -2.251 & -0.105 \\ \mathrm{H} & 1.303 & -1.329 & -1.375 \\ \mathrm{~N} & -0.061 & 0.268 & 0.371 \\ \mathrm{O} & 2.624 & -0.292 & -0.175 \\ \mathrm{H} & -1.521 & 1.555 & -0.107 \\ \mathrm{H} & -4.000 & 0.807 & 0.509 \\ \mathrm{H} & -2.384 & -1.107 & 0.856\end{array}$

\section{En10}

$\begin{array}{lrrr}C & -0.350 & -0.309 & -0.017 \\ C & 0.588 & 0.694 & -0.603\end{array}$




$\begin{array}{lrrr}\text { H } & 0.311 & 1.684 & -0.225 \\ \mathrm{C} & 2.021 & 0.370 & -0.262 \\ \mathrm{C} & 2.709 & -0.585 & -1.003 \\ \mathrm{C} & 2.647 & 0.992 & 0.812 \\ \mathrm{C} & 4.014 & -0.918 & -0.670 \\ \mathrm{H} & 2.233 & -1.061 & -1.853 \\ \mathrm{C} & 3.953 & 0.659 & 1.144 \\ \mathrm{H} & 2.120 & 1.746 & 1.385 \\ \mathrm{C} & 4.636 & -0.296 & 0.405 \\ \mathrm{H} & 4.547 & -1.656 & -1.254 \\ \mathrm{H} & 4.438 & 1.152 & 1.976 \\ \mathrm{H} & 5.655 & -0.551 & 0.661 \\ \mathrm{C} & -2.357 & 0.555 & -1.099 \\ \mathrm{C} & -2.469 & -1.402 & 0.387 \\ \mathrm{C} & -3.495 & 1.154 & -0.274 \\ \mathrm{H} & -2.764 & -0.025 & -1.929 \\ \mathrm{H} & -1.691 & 1.324 & -1.478 \\ \mathrm{C} & -3.598 & -0.695 & 1.130 \\ \mathrm{H} & -2.878 & -2.017 & -0.415 \\ \mathrm{H} & -1.863 & -2.014 & 1.052 \\ \mathrm{H} & -4.115 & 1.774 & -0.918 \\ \mathrm{H} & -3.084 & 1.778 & 0.530 \\ \mathrm{H} & -4.295 & -1.435 & 1.517 \\ \mathrm{H} & -3.191 & -0.118 & 1.970 \\ \mathrm{~N} & -1.606 & -0.383 & -0.245 \\ \mathrm{O} & -4.308 & 0.141 & 0.253 \\ \mathrm{H} & 0.059 & -1.056 & 0.659 \\ \mathrm{H} & 0.440 & 0.723 & -1.687 \\ & & & \end{array}$

\section{En11}

$\begin{array}{lrrr}\text { C } & -0.349 & -0.271 & 0.385 \\ \text { C } & 0.594 & -0.672 & -0.702 \\ \text { H } & 0.260 & -0.220 & -1.642 \\ \text { C } & 2.010 & -0.264 & -0.382 \\ \text { C } & 2.837 & -1.118 & 0.340 \\ \text { C } & 2.480 & 0.985 & -0.769 \\ \text { C } & 4.125 & -0.722 & 0.674 \\ \text { H } & 2.482 & -2.099 & 0.633 \\ \text { C } & 3.768 & 1.380 & -0.436 \\ \text { H } & 1.846 & 1.650 & -1.346 \\ \text { C } & 4.590 & 0.527 & 0.288 \\ \text { H } & 4.767 & -1.393 & 1.229 \\ \text { H } & 4.131 & 2.350 & -0.747 \\ \text { H } & 5.595 & 0.833 & 0.543 \\ \text { C } & -2.367 & -1.029 & -0.744 \\ \text { C } & -2.472 & 0.038 & 1.488 \\ \text { C } & -3.359 & -0.008 & -1.294 \\ \text { H } & -2.892 & -1.894 & -0.331 \\ \text { H } & -1.668 & -1.376 & -1.498 \\ \text { C } & -3.469 & 1.068 & 0.969 \\ \text { H } & -2.989 & -0.842 & 1.875\end{array}$

$\begin{array}{lrrr}\mathrm{H} & -1.826 & 0.439 & 2.267 \\ \mathrm{H} & -3.926 & -0.486 & -2.092 \\ \mathrm{H} & -2.805 & 0.821 & -1.742 \\ \mathrm{H} & -4.115 & 1.359 & 1.797 \\ \mathrm{H} & -2.925 & 1.962 & 0.653 \\ \mathrm{~N} & -1.615 & -0.430 & 0.377 \\ \mathrm{H} & 0.070 & 0.201 & 1.272 \\ \mathrm{C} & -4.281 & 0.504 & -0.192 \\ \mathrm{H} & -4.947 & 1.269 & -0.589 \\ \mathrm{H} & -4.913 & -0.314 & 0.165 \\ \mathrm{H} & 0.521 & -1.757 & -0.837\end{array}$

\section{En12}

$\begin{array}{lrrr}\text { N } & -1.745 & 0.527 & -0.005 \\ \mathrm{C} & -2.283 & -0.487 & -0.953 \\ \mathrm{C} & -2.654 & 0.677 & 1.165 \\ \mathrm{C} & -3.662 & -0.816 & -0.389 \\ \mathrm{H} & -2.299 & -0.075 & -1.959 \\ \mathrm{C} & -3.494 & -0.590 & 1.114 \\ \mathrm{H} & -4.407 & -0.131 & -0.795 \\ \mathrm{H} & -4.440 & -0.467 & 1.636 \\ \mathrm{H} & -1.614 & -1.348 & -0.926 \\ \mathrm{H} & -3.962 & -1.831 & -0.639 \\ \mathrm{H} & -2.953 & -1.419 & 1.572 \\ \mathrm{H} & -3.264 & 1.566 & 0.995 \\ \mathrm{C} & -0.644 & 1.157 & -0.134 \\ \mathrm{C} & 0.346 & 0.970 & -1.222 \\ \mathrm{H} & 0.596 & 1.950 & -1.632 \\ \mathrm{H} & -2.062 & 0.807 & 2.067 \\ \mathrm{C} & 1.571 & 0.347 & -0.568 \\ \mathrm{C} & 1.716 & -1.036 & -0.546 \\ \mathrm{C} & 2.524 & 1.153 & 0.047 \\ \mathrm{C} & 2.816 & -1.610 & 0.076 \\ \mathrm{H} & 0.989 & -1.668 & -1.043 \\ \mathrm{C} & 3.620 & 0.577 & 0.672 \\ \mathrm{H} & 2.422 & 2.232 & 0.021 \\ \mathrm{C} & 3.765 & -0.803 & 0.688 \\ \mathrm{H} & 2.934 & -2.685 & 0.076 \\ \mathrm{H} & 4.365 & 1.208 & 1.138 \\ \mathrm{H} & -0.388 & 1.847 & 0.667 \\ \mathrm{H} & 4.624 & -1.251 & 1.170 \\ \mathrm{H} & -0.039 & 0.342 & -2.022\end{array}$

\section{En13}

$\begin{array}{lrrr}\text { C } & -0.593 & -0.424 & 0.168 \\ C & 0.241 & -1.633 & -0.205 \\ \text { H } & -0.097 & -1.941 & -1.202 \\ \text { C } & 0.022 & 0.918 & 0.179 \\ C & -0.366 & 1.842 & -0.791 \\ C & 1.020 & 1.240 & 1.097\end{array}$




$\begin{array}{lrrr}\mathrm{C} & 0.246 & 3.084 & -0.843 \\ \mathrm{H} & -1.127 & 1.580 & -1.518 \\ \mathrm{C} & 1.604 & 2.496 & 1.056 \\ \mathrm{H} & 1.336 & 0.518 & 1.837 \\ \mathrm{C} & 1.226 & 3.413 & 0.084 \\ \mathrm{H} & -0.043 & 3.794 & -1.606 \\ \mathrm{H} & 2.367 & 2.753 & 1.779 \\ \mathrm{H} & 1.698 & 4.385 & 0.047 \\ \mathrm{C} & 1.731 & -1.413 & -0.222 \\ \mathrm{C} & 2.509 & -1.859 & 0.839 \\ \mathrm{C} & 2.338 & -0.757 & -1.288 \\ \mathrm{C} & 3.880 & -1.637 & 0.845 \\ \mathrm{H} & 2.046 & -2.386 & 1.666 \\ \mathrm{C} & 3.706 & -0.534 & -1.283 \\ \mathrm{H} & 1.740 & -0.415 & -2.125 \\ \mathrm{C} & 4.478 & -0.970 & -0.213 \\ \mathrm{H} & 4.479 & -1.992 & 1.673 \\ \mathrm{H} & 4.172 & -0.026 & -2.117 \\ \mathrm{H} & 5.546 & -0.800 & -0.212 \\ \mathrm{C} & -2.611 & -1.817 & 0.265 \\ \mathrm{C} & -2.742 & 0.527 & 0.895 \\ \mathrm{C} & -3.786 & -1.559 & -0.678 \\ \mathrm{H} & -2.980 & -2.103 & 1.251 \\ \mathrm{H} & -1.977 & -2.603 & -0.128 \\ \mathrm{C} & -3.913 & 0.678 & -0.067 \\ \mathrm{H} & -3.115 & 0.211 & 1.871 \\ \mathrm{H} & -2.178 & 1.447 & 0.999 \\ \mathrm{H} & -4.390 & -2.461 & -0.752 \\ \mathrm{H} & -3.406 & -1.306 & -1.677 \\ \mathrm{H} & -4.609 & 1.417 & 0.325 \\ \mathrm{H} & -3.554 & 1.016 & -1.048 \\ \mathrm{~N} & -1.854 & -0.559 & 0.423 \\ \mathrm{O} & -4.610 & -0.536 & -0.190 \\ \mathrm{H} & -0.004 & -2.453 & 0.473\end{array}$

$\begin{array}{lrrr}\text { C } & 2.551 & -1.815 & 0.868 \\ \text { C } & 2.247 & -0.823 & -1.298 \\ \text { C } & 3.918 & -1.587 & 0.781 \\ \text { H } & 2.142 & -2.301 & 1.747 \\ \text { C } & 3.611 & -0.594 & -1.386 \\ \text { H } & 1.598 & -0.528 & -2.114 \\ \text { C } & 4.448 & -0.971 & -0.343 \\ \text { H } & 4.568 & -1.896 & 1.589 \\ \text { H } & 4.022 & -0.126 & -2.270 \\ \text { H } & 5.513 & -0.796 & -0.414 \\ \text { C } & -2.632 & -1.794 & 0.382 \\ \text { C } & -2.751 & 0.600 & 0.875 \\ \text { C } & -3.712 & -1.645 & -0.688 \\ \text { H } & -3.082 & -1.971 & 1.361 \\ \text { H } & -1.963 & -2.615 & 0.157 \\ \text { C } & -3.845 & 0.802 & -0.166 \\ \text { H } & -3.192 & 0.314 & 1.833 \\ \text { H } & -2.145 & 1.487 & 1.020 \\ \text { C } & -4.644 & -0.481 & -0.371 \\ \text { H } & -4.262 & -2.585 & -0.745 \\ \text { H } & -3.230 & -1.494 & -1.658 \\ \text { H } & -4.489 & 1.614 & 0.173 \\ \text { H } & -3.393 & 1.121 & -1.108 \\ \text { H } & -5.369 & -0.351 & -1.173 \\ \text { H } & -5.210 & -0.707 & 0.537 \\ \text { N } & -1.873 & -0.530 & 0.488 \\ \text { H } & 0.021 & -2.408 & 0.711\end{array}$

$\begin{array}{lrrr}\text { En14 } & & & \\ \text { C } & -0.607 & -0.421 & 0.253 \\ \text { C } & 0.223 & -1.653 & -0.053 \\ \text { H } & -0.161 & -2.058 & -0.997 \\ \text { C } & 0.033 & 0.911 & 0.225 \\ \text { C } & -0.308 & 1.806 & -0.786 \\ \text { C } & 1.019 & 1.241 & 1.153 \\ \text { C } & 0.337 & 3.030 & -0.869 \\ \text { H } & -1.058 & 1.537 & -1.520 \\ \text { C } & 1.638 & 2.478 & 1.081 \\ \text { H } & 1.299 & 0.538 & 1.926 \\ \text { C } & 1.304 & 3.368 & 0.068 \\ \text { H } & 0.083 & 3.719 & -1.663 \\ \text { H } & 2.392 & 2.742 & 1.810 \\ \text { H } & 1.803 & 4.326 & 0.006 \\ \text { C } & 1.708 & -1.427 & -0.166\end{array}$

En15

$\begin{array}{lrrr}\text { N } & -2.097 & -0.528 & -0.087 \\ \mathrm{C} & -2.770 & -1.854 & -0.148 \\ \mathrm{C} & -3.118 & 0.553 & -0.045 \\ \mathrm{C} & -4.257 & -1.523 & -0.233 \\ \mathrm{H} & -2.397 & -2.416 & -1.002 \\ \mathrm{C} & -4.357 & -0.168 & 0.462 \\ \mathrm{H} & -4.563 & -1.436 & -1.276 \\ \mathrm{H} & -5.267 & 0.375 & 0.217 \\ \mathrm{H} & -2.524 & -2.400 & 0.764 \\ \mathrm{H} & -4.864 & -2.295 & 0.233 \\ \mathrm{H} & -4.308 & -0.284 & 1.546 \\ \mathrm{H} & -3.273 & 0.923 & -1.059 \\ \mathrm{C} & -0.822 & -0.372 & 0.052 \\ \mathrm{C} & 0.044 & -1.602 & 0.181 \\ \mathrm{H} & -0.316 & -2.342 & -0.538 \\ \mathrm{H} & -2.759 & 1.366 & 0.580 \\ \mathrm{C} & -0.235 & 0.974 & 0.109 \\ \mathrm{C} & -0.506 & 1.910 & -0.889 \\ \mathrm{C} & 0.639 & 1.294 & 1.150 \\ \mathrm{C} & 0.092 & 3.158 & -0.845 \\ \mathrm{H} & -1.151 & 1.651 & -1.719 \\ \mathrm{C} & 1.203 & 2.556 & 1.207 \\ & & & \end{array}$




$\begin{array}{lrrr}\mathrm{H} & 0.869 & 0.562 & 1.913 \\ \mathrm{C} & 0.937 & 3.484 & 0.208 \\ \mathrm{H} & -0.100 & 3.875 & -1.631 \\ \mathrm{H} & 1.864 & 2.811 & 2.025 \\ \mathrm{H} & 1.397 & 4.463 & 0.246 \\ \mathrm{C} & 1.525 & -1.396 & -0.007 \\ \mathrm{C} & 2.413 & -1.693 & 1.019 \\ \mathrm{C} & 2.016 & -0.926 & -1.221 \\ \mathrm{C} & 3.778 & -1.512 & 0.839 \\ \mathrm{H} & 2.040 & -2.071 & 1.964 \\ \mathrm{C} & 3.377 & -0.741 & -1.401 \\ \mathrm{H} & 1.332 & -0.700 & -2.032 \\ \mathrm{C} & 4.261 & -1.032 & -0.368 \\ \mathrm{H} & 4.462 & -1.750 & 1.643 \\ \mathrm{H} & 3.751 & -0.375 & -2.348 \\ \mathrm{H} & 5.324 & -0.891 & -0.510 \\ \mathrm{H} & -0.160 & -2.033 & 1.169\end{array}$

\section{En16}

$\begin{array}{lrrr}\text { N } & -2.666 & -0.693 & -0.036 \\ \mathrm{C} & -3.232 & -2.047 & -0.272 \\ \mathrm{C} & -3.720 & 0.216 & 0.495 \\ \mathrm{C} & -4.729 & -1.866 & -0.053 \\ \mathrm{H} & -2.960 & -2.390 & -1.269 \\ \mathrm{C} & -4.790 & -0.747 & 0.985 \\ \mathrm{H} & -5.211 & -1.550 & -0.980 \\ \mathrm{H} & -5.764 & -0.268 & 1.044 \\ \mathrm{H} & -2.800 & -2.727 & 0.464 \\ \mathrm{H} & -5.199 & -2.789 & 0.276 \\ \mathrm{H} & -4.533 & -1.127 & 1.975 \\ \mathrm{H} & -4.092 & 0.830 & -0.325 \\ \mathrm{C} & -1.426 & -0.384 & -0.208 \\ \mathrm{C} & -0.469 & -1.454 & -0.680 \\ \mathrm{H} & -0.718 & -1.613 & -1.738 \\ \mathrm{H} & -3.286 & 0.864 & 1.252 \\ \mathrm{C} & -0.990 & 1.011 & -0.014 \\ \mathrm{C} & -1.540 & 2.010 & -0.820 \\ \mathrm{C} & -0.017 & 1.330 & 0.932 \\ \mathrm{C} & -1.113 & 3.320 & -0.680 \\ \mathrm{H} & -2.274 & 1.758 & -1.576 \\ \mathrm{C} & 0.377 & 2.649 & 1.089 \\ \mathrm{H} & 0.427 & 0.554 & 1.541 \\ \mathrm{C} & -0.163 & 3.640 & 0.280 \\ \mathrm{H} & -1.523 & 4.090 & -1.320 \\ \mathrm{H} & 1.119 & 2.899 & 1.834 \\ \mathrm{H} & 0.163 & 4.666 & 0.394 \\ \mathrm{C} & 0.995 & -1.145 & -0.533 \\ \mathrm{C} & 1.730 & -1.706 & 0.497 \\ \mathrm{C} & 1.637 & -0.275 & -1.416 \\ \mathrm{C} & 3.078 & -1.408 & 0.671 \\ \mathrm{H} & 1.256 & -2.396 & 1.187\end{array}$

$\begin{array}{lrrr}\mathrm{C} & 2.969 & 0.033 & -1.261 \\ \mathrm{H} & 1.082 & 0.171 & -2.235 \\ \mathrm{C} & 3.703 & -0.530 & -0.210 \\ \mathrm{H} & 3.624 & -1.870 & 1.480 \\ \mathrm{H} & 3.478 & 0.702 & -1.942 \\ \mathrm{O} & 4.996 & -0.173 & -0.141 \\ \mathrm{C} & 5.796 & -0.741 & 0.880 \\ \mathrm{H} & 5.833 & -1.828 & 0.787 \\ \mathrm{H} & 6.792 & -0.330 & 0.745 \\ \mathrm{H} & 5.420 & -0.468 & 1.869 \\ \mathrm{H} & -0.710 & -2.392 & -0.175\end{array}$

\section{En17}

$\begin{array}{lrrr}\text { N } & -1.382 & -1.448 & 0.041 \\ \text { C } & -0.690 & -2.730 & 0.348 \\ \text { C } & -2.473 & -1.687 & -0.942 \\ \text { C } & -1.321 & -3.735 & -0.609 \\ \text { H } & 0.382 & -2.598 & 0.219 \\ \text { C } & -2.729 & -3.180 & -0.812 \\ \text { H } & -0.779 & -3.747 & -1.556 \\ \text { H } & -3.227 & -3.579 & -1.692 \\ \text { H } & -0.899 & -2.986 & 1.388 \\ \text { H } & -1.307 & -4.740 & -0.197 \\ \text { H } & -3.354 & -3.383 & 0.059 \\ \text { H } & -2.101 & -1.448 & -1.938 \\ \text { C } & -1.115 & -0.326 & 0.618 \\ \text { C } & -0.044 & -0.255 & 1.671 \\ \text { H } & -0.273 & 0.577 & 2.336 \\ \text { H } & -3.316 & -1.040 & -0.711 \\ \text { C } & -1.805 & 0.915 & 0.230 \\ \text { C } & -1.863 & 1.308 & -1.109 \\ \text { C } & -2.354 & 1.733 & 1.220 \\ \text { C } & -2.470 & 2.504 & -1.451 \\ \text { H } & -1.394 & 0.704 & -1.875 \\ \text { C } & -2.993 & 2.910 & 0.866 \\ \text { H } & -2.310 & 1.440 & 2.261 \\ \text { C } & -3.046 & 3.298 & -0.466 \\ \text { H } & -2.492 & 2.819 & -2.485 \\ \text { H } & -3.440 & 3.529 & 1.631 \\ \text { H } & -3.529 & 4.228 & -0.738 \\ \text { C } & 1.310 & -0.021 & 1.016 \\ \text { C } & 1.555 & 1.149 & 0.301 \\ \text { C } & 2.321 & -0.969 & 1.146 \\ \text { C } & 2.786 & 1.362 & -0.292 \\ \text { H } & 0.786 & 1.909 & 0.218 \\ \text { C } & 3.559 & -0.762 & 0.559 \\ \text { H } & 2.152 & -1.866 & 1.730 \\ \text { C } & 3.789 & 0.401 & -0.168 \\ \text { H } & 2.982 & 2.272 & -0.843 \\ \text { H } & 4.348 & -1.494 & 0.667 \\ \text { C } & 5.066 & 0.617 & -0.789\end{array}$




$\begin{array}{lrrrrrrr}\text { N } & -6.082 & 0.787 & -1.292 & \text { C } & -3.384 & 0.137 & -1.155 \\ \text { H } & -0.016 & -1.167 & 2.264 & \mathrm{C} & -3.115 & -1.011 & 0.946 \\ & & & & \mathrm{H} & -1.231 & -0.744 & 1.932 \\ \text { En18 } & & & & \mathrm{C} & -3.886 & -0.699 & -0.166 \\ \mathrm{~N} & -1.779 & -1.426 & 0.012 & \mathrm{H} & -3.986 & 0.381 & -2.019 \\ \mathrm{C} & -1.157 & -2.746 & 0.310 & \mathrm{H} & -3.515 & -1.645 & 1.725 \\ \mathrm{C} & -2.852 & -1.592 & -1.006 & \mathrm{H} & -4.886 & -1.101 & -0.258 \\ \mathrm{C} & -1.808 & -3.699 & -0.685 & \mathrm{H} & -1.730 & 1.337 & -1.795 \\ \mathrm{H} & -0.077 & -2.663 & 0.213 & \mathrm{C} & 0.014 & 0.937 & 0.222 \\ \mathrm{C} & -3.183 & -3.073 & -0.913 & \mathrm{C} & 0.114 & 2.428 & 0.133 \\ \mathrm{H} & -1.241 & -3.719 & -1.616 & \mathrm{H} & -0.796 & 2.830 & -0.301 \\ \mathrm{H} & -3.674 & -3.431 & -1.815 & \mathrm{H} & 0.204 & 2.845 & 1.140 \\ \mathrm{H} & -1.408 & -3.012 & 1.338 & \mathrm{C} & 2.385 & 0.792 & 0.783 \\ \mathrm{H} & -1.854 & -4.713 & -0.294 & \mathrm{C} & 1.116 & -1.259 & 0.338 \\ \mathrm{H} & -3.841 & -3.263 & -0.064 & \mathrm{C} & 3.382 & 0.474 & -0.325 \\ \mathrm{H} & -2.440 & -1.353 & -1.986 & \mathrm{H} & 2.696 & 0.318 & 1.717 \\ \mathrm{C} & -1.476 & -0.329 & 0.619 & \mathrm{H} & 2.288 & 1.856 & 0.964 \\ \mathrm{C} & -0.434 & -0.330 & 1.705 & \mathrm{C} & 2.102 & -1.630 & -0.767 \\ \mathrm{H} & -0.650 & 0.492 & 2.386 & \mathrm{H} & 1.441 & -1.653 & 1.304 \\ \mathrm{H} & -3.669 & -0.910 & -0.785 & \mathrm{H} & 0.126 & -1.638 & 0.117 \\ \mathrm{C} & -2.094 & 0.951 & 0.238 & \mathrm{C} & 3.483 & -1.034 & -0.526 \\ \mathrm{C} & -2.093 & 1.373 & -1.093 & \mathrm{H} & 4.346 & 0.902 & -0.051 \\ \mathrm{C} & -2.632 & 1.775 & 1.229 & \mathrm{H} & 3.060 & 0.962 & -1.249 \\ \mathrm{C} & -2.629 & 2.604 & -1.427 & \mathrm{H} & 2.148 & -2.718 & -0.819 \\ \mathrm{H} & -1.631 & 0.761 & -1.858 & \mathrm{H} & 1.701 & -1.280 & -1.722 \\ \mathrm{C} & -3.201 & 2.989 & 0.881 & \mathrm{H} & 4.142 & -1.260 & -1.364 \\ \mathrm{H} & -2.634 & 1.460 & 2.264 & \mathrm{H} & 3.930 & -1.488 & 0.363 \\ \mathrm{C} & -3.194 & 3.407 & -0.443 & & 1.060 & 0.216 & 0.464 \\ \mathrm{H} & -2.604 & 2.940 & -2.455 & 0.974 & 2.751 & -0.450\end{array}$

\section{En20}

C

C

C

$\begin{array}{lll}2.513 & 1.239 & -0.122\end{array}$

$\begin{array}{lll}0.522 & 1.847 & 0.358\end{array}$

$\begin{array}{lll}3.174 & -0.954 & 0.672\end{array}$

$\begin{array}{lll}1.688 & -2.035 & 1.764\end{array}$

$\begin{array}{lll}3.444 & 0.224 & 0.005\end{array}$

$\begin{array}{lll}2.781 & 2.150 & -0.637\end{array}$

$\begin{array}{lll}3.942 & -1.708 & 0.760\end{array}$

$\begin{array}{lll}4.786 & 0.413 & -0.591\end{array}$

$\begin{array}{lll}5.576 & -0.490 & -0.460\end{array}$

$\begin{array}{lll}4.989 & 1.453 & -1.167\end{array}$

$\begin{array}{lll}-0.459 & -1.259 & 2.272\end{array}$

$\begin{array}{lrrr}\text { En19 } & & & \\ \text { C } & -1.323 & 0.336 & 0.070 \\ \text { C } & -2.114 & 0.675 & -1.028 \\ \text { C } & -1.831 & -0.508 & 1.061\end{array}$

$\begin{array}{rrr}1.325 & 0.335 & -0.076 \\ 2.157 & 0.745 & 0.967 \\ 1.787 & -0.590 & -1.016 \\ 3.422 & 0.197 & 1.093 \\ 3.067 & -1.104 & -0.904 \\ 1.158 & -0.881 & -1.848 \\ 3.879 & -0.721 & 0.156 \\ 4.057 & 0.496 & 1.917 \\ 3.432 & -1.802 & -1.645 \\ 4.876 & -1.132 & 0.247 \\ 1.809 & 1.471 & 1.692 \\ -0.006 & 0.945 & -0.227 \\ -0.096 & 2.440 & -0.206 \\ 0.850 & 2.861 & 0.118 \\ -0.289 & 2.806 & -1.218 \\ -2.395 & 0.783 & -0.738 \\ -1.134 & -1.245 & -0.250 \\ -3.399 & 0.364 & 0.329 \\ -2.685 & 0.352 & -1.698 \\ -2.342 & 1.861 & -0.837\end{array}$




$\begin{array}{lrrr}\mathrm{C} & -2.206 & -1.567 & 0.789 \\ \mathrm{H} & -1.399 & -1.677 & -1.216 \\ \mathrm{H} & -0.171 & -1.624 & 0.072 \\ \mathrm{H} & -4.391 & 0.713 & 0.049 \\ \mathrm{H} & -3.127 & 0.809 & 1.296 \\ \mathrm{H} & -2.318 & -2.648 & 0.857 \\ \mathrm{H} & -1.893 & -1.183 & 1.768 \\ \mathrm{~N} & -1.066 & 0.224 & -0.415 \\ \mathrm{H} & -0.896 & 2.793 & 0.444 \\ \mathrm{O} & -3.451 & -1.034 & 0.426\end{array}$

En21

$\begin{array}{lrrr}\mathrm{C} & -0.260 & 2.036 & -0.403 \\ \mathrm{C} & -0.351 & 0.540 & -0.252 \\ \mathrm{C} & 0.881 & -0.240 & -0.169 \\ \mathrm{C} & 2.018 & 0.398 & 0.364 \\ \mathrm{C} & 1.957 & 1.858 & 0.713 \\ \mathrm{C} & 1.164 & 2.573 & -0.375 \\ \mathrm{H} & 0.140 & -2.032 & -1.124 \\ \mathrm{H} & -0.779 & 2.323 & -1.321 \\ \mathrm{C} & 0.977 & -1.561 & -0.632 \\ \mathrm{C} & 3.191 & -0.331 & 0.499 \\ \mathrm{H} & 1.475 & 2.006 & 1.685 \\ \mathrm{H} & 1.648 & 2.412 & -1.340 \\ \mathrm{C} & 3.265 & -1.647 & 0.071 \\ \mathrm{C} & 2.164 & -2.256 & -0.521 \\ \mathrm{H} & 4.064 & 0.149 & 0.924 \\ \mathrm{H} & 4.194 & -2.193 & 0.172 \\ \mathrm{H} & 2.235 & -3.267 & -0.898 \\ \mathrm{H} & 2.966 & 2.259 & 0.794 \\ \mathrm{H} & 1.133 & 3.648 & -0.200 \\ \mathrm{~N} & -1.536 & 0.019 & -0.149 \\ \mathrm{C} & -1.876 & -1.377 & 0.225 \\ \mathrm{C} & -2.782 & 0.826 & -0.246 \\ \mathrm{C} & -3.263 & -1.244 & 0.833 \\ \mathrm{H} & -1.920 & -1.985 & -0.679 \\ \mathrm{C} & -3.906 & -0.185 & -0.058 \\ \mathrm{H} & -2.774 & 1.565 & 0.557 \\ \mathrm{H} & -3.795 & -2.192 & 0.830 \\ \mathrm{H} & -4.190 & -0.619 & -1.017 \\ \mathrm{H} & -1.112 & -1.773 & 0.889 \\ \mathrm{H} & -3.192 & -0.891 & 1.864 \\ \mathrm{H} & -4.787 & 0.276 & 0.381 \\ \mathrm{H} & -2.808 & 1.350 & -1.199 \\ \mathrm{H} & -0.834 & 2.479 & 0.419\end{array}$

En22

$\begin{array}{lrrr}C & -0.979 & -0.161 & -0.031 \\ C & -2.020 & 0.775 & 0.063 \\ C & -3.610 & -1.001 & -0.011\end{array}$

$\begin{array}{lrrr}\text { C } & -2.578 & -1.937 & -0.122 \\ \mathrm{C} & -1.260 & -1.532 & -0.133 \\ \mathrm{C} & 0.274 & 0.567 & -0.037 \\ \mathrm{C} & 0.003 & 2.045 & -0.068 \\ \mathrm{H} & -4.636 & -1.343 & -0.004 \\ \mathrm{H} & -2.817 & -2.988 & -0.206 \\ \mathrm{H} & -0.484 & -2.274 & -0.240 \\ \mathrm{H} & -1.976 & 2.820 & -0.614 \\ \mathrm{H} & 0.316 & 2.438 & -1.039 \\ \mathrm{H} & -4.146 & 1.074 & 0.153 \\ \mathrm{C} & -3.340 & 0.355 & 0.078 \\ \mathrm{C} & -1.511 & 2.184 & 0.139 \\ \mathrm{H} & -1.738 & 2.622 & 1.113 \\ \mathrm{C} & 2.682 & 0.969 & -0.055 \\ \mathrm{C} & 1.864 & -1.323 & 0.097 \\ \mathrm{C} & 3.816 & -0.009 & -0.328 \\ \mathrm{H} & 2.792 & 1.455 & 0.917 \\ \mathrm{H} & 2.557 & 1.733 & -0.819 \\ \mathrm{C} & 3.358 & -1.271 & 0.399 \\ \mathrm{H} & 1.661 & -1.810 & -0.859 \\ \mathrm{H} & 1.276 & -1.799 & 0.879 \\ \mathrm{H} & 3.901 & -0.194 & -1.400 \\ \mathrm{H} & 4.770 & 0.370 & 0.029 \\ \mathrm{H} & 3.867 & -2.171 & 0.062 \\ \mathrm{H} & 3.517 & -1.170 & 1.473 \\ \mathrm{~N} & 1.479 & 0.102 & -0.010 \\ \mathrm{H} & 0.596 & 2.563 & 0.687\end{array}$

\section{En23}

$\begin{array}{lrrr}\text { C } & -1.302 & -1.444 & -0.023 \\ \text { C } & -0.709 & 0.723 & -1.013 \\ \text { C } & -1.831 & 1.330 & -0.170 \\ \text { C } & -2.388 & -0.720 & 0.767 \\ \text { H } & 0.104 & 1.429 & -1.149 \\ \text { H } & -1.704 & -1.792 & -0.975 \\ \text { H } & -0.887 & -2.283 & 0.532 \\ \text { H } & -1.425 & 1.689 & 0.784 \\ \text { H } & -2.264 & 2.171 & -0.707 \\ \text { H } & -1.998 & -0.413 & 1.746 \\ \text { H } & -3.232 & -1.389 & 0.919 \\ \text { H } & -1.098 & 0.412 & -1.983 \\ \text { O } & -2.850 & 0.393 & 0.047 \\ \text { N } & -0.218 & -0.490 & -0.332 \\ \text { C } & 0.994 & -0.695 & 0.021 \\ \text { H } & 1.172 & -1.623 & 0.562 \\ \text { C } & 2.159 & 0.203 & -0.193 \\ \text { C } & 2.235 & 1.165 & 1.008 \\ \text { H } & 3.107 & 1.807 & 0.890 \\ \text { H } & 1.349 & 1.796 & 1.082 \\ \text { H } & 2.347 & 0.613 & 1.942 \\ \text { C } & 3.434 & -0.632 & -0.321\end{array}$




$\begin{array}{rrrr}\mathrm{H} & 4.284 & 0.028 & -0.488 \\ \mathrm{H} & 3.621 & -1.195 & 0.596 \\ \mathrm{H} & 3.380 & -1.330 & -1.155 \\ \mathrm{H} & 2.016 & 0.782 & -1.106\end{array}$

$\begin{array}{rrrr}\mathrm{H} & 4.122 & 0.163 & -0.015 \\ \mathrm{H} & -1.641 & -1.573 & 1.164\end{array}$

En26

En24

$\begin{array}{lrrr}\text { N } & -1.031 & -0.704 & 0.089 \\ \mathrm{C} & -2.185 & 0.195 & -0.134 \\ \mathrm{H} & -1.206 & -1.683 & -0.122 \\ \mathrm{O} & -2.005 & 1.360 & -0.234 \\ \mathrm{C} & -3.474 & -0.549 & -0.234 \\ \mathrm{H} & -3.438 & -1.248 & -1.073 \\ \mathrm{H} & -3.648 & -1.123 & 0.678 \\ \mathrm{H} & -4.278 & 0.162 & -0.388 \\ \mathrm{C} & 0.166 & -0.402 & 0.506 \\ \mathrm{C} & 0.617 & 0.955 & 0.875 \\ \mathrm{C} & 1.216 & -1.447 & 0.476 \\ \mathrm{C} & 1.619 & 1.397 & -0.232 \\ \mathrm{H} & -0.189 & 1.669 & 0.988 \\ \mathrm{C} & 2.231 & -1.015 & -0.622 \\ \mathrm{H} & 1.727 & -1.467 & 1.440 \\ \mathrm{H} & 0.809 & -2.437 & 0.267 \\ \mathrm{C} & 2.757 & 0.391 & -0.362 \\ \mathrm{H} & 1.988 & 2.385 & 0.038 \\ \mathrm{H} & 1.080 & 1.499 & -1.177 \\ \mathrm{H} & 3.036 & -1.748 & -0.633 \\ \mathrm{H} & 1.738 & -1.060 & -1.596 \\ \mathrm{H} & 3.418 & 0.687 & -1.176 \\ \mathrm{H} & 3.356 & 0.396 & 0.552 \\ \mathrm{H} & 1.173 & 0.863 & 1.812\end{array}$

\section{En27}

En25

$\begin{array}{lrrr}\mathrm{C} & -2.431 & 0.917 & 0.237 \\ \mathrm{C} & -0.918 & 1.069 & 0.029 \\ \mathrm{C} & -0.406 & -0.325 & 0.058 \\ \mathrm{C} & -1.531 & -1.299 & 0.104 \\ \mathrm{C} & -2.743 & -0.467 & -0.337 \\ \mathrm{H} & -2.989 & 1.716 & -0.243 \\ \mathrm{H} & -0.391 & 1.724 & 0.722 \\ \mathrm{H} & -0.681 & 1.461 & -0.970 \\ \mathrm{H} & -1.333 & -2.214 & -0.454 \\ \mathrm{H} & -3.678 & -0.885 & 0.025 \\ \mathrm{H} & -2.788 & -0.430 & -1.427 \\ \mathrm{H} & -2.661 & 0.943 & 1.304 \\ \mathrm{~N} & 0.836 & -0.700 & 0.032 \\ \mathrm{C} & 2.013 & 0.192 & -0.033 \\ \mathrm{H} & 1.028 & -1.699 & 0.044 \\ \mathrm{O} & 1.836 & 1.357 & -0.135 \\ \mathrm{C} & 3.305 & -0.550 & 0.032 \\ \mathrm{H} & 3.363 & -1.122 & 0.961 \\ \mathrm{H} & 3.376 & -1.250 & -0.804\end{array}$

$\begin{array}{lrrr}\text { N } & 1.568 & -0.625 & -0.057 \\ \mathrm{C} & 2.993 & -0.332 & -0.094 \\ \mathrm{H} & 1.335 & -1.599 & -0.206 \\ \mathrm{O} & 3.382 & 0.788 & -0.035 \\ \mathrm{C} & 3.826 & -1.568 & -0.220 \\ \mathrm{H} & 3.649 & -2.231 & 0.628 \\ \mathrm{H} & 3.568 & -2.106 & -1.135 \\ \mathrm{H} & 4.873 & -1.281 & -0.249 \\ \mathrm{C} & 0.556 & 0.212 & 0.094 \\ \mathrm{C} & 0.796 & 1.677 & 0.218 \\ \mathrm{C} & -0.470 & 2.473 & 0.499 \\ \mathrm{H} & 1.565 & 1.841 & 0.975 \\ \mathrm{C} & -1.592 & 2.020 & -0.420 \\ \mathrm{H} & -0.256 & 3.532 & 0.362 \\ \mathrm{H} & -0.771 & 2.334 & 1.540 \\ \mathrm{H} & -2.507 & 2.580 & -0.234 \\ \mathrm{H} & -1.319 & 2.199 & -1.466 \\ \mathrm{C} & -0.786 & -0.320 & 0.090 \\ \mathrm{C} & -1.850 & 0.556 & -0.212 \\ \mathrm{C} & -1.043 & -1.685 & 0.333\end{array}$




$\begin{array}{lrrr}\text { C } & -3.131 & 0.028 & -0.316 \\ \text { C } & -2.322 & -2.178 & 0.251 \\ \text { H } & -0.252 & -2.356 & 0.647 \\ \text { C } & -3.367 & -1.317 & -0.092 \\ \text { H } & -3.954 & 0.687 & -0.560 \\ \text { H } & -2.520 & -3.219 & 0.461 \\ \text { H } & -4.376 & -1.703 & -0.163 \\ \text { H } & 1.270 & 2.000 & -0.718\end{array}$

\section{En28}

$\begin{array}{lrrr}\text { N } & 0.816 & -0.385 & 0.000 \\ \mathrm{C} & 2.238 & 0.007 & 0.000 \\ \mathrm{H} & 0.648 & -1.386 & 0.000 \\ \mathrm{O} & 2.531 & 1.154 & 0.001 \\ \mathrm{C} & 3.151 & -1.173 & 0.000 \\ \mathrm{H} & 2.970 & -1.788 & 0.884 \\ \mathrm{H} & 2.971 & -1.787 & -0.885 \\ \mathrm{H} & 4.178 & -0.822 & 0.000 \\ \mathrm{C} & -0.236 & 0.385 & 0.000 \\ \mathrm{C} & -0.103 & 1.860 & 0.000 \\ \mathrm{H} & -1.076 & 2.337 & -0.001 \\ \mathrm{H} & 0.474 & 2.183 & 0.868 \\ \mathrm{H} & 0.475 & 2.183 & -0.869 \\ \mathrm{C} & -1.600 & -0.254 & 0.000 \\ \mathrm{C} & -1.550 & -1.781 & 0.001 \\ \mathrm{H} & -1.066 & -2.181 & 0.895 \\ \mathrm{H} & -2.569 & -2.163 & 0.001 \\ \mathrm{H} & -1.066 & -2.182 & -0.894 \\ \mathrm{C} & -2.339 & 0.232 & 1.265 \\ \mathrm{H} & -2.467 & 1.312 & 1.285 \\ \mathrm{H} & -3.330 & -0.221 & 1.268 \\ \mathrm{H} & -1.821 & -0.079 & 2.173 \\ \mathrm{C} & -2.339 & 0.231 & -1.265 \\ \mathrm{H} & -1.821 & -0.081 & -2.173 \\ \mathrm{H} & -3.330 & -0.222 & -1.268 \\ \mathrm{H} & -2.467 & 1.311 & -1.286\end{array}$

\section{En29}

$\begin{array}{lrrr}\mathrm{N} & -0.035 & 0.432 & 0.061 \\ \mathrm{C} & 1.276 & 1.075 & 0.160 \\ \mathrm{H} & -0.030 & -0.578 & 0.143 \\ \mathrm{O} & 1.332 & 2.244 & 0.384 \\ \mathrm{C} & -2.385 & 0.168 & 0.023 \\ \mathrm{C} & -3.531 & 0.476 & -0.721 \\ \mathrm{C} & -2.380 & -0.970 & 0.846 \\ \mathrm{C} & -4.635 & -0.355 & -0.665 \\ \mathrm{C} & -3.498 & -1.776 & 0.920 \\ \mathrm{H} & -1.530 & -1.187 & 1.483 \\ \mathrm{C} & -4.621 & -1.475 & 0.157 \\ \mathrm{H} & -5.509 & -0.128 & -1.260\end{array}$

$\begin{array}{lrrr}\text { H } & -3.501 & -2.633 & 1.580 \\ \mathrm{H} & -5.494 & -2.113 & 0.211 \\ \mathrm{H} & -3.548 & 1.335 & -1.376 \\ \mathrm{C} & -1.204 & 1.013 & -0.068 \\ \mathrm{C} & -1.314 & 2.475 & -0.280 \\ \mathrm{H} & -2.328 & 2.752 & -0.545 \\ \mathrm{H} & -1.052 & 2.970 & 0.660 \\ \mathrm{C} & 2.414 & 0.155 & 0.004 \\ \mathrm{C} & 3.644 & 0.591 & 0.499 \\ \mathrm{C} & 2.310 & -1.083 & -0.634 \\ \mathrm{C} & 4.758 & -0.219 & 0.375 \\ \mathrm{H} & 3.706 & 1.558 & 0.979 \\ \mathrm{C} & 3.432 & -1.882 & -0.765 \\ \mathrm{H} & 1.379 & -1.419 & -1.077 \\ \mathrm{C} & 4.651 & -1.454 & -0.255 \\ \mathrm{H} & 5.710 & 0.111 & 0.765 \\ \mathrm{H} & 3.359 & -2.835 & -1.272 \\ \mathrm{H} & 5.525 & -2.084 & -0.354 \\ \mathrm{H} & -0.596 & 2.833 & -1.016\end{array}$

\section{En30}

$\begin{array}{lrrr}\text { N } & 1.349 & -0.360 & -0.115 \\ \mathrm{C} & 2.805 & -0.237 & -0.078 \\ \mathrm{H} & 0.999 & -1.280 & -0.359 \\ \mathrm{O} & 3.308 & 0.808 & 0.165 \\ \mathrm{C} & 3.495 & -1.529 & -0.375 \\ \mathrm{H} & 3.209 & -2.286 & 0.359 \\ \mathrm{H} & 3.210 & -1.889 & -1.365 \\ \mathrm{H} & 4.567 & -1.369 & -0.337 \\ \mathrm{C} & -0.951 & 0.180 & 0.064 \\ \mathrm{C} & -1.922 & 1.102 & -0.352 \\ \mathrm{C} & -1.342 & -1.122 & 0.419 \\ \mathrm{C} & -3.246 & 0.716 & -0.445 \\ \mathrm{C} & -2.670 & -1.488 & 0.356 \\ \mathrm{H} & -0.621 & -1.829 & 0.814 \\ \mathrm{C} & -3.621 & -0.572 & -0.087 \\ \mathrm{H} & -3.988 & 1.422 & -0.791 \\ \mathrm{H} & -2.972 & -2.480 & 0.661 \\ \mathrm{H} & -4.661 & -0.864 & -0.142 \\ \mathrm{H} & -1.638 & 2.103 & -0.647 \\ \mathrm{C} & 0.446 & 0.574 & 0.100 \\ \mathrm{C} & 0.848 & 1.973 & 0.380 \\ \mathrm{H} & -0.019 & 2.595 & 0.560 \\ \mathrm{H} & 1.492 & 1.986 & 1.262 \\ \mathrm{H} & 1.460 & 2.371 & -0.431 \\ \mathrm{C} & -0.260 & 2.036 & -0.403 \\ \mathrm{C} & -0.351 & 0.540 & -0.252 \\ \mathrm{C} & 0.881 & -0.240 & -0.169 \\ \mathrm{C} & 2.018 & 0.398 & 0.364 \\ \mathrm{C} & 1.957 & 1.858 & 0.713 \\ \mathrm{C} & 1.164 & 2.573 & -0.375\end{array}$




$\begin{array}{lrrr}\text { H } & 0.140 & -2.032 & -1.124 \\ \text { H } & -0.779 & 2.323 & -1.321 \\ \mathrm{C} & 0.977 & -1.561 & -0.632 \\ \mathrm{C} & 3.191 & -0.331 & 0.499 \\ \mathrm{H} & 1.475 & 2.006 & 1.685 \\ \mathrm{H} & 1.648 & 2.412 & -1.340 \\ \mathrm{C} & 3.265 & -1.647 & 0.071 \\ \mathrm{C} & 2.164 & -2.256 & -0.521 \\ \mathrm{H} & 4.064 & 0.149 & 0.924 \\ \mathrm{H} & 4.194 & -2.193 & 0.172 \\ \mathrm{H} & 2.235 & -3.267 & -0.898 \\ \mathrm{H} & 2.966 & 2.259 & 0.794 \\ \mathrm{H} & 1.133 & 3.648 & -0.200 \\ \mathrm{~N} & -1.536 & 0.019 & -0.149 \\ \mathrm{C} & -1.876 & -1.377 & 0.225 \\ \mathrm{C} & -2.782 & 0.826 & -0.246 \\ \mathrm{C} & -3.263 & -1.244 & 0.833 \\ \mathrm{H} & -1.920 & -1.985 & -0.679 \\ \mathrm{C} & -3.906 & -0.185 & -0.058 \\ \mathrm{H} & -2.774 & 1.565 & 0.557 \\ \mathrm{H} & -3.795 & -2.192 & 0.830 \\ \mathrm{H} & -4.190 & -0.619 & -1.017 \\ \mathrm{H} & -1.112 & -1.773 & 0.889 \\ \mathrm{H} & -3.192 & -0.891 & 1.864 \\ \mathrm{H} & -4.787 & 0.276 & 0.381 \\ \mathrm{H} & -2.808 & 1.350 & -1.199 \\ \mathrm{H} & -0.834 & 2.479 & 0.419 \\ & & & \end{array}$

\section{En31}

$\begin{array}{lrrr}\mathrm{N} & 2.144 & -0.404 & -0.109 \\ \mathrm{C} & 3.605 & -0.415 & -0.090 \\ \mathrm{H} & 1.713 & -1.293 & -0.333 \\ \mathrm{O} & 4.207 & 0.584 & 0.128 \\ \mathrm{C} & 4.170 & -1.770 & -0.369 \\ \mathrm{H} & 3.829 & -2.483 & 0.384 \\ \mathrm{H} & 3.837 & -2.122 & -1.348 \\ \mathrm{H} & 5.253 & -1.707 & -0.351 \\ \mathrm{C} & -0.092 & 0.354 & 0.086 \\ \mathrm{C} & -0.988 & 1.363 & -0.301 \\ \mathrm{C} & -0.603 & -0.909 & 0.439 \\ \mathrm{C} & -2.341 & 1.111 & -0.372 \\ \mathrm{C} & -1.955 & -1.155 & 0.404 \\ \mathrm{H} & 0.050 & -1.688 & 0.815 \\ \mathrm{C} & -2.823 & -0.145 & -0.015 \\ \mathrm{H} & -3.030 & 1.878 & -0.697 \\ \mathrm{H} & -2.354 & -2.113 & 0.707 \\ \mathrm{H} & -0.625 & 2.337 & -0.598 \\ \mathrm{C} & 1.332 & 0.615 & 0.095 \\ \mathrm{C} & 1.869 & 1.975 & 0.335 \\ \mathrm{H} & 1.070 & 2.684 & 0.512 \\ \mathrm{H} & 2.524 & 1.947 & 1.209\end{array}$

$\begin{array}{lrrr}\mathrm{Cl} & -4.499 & -0.454 & -0.078 \\ \mathrm{H} & 2.504 & 2.295 & -0.493\end{array}$

En32

$\begin{array}{lrrr}\text { N } & 1.777 & -0.389 & -0.108 \\ \mathrm{C} & 3.233 & -0.358 & -0.078 \\ \mathrm{H} & 1.373 & -1.291 & -0.332 \\ \mathrm{O} & 3.808 & 0.657 & 0.142 \\ \mathrm{C} & 3.840 & -1.697 & -0.351 \\ \mathrm{H} & 3.514 & -2.418 & 0.401 \\ \mathrm{H} & 3.525 & -2.060 & -1.331 \\ \mathrm{H} & 4.920 & -1.602 & -0.324 \\ \mathrm{C} & -0.482 & 0.302 & 0.073 \\ \mathrm{C} & -1.408 & 1.287 & -0.303 \\ \mathrm{C} & -0.958 & -0.981 & 0.404 \\ \mathrm{C} & -2.753 & 0.986 & -0.380 \\ \mathrm{C} & -2.303 & -1.258 & 0.356 \\ \mathrm{H} & -0.284 & -1.747 & 0.769 \\ \mathrm{C} & -3.227 & -0.283 & -0.046 \\ \mathrm{H} & -3.451 & 1.747 & -0.701 \\ \mathrm{H} & -2.659 & -2.239 & 0.644 \\ \mathrm{H} & -1.075 & 2.275 & -0.587 \\ \mathrm{C} & 0.930 & 0.605 & 0.089 \\ \mathrm{C} & 1.429 & 1.981 & 0.331 \\ \mathrm{H} & 0.609 & 2.667 & 0.501 \\ \mathrm{H} & 2.078 & 1.972 & 1.210 \\ \mathrm{H} & 2.061 & 2.316 & -0.493 \\ \mathrm{C} & -4.688 & -0.599 & -0.081 \\ \mathrm{H} & -5.085 & -0.620 & 0.937 \\ \mathrm{H} & -5.245 & 0.145 & -0.646 \\ \mathrm{H} & -4.863 & -1.583 & -0.517\end{array}$

EE1

$\begin{array}{lrrr}\text { C } & -0.732 & -0.447 & 0.513 \\ \text { C } & -1.108 & 0.958 & 0.789 \\ \text { C } & -2.168 & 1.371 & -0.269 \\ \text { C } & -3.341 & 0.399 & -0.274 \\ \text { C } & -2.871 & -1.029 & -0.523 \\ \text { C } & -1.810 & -1.458 & 0.527 \\ \text { H } & -2.490 & 2.386 & -0.039 \\ \text { H } & -1.576 & 0.992 & 1.777 \\ \text { H } & -3.868 & 0.454 & 0.682 \\ \text { H } & -4.054 & 0.690 & -1.046 \\ \text { H } & -3.697 & -1.736 & -0.471 \\ \text { H } & -2.435 & -1.114 & -1.521 \\ \text { H } & -2.279 & -1.447 & 1.516 \\ \text { H } & -1.405 & -2.447 & 0.325 \\ \text { H } & -1.695 & 1.397 & -1.255 \\ \text { O } & 0.417 & -0.800 & 0.165 \\ \text { Si } & 1.977 & 0.041 & -0.106\end{array}$




$\begin{array}{lrrr}\mathrm{C} & 1.625 & 1.279 & -1.436 \\ \mathrm{H} & 1.151 & 0.806 & -2.298 \\ \mathrm{H} & 1.006 & 2.114 & -1.107 \\ \mathrm{H} & 2.574 & 1.697 & -1.780 \\ \mathrm{C} & 2.450 & 0.741 & 1.538 \\ \mathrm{H} & 2.443 & -0.031 & 2.310 \\ \mathrm{H} & 3.473 & 1.120 & 1.470 \\ \mathrm{H} & 1.820 & 1.568 & 1.865 \\ \mathrm{C} & 3.033 & -1.362 & -0.654 \\ \mathrm{H} & 4.044 & -1.006 & -0.863 \\ \mathrm{H} & 3.103 & -2.130 & 0.117 \\ \mathrm{H} & 2.646 & -1.820 & -1.565 \\ \mathrm{H} & -0.247 & 1.621 & 0.782\end{array}$

$\begin{array}{lrrr}\mathrm{O} & -0.554 & -0.646 & 0.006 \\ \mathrm{Si} & 1.134 & -0.013 & -0.004 \\ \mathrm{C} & 1.372 & 0.599 & -1.730 \\ \mathrm{H} & 1.161 & -0.184 & -2.460 \\ \mathrm{H} & 0.771 & 1.476 & -1.972 \\ \mathrm{H} & 2.421 & 0.884 & -1.853 \\ \mathrm{C} & 1.192 & 1.280 & 1.318 \\ \mathrm{H} & 0.741 & 0.927 & 2.247 \\ \mathrm{H} & 2.242 & 1.491 & 1.536 \\ \mathrm{H} & 0.727 & 2.223 & 1.032 \\ \mathrm{C} & 2.069 & -1.543 & 0.404 \\ \mathrm{H} & 3.139 & -1.329 & 0.414 \\ \mathrm{H} & 1.797 & -1.927 & 1.388 \\ \mathrm{H} & 1.891 & -2.325 & -0.335 \\ \mathrm{H} & -2.886 & 1.573 & -0.418 \\ \mathrm{H} & -3.595 & -0.691 & 0.739 \\ \mathrm{H} & -1.878 & 1.661 & 1.027\end{array}$

\section{EE2}

$\begin{array}{lrrr}\mathrm{O} & 0.191 & 0.798 & -0.044 \\ \mathrm{Si} & 1.778 & -0.045 & 0.002 \\ \mathrm{C} & 1.655 & -1.291 & 1.364 \\ \mathrm{H} & 1.251 & -0.853 & 2.279 \\ \mathrm{H} & 1.071 & -2.172 & 1.100 \\ \mathrm{H} & 2.667 & -1.633 & 1.598 \\ \mathrm{C} & 1.940 & -0.753 & -1.697 \\ \mathrm{H} & 1.882 & 0.026 & -2.457 \\ \mathrm{H} & 2.919 & -1.229 & -1.789 \\ \mathrm{H} & 1.190 & -1.514 & -1.916 \\ \mathrm{C} & 2.908 & 1.359 & 0.368 \\ \mathrm{H} & 3.941 & 1.004 & 0.394 \\ \mathrm{H} & 2.839 & 2.133 & -0.397 \\ \mathrm{H} & 2.686 & 1.808 & 1.337 \\ \mathrm{C} & -0.988 & 0.399 & -0.021 \\ \mathrm{C} & -2.142 & 1.326 & -0.122 \\ \mathrm{C} & -1.448 & -1.012 & 0.106 \\ \mathrm{C} & -3.349 & 0.460 & 0.256 \\ \mathrm{H} & -1.980 & 2.245 & 0.441 \\ \mathrm{H} & -2.186 & 1.608 & -1.184 \\ \mathrm{C} & -2.944 & -0.939 & -0.229 \\ \mathrm{H} & -0.843 & -1.708 & -0.475 \\ \mathrm{H} & -4.271 & 0.815 & -0.195 \\ \mathrm{H} & -3.482 & 0.462 & 1.339 \\ \mathrm{H} & -3.511 & -1.737 & 0.243 \\ \mathrm{H} & -3.083 & -1.021 & -1.308 \\ \mathrm{H} & -1.304 & -1.267 & 1.167\end{array}$

EE3

$\begin{array}{lrrr}\mathrm{C} & -1.695 & -0.142 & -0.002 \\ \mathrm{C} & -1.906 & 1.325 & -0.017 \\ \mathrm{C} & -2.858 & -1.052 & 0.020 \\ \mathrm{H} & -1.119 & 1.844 & -0.559 \\ \mathrm{H} & -2.564 & -2.076 & 0.225 \\ \mathrm{H} & -3.337 & -0.992 & -0.964\end{array}$

\section{EE4}

$\begin{array}{lrrr}\text { C } & -0.566 & 2.281 & 0.133 \\ \text { H } & -1.379 & 2.283 & -0.599 \\ \mathrm{C} & 0.068 & 0.930 & 0.081 \\ \mathrm{C} & 0.397 & 3.437 & -0.079 \\ \mathrm{H} & 0.876 & 3.376 & -1.055 \\ \mathrm{H} & -0.146 & 4.377 & -0.022 \\ \mathrm{H} & 1.174 & 3.443 & 0.683 \\ \mathrm{O} & 1.326 & 0.847 & 0.065 \\ \mathrm{O} & -0.621 & -0.139 & 0.071 \\ \mathrm{Si} & 2.347 & -0.611 & 0.000 \\ \mathrm{Si} & -2.339 & -0.536 & -0.019 \\ \mathrm{C} & -2.804 & -0.212 & -1.779 \\ \mathrm{H} & -2.751 & 0.844 & -2.048 \\ \mathrm{H} & -2.171 & -0.777 & -2.465 \\ \mathrm{H} & -3.835 & -0.535 & -1.941 \\ \mathrm{C} & -3.233 & 0.514 & 1.216 \\ \mathrm{H} & -4.230 & 0.089 & 1.362 \\ \mathrm{H} & -2.739 & 0.508 & 2.189 \\ \mathrm{H} & -3.368 & 1.546 & 0.891 \\ \mathrm{C} & -2.306 & -2.323 & 0.434 \\ \mathrm{H} & -1.957 & -2.468 & 1.457 \\ \mathrm{H} & -3.310 & -2.745 & 0.364 \\ \mathrm{H} & -1.659 & -2.891 & -0.237 \\ \mathrm{C} & 4.031 & 0.137 & 0.027 \\ \mathrm{H} & 4.196 & 0.710 & 0.941 \\ \mathrm{H} & 4.788 & -0.649 & -0.015 \\ \mathrm{H} & 4.187 & 0.797 & -0.828 \\ \mathrm{C} & 1.926 & -1.587 & 1.512 \\ \mathrm{H} & 2.622 & -2.425 & 1.602 \\ \mathrm{H} & 2.024 & -0.985 & 2.417 \\ \mathrm{H} & 0.917 & -1.998 & 1.467 \\ \mathrm{C} & 1.912 & -1.440 & -1.595\end{array}$




$\begin{array}{rrrr}H & 0.895 & -1.835 & -1.589 \\ \mathrm{H} & 2.023 & -0.760 & -2.442 \\ \mathrm{H} & 2.591 & -2.279 & -1.760 \\ \mathrm{H} & -1.053 & 2.340 & 1.113\end{array}$

EE5

$\begin{array}{lrrr}\text { C } & -3.180 & -0.916 & 0.270 \\ \text { C } & -3.328 & 0.454 & -0.378 \\ \text { C } & -2.269 & 1.382 & 0.159 \\ \text { C } & -0.713 & -0.467 & 0.002 \\ \text { C } & -1.806 & -1.478 & -0.076 \\ \text { H } & -2.178 & 2.309 & -0.399 \\ \text { H } & -3.245 & 0.376 & -1.465 \\ \text { H } & -4.300 & 0.896 & -0.158 \\ \text { H } & -3.288 & -0.830 & 1.353 \\ \text { H } & -3.949 & -1.602 & -0.077 \\ \text { H } & -1.506 & -2.324 & 0.543 \\ \text { H } & -2.412 & 1.607 & 1.216 \\ \text { O } & -0.921 & 0.794 & 0.077 \\ \text { O } & 0.479 & -0.865 & -0.034 \\ \text { Si } & 2.015 & 0.053 & -0.007 \\ \text { C } & 2.004 & 0.971 & 1.598 \\ \text { H } & 1.243 & 1.750 & 1.625 \\ \text { H } & 1.852 & 0.296 & 2.442 \\ \text { H } & 2.975 & 1.452 & 1.737 \\ \mathrm{C} & 3.238 & -1.321 & -0.088 \\ \text { H } & 3.119 & -1.906 & -1.000 \\ \text { H } & 4.252 & -0.914 & -0.081 \\ \text { H } & 3.142 & -1.989 & 0.769 \\ \mathrm{C} & 1.966 & 1.123 & -1.513 \\ \text { H } & 1.803 & 0.533 & -2.417 \\ \text { H } & 1.200 & 1.895 & -1.450 \\ \text { H } & 2.931 & 1.624 & -1.623 \\ \text { H } & -1.777 & -1.839 & -1.111\end{array}$

$\begin{array}{lrrr}\text { EE6 } & & & \\ \text { O } & -0.183 & 0.856 & 0.046 \\ \mathrm{Si} & -1.736 & -0.058 & -0.018 \\ \mathrm{C} & -1.724 & -1.094 & 1.511 \\ \mathrm{H} & -0.940 & -1.850 & 1.491 \\ \mathrm{H} & -1.610 & -0.484 & 2.408 \\ \mathrm{H} & -2.681 & -1.615 & 1.592 \\ \mathrm{C} & -2.948 & 1.327 & -0.007 \\ \mathrm{H} & -2.815 & 1.979 & -0.872 \\ \mathrm{H} & -3.964 & 0.928 & -0.043 \\ \mathrm{H} & -2.858 & 1.928 & 0.899 \\ \mathrm{C} & -1.650 & -1.004 & -1.601 \\ \mathrm{H} & -1.482 & -0.344 & -2.454 \\ \mathrm{H} & -0.872 & -1.768 & -1.584 \\ \mathrm{H} & -2.604 & -1.510 & -1.764\end{array}$

$\begin{array}{lrrr}\text { C } & 0.992 & 0.443 & 0.063 \\ \text { C } & 2.206 & 1.303 & 0.152 \\ \text { C } & 2.717 & -1.029 & 0.105 \\ \text { C } & 3.318 & 0.329 & -0.249 \\ \text { H } & 2.950 & -1.831 & -0.587 \\ \text { H } & 2.914 & -1.343 & 1.128 \\ \text { H } & 4.250 & 0.508 & 0.280 \\ \text { H } & 3.510 & 0.394 & -1.318 \\ \text { O } & 1.264 & -0.814 & 0.017 \\ \text { H } & 2.103 & 2.190 & -0.469 \\ \text { H } & 2.285 & 1.634 & 1.193\end{array}$

EE7

$\begin{array}{lrrr}\mathrm{C} & -0.147 & 2.105 & -0.033 \\ \mathrm{H} & -1.215 & 2.259 & -0.145 \\ \mathrm{H} & 0.182 & 2.555 & 0.907 \\ \mathrm{C} & 0.203 & 0.658 & -0.027 \\ \mathrm{O} & -0.706 & -0.220 & -0.020 \\ \mathrm{Si} & -2.479 & -0.254 & 0.009 \\ \mathrm{C} & -2.977 & 0.641 & 1.550 \\ \mathrm{H} & -4.047 & 0.487 & 1.709 \\ \mathrm{H} & -2.810 & 1.718 & 1.507 \\ \mathrm{H} & -2.459 & 0.240 & 2.423 \\ \mathrm{C} & -2.790 & -2.069 & 0.079 \\ \mathrm{H} & -3.864 & -2.262 & 0.103 \\ \mathrm{H} & -2.351 & -2.513 & 0.973 \\ \mathrm{H} & -2.381 & -2.575 & -0.797 \\ \mathrm{C} & -3.031 & 0.528 & -1.574 \\ \mathrm{H} & -4.104 & 0.352 & -1.689 \\ \mathrm{H} & -2.536 & 0.070 & -2.432 \\ \mathrm{H} & -2.875 & 1.606 & -1.615 \\ \mathrm{C} & 1.573 & 0.209 & -0.018 \\ \mathrm{C} & 1.853 & -1.168 & -0.029 \\ \mathrm{C} & 2.627 & 1.136 & 0.010 \\ \mathrm{C} & 3.160 & -1.603 & -0.015 \\ \mathrm{H} & 1.037 & -1.878 & -0.050 \\ \mathrm{C} & 3.934 & 0.691 & 0.028 \\ \mathrm{H} & 2.430 & 2.199 & 0.023 \\ \mathrm{C} & 4.198 & -0.675 & 0.014 \\ \mathrm{H} & 3.380 & -2.661 & -0.026 \\ \mathrm{H} & 4.748 & 1.402 & 0.052 \\ \mathrm{H} & 5.224 & -1.020 & 0.025 \\ \mathrm{H} & 0.379 & 2.614 & -0.841 \\ & & & \end{array}$

EE8

$\begin{array}{llrr}\mathrm{C} & 1.254 & -1.917 & 0.012 \\ \mathrm{H} & 1.237 & -2.231 & -1.039 \\ \mathrm{H} & 2.098 & -2.412 & 0.488 \\ \mathrm{C} & 1.431 & -0.445 & 0.026 \\ \mathrm{O} & 0.447 & 0.339 & 0.027\end{array}$




$\begin{array}{lrrr}\mathrm{Si} & -1.329 & 0.148 & 0.000 \\ \mathrm{C} & -1.694 & -1.005 & -1.401 \\ \mathrm{H} & -2.762 & -0.942 & -1.623 \\ \mathrm{H} & -1.472 & -2.049 & -1.179 \\ \mathrm{H} & -1.162 & -0.714 & -2.309 \\ \mathrm{C} & -1.854 & 1.885 & -0.313 \\ \mathrm{H} & -2.945 & 1.941 & -0.343 \\ \mathrm{H} & -1.477 & 2.251 & -1.269 \\ \mathrm{H} & -1.510 & 2.553 & 0.478 \\ \mathrm{C} & -1.768 & -0.482 & 1.681 \\ \mathrm{H} & -2.857 & -0.482 & 1.775 \\ \mathrm{H} & -1.374 & 0.170 & 2.462 \\ \mathrm{H} & -1.429 & -1.500 & 1.872 \\ \mathrm{C} & 2.769 & 0.111 & 0.002 \\ \mathrm{H} & 3.590 & -0.592 & -0.008 \\ \mathrm{C} & 2.971 & 1.431 & -0.004 \\ \mathrm{H} & 2.141 & 2.127 & 0.011 \\ \mathrm{H} & 3.972 & 1.842 & -0.021 \\ \mathrm{H} & 0.318 & -2.221 & 0.474\end{array}$

\section{EE9}

$\begin{array}{lrrr}\text { C } & 0.416 & 2.335 & 0.008 \\ \text { H } & -0.292 & 3.156 & 0.011 \\ \text { H } & 1.065 & 2.377 & 0.884 \\ \text { C } & -0.318 & 1.047 & 0.003 \\ \text { O } & -1.569 & 1.034 & 0.004 \\ \text { O } & 0.288 & -0.093 & -0.001 \\ \text { C } & 1.710 & -0.167 & -0.001 \\ \text { C } & 4.428 & -0.399 & -0.001 \\ \text { C } & 2.357 & -0.236 & 1.215 \\ \text { C } & 2.357 & -0.227 & -1.217 \\ \text { C } & 3.741 & -0.341 & -1.206 \\ \text { C } & 3.741 & -0.350 & 1.204 \\ \text { H } & 1.796 & -0.212 & 2.141 \\ \text { H } & 1.796 & -0.196 & -2.142 \\ \text { H } & 4.278 & -0.391 & -2.143 \\ \text { H } & 4.278 & -0.407 & 2.141 \\ \text { H } & 5.506 & -0.492 & -0.001 \\ \text { Si } & -2.714 & -0.354 & -0.001 \\ \text { C } & -2.345 & -1.269 & -1.563 \\ \text { H } & -3.093 & -2.054 & -1.697 \\ \text { H } & -1.366 & -1.747 & -1.541 \\ \text { H } & -2.404 & -0.613 & -2.433 \\ \text { C } & -4.325 & 0.538 & 0.001 \\ \text { H } & -5.146 & -0.182 & -0.001 \\ \text { H } & -4.432 & 1.168 & -0.882 \\ \text { H } & -4.432 & 1.163 & 0.889 \\ \mathrm{C} & -2.345 & -1.279 & 1.555 \\ \text { H } & -3.093 & -2.065 & 1.684 \\ \text { H } & -2.404 & -0.629 & 2.429 \\ \text { H } & -1.366 & -1.757 & 1.530\end{array}$

\section{EE10}

$\begin{array}{lrrr}\text { C } & 0.801 & -1.885 & 0.005 \\ \text { H } & -0.169 & -2.294 & 0.269 \\ \text { H } & 1.083 & -2.214 & -1.004 \\ \text { C } & 0.807 & -0.415 & -0.006 \\ \text { O } & -0.172 & 0.325 & -0.007 \\ \text { Si } & -2.005 & 0.101 & 0.001 \\ \text { C } & -2.307 & -0.993 & -1.454 \\ \text { H } & -3.385 & -1.017 & -1.639 \\ \text { H } & -1.985 & -2.023 & -1.307 \\ \text { H } & -1.839 & -0.596 & -2.357 \\ \text { C } & -2.533 & 1.846 & -0.209 \\ \text { H } & -3.624 & 1.898 & -0.214 \\ \text { H } & -2.175 & 2.261 & -1.152 \\ \text { H } & -2.173 & 2.471 & 0.608 \\ \text { C } & -2.320 & -0.630 & 1.665 \\ \text { H } & -3.402 & -0.672 & 1.816 \\ \text { H } & -1.908 & -0.004 & 2.458 \\ \text { H } & -1.940 & -1.645 & 1.779 \\ \text { C } & 2.185 & 0.294 & -0.008 \\ \text { F } & 2.965 & -0.293 & -0.900 \\ \text { F } & 2.705 & 0.141 & 1.202 \\ \text { F } & 2.062 & 1.569 & -0.285 \\ \text { H } & 1.584 & -2.248 & 0.674\end{array}$

\section{EE11}

$\begin{array}{lrrr}\mathrm{C} & 0.675 & -1.876 & 0.002 \\ \mathrm{H} & -0.237 & -2.189 & -0.499 \\ \mathrm{H} & 1.542 & -2.356 & -0.443 \\ \mathrm{C} & 0.814 & -0.398 & 0.014 \\ \mathrm{O} & -0.200 & 0.334 & 0.018 \\ \mathrm{Si} & -1.975 & 0.097 & 0.001 \\ \mathrm{C} & -2.351 & -0.488 & -1.711 \\ \mathrm{H} & -3.437 & -0.515 & -1.837 \\ \mathrm{H} & -1.978 & -1.489 & -1.926 \\ \mathrm{H} & -1.956 & 0.201 & -2.458 \\ \mathrm{C} & -2.548 & 1.809 & 0.359 \\ \mathrm{H} & -3.640 & 1.841 & 0.366 \\ \mathrm{H} & -2.198 & 2.510 & -0.400 \\ \mathrm{H} & -2.198 & 2.150 & 1.334 \\ \mathrm{C} & -2.338 & -1.111 & 1.355 \\ \mathrm{H} & -3.411 & -1.078 & 1.562 \\ \mathrm{H} & -1.828 & -0.840 & 2.281 \\ \mathrm{H} & -2.090 & -2.142 & 1.102 \\ \mathrm{C} & 2.156 & 0.258 & 0.021 \\ \mathrm{H} & 0.621 & -2.199 & 1.048 \\ \mathrm{C} & 2.046 & 1.741 & 0.366 \\ \mathrm{H} & 1.429 & 2.277 & -0.353\end{array}$




$\begin{array}{rrrr}\mathrm{H} & 1.624 & 1.887 & 1.361 \\ \mathrm{H} & 3.043 & 2.180 & 0.353 \\ \mathrm{C} & 3.090 & -0.463 & 1.006 \\ \mathrm{H} & 3.247 & -1.509 & 0.748 \\ \mathrm{H} & 4.060 & 0.033 & 0.978 \\ \mathrm{H} & 2.713 & -0.402 & 2.028 \\ \mathrm{C} & 2.692 & 0.094 & -1.424 \\ \mathrm{H} & 3.665 & 0.585 & -1.468 \\ \mathrm{H} & 2.830 & -0.951 & -1.701 \\ \mathrm{H} & 2.036 & 0.575 & -2.151\end{array}$

\section{EE12}

$\begin{array}{lrrr}\text { C } & -1.250 & 0.391 & 0.167 \\ \text { C } & -1.937 & -0.934 & 0.215 \\ \text { C } & -2.111 & -1.421 & -1.235 \\ \text { H } & -1.144 & -1.593 & -1.709 \\ \text { H } & -2.654 & -2.365 & -1.218 \\ \text { H } & -2.681 & -0.710 & -1.833 \\ \mathrm{C} & -1.196 & -1.948 & 1.078 \\ \text { H } & -1.817 & -2.837 & 1.181 \\ \text { H } & -0.260 & -2.256 & 0.614 \\ \text { H } & -0.988 & -1.564 & 2.077 \\ \text { O } & -2.021 & 1.414 & 0.190 \\ \text { O } & -0.011 & 0.574 & 0.070 \\ \mathrm{C} & -1.455 & 2.744 & 0.079 \\ \text { H } & -0.915 & 2.828 & -0.860 \\ \text { H } & -2.307 & 3.412 & 0.104 \\ \text { H } & -0.791 & 2.921 & 0.922 \\ \text { Si } & 1.639 & -0.067 & -0.061 \\ \mathrm{C} & 2.080 & -0.665 & 1.629 \\ \text { H } & 1.848 & 0.086 & 2.386 \\ \text { H } & 1.596 & -1.601 & 1.903 \\ \text { H } & 3.160 & -0.830 & 1.659 \\ \mathrm{C} & 2.545 & 1.465 & -0.546 \\ \text { H } & 3.609 & 1.247 & -0.659 \\ \text { H } & 2.187 & 1.859 & -1.499 \\ \text { H } & 2.447 & 2.243 & 0.212 \\ \mathrm{C} & 1.643 & -1.343 & -1.399 \\ \text { H } & 2.688 & -1.588 & -1.612 \\ \text { H } & 1.142 & -2.274 & -1.136 \\ \text { H } & 1.212 & -0.954 & -2.323 \\ \text { H } & -2.923 & -0.734 & 0.633\end{array}$

EE13

$\begin{array}{lrrr}\text { O } & -0.267 & 0.643 & 0.206 \\ \text { Si } & -1.929 & -0.005 & -0.028 \\ \text { C } & -2.136 & -1.250 & 1.320 \\ \text { H } & -1.473 & -2.107 & 1.196 \\ \text { H } & -1.968 & -0.808 & 2.303 \\ \text { H } & -3.163 & -1.624 & 1.303\end{array}$

$\begin{array}{lrrr}\mathrm{C} & -2.927 & 1.528 & 0.176 \\ \mathrm{H} & -2.667 & 2.275 & -0.575 \\ \mathrm{H} & -3.988 & 1.296 & 0.061 \\ \mathrm{H} & -2.787 & 1.965 & 1.165 \\ \mathrm{C} & -1.920 & -0.703 & -1.738 \\ \mathrm{H} & -1.624 & 0.045 & -2.474 \\ \mathrm{H} & -1.263 & -1.569 & -1.826 \\ \mathrm{H} & -2.931 & -1.029 & -1.993 \\ \mathrm{C} & 0.835 & 0.058 & 0.176 \\ \mathrm{C} & 2.158 & 0.712 & 0.417 \\ \mathrm{C} & 2.324 & -1.636 & -0.013 \\ \mathrm{C} & 3.112 & -0.341 & -0.161 \\ \mathrm{H} & 2.452 & -2.355 & -0.816 \\ \mathrm{H} & 2.454 & -2.122 & 0.952 \\ \mathrm{H} & 4.064 & -0.382 & 0.361 \\ \mathrm{H} & 3.303 & -0.127 & -1.213 \\ \mathrm{O} & 0.918 & -1.202 & -0.067 \\ \mathrm{C} & 2.261 & 2.121 & -0.147 \\ \mathrm{H} & 2.133 & 2.113 & -1.230 \\ \mathrm{H} & 1.506 & 2.772 & 0.291 \\ \mathrm{H} & 3.243 & 2.533 & 0.079 \\ \mathrm{H} & 2.254 & 0.744 & 1.511\end{array}$

\section{EE14}

$\begin{array}{lrrr}\text { C } & -0.823 & 3.416 & 0.077 \\ \text { C } & -1.613 & 2.117 & -0.018 \\ \text { C } & -0.919 & 1.093 & -0.946 \\ \text { C } & 0.519 & 0.940 & -0.539 \\ \text { C } & 1.331 & 2.169 & -0.345 \\ \text { C } & 0.589 & 3.154 & 0.583 \\ \text { H } & -1.725 & 1.677 & 0.976 \\ \text { H } & -2.614 & 2.277 & -0.417 \\ \text { H } & -0.782 & 3.902 & -0.902 \\ \text { H } & -1.335 & 4.104 & 0.749 \\ \text { H } & 2.325 & 1.925 & 0.023 \\ \text { H } & 1.440 & 2.632 & -1.333 \\ \text { H } & 0.552 & 2.735 & 1.593 \\ \text { H } & 1.170 & 4.072 & 0.638 \\ \text { O } & -1.558 & -0.113 & -1.084 \\ \text { O } & 0.963 & -0.213 & -0.384 \\ \text { Si } & 2.527 & -0.945 & 0.079 \\ \text { Si } & -2.322 & -1.087 & 0.056 \\ \text { C } & 2.182 & -2.731 & -0.202 \\ \text { H } & 3.083 & -3.315 & -0.001 \\ \text { H } & 1.392 & -3.105 & 0.450 \\ \text { H } & 1.892 & -2.914 & -1.238 \\ \text { C } & 3.784 & -0.260 & -1.094 \\ \text { H } & 3.430 & -0.313 & -2.125 \\ \text { H } & 4.083 & 0.765 & -0.877 \\ \text { H } & 4.681 & -0.880 & -1.032 \\ \mathrm{C} & 2.733 & -0.462 & 1.852\end{array}$




$\begin{array}{lrrr}\mathrm{H} & 2.869 & 0.612 & 1.988 \\ \mathrm{H} & 1.875 & -0.784 & 2.445 \\ \mathrm{H} & 3.619 & -0.956 & 2.258 \\ \mathrm{C} & -1.250 & -1.216 & 1.589 \\ \mathrm{H} & -0.358 & -1.812 & 1.393 \\ \mathrm{H} & -0.935 & -0.248 & 1.986 \\ \mathrm{H} & -1.811 & -1.715 & 2.382 \\ \mathrm{C} & -2.476 & -2.728 & -0.785 \\ \mathrm{H} & -2.956 & -3.456 & -0.129 \\ \mathrm{H} & -3.079 & -2.645 & -1.691 \\ \mathrm{H} & -1.497 & -3.118 & -1.066 \\ \mathrm{C} & -3.982 & -0.369 & 0.489 \\ \mathrm{H} & -4.552 & -0.139 & -0.413 \\ \mathrm{H} & -4.557 & -1.098 & 1.063 \\ \mathrm{H} & -3.914 & 0.538 & 1.091 \\ \mathrm{H} & -0.851 & 1.571 & -1.938\end{array}$

$\begin{array}{lrrr}\text { C } & 1.051 & -0.378 & -0.494 \\ \text { O } & 0.133 & 0.305 & -0.020 \\ \text { Si } & -1.649 & -0.018 & 0.050 \\ \text { C } & -1.858 & -1.665 & -0.763 \\ \text { H } & -2.923 & -1.914 & -0.748 \\ \text { H } & -1.341 & -2.469 & -0.236 \\ \text { H } & -1.551 & -1.665 & -1.811 \\ \text { C } & -2.321 & 1.412 & -0.895 \\ \text { H } & -3.413 & 1.371 & -0.886 \\ \text { H } & -1.998 & 1.399 & -1.937 \\ \text { H } & -2.021 & 2.359 & -0.445 \\ \text { C } & -1.958 & 0.002 & 1.864 \\ \text { H } & -3.026 & -0.130 & 2.051 \\ \text { H } & -1.660 & 0.954 & 2.306 \\ \text { H } & -1.431 & -0.804 & 2.376 \\ \text { H } & 0.799 & -1.343 & -0.952 \\ \text { H } & 2.822 & -0.061 & -1.465 \\ \text { C } & 3.195 & -1.056 & 0.392 \\ \text { H } & 3.044 & -2.057 & -0.013 \\ \text { H } & 4.260 & -0.834 & 0.362 \\ \text { H } & 2.868 & -1.035 & 1.431 \\ \mathrm{C} & 2.668 & 1.435 & 0.109 \\ \text { H } & 2.132 & 2.178 & -0.481 \\ \text { H } & 2.328 & 1.505 & 1.142 \\ \text { H } & 3.728 & 1.678 & 0.083\end{array}$

\section{EE15}

$\begin{array}{lrrr}\text { C } & -1.170 & -0.411 & 0.103 \\ \mathrm{C} & -0.702 & 1.023 & -0.127 \\ \mathrm{C} & 0.820 & 0.944 & 0.095 \\ \mathrm{C} & 1.088 & -0.493 & -0.046 \\ \mathrm{O} & 0.079 & -1.220 & -0.057 \\ \mathrm{H} & -1.507 & -0.632 & 1.113 \\ \mathrm{H} & -0.918 & 1.341 & -1.144 \\ \mathrm{H} & 1.138 & 1.223 & 1.110 \\ \mathrm{H} & 2.053 & -0.992 & -0.119 \\ \mathrm{H} & -1.182 & 1.713 & 0.561 \\ \mathrm{H} & -1.866 & -0.810 & -0.628 \\ \mathrm{H} & 1.433 & 1.535 & -0.588\end{array}$

EE18

\section{EE16}

$\begin{array}{lrrr}\mathrm{C} & -1.079 & 0.261 & -0.300 \\ \mathrm{H} & -0.946 & 0.887 & -1.188 \\ \mathrm{C} & -2.381 & -0.306 & 0.027 \\ \mathrm{H} & -2.721 & -0.896 & -0.831 \\ \mathrm{H} & -2.354 & -0.904 & 0.933 \\ \mathrm{O} & -0.090 & 0.049 & 0.419 \\ \mathrm{C} & 1.255 & 0.601 & 0.103 \\ \mathrm{H} & 1.132 & 1.271 & -0.748 \\ \mathrm{H} & 1.510 & 1.164 & 0.997 \\ \mathrm{C} & 2.186 & -0.550 & -0.157 \\ \mathrm{H} & 1.891 & -1.110 & -1.043 \\ \mathrm{H} & 3.183 & -0.143 & -0.327 \\ \mathrm{H} & 2.231 & -1.217 & 0.701 \\ \mathrm{H} & -3.097 & 0.520 & 0.117\end{array}$




$\begin{array}{lrrr}\text { C } & -2.329 & -0.826 & 0.006 \\ \mathrm{C} & -4.142 & 0.714 & 0.019 \\ \mathrm{C} & -3.258 & 1.806 & -0.015 \\ \mathrm{C} & -1.901 & 1.596 & -0.039 \\ \mathrm{C} & -0.119 & -0.224 & -0.052 \\ \mathrm{C} & -0.106 & -1.721 & -0.074 \\ \mathrm{H} & -5.207 & 0.908 & 0.037 \\ \mathrm{H} & -3.656 & 2.811 & -0.022 \\ \mathrm{H} & -1.195 & 2.416 & -0.067 \\ \mathrm{H} & -1.788 & -2.677 & 0.947 \\ \mathrm{H} & 0.359 & -2.049 & -1.008 \\ \mathrm{H} & -4.400 & -1.420 & 0.058 \\ \mathrm{C} & -3.696 & -0.599 & 0.030 \\ \mathrm{C} & -1.584 & -2.131 & 0.025 \\ \mathrm{H} & -1.888 & -2.773 & -0.801 \\ \mathrm{O} & 0.899 & 0.523 & -0.057 \\ \mathrm{Si} & 2.638 & 0.220 & 0.018 \\ \mathrm{C} & 2.952 & -0.192 & 1.794 \\ \mathrm{H} & 2.458 & -1.113 & 2.105 \\ \mathrm{H} & 4.025 & -0.332 & 1.946 \\ \mathrm{H} & 2.628 & 0.616 & 2.451 \\ \mathrm{C} & 3.319 & 1.845 & -0.520 \\ \mathrm{H} & 2.979 & 2.651 & 0.131 \\ \mathrm{H} & 4.410 & 1.823 & -0.484 \\ \mathrm{H} & 3.025 & 2.080 & -1.544 \\ \mathrm{C} & 3.017 & -1.163 & -1.155 \\ \mathrm{H} & 2.749 & -2.146 & -0.768 \\ \mathrm{H} & 2.541 & -1.018 & -2.126 \\ \mathrm{H} & 4.097 & -1.170 & -1.328 \\ \mathrm{H} & 0.518 & -2.106 & 0.736\end{array}$

$\begin{array}{lrrr}\text { CX1 } & & & \\ \text { C } & -1.047 & 0.648 & -0.093 \\ \text { H } & -1.046 & 1.129 & 0.887 \\ \text { C } & -0.010 & -0.481 & -0.192 \\ \text { C } & 1.411 & -0.128 & -0.069 \\ \text { C } & 2.350 & -1.158 & -0.169 \\ \text { C } & 1.832 & 1.186 & 0.141 \\ \text { C } & 3.697 & -0.870 & -0.060 \\ \text { H } & 2.005 & -2.170 & -0.335 \\ \text { C } & 3.182 & 1.469 & 0.252 \\ \text { H } & 1.119 & 1.998 & 0.222 \\ \text { C } & 4.112 & 0.441 & 0.151 \\ \text { H } & 4.428 & -1.663 & -0.139 \\ \text { H } & 3.512 & 2.485 & 0.416 \\ \text { O } & -0.425 & -1.603 & -0.361 \\ \text { S } & -2.669 & -0.075 & -0.407 \\ \text { C } & -3.005 & -1.000 & 1.092 \\ \text { H } & -2.379 & -1.887 & 1.063 \\ \text { H } & -2.792 & -0.382 & 1.963 \\ \text { H } & -4.058 & -1.275 & 1.059\end{array}$

$\begin{array}{lrrr}\mathrm{C} & -3.718 & 1.362 & -0.169 \\ \mathrm{H} & -3.528 & 1.819 & 0.800 \\ \mathrm{H} & -3.512 & 2.059 & -0.979 \\ \mathrm{H} & -4.749 & 1.020 & -0.240 \\ \mathrm{H} & 5.168 & 0.664 & 0.236 \\ \mathrm{H} & -0.893 & 1.407 & -0.863\end{array}$

\section{CX2}

C

$\begin{array}{llr}2.553 & -0.646 & -0.107 \\ 2.490 & -1.144 & 0.862 \\ 1.616 & 0.568 & -0.202\end{array}$

C

C

C

C

$\mathrm{H}$

C

$\mathrm{H}$

C

$\mathrm{H}$

$\mathrm{H}$

O

$\mathrm{S}$

C

$\mathrm{H}$

$\mathrm{H}$

$\mathrm{H}$

C

$\mathrm{H}$

$\mathrm{H}$

$\mathrm{H}$

$\mathrm{Br}$

0.170

$\begin{array}{ll}0.368 & -0.125\end{array}$

C

$-0.680$

$1.438 \quad-0.227$

$\begin{array}{lll}-0.370 & -0.943 & 0.042\end{array}$

C

-2.048
-0.251

1.271

$-0.164$

$-1.740$

2.423

$-0.357$

$-1.740$

$-1.119$

0.107

$\begin{array}{lll}0.265 & -1.817 & 0.124\end{array}$

$-2.571$

$-0.009$

0.002

$-2.713$

2.119

$-0.244$

$-2.166$

$-2.104$

0.237

2.126

1.656

$-0.331$

4.236

$-0.054$

$-0.370$

4.614

0.805

1.158

4.059

1.738

1.142

4.339

0.184

2.009

5.686

0.999

1.149

5.156

$-1.579$

$-0.149$

4.906

$-2.044$

0.802

4.913

$-2.236$

$-0.982$

6.213

$-1.322$

$-0.189$

$-4.431$

$-0.243$

0.083

$\mathrm{H}$

$$
2.354
$$

$-1.373$

$-0.896$

cx3

$\begin{array}{lrrr}\mathrm{C} & -2.209 & 0.651 & -0.127 \\ \mathrm{H} & -2.157 & 1.174 & 0.829 \\ \mathrm{C} & -1.260 & -0.559 & -0.198 \\ \mathrm{C} & 0.161 & -0.313 & -0.123 \\ \mathrm{C} & 1.040 & -1.407 & -0.180 \\ \mathrm{C} & 0.710 & 0.971 & 0.005 \\ \mathrm{C} & 2.396 & -1.234 & -0.115 \\ \mathrm{H} & 0.624 & -2.401 & -0.281 \\ \mathrm{C} & 2.066 & 1.166 & 0.074 \\ \mathrm{H} & 0.071 & 1.845 & 0.052 \\ \mathrm{C} & 2.958 & 0.064 & 0.015 \\ \mathrm{H} & 3.037 & -2.100 & -0.164 \\ \mathrm{H} & 2.447 & 2.170 & 0.171 \\ \mathrm{O} & -1.784 & -1.652 & -0.309 \\ \mathrm{~S} & -3.879 & 0.020 & -0.370\end{array}$




$\begin{array}{lrrr}\mathrm{C} & -4.236 & -0.811 & 1.178 \\ \mathrm{H} & -3.664 & -1.734 & 1.176 \\ \mathrm{H} & -3.962 & -0.167 & 2.012 \\ \mathrm{H} & -5.304 & -1.019 & 1.184 \\ \mathrm{C} & -4.829 & 1.530 & -0.170 \\ \mathrm{H} & -4.582 & 2.017 & 0.771 \\ \mathrm{H} & -4.604 & 2.177 & -1.015 \\ \mathrm{H} & -5.882 & 1.254 & -0.196 \\ \mathrm{~N} & 4.292 & 0.243 & 0.080 \\ \mathrm{C} & 5.189 & -0.900 & 0.013 \\ \mathrm{H} & 6.214 & -0.549 & 0.080 \\ \mathrm{H} & 5.010 & -1.592 & 0.839 \\ \mathrm{H} & 5.072 & -1.440 & -0.929 \\ \mathrm{C} & 4.847 & 1.579 & 0.211 \\ \mathrm{H} & 4.572 & 2.207 & -0.640 \\ \mathrm{H} & 4.503 & 2.061 & 1.129 \\ \mathrm{H} & 5.929 & 1.511 & 0.247 \\ \mathrm{H} & -2.030 & 1.363 & -0.935\end{array}$

CX5

$\begin{array}{lrrr}\text { CX4 } & & & \\ \text { C } & -1.892 & 0.645 & -0.098 \\ \text { H } & -1.876 & 1.131 & 0.879 \\ \text { C } & -0.871 & -0.500 & -0.203 \\ \text { C } & 0.542 & -0.167 & -0.105 \\ \text { C } & 1.476 & -1.202 & -0.207 \\ \text { C } & 0.999 & 1.146 & 0.087 \\ \text { C } & 2.828 & -0.948 & -0.123 \\ \text { H } & 1.119 & -2.212 & -0.358 \\ \text { C } & 2.342 & 1.412 & 0.175 \\ \text { H } & 0.303 & 1.973 & 0.171 \\ \text { C } & 3.271 & 0.367 & 0.070 \\ \text { H } & 3.531 & -1.763 & -0.207 \\ \text { H } & 2.714 & 2.416 & 0.323 \\ \text { O } & -1.315 & -1.618 & -0.359 \\ \text { S } & -3.524 & -0.064 & -0.386 \\ \text { C } & -3.852 & -0.973 & 1.124 \\ \text { H } & -3.234 & -1.865 & 1.094 \\ \text { H } & -3.622 & -0.350 & 1.986 \\ \text { H } & -4.908 & -1.237 & 1.107 \\ \text { C } & -4.555 & 1.385 & -0.145 \\ \text { H } & -4.348 & 1.847 & 0.818 \\ \text { H } & -4.353 & 2.075 & -0.962 \\ \text { H } & -5.591 & 1.054 & -0.200 \\ \text { C } & 5.554 & -0.282 & 0.064 \\ \text { H } & 6.501 & 0.236 & 0.168 \\ \text { H } & 5.446 & -1.018 & 0.863 \\ \text { H } & 5.509 & -0.776 & -0.908 \\ \text { O } & 4.551 & 0.720 & 0.165 \\ \text { H } & -1.742 & 1.398 & -0.874\end{array}$

$\begin{array}{lrrr}\text { S } & 0.680 & 0.030 & -0.523 \\ \mathrm{C} & 0.484 & 1.607 & 0.308 \\ \mathrm{H} & 0.545 & 1.473 & 1.386 \\ \mathrm{H} & 1.280 & 2.254 & -0.058 \\ \mathrm{H} & -0.485 & 2.006 & 0.009 \\ \mathrm{C} & 2.183 & -0.621 & 0.205 \\ \mathrm{H} & 2.131 & -0.565 & 1.290 \\ \mathrm{H} & 2.294 & -1.648 & -0.138 \\ \mathrm{H} & 3.005 & -0.019 & -0.180 \\ \mathrm{C} & -0.579 & -0.984 & 0.318 \\ \mathrm{H} & -0.527 & -1.980 & -0.122 \\ \mathrm{C} & -1.877 & -0.371 & 0.089 \\ \mathrm{~N} & -2.864 & 0.176 & -0.103 \\ \mathrm{H} & -0.347 & -1.029 & 1.383\end{array}$

CX6

C

$\mathrm{H}$

$\mathrm{H}$

$\mathrm{H}$

C

$\mathrm{H}$

$\mathrm{H}$

$\mathrm{H}$

C

$\mathrm{H}$

$\mathrm{H}$

C

O

0

C

$\mathrm{H}$

$\mathrm{H}$

C

$\mathrm{H}$

$\mathrm{H}$

$\mathrm{H}$

CX7

$\begin{array}{lrrr}\mathrm{S} & -2.515 & -0.262 & 0.531 \\ \mathrm{C} & -2.186 & 1.439 & 0.981 \\ \mathrm{H} & -2.383 & 2.093 & 0.133 \\ \mathrm{H} & -2.831 & 1.681 & 1.824 \\ \mathrm{H} & -1.142 & 1.493 & 1.285 \\ \mathrm{C} & -4.183 & -0.166 & -0.121 \\ \mathrm{H} & -4.244 & 0.616 & -0.875 \\ \mathrm{H} & -4.422 & -1.139 & -0.545\end{array}$




$\begin{array}{lrrr}\text { H } & -4.849 & 0.042 & 0.714 \\ \mathrm{C} & -1.518 & -0.455 & -0.985 \\ \mathrm{H} & -1.771 & -1.452 & -1.349 \\ \mathrm{C} & -0.063 & -0.311 & -0.652 \\ \mathrm{C} & 0.615 & 0.854 & -0.998 \\ \mathrm{C} & 0.607 & -1.335 & 0.014 \\ \mathrm{C} & 1.956 & 0.999 & -0.683 \\ \mathrm{H} & 0.104 & 1.646 & -1.532 \\ \mathrm{C} & 1.945 & -1.197 & 0.335 \\ \mathrm{H} & 0.087 & -2.250 & 0.274 \\ \mathrm{C} & 2.619 & -0.026 & -0.012 \\ \mathrm{H} & 2.493 & 1.896 & -0.958 \\ \mathrm{H} & 2.474 & -1.989 & 0.847 \\ \mathrm{C} & 4.007 & 0.123 & 0.323 \\ \mathrm{~N} & 5.114 & 0.245 & 0.595 \\ \mathrm{H} & -1.865 & 0.295 & -1.697\end{array}$

\section{CX8}

$\begin{array}{lrrr}\text { S } & -2.900 & -0.345 & 0.489 \\ \mathrm{C} & -2.563 & 1.257 & 1.213 \\ \mathrm{H} & -2.770 & 2.044 & 0.489 \\ \mathrm{H} & -3.197 & 1.354 & 2.092 \\ \mathrm{H} & -1.516 & 1.262 & 1.509 \\ \mathrm{C} & -4.575 & -0.143 & -0.116 \\ \mathrm{H} & -4.645 & 0.753 & -0.730 \\ \mathrm{H} & -4.822 & -1.033 & -0.692 \\ \mathrm{H} & -5.230 & -0.076 & 0.750 \\ \mathrm{C} & -1.925 & -0.280 & -1.051 \\ \mathrm{H} & -2.185 & -1.200 & -1.576 \\ \mathrm{C} & -0.463 & -0.200 & -0.719 \\ \mathrm{C} & 0.216 & 1.005 & -0.877 \\ \mathrm{C} & 0.207 & -1.325 & -0.244 \\ \mathrm{C} & 1.564 & 1.092 & -0.564 \\ \mathrm{H} & -0.299 & 1.877 & -1.264 \\ \mathrm{C} & 1.552 & -1.250 & 0.076 \\ \mathrm{H} & -0.317 & -2.268 & -0.133 \\ \mathrm{C} & 2.199 & -0.039 & -0.090 \\ \mathrm{H} & 2.121 & 2.009 & -0.685 \\ \mathrm{H} & 2.102 & -2.106 & 0.440 \\ \mathrm{H} & -2.276 & 0.580 & -1.621 \\ \mathrm{~N} & 3.640 & 0.048 & 0.252 \\ \mathrm{O} & 4.166 & -0.959 & 0.655 \\ \mathrm{O} & 4.171 & 1.120 & 0.104\end{array}$

\section{CX9}

$\begin{array}{lrrr}C & -0.109 & 0.001 & -0.484 \\ C & 0.539 & 1.213 & -0.258 \\ C & 0.538 & -1.212 & -0.260 \\ C & 1.848 & 1.207 & 0.195 \\ C & 1.846 & -1.208 & 0.193\end{array}$

$\begin{array}{lrrr}\mathrm{H} & 0.030 & -2.151 & -0.450 \\ \mathrm{C} & 2.496 & -0.001 & 0.420 \\ \mathrm{H} & 2.363 & 2.142 & 0.364 \\ \mathrm{H} & 2.361 & -2.144 & 0.361 \\ \mathrm{H} & 3.520 & -0.002 & 0.770 \\ \mathrm{H} & 0.033 & 2.153 & -0.447 \\ \mathrm{C} & -1.510 & 0.002 & -0.985 \\ \mathrm{H} & -1.821 & 0.903 & -1.513 \\ \mathrm{~N} & -3.049 & -0.002 & 1.134 \\ \mathrm{~N} & -2.459 & 0.000 & 0.224 \\ \mathrm{H} & -1.821 & -0.897 & -1.517\end{array}$

\section{CX10}

$\begin{array}{lrrr}\mathrm{C} & -0.987 & 0.000 & 0.103 \\ \mathrm{C} & -0.314 & -1.213 & 0.225 \\ \mathrm{C} & 1.047 & -1.211 & 0.465 \\ \mathrm{C} & 1.728 & 0.000 & 0.586 \\ \mathrm{C} & 1.047 & 1.211 & 0.465 \\ \mathrm{C} & -0.314 & 1.213 & 0.225 \\ \mathrm{H} & 1.572 & -2.153 & 0.571 \\ \mathrm{H} & 1.572 & 2.153 & 0.571 \\ \mathrm{H} & -0.856 & 2.144 & 0.138 \\ \mathrm{H} & -0.856 & -2.144 & 0.138 \\ \mathrm{C} & 3.182 & 0.000 & 0.860 \\ \mathrm{H} & 3.580 & 0.901 & 1.327 \\ \mathrm{Br} & -2.832 & 0.000 & -0.219 \\ \mathrm{~N} & 3.943 & 0.000 & -0.500 \\ \mathrm{~N} & 4.370 & 0.000 & -1.498 \\ \mathrm{H} & 3.580 & -0.900 & 1.327\end{array}$

\section{CX11}

$\begin{array}{lrrr}\text { C } & -1.791 & 0.000 & -0.017 \\ \text { C } & -1.118 & -1.213 & 0.127 \\ \text { C } & 0.235 & -1.212 & 0.408 \\ \text { C } & 0.905 & 0.001 & 0.547 \\ \text { C } & 0.235 & 1.213 & 0.406 \\ \text { C } & -1.118 & 1.214 & 0.125 \\ \text { H } & 0.759 & -2.152 & 0.532 \\ \text { H } & 0.759 & 2.154 & 0.528 \\ \text { H } & -1.659 & 2.144 & 0.020 \\ \text { H } & -1.659 & -2.144 & 0.023 \\ \text { C } & 2.364 & 0.001 & 0.866 \\ \text { H } & 2.733 & 0.900 & 1.360 \\ \text { N } & 3.147 & 0.000 & -0.438 \\ \text { N } & 3.635 & -0.002 & -1.408 \\ \text { C } & -3.197 & 0.000 & -0.309 \\ \text { N } & -4.319 & -0.001 & -0.545 \\ \text { H } & 2.733 & -0.898 & 1.361\end{array}$




$\begin{array}{lrrr}\mathrm{C} & -1.374 & 0.000 & 0.051 \\ \mathrm{C} & -0.730 & -1.217 & 0.177 \\ \mathrm{C} & 0.631 & -1.214 & 0.434 \\ \mathrm{C} & 1.300 & 0.001 & 0.560 \\ \mathrm{C} & 0.630 & 1.215 & 0.433 \\ \mathrm{C} & -0.730 & 1.217 & 0.175 \\ \mathrm{H} & 1.158 & -2.153 & 0.549 \\ \mathrm{H} & 1.158 & 2.154 & 0.546 \\ \mathrm{H} & -1.292 & 2.135 & 0.076 \\ \mathrm{H} & -1.292 & -2.135 & 0.079 \\ \mathrm{C} & 2.767 & 0.001 & 0.854 \\ \mathrm{H} & 3.142 & 0.900 & 1.344 \\ \mathrm{~N} & 3.526 & 0.000 & -0.459 \\ \mathrm{~N} & 4.005 & -0.001 & -1.432 \\ \mathrm{~N} & -2.836 & 0.000 & -0.226 \\ \mathrm{O} & -3.366 & 1.076 & -0.327 \\ \mathrm{O} & -3.365 & -1.077 & -0.327 \\ \mathrm{H} & 3.142 & -0.897 & 1.346\end{array}$

CX13

$\begin{array}{lrrr}\mathrm{C} & 0.829 & 0.216 & 0.000 \\ \mathrm{O} & 0.433 & 1.333 & 0.000 \\ \mathrm{C} & 2.237 & -0.272 & 0.000 \\ \mathrm{H} & 2.905 & 0.585 & 0.000 \\ \mathrm{H} & 2.419 & -0.889 & 0.883 \\ \mathrm{H} & 2.419 & -0.889 & -0.883 \\ \mathrm{C} & -0.242 & -0.959 & 0.000 \\ \mathrm{H} & -0.198 & -1.576 & -0.899 \\ \mathrm{~N} & -1.534 & -0.322 & 0.000 \\ \mathrm{~N} & -2.431 & 0.288 & 0.000 \\ \mathrm{H} & -0.198 & -1.576 & 0.899\end{array}$

$\begin{array}{lrrr}\text { CX14 } & & & \\ \text { C } & 2.454 & 0.602 & 0.000 \\ \text { C } & 1.363 & 1.455 & 0.000 \\ \text { C } & 2.246 & -0.774 & 0.000 \\ \text { C } & 0.089 & 0.926 & 0.000 \\ \text { H } & 1.481 & 2.529 & 0.000 \\ \text { C } & 0.960 & -1.253 & 0.000 \\ \text { N } & -0.090 & -0.405 & 0.000 \\ \text { H } & -0.801 & 1.540 & 0.000 \\ \text { H } & 0.724 & -2.309 & 0.000 \\ \text { C } & -1.444 & -1.025 & 0.000 \\ \text { H } & -1.529 & -1.651 & 0.889 \\ \text { H } & 3.460 & 1.001 & 0.000 \\ \text { H } & 3.069 & -1.473 & 0.000 \\ \text { C } & -2.505 & -0.029 & 0.000 \\ \text { N } & -3.318 & 0.776 & 0.000 \\ \text { H } & -1.529 & -1.652 & -0.888\end{array}$

\section{CX15}

$\begin{array}{lrrr}\text { C } & -4.236 & 0.294 & 0.334 \\ \mathrm{C} & -3.495 & 1.154 & -0.461 \\ \mathrm{C} & -3.671 & -0.9 & 0.762 \\ \mathrm{C} & -2.209 & 0.805 & -0.807 \\ \mathrm{H} & -3.896 & 2.095 & -0.807 \\ \mathrm{C} & -2.385 & -1.203 & 0.382 \\ \mathrm{~N} & -1.685 & -0.363 & -0.4 \\ \mathrm{H} & -1.568 & 1.434 & -1.406 \\ \mathrm{H} & -1.884 & -2.111 & 0.683 \\ \mathrm{C} & -0.286 & -0.656 & -0.732 \\ \mathrm{H} & -0.157 & -0.545 & -1.808 \\ \mathrm{C} & 0.618 & 0.339 & 0.041 \\ \mathrm{O} & 0.075 & 1.234 & 0.661 \\ \mathrm{H} & -5.247 & 0.552 & 0.622 \\ \mathrm{H} & -4.215 & -1.594 & 1.386 \\ \mathrm{C} & 2.558 & -0.995 & -0.725 \\ \mathrm{C} & 3.079 & -2.038 & 0.253 \\ \mathrm{H} & 3.37 & -0.618 & -1.346 \\ \mathrm{H} & 1.845 & -1.441 & -1.415 \\ \mathrm{H} & 3.524 & -2.871 & -0.289 \\ \mathrm{H} & 3.845 & -1.615 & 0.904 \\ \mathrm{H} & 2.276 & -2.423 & 0.883 \\ \mathrm{C} & 2.816 & 1.028 & 0.739 \\ \mathrm{C} & 4.09 & 1.414 & 0.008 \\ \mathrm{H} & 3.051 & 0.532 & 1.684 \\ \mathrm{H} & 2.23 & 1.911 & 0.976 \\ \mathrm{H} & 4.614 & 2.168 & 0.594 \\ \mathrm{H} & 4.771 & 0.573 & -0.122 \\ \mathrm{H} & 3.869 & 1.841 & -0.97 \\ \mathrm{~N} & 1.94 & 0.146 & -0.052 \\ \mathrm{H} & -0.083 & -1.689 & -0.462\end{array}$

CX16

$\begin{array}{lrrr}\mathrm{C} & -3.734 & 0.269 & 0.43 \\ \mathrm{C} & -2.964 & 1.223 & -0.218 \\ \mathrm{C} & -3.22 & -1.005 & 0.63 \\ \mathrm{C} & -1.702 & 0.885 & -0.648 \\ \mathrm{H} & -3.328 & 2.226 & -0.388 \\ \mathrm{C} & -1.954 & -1.292 & 0.178 \\ \mathrm{~N} & -1.224 & -0.357 & -0.456 \\ \mathrm{H} & -1.042 & 1.581 & -1.146 \\ \mathrm{H} & -1.491 & -2.261 & 0.303 \\ \mathrm{C} & 0.148 & -0.657 & -0.881 \\ \mathrm{H} & 0.27 & -0.379 & -1.928 \\ \mathrm{C} & 1.142 & 0.138 & -0.03 \\ \mathrm{O} & 0.809 & 1.036 & 0.689\end{array}$




$\begin{array}{rrrr}\mathrm{H} & -4.729 & 0.516 & 0.778 \\ \mathrm{H} & -3.788 & -1.774 & 1.132 \\ \mathrm{O} & 2.358 & -0.304 & -0.242 \\ \mathrm{C} & 3.431 & 0.386 & 0.463 \\ \mathrm{C} & 4.725 & -0.277 & 0.076 \\ \mathrm{H} & 3.223 & 0.314 & 1.53 \\ \mathrm{H} & 3.393 & 1.436 & 0.174 \\ \mathrm{H} & 5.548 & 0.22 & 0.589 \\ \mathrm{H} & 4.726 & -1.327 & 0.363 \\ \mathrm{H} & 4.895 & -0.203 & -0.997 \\ \mathrm{H} & 0.332 & -1.723 & -0.787\end{array}$

\section{CX17}

$\begin{array}{lrrr}\text { C } & 2.86 & 0.237 & -0.311 \\ \text { C } & 2.066 & 1.232 & 0.24 \\ \text { C } & 2.34 & -1.04 & -0.477 \\ \text { C } & 0.776 & 0.93 & 0.61 \\ \text { H } & 2.433 & 2.238 & 0.382 \\ \text { C } & 1.046 & -1.289 & -0.088 \\ \text { N } & 0.293 & -0.315 & 0.455 \\ \text { H } & 0.099 & 1.658 & 1.034 \\ \text { H } & 0.578 & -2.258 & -0.192 \\ \text { C } & -1.112 & -0.565 & 0.785 \\ \text { H } & -1.313 & -0.206 & 1.796 \\ \mathrm{C} & -2.037 & 0.172 & -0.208 \\ \text { O } & -1.57 & 0.977 & -0.961 \\ \text { H } & 3.877 & 0.454 & -0.61 \\ \text { H } & 2.926 & -1.84 & -0.905 \\ \mathrm{C} & -3.485 & -0.195 & -0.125 \\ \text { H } & -3.822 & -0.217 & 0.913 \\ \text { H } & -3.622 & -1.2 & -0.534 \\ \text { H } & -4.075 & 0.511 & -0.702 \\ \text { H } & -1.3 & -1.637 & 0.767\end{array}$

\section{CX18}

$\begin{array}{lrrr}\text { C } & 4.462 & -0.314 & 0.195 \\ \mathrm{C} & 3.724 & -0.826 & -0.861 \\ \mathrm{C} & 3.881 & 0.615 & 1.048 \\ \mathrm{C} & 2.428 & -0.4 & -1.04 \\ \mathrm{H} & 4.138 & -1.555 & -1.542 \\ \mathrm{C} & 2.584 & 1.01 & 0.821 \\ \mathrm{~N} & 1.887 & 0.514 & -0.216 \\ \mathrm{H} & 1.79 & -0.768 & -1.831 \\ \mathrm{H} & 2.07 & 1.724 & 1.449 \\ \mathrm{C} & 0.477 & 0.869 & -0.394 \\ \mathrm{H} & 0.307 & 1.129 & -1.44 \\ \mathrm{C} & -0.409 & -0.335 & 0.006 \\ \mathrm{O} & 0.133 & -1.376 & 0.274\end{array}$

$\begin{array}{lrrr}\text { C } & -1.867 & -0.127 & 0.018 \\ \text { C } & -2.447 & 1.085 & -0.362 \\ \text { C } & -2.675 & -1.194 & 0.417 \\ \text { C } & -3.825 & 1.227 & -0.343 \\ \text { H } & -1.842 & 1.924 & -0.68 \\ \text { C } & -4.049 & -1.046 & 0.438 \\ \text { H } & -2.207 & -2.125 & 0.706 \\ \text { C } & -4.623 & 0.163 & 0.058 \\ \text { H } & -4.277 & 2.163 & -0.64 \\ \text { H } & -4.677 & -1.869 & 0.749 \\ \text { H } & -5.699 & 0.277 & 0.075 \\ \text { H } & 5.482 & -0.638 & 0.356 \\ \text { H } & 4.422 & 1.034 & 1.884 \\ \text { H } & 0.256 & 1.74 & 0.217\end{array}$

CX19

$\begin{array}{lrrr}\text { C } & 3.737 & 0.994 & -0.030 \\ \text { C } & 2.834 & 1.952 & 0.397 \\ \text { C } & 3.335 & -0.335 & -0.090 \\ \text { C } & 1.559 & 1.573 & 0.753 \\ \text { H } & 3.110 & 2.995 & 0.449 \\ \text { C } & 2.048 & -0.668 & 0.281 \\ \text { N } & 1.199 & 0.281 & 0.706 \\ \text { H } & 0.803 & 2.272 & 1.078 \\ \text { H } & 1.683 & -1.684 & 0.248 \\ \text { C } & -0.194 & -0.070 & 1.001 \\ \text { H } & -0.485 & 0.403 & 1.940 \\ \text { C } & -1.099 & 0.428 & -0.152 \\ \text { O } & -0.599 & 1.098 & -1.018 \\ \text { C } & -2.523 & 0.054 & -0.102 \\ \text { C } & -3.061 & -0.678 & 0.958 \\ \text { C } & -3.342 & 0.466 & -1.155 \\ \text { C } & -4.409 & -0.996 & 0.963 \\ \text { H } & -2.446 & -1.003 & 1.788 \\ \text { C } & -4.686 & 0.142 & -1.147 \\ \text { H } & -2.907 & 1.034 & -1.967 \\ \text { C } & -5.218 & -0.587 & -0.089 \\ \text { H } & -4.829 & -1.560 & 1.784 \\ \text { H } & -5.323 & 0.457 & -1.963 \\ \text { H } & -6.271 & -0.838 & -0.084 \\ \text { Cl } & 4.394 & -1.565 & -0.612 \\ \text { H } & 4.745 & 1.264 & -0.319 \\ \text { H } & -0.265 & -1.148 & 1.122\end{array}$

\section{CX20}

$\begin{array}{lrrr}C & -3.335 & 0.067 & 0.030 \\ C & -2.550 & -0.649 & 0.976 \\ C & -2.667 & 1.101 & -0.682\end{array}$




$\begin{array}{lrrr}\text { C } & -1.240 & -0.335 & 1.154 \\ \text { H } & -2.963 & -1.457 & 1.557 \\ \text { C } & -1.354 & 1.358 & -0.442 \\ \text { H } & -3.175 & 1.696 & -1.423 \\ \text { N } & -0.642 & 0.670 & 0.476 \\ \text { H } & -0.613 & -0.876 & 1.849 \\ \text { H } & -0.821 & 2.132 & -0.976 \\ \text { C } & 0.791 & 0.886 & 0.631 \\ \text { H } & 1.036 & 0.931 & 1.693 \\ \text { C } & 1.575 & -0.257 & -0.043 \\ \text { O } & 0.958 & -1.185 & -0.501 \\ \text { C } & 3.049 & -0.152 & -0.074 \\ \text { C } & 3.728 & 0.918 & 0.510 \\ \text { C } & 3.764 & -1.169 & -0.708 \\ \text { C } & 5.112 & 0.969 & 0.461 \\ \text { H } & 3.194 & 1.716 & 1.011 \\ \text { C } & 5.145 & -1.113 & -0.758 \\ \text { H } & 3.220 & -1.991 & -1.154 \\ \text { C } & 5.817 & -0.045 & -0.174 \\ \text { H } & 5.639 & 1.796 & 0.915 \\ \text { H } & 5.701 & -1.899 & -1.250 \\ \text { H } & 6.898 & -0.003 & -0.213 \\ \text { N } & -4.619 & -0.216 & -0.177 \\ \text { C } & -5.265 & -1.291 & 0.568 \\ \text { H } & -5.233 & -1.093 & 1.641 \\ \text { H } & -4.784 & -2.249 & 0.364 \\ \text { C } & -5.387 & 0.535 & -1.164 \\ \text { H } & -4.960 & 0.418 & -2.162 \\ \text { H } & -5.417 & 1.596 & -0.908 \\ \text { H } & -6.303 & -1.356 & 0.263 \\ \text { H } & -6.404 & 0.158 & -1.180 \\ \text { H } & 1.050 & 1.846 & 0.188\end{array}$

CX21

$\begin{array}{lrrr}\text { C } & -5.371 & -0.569 & 0.311 \\ \text { C } & -4.667 & 0.532 & 0.716 \\ \text { C } & -3.311 & 0.663 & 0.358 \\ \text { C } & -2.705 & -0.356 & -0.422 \\ \text { C } & -3.453 & -1.486 & -0.828 \\ \text { C } & -4.765 & -1.585 & -0.464 \\ \text { H } & -2.932 & 2.564 & 1.34 \\ \text { H } & -6.413 & -0.671 & 0.587 \\ \text { H } & -5.137 & 1.305 & 1.311 \\ \text { C } & -2.512 & 1.767 & 0.742 \\ \text { C } & -1.358 & -0.216 & -0.767 \\ \text { H } & -2.976 & -2.259 & -1.417 \\ \text { H } & -5.349 & -2.443 & -0.765 \\ \text { C } & -1.213 & 1.836 & 0.365 \\ \text { H } & -0.84 & -0.967 & -1.348\end{array}$

$\begin{array}{lrrr}\text { H } & -0.559 & 2.651 & 0.632 \\ \text { N } & -0.655 & 0.844 & -0.402 \\ \mathrm{C} & 0.774 & 0.887 & -0.71 \\ \mathrm{C} & 1.519 & -0.193 & 0.109 \\ \mathrm{O} & 0.869 & -1.003 & 0.716 \\ \mathrm{C} & 2.994 & -0.173 & 0.072 \\ \mathrm{C} & 3.678 & -1.112 & 0.848 \\ \mathrm{C} & 3.706 & 0.734 & -0.713 \\ \mathrm{C} & 5.06 & -1.136 & 0.839 \\ \mathrm{H} & 3.108 & -1.809 & 1.448 \\ \mathrm{C} & 5.091 & 0.702 & -0.723 \\ \mathrm{H} & 3.197 & 1.466 & -1.328 \\ \mathrm{C} & 5.765 & -0.23 & 0.054 \\ \mathrm{H} & 5.591 & -1.86 & 1.442 \\ \mathrm{H} & 5.644 & 1.403 & -1.334 \\ \mathrm{H} & 6.847 & -0.252 & 0.048 \\ \mathrm{H} & 1.149 & 1.882 & -0.482 \\ \mathrm{H} & 0.919 & 0.704 & -1.775\end{array}$

CX22

$\begin{array}{lrrr}\text { C } & 0.283 & -0.889 & 0.822 \\ \mathrm{C} & 1.102 & -0.391 & -0.388 \\ \mathrm{O} & 0.54 & -0.263 & -1.444 \\ \mathrm{C} & 2.536 & -0.115 & -0.172 \\ \mathrm{C} & 3.149 & -0.301 & 1.068 \\ \mathrm{C} & 3.284 & 0.34 & -1.26 \\ \mathrm{C} & 4.5 & -0.034 & 1.218 \\ \mathrm{H} & 2.59 & -0.659 & 1.924 \\ \mathrm{C} & 4.631 & 0.608 & -1.105 \\ \mathrm{H} & 2.791 & 0.475 & -2.213 \\ \mathrm{C} & 5.238 & 0.421 & 0.133 \\ \mathrm{H} & 4.978 & -0.18 & 2.177 \\ \mathrm{H} & 5.213 & 0.96 & -1.945 \\ \mathrm{H} & 6.294 & 0.63 & 0.252 \\ \mathrm{C} & -1.892 & 0.143 & 0.314 \\ \mathrm{C} & -1.621 & -2.199 & 0.167 \\ \mathrm{C} & -1.365 & 1.424 & 0.559 \\ \mathrm{C} & -3.243 & 0 & -0.088 \\ \mathrm{C} & -2.934 & -2.384 & -0.244 \\ \mathrm{H} & -0.94 & -3.032 & 0.278 \\ \mathrm{C} & -2.177 & 2.518 & 0.421 \\ \mathrm{H} & -0.33 & 1.565 & 0.831 \\ \mathrm{C} & -4.049 & 1.156 & -0.216 \\ \mathrm{C} & -3.744 & -1.285 & -0.363 \\ \mathrm{H} & -3.284 & -3.383 & -0.456 \\ \mathrm{C} & -3.528 & 2.39 & 0.036 \\ \mathrm{H} & -1.768 & 3.502 & 0.604 \\ \mathrm{H} & -5.08 & 1.036 & -0.522 \\ \mathrm{H} & -4.776 & -1.39 & -0.675\end{array}$




$\begin{array}{rrrr}\mathrm{H} & -4.141 & 3.275 & -0.063 \\ \mathrm{~N} & -1.129 & -0.999 & 0.451 \\ \mathrm{H} & 0.637 & -1.874 & 1.121 \\ \mathrm{H} & 0.381 & -0.229 & 1.68\end{array}$

\section{CX23}

$\begin{array}{lrrr}\text { S } & 0.000 & 0.000 & 0.155 \\ \mathrm{O} & 0.000 & 0.001 & 1.588 \\ \mathrm{C} & -1.450 & 0.737 & -0.525 \\ \mathrm{H} & -1.488 & 1.758 & -0.147 \\ \mathrm{H} & -1.405 & 0.715 & -1.613 \\ \mathrm{H} & -2.298 & 0.165 & -0.149 \\ \mathrm{C} & 1.364 & 0.887 & -0.525 \\ \mathrm{H} & 1.292 & 1.907 & -0.149 \\ \mathrm{H} & 2.267 & 0.409 & -0.146 \\ \mathrm{H} & 1.323 & 0.858 & -1.613 \\ \mathrm{C} & 0.087 & -1.625 & -0.525 \\ \mathrm{H} & 0.083 & -1.575 & -1.612 \\ \mathrm{H} & 1.006 & -2.072 & -0.147 \\ \mathrm{H} & -0.779 & -2.168 & -0.146\end{array}$

CX24

$\begin{array}{lrrr}\mathrm{C} & -2.589 & 0.260 & 0.076 \\ \mathrm{H} & -2.308 & 1.220 & 0.501 \\ \mathrm{H} & -2.977 & 0.392 & -0.939 \\ \mathrm{~N} & -1.502 & -0.690 & -0.032 \\ \mathrm{C} & 0.146 & 1.125 & 0.044 \\ \mathrm{C} & 0.787 & -1.241 & -0.166 \\ \mathrm{C} & 1.644 & 0.985 & -0.202 \\ \mathrm{H} & -0.080 & 1.471 & 1.055 \\ \mathrm{H} & -0.391 & 1.737 & -0.678 \\ \mathrm{C} & 1.969 & -0.419 & 0.318 \\ \mathrm{H} & 0.865 & -1.486 & -1.227 \\ \mathrm{H} & 0.536 & -2.134 & 0.398 \\ \mathrm{H} & 1.855 & 1.054 & -1.270 \\ \mathrm{H} & 2.201 & 1.767 & 0.306 \\ \mathrm{H} & 2.908 & -0.804 & -0.069 \\ \mathrm{H} & 2.018 & -0.428 & 1.408 \\ \mathrm{~N} & -0.355 & -0.285 & -0.049 \\ \mathrm{H} & -3.370 & -0.223 & 0.660\end{array}$

\section{CX25}

$\begin{array}{lrrr}\mathrm{C} & -1.909 & -0.209 & -0.005 \\ \mathrm{H} & -1.988 & 0.670 & -0.644 \\ \mathrm{H} & -2.599 & -0.986 & -0.318 \\ \mathrm{~N} & -0.582 & -0.792 & -0.006 \\ \mathrm{~N} & 0.391 & -0.053 & -0.002 \\ \mathrm{C} & 0.341 & 1.424 & 0.002 \\ \mathrm{H} & -0.346 & 1.756 & 0.778\end{array}$

$\begin{array}{lrrr}\mathrm{H} & 1.342 & 1.795 & 0.193 \\ \mathrm{H} & -0.004 & 1.763 & -0.975 \\ \mathrm{C} & 1.728 & -0.673 & 0.001 \\ \mathrm{H} & 2.272 & -0.293 & -0.864 \\ \mathrm{H} & 2.236 & -0.367 & 0.915 \\ \mathrm{H} & 1.600 & -1.748 & -0.045 \\ \mathrm{H} & -2.143 & 0.070 & 1.028\end{array}$

\section{NHC1}

C

C

C

$\mathrm{H}$

$\mathrm{H}$

C

C

C

C

C

C

$\mathrm{H}$

$\mathrm{H}$

C

C

C

C

C

C

$\mathrm{H}$

$\mathrm{H}$

C

$\mathrm{H}$

$\mathrm{H}$

$\mathrm{H}$

C

C

$\mathrm{H}$

$\mathrm{H}$

$\mathrm{H}$

C

$\mathrm{H}$

$\mathrm{H}$

$\mathrm{H}$

C

$\mathrm{H}$

$\mathrm{H}$

$\mathrm{H}$

C

$\mathrm{H}$

$\mathrm{H}$

$\mathrm{H}$

C

$\begin{array}{rrr}-0.001 & 0.091 & -0.097 \\ -0.764 & 1.017 & 1.843 \\ 0.777 & 0.937 & 1.873 \\ -1.133 & 2.042 & 1.838 \\ 1.251 & 1.905 & 2.021 \\ 2.438 & 0.111 & 0.102 \\ 3.292 & 1.164 & -0.226 \\ 2.845 & -1.225 & 0.065 \\ 4.587 & 0.844 & -0.614 \\ 4.149 & -1.489 & -0.330 \\ 5.033 & -0.471 & -0.675 \\ 5.265 & 1.648 & -0.879 \\ 4.487 & -2.519 & -0.362 \\ -2.439 & 0.110 & 0.119 \\ -3.208 & 1.162 & -0.375 \\ -2.932 & -1.191 & 0.239 \\ -4.510 & 0.875 & -0.768 \\ -4.237 & -1.425 & -0.167 \\ -5.041 & -0.406 & -0.674 \\ -5.125 & 1.677 & -1.161 \\ -4.642 & -2.427 & -0.081 \\ -2.077 & -2.302 & 0.786 \\ -1.293 & -2.588 & 0.081 \\ -1.589 & -2.014 & 1.719 \\ -2.679 & -3.186 & 0.980 \\ -2.674 & 2.565 & -0.473 \\ -2.835 & 3.110 & 0.461 \\ -1.606 & 2.585 & -0.694 \\ -3.189 & 3.113 & -1.259 \\ 2.850 & 2.600 & -0.161 \\ 1.792 & 2.715 & -0.396 \\ 3.023 & 3.018 & 0.834 \\ 3.419 & 3.204 & -0.865 \\ 1.913 & -2.348 & 0.430 \\ 1.291 & -2.103 & 1.293 \\ 1.247 & -2.599 & -0.400 \\ 2.479 & -3.246 & 0.670 \\ -6.441 & -0.698 & -1.128 \\ -7.036 & 0.211 & -1.190 \\ -6.429 & -1.160 & -2.117 \\ -6.936 & -1.392 & -0.448 \\ 6.429 & -0.791 & -1.125\end{array}$




$\begin{array}{rrrr}\mathrm{H} & 6.436 & -1.029 & -2.191 \\ \mathrm{H} & 7.099 & 0.051 & -0.967 \\ \mathrm{H} & 6.825 & -1.655 & -0.592 \\ \mathrm{~N} & -1.098 & 0.372 & 0.559 \\ \mathrm{~N} & 1.102 & 0.413 & 0.531 \\ \mathrm{H} & -1.239 & 0.476 & 2.659 \\ \mathrm{H} & 1.154 & 0.241 & 2.623 \\ \mathrm{H} & -0.006 & -0.362 & -1.080\end{array}$

$\begin{array}{lrrr}\mathrm{C} & 6.475 & -0.428 & -1.129 \\ \mathrm{H} & 6.512 & -0.428 & -2.221 \\ \mathrm{H} & 7.107 & 0.386 & -0.778 \\ \mathrm{H} & 6.896 & -1.370 & -0.782 \\ \mathrm{~N} & -1.081 & 0.234 & 0.595 \\ \mathrm{~N} & 1.081 & 0.234 & 0.595 \\ \mathrm{H} & 0.000 & -0.348 & -1.161\end{array}$

NCH3

\section{NHC2}

$\begin{array}{lrrr}\text { C } & 0.000 & -0.011 & -0.138 \\ \text { C } & -0.678 & 0.653 & 1.843 \\ \mathrm{C} & 0.678 & 0.652 & 1.843 \\ \mathrm{H} & -1.392 & 0.901 & 2.608 \\ \mathrm{H} & 1.392 & 0.900 & 2.608 \\ \mathrm{C} & 2.446 & 0.065 & 0.155 \\ \mathrm{C} & 3.180 & 1.204 & -0.169 \\ \mathrm{C} & 2.963 & -1.226 & 0.094 \\ \mathrm{C} & 4.493 & 1.013 & -0.576 \\ \mathrm{C} & 4.283 & -1.359 & -0.319 \\ \mathrm{C} & 5.060 & -0.256 & -0.659 \\ \mathrm{H} & 5.090 & 1.879 & -0.836 \\ \mathrm{H} & 4.716 & -2.351 & -0.370 \\ \mathrm{C} & -2.446 & 0.067 & 0.155 \\ \mathrm{C} & -3.177 & 1.206 & -0.174 \\ \mathrm{C} & -2.966 & -1.224 & 0.100 \\ \mathrm{C} & -4.491 & 1.015 & -0.582 \\ \mathrm{C} & -4.285 & -1.356 & -0.313 \\ \mathrm{C} & -5.060 & -0.253 & -0.659 \\ \mathrm{H} & -5.086 & 1.881 & -0.845 \\ \mathrm{H} & -4.721 & -2.347 & -0.359 \\ \mathrm{C} & -2.139 & -2.425 & 0.468 \\ \mathrm{H} & -1.421 & -2.669 & -0.319 \\ \mathrm{H} & -1.577 & -2.262 & 1.390 \\ \mathrm{H} & -2.775 & -3.295 & 0.611 \\ \mathrm{C} & -2.577 & 2.581 & -0.087 \\ \mathrm{H} & -2.460 & 2.899 & 0.951 \\ \mathrm{H} & -1.592 & 2.622 & -0.556 \\ \mathrm{H} & -3.217 & 3.306 & -0.584 \\ \mathrm{C} & 2.582 & 2.580 & -0.076 \\ \mathrm{H} & 1.598 & 2.625 & -0.546 \\ \mathrm{H} & 2.463 & 2.894 & 0.963 \\ \mathrm{H} & -6.224 & 3.307 & -0.568 \\ \mathrm{H} & -6.497 & -0.556 & -2.214 \\ \mathrm{C} & -6.932 & -1.311 & -0.684\end{array}$

$\begin{array}{lrrr}\mathrm{C} & 0.867 & 0.747 & 0.000 \\ \mathrm{C} & 1.787 & -1.244 & 0.000 \\ \mathrm{C} & 0.441 & -1.400 & 0.000 \\ \mathrm{H} & 2.582 & -1.969 & 0.000 \\ \mathrm{H} & -0.155 & -2.294 & 0.000 \\ \mathrm{~N} & 2.033 & 0.107 & 0.000 \\ \mathrm{~N} & -0.118 & -0.143 & 0.000 \\ \mathrm{C} & 3.354 & 0.731 & 0.000 \\ \mathrm{H} & 3.897 & 0.426 & -0.891 \\ \mathrm{H} & 3.232 & 1.810 & 0.000 \\ \mathrm{H} & 3.897 & 0.426 & 0.891 \\ \mathrm{C} & -1.594 & 0.137 & 0.000 \\ \mathrm{C} & -2.181 & -0.489 & -1.261 \\ \mathrm{H} & -2.045 & -1.570 & -1.275 \\ \mathrm{H} & -3.252 & -0.289 & -1.290 \\ \mathrm{H} & -1.727 & -0.065 & -2.157 \\ \mathrm{C} & -2.181 & -0.489 & 1.261 \\ \mathrm{H} & -1.727 & -0.065 & 2.157 \\ \mathrm{H} & -3.252 & -0.289 & 1.290 \\ \mathrm{H} & -2.045 & -1.570 & 1.275 \\ \mathrm{C} & -1.810 & 1.644 & 0.000 \\ \mathrm{H} & -1.393 & 2.114 & -0.893 \\ \mathrm{H} & -2.881 & 1.834 & 0.000 \\ \mathrm{H} & -1.393 & 2.114 & 0.893 \\ \mathrm{H} & 0.755 & 1.817 & 0.000\end{array}$

\section{NHC4}

$\begin{array}{lrrr}\mathrm{C} & 2.302 & 0.636 & -0.383 \\ \mathrm{C} & 3.409 & -1.051 & 0.482 \\ \mathrm{C} & 2.087 & -1.279 & 0.672 \\ \mathrm{H} & 4.273 & -1.628 & 0.763 \\ \mathrm{H} & 1.567 & -2.083 & 1.161 \\ \mathrm{C} & -0.014 & -0.033 & 0.103 \\ \mathrm{C} & -0.820 & -1.071 & -0.326 \\ \mathrm{C} & -0.559 & 1.178 & 0.515 \\ \mathrm{C} & -2.197 & -0.907 & -0.349 \\ \mathrm{C} & -1.924 & 1.348 & 0.482 \\ \mathrm{C} & -2.756 & 0.307 & 0.052 \\ \mathrm{H} & -2.819 & -1.722 & -0.689 \\ \mathrm{H} & -2.381 & 2.275 & 0.800 \\ \mathrm{~N} & 3.521 & 0.152 & -0.174\end{array}$




$\begin{array}{lrrr}\mathrm{N} & 1.411 & -0.212 & 0.124 \\ \mathrm{C} & 4.775 & 0.782 & -0.582 \\ \mathrm{H} & 5.304 & 0.122 & -1.264 \\ \mathrm{H} & 4.548 & 1.719 & -1.083 \\ \mathrm{H} & 5.383 & 0.976 & 0.298 \\ \mathrm{H} & 2.064 & 1.552 & -0.896 \\ \mathrm{H} & -0.381 & -2.002 & -0.663 \\ \mathrm{H} & 0.081 & 1.972 & 0.881 \\ \mathrm{O} & -4.067 & 0.568 & 0.063 \\ \mathrm{C} & -4.968 & -0.448 & -0.350 \\ \mathrm{H} & -5.961 & -0.022 & -0.253 \\ \mathrm{H} & -4.788 & -0.726 & -1.390 \\ \mathrm{H} & -4.882 & -1.327 & 0.292\end{array}$

$\begin{array}{lrrr}\text { C } & -1.291 & -0.702 & 0.000 \\ \mathrm{C} & 0.000 & -1.482 & 0.000 \\ \mathrm{C} & 1.291 & -0.702 & 0.000 \\ \mathrm{~N} & 1.168 & 0.674 & 0.000 \\ \mathrm{H} & 0.000 & -2.139 & 0.871 \\ \mathrm{O} & -2.369 & -1.232 & 0.000 \\ \mathrm{O} & 2.369 & -1.232 & 0.000 \\ \mathrm{O} & 0.000 & 2.618 & 0.000 \\ \mathrm{H} & -2.024 & 1.213 & 0.000 \\ \mathrm{H} & 2.024 & 1.213 & 0.000 \\ \mathrm{H} & 0.000 & -2.139 & -0.871\end{array}$

\section{CA2}

\begin{tabular}{lrrr} 
NHC5 & & & \\
C & 1.802 & 0.209 & -0.605 \\
C & 2.718 & -1.135 & 0.871 \\
C & 1.382 & -1.134 & 1.085 \\
H & 3.513 & -1.658 & 1.372 \\
H & 0.775 & -1.656 & 1.801 \\
C & -0.560 & 0.041 & 0.030 \\
C & -1.496 & -0.992 & -0.080 \\
C & -0.950 & 1.383 & 0.040 \\
C & -2.845 & -0.671 & -0.176 \\
C & -2.302 & 1.692 & -0.056 \\
C & -3.223 & 0.662 & -0.161 \\
H & -3.592 & -1.445 & -0.263 \\
H & -2.637 & 2.718 & -0.049 \\
N & 2.958 & -0.289 & -0.190 \\
N & 0.826 & -0.287 & 0.150 \\
H & -4.274 & 0.908 & -0.236 \\
O & -0.996 & -2.241 & -0.081 \\
O & 0.043 & 2.289 & 0.139 \\
C & -1.906 & -3.324 & -0.236 \\
H & -2.615 & -3.353 & 0.593 \\
H & -2.441 & -3.244 & -1.183 \\
H & -1.300 & -4.224 & -0.232 \\
C & -0.318 & 3.661 & 0.251 \\
H & -0.831 & 4.000 & -0.649 \\
H & -0.950 & 3.821 & 1.125 \\
H & 0.614 & 4.206 & 0.369 \\
C & 4.271 & 0.010 & -0.754 \\
H & 4.728 & -0.910 & -1.110 \\
H & 4.147 & 0.700 & -1.583 \\
H & 4.897 & 0.465 & 0.011 \\
H & 1.668 & 0.910 & -1.410 \\
& & & \\
\hline
\end{tabular}

\section{CA1}

$\begin{array}{lrrr}\mathrm{C} & 0.000 & 1.417 & 0.000 \\ \mathrm{~N} & -1.168 & 0.674 & 0.000\end{array}$

\author{
C
}

N

C

C

C

N

$\mathrm{H}$

O

O

$\mathrm{H}$

$\mathrm{H}$

$S$

$\mathrm{H}$

CA3

$$
\mathrm{C}
$$

$\mathrm{N}$

C

C

C

N

$\mathrm{H}$

O

O

O

C

$\mathrm{H}$

$\mathrm{H}$

$\mathrm{H}$

C

$\mathrm{H}$

$\mathrm{H}$

$\mathrm{H}$

$\mathrm{H}$

$\begin{array}{rrr}1.035 & 0.000 & 0.000 \\ 0.299 & 1.157 & 0.000 \\ -1.081 & 1.286 & 0.000 \\ -1.866 & 0.000 & 0.000 \\ -1.081 & -1.286 & 0.000 \\ 0.299 & -1.157 & 0.000 \\ -2.523 & 0.000 & 0.872 \\ -1.599 & 2.368 & 0.000 \\ -1.599 & -2.368 & 0.000 \\ 0.831 & 2.017 & 0.000 \\ 0.831 & -2.017 & 0.000 \\ 2.670 & 0.000 & 0.000 \\ -2.523 & 0.000 & -0.872\end{array}$

$\begin{array}{rrr}0.000 & 1.088 & 0.012 \\ -1.196 & 0.377 & 0.006 \\ -1.293 & -1.003 & -0.001 \\ 0.000 & -1.768 & 0.054 \\ 1.293 & -1.003 & -0.001 \\ 1.196 & 0.377 & 0.006 \\ 0.000 & -2.350 & 0.978 \\ -2.353 & -1.573 & -0.035 \\ 2.353 & -1.573 & -0.035 \\ 0.000 & 2.293 & 0.016 \\ -2.414 & 1.185 & -0.013 \\ -2.430 & 1.805 & -0.906 \\ -3.257 & 0.504 & -0.006 \\ -2.435 & 1.833 & 0.861 \\ 2.414 & 1.185 & -0.013 \\ 2.430 & 1.805 & -0.906 \\ 2.435 & 1.833 & 0.861 \\ 3.257 & 0.504 & -0.006 \\ 0.000 & -2.497 & -0.756\end{array}$




$\begin{array}{lrrr}\text { CA4 } & & & \\ \text { C } & -0.984 & -1.265 & -0.154 \\ \text { C } & -1.754 & 0.000 & -0.494 \\ \text { C } & -0.984 & 1.265 & -0.154 \\ \text { H } & -1.891 & 0.000 & -1.583 \\ \text { O } & -1.513 & -2.197 & 0.390 \\ \text { O } & -1.513 & 2.196 & 0.390 \\ \text { C } & 0.478 & 1.254 & -0.536 \\ \text { H } & 0.942 & 2.164 & -0.156 \\ \text { H } & 0.544 & 1.265 & -1.631 \\ \text { C } & 0.478 & -1.254 & -0.536 \\ \text { H } & 0.544 & -1.264 & -1.631 \\ \text { H } & 0.942 & -2.164 & -0.156 \\ \text { C } & 1.194 & 0.000 & -0.003 \\ \text { C } & 1.158 & 0.000 & 1.527 \\ \text { H } & 1.661 & -0.885 & 1.921 \\ \text { H } & 1.660 & 0.885 & 1.921 \\ \text { H } & 0.136 & 0.000 & 1.915 \\ \text { C } & 2.641 & 0.000 & -0.485 \\ \text { H } & 3.168 & 0.884 & -0.120 \\ \text { H } & 3.168 & -0.883 & -0.120 \\ \text { H } & 2.691 & 0.000 & -1.576 \\ \text { H } & -2.728 & 0.000 & -0.013\end{array}$

$\begin{array}{lrrr}\text { C } & 1.826 & 0.967 & -0.520 \\ \text { C } & 2.011 & -1.336 & 0.114 \\ \text { C } & 3.130 & 1.186 & -0.104 \\ \text { H } & 1.239 & 1.786 & -0.916 \\ \text { C } & 3.318 & -1.121 & 0.530 \\ \text { H } & 1.575 & -2.325 & 0.200 \\ \text { C } & 3.881 & 0.141 & 0.422 \\ \text { H } & 3.562 & 2.174 & -0.189 \\ \text { H } & 3.895 & -1.941 & 0.939 \\ \text { H } & 4.899 & 0.313 & 0.745 \\ \text { C } & -1.201 & 0.232 & -0.092 \\ \text { O } & -1.011 & 1.234 & 0.536 \\ \text { O } & -2.408 & -0.336 & -0.227 \\ \text { C } & -3.493 & 0.345 & 0.418 \\ \text { H } & -3.551 & 1.361 & 0.025 \\ \text { H } & -3.276 & 0.415 & 1.484 \\ \mathrm{C} & -4.751 & -0.442 & 0.144 \\ \text { H } & -5.602 & 0.045 & 0.619 \\ \text { H } & -4.941 & -0.504 & -0.927 \\ \text { H } & -4.665 & -1.454 & 0.540 \\ \text { H } & -0.412 & -1.601 & -0.826\end{array}$

\section{CA5}

$\begin{array}{lrrr}\mathrm{C} & -0.914 & -1.273 & -0.085 \\ \mathrm{C} & -1.593 & 0.000 & 0.361 \\ \mathrm{C} & -0.914 & 1.273 & -0.086 \\ \mathrm{H} & -2.617 & 0.000 & -0.001 \\ \mathrm{O} & -1.484 & -2.307 & -0.231 \\ \mathrm{O} & -1.484 & 2.307 & -0.231 \\ \mathrm{C} & 1.125 & 0.000 & 0.015 \\ \mathrm{C} & 1.418 & 0.000 & 1.505 \\ \mathrm{H} & 1.993 & -0.889 & 1.759 \\ \mathrm{H} & 1.993 & 0.888 & 1.759 \\ \mathrm{H} & 0.502 & 0.000 & 2.096 \\ \mathrm{C} & 2.379 & 0.000 & -0.824 \\ \mathrm{H} & 2.965 & 0.890 & -0.603 \\ \mathrm{H} & 2.965 & -0.890 & -0.602 \\ \mathrm{H} & 2.107 & 0.000 & -1.878 \\ \mathrm{O} & 0.405 & 1.175 & -0.350 \\ \mathrm{O} & 0.405 & -1.175 & -0.350 \\ \mathrm{H} & -1.643 & 0.000 & 1.453\end{array}$

\section{CA6}

$\begin{array}{llll}\mathrm{C} & -0.157 & -0.543 & -0.871 \\ \mathrm{H} & -0.283 & -0.239 & -1.915 \\ \mathrm{C} & 1.254 & -0.298 & -0.415\end{array}$

CA7

$\begin{array}{lrrr}\text { C } & -1.866 & 0.699 & 0.907 \\ \text { H } & -2.071 & 0.516 & 1.966 \\ \mathrm{C} & -0.410 & 0.479 & 0.607 \\ \mathrm{C} & 0.203 & -0.738 & 0.891 \\ \mathrm{C} & 0.352 & 1.494 & 0.042 \\ \mathrm{C} & 1.547 & -0.938 & 0.622 \\ \mathrm{H} & -0.381 & -1.544 & 1.316 \\ \mathrm{C} & 1.701 & 1.311 & -0.233 \\ \mathrm{H} & -0.108 & 2.447 & -0.188 \\ \mathrm{C} & 2.285 & 0.093 & 0.061 \\ \mathrm{H} & 2.018 & -1.885 & 0.844 \\ \mathrm{H} & 2.288 & 2.106 & -0.671 \\ \mathrm{C} & -2.798 & -0.222 & 0.144 \\ \mathrm{O} & -2.506 & -1.289 & -0.317 \\ \mathrm{O} & -4.030 & 0.299 & 0.083 \\ \mathrm{C} & -5.020 & -0.514 & -0.563 \\ \mathrm{H} & -5.084 & -1.469 & -0.038 \\ \mathrm{H} & -4.692 & -0.718 & -1.582 \\ \mathrm{C} & -6.325 & 0.243 & -0.525 \\ \mathrm{H} & -7.106 & -0.343 & -1.009 \\ \mathrm{H} & -6.626 & 0.442 & 0.503 \\ \mathrm{H} & -6.232 & 1.194 & -1.049 \\ \mathrm{H} & -2.161 & 1.729 & 0.709 \\ \mathrm{Br} & 4.127 & -0.172 & -0.304\end{array}$




$\begin{array}{lrrr}\text { CA8 } & & & \\ \text { C } & -0.943 & 0.669 & 0.881 \\ \text { H } & -1.121 & 0.464 & 1.942 \\ \text { C } & 0.499 & 0.430 & 0.537 \\ \text { C } & 1.091 & -0.810 & 0.775 \\ \text { C } & 1.265 & 1.448 & -0.021 \\ \text { C } & 2.420 & -1.027 & 0.465 \\ \text { H } & 0.499 & -1.612 & 1.194 \\ \text { C } & 2.598 & 1.244 & -0.336 \\ \text { H } & 0.814 & 2.414 & -0.212 \\ \text { C } & 3.178 & 0.003 & -0.092 \\ \text { H } & 2.879 & -1.989 & 0.648 \\ \text { H } & 3.192 & 2.038 & -0.768 \\ \text { C } & -1.909 & -0.221 & 0.123 \\ \text { O } & -1.639 & -1.273 & -0.383 \\ \text { O } & -3.135 & 0.311 & 0.122 \\ \text { C } & -4.157 & -0.472 & -0.517 \\ \text { H } & -4.211 & -1.443 & -0.021 \\ \text { H } & -3.865 & -0.644 & -1.553 \\ \text { C } & -5.451 & 0.295 & -0.409 \\ \text { H } & -6.254 & -0.269 & -0.883 \\ \text { H } & -5.715 & 0.462 & 0.635 \\ \text { H } & -5.368 & 1.262 & -0.905 \\ \text { H } & -1.226 & 1.707 & 0.716 \\ \text { C } & 4.560 & -0.217 & -0.412 \\ \text { N } & 5.666 & -0.391 & -0.667\end{array}$

\section{CA9}

$\begin{array}{lrrr}\mathrm{C} & -1.384 & 0.615 & 0.942 \\ \mathrm{H} & -1.578 & 0.330 & 1.980 \\ \mathrm{C} & 0.065 & 0.409 & 0.605 \\ \mathrm{C} & 0.656 & -0.845 & 0.754 \\ \mathrm{C} & 0.835 & 1.472 & 0.145 \\ \mathrm{C} & 1.992 & -1.036 & 0.453 \\ \mathrm{H} & 0.059 & -1.679 & 1.096 \\ \mathrm{C} & 2.176 & 1.301 & -0.162 \\ \mathrm{H} & 0.383 & 2.449 & 0.024 \\ \mathrm{C} & 2.728 & 0.045 & 0.000 \\ \mathrm{H} & 2.468 & -1.999 & 0.561 \\ \mathrm{H} & 2.788 & 2.115 & -0.520 \\ \mathrm{C} & -2.332 & -0.217 & 0.099 \\ \mathrm{O} & -2.042 & -1.215 & -0.498 \\ \mathrm{O} & -3.565 & 0.296 & 0.137 \\ \mathrm{C} & -4.571 & -0.440 & -0.578 \\ \mathrm{H} & -4.613 & -1.453 & -0.176 \\ \mathrm{H} & -4.271 & -0.510 & -1.624 \\ \mathrm{C} & -5.878 & 0.295 & -0.409 \\ \mathrm{H} & -6.669 & -0.235 & -0.940\end{array}$

$\begin{array}{lrrr}\mathrm{H} & -6.150 & 0.359 & 0.644 \\ \mathrm{H} & -5.807 & 1.305 & -0.811 \\ \mathrm{H} & -1.669 & 1.662 & 0.851 \\ \mathrm{~N} & 4.154 & -0.149 & -0.320 \\ \mathrm{O} & 4.617 & -1.255 & -0.161 \\ \mathrm{O} & 4.773 & 0.810 & -0.721\end{array}$

CA10

$\begin{array}{lrrr}\text { C } & -0.149 & 0.577 & 0.848 \\ \text { H } & -0.263 & 0.314 & 1.904 \\ \text { C } & 1.258 & 0.309 & 0.396 \\ \text { C } & 1.828 & -0.954 & 0.501 \\ \text { C } & 2.041 & 1.324 & -0.136 \\ \text { C } & 3.135 & -1.136 & 0.078 \\ \text { H } & 1.260 & -1.786 & 0.893 \\ \text { C } & 3.342 & 1.039 & -0.529 \\ \text { H } & 1.650 & 2.328 & -0.244 \\ \text { H } & 3.599 & -2.114 & 0.150 \\ \text { H } & 3.968 & 1.821 & -0.945 \\ \text { C } & -1.197 & -0.230 & 0.107 \\ \text { O } & -1.006 & -1.260 & -0.474 \\ \text { O } & -2.398 & 0.348 & 0.217 \\ \text { C } & -3.489 & -0.357 & -0.396 \\ \text { H } & -3.551 & -1.353 & 0.045 \\ \text { H } & -3.271 & -0.478 & -1.458 \\ \text { C } & -4.741 & 0.450 & -0.159 \\ \text { H } & -5.595 & -0.054 & -0.611 \\ \text { H } & -4.931 & 0.564 & 0.907 \\ \text { H } & -4.650 & 1.441 & -0.603 \\ \text { H } & -0.400 & 1.633 & 0.762 \\ \text { N } & 3.892 & -0.165 & -0.429\end{array}$

CA11

$\begin{array}{lrrr}\text { C } & -3.179 & 0.850 & -0.008 \\ \text { C } & -1.699 & 0.521 & -0.004 \\ \text { C } & -1.225 & -0.781 & -0.009 \\ \text { C } & -0.793 & 1.581 & 0.002 \\ \text { C } & 0.143 & -1.029 & -0.007 \\ \text { H } & -1.920 & -1.611 & -0.013 \\ \text { C } & 0.566 & 1.341 & 0.004 \\ \text { H } & -1.159 & 2.601 & 0.006 \\ \text { C } & 1.032 & 0.030 & -0.001 \\ \text { H } & 0.511 & -2.045 & -0.011 \\ \text { H } & 1.269 & 2.164 & 0.008 \\ \text { C } & -4.046 & -0.324 & 0.004 \\ \text { N } & -4.714 & -1.255 & 0.013 \\ \text { H } & -3.427 & 1.462 & 0.863 \\ \text { C } & 2.517 & -0.201 & 0.001\end{array}$




$\begin{array}{rrrr}F & 2.831 & -1.498 & -0.005 \\ F & 3.096 & 0.344 & 1.080 \\ F & 3.102 & 0.357 & -1.068 \\ H & -3.427 & 1.443 & -0.891\end{array}$

$\begin{array}{lrrr}\mathrm{C} & 2.796 & 0.868 & 0.001 \\ \mathrm{C} & 1.321 & 0.520 & 0.000 \\ \mathrm{C} & 0.867 & -0.792 & 0.000 \\ \mathrm{C} & 0.403 & 1.568 & 0.000 \\ \mathrm{C} & -0.494 & -1.062 & 0.000 \\ \mathrm{H} & 1.574 & -1.612 & 0.000 \\ \mathrm{C} & -0.956 & 1.316 & 0.000 \\ \mathrm{H} & 0.756 & 2.593 & 0.000 \\ \mathrm{C} & -1.378 & -0.002 & 0.000 \\ \mathrm{H} & -0.871 & -2.074 & 0.000 \\ \mathrm{H} & -1.684 & 2.113 & 0.000 \\ \mathrm{C} & 3.679 & -0.295 & 0.000 \\ \mathrm{~N} & 4.357 & -1.218 & -0.001 \\ \mathrm{H} & 3.034 & 1.475 & -0.876 \\ \mathrm{~N} & -2.827 & -0.282 & 0.000 \\ \mathrm{O} & -3.577 & 0.666 & -0.001 \\ \mathrm{O} & -3.171 & -1.441 & 0.001 \\ \mathrm{H} & 3.034 & 1.473 & 0.878\end{array}$

CA15

$\begin{array}{lrrr}\text { CA12 } & & & \\ \text { C } & 2.391 & 0.864 & 0.000 \\ \text { C } & 0.921 & 0.494 & 0.000 \\ \text { C } & 0.485 & -0.824 & 0.000 \\ \text { C } & -0.014 & 1.527 & 0.000 \\ \text { C } & -0.871 & -1.111 & 0.000 \\ \text { H } & 1.204 & -1.633 & 0.000 \\ \text { C } & -1.368 & 1.251 & 0.000 \\ \text { H } & 0.322 & 2.557 & 0.000 \\ \text { C } & -1.799 & -0.075 & 0.000 \\ \text { H } & -1.214 & -2.137 & 0.000 \\ \text { H } & -2.094 & 2.052 & 0.000 \\ \text { C } & 3.290 & -0.287 & 0.000 \\ \text { N } & 3.981 & -1.200 & 0.000 \\ \text { H } & 2.621 & 1.473 & -0.877 \\ \text { H } & 2.621 & 1.473 & 0.877 \\ \text { C } & -3.205 & -0.369 & 0.000 \\ \text { N } & -4.329 & -0.600 & 0.000\end{array}$

\section{CA13}

$\begin{array}{lrrr}\mathrm{C} & -2.169 & 0.526 & 0.225 \\ \mathrm{C} & -0.674 & 0.272 & 0.181 \\ \mathrm{C} & -0.146 & -0.930 & -0.274 \\ \mathrm{C} & 0.188 & 1.301 & 0.552 \\ \mathrm{C} & 1.225 & -1.104 & -0.362 \\ \mathrm{H} & -0.808 & -1.741 & -0.551 \\ \mathrm{C} & 1.558 & 1.138 & 0.470 \\ \mathrm{H} & -0.219 & 2.238 & 0.914 \\ \mathrm{C} & 2.080 & -0.070 & 0.009 \\ \mathrm{H} & 1.639 & -2.040 & -0.713 \\ \mathrm{H} & 2.228 & 1.935 & 0.763 \\ \mathrm{C} & 3.502 & -0.246 & -0.078 \\ \mathrm{~N} & 4.639 & -0.384 & -0.149 \\ \mathrm{C} & -2.639 & 1.274 & -1.035 \\ \mathrm{H} & -2.100 & 2.217 & -1.117 \\ \mathrm{H} & -2.432 & 0.677 & -1.922 \\ \mathrm{H} & -3.708 & 1.476 & -0.986 \\ \mathrm{C} & -2.928 & -0.720 & 0.372 \\ \mathrm{~N} & -3.524 & -1.695 & 0.455 \\ \mathrm{H} & -2.392 & 1.137 & 1.103\end{array}$

CA14

$\begin{array}{lrrr}\mathrm{C} & -2.566 & 0.541 & 0.190 \\ \mathrm{C} & -1.068 & 0.301 & 0.166 \\ \mathrm{C} & -0.527 & -0.928 & -0.196 \\ \mathrm{C} & -0.220 & 1.366 & 0.461 \\ \mathrm{C} & 0.847 & -1.096 & -0.269 \\ \mathrm{H} & -1.181 & -1.764 & -0.411 \\ \mathrm{C} & 1.153 & 1.215 & 0.393 \\ \mathrm{H} & -0.639 & 2.323 & 0.750 \\ \mathrm{C} & 1.660 & -0.018 & 0.026 \\ \mathrm{H} & 1.289 & -2.041 & -0.545 \\ \mathrm{H} & 1.829 & 2.026 & 0.622 \\ \mathrm{C} & -3.039 & 1.197 & -1.119 \\ \mathrm{H} & -2.510 & 2.138 & -1.264 \\ \mathrm{H} & -2.824 & 0.543 & -1.963 \\ \mathrm{H} & -4.110 & 1.391 & -1.086 \\ \mathrm{C} & -3.313 & -0.699 & 0.421 \\ \mathrm{~N} & -3.900 & -1.672 & 0.570 \\ \mathrm{~N} & 3.124 & -0.190 & -0.048 \\ \mathrm{O} & 3.811 & 0.768 & 0.222 \\ \mathrm{O} & 3.542 & -1.276 & -0.375 \\ \mathrm{H} & -2.797 & 1.208 & 1.024\end{array}$

\section{CA16}

$\begin{array}{lrrr}C & -1.387 & 0.617 & 0.247 \\ C & 0.073 & 0.208 & 0.159 \\ C & 0.460 & -1.026 & -0.348 \\ C & 1.048 & 1.125 & 0.539 \\ C & 1.808 & -1.337 & -0.474\end{array}$




$\begin{array}{lrrr}\mathrm{H} & -0.292 & -1.752 & -0.632 \\ \mathrm{C} & 2.393 & 0.815 & 0.412 \\ \mathrm{H} & 0.750 & 2.088 & 0.940 \\ \mathrm{C} & 2.776 & -0.419 & -0.096 \\ \mathrm{H} & 2.099 & -2.303 & -0.865 \\ \mathrm{H} & 3.142 & 1.534 & 0.716 \\ \mathrm{C} & -1.817 & 1.416 & -0.994 \\ \mathrm{H} & -1.184 & 2.297 & -1.091 \\ \mathrm{H} & -1.700 & 0.805 & -1.888 \\ \mathrm{H} & -2.857 & 1.729 & -0.914 \\ \mathrm{C} & -2.269 & -0.542 & 0.417 \\ \mathrm{~N} & -2.967 & -1.445 & 0.518 \\ \mathrm{H} & 3.825 & -0.665 & -0.192 \\ \mathrm{H} & -1.519 & 1.242 & 1.133\end{array}$

\section{CA17}

$\begin{array}{lrrr}\mathrm{C} & -1.739 & 0.005 & 0.999 \\ \mathrm{H} & -2.025 & -0.891 & 1.542 \\ \mathrm{C} & -0.286 & 0.004 & 0.624 \\ \mathrm{C} & 0.390 & -1.197 & 0.435 \\ \mathrm{C} & 0.391 & 1.202 & 0.431 \\ \mathrm{C} & 1.724 & -1.194 & 0.064 \\ \mathrm{H} & -0.134 & -2.134 & 0.577 \\ \mathrm{C} & 1.726 & 1.197 & 0.060 \\ \mathrm{H} & -0.131 & 2.141 & 0.570 \\ \mathrm{C} & 2.413 & 0.001 & -0.132 \\ \mathrm{H} & 2.243 & -2.136 & -0.075 \\ \mathrm{H} & 2.245 & 2.137 & -0.082 \\ \mathrm{O} & -2.859 & 1.071 & -0.734 \\ \mathrm{O} & -2.852 & -1.080 & -0.726 \\ \mathrm{~N} & -2.568 & -0.002 & -0.262 \\ \mathrm{H} & -2.025 & 0.907 & 1.532 \\ \mathrm{C} & 3.852 & -0.004 & -0.564 \\ \mathrm{H} & 4.391 & -0.846 & -0.130 \\ \mathrm{H} & 3.924 & -0.091 & -1.650 \\ \mathrm{H} & 4.356 & 0.916 & -0.271\end{array}$

\section{CA18}

$\begin{array}{lrrr}\mathrm{C} & -1.128 & -0.849 & 0.000 \\ \mathrm{H} & -1.302 & -1.475 & -0.875 \\ \mathrm{C} & 0.283 & -0.311 & 0.000 \\ \mathrm{C} & 0.683 & 1.020 & -0.001 \\ \mathrm{C} & 1.262 & -1.307 & 0.000 \\ \mathrm{C} & 2.038 & 1.336 & -0.001 \\ \mathrm{H} & -0.045 & 1.812 & -0.001 \\ \mathrm{C} & 2.608 & -0.988 & 0.000 \\ \mathrm{H} & 0.962 & -2.350 & 0.000 \\ \mathrm{C} & 3.003 & 0.343 & 0.000\end{array}$

$\begin{array}{lrrr}\mathrm{H} & 2.334 & 2.377 & -0.001 \\ \mathrm{H} & 3.347 & -1.779 & 0.000 \\ \mathrm{O} & -2.159 & 1.272 & 0.002 \\ \mathrm{O} & -3.384 & -0.485 & 0.000 \\ \mathrm{~N} & -2.314 & 0.077 & 0.000 \\ \mathrm{H} & -1.302 & -1.474 & 0.875 \\ \mathrm{H} & 4.054 & 0.601 & 0.000\end{array}$

CA19

$\begin{array}{lrrr}\mathrm{C} & 1.927 & 0.813 & 0.000 \\ \mathrm{H} & 2.150 & 1.422 & -0.875 \\ \mathrm{C} & 0.477 & 0.395 & 0.000 \\ \mathrm{C} & -0.032 & -0.899 & 0.000 \\ \mathrm{C} & -0.416 & 1.471 & 0.000 \\ \mathrm{C} & -1.404 & -1.107 & 0.000 \\ \mathrm{H} & 0.628 & -1.749 & 0.000 \\ \mathrm{C} & -1.781 & 1.272 & 0.000 \\ \mathrm{H} & -0.031 & 2.485 & 0.001 \\ \mathrm{C} & -2.281 & -0.029 & 0.000 \\ \mathrm{H} & -1.797 & -2.115 & 0.000 \\ \mathrm{H} & -2.461 & 2.112 & 0.000 \\ \mathrm{O} & 2.767 & -1.389 & 0.001 \\ \mathrm{O} & 4.140 & 0.256 & -0.002 \\ \mathrm{C} & -3.700 & -0.252 & 0.000 \\ \mathrm{~N} & -4.834 & -0.428 & 0.000 \\ \mathrm{~N} & 3.028 & -0.212 & 0.001 \\ \mathrm{H} & 2.150 & 1.422 & 0.876\end{array}$

\section{CA20}

$\begin{array}{lrrr}\mathrm{C} & 2.337 & 0.820 & 0.000 \\ \mathrm{H} & 2.565 & 1.427 & -0.875 \\ \mathrm{C} & 0.883 & 0.415 & 0.000 \\ \mathrm{C} & 0.365 & -0.876 & 0.000 \\ \mathrm{C} & 0.002 & 1.501 & 0.000 \\ \mathrm{C} & -1.010 & -1.074 & 0.000 \\ \mathrm{H} & 1.019 & -1.731 & 0.000 \\ \mathrm{C} & -1.366 & 1.317 & 0.000 \\ \mathrm{H} & 0.397 & 2.510 & 0.000 \\ \mathrm{C} & -1.848 & 0.020 & 0.000 \\ \mathrm{H} & -1.429 & -2.069 & -0.001 \\ \mathrm{H} & -2.054 & 2.149 & 0.000 \\ \mathrm{O} & 3.159 & -1.389 & 0.001 \\ \mathrm{O} & 4.545 & 0.245 & 0.000 \\ \mathrm{~N} & 3.430 & -0.214 & 0.000 \\ \mathrm{~N} & -3.308 & -0.196 & 0.000 \\ \mathrm{O} & -4.015 & 0.785 & 0.000 \\ \mathrm{O} & -3.703 & -1.338 & 0.000 \\ \mathrm{H} & 2.565 & 1.427 & 0.876\end{array}$




\section{CA21}

$\begin{array}{lrrr}\mathrm{C} & 0.000 & 0.837 & 0.000 \\ \mathrm{C} & -1.217 & 0.023 & 0.000 \\ \mathrm{~N} & -2.183 & -0.590 & 0.000 \\ \mathrm{H} & 0.000 & 1.480 & 0.882 \\ \mathrm{C} & 1.217 & 0.023 & 0.000 \\ \mathrm{~N} & 2.183 & -0.590 & 0.000 \\ \mathrm{H} & 0.000 & 1.480 & -0.882\end{array}$

C

$-1.834$

0.000

$-0.495$

$\mathrm{H}$

$-2.786$

0.000

0.031

$\mathrm{H}$

$-1.943$

0.000

$-1.576$

\section{CA24}

\section{CA22}

$\begin{array}{lrrr}\mathrm{C} & -1.147 & 0.665 & -0.988 \\ \mathrm{C} & -0.676 & -0.336 & -0.149 \\ \mathrm{C} & -1.482 & -0.921 & 0.819 \\ \mathrm{C} & -2.791 & -0.484 & 0.952 \\ \mathrm{C} & -3.274 & 0.521 & 0.123 \\ \mathrm{C} & -2.458 & 1.092 & -0.845 \\ \mathrm{H} & -0.492 & 1.082 & -1.741 \\ \mathrm{H} & -1.088 & -1.719 & 1.435 \\ \mathrm{H} & -3.436 & -0.933 & 1.695 \\ \mathrm{H} & -4.297 & 0.858 & 0.228 \\ \mathrm{H} & -2.844 & 1.868 & -1.492 \\ \mathrm{~S} & 0.994 & -0.880 & -0.312 \\ \mathrm{O} & 1.460 & -0.552 & -1.626 \\ \mathrm{O} & 1.102 & -2.216 & 0.206 \\ \mathrm{C} & 1.901 & 0.125 & 0.872 \\ \mathrm{H} & 2.931 & -0.220 & 0.801 \\ \mathrm{Cl} & 1.818 & 1.845 & 0.521 \\ \mathrm{H} & 1.492 & -0.074 & 1.859\end{array}$

CA23

$\begin{array}{lrrr}\text { C } & 0.700 & 0.690 & -0.005 \\ \text { C } & 0.701 & -0.690 & -0.005 \\ \text { C } & 1.886 & -1.407 & 0.009 \\ \text { C } & 3.076 & -0.696 & 0.034 \\ \text { C } & 3.075 & 0.697 & 0.034 \\ \text { C } & 1.885 & 1.408 & 0.009 \\ \text { H } & 1.872 & -2.489 & -0.010 \\ \text { H } & 4.016 & -1.231 & 0.045 \\ \text { H } & 4.015 & 1.233 & 0.044 \\ \text { H } & 1.871 & 2.489 & -0.010 \\ \text { S } & -0.896 & -1.459 & -0.009 \\ \text { S } & -0.896 & 1.458 & -0.009 \\ \text { O } & -0.953 & -2.427 & -1.061 \\ \text { O } & -1.252 & -1.818 & 1.328 \\ \text { O } & -0.954 & 2.427 & -1.060 \\ \text { O } & -1.253 & 1.817 & 1.328\end{array}$

C
C
C
C
C
C

$\mathrm{C}$

$\mathrm{H}$

$\mathrm{H}$

$\mathrm{H}$

$\mathrm{H}$

$\mathrm{S}$

$\mathrm{S}$

0

0

0

O

C

$\mathrm{H}$

$\mathrm{F}$

N1

C

C

C

C

$\mathrm{N}$

$\mathrm{H}$

$\mathrm{H}$

$\mathrm{H}$

$\mathrm{H}$

$\mathrm{H}$

$\mathrm{H}$

N2

C

C

C

C

C

N

$\mathrm{H}$

$\begin{array}{rrr}-0.837 & -0.691 & 0.045 \\ -0.837 & 0.691 & 0.045 \\ -2.016 & 1.407 & -0.071 \\ -3.203 & 0.696 & -0.170 \\ -3.203 & -0.696 & -0.170 \\ -2.016 & -1.407 & -0.071 \\ -1.999 & 2.489 & -0.095 \\ -4.138 & 1.231 & -0.262 \\ -4.138 & -1.231 & -0.262 \\ -1.999 & -2.489 & -0.095 \\ 0.751 & 1.459 & 0.203 \\ 0.751 & -1.459 & 0.203 \\ 0.889 & 2.485 & -0.779 \\ 1.037 & 1.701 & 1.582 \\ 0.889 & -2.485 & -0.779 \\ 1.037 & -1.701 & 1.582 \\ 1.733 & 0.000 & -0.301 \\ 2.681 & 0.000 & 0.238 \\ 1.894 & 0.000 & -1.636\end{array}$

$\begin{array}{lll}0.663 & -1.182 & 0.000\end{array}$

$\begin{array}{lll}-0.713 & -1.205 & 0.000\end{array}$

$\begin{array}{lll}-1.406 & 0.000 & 0.000\end{array}$

$\begin{array}{lll}-0.713 & 1.205 & 0.000\end{array}$

$\begin{array}{lll}0.663 & 1.182 & 0.000\end{array}$

$\begin{array}{lll}1.300 & 0.000 & 0.000\end{array}$

$\begin{array}{lll}-2.488 & 0.000 & 0.000\end{array}$

$\begin{array}{lll}1.281 & -2.068 & 0.000\end{array}$

$\begin{array}{lll}-1.228 & -2.154 & 0.000\end{array}$

$\begin{array}{lll}-1.228 & 2.154 & 0.000\end{array}$

$\begin{array}{lll}1.281 & 2.068 & 0.000\end{array}$

$\begin{array}{lll}2.315 & 0.000 \quad 0.000\end{array}$

$\begin{array}{rrr}1.511 & -1.180 & 0.000 \\ 0.141 & -1.210 & 0.000 \\ -0.556 & 0.000 & 0.000 \\ 0.141 & 1.210 & 0.000 \\ 1.511 & 1.180 & 0.000 \\ 2.155 & 0.000 & 0.000 \\ 2.124 & -2.070 & 0.000\end{array}$




$\begin{array}{lrrr}\mathrm{H} & -0.384 & -2.154 & 0.000 \\ \mathrm{H} & -0.384 & 2.154 & 0.000 \\ \mathrm{H} & 2.124 & 2.070 & 0.000 \\ \mathrm{Cl} & -2.248 & 0.000 & 0.000 \\ \mathrm{H} & 3.169 & 0.000 & 0.000\end{array}$

N3

$\begin{array}{lrrr}\text { C } & -2.573 & -1.175 & -0.082 \\ \text { C } & -1.218 & -1.212 & -0.083 \\ \text { C } & -0.468 & 0.000 & 0.000 \\ \text { C } & -1.218 & 1.212 & 0.083 \\ \text { C } & -2.573 & 1.175 & 0.082 \\ \text { N } & -3.238 & 0.000 & 0.000 \\ \text { H } & -3.180 & -2.067 & -0.149 \\ \text { H } & -0.724 & -2.168 & -0.161 \\ \text { H } & -0.724 & 2.168 & 0.161 \\ \text { H } & -3.180 & 2.067 & 0.149 \\ \text { N } & 0.852 & 0.000 & 0.000 \\ \mathrm{C} & 1.678 & -1.219 & 0.000 \\ \mathrm{C} & 1.678 & 1.219 & 0.000 \\ \mathrm{C} & 3.076 & -0.697 & 0.313 \\ \mathrm{H} & 1.312 & -1.923 & 0.746 \\ \mathrm{C} & 3.076 & 0.697 & -0.313 \\ \mathrm{H} & 1.312 & 1.923 & -0.746 \\ \mathrm{H} & 3.852 & -1.346 & -0.084 \\ \mathrm{H} & 3.211 & 0.626 & -1.393 \\ \mathrm{H} & 1.633 & -1.692 & -0.985 \\ \mathrm{H} & 3.210 & -0.626 & 1.393 \\ \mathrm{H} & 3.852 & 1.346 & 0.084 \\ \mathrm{H} & 1.632 & 1.692 & 0.985 \\ \mathrm{H} & -4.248 & 0.000 & 0.000\end{array}$

N4

N6

$\begin{array}{lrrr}\text { C } & -1.872 & -1.176 & 0.000 \\ \text { C } & -0.516 & -1.213 & 0.000 \\ \text { C } & 0.235 & 0.000 & 0.000 \\ \text { C } & -0.516 & 1.213 & 0.000 \\ \text { C } & -1.872 & 1.176 & 0.000 \\ \text { N } & -2.537 & 0.000 & 0.000 \\ \text { H } & -2.478 & -2.071 & 0.000 \\ \text { H } & -0.030 & -2.176 & 0.000 \\ \text { H } & -0.030 & 2.176 & 0.000 \\ \text { H } & -2.478 & 2.071 & 0.000 \\ \text { C } & 2.302 & -1.261 & 0.000 \\ \text { H } & 3.364 & -1.044 & -0.001 \\ \text { H } & 2.069 & -1.846 & 0.891 \\ \text { H } & 2.068 & -1.846 & -0.890 \\ \text { N } & 1.563 & 0.000 & 0.000 \\ \text { C } & 2.302 & 1.261 & 0.000 \\ \text { H } & 2.069 & 1.846 & -0.890 \\ \text { H } & 2.068 & 1.846 & 0.891 \\ \text { H } & 3.364 & 1.044 & 0.001 \\ \text { H } & -3.547 & 0.000 & 0.000\end{array}$

N5

$\begin{array}{lrrr}\text { C } & -0.227 & 0.698 & 0.000 \\ \text { C } & -0.227 & -0.698 & 0.000 \\ \text { C } & 0.955 & -1.443 & 0.000 \\ \text { C } & 2.114 & -0.708 & 0.000 \\ \text { C } & 2.114 & 0.708 & 0.000 \\ \text { C } & 0.955 & 1.443 & 0.000 \\ \text { H } & 0.956 & -2.524 & 0.000 \\ \text { H } & 3.065 & -1.224 & 0.000 \\ \text { H } & 3.065 & 1.224 & 0.000 \\ \text { H } & 0.956 & 2.524 & 0.000 \\ \text { H } & -1.987 & 1.949 & 0.000 \\ \text { N } & -1.557 & -1.031 & 0.000 \\ \text { N } & -2.339 & 0.000 & 0.000 \\ \text { N } & -1.557 & 1.031 & 0.000 \\ \text { H } & -1.987 & -1.949 & 0.000 \\ & & & \\ \text { N7 } & & & \\ \text { C } & & & \\ \text { C } & -0.138 & -0.377 & 0.000 \\ \text { C } & 0.397 & 0.910 & 0.000 \\ \text { C } & 1.775 & 1.142 & 0.000 \\ \text { C } & 2.563 & 0.017 & 0.000 \\ & 2.019 & -1.291 & 0.000\end{array}$




$\begin{array}{lrrr}\mathrm{C} & 0.666 & -1.520 & 0.000 \\ \mathrm{H} & 2.193 & 2.138 & 0.000 \\ \mathrm{H} & 3.639 & 0.129 & 0.000 \\ \mathrm{H} & 2.698 & -2.133 & 0.000 \\ \mathrm{H} & 0.250 & -2.518 & 0.000 \\ \mathrm{~N} & -0.704 & 1.723 & 0.000 \\ \mathrm{~N} & -1.499 & -0.194 & 0.000 \\ \mathrm{C} & -2.534 & -1.224 & 0.000 \\ \mathrm{H} & -2.422 & -1.834 & -0.893 \\ \mathrm{H} & -2.422 & -1.834 & 0.894 \\ \mathrm{H} & -3.497 & -0.722 & 0.000 \\ \mathrm{~N} & -1.821 & 1.057 & 0.000 \\ \mathrm{H} & -0.756 & 2.734 & 0.000\end{array}$

$\begin{array}{lrrr}\mathrm{C} & 0.144 & -1.069 & 0.000 \\ \mathrm{C} & 1.444 & 0.715 & 0.000 \\ \mathrm{C} & 0.154 & 1.129 & 0.000 \\ \mathrm{~N} & -0.636 & 0.000 & 0.000 \\ \mathrm{H} & -0.190 & -2.093 & 0.000 \\ \mathrm{H} & 2.365 & 1.270 & 0.000 \\ \mathrm{H} & -0.269 & 2.118 & 0.000 \\ \mathrm{~N} & 1.408 & -0.658 & 0.000 \\ \mathrm{C} & -2.101 & -0.006 & 0.000 \\ \mathrm{H} & -2.458 & 0.500 & 0.893 \\ \mathrm{H} & -2.459 & 0.504 & -0.891 \\ \mathrm{H} & -2.446 & -1.036 & -0.002 \\ \mathrm{H} & 2.212 & -1.270 & 0.000\end{array}$

$\begin{array}{lrrr}\text { C } & -0.135 & -0.368 & 0.000 \\ \text { C } & 0.405 & 0.917 & 0.000 \\ \text { C } & 1.778 & 1.137 & 0.000 \\ \text { C } & 2.573 & 0.010 & 0.000 \\ \text { C } & 2.029 & -1.286 & 0.000 \\ \text { C } & 0.667 & -1.504 & 0.000 \\ \text { C } & -1.804 & 1.069 & 0.000 \\ \text { H } & 2.199 & 2.133 & 0.000 \\ \text { H } & 3.648 & 0.126 & 0.000 \\ \text { H } & 2.699 & -2.135 & 0.000 \\ \text { H } & 0.249 & -2.501 & 0.000 \\ \text { H } & -2.801 & 1.481 & 0.000 \\ \text { N } & -0.682 & 1.782 & 0.000 \\ \text { N } & -1.519 & -0.221 & 0.000 \\ \text { C } & -2.479 & -1.320 & 0.000 \\ \text { H } & -2.329 & -1.926 & -0.891 \\ \text { H } & -2.329 & -1.925 & 0.892 \\ \text { H } & -3.485 & -0.911 & 0.000 \\ \text { H } & -0.645 & 2.791 & 0.000\end{array}$

C

C

C

C

C

C

H

$\mathrm{H}$

$\mathrm{H}$

$\mathrm{H}$

$\mathrm{H}$

$\mathrm{N}$

C

C

$\mathrm{H}$

C

$\mathrm{H}$

$\mathrm{H}$

$\mathrm{N}$

$\mathrm{H}$

$\begin{array}{rrr}1.139 & -1.161 & 0.351 \\ 0.472 & 0.003 & 0.005 \\ 1.141 & 1.165 & -0.342 \\ 2.528 & 1.154 & -0.340 \\ 3.217 & -0.004 & -0.006 \\ 2.526 & -1.158 & 0.336 \\ 0.590 & -2.043 & 0.654 \\ 0.592 & 2.053 & -0.628 \\ 3.068 & 2.051 & -0.611 \\ 4.299 & -0.006 & -0.010 \\ 3.064 & -2.056 & 0.607 \\ -0.968 & 0.009 & 0.005 \\ -1.739 & -0.910 & -0.562 \\ -1.772 & 0.966 & 0.591 \\ -1.388 & -1.765 & -1.115 \\ -3.056 & 0.601 & 0.367 \\ -1.354 & 1.798 & 1.129 \\ -3.983 & 1.061 & 0.659 \\ -3.006 & -0.570 & -0.350 \\ -3.804 & -1.094 & -0.680\end{array}$

N9

$\begin{array}{lrrr}\mathrm{C} & 0.007 & -1.134 & 0.000 \\ \mathrm{C} & -0.683 & 0.969 & 0.000 \\ \mathrm{C} & 0.671 & 0.977 & 0.000 \\ \mathrm{~N} & 1.069 & -0.341 & 0.000 \\ \mathrm{H} & 2.027 & -0.667 & 0.000 \\ \mathrm{H} & 0.013 & -2.211 & 0.000 \\ \mathrm{H} & -1.395 & 1.775 & 0.000 \\ \mathrm{H} & 1.373 & 1.791 & 0.000 \\ \mathrm{~N} & -1.065 & -0.354 & 0.000 \\ \mathrm{H} & -2.019 & -0.690 & 0.000\end{array}$

N10

N12

$\begin{array}{lrrr}\text { C } & 1.394 & 1.063 & 0.000 \\ \text { C } & 1.399 & -1.122 & 0.000 \\ \text { C } & 2.692 & -0.720 & 0.000 \\ \text { N } & 2.661 & 0.654 & 0.000 \\ \text { H } & 1.075 & 2.091 & 0.000 \\ \text { H } & 0.987 & -2.116 & 0.000 \\ \text { H } & 3.612 & -1.277 & 0.000 \\ \text { N } & 0.597 & 0.004 & 0.000 \\ \text { Si } & -1.259 & 0.001 & 0.000 \\ \text { C } & -1.716 & -0.896 & -1.550 \\ \text { H } & -1.343 & -1.922 & -1.554\end{array}$




$\begin{array}{lrrr}\mathrm{H} & -2.804 & -0.947 & -1.634 \\ \mathrm{H} & -1.337 & -0.387 & -2.437 \\ \mathrm{C} & -1.716 & -0.892 & 1.553 \\ \mathrm{H} & -1.338 & -0.380 & 2.438 \\ \mathrm{H} & -2.804 & -0.944 & 1.637 \\ \mathrm{H} & -1.342 & -1.917 & 1.559 \\ \mathrm{C} & -1.701 & 1.799 & -0.002 \\ \mathrm{H} & -1.340 & 2.316 & -0.893 \\ \mathrm{H} & -2.790 & 1.889 & -0.003 \\ \mathrm{H} & -1.341 & 2.319 & 0.887 \\ \mathrm{H} & 3.465 & 1.264 & 0.000\end{array}$

N13

$\begin{array}{lrrr}\text { C } & -1.094 & -0.101 & 0.000 \\ \text { C } & 1.150 & -0.025 & 0.000 \\ \text { C } & 0.680 & 1.246 & 0.000 \\ \text { N } & -0.699 & 1.168 & 0.000 \\ \text { H } & -1.329 & 1.958 & 0.000 \\ \text { H } & 1.200 & 2.187 & 0.000 \\ \text { N } & 0.022 & -0.829 & 0.000 \\ \text { C } & -2.489 & -0.602 & 0.000 \\ \text { H } & -3.018 & -0.254 & 0.887 \\ \text { H } & -3.020 & -0.248 & -0.884 \\ \text { H } & -2.497 & -1.689 & -0.003 \\ \text { C } & 2.534 & -0.574 & 0.000 \\ \text { H } & 3.078 & -0.245 & -0.884 \\ \text { H } & 3.079 & -0.241 & 0.882 \\ \text { H } & 2.519 & -1.663 & 0.003 \\ \text { H } & 0.035 & -1.840 & -0.001\end{array}$

N14

$\begin{array}{lrrr}\mathrm{C} & 1.961 & -1.318 & 0.058 \\ \mathrm{C} & 0.445 & -1.566 & -0.063 \\ \mathrm{H} & 2.369 & -1.662 & 1.007 \\ \mathrm{H} & 2.525 & -1.763 & -0.758 \\ \mathrm{H} & 0.182 & -2.029 & -1.013 \\ \mathrm{H} & 0.061 & -2.177 & 0.749 \\ \mathrm{~N} & 2.026 & 0.145 & -0.010 \\ \mathrm{~N} & -0.124 & -0.202 & 0.001 \\ \mathrm{C} & 0.851 & 0.719 & -0.008 \\ \mathrm{C} & -1.531 & 0.095 & 0.007 \\ \mathrm{O} & -1.892 & 1.231 & 0.019 \\ \mathrm{C} & -2.423 & -1.106 & 0.008 \\ \mathrm{H} & -2.283 & -1.681 & 0.926 \\ \mathrm{H} & -3.451 & -0.762 & -0.048 \\ \mathrm{H} & -2.205 & -1.759 & -0.838 \\ \mathrm{C} & 0.678 & 2.188 & -0.013 \\ \mathrm{H} & 0.087 & 2.487 & -0.878\end{array}$

$\begin{array}{lllr}\mathrm{H} & 0.113 & 2.494 & 0.867 \\ \mathrm{H} & 1.650 & 2.674 & -0.029 \\ \mathrm{H} & 2.887 & 0.672 & 0.001\end{array}$

N15

$\begin{array}{lrrr}\mathrm{C} & -1.469 & -0.774 & 0.007 \\ \mathrm{C} & -1.469 & 0.774 & 0.007 \\ \mathrm{C} & 0.713 & 0.000 & -0.014 \\ \mathrm{~N} & -0.032 & -1.083 & -0.010 \\ \mathrm{H} & 0.348 & -2.017 & -0.017 \\ \mathrm{H} & -1.952 & -1.190 & -0.874 \\ \mathrm{H} & -1.953 & 1.190 & -0.874 \\ \mathrm{C} & 2.195 & 0.000 & 0.008 \\ \mathrm{H} & 2.530 & 0.004 & 1.047 \\ \mathrm{H} & 2.586 & -0.890 & -0.480 \\ \mathrm{H} & 2.586 & 0.886 & -0.487 \\ \mathrm{~N} & -0.032 & 1.083 & -0.010 \\ \mathrm{H} & -1.932 & 1.189 & 0.900 \\ \mathrm{H} & -1.932 & -1.189 & 0.899 \\ \mathrm{H} & 0.348 & 2.017 & -0.016\end{array}$

N16

$\begin{array}{lrrr}\text { C } & -1.402 & -0.809 & 0.000 \\ \text { C } & -1.489 & 0.730 & 0.000 \\ \text { C } & 0.696 & 0.020 & 0.000 \\ \text { H } & -1.816 & -1.270 & -0.892 \\ \text { H } & -1.972 & 1.129 & -0.889 \\ \text { C } & 2.170 & -0.011 & 0.000 \\ \text { H } & 2.506 & -0.560 & 0.881 \\ \text { H } & 2.506 & -0.560 & -0.881 \\ \text { H } & 2.589 & 0.991 & 0.000 \\ \text { N } & -0.061 & 1.075 & 0.000 \\ \text { H } & -1.972 & 1.129 & 0.890 \\ \text { H } & -1.816 & -1.270 & 0.891 \\ \text { O } & 0.032 & -1.089 & 0.000 \\ \text { H } & 0.299 & 2.019 & 0.000\end{array}$

N17

$\begin{array}{lrrr}\mathrm{C} & 1.530 & 0.685 & 0.171 \\ \mathrm{C} & 1.438 & -0.824 & -0.089 \\ \mathrm{C} & -0.744 & -0.006 & -0.008 \\ \mathrm{H} & 2.291 & 1.156 & -0.445 \\ \mathrm{H} & 1.756 & -1.114 & -1.091 \\ \mathrm{C} & -2.217 & -0.009 & 0.048 \\ \mathrm{H} & -2.534 & 0.531 & 0.943 \\ \mathrm{H} & -2.606 & 0.547 & -0.808 \\ \mathrm{H} & -2.637 & -1.012 & 0.058\end{array}$




$\begin{array}{lrrr}\text { N } & -0.017 & -1.064 & 0.018 \\ \mathrm{H} & 1.963 & -1.435 & 0.641 \\ \mathrm{H} & 1.778 & 0.868 & 1.215 \\ \mathrm{H} & -0.412 & -1.998 & 0.057 \\ \mathrm{C} & 0.116 & 1.208 & -0.132 \\ \mathrm{H} & -0.224 & 2.006 & 0.527 \\ \mathrm{H} & 0.007 & 1.576 & -1.158 \\ & & & \\ \mathrm{~N} 18 & & & \\ & & & \\ \mathrm{C} & -1.664 & -0.333 & 0.210 \\ \mathrm{C} & -1.353 & 1.119 & -0.145 \\ \mathrm{C} & 0.806 & 0.173 & 0.005 \\ \mathrm{H} & -2.451 & -0.749 & -0.412 \\ \mathrm{H} & -1.586 & 1.345 & -1.187 \\ \mathrm{C} & 2.288 & 0.162 & 0.048 \\ \mathrm{H} & 2.609 & -0.195 & 1.030 \\ \mathrm{H} & 2.675 & -0.533 & -0.697 \\ \mathrm{H} & 2.699 & 1.154 & -0.128 \\ \mathrm{~N} & 0.098 & 1.262 & 0.040 \\ \mathrm{H} & -1.880 & 1.817 & 0.501 \\ \mathrm{H} & -1.923 & -0.458 & 1.259 \\ \mathrm{H} & 0.540 & 2.174 & 0.064 \\ \mathrm{~S} & -0.115 & -1.257 & -0.089 \\ & & & \end{array}$

$\begin{array}{lrrr}\mathrm{C} & -0.264 & -0.674 & 0.001 \\ \mathrm{C} & 2.148 & -0.736 & 0.226 \\ \mathrm{C} & 2.105 & 0.653 & -0.393 \\ \mathrm{C} & 0.942 & 1.459 & 0.172 \\ \mathrm{C} & -1.622 & 1.174 & -0.046 \\ \mathrm{C} & -2.488 & -0.092 & 0.055 \\ \mathrm{H} & 2.905 & -1.351 & -0.255 \\ \mathrm{H} & 1.998 & 0.561 & -1.475 \\ \mathrm{H} & 1.140 & 1.781 & 1.196 \\ \mathrm{H} & -1.691 & 1.644 & -1.029 \\ \mathrm{H} & -3.279 & -0.114 & -0.688 \\ \mathrm{H} & -2.920 & -0.204 & 1.050 \\ \mathrm{H} & -1.721 & -2.126 & -0.077 \\ \mathrm{~N} & -0.270 & 0.644 & 0.151 \\ \mathrm{~N} & 0.848 & -1.388 & 0.028 \\ \mathrm{~N} & -1.501 & -1.151 & -0.198 \\ \mathrm{H} & 2.373 & -0.684 & 1.293 \\ \mathrm{H} & 3.040 & 1.173 & -0.191 \\ \mathrm{H} & 0.759 & 2.348 & -0.432 \\ \mathrm{H} & -1.864 & 1.909 & 0.719 \\ \mathrm{H} & 0.794 & -2.389 & -0.069\end{array}$

$\begin{array}{lrrr}\text { N19 } & & & \\ \text { C } & 0.756 & 1.558 & 0.118 \\ \text { C } & -0.756 & 1.558 & -0.118 \\ \text { H } & 1.294 & 2.201 & -0.575 \\ \text { H } & 1.011 & 1.836 & 1.143 \\ \text { H } & -1.294 & 2.202 & 0.574 \\ \text { H } & -1.011 & 1.835 & -1.143 \\ \text { N } & 1.099 & 0.151 & -0.119 \\ \text { N } & -1.099 & 0.151 & 0.119 \\ \text { C } & 0.000 & -0.592 & 0.000 \\ \text { H } & -0.861 & -2.439 & 0.008 \\ \text { C } & 2.455 & -0.350 & -0.003 \\ \text { H } & 2.590 & -1.226 & -0.636 \\ \text { H } & 3.133 & 0.419 & -0.364 \\ \text { H } & 2.711 & -0.592 & 1.032 \\ \mathrm{C} & -2.455 & -0.350 & 0.002 \\ \text { H } & -2.590 & -1.227 & 0.634 \\ \text { H } & -3.133 & 0.418 & 0.365 \\ \text { H } & -2.711 & -0.590 & -1.032 \\ \text { N } & 0.000 & -1.921 & 0.000 \\ \text { H } & 0.862 & -2.439 & -0.008\end{array}$

N21

$\begin{array}{lrrr}\text { C } & 0.000 & -0.489 & 0.000 \\ \text { N } & -1.165 & 0.162 & -0.006 \\ \text { N } & 1.166 & 0.162 & 0.006 \\ \text { N } & 0.000 & -1.828 & 0.000 \\ \text { H } & -0.819 & -2.348 & -0.266 \\ \mathrm{C} & -2.407 & -0.509 & 0.371 \\ \mathrm{H} & -2.220 & -1.239 & 1.155 \\ \mathrm{H} & -3.092 & 0.242 & 0.759 \\ \mathrm{H} & -2.877 & -0.992 & -0.490 \\ \mathrm{C} & -1.330 & 1.462 & -0.656 \\ \mathrm{H} & -1.555 & 2.241 & 0.072 \\ \mathrm{H} & -0.433 & 1.723 & -1.210 \\ \mathrm{H} & -2.160 & 1.388 & -1.359 \\ \mathrm{C} & 1.330 & 1.462 & 0.656 \\ \mathrm{H} & 1.555 & 2.241 & -0.072 \\ \mathrm{H} & 0.433 & 1.723 & 1.210 \\ \mathrm{H} & 2.160 & 1.387 & 1.359 \\ \mathrm{C} & 2.407 & -0.509 & -0.370 \\ \mathrm{H} & 2.878 & -0.992 & 0.490 \\ \mathrm{H} & 2.220 & -1.240 & -1.154 \\ \mathrm{H} & 3.091 & 0.242 & -0.759 \\ \mathrm{H} & 0.819 & -2.348 & 0.266\end{array}$

N22

N20

$\begin{array}{llll}\text { C } & 0.169 & -1.216 & -0.006\end{array}$ 


$\begin{array}{lrrr}\mathrm{C} & -1.219 & -1.206 & 0.001 \\ \mathrm{C} & -1.907 & 0.000 & 0.006 \\ \mathrm{C} & -1.220 & 1.206 & 0.001 \\ \mathrm{C} & 0.168 & 1.216 & -0.006 \\ \mathrm{C} & 0.822 & 0.000 & -0.009 \\ \mathrm{H} & 0.714 & -2.152 & -0.010 \\ \mathrm{H} & -1.758 & -2.143 & 0.001 \\ \mathrm{H} & -2.989 & -0.001 & 0.009 \\ \mathrm{H} & -1.759 & 2.143 & 0.001 \\ \mathrm{H} & 0.713 & 2.153 & -0.009 \\ \mathrm{~N} & 2.309 & 0.000 & 0.007 \\ \mathrm{H} & 2.682 & -0.800 & -0.511 \\ \mathrm{H} & 2.679 & 0.850 & -0.426 \\ \mathrm{H} & 2.677 & -0.048 & 0.963\end{array}$

N23

$\begin{array}{lrrr}\mathrm{C} & 1.039 & -1.214 & -0.007 \\ \mathrm{C} & -0.346 & -1.210 & -0.002 \\ \mathrm{C} & -1.031 & 0.000 & 0.001 \\ \mathrm{C} & -0.346 & 1.210 & -0.002 \\ \mathrm{C} & 1.039 & 1.214 & -0.007 \\ \mathrm{C} & 1.698 & 0.000 & -0.009 \\ \mathrm{H} & 1.579 & -2.153 & -0.009 \\ \mathrm{H} & -0.896 & -2.141 & -0.004 \\ \mathrm{H} & -0.896 & 2.141 & -0.004 \\ \mathrm{H} & 1.578 & 2.153 & -0.009 \\ \mathrm{~N} & 3.183 & 0.000 & 0.011 \\ \mathrm{H} & 3.557 & -0.800 & -0.508 \\ \mathrm{H} & 3.555 & 0.849 & -0.423 \\ \mathrm{Cl} & -2.743 & 0.000 & 0.004 \\ \mathrm{H} & 3.552 & -0.049 & 0.967\end{array}$

$\begin{array}{lrrr}\text { N24 } & & & \\ \text { C } & -0.659 & -1.212 & 0.005 \\ \text { C } & 0.725 & -1.197 & -0.007 \\ \text { C } & 1.437 & 0.004 & -0.012 \\ \text { C } & 0.724 & 1.201 & -0.007 \\ \text { C } & -0.662 & 1.212 & 0.005 \\ \text { C } & -1.321 & 0.000 & 0.011 \\ \text { H } & -1.199 & -2.152 & 0.007 \\ \text { H } & 1.263 & -2.137 & -0.012 \\ \text { H } & 1.258 & 2.142 & -0.012 \\ \text { H } & -1.204 & 2.151 & 0.007 \\ \text { N } & -2.807 & -0.003 & -0.004 \\ \text { H } & -3.179 & -0.773 & 0.558 \\ \text { H } & -3.176 & 0.869 & 0.385 \\ \text { H } & -3.177 & -0.102 & -0.955 \\ \text { C } & 2.937 & -0.001 & 0.004\end{array}$

$\begin{array}{rrrr}H & 3.299 & -0.152 & 1.023 \\ \mathrm{H} & 3.332 & -0.810 & -0.609 \\ \mathrm{H} & 3.340 & 0.942 & -0.359\end{array}$

N25

$\begin{array}{lrrr}\text { C } & 0.906 & -1.195 & 0.000 \\ \text { C } & -0.470 & -1.013 & 0.000 \\ \text { C } & -0.996 & 0.280 & 0.000 \\ \text { C } & -0.132 & 1.387 & 0.000 \\ \text { C } & 1.231 & 1.206 & 0.000 \\ \text { C } & 1.728 & -0.090 & 0.000 \\ \text { H } & 1.312 & -2.200 & 0.000 \\ \text { H } & -1.115 & -1.878 & 0.000 \\ \text { H } & -0.564 & 2.378 & 0.000 \\ \text { H } & 1.894 & 2.064 & 0.000 \\ \text { N } & 3.198 & -0.287 & 0.000 \\ \text { H } & 3.426 & -1.284 & -0.007 \\ \text { H } & 3.638 & 0.135 & -0.823 \\ \text { H } & 3.635 & 0.122 & 0.832 \\ \text { O } & -2.296 & 0.565 & 0.000 \\ \text { C } & -3.234 & -0.504 & 0.000 \\ \text { H } & -3.118 & -1.118 & 0.896 \\ \text { H } & -4.213 & -0.038 & 0.001 \\ \text { H } & -3.119 & -1.118 & -0.895\end{array}$

N26

$\begin{array}{lrrr}\mathrm{N} & 1.858 & 0.113 & 0.000 \\ \mathrm{H} & 1.976 & 0.708 & -0.824 \\ \mathrm{H} & 1.976 & 0.709 & 0.824 \\ \mathrm{C} & 0.487 & -0.534 & 0.000 \\ \mathrm{H} & 0.446 & -1.165 & 0.887 \\ \mathrm{H} & 0.447 & -1.165 & -0.886 \\ \mathrm{C} & -0.595 & 0.526 & 0.000 \\ \mathrm{H} & -0.484 & 1.163 & 0.882 \\ \mathrm{H} & -0.484 & 1.163 & -0.882 \\ \mathrm{C} & -1.976 & -0.123 & 0.000 \\ \mathrm{H} & -2.118 & -0.744 & 0.884 \\ \mathrm{H} & -2.749 & 0.642 & 0.000 \\ \mathrm{H} & -2.118 & -0.744 & -0.884 \\ \mathrm{H} & 2.612 & -0.578 & 0.000\end{array}$

N27

$\begin{array}{lrrr}\mathrm{C} & 0.405 & 0.300 & 0.000 \\ \mathrm{C} & -1.117 & 0.286 & 0.000 \\ \mathrm{H} & -1.498 & 0.769 & 0.897 \\ \mathrm{H} & -1.498 & 0.769 & -0.897 \\ \mathrm{~N} & -1.548 & -1.150 & 0.000\end{array}$




$\begin{array}{lrrr}H & -1.187 & -1.638 & -0.828 \\ H & -1.187 & -1.638 & 0.828 \\ F & 0.854 & -0.348 & -1.077 \\ F & 0.854 & -0.348 & 1.077 \\ F & 0.854 & 1.530 & 0.000 \\ H & -2.569 & -1.238 & 0.000\end{array}$

N28

$\begin{array}{lrrr}\mathrm{C} & 0.000 & 0.000 & -0.088 \\ \mathrm{C} & -0.733 & -1.261 & -0.513 \\ \mathrm{H} & -1.765 & -1.268 & -0.155 \\ \mathrm{H} & -0.762 & -1.301 & -1.601 \\ \mathrm{H} & -0.225 & -2.161 & -0.162 \\ \mathrm{C} & 1.456 & -0.003 & -0.521 \\ \mathrm{H} & 1.499 & -0.003 & -1.610 \\ \mathrm{H} & 1.983 & 0.887 & -0.170 \\ \mathrm{H} & 1.980 & -0.894 & -0.170 \\ \mathrm{C} & -0.729 & 1.264 & -0.513 \\ \mathrm{H} & -1.760 & 1.275 & -0.155 \\ \mathrm{H} & -0.217 & 2.162 & -0.162 \\ \mathrm{H} & -0.757 & 1.304 & -1.601 \\ \mathrm{~N} & 0.006 & 0.000 & 1.450 \\ \mathrm{H} & 0.484 & -0.829 & 1.813 \\ \mathrm{H} & 0.487 & 0.827 & 1.813 \\ \mathrm{H} & -0.949 & 0.002 & 1.819\end{array}$

N29

$\begin{array}{lrrr}\mathrm{C} & 2.315 & 0.000 & -0.257 \\ \mathrm{C} & 1.643 & 1.205 & -0.100 \\ \mathrm{C} & 0.292 & 1.207 & 0.211 \\ \mathrm{C} & -0.387 & 0.000 & 0.363 \\ \mathrm{C} & 0.291 & -1.207 & 0.211 \\ \mathrm{C} & 1.642 & -1.205 & -0.100 \\ \mathrm{H} & 3.371 & -0.001 & -0.492 \\ \mathrm{H} & 2.173 & 2.141 & -0.207 \\ \mathrm{H} & -0.227 & 2.148 & 0.357 \\ \mathrm{H} & -0.228 & -2.148 & 0.358 \\ \mathrm{H} & 2.172 & -2.142 & -0.207 \\ \mathrm{C} & -1.857 & 0.001 & 0.637 \\ \mathrm{H} & -2.191 & 0.891 & 1.167 \\ \mathrm{H} & -2.191 & -0.888 & 1.168 \\ \mathrm{~N} & -2.603 & 0.000 & -0.695 \\ \mathrm{H} & -2.334 & -0.823 & -1.242 \\ \mathrm{H} & -3.620 & -0.002 & -0.581 \\ \mathrm{H} & -2.336 & 0.823 & -1.242\end{array}$

N30

$\begin{array}{lrrr}\mathrm{N} & 0.000 & -0.262 & 0.000 \\ \mathrm{H} & 0.000 & -0.882 & -0.815 \\ \mathrm{C} & 1.268 & 0.547 & 0.000 \\ \mathrm{H} & 1.224 & 1.182 & 0.884 \\ \mathrm{H} & 1.224 & 1.182 & -0.884 \\ \mathrm{C} & 2.482 & -0.355 & 0.000 \\ \mathrm{H} & 3.381 & 0.258 & 0.000 \\ \mathrm{H} & 2.513 & -0.987 & 0.888 \\ \mathrm{H} & 2.513 & -0.987 & -0.889 \\ \mathrm{C} & -1.268 & 0.547 & 0.000 \\ \mathrm{H} & -1.224 & 1.182 & 0.884 \\ \mathrm{H} & -1.224 & 1.182 & -0.884 \\ \mathrm{C} & -2.482 & -0.355 & 0.000 \\ \mathrm{H} & -3.381 & 0.258 & 0.000 \\ \mathrm{H} & -2.513 & -0.987 & -0.889 \\ \mathrm{H} & -2.513 & -0.987 & 0.888 \\ \mathrm{H} & 0.000 & -0.882 & 0.815\end{array}$

N31

$\begin{array}{lrrr}\text { C } & 1.043 & -0.719 & -0.257 \\ \text { C } & -0.333 & -1.226 & 0.145 \\ \text { C } & -0.333 & 1.226 & -0.146 \\ \text { C } & 1.043 & 0.719 & 0.257 \\ \text { H } & 1.828 & -1.331 & 0.182 \\ \text { H } & 1.161 & -0.742 & -1.341 \\ \text { H } & -0.365 & -1.517 & 1.193 \\ \text { H } & -0.733 & -2.030 & -0.466 \\ \text { H } & -0.365 & 1.517 & -1.193 \\ \text { H } & -0.733 & 2.030 & 0.465 \\ \text { H } & 1.828 & 1.331 & -0.182 \\ \text { H } & 1.160 & 0.743 & 1.341 \\ \text { N } & -1.232 & 0.000 & 0.000 \\ \text { H } & -1.843 & 0.091 & 0.811 \\ \text { H } & -1.844 & -0.091 & -0.810\end{array}$

N32

$\begin{array}{lrrr}\text { C } & -1.518 & 0.775 & -0.034 \\ \text { C } & -0.061 & 1.182 & 0.173 \\ \text { C } & -0.061 & -1.182 & 0.173 \\ \text { C } & -1.518 & -0.775 & -0.034 \\ \text { H } & -2.141 & 1.190 & 0.755 \\ \text { H } & -1.894 & 1.161 & -0.980 \\ \text { H } & 0.198 & 1.283 & 1.227 \\ \text { H } & 0.254 & 2.076 & -0.361 \\ \text { H } & 0.254 & -2.076 & -0.360 \\ \text { H } & 0.197 & -1.282 & 1.227 \\ \text { H } & -1.894 & -1.160 & -0.980 \\ \text { H } & -2.141 & -1.190 & 0.754\end{array}$




$\begin{array}{lrrr}\mathrm{N} & 0.721 & 0.000 & -0.330 \\ \mathrm{C} & 2.157 & 0.000 & 0.048 \\ \mathrm{H} & 2.630 & -0.892 & -0.354 \\ \mathrm{H} & 2.630 & 0.892 & -0.354 \\ \mathrm{H} & 2.220 & 0.000 & 1.134 \\ \mathrm{H} & 0.658 & 0.000 & -1.351\end{array}$

$\begin{array}{lrrr}\text { N33 } & & & \\ \text { C } & 0.696 & 1.256 & 0.247 \\ \text { C } & 0.696 & -1.256 & 0.247 \\ \text { C } & -0.744 & -1.256 & -0.231 \\ \text { C } & -0.744 & 1.256 & -0.231 \\ \text { H } & 1.268 & -2.101 & -0.133 \\ \text { H } & 0.762 & 1.240 & 1.335 \\ \text { H } & 1.268 & 2.101 & -0.133 \\ \text { H } & -0.765 & -1.330 & -1.323 \\ \text { H } & -1.225 & -2.156 & 0.151 \\ \text { H } & -0.765 & 1.330 & -1.323 \\ \text { H } & -1.225 & 2.156 & 0.151 \\ \text { H } & 0.762 & -1.240 & 1.335 \\ \text { N } & 1.384 & 0.000 & -0.229 \\ \text { H } & 1.399 & 0.000 & -1.253 \\ \text { C } & -1.480 & 0.000 & 0.229 \\ \text { H } & -2.496 & 0.000 & -0.163 \\ \text { H } & -1.560 & 0.000 & 1.320 \\ \text { H } & 2.358 & 0.000 & 0.078\end{array}$

N34

$\begin{array}{lrrr}\mathrm{C} & -0.278 & -1.242 & 0.206 \\ \mathrm{C} & -0.278 & 1.242 & 0.206 \\ \mathrm{C} & 1.179 & 1.252 & -0.218 \\ \mathrm{C} & 1.179 & -1.252 & -0.218 \\ \mathrm{H} & -0.833 & 2.094 & -0.187 \\ \mathrm{H} & -0.384 & -1.224 & 1.292 \\ \mathrm{H} & -0.833 & -2.094 & -0.187 \\ \mathrm{H} & 1.241 & 1.328 & -1.309 \\ \mathrm{H} & 1.639 & 2.155 & 0.183 \\ \mathrm{H} & 1.241 & -1.328 & -1.309 \\ \mathrm{H} & 1.639 & -2.155 & 0.183 \\ \mathrm{H} & -0.384 & 1.224 & 1.292 \\ \mathrm{~N} & -0.968 & 0.000 & -0.293 \\ \mathrm{C} & 1.904 & 0.000 & 0.266 \\ \mathrm{H} & 2.932 & 0.000 & -0.093 \\ \mathrm{H} & 1.947 & 0.000 & 1.359 \\ \mathrm{C} & -2.417 & 0.000 & 0.049 \\ \mathrm{H} & -2.880 & 0.892 & -0.365 \\ \mathrm{H} & -2.880 & -0.892 & -0.365 \\ \mathrm{H} & -2.509 & 0.000 & 1.133\end{array}$

$\mathrm{H}$

$-0.884 \quad 0.000$

$-1.313$

N35

$\begin{array}{lrrr}\mathrm{C} & 1.266 & 0.621 & -0.236 \\ \mathrm{C} & -1.226 & 0.697 & -0.236 \\ \mathrm{C} & -1.188 & -0.756 & 0.206 \\ \mathrm{C} & 1.140 & -0.827 & 0.206 \\ \mathrm{H} & -2.071 & 1.239 & 0.184 \\ \mathrm{H} & 1.279 & 0.697 & -1.322 \\ \mathrm{H} & 2.143 & 1.110 & 0.184 \\ \mathrm{H} & -1.219 & -0.823 & 1.302 \\ \mathrm{H} & -2.058 & -1.275 & -0.192 \\ \mathrm{H} & 1.166 & -0.896 & 1.303 \\ \mathrm{H} & 1.977 & -1.398 & -0.191 \\ \mathrm{H} & -1.234 & 0.774 & -1.322 \\ \mathrm{O} & -0.043 & -1.393 & -0.296 \\ \mathrm{~N} & 0.042 & 1.368 & 0.232 \\ \mathrm{H} & 0.043 & 1.405 & 1.255 \\ \mathrm{H} & 0.071 & 2.335 & -0.097\end{array}$

N36

\author{
C
}

C

C

C

$\mathrm{H}$

$\mathrm{H}$

$\mathrm{H}$

$\mathrm{H}$

$\mathrm{H}$

$\mathrm{H}$

$\mathrm{H}$

$\mathrm{H}$

$\mathrm{O}$

N

C

$\mathrm{H}$

$\mathrm{H}$

$\mathrm{H}$

$\mathrm{H}$

N37

$\begin{array}{llrr}\mathrm{N} & 0.000 & 0.000 & -0.345 \\ \mathrm{C} & 0.881 & -1.119 & 0.104 \\ \mathrm{H} & 0.483 & -2.056 & -0.276 \\ \mathrm{H} & 1.886 & -0.951 & -0.277 \\ \mathrm{H} & 0.888 & -1.126 & 1.191\end{array}$




$\begin{array}{lrrr}\mathrm{C} & -1.410 & -0.204 & 0.104 \\ \mathrm{H} & -2.022 & 0.610 & -0.276 \\ \mathrm{H} & -1.767 & -1.157 & -0.277 \\ \mathrm{H} & -1.419 & -0.206 & 1.191 \\ \mathrm{C} & 0.528 & 1.323 & 0.104 \\ \mathrm{H} & 1.539 & 1.446 & -0.276 \\ \mathrm{H} & -0.119 & 2.109 & -0.277 \\ \mathrm{H} & 0.531 & 1.332 & 1.191 \\ \mathrm{H} & 0.000 & 0.000 & -1.367\end{array}$

$\begin{array}{lrrr}\mathrm{H} & 1.151 & -1.285 & -1.659 \\ \mathrm{H} & 1.149 & -2.098 & -0.085 \\ \mathrm{C} & -0.815 & -1.225 & -0.633 \\ \mathrm{H} & -1.208 & -1.271 & -1.647 \\ \mathrm{H} & -1.209 & -2.078 & -0.083 \\ \mathrm{~N} & 1.218 & -0.001 & 0.001 \\ \mathrm{~N} & -1.281 & 0.001 & -0.002 \\ \mathrm{H} & 2.237 & -0.001 & 0.003\end{array}$

\section{NA1}

N38

$\begin{array}{lrrr}\text { C } & -0.750 & 0.041 & -1.428 \\ \mathrm{C} & 1.306 & 0.000 & 0.000 \\ \mathrm{C} & 0.790 & 0.043 & -1.441 \\ \mathrm{H} & -1.176 & -0.829 & -1.924 \\ \mathrm{H} & -1.177 & 0.938 & -1.873 \\ \mathrm{H} & 1.156 & -0.818 & -1.998 \\ \mathrm{H} & 1.155 & 0.937 & -1.945 \\ \mathrm{C} & -0.750 & -1.257 & 0.678 \\ \mathrm{H} & -1.177 & -1.251 & 1.679 \\ \mathrm{H} & -1.178 & -2.091 & 0.124 \\ \mathrm{C} & 0.789 & -1.269 & 0.683 \\ \mathrm{H} & 1.155 & -1.321 & 1.708 \\ \mathrm{H} & 1.154 & -2.153 & 0.162 \\ \mathrm{H} & 2.394 & 0.000 & 0.001 \\ \mathrm{H} & -2.244 & 0.000 & 0.000 \\ \mathrm{C} & 0.790 & 1.226 & 0.758 \\ \mathrm{H} & 1.154 & 1.216 & 1.784 \\ \mathrm{H} & 1.156 & 2.139 & 0.291 \\ \mathrm{C} & -0.750 & 1.216 & 0.750 \\ \mathrm{H} & -1.178 & 1.153 & 1.748 \\ \mathrm{H} & -1.176 & 2.080 & 0.243 \\ \mathrm{~N} & -1.224 & 0.000 & 0.000\end{array}$

N39

$\begin{array}{lrrr}\mathrm{C} & -0.817 & 0.066 & 1.376 \\ \mathrm{C} & 0.734 & 0.067 & 1.425 \\ \mathrm{H} & -1.210 & 0.969 & 1.839 \\ \mathrm{H} & -1.212 & -0.789 & 1.923 \\ \mathrm{H} & 1.148 & 0.974 & 1.861 \\ \mathrm{H} & 1.147 & -0.797 & 1.944 \\ \mathrm{C} & -0.814 & 1.161 & -0.746 \\ \mathrm{H} & -1.206 & 1.110 & -1.761 \\ \mathrm{H} & -1.208 & 2.062 & -0.279 \\ \mathrm{C} & 0.737 & 1.200 & -0.769 \\ \mathrm{H} & 1.153 & 1.123 & -1.772 \\ \mathrm{H} & 1.151 & 2.080 & -0.280 \\ \mathrm{C} & 0.736 & -1.267 & -0.653\end{array}$

$\begin{array}{lrrr}\text { C } & -1.059 & 0.059 & 0.000 \\ \text { C } & -0.246 & 1.260 & 0.000 \\ \text { C } & 1.106 & 1.197 & 0.000 \\ \text { C } & 1.795 & -0.050 & 0.000 \\ \text { C } & 1.054 & -1.181 & 0.000 \\ \text { N } & -0.303 & -1.117 & 0.000 \\ \text { H } & -0.782 & 2.198 & 0.000 \\ \text { H } & 2.873 & -0.103 & 0.000 \\ \text { H } & 1.482 & -2.173 & 0.000 \\ \text { H } & 1.683 & 2.115 & 0.000 \\ \text { O } & -2.273 & 0.004 & 0.000 \\ \text { H } & -0.853 & -1.964 & 0.000\end{array}$

NA2

$\begin{array}{lrrr}\mathrm{C} & -1.040 & -1.186 & 0.000 \\ \mathrm{C} & 0.306 & -1.225 & 0.000 \\ \mathrm{C} & 1.104 & 0.000 & 0.000 \\ \mathrm{C} & 0.306 & 1.225 & 0.000 \\ \mathrm{C} & -1.040 & 1.186 & 0.000 \\ \mathrm{~N} & -1.718 & 0.000 & 0.000 \\ \mathrm{H} & -1.655 & -2.075 & 0.001 \\ \mathrm{H} & 0.825 & -2.173 & 0.000 \\ \mathrm{H} & 0.825 & 2.173 & 0.000 \\ \mathrm{H} & -1.655 & 2.075 & 0.000 \\ \mathrm{O} & 2.325 & 0.000 & 0.000 \\ \mathrm{H} & -2.722 & 0.000 & 0.000\end{array}$

NA3

$\begin{array}{rrrr}\text { C } & 0.179 & -0.514 & 0.000 \\ \text { C } & 0.703 & 0.771 & 0.000 \\ \text { C } & -1.292 & 1.688 & 0.000 \\ \text { C } & -1.222 & -0.607 & 0.000 \\ \text { C } & 2.277 & -0.766 & 0.000 \\ \text { H } & 3.274 & -1.177 & 0.000 \\ \text { N } & -1.938 & 0.517 & 0.000 \\ \text { N } & 0.013 & 1.912 & 0.000 \\ \text { N } & 1.179 & -1.463 & 0.000\end{array}$




$\begin{array}{lrrr}\mathrm{N} & 2.061 & 0.589 & 0.000 \\ \mathrm{H} & -1.927 & 2.567 & 0.000 \\ \mathrm{~N} & -1.855 & -1.795 & 0.000 \\ \mathrm{H} & -1.324 & -2.647 & 0.000 \\ \mathrm{H} & -2.859 & -1.814 & 0.000 \\ \mathrm{H} & 2.753 & 1.318 & 0.000\end{array}$

NA4

$\begin{array}{lrrr}\text { C } & -0.816 & 0.429 & 0.010 \\ \mathrm{C} & -0.519 & -0.921 & -0.001 \\ \mathrm{C} & 1.679 & -0.534 & -0.004 \\ \mathrm{C} & 0.190 & 1.438 & 0.003 \\ \mathrm{C} & -2.634 & -0.766 & 0.000 \\ \mathrm{H} & -3.687 & -0.997 & -0.003 \\ \mathrm{~N} & 1.453 & 0.822 & -0.006 \\ \mathrm{~N} & 0.748 & -1.429 & 0.007 \\ \mathrm{O} & 0.072 & 2.646 & -0.004 \\ \mathrm{~N} & -2.184 & 0.512 & 0.008 \\ \mathrm{~N} & -1.670 & -1.655 & -0.005 \\ \mathrm{H} & 2.233 & 1.460 & -0.085 \\ \mathrm{~N} & 3.000 & -0.911 & -0.068 \\ \mathrm{H} & 3.666 & -0.337 & 0.425 \\ \mathrm{H} & 3.122 & -1.902 & 0.078 \\ \mathrm{H} & -2.727 & 1.359 & 0.011\end{array}$

$\begin{array}{lrrr}\mathrm{C} & -2.080 & 0.718 & 0.000 \\ \mathrm{C} & -0.906 & 1.436 & 0.000 \\ \mathrm{H} & -0.929 & -2.505 & 0.000 \\ \mathrm{H} & -3.050 & -1.204 & 0.000 \\ \mathrm{H} & -3.022 & 1.250 & 0.000 \\ \mathrm{H} & -0.903 & 2.517 & 0.000 \\ \mathrm{H} & 2.059 & 1.883 & 0.000 \\ \mathrm{~N} & 1.577 & -1.147 & 0.000 \\ \mathrm{~N} & 2.345 & -0.129 & 0.000 \\ \mathrm{~N} & 1.599 & 0.989 & 0.000\end{array}$

NA7

$\begin{array}{lrrr}\mathrm{C} & -1.159 & -0.175 & 0.000 \\ \mathrm{C} & 0.707 & 1.245 & 0.000 \\ \mathrm{C} & 1.160 & -0.214 & 0.000 \\ \mathrm{~N} & 0.007 & -0.957 & 0.000 \\ \mathrm{H} & 1.099 & 1.743 & -0.888 \\ \mathrm{~N} & -0.730 & 1.119 & 0.000 \\ \mathrm{O} & 2.288 & -0.618 & 0.000 \\ \mathrm{O} & -2.285 & -0.591 & 0.000 \\ \mathrm{H} & -1.389 & 1.877 & 0.000 \\ \mathrm{H} & 1.099 & 1.743 & 0.888 \\ \mathrm{H} & -0.022 & -1.964 & 0.000\end{array}$

\section{NA8}

$\begin{array}{lrrr}\text { NA5 } & & & \\ \text { C } & 0.000 & 0.732 & 0.000 \\ \text { C } & 0.501 & -0.580 & 0.000 \\ \text { C } & -0.381 & -1.661 & 0.000 \\ \text { C } & -1.734 & -1.391 & 0.000 \\ \text { C } & -2.218 & -0.072 & 0.000 \\ \text { C } & -1.363 & 1.012 & 0.000 \\ \text { C } & 2.203 & 0.692 & 0.000 \\ \text { H } & 0.000 & -2.673 & 0.000 \\ \text { H } & -2.443 & -2.209 & 0.000 \\ \text { H } & -3.287 & 0.097 & 0.000 \\ \text { H } & -1.738 & 2.027 & 0.000 \\ \text { H } & 3.211 & 1.079 & 0.000 \\ \text { N } & 1.122 & 1.531 & 0.000 \\ \text { N } & 1.886 & -0.565 & 0.000 \\ \text { H } & 1.149 & 2.535 & 0.000\end{array}$

$\begin{array}{lrrr}\text { NA6 } & & & \\ \text { C } & 0.274 & 0.687 & 0.000 \\ \text { C } & 0.272 & -0.710 & 0.000 \\ \text { C } & -0.931 & -1.424 & 0.000 \\ \text { C } & -2.096 & -0.694 & 0.000\end{array}$

$\begin{array}{lrrr}\mathrm{C} & -1.149 & -0.160 & 0.000 \\ \mathrm{C} & -0.663 & 1.263 & 0.000 \\ \mathrm{C} & 0.663 & 1.263 & 0.000 \\ \mathrm{C} & 1.149 & -0.160 & 0.000 \\ \mathrm{~N} & 0.000 & -0.941 & 0.000 \\ \mathrm{H} & 1.350 & 2.094 & 0.000 \\ \mathrm{O} & -2.278 & -0.556 & 0.000 \\ \mathrm{O} & 2.278 & -0.556 & 0.000 \\ \mathrm{H} & -1.350 & 2.094 & 0.000 \\ \mathrm{H} & 0.000 & -1.948 & 0.000\end{array}$

NA9

$\begin{array}{lrrr}\mathrm{C} & -1.167 & -0.214 & 0.000 \\ \mathrm{C} & -0.765 & 1.252 & 0.000 \\ \mathrm{C} & 0.765 & 1.253 & 0.000 \\ \mathrm{C} & 1.167 & -0.214 & 0.000 \\ \mathrm{~N} & 0.000 & -0.959 & 0.000 \\ \mathrm{H} & -1.203 & 1.727 & -0.877 \\ \mathrm{H} & 1.202 & 1.726 & 0.877 \\ \mathrm{O} & -2.276 & -0.668 & 0.000 \\ \mathrm{O} & 2.276 & -0.668 & 0.000 \\ \mathrm{H} & -1.201 & 1.726 & 0.878\end{array}$




$\begin{array}{rrrrrrrr}\mathrm{H} & 1.202 & 1.726 & -0.878 & \mathrm{H} & -2.161 & -0.600 & 0.000 \\ \mathrm{H} & 0.000 & -1.969 & 0.000 & \mathrm{H} & -1.093 & 1.936 & 0.000 \\ & & & & \mathrm{~N} & -0.111 & -1.220 & 0.000\end{array}$

NA10

$\begin{array}{lrrr}\text { C } & 0.000 & -0.693 & -0.179 \\ \text { C } & 0.000 & 0.693 & -0.179 \\ \text { C } & 0.000 & 1.417 & -1.352 \\ \text { C } & 0.000 & 0.697 & -2.543 \\ \text { C } & 0.000 & -0.697 & -2.543 \\ \text { C } & 0.000 & -1.417 & -1.352 \\ \text { C } & 0.000 & -1.165 & 1.240 \\ \text { C } & 0.000 & 1.165 & 1.240 \\ \text { H } & 0.000 & 2.499 & -1.338 \\ \text { H } & 0.000 & 1.225 & -3.488 \\ \text { H } & 0.000 & -1.225 & -3.488 \\ \text { H } & 0.000 & -2.499 & -1.338 \\ \text { O } & 0.000 & -2.286 & 1.663 \\ \text { O } & 0.000 & 2.286 & 1.663 \\ \text { N } & 0.000 & 0.000 & 2.006 \\ \text { H } & 0.000 & 0.000 & 3.014\end{array}$

NA13

$\begin{array}{lrrr}\text { C } & 0.448 & 0.633 & 0.000 \\ \text { C } & 0.000 & -0.694 & 0.000 \\ \text { C } & -2.112 & -0.070 & 0.000 \\ \text { C } & -0.546 & 1.601 & 0.000 \\ \text { C } & 2.190 & -0.567 & 0.000 \\ \text { H } & 3.212 & -0.914 & 0.000 \\ \text { N } & -1.822 & 1.236 & 0.000 \\ \text { N } & -1.261 & -1.090 & 0.000 \\ \text { N } & 1.830 & 0.679 & 0.000 \\ \text { N } & 1.140 & -1.452 & 0.000 \\ \text { H } & -0.319 & 2.662 & 0.000 \\ \text { H } & -3.165 & -0.324 & 0.000 \\ \text { H } & 1.186 & -2.457 & 0.000\end{array}$

NA14

$\begin{array}{lrrr}\text { NA11 } & & & \\ \text { C } & 0.309 & -0.585 & 0.000 \\ \text { C } & 0.809 & 0.703 & 0.000 \\ \text { C } & 2.174 & 0.925 & 0.000 \\ \text { C } & 3.014 & -0.180 & 0.000 \\ \text { C } & 2.496 & -1.474 & 0.000 \\ \text { C } & 1.125 & -1.698 & 0.000 \\ \text { C } & -0.258 & 1.745 & 0.000 \\ \text { H } & 2.555 & 1.938 & 0.000 \\ \text { H } & 4.087 & -0.038 & 0.000 \\ \text { H } & 3.172 & -2.319 & 0.000 \\ \text { H } & 0.714 & -2.698 & 0.000 \\ \text { S } & -1.454 & -0.573 & 0.000 \\ \text { N } & -1.484 & 1.099 & 0.000 \\ \text { O } & -0.105 & 2.934 & 0.000 \\ \text { O } & -1.981 & -1.069 & 1.229 \\ \text { O } & -1.981 & -1.069 & -1.229 \\ \text { H } & -2.354 & 1.611 & 0.000\end{array}$

\section{NA12}

$\begin{array}{lrrr}\text { C } & 0.991 & -0.522 & 0.000 \\ \text { C } & -1.127 & -0.299 & 0.000 \\ \text { C } & -0.624 & 0.969 & 0.000 \\ \text { N } & 0.739 & 0.813 & 0.000 \\ \text { H } & 1.423 & 1.549 & 0.000 \\ \text { H } & 1.994 & -0.919 & 0.000\end{array}$

N

C

C

$\mathrm{N}$

$\mathrm{H}$

$\mathrm{H}$

O

0

$\mathrm{H}$

C

$\mathrm{H}$

$\mathrm{H}$

$\mathrm{H}$

NA15

C

N

C

C

C

$\mathrm{N}$

$\mathrm{H}$

$\mathrm{H}$

$\mathrm{H}$

O

$\begin{array}{rrr}1.670 & 0.028 & 0.000 \\ 0.723 & 1.028 & 0.000 \\ -0.666 & 0.892 & 0.000 \\ -1.148 & -0.492 & 0.000 \\ -0.237 & -1.474 & 0.000 \\ 1.117 & -1.235 & 0.000 \\ -0.519 & -2.519 & 0.000 \\ 1.083 & 1.973 & 0.000 \\ -1.379 & 1.868 & 0.000 \\ 2.859 & 0.229 & 0.000 \\ 1.780 & -1.993 & 0.000 \\ -2.626 & -0.709 & 0.000 \\ -3.080 & -0.243 & 0.875 \\ -3.080 & -0.243 & -0.875 \\ -2.864 & -1.771 & 0.000\end{array}$

$\begin{array}{rrrr}C & -1.214 & -0.401 & 0.000 \\ N & 0.032 & -0.985 & 0.000 \\ C & 1.281 & -0.351 & 0.000 \\ C & 1.199 & 1.105 & 0.000 \\ C & 0.000 & 1.699 & 0.000 \\ N & -1.168 & 0.982 & 0.000 \\ H & 2.119 & 1.667 & 0.000 \\ H & -0.118 & 2.773 & 0.000 \\ H & 0.041 & -1.996 & 0.000 \\ & 2.296 & -1.003 & 0.000\end{array}$




$\begin{array}{lrrrrrrr}\mathrm{O} & -2.249 & -1.019 & 0.000 & \mathrm{H} & -0.763 & -2.153 & 0.000 \\ \mathrm{H} & -2.065 & 1.439 & 0.000 & \mathrm{H} & 1.719 & -2.113 & 0.000 \\ & & & & \mathrm{O} & -2.292 & -0.111 & 0.000 \\ \text { NA16 } & & & & \mathrm{H} & -2.673 & 0.773 & 0.000\end{array}$

$\begin{array}{lrrr}\mathrm{C} & -0.918 & -0.806 & 0.000 \\ \mathrm{C} & -1.038 & 0.557 & 0.000 \\ \mathrm{C} & 1.324 & 0.805 & 0.000 \\ \mathrm{C} & 0.336 & -1.496 & 0.000 \\ \mathrm{C} & -3.010 & -0.193 & 0.000 \\ \mathrm{H} & -4.085 & -0.270 & 0.000 \\ \mathrm{~N} & 1.408 & -0.593 & 0.000 \\ \mathrm{~N} & 0.065 & 1.368 & 0.000 \\ \mathrm{O} & 0.481 & -2.701 & 0.000 \\ \mathrm{O} & 2.323 & 1.490 & 0.000 \\ \mathrm{C} & 2.766 & -1.128 & 0.000 \\ \mathrm{H} & 3.298 & -0.780 & -0.883 \\ \mathrm{H} & 3.299 & -0.779 & 0.882 \\ \mathrm{H} & 2.691 & -2.210 & 0.001 \\ \mathrm{C} & -0.037 & 2.820 & 0.000 \\ \mathrm{H} & 0.453 & 3.226 & 0.883 \\ \mathrm{H} & 0.453 & 3.225 & -0.884 \\ \mathrm{H} & -1.092 & 3.076 & 0.000 \\ \mathrm{~N} & -2.209 & -1.276 & 0.000 \\ \mathrm{~N} & -2.338 & 0.943 & 0.000 \\ \mathrm{H} & -2.478 & -2.246 & -0.001\end{array}$

\section{OA2}

$\begin{array}{lrrr}\text { C } & 0.927 & 0.284 & 0.000 \\ \text { C } & 0.403 & -1.001 & 0.000 \\ \text { C } & -0.978 & -1.187 & 0.000 \\ \text { C } & -1.835 & -0.101 & 0.000 \\ \text { C } & -1.307 & 1.188 & 0.000 \\ \text { C } & 0.059 & 1.376 & 0.000 \\ \text { H } & 1.047 & -1.868 & 0.000 \\ \text { H } & -1.381 & -2.193 & 0.000 \\ \text { H } & -1.986 & 2.031 & 0.000 \\ \text { H } & 0.484 & 2.371 & 0.000 \\ \text { O } & -3.195 & -0.224 & 0.000 \\ \text { O } & 2.257 & 0.576 & 0.000 \\ \text { C } & 3.157 & -0.504 & 0.000 \\ \text { H } & 3.032 & -1.126 & -0.891 \\ \text { H } & 4.155 & -0.072 & 0.000 \\ \text { H } & 3.032 & -1.126 & 0.891 \\ \text { H } & -3.434 & -1.156 & 0.000\end{array}$

\section{OA3}

NA17

C

C

$\begin{array}{lrrr}\mathrm{C} & -0.941 & 0.206 & 0.000 \\ \mathrm{O} & -1.418 & 1.307 & 0.000 \\ \mathrm{~N} & -1.620 & -0.959 & 0.000 \\ \mathrm{H} & -1.154 & -1.849 & 0.000 \\ \mathrm{C} & 0.593 & 0.001 & 0.000 \\ \mathrm{~F} & 0.972 & -0.699 & -1.077 \\ \mathrm{~F} & 1.229 & 1.152 & 0.000 \\ \mathrm{~F} & 0.972 & -0.698 & 1.077 \\ \mathrm{H} & -2.626 & -0.928 & 0.000\end{array}$

OA1

$\begin{array}{lrrr}\text { C } & 1.846 & 0.029 & 0.000 \\ \text { C } & 1.124 & 1.213 & 0.000 \\ \text { C } & -0.264 & 1.192 & 0.000 \\ \text { C } & -0.934 & -0.026 & 0.000 \\ \text { C } & -0.219 & -1.217 & 0.000 \\ \text { C } & 1.166 & -1.183 & 0.000 \\ \text { H } & 2.927 & 0.049 & 0.000 \\ \text { H } & 1.640 & 2.164 & 0.000 \\ \text { H } & -0.827 & 2.118 & 0.000\end{array}$

$\begin{array}{lll}0.373 & -1.158 & 0.000\end{array}$

$\begin{array}{lll}1.758 & -1.177 & 0.000\end{array}$

$\begin{array}{lll}2.465 & 0.019 & 0.000\end{array}$

$\begin{array}{lll}1.774 & 1.220 & 0.000\end{array}$

$\begin{array}{lll}0.386 & 1.218 & 0.000\end{array}$

$\begin{array}{lll}-0.152 & -2.106 & 0.000\end{array}$

$\begin{array}{lll}2.290 & -2.121 & 0.000\end{array}$

$2.332 \quad 2.146 \quad 0.000$

$\begin{array}{lll}-0.121 & 2.173 \quad 0.000\end{array}$

$\begin{array}{lll}3.827 & 0.067 & 0.000\end{array}$

$\begin{array}{lll}-1.877 & -0.001 & 0.000\end{array}$

$\begin{array}{lll}-2.372 & -0.739 & 1.251\end{array}$

$\begin{array}{lll}-2.033 & -0.232 & 2.157\end{array}$

$\begin{array}{lll}-3.464 & -0.772 & 1.263\end{array}$

$\begin{array}{lll}-2.005 & -1.766 & 1.282\end{array}$

$\begin{array}{lll}-2.372 & -0.739 & -1.251\end{array}$

$\begin{array}{lll}-3.464 & -0.772 & -1.262\end{array}$

$\begin{array}{lll}-2.033 & -0.233 & -2.157\end{array}$

$-2.005 \quad-1.766 \quad-1.282$

$\begin{array}{llll}\mathrm{C} & -2.484 & 1.402 & 0.000 \\ \mathrm{H} & -2.188 & 1.967 & 0.886\end{array}$

$\begin{array}{llll}H & -2.188 & 1.967 & -0.886\end{array}$ 


$\begin{array}{rrrr}H & -3.573 & 1.327 & 0.000 \\ H & 4.181 & -0.827 & 0.000\end{array}$

$\begin{array}{lrrr}\mathrm{O} & 3.054 & 0.084 & 0.000 \\ \mathrm{C} & -2.505 & -0.009 & 0.000 \\ \mathrm{~N} & -3.653 & -0.017 & 0.000 \\ \mathrm{H} & 3.433 & -0.800 & 0.000\end{array}$

\section{OA4}

$\begin{array}{lrrr}\text { C } & -1.371 & -0.013 & 0.000 \\ \text { C } & -0.641 & -1.194 & 0.000 \\ \text { C } & 0.748 & -1.187 & 0.000 \\ \text { C } & 1.432 & 0.019 & 0.000 \\ \text { C } & 0.721 & 1.214 & 0.000 \\ \text { C } & -0.661 & 1.188 & 0.000 \\ \text { H } & -1.163 & -2.144 & 0.000 \\ \text { H } & 1.298 & -2.122 & 0.000 \\ \text { H } & 1.268 & 2.147 & 0.000 \\ \text { H } & -1.207 & 2.125 & 0.000 \\ \text { O } & 2.792 & 0.095 & 0.000 \\ \text { C } & -2.876 & -0.016 & 0.000 \\ \text { H } & -3.267 & -1.032 & 0.000 \\ \text { H } & -3.270 & 0.496 & 0.880 \\ \text { H } & -3.270 & 0.496 & -0.879 \\ \text { H } & 3.165 & -0.791 & 0.000\end{array}$

OA5

$\begin{array}{lrrr}\mathrm{C} & 0.958 & -0.002 & 0.000 \\ \mathrm{C} & 0.266 & -1.200 & 0.000 \\ \mathrm{C} & -1.121 & -1.190 & 0.000 \\ \mathrm{C} & -1.809 & 0.017 & 0.000 \\ \mathrm{C} & -1.107 & 1.216 & 0.000 \\ \mathrm{C} & 0.277 & 1.206 & 0.000 \\ \mathrm{H} & 0.808 & -2.135 & 0.000 \\ \mathrm{H} & -1.667 & -2.127 & 0.000 \\ \mathrm{H} & -1.658 & 2.146 & 0.000 \\ \mathrm{H} & 0.831 & 2.135 & 0.000 \\ \mathrm{O} & -3.167 & 0.086 & 0.000 \\ \mathrm{Cl} & 2.693 & -0.011 & 0.000 \\ \mathrm{H} & -3.541 & -0.800 & 0.000\end{array}$

\section{OA6}

$\begin{array}{lrrr}\text { C } & -1.072 & -0.001 & 0.000 \\ \mathrm{C} & -0.367 & -1.202 & 0.000 \\ \mathrm{C} & 1.016 & -1.195 & 0.000 \\ \mathrm{C} & 1.703 & 0.016 & 0.000 \\ \mathrm{C} & 1.003 & 1.220 & 0.000 \\ \mathrm{C} & -0.376 & 1.210 & 0.000 \\ \mathrm{H} & -0.907 & -2.139 & 0.000 \\ \mathrm{H} & 1.565 & -2.130 & 0.000 \\ \mathrm{H} & 1.560 & 2.147 & 0.000 \\ \mathrm{H} & -0.927 & 2.140 & 0.000\end{array}$

OA7

$\begin{array}{lrrr}\text { C } & -0.365 & -0.024 & -0.005 \\ \text { C } & 0.343 & -1.214 & -0.003 \\ \text { C } & 1.730 & -1.195 & -0.001 \\ \text { C } & 2.405 & 0.019 & 0.001 \\ \text { C } & 1.694 & 1.217 & -0.001 \\ \text { C } & 0.315 & 1.191 & -0.004 \\ \text { H } & -0.184 & -2.158 & -0.005 \\ \text { H } & 2.287 & -2.125 & 0.000 \\ \text { H } & 2.241 & 2.150 & 0.000 \\ \text { H } & -0.244 & 2.118 & -0.006 \\ \text { O } & 3.757 & 0.103 & 0.003 \\ \text { C } & -1.863 & -0.011 & 0.000 \\ \text { F } & -2.358 & 0.683 & -1.035 \\ \text { F } & -2.384 & -1.241 & -0.065 \\ \text { F } & -2.353 & 0.566 & 1.108 \\ \text { H } & 4.143 & -0.779 & 0.003\end{array}$

OA8

$\begin{array}{lrrr}\text { C } & 2.028 & -0.501 & 0.000 \\ \text { C } & 0.680 & -0.847 & 0.000 \\ \text { C } & -0.270 & 0.156 & 0.000 \\ \text { C } & 0.092 & 1.499 & 0.000 \\ \text { C } & 1.436 & 1.828 & 0.000 \\ \text { C } & 2.406 & 0.834 & 0.000 \\ \text { H } & -0.669 & 2.267 & 0.000 \\ \text { H } & 1.738 & 2.867 & 0.000 \\ \text { H } & 3.458 & 1.098 & 0.000 \\ \text { C } & -1.734 & -0.185 & 0.000 \\ \text { F } & -2.355 & 0.321 & 1.074 \\ \text { F } & -2.356 & 0.322 & -1.074 \\ \text { F } & -1.955 & -1.502 & -0.001 \\ \text { O } & 2.926 & -1.519 & 0.000 \\ \text { H } & 3.822 & -1.168 & 0.000 \\ \text { H } & 0.401 & -1.891 & 0.000\end{array}$

OA9

$\begin{array}{llll}C & -2.135 & 0.937 & 0.000 \\ C & -0.746 & 0.957 & 0.000 \\ C & -0.040 & -0.248 & 0.000 \\ C & -0.729 & -1.450 & 0.000 \\ C & -2.116 & -1.470 & 0.000\end{array}$




$\begin{array}{lrrr}\text { C } & -2.813 & -0.272 & 0.000 \\ \text { H } & -2.680 & 1.874 & 0.000 \\ \text { H } & -0.169 & -2.374 & 0.000 \\ \text { H } & -2.645 & -2.413 & 0.000 \\ \text { H } & -3.895 & -0.272 & 0.000 \\ \text { C } & 1.464 & -0.222 & 0.000 \\ \text { F } & 1.957 & 0.401 & 1.076 \\ \text { F } & 1.975 & -1.460 & 0.000 \\ \text { F } & 1.956 & 0.400 & -1.076 \\ \text { O } & -0.035 & 2.107 & 0.000 \\ \text { H } & -0.630 & 2.864 & 0.000\end{array}$

\section{OA10}

$\begin{array}{lrrr}\mathrm{C} & 1.055 & 1.837 & 0.000 \\ \mathrm{C} & -0.574 & 0.120 & 0.000 \\ \mathrm{C} & 2.054 & 0.874 & 0.000 \\ \mathrm{H} & 1.325 & 2.884 & 0.000 \\ \mathrm{C} & 0.389 & -0.868 & 0.000 \\ \mathrm{C} & 1.722 & -0.478 & 0.000 \\ \mathrm{H} & 3.096 & 1.170 & 0.000 \\ \mathrm{H} & 0.118 & -1.913 & 0.000 \\ \mathrm{C} & -0.281 & 1.472 & 0.000 \\ \mathrm{O} & 2.654 & -1.462 & 0.000 \\ \mathrm{~N} & -1.994 & -0.296 & 0.000 \\ \mathrm{O} & -2.829 & 0.578 & 0.000 \\ \mathrm{O} & -2.229 & -1.482 & 0.000 \\ \mathrm{H} & -1.079 & 2.198 & 0.000 \\ \mathrm{H} & 3.538 & -1.082 & 0.001\end{array}$

OA11

$\begin{array}{lrrr}\text { C } & 0.561 & 1.774 & 0.000 \\ \text { C } & -0.764 & -0.220 & 0.000 \\ \text { C } & 1.724 & 1.025 & 0.000 \\ \text { H } & 0.627 & 2.854 & 0.000 \\ \text { C } & 0.395 & -0.989 & 0.000 \\ \mathrm{C} & 1.630 & -0.366 & 0.000 \\ \text { H } & 2.694 & 1.507 & 0.000 \\ \text { H } & 0.327 & -2.067 & 0.000 \\ \mathrm{C} & -0.691 & 1.172 & 0.000 \\ \mathrm{O} & 2.730 & -1.165 & 0.000 \\ \mathrm{H} & -1.580 & 1.783 & 0.000 \\ \mathrm{O} & -1.926 & -0.917 & 0.000 \\ \mathrm{C} & -3.132 & -0.188 & 0.000 \\ \mathrm{H} & -3.215 & 0.439 & -0.891 \\ \mathrm{H} & -3.215 & 0.439 & 0.892 \\ \mathrm{H} & -3.932 & -0.924 & 0.001 \\ \mathrm{H} & 3.523 & -0.621 & 0.000\end{array}$

OA12

$\begin{array}{lrrr}\mathrm{C} & -0.682 & 0.001 & 0.000 \\ \mathrm{C} & -0.005 & -1.206 & 0.000 \\ \mathrm{C} & 1.377 & -1.196 & 0.000 \\ \mathrm{C} & 2.063 & 0.016 & 0.000 \\ \mathrm{C} & 1.365 & 1.223 & 0.000 \\ \mathrm{C} & -0.015 & 1.216 & 0.000 \\ \mathrm{H} & -0.564 & -2.131 & 0.000 \\ \mathrm{H} & 1.927 & -2.130 & 0.000 \\ \mathrm{H} & 1.923 & 2.149 & 0.000 \\ \mathrm{H} & -0.582 & 2.135 & 0.000 \\ \mathrm{O} & 3.412 & 0.085 & 0.000 \\ \mathrm{~N} & -2.149 & -0.007 & 0.000 \\ \mathrm{O} & -2.705 & -1.083 & 0.000 \\ \mathrm{O} & -2.716 & 1.062 & 0.000 \\ \mathrm{H} & 3.792 & -0.800 & 0.000\end{array}$

OA13

\begin{tabular}{lrrr} 
C & -1.261 & 0.433 & -0.002 \\
C & -0.041 & -0.284 & -0.014 \\
C & 1.207 & 0.358 & -0.010 \\
C & 1.193 & 1.757 & -0.014 \\
C & 0.025 & 2.484 & -0.010 \\
C & -1.192 & 1.817 & 0.001 \\
H & 2.134 & 2.292 & -0.019 \\
H & 0.055 & 3.565 & -0.012 \\
H & -2.099 & 2.402 & 0.009 \\
C & 2.611 & -0.291 & 0.004 \\
C & 3.376 & 0.151 & -1.254 \\
C & 3.364 & 0.191 & 1.256 \\
C & 2.678 & -1.826 & 0.035 \\
H & 2.859 & -0.184 & -2.155 \\
H & 3.476 & 1.234 & -1.309 \\
H & 4.380 & -0.280 & -1.252 \\
H & 2.840 & -0.121 & 2.162 \\
H & 4.368 & -0.237 & 1.275 \\
H & 3.461 & 1.275 & 1.282 \\
H & 3.727 & -2.124 & 0.073 \\
H & 2.204 & -2.249 & 0.924 \\
H & 2.273 & -2.283 & -0.873 \\
C & -2.614 & -0.292 & 0.005 \\
C & -2.778 & -1.145 & -1.264 \\
C & -2.753 & -1.169 & 1.260 \\
C & -3.781 & 0.701 & 0.026 \\
H & -2.704 & -0.516 & -2.154 \\
H & -2.028 & -1.928 & -1.328 \\
H & -3.766 & -1.612 & -1.263 \\
H & -2.665 & -0.557 & 2.160 \\
& & & \\
\hline
\end{tabular}




$\begin{array}{lrrr}\mathrm{H} & -3.738 & -1.641 & 1.267 \\ \mathrm{H} & -1.998 & -1.950 & 1.297 \\ \mathrm{H} & -4.718 & 0.142 & 0.035 \\ \mathrm{H} & -3.763 & 1.332 & 0.917 \\ \mathrm{H} & -3.786 & 1.343 & -0.856 \\ \mathrm{O} & -0.174 & -1.644 & -0.023 \\ \mathrm{H} & 0.680 & -2.064 & -0.120\end{array}$

$\begin{array}{lrrr}\mathrm{H} & -1.272 & -2.131 & 0.000 \\ \mathrm{H} & -1.325 & 2.136 & 0.000 \\ \mathrm{~N} & -2.870 & -0.019 & 0.000 \\ \mathrm{O} & -3.440 & 1.047 & 0.000 \\ \mathrm{O} & -3.412 & -1.099 & 0.000 \\ \mathrm{H} & 4.369 & -0.963 & 0.000\end{array}$

\section{OA14}

$\begin{array}{rrrr}\text { C } & -0.090 & 0.126 & 0.000 \\ \text { O } & -0.624 & 1.198 & 0.000 \\ \text { O } & -0.788 & -1.027 & 0.000 \\ \text { C } & 1.388 & -0.123 & 0.000 \\ \text { H } & 1.657 & -0.708 & 0.879 \\ \text { H } & 1.657 & -0.708 & -0.879 \\ \text { H } & 1.915 & 0.825 & 0.000 \\ \text { H } & -1.727 & -0.791 & 0.000\end{array}$

\section{OA17}

OA15

$\begin{array}{lrrr}\text { C } & 1.698 & 0.123 & 0.000 \\ \text { O } & 2.320 & 1.150 & 0.000 \\ \text { O } & 2.302 & -1.081 & 0.000 \\ \text { C } & 0.214 & 0.030 & 0.000 \\ \text { C } & -0.444 & -1.196 & 0.000 \\ \text { C } & -0.511 & 1.217 & 0.000 \\ \text { C } & -1.830 & -1.230 & 0.000 \\ \text { H } & 0.131 & -2.111 & 0.000 \\ \text { C } & -1.895 & 1.179 & 0.000 \\ \text { H } & 0.027 & 2.156 & 0.000 \\ \text { C } & -2.554 & -0.045 & 0.000 \\ \text { H } & -2.346 & -2.181 & 0.000 \\ \text { H } & -2.462 & 2.100 & 0.000 \\ \text { H } & -3.636 & -0.075 & 0.000 \\ \text { H } & 3.254 & -0.913 & 0.000\end{array}$

\section{OA16}

$\begin{array}{lrrr}\text { C } & 2.831 & 0.106 & 0.000 \\ \mathrm{O} & 3.458 & 1.128 & 0.000 \\ \mathrm{O} & 3.411 & -1.105 & 0.000 \\ \mathrm{C} & 1.341 & 0.034 & 0.000 \\ \mathrm{C} & 0.669 & -1.184 & 0.000 \\ \mathrm{C} & 0.638 & 1.234 & 0.000 \\ \mathrm{C} & -0.716 & -1.206 & 0.000 \\ \mathrm{H} & 1.230 & -2.108 & 0.000 \\ \mathrm{C} & -0.746 & 1.225 & 0.000 \\ \mathrm{H} & 1.190 & 2.164 & 0.000 \\ \mathrm{C} & -1.391 & 0.001 & 0.000\end{array}$

$\begin{array}{lrrr}\mathrm{C} & -2.745 & -0.775 & 0.000 \\ \mathrm{O} & -3.103 & -1.917 & 0.000 \\ \mathrm{O} & -3.586 & 0.267 & -0.001 \\ \mathrm{C} & -1.313 & -0.352 & 0.000 \\ \mathrm{C} & -0.948 & 0.988 & 0.000 \\ \mathrm{C} & -0.345 & -1.349 & 0.000 \\ \mathrm{C} & 0.398 & 1.302 & 0.000 \\ \mathrm{H} & -1.688 & 1.774 & 0.000 \\ \mathrm{C} & 0.984 & -0.977 & 0.000 \\ \mathrm{H} & -0.631 & -2.392 & 0.000 \\ \mathrm{C} & 1.392 & 0.344 & 0.000 \\ \mathrm{H} & 2.438 & 0.612 & 0.000 \\ \mathrm{~N} & 2.021 & -2.032 & 0.000 \\ \mathrm{O} & 1.641 & -3.177 & 0.000 \\ \mathrm{O} & 3.173 & -1.671 & 0.001 \\ \mathrm{~N} & 0.796 & 2.728 & 0.000 \\ \mathrm{O} & 1.978 & 2.970 & -0.001 \\ \mathrm{O} & -0.091 & 3.546 & 0.001 \\ \mathrm{H} & -4.488 & -0.085 & 0.000\end{array}$

OA18

$\begin{array}{lrrr}\text { C } & -0.491 & 0.089 & 0.000 \\ \mathrm{O} & -1.599 & -0.658 & 0.000 \\ \mathrm{O} & -0.470 & 1.288 & 0.000 \\ \mathrm{O} & 0.564 & -0.711 & 0.000 \\ \mathrm{C} & 1.821 & -0.034 & 0.000 \\ \mathrm{H} & 1.913 & 0.590 & 0.888 \\ \mathrm{H} & 2.574 & -0.815 & 0.000 \\ \mathrm{H} & 1.913 & 0.590 & -0.888 \\ \mathrm{H} & -2.344 & -0.045 & 0.001\end{array}$

SA1

$\begin{array}{lrrr}\text { C } & -0.937 & 0.101 & 0.004 \\ \text { S } & -1.721 & -1.491 & -0.068 \\ \text { S } & -1.799 & 1.512 & 0.072 \\ \text { H } & -2.952 & -0.976 & -0.024 \\ \text { C } & 1.185 & -1.203 & -0.025 \\ \text { C } & 1.239 & 1.228 & -0.005 \\ \text { C } & 2.591 & -0.721 & 0.321\end{array}$




$\begin{array}{lrrr}\mathrm{H} & 1.147 & -1.659 & -1.019 \\ \mathrm{H} & 0.792 & -1.920 & 0.697 \\ \mathrm{C} & 2.632 & 0.678 & -0.294 \\ \mathrm{H} & 1.173 & 1.714 & 0.973 \\ \mathrm{H} & 0.875 & 1.936 & -0.747 \\ \mathrm{H} & 2.700 & -0.657 & 1.405 \\ \mathrm{H} & 3.361 & -1.388 & -0.062 \\ \mathrm{H} & 3.417 & 1.306 & 0.121 \\ \mathrm{H} & 2.786 & 0.607 & -1.372 \\ \mathrm{~N} & 0.393 & 0.030 & -0.008\end{array}$

$\begin{array}{llll}\mathrm{H} & 2.717 & -2.046 & -0.272 \\ \mathrm{H} & 1.671 & -1.196 & -1.436 \\ \mathrm{~N} & 0.144 & 0.025 & 0.440 \\ \mathrm{O} & 2.878 & -0.036 & -0.230\end{array}$

\section{SA2}

SA4

$\begin{array}{lrrr}\mathrm{C} & -0.286 & 0.133 & 0.000 \\ \mathrm{~N} & 1.032 & -0.110 & 0.001 \\ \mathrm{C} & 1.952 & 1.020 & 0.000 \\ \mathrm{H} & 1.784 & 1.643 & 0.879 \\ \mathrm{H} & 2.970 & 0.641 & 0.002 \\ \mathrm{H} & 1.787 & 1.640 & -0.881 \\ \mathrm{C} & 1.563 & -1.467 & 0.000 \\ \mathrm{H} & 1.238 & -2.012 & -0.889 \\ \mathrm{H} & 2.647 & -1.422 & 0.001 \\ \mathrm{H} & 1.236 & -2.014 & 0.886 \\ \mathrm{~S} & -0.960 & 1.642 & 0.000 \\ \mathrm{~S} & -1.280 & -1.340 & 0.000 \\ \mathrm{H} & -2.425 & -0.653 & 0.000\end{array}$

SA5

$\begin{array}{llll}\text { C } & 1.895 & -1.278 & -0.462\end{array}$

$\begin{array}{llll}\mathrm{H} & 1.449 & -1.208 & 1.653\end{array}$

$\begin{array}{llll}\mathrm{H} & 0.250 & -2.082 & 0.716\end{array}$

$\begin{array}{llll}\text { C } & 2.795 & -0.042 & -0.461\end{array}$

$\begin{array}{llll}\mathrm{H} & 2.620 & 2.114 & -0.305\end{array}$

$\begin{array}{llll}\mathrm{H} & 1.397 & 1.367 & -1.331\end{array}$

$\begin{array}{llll}H & 2.490 & -2.190 & -0.390\end{array}$

$\begin{array}{llll}\mathrm{H} & 1.325 & -1.329 & -1.394\end{array}$

$\begin{array}{llll}\text { H } & 3.441 & -0.045 & -1.340\end{array}$

$\begin{array}{llll}\mathrm{H} & 3.451 & -0.082 & 0.415\end{array}$

$\begin{array}{llll}\mathrm{N} & 0.135 & 0.019 & 0.625\end{array}$

SA3

$\begin{array}{lrrr}\text { C } & 0.546 & 0.044 & 0.000 \\ \text { S } & 1.721 & -1.265 & 0.000 \\ \text { H } & 2.780 & -0.450 & 0.000 \\ \text { S } & 0.888 & 1.637 & 0.000 \\ \text { O } & -0.655 & -0.509 & 0.000 \\ \text { C } & -1.814 & 0.341 & 0.000 \\ \text { C } & -3.024 & -0.559 & 0.000 \\ \text { H } & -1.769 & 0.981 & -0.882 \\ \text { H } & -1.768 & 0.981 & 0.882 \\ \text { H } & -3.928 & 0.049 & 0.000 \\ \text { H } & -3.033 & -1.194 & -0.885 \\ \text { H } & -3.033 & -1.194 & 0.886\end{array}$

$\begin{array}{lrrr}\mathrm{C} & -1.146 & 0.105 & 0.083 \\ \mathrm{~S} & -1.948 & -1.471 & -0.113 \\ \mathrm{~S} & -1.990 & 1.507 & -0.167 \\ \mathrm{H} & -3.118 & -0.913 & -0.428 \\ \mathrm{C} & 0.982 & 1.210 & 0.614 \\ \mathrm{C} & 0.922 & -1.202 & 0.580 \\ \mathrm{C} & 2.129 & 1.153 & -0.382 \\ \mathrm{H} & 1.378 & 1.199 & 1.633 \\ \mathrm{H} & 0.376 & 2.098 & 0.469 \\ \mathrm{C} & 2.075 & -1.178 & -0.416 \\ \mathrm{H} & 1.319 & -1.243 & 1.598 \\ \mathrm{H} & 0.306 & -2.082 & 0.418 \\ \mathrm{H} & 2.816 & 1.982 & -0.218 \\ \mathrm{H} & 1.728 & 1.217 & -1.402\end{array}$

SA6

$\begin{array}{lrrr}\mathrm{C} & 1.398 & -0.932 & 0.000 \\ \mathrm{H} & 1.195 & -1.579 & -0.854 \\ \mathrm{H} & 1.253 & -1.519 & 0.908 \\ \mathrm{H} & 2.417 & -0.557 & -0.044 \\ \mathrm{C} & 0.446 & 0.236 & -0.002 \\ \mathrm{O} & 0.781 & 1.383 & 0.000 \\ \mathrm{~S} & -1.278 & -0.264 & -0.001 \\ \mathrm{H} & -1.735 & 0.993 & 0.010\end{array}$

SA7

$\begin{array}{llll}0 & -2.150 & 1.252 & 0.296\end{array}$ 


$\begin{array}{lrrr}\text { S } & -1.656 & 0.000 & -0.193 \\ \mathrm{O} & -2.150 & -1.252 & 0.297 \\ \mathrm{C} & 0.104 & 0.000 & -0.084 \\ \mathrm{C} & 0.777 & -1.214 & -0.044 \\ \mathrm{C} & 0.777 & 1.213 & -0.044 \\ \mathrm{C} & 2.161 & -1.207 & 0.030 \\ \mathrm{H} & 0.215 & -2.138 & -0.051 \\ \mathrm{C} & 2.161 & 1.207 & 0.030 \\ \mathrm{H} & 0.215 & 2.138 & -0.051 \\ \mathrm{C} & 2.849 & 0.000 & 0.064 \\ \mathrm{H} & 2.703 & -2.142 & 0.069 \\ \mathrm{H} & 2.703 & 2.142 & 0.069 \\ \mathrm{H} & 3.929 & 0.000 & 0.124 \\ \mathrm{H} & -1.846 & 0.000 & -1.535\end{array}$




\section{References}

1. Gaussian 16, Revision C.01, Frisch, M. J.; Trucks, G. W.; Schlegel, H. B.; Scuseria, G. E.; Robb, M. A.; Cheeseman, J. R.; Scalmani, G.; Barone, V.; Petersson, G. A.; Nakatsuji, H.; Li, X.; Caricato, M.; Marenich, A. V.; Bloino, J.; Janesko, B. G.; Gomperts, R.; Mennucci, B.; Hratchian, H. P.; Ortiz, J. V.; Izmaylov, A. F.; Sonnenberg, J. L.; Williams-Young, D.; Ding, F.; Lipparini, F.; Egidi, F.; Goings, J.; Peng, B.; Petrone, A.; Henderson, T.; Ranasinghe, D.; Zakrzewski, V. G.; Gao, J.; Rega, N.; Zheng, G.; Liang, W.; Hada, M.; Ehara, M.; Toyota, K.; Fukuda, R.; Hasegawa, J.; Ishida, M.; Nakajima, T.; Honda, Y.; Kitao, O.; Nakai, H.; Vreven, T.; Throssell, K.; Montgomery, J. A., Jr.; Peralta, J. E.; Ogliaro, F.; Bearpark, M. J.; Heyd, J. J.; Brothers, E. N.; Kudin, K. N.; Staroverov, V. N.; Keith, T. A.; Kobayashi, R.; Normand, J.; Raghavachari, K.; Rendell, A. P.; Burant, J. C.; lyengar, S. S.; Tomasi, J.; Cossi, M.; Millam, J. M.; Klene, M.; Adamo, C.; Cammi, R.; Ochterski, J. W.; Martin, R. L.; Morokuma, K.; Farkas, O.; Foresman, J. B.; Fox, D. J. Gaussian, Inc., Wallingford CT, 2016.

2. a) F. Weigend, R. Ahlrichs, Phys. Chem. Chem. Phys. 2005, 7, 3297-3305. b) R. Valero, J. R. B. Gomes, D. G. Truhlar, F. Illas, J. Chem. Phys. 2008, 129, 124710. c) Y. Zhao, D. G. Truhlar, Theor. Chem. Acc. 2008, 120, 215-241.

3. a) Yamamoto, E.; Hilton, M. J.; Orlandi, M.; Saini, V.; Toste, F. D.; Sigman, M. S., Development and Analysis of a $\mathrm{Pd}(0)$-Catalyzed Enantioselective 1,1-Diarylation of Acrylates Enabled by Chiral Anion Phase Transfer. J. Am. Chem. Soc. 2016, 138 (49), 15877-15880. b) Biswas, S.; Kubota, K.; Orlandi, M.; Turberg, M.; Miles, D. H.; Sigman, M. S.; Toste, F. D., Enantioselective Synthesis of N,S-Acetals by an Oxidative Pummerer-Type Transformation using Phase-Transfer Catalysis. Ang. Chem. Int. Ed. 2018, 57, 589-593. c) Orlandi, M.; Coelho, J. A. S.; Hilton, M. J.; Toste, F. D.; Sigman, M. S., Parametrization of Non-covalent Interactions for Transition State Interrogation Applied to Asymmetric Catalysis. J. Am. Chem. Soc. 2017, 139, 6803-6806. d) Orlandi, M.; Hilton, M. J.; Yamamoto, E.; Toste, F. D.; Sigman, M. S., Mechanistic Investigations of the Pd(0)-Catalyzed Enantioselective 1,1-Diarylation of Benzyl Acrylates. J. Am. Chem. Soc. 2017, 139, 12688-12695. e) Orlandi, M.; Toste, F. D.; Sigman, M. S., Multidimensional Correlations in Asymmetric Catalysis through Parameterization of Uncatalyzed Transition States. Ang. Chem. Int. Ed. 2017, 56, 14080-14084. f) Woods, B. P.; Orlandi, M.; Huang, C.-Y.; Sigman, M. S.; Doyle, A. G., Nickel-Catalyzed Enantioselective Reductive CrossCoupling of Styrenyl Aziridines. J. Am. Chem. Soc. 2017, 139, 5688-5691. g) Coelho, J. A. S.; Matsumoto, A.; Orlandi, M.; Hilton, M. J.; Sigman, M. S.; Toste, F. D., Enantioselective fluorination of homoallylic alcohols enabled by the tuning of non-covalent interactions. Chem. Sci. 2018, 9, 7153-7158.

4. NBO Version 3.1, E. D. Glendening, A. E. Reed, J. E. Carpenter, and F. Weinhold.

5. Falivene, L.; Cao, Z.; Petta, A.; Serra, L.; Poater, A.; Oliva, R.; Scarano, V.; Cavallo, L., Towards the online computer-aided design of catalytic pockets. Nat. Chem. 2019, 11, 872-879.

6. MATLAB and Statistics Toolbox Release R2020a, The MathWorks, Inc., Natick, Massachusetts, United States.

7. For example, see: Santiago, C. B.; Guo, J.-Y.; Sigman, M. S., Predictive and mechanistic multivariate linear regression models for reaction development. Chem. Sci. 2018, 9 , 2398-2412.

8. Mayr, H., Reactivity scales for quantifying polar organic reactivity: the benzhydrylium methodology. Tetrahedron 2015, 71, 5095-5111. 